INFLUENCE OF COMPOUNDING AND MIXING ON FILLER DISPERSION AND CURING BEHAVIOR OF SILICA COMPOUNDS

Jungmin Jin 
This research is a joint project between HANKOOKTIRE. Co., Ltd. and Elastomer Technology and Engineering (ETE) group of the University of Twente.

\section{Graduation Committee:}

Chairman / secretary: Prof. Dr. G.P.M.R. Dewulf

Supervisors:

Committee Members:

Dr. M. de Rooij

Prof. Dr. J.E. ten Elshof

Prof. Dr. I. Hudec

Prof. Dr. C. Kummerlöwe
University of Twente, ET, the Netherlands

University of Twente, ET, the Netherlands University of Twente, ET, the Netherlands

University of Twente, ET, the Netherlands University of Twente, TNW, the Netherlands Slovak University, Faculty of Chemical and Food Technology, Slovakia University of Applied Science Osnabrück, Faculty of Engineering and Computer Science, Germany

Evonik Industries AG, Germany

Referee:

Dr. J. Kiesewetter 


\title{
INFLUENCE OF COMPOUNDING AND MIXING ON FILLER DISPERSION AND CURING BEHAVIOR OF SILICA COMPOUNDS
}

\author{
DISSERTATION \\ to obtain \\ the degree of doctor at the University of Twente, \\ on the authority of the rector magnificus, \\ Prof. Dr. T.T.M. Palstra, \\ on account of the decision of the graduation committee \\ to be publicly defended \\ on Wednesday, $11^{\text {th }}$ of March, 2020 at 14.45 hour
}

by

Jungmin Jin

born on the $8^{\text {th }}$ of January, 1982

in Busan, Republic of Korea 
This dissertation has been approved by:

supervisors

Associate Prof. Dr. W.K. Dierkes

Prof. Dr. A. Blume

Cover design: Jungmin Jin

Printed by: IPSKAMP printing, Auke Vleerstaat 145, $7547 \mathrm{PH}$, Enschede, the Netherlands Lay-out: Jungmin Jin

ISBN: 978-90-365-4926-4

DOI (URL): 10.3990/1.9789036549264 (https://doi.org/10.3990/1.9789036549264)

(C) 2020 Jungmin Jin, The Netherlands. All rights reserved. No parts of this thesis may be reproduced, stored in a retrieval system or transmitted in any form or by any means without permission of the author. Alle rechten voorbehouden. Niets uit deze uitgave mag worden vermenigvuldigd, in enige vorm of op enige wijze, zonder voorafgaande schriftelijke toestemming van de auteur. 


\section{Table of Contents}

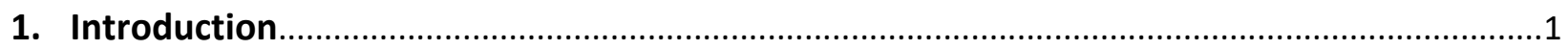

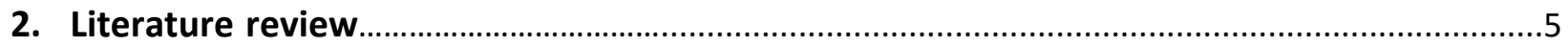

3. Investigation on the origin of marching modulus of silica filled tire tread compounds and

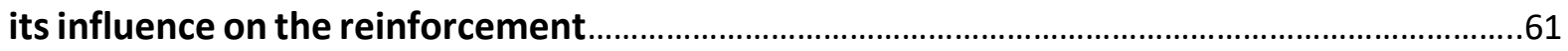

3.1. Investigation of the origin of marching modulus of silica filled tire tread compounds.................63

3.2. The effect of marching modulus phenomenon on the mechanical properties alteration of silica

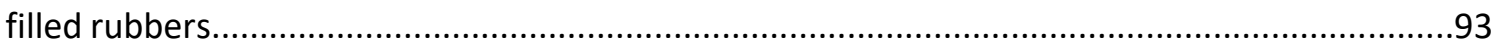

4. The effect of mixing parameters on marching modulus of silica filled tire tread

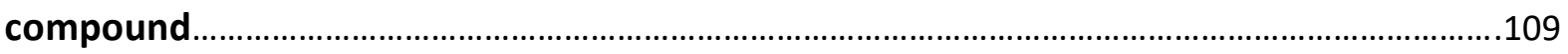

4.1. The effect of silanization temperature and time on marching modulus.......................................111

4.2. The effect of zinc oxide concentration in the early and later mixing stage on marching modulus.

4.3. The effect of 1,3-diphenylguanidine concentration in the earlier and later mixing stages on marching modulus..

4.4. Model compound study: effect of $\mathrm{ZnO}$, stearic acid and DPG on the silane chemistry.

5. The effect of polymer and silica characteristics on marching modulus of silica filled tire tread compounds.

5.1. The effect of the SBR/BR blend ratio on marching modulus.

5.2. The effect the dispersibility of silica filler on marching modulus.

6. Scale up: production scale mixing trials

7. The relation between macro-/micro-dispersion of silica in rubber matrix and their effect on rubber reinforcement

8. Summary

Samenvatting.

Bibliography 



\section{Chapter 1 Introduction}

\subsection{History of rubber technology}

Rubber is one of the most widely used organic materials in the world. It has been 3,500 years since humankind used rubber. ${ }^{[1]}$ Rubber was first introduced in Europe at the end of the 15th century. In 1493, Christopher Columbus, who is well known as first discoverer of the new continent, saw a ball game which was played by natives of Haiti. ${ }^{[2]}$ The ball was made with latex from the Para rubber tree which was called "caa (tree) o-chu (weeping)" by the aboriginals of the Amazon basin. ${ }^{[3]}$

However, until the 18th century there were not many applications for rubber. In 1770, Joseph Priestley, a chemist of England, found a new application for rubber: the eraser. The English word "rubber" for elastomers is originating from the word "rub". The next application was for water-proof raincoats, which was invented by Charles Macintosh in 1823. ${ }^{[4]}$

At 1839 , the most important invention of the rubber industries was made by Charles Goodyear by serendipity. It was called vulcanization, named after the Roman God of Fire "Vulcan". After the invention of the vulcanization process, rubber industries could lay the foundation of rubber technology and still it is one of the key processes in elastomer processing. ${ }^{[3,4]}$

Rubber tires for automobiles were first invented by R. W. Thompson, patented in $1848 .{ }^{[5]}$ Later on, the pneumatic tire for vehicles, which is basically still the same today, was first invented by J. B. Dunlop, a veterinarian, in $1888 .{ }^{[3]}$

\subsection{Background and aim of this investigation}

The market requires to manufacture tires which are durable (low fuel consumption, high abrasion resistance) and safe (good snow, ice, wet traction). The start of the Europe Union Labeling regulation in 2012 and tire grading on the above-mentioned characteristics via reliable automotive magazines are a good example of recent tire market trends.

Due to these world tire market trends, various innovative raw materials and additives such as functionalized Styrene Butadiene Rubber (SBR), silica filler systems, mercapto silanes, resins and etc. were developed in order to satisfy those demands. These novel materials provide a large contribution to tire performance improvement but often have poor processability. In spite of the disadvantages in processing, compound designers prefer to use these materials as much as they can in order to survive in the tire market. Additionally, the complexity of the tire structure design is increasing and thus induces many 
problems in extrusion processes as well as green tire assembling. For these reasons, process technology became the new champion for tire evolution, as shown in Figure 1.1.

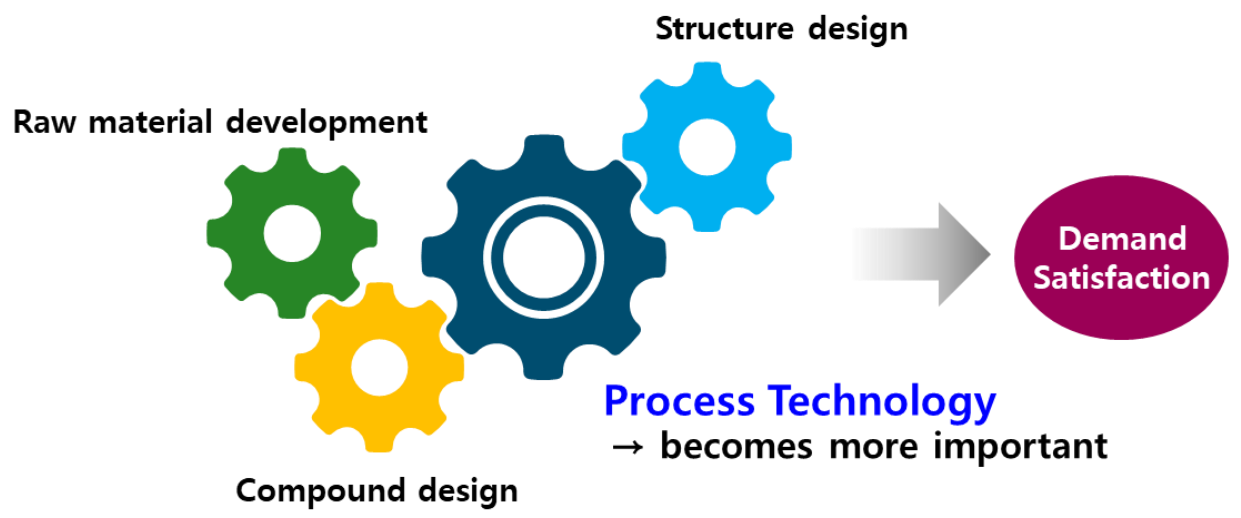

Figure 1.1 Increasing dependency on process technology.

Especially, mixing is one of the most important processes in tire manufacturing. It can never be independent from further processing steps, because it directly affects the processability of downstream processes of tire manufacturing. ${ }^{[6]}$ In addition, "Quality starts in mixing" is well known to all tire producers and quoted frequently. ${ }^{[7]}$

The mixing paradigm has changed since silica was used as a filler for passenger car tire tread compounds. Mixing of a silica filled rubber compound is more complicated compared to mixing of a carbon black compound due to the high polarity difference between silica and the elastomer. Not only the mechanical dispersion of silica, but also control of the reaction between coupling agent and silica or polymer is very important in order to achieve the desired performance and processability. Many studies were done to find out the optimum mixing conditions for silica compounds, but experience shows that still there is much more to be done.

\subsection{Aim of this thesis}

Silica reinforced Solution-Styrene Butadiene Rubber (S-SBR)/Butadiene Rubber (BR) tire tread compounds often show characteristic vulcanization profiles that do not exhibit a distinct maximum in the cure curve, nor a plateau profile within acceptable time scales: marching modulus. In such a situation, it is difficult to determine the optimum curing time, and as a consequence the physical properties of the rubber compounds may vary. It is neither productive nor efficient to wait for a prolonged time in order to obtain a plateau in the vulcanization profile. Therefore, the marching modulus phenomenon should be avoided 
or at least minimized. However, there is no clear solution because the reaction mechanism is not clearly defined yet. Therefore, the physicochemical mechanism underlying the marching modulus phenomenon found in silica compounds will be investigated, and basic requirements for minimizing the marching phenomenon will be developed in this study. Finally, possible guidelines for a tailored mixing process and compound would be provided in order to obtain consistent quality of the compound with enhanced productivity as well as better processability.

\subsection{Structure of this thesis}

The research in this thesis deals with the effect of mixing process parameters and compound formulations on filler dispersion and curing behavior of silica compounds.

First of all, an overview about basic rubber technologies and theories related to the topic will be provided in Chapter 2.

Chapter 3: The origin of marching modulus of silica filled tire tread compounds is elaborated. The silica compounds with various types of silane coupling agent having different sulfidic bridges and functions are compared in terms of filler-filler interaction, filler-polymer interaction and sulfur donation effect on the marching modulus.

Chapter 4: Based on the results of Chapter 3, the influence of mixing parameters which are related to the degree of silanization as well as filler-polymer coupling reaction are investigated. Additionally, the essential ingredients for silica compounds which are possible to affect those reactions are investigated by compound mixing trials as well as model compound studies.

Chapter 5: The influence of compound formulations on the marching modulus phenomenon are described. The studies are performed by changing S-SBR/BR blending ratio and using silicas having different dispersibility.

Chapter 6: Several series of compounds are mixed in plant scale mixers (larger than 250L) in order to confirm the basic mechanism would be still valid regardless of the mixer scale. The effect of silanization time and temperature as well as rotor geometry on compound quality are compared in terms of influencing factors on marching modulus as well as filler dispersion quality. 
Chapter 7: The relation between micro- and macro-dispersion of silica and their effect on rubber reinforcement is investigated. Additionally, the major influencing factors on micro- and macro-dispersion of silica are identified.

Chapter 8: An overall summary of this thesis is given.

\section{References}

[1] J. Kennedy, Science vol. 213, 757 (1981).

[2] J. A. Brydson, "Rubbery Materials and their Compounds", Elsevier Science Publishers Ltd., Essex (1988).

[3] "Rubber Technology and Manufacture, second edition", C. M. Blow, C. Hepburn, Butterworths, London (1982).

[4] "Rubber Technology handbook", H.F. Mark, Hanser Publishers, Munich (1996).

[5] R. W. Thompson, (Improvement in carriage-wheels), US. Pat. US5104A (May. 8, 1847).

[6] "The mixing of rubber", R. F. Grossman, Champman \& Hall (1997).

[7] D. Beach, J. Schroeder, Rubber World 222, 6 (2000). 


\section{Chapter 2 Literature review}

The color of a tire is traditionally black, and this is related to the components which are added to the elastomer in order to enhance the mechanical properties of the a compound. The pure elastomer is easily destroyed under severe conditions such as high friction, heat and pressure like a rolling tire on the road is suffering. Thus, reinforcing fillers are applied in order to enhance the strength and durability of rubber.

Since silica/silane filler systems are used for tire tread compounds, mixing became a more critical step of the tire manufacturing process. Due to the characteristics of silica, fundamentally different mixing concepts must be applied. For example, the mixer should provide not only mechanical shear forces, but also appropriate conditions for the reaction between the silane and the silica. To approach the optimal mixing conditions, background knowledge in and understanding of rubber technology is required. This chapter gives an overview of the fundamentals of rubber reinforcement and rubber mixing, from a theoretical as well as a practical point of view. 


\subsection{Dynamic mechanical properties and tire performance}

Tire development is mainly focused on three major performances: rolling resistance, wet grip, and abrasion resistance. These performance indicators are well known as the "magic triangle" of the tire. They are conflicting with each other: to reduce rolling resistance, the hysteresis of a rubber compound should be minimized, whereas it should be maximized for wet grip. Thus, these properties cannot all be improved at the same time. ${ }^{[1-3]}$

The performance of a tire is strongly related to the viscoelastic properties of the rubber compound. Studying the basic theory of viscoelasticity is necessary in order to understand the relationship between tire performance and dynamic mechanical properties. Viscoelasticity is the most representative property of an elastomer that exhibits both, viscous liquid-like behavior and elastic solid-like characteristics when undergoing deformation. This means that a part of the deformation energy can be stored elastically, whereas the rest is dissipated as heat.

The dynamic properties of elastomers can well be visualized in terms of a specimen undergoing uniform sinusoidal shear deformation. When a stimulation is applied to a viscoelastic material, rearrangements take place on molecular scale as response to the excitation. A sinusoidal strain deformation $\gamma$ is applied to a viscoelastic material with angular oscillation frequency $\omega:{ }^{[4,5]}$

$$
\gamma=\gamma_{0} \sin (\omega t)
$$

Where $\nu_{0}$ is the amplitude of the applied strain and $t$ is time, respectively.

The shear stress response $\sigma$ is also sinusoidal, but out of phase with strain:

$$
\sigma=\sigma_{0} \sin (\omega t+\delta)=\left(\sigma_{0} \cos \delta\right) \sin \omega t+\left(\sigma_{0} \sin \delta\right) \cos \omega t
$$

Where $\sigma_{0}$ is amplitude of the stress response and $\delta$ is the phase angle.

The phase angle is illustrated in Figure 2.1. The shear stress response $\sigma$ can be separated into two components: one is in phase $\left(\sigma_{0} \cos \delta\right)$ and the other is $90^{\circ}$ out of phase $\left(\sigma_{0} \sin \delta\right)$ with the applied strain. 


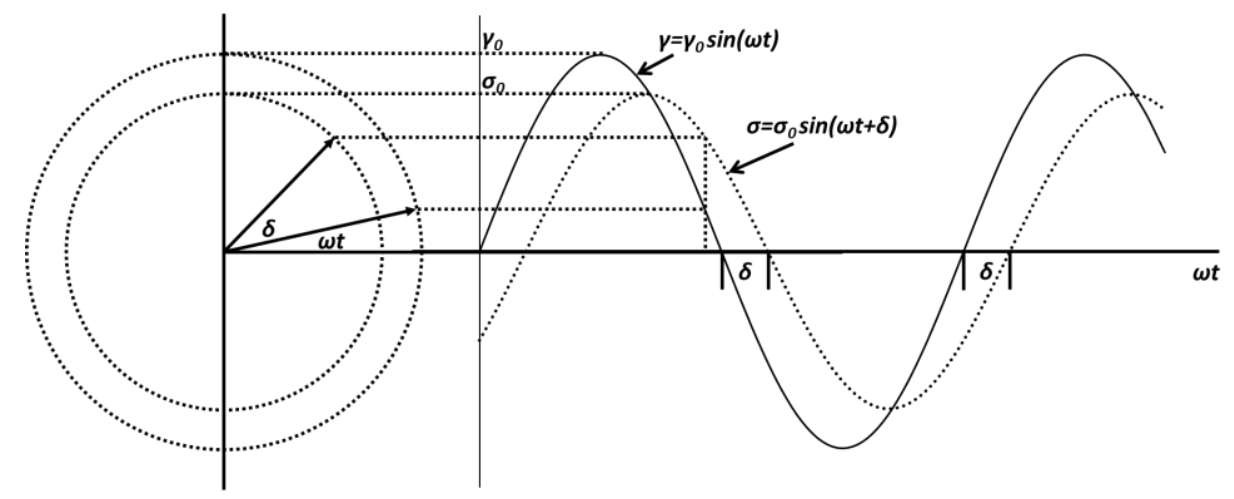

Figure 2.1 Illustration of the phase angle for the delay of the stress response on sinusoidal deformation.

These two components can be expressed by the storage modulus $\mathrm{G}^{\prime}$ (in phase modulus), and the loss modulus $\mathrm{G}^{\prime \prime}\left(90^{\circ}\right.$ out of phase modulus) with oscillating strain given as:

$$
\begin{aligned}
& G^{\prime}=\frac{\sigma_{0}}{\gamma_{0}} \cos \delta \\
& G^{\prime \prime}=\frac{\sigma_{0}}{\gamma_{0}} \sin \delta
\end{aligned}
$$

By this substitution, Equation 2.2 can be derived as Equation 2.5:

$$
\sigma=\gamma_{0}\left[G^{\prime} \sin \omega t+G^{\prime \prime} \cos \omega t\right]
$$

The shear modulus $\mathrm{G}^{*}$ can be described in a complex form with two components: the real and the imaginary part. The storage modulus $\left(G^{\prime}\right)$ and the loss modulus $\left(G^{\prime \prime}\right)$ correspond to the real part and imaginary part, respectively: Equations 2.6 and 2.7.

$$
\begin{gathered}
G^{*}=G^{\prime}+i G^{\prime \prime} \\
G^{*^{2}}=G^{2}+G^{\prime 2}
\end{gathered}
$$

Using these two moduli, the phase or loss angle is given by: 


$$
\tan \delta=\frac{G^{\prime \prime}}{G^{\prime}}
$$

The loss angle tan $\delta$ is a useful parameter in dynamic mechanical analysis. ${ }^{[4,5]}$ The dynamic properties $\mathrm{G}^{\prime}$ and G" are strongly affected by frequency, temperature and strain. This is due to the intrinsic mobility of chain segments in the material. Figure 2.2 shows the frequency-dependence of the dynamic moduli for a typical non-vulcanized viscoelastic material.

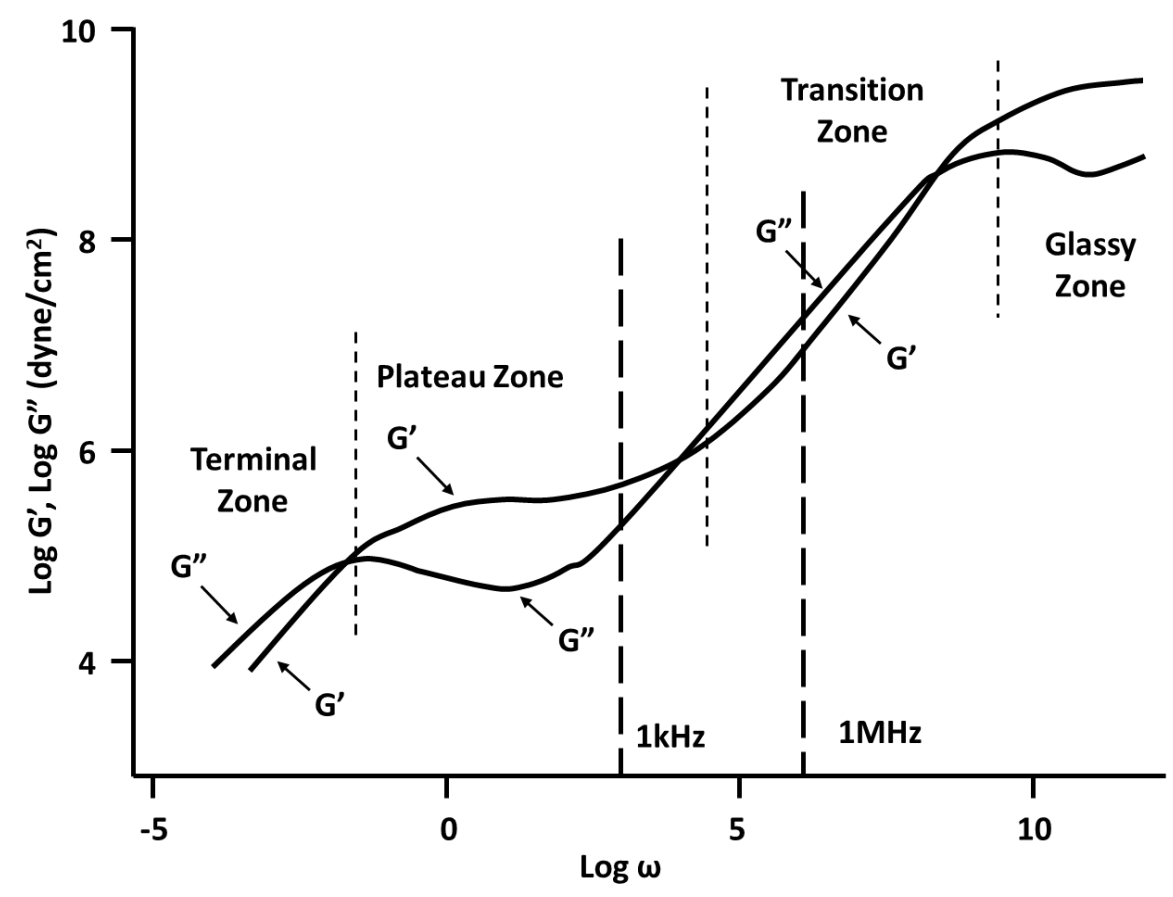

Figure 2.2 Frequency-dependence of the storage modulus $G^{\prime}$ and the loss modulus $G^{\prime \prime} .{ }^{[4]}$

Depending on the molecular motion of the elastomer, by varying the applied deformation frequency to the viscoelastic material, four different regions are evident: the terminal, the plateau, the transition, and the glassy zone. In the terminal zone, the polymer behaves like a viscoelastic liquid. The regular interval between excitations is long enough for the molecules to rearrange their original configurations completely. $\mathrm{G}^{\prime}$ and $\mathrm{G}^{\prime \prime}$ are proportional to the frequency of oscillation in this region. In the plateau zone, the polymer behaves like a rubbery solid. G' changes little and G" passes through a minimum with increasing frequency. In this region, the losses are small because the period of oscillation is long enough for relaxation of entanglement loci, but at the same time short in relation to any relaxation times for motion involving entanglement slippage. Thus, the entanglements act as temporary crosslinks. In the transition zone, the 
period of oscillation is too short so that large segments of the chain cannot respond to the applied deformation. Finally, at higher frequencies than the transition zone, the polymer chains have no chance to rearrange in their original configuration within the period of oscillation. Therefore, most of the motion of chain segments is frozen in the glassy zone. ${ }^{[6-10]}$

The frequency regions of rolling resistance and wet grip of a tire are shown in Figure 2.3. Two major performances of the tire are strongly related to special ranges of the deformation frequency. ${ }^{[2,6,11]}$

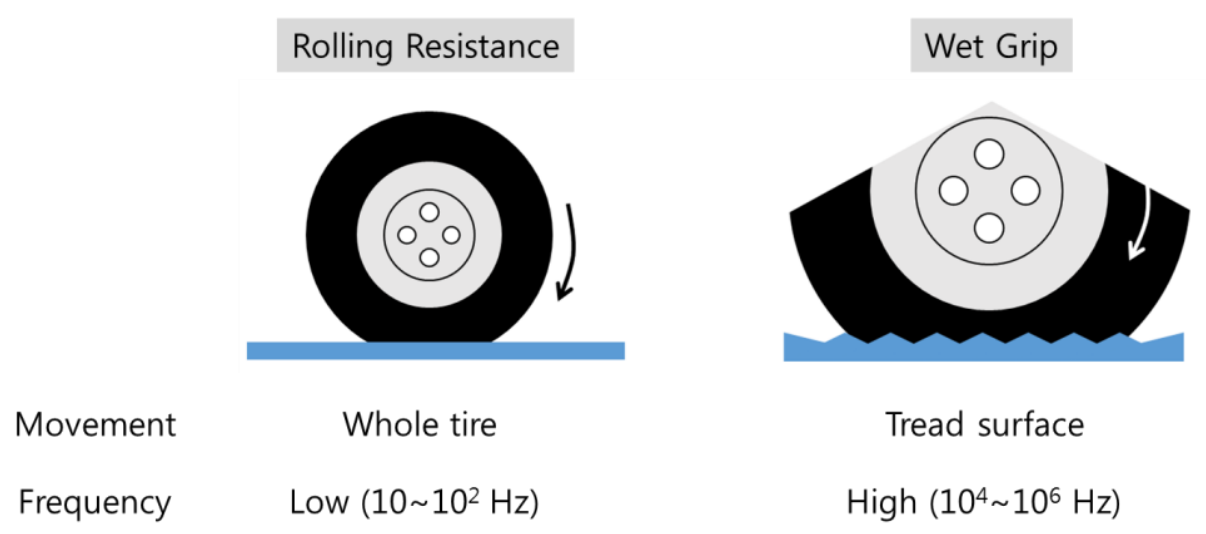

Figure 2.3 Movement of tread rubber as related to the frequency. ${ }^{[11]}$

Similar to the frequency-dependent characteristics of the viscoelastic material, the properties pass from glassy state (at low temperatures) to a rubbery state within a wide temperature range. According to the temperature-frequency shift, measuring the loss angle tan $\delta$ at low temperatures and frequencies can be correlated with the properties measured at ambient temperature and high frequencies. Therefore, it is possible to predict the tire performances by measuring tan $\delta$ at varying temperatures. ${ }^{[12-14]}$ Figure 2.4 shows the tan $\delta$ curve related to several important performances of the tire tread compound. 


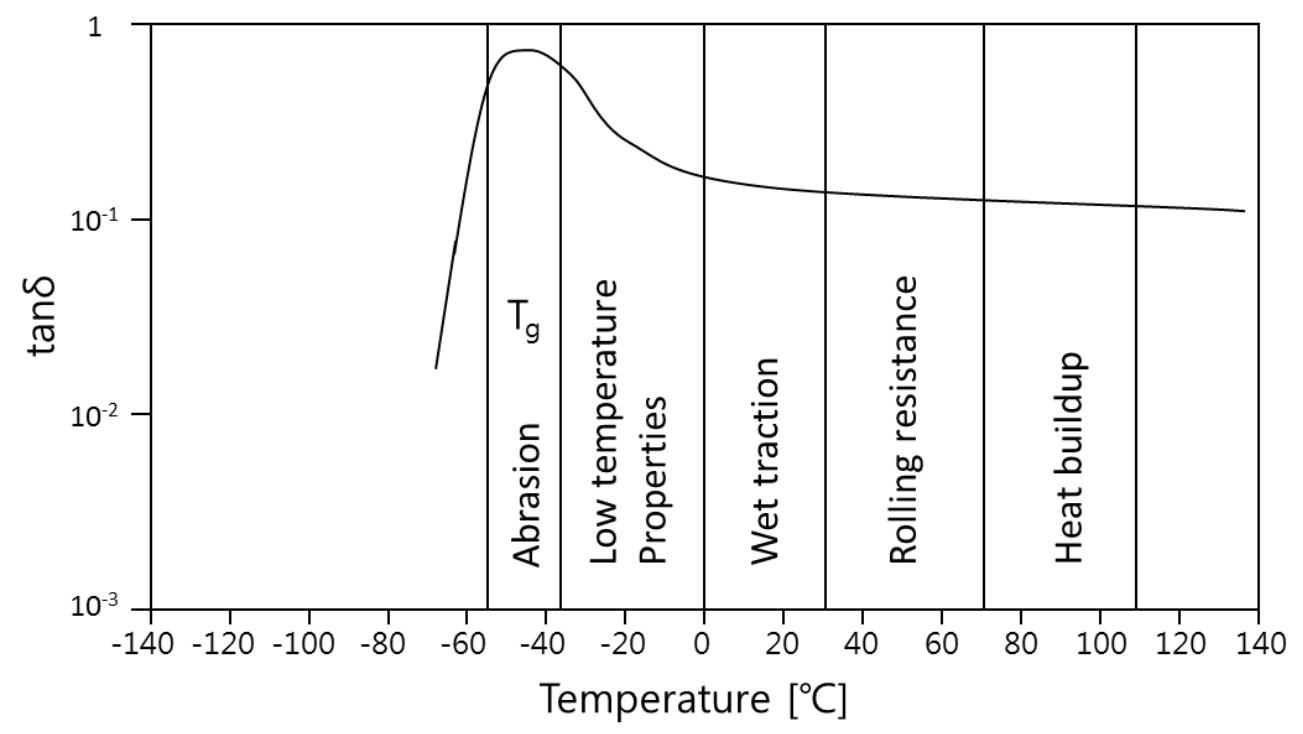

Figure 2.4 Tan $\delta$ curve and representative performances of the tire tread compound. ${ }^{[15]}$

It is known that the dynamic properties of compounds can be influenced by filler loading and interaction between filler and polymer: surface area and activity, particle size and structure. ${ }^{[14]}$ Silica has become more important as reinforcing filler of passenger tire treads since Michelin introduced this technology in the Nineties of the last century. Typical tan $\delta$ curves for carbon black and silica filled compounds are shown in Figure 2.5.

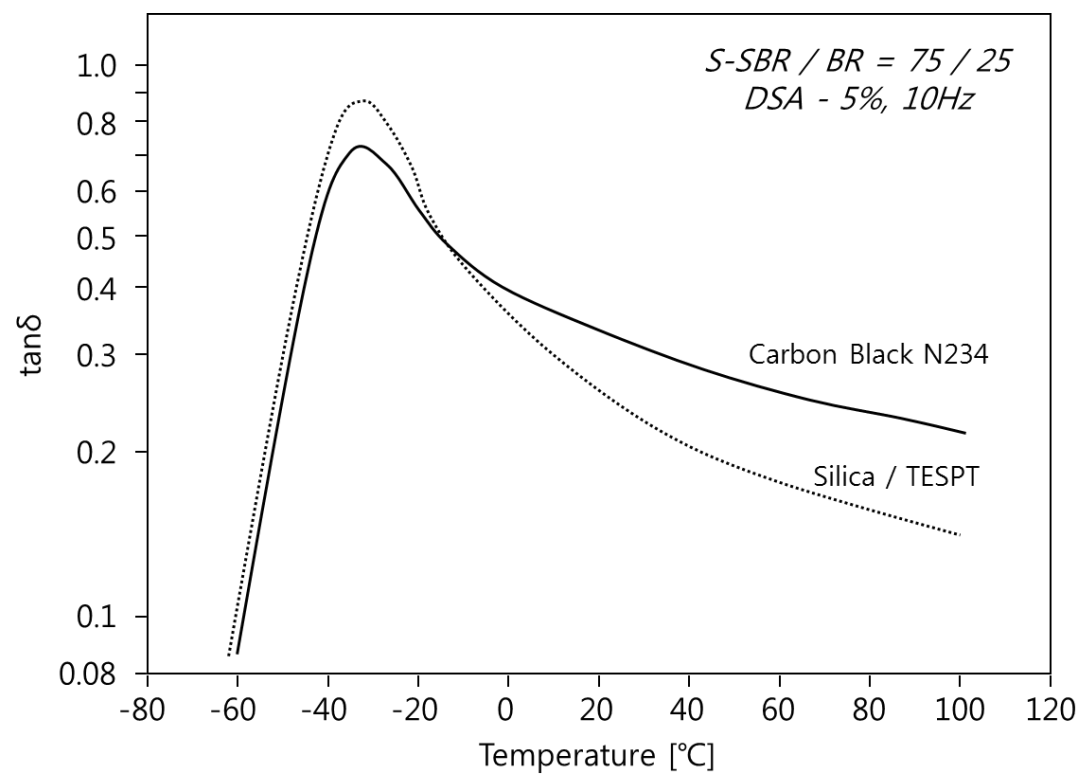

Figure 2.5 Temperature dependence of tan $\delta$ for carbon black and silica filled rubber compounds. ${ }^{[14]}$ 
Compared to carbon black filled rubber compounds, lower tan $\delta$ values can be seen in the higher temperature region, and higher $\tan \delta$ values can be seen in the low temperature region for silica filled material. This can leads to advantages in terms of rolling resistance and wet traction performance compared to carbon black filled rubber. This is mainly due to the difference in surface characteristics between silica and carbon black.

\subsection{Reinforcement of rubber}

With exception of vulcanization, no rubber manufacturing process is more important than the reinforcement of elastomers by fillers. ${ }^{[16-18]}$ The interaction between polymer and filler, which leads to adsorption of polymer chains onto the filler particle surface, can be controlled by varying the nature of the polymer-filler interface. ${ }^{[19,20]}$ In case of carbon black, physical bonding mainly governs polymer-filler interaction, whereas chemical bonding via coupling agents dominates in silica filled rubber compounds. When an elastomer is reinforced with active fillers, filler-filler interaction occurs besides filler-polymer interaction. Applied energy can partly be dissipated in filler-filler interactions during deformation, as expressed by the Payne effect. Figure 2.6 shows the typical behavior of the complex shear modulus of filled rubber versus dynamic shear deformation. ${ }^{[20]}$

There are four contributing parameters to the complex shear modulus: the hydrodynamic effect, the polymer network, the filler-polymer interaction, and the filler-filler interaction. Except for the effect of filler-filler interaction, the other three contributions to the complex shear modulus are strain-independent.

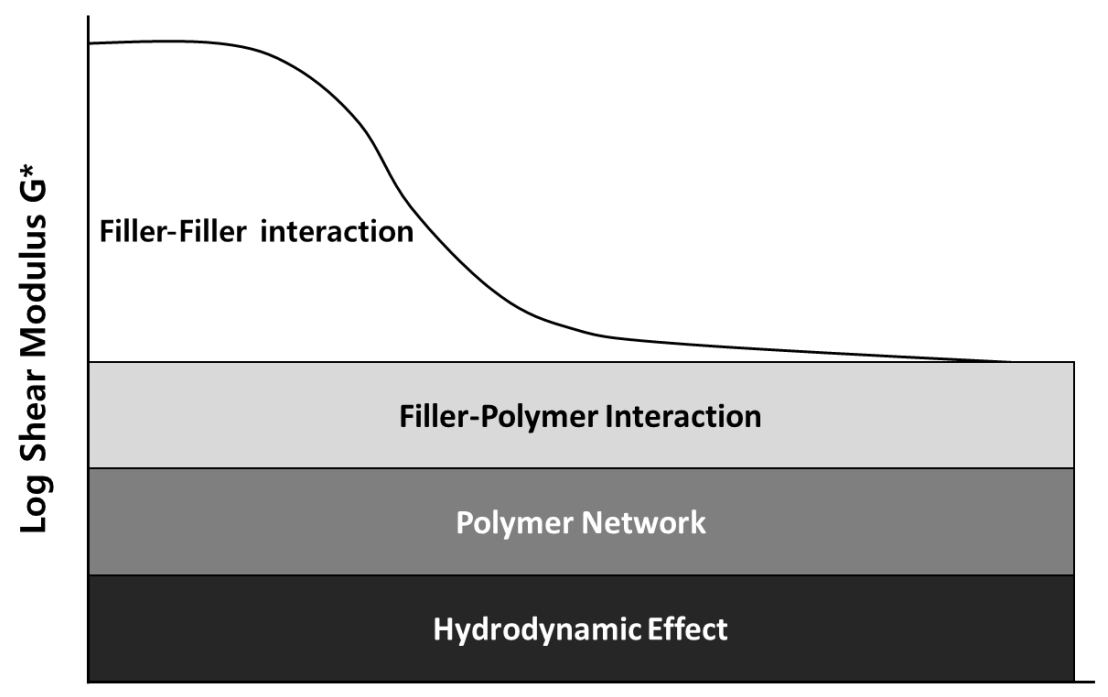

Log Shear Deformation

Figure 2.6 Contributions to the shear modulus for a typical rubber compound. ${ }^{[14]}$ 


\subsubsection{Hydrodynamic effect}

The inclusion of rigid filler particles in viscous fluids leads to an increase of the viscosity of the fluid: the hydrodynamic effect. ${ }^{[21,22]}$ Einstein ${ }^{[21,22]}$ modeled this phenomenon at the beginning of the last century based on a suspension liquid with dispersed rigid spherical particles. The viscosity change was described as follow: ${ }^{[16,21,22]}$

$$
\eta=\eta_{0}(1+2.5 \varphi)
$$

Where $\eta$ is the viscosity of the mixture, $\eta_{0}$ is the viscosity of the pure liquid and $\phi$ is the volume fraction of particles in the mixture. However, this equation is not applicable to filled elastomers because it is valid only when

- the concentration of particles is low and the system is dilute,

- the suspended colloidal particles are spherical and uniform,

- wettability of the particle surface is perfect, and

- $\quad$ no interactions between the particles occur.

Guth and Gold ${ }^{[23]}$ have generalized Einstein's theory and took into account the mutual interaction between pairs of particles by adding a second order term $\varphi^{2}$. ${ }^{[16,18,23,24]}$

$$
\eta=\eta_{0}\left(1+2.5 \varphi+14.1 \varphi^{2}\right)
$$

For an elastic material which is filled with colloidal particles, the viscosity can be replaced by a modulus: ${ }^{[24-26]}$

$$
G=G_{0}\left(1+2.5 \varphi+14.1 \varphi^{2}\right)
$$

where $G_{\text {and }} \mathrm{G}_{0}$ are the shear moduli of the filled and unfilled systems.

If the concentration of the colloidal particles increases above a certain threshold, the colloidal particles form rod-like structures which leads to a rapid increase of the stiffness of the material and the linear 
relationship no longer holds. Therefore, Guth ${ }^{[24]}$ took into account the effect of primary aggregate morphology and modified Equation 2.11 as follows: ${ }^{[24,26]}$

$$
G=G_{0}\left(1+0.67 f \varphi+1.62 f^{2} \varphi^{2}\right)
$$

Where $\mathrm{f}$ is the shape factor depending upon the ratio of particle length to width. Equation 2.12 is useful to express the filler concentration effect on mechanical properties of filled elastomers at low extensions. ${ }^{[27]}$

\subsubsection{Polymer network}

The polymer network can be defined in terms of the number of crosslinks introduced between the independent molecules. The polymer network contribution depends on the crosslink density of the matrix and the nature of the polymer. ${ }^{[20,28]}$ The shear modulus is described as a function of concentration of elastically active network chains, the absolute temperature and the Boltzmann constant.

$$
G=v k T
$$

where $\mathrm{v}$ is the density of the entanglements, $\mathrm{k}$ is Boltzmann's constant and $\mathrm{T}$ is the absolute temperature. Equation 2.13 can be expressed alternatively in terms of the mean molecular weight of the chain between cross links $M_{c}{ }^{[28]}$

$$
G=v k T=\rho R T / M_{c}
$$

Where $\rho$ is the density of the elastomer and $R$ is the gas constant, respectively.

\subsubsection{Filler-polymer interaction}

The filler-polymer interaction is one of the strain-independent contributions to the filler reinforcement effect and it plays an important role for the mechanical properties of rubber goods. This effect arises from molecular interactions between the rubber and the filler by formation of bound rubber. ${ }^{[19]}$ The bound rubber is generally defined as the remaining rubber attached to the filler surface after a thorough solvent 
extraction process of an uncured rubber sample. In the extraction process, all free unbound rubber is removed. ${ }^{[18]}$ Morphology and the physicochemical nature of reinforcing fillers such as specific surface area, degree of structure and surface activity are crucially important, because they are directly related to formation and amount of bound rubber. ${ }^{[29-33]}$

\subsubsection{General bound rubber model}

Many researchers have suggested and developed models for bound rubber. ${ }^{[34-44]}$ Medalia ${ }^{[35]}$ and Kraus [36] proposed the 'occluded rubber model'. In this model, rubber is partly trapped in the filler aggregates and the amount of occluded rubber can vary according to the surface area of the filler.

Smit ${ }^{[34]}$ and Pliskin ${ }^{[37]}$ proposed the 'shell rubber model', which is based on chemical adsorption of the polymer onto the filler surface. $\mathrm{O}^{\prime}$ Brien et al. ${ }^{[38]}$ further developed this model by using NMR. According to this model, a double layer structure - a glassy state and a rubbery state layer - of the polymer is formed on the carbon black particle surface as shown in Figure 2.7.

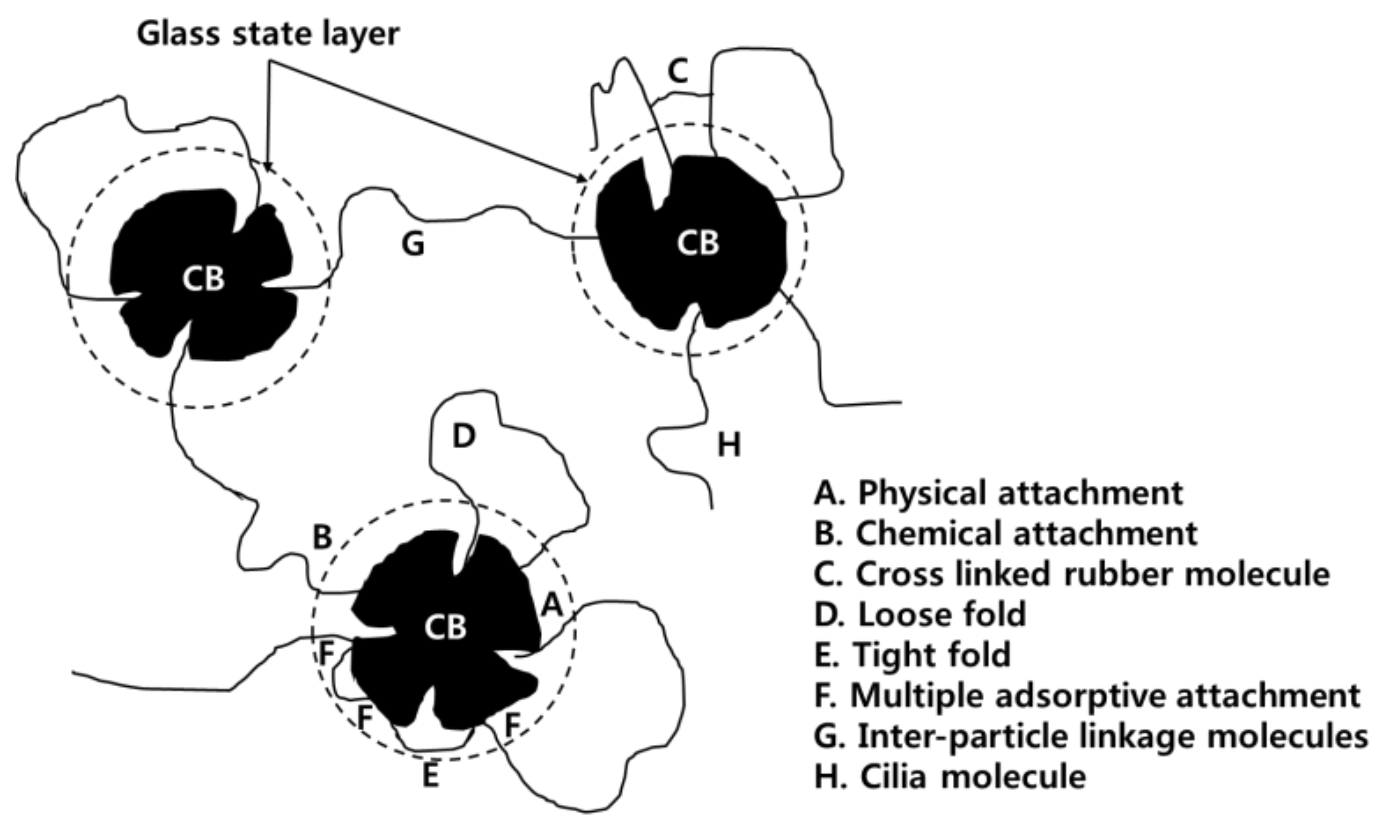

Figure 2.7 O'Brien's bound rubber model. ${ }^{[38]}$

In Leblanc's ${ }^{[39]}$ bound rubber model, bound rubber consists of two components as shown in Figure 2.8: tightly bound rubber and loosely bound rubber. ${ }^{[39-41]}$ 


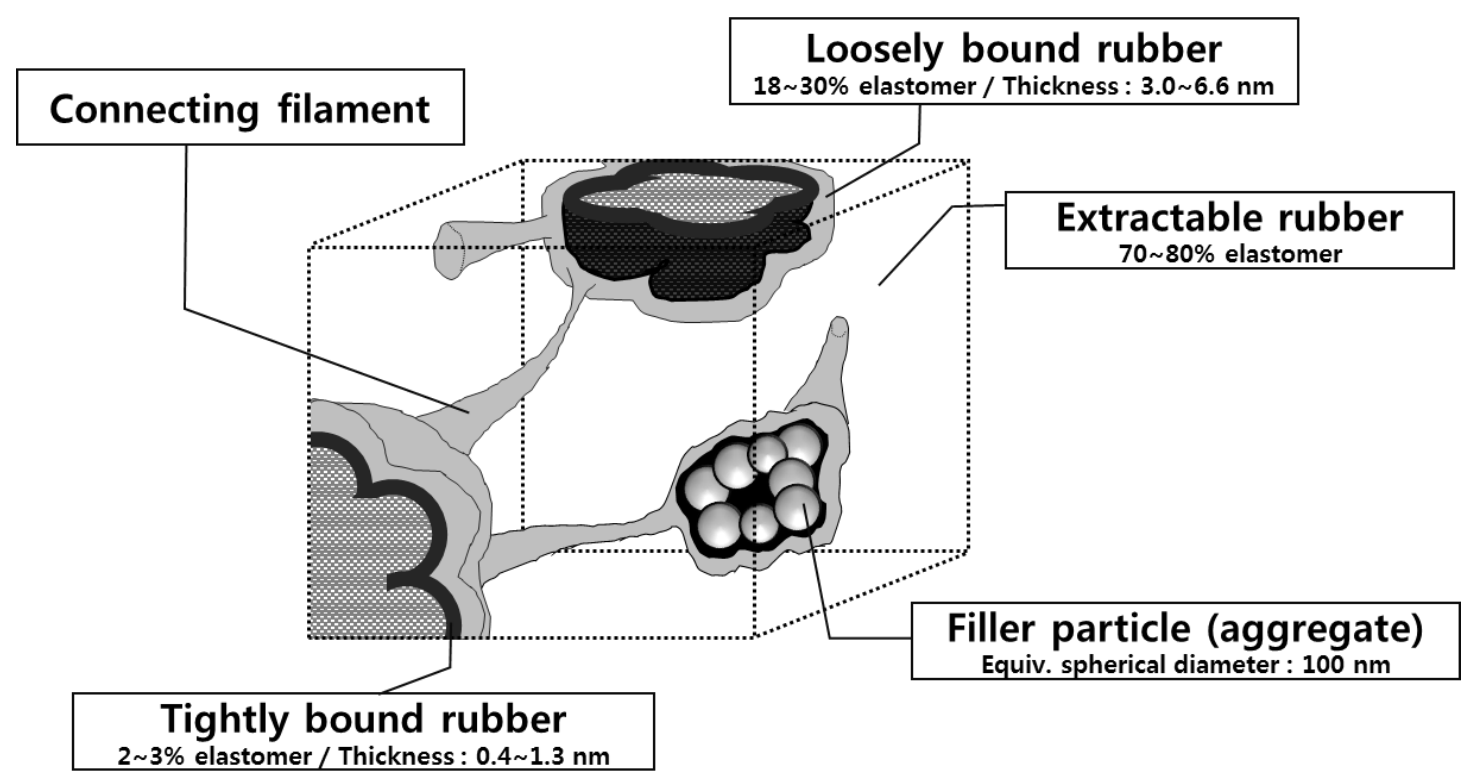

Figure 2.8 Leblanc's bound rubber model. ${ }^{[41]}$

Tightly bound rubber, which is located very close to the filler particles, behaves exactly the same way as the filler aggregate. Loosely bound rubber, in which the polymer chains are attached to the particle surface through the tightly bound rubber layer, is able to undergo very large deformations during flow. The loosely bound rubber layers of different filler particles are connected by filaments. As shown in Figure 2.9, by microscopic analysis, the connecting filaments look clearly different from the other extractable rubber. ${ }^{[42]}$ However, the connecting filament rubber can be extracted easily by a solvent, which means that this rubber region has not a direct interaction between rubber and filler particles. ${ }^{[41]}$ 


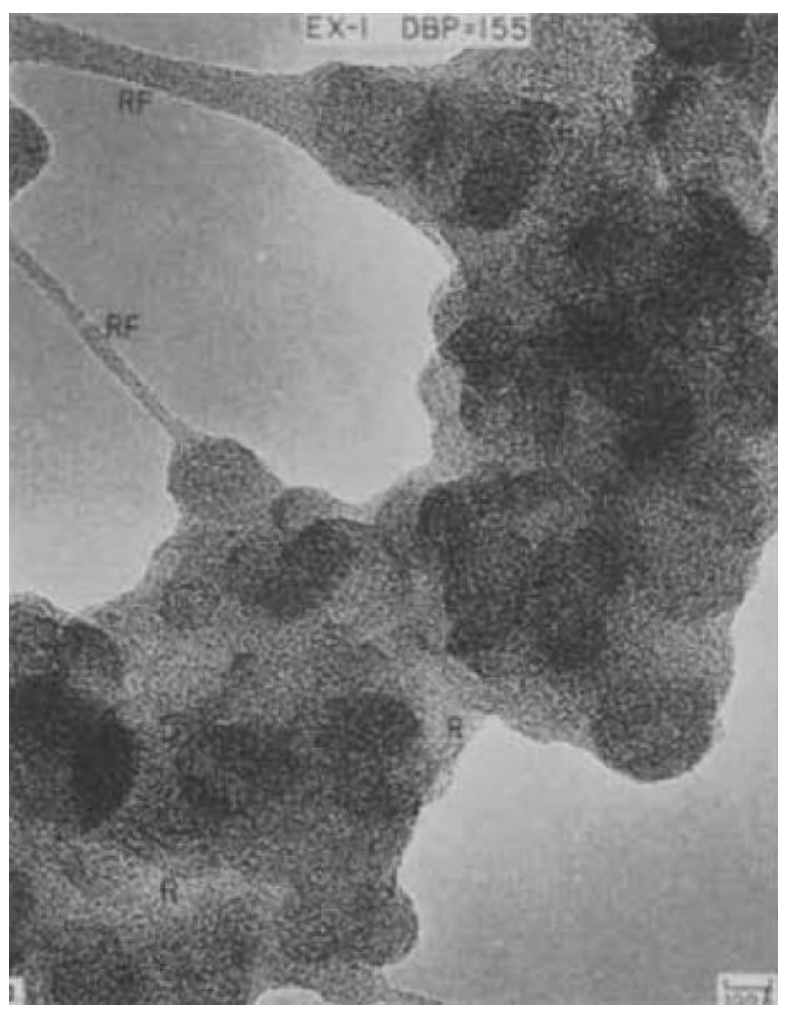

Figure 2.9 High resolution image of carbon-rubber gel network; RF designates connecting filaments. ${ }^{[42]}$

Recently, Fukahori ${ }^{[43]}$ proposed a new bound rubber model based on stress analysis. In this model, bound rubber consists of a double-layer: an inner polymer layer (glassy hard, GH) and an outer polymer layer (sticky hard, $\mathrm{SH}$ ) as show in Figure 2.10.

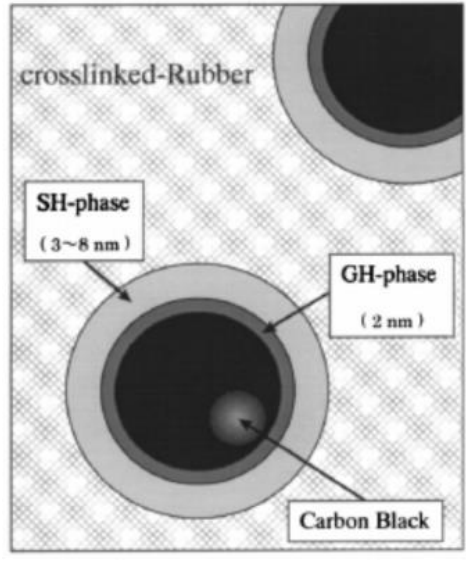

(a)

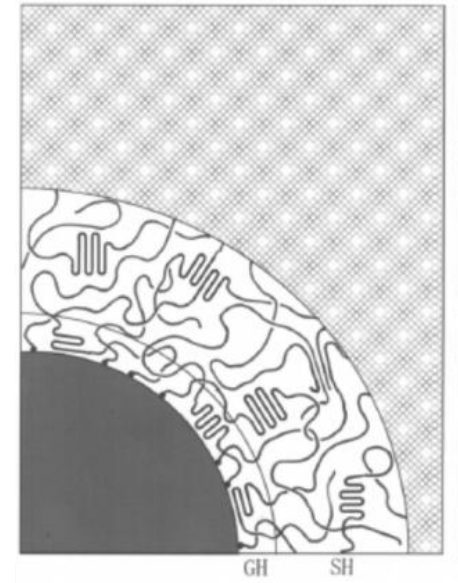

(b)

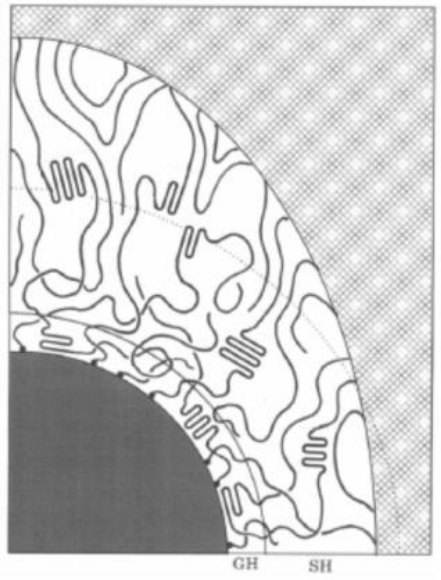

(c)

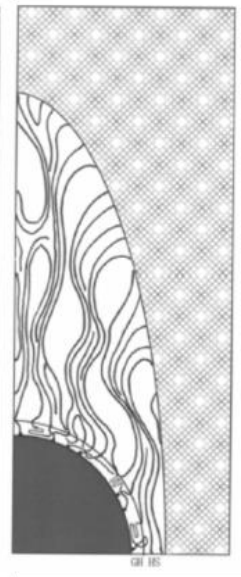

(d)

Figure 2.10 Fukahori's bound rubber model ${ }^{[43]}$; (a): double-layer interface consisting of a $\mathrm{GH}$ - and SH-layer; (b): no deformation; (c): under small extension; (d): under large extension. 
In the GH layer, molecular mobility is significantly restricted resulting in a higher modulus at small extensions, scarcely affecting the great increase in stress at large extensions. The SH layer consists of the polymer which is extended from the GH layer or entangled with polymers extended from the GH layer. This layer is positioned between the GH layer and the matrix rubber. The thickness of the SH layer is much larger and it is softer than the GH layer. Because of these characteristics, the SH layer plays a very important role in large extension. ${ }^{[43,44]}$

\subsubsection{Bound rubber model for silica filled rubber}

Bound rubber formation of a silica filled rubber compound does not differ much from the bound rubber models mentioned above. However, taking into account the silanization reaction, the rubber-filler mesophase is more complex. ${ }^{[41]}$

In general it is assumed that the silica-rubber coupling via a coupling agent takes place during vulcanization. ${ }^{[45]}$ But according to Mihara's ${ }^{[46]}$ results, chemically bound rubber can be partially formed on the silica surface during mixing. Qu et al. ${ }^{[47]}$ reported that the amount of bound rubber can be varied by the amount of coupling agent. These studies indicate that the chemical reaction between silica-silanerubber plays an important role for the bound rubber formation of silica filled rubber.

Luginsland et al. ${ }^{[48]}$ proposed a bound rubber model of silica reinforced rubber compounds with coupling agent based on the model of Medalia as shown in Figure 2.11.

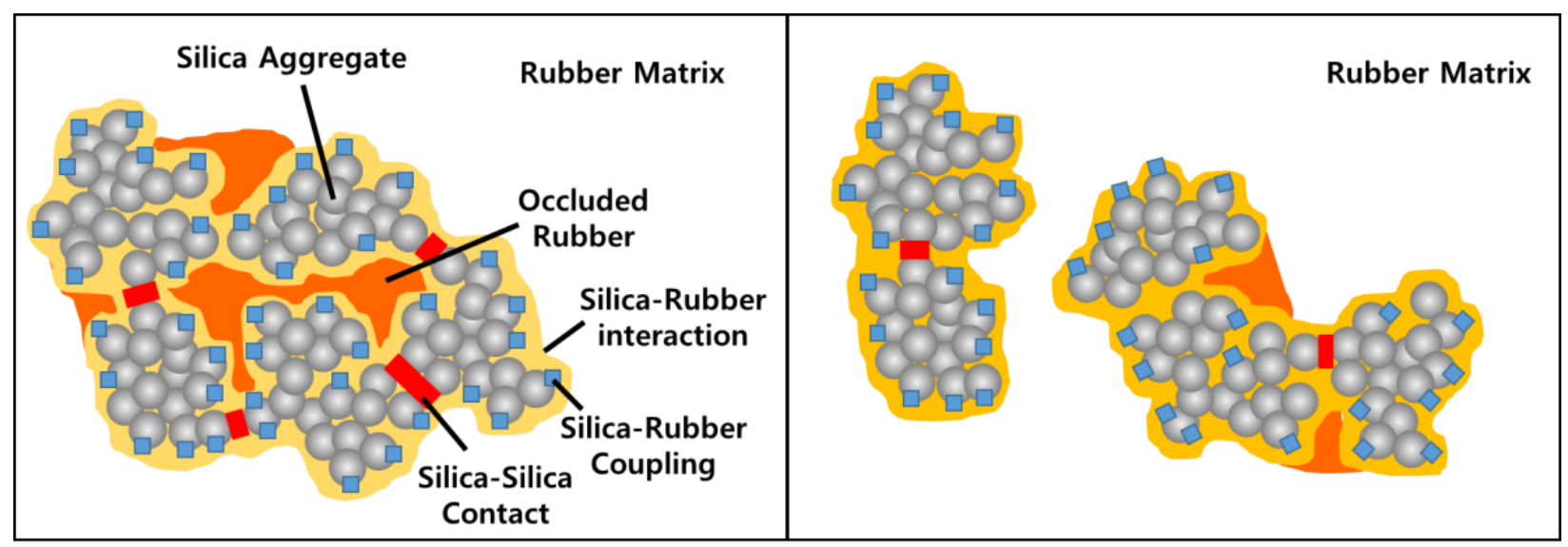

(a)

(b)

Figure 2.11 Bound rubber model for a silica/silane reinforced rubber compound ${ }^{[48]}$;

(a): Before deformation; (b): After deformation. 
Due to the surface polarity characteristics of silica, a filler-filler network can be easily formed within the rubber matrix. A part of the rubber can be easily occluded within the filler-filler network and be immobilized by physicochemical interactions which contribute to the low strain modulus. Under high deformation, the filler network is destroyed and a part of the occluded rubber is released. But due to the chemical bonding by the silane, a part of the occluded rubber and the rubber layer on the silica surface remains immobilized, and therefore still contributes to the modulus at high strain. ${ }^{[48]}$ Furthermore, the amount of this immobilized rubber can affect the $T_{g}$ and $\tan \delta$ due to its limited mobility. ${ }^{[47]}$

Choi and Ko ${ }^{[49]}$ made a more detailed categorization of bound rubber in silica filled rubber. According to their study, three major components of bound rubber can be obtained by using extraction and sonification at various temperatures: core shell, primary layer, secondary layer. They also proposed possible components of the bound rubber layer, which are in direct contact with the silica surface as shown in Figure 2.12. The core shell mainly consists of chemically bound rubber with a strong contact of the rubber to the filler surface. ${ }^{[49]}$

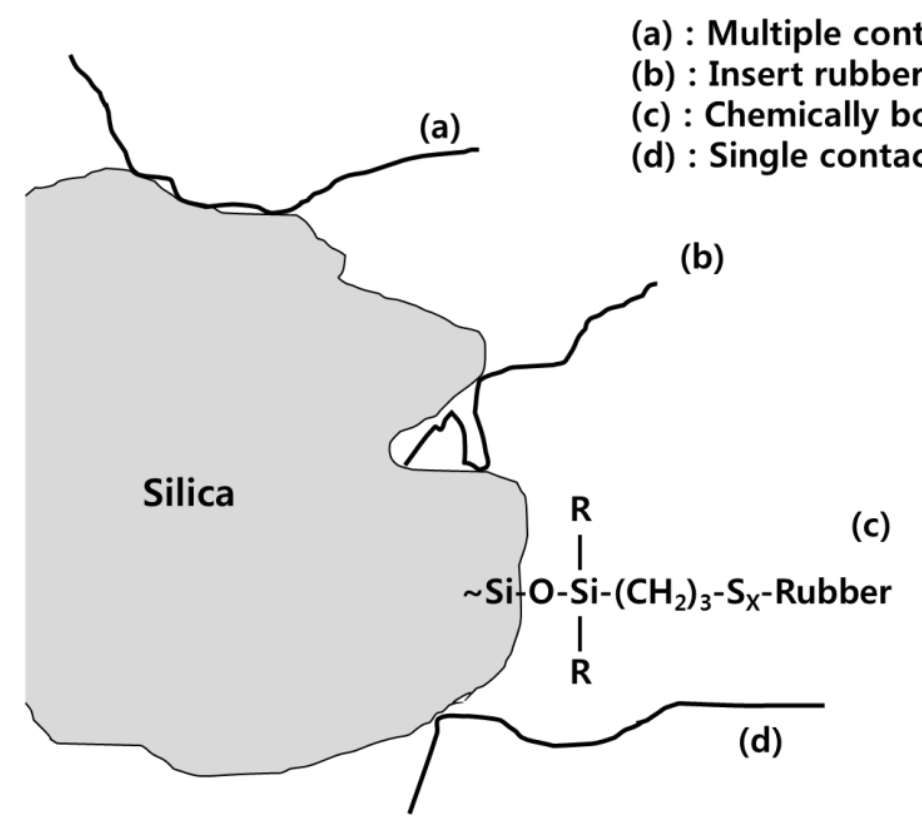

Figure 2.12 Directly contacted bound rubber components. ${ }^{[49]}$

\subsubsection{Filler-filler interaction : Payne effect}

It is well known that the Payne effect is attributed to breakage of the filler-filler interaction. The filler-filler interaction is mainly based on London and van der Waals forces. ${ }^{[16,50]}$ When active fillers are applied in a rubber matrix, they form a filler network which contributes to an increase of the modulus. At very low strain ranges, the storage modulus decreases with increasing amplitude of oscillation. Payne ${ }^{[61]}$ reported 
that the storage modulus has a limiting value at low strains $\left(\mathrm{G}^{\prime}{ }_{0}\right)$ and at high strains $\left(\mathrm{G}_{\infty}{ }_{\infty}\right)$ when there is no further change of modulus with increasing strains. And the loss modulus has a local maximum value at the strain where the storage modulus changes most. ${ }^{[16,51]}$

As shown in Figure 2.13, the Payne effect can be used in order to estimate the filler dispersion status within a rubber matrix by measuring $G_{0}^{\prime}$ and $G_{\infty}^{\prime}{ }^{[16,52,53]}$ a larger $\Delta G^{\prime}$ represents a continuous network between filler aggregates which means poor dispersion. Therefore, this effect plays an important role in order to understand the reinforcement mechanism of filled rubber. ${ }^{[20]}$

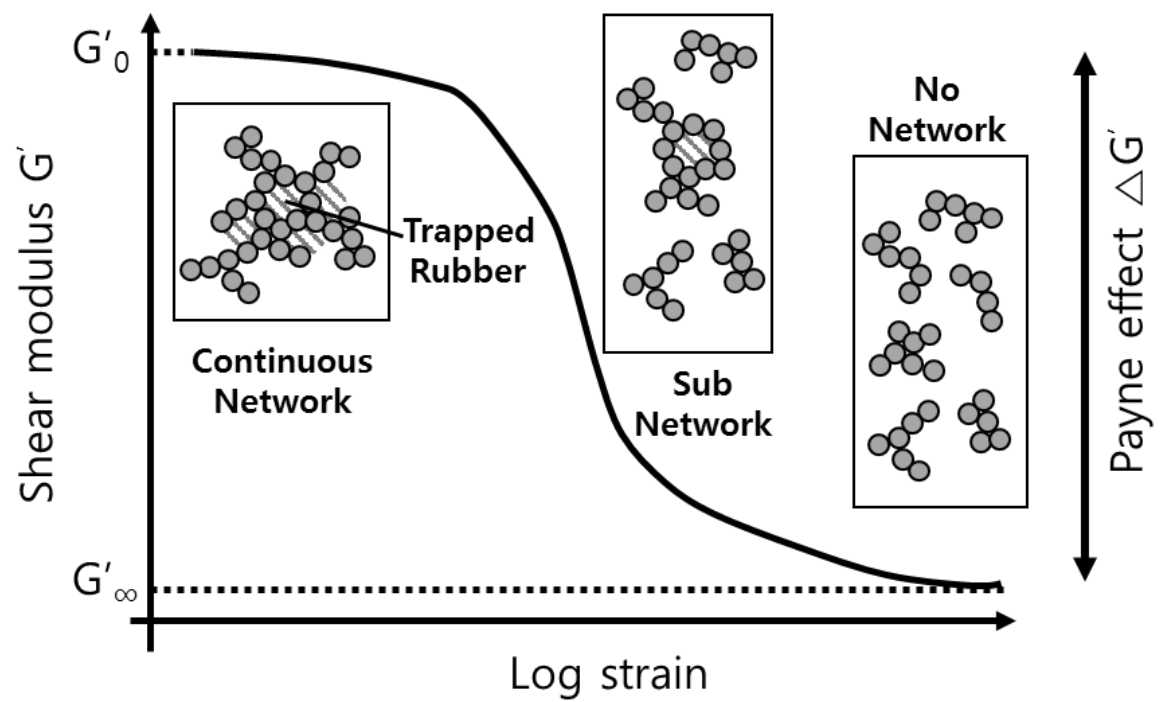

Figure 2.13 Strain-dependent breakdown of the filler network (Payne effect). ${ }^{[54]}$

Many researchers made an approach to explain the Payne effect. ${ }^{[55-61]}$ The first quantitative model was proposed by Kraus based on van der Waals and Lennard-Jones forces. ${ }^{[55,58,60,61]}$ In this model, when a filled elastomer undergoes sinusoidal deformation, filler-filler contacts are continuously broken and recombined as a function of the strain amplitude $y_{0}$. The rate of filler contact breakage $R_{b}$ is proportional to the number of remaining filler contacts $N$ and to $k_{b}$ :

$$
R_{b}=k_{b} \cdot \gamma_{0}^{m} \cdot N
$$

Where $k_{b}$ is the filler-filler contact breakage rate constant and $\mathrm{m}$ is related to filler agglomerate structures and dimensions. The $\mathrm{m}$ parameter gives information as the shear strain sensitivity of filler network 
breakage. The value of $m$ has been found experimentally to be universal $(m \approx 0.6)$, but the universality is still under discussion. ${ }^{[55,58,59]}$

The corresponding network reformation rate $R_{r}$ is proportional to $N_{o}-N$, where $N_{o}$ is the number of fillerfiller contacts at zero deformation: ${ }^{[55,58]}$

$$
R_{r}=k_{r} \cdot \gamma_{0}^{-m} \cdot\left(N_{0}-N\right)
$$

Where $k_{r}$ is the filler-filler contact reformation rate constant. At equilibrium, $R_{b}$ and $R_{r}$ are equal, which gives $N$ as:

$$
N=\frac{N_{0}}{1+\left(\frac{\gamma_{0}}{\gamma_{c}}\right)^{2 m}}
$$

Where $\gamma_{c}$ is a specific strain which corresponds to the breakage of half of the filler-filler contacts, given by

$$
\gamma_{c}=\left(\frac{k_{r}}{k_{b}}\right)^{\frac{1}{2 m}}
$$

At a strain amplitude $\nu_{0}, \mathrm{G}^{\prime}\left(\gamma_{0}\right)-\mathrm{G}_{\infty}^{\prime}$ over $\mathrm{G}_{0}^{\prime} \mathrm{G}^{\prime}{ }_{\infty}$ is taken as being proportional to the remaining number of contacts $N$, therefore $G^{\prime}$ at a strain $\gamma_{0}$ can be expressed as follows:

$$
\frac{G^{\prime}\left(\gamma_{0}\right)-G_{\infty}^{\prime}}{G_{0}^{\prime}-G_{\infty}^{\prime}}=\frac{N}{N_{0}}=\frac{1}{1+\left(\frac{\gamma_{0}}{\gamma_{c}}\right)^{2 m}}
$$

While the excess loss modulus is proportional to $R_{b}$ :

$$
G^{\prime \prime}\left(\gamma_{0}\right)-G_{\infty}^{\prime \prime} \propto k_{b} \cdot \gamma_{0}^{m} \cdot N \propto \frac{\gamma_{0}^{m}\left(G^{\prime}{ }_{0}-G^{\prime}{ }_{\infty}\right)}{1+\left(\frac{\gamma_{0}}{\gamma_{c}}\right)^{2 m}}
$$


Since $G^{\prime \prime}\left(\gamma_{0}\right)$ has a maximum value $G^{\prime \prime}{ }_{m}$ when $\gamma_{0}$ reaches $\gamma_{c}, G "\left(\nu_{0}\right)-G^{\prime \prime}{ }_{\infty}$ over $G{ }_{m}-G^{\prime \prime}{ }_{\infty}$ can be written as follows:

$$
\frac{G^{\prime \prime}\left(\gamma_{0}\right)-G_{\infty}^{\prime \prime}}{G^{\prime \prime}-G_{\infty}^{\prime \prime}}=\frac{2\left(\frac{\gamma_{0}}{\gamma_{c}}\right)^{m}}{1+\left(\frac{\gamma_{0}}{\gamma_{c}}\right)^{2 m}}
$$

Finally, the loss tangent tan $\delta$ becomes:

$$
\tan \delta\left(\gamma_{0}\right)=\frac{G_{\infty}^{\prime \prime}\left[\left(\frac{\gamma_{0}}{\gamma_{c}}\right)^{m / 2}-\left(\frac{\gamma_{0}}{\gamma_{c}}\right)^{-m / 2}\right]^{2}+2 G_{m}^{\prime \prime}}{G_{\infty}^{\prime}\left(\frac{\gamma_{0}}{\gamma_{c}}\right)^{m}+G^{\prime}{ }_{0}\left(\frac{\gamma_{0}}{\gamma_{c}}\right)^{-m}}
$$

The Kraus model has been successfully applied several times to the empirical data of Payne. However, the effects of temperature, frequency, filler type and concentration were not considered in this model. ${ }^{[55,58,59]}$ Recently, Huber and Vilgis ${ }^{[57]}$ extended the Kraus model by taking into account the fractal nature of the filler surface. They proposed a theoretical model which establishes a connection between the Payne effect and the structural properties of the filler network. In this model, it was assumed that at very small deformation amplitudes, the energetically elastic contribution of the rigid filler network is dominant, whereas at higher strains the hydrodynamic effect and rubber-filler interactions dominate. ${ }^{[57,58]}$ The expressions of Huber and Vilgis are as follow: ${ }^{[57]}$

$$
\begin{gathered}
\frac{G^{\prime}\left(\gamma_{0}\right)-G_{\infty}^{\prime}}{G_{0}^{\prime}-G_{\infty}^{\prime}}=\frac{1}{1+K^{2} \gamma_{0}^{2 m}} \\
\frac{G^{\prime \prime}\left(\gamma_{0}\right)}{G_{0}^{\prime \prime}-G_{\infty}^{\prime \prime}}=\frac{K \gamma_{0}^{m}}{1+K^{2} \gamma_{0}^{2 m}} \\
m=\frac{1}{C-d_{f}+2}
\end{gathered}
$$


Where $d_{f}$ and $\mathrm{C}$ are fractal exponents and $\mathrm{K}$ is a constant which collects the remaining system parameters. ${ }^{[57,58]}$ For carbon black, the filler network is best described by the model of kinetic clustercluster aggregation (CCA). ${ }^{[62]}$ In this case, the fractal exponents take the values $d_{f} \approx 1.8$, and $C \approx 1.3$. Inserting these values for CCA clusters lead to $m \approx 0.66$, which is in good agreement with the experimental value of the Kraus model $(m \approx 0.6)$. ${ }^{[57,58]}$

\subsection{Silica}

\subsubsection{The manufacturing process of precipitated silica}

Wagner ${ }^{[45]}$ indicated several important silica characteristics which can affect processing and properties of rubber compounds wherein it is applied as reinforcing agent: surface area, structure, chemical composition, water content, $\mathrm{pH}$ level. These properties of silica can be varied within the silica manufacturing process. ${ }^{[63,64]}$

Precipitated silica manufacturing can be separated into four main stages: precipitation, filtration, drying, milling and granulation. ${ }^{[54,65]}$ Additional milling and granulation can be applied especially for silica use in the tire industry. ${ }^{[54,65]}$ Even a small change in process parameters results in major changes of silica properties and rubber behavior ${ }^{[63]}$ as shown in Table 2.1 and 2.2. Therefore, applying appropriate process parameters and control is crucially important.

Table 2.1 Silica manufacturing process and corresponding behavior in rubber ${ }^{[64]}$

\begin{tabular}{cc}
\hline Manufacturing stage & Corresponding behavior in rubber \\
\hline Precipitation & Reinforcement \\
Precipitation, Drying, Granulation & Visible dispersion \\
Drying, Granulation & Dustiness \\
\hline
\end{tabular}

Table 2.2 Process variation-change in properties ${ }^{[63]}$

\begin{tabular}{cccc}
\hline Precipitation & Drying & Dipsersibility & Product \\
\hline High pH & Long time & Poor & Conventional Silica \\
Lower $\mathrm{pH}$ & Long time & Medium & Semi HD silica \\
High pH & Short time & Good & HD Silica \\
Optimized & Short time (different type) & Excellent & HD Silica \\
\hline
\end{tabular}


The most important properties of the silica: surface area, structure, silanol group density, particle size distribution, dispersibility, are more or less fixed during the precipitation stage. ${ }^{[63-66]}$ In this stage, the chemical reaction between sodium silicate (waterglass) and an acid $[54,63,64,66]$ occurs as shown in Figure 2.14.

\section{$\begin{gathered}\text { Sodium Silicate } \\ \left(\mathrm{Na}_{2} \mathrm{O} \cdot 3.5 \mathrm{SiO}_{2}\right)\end{gathered}+\begin{gathered}\text { Sulfuric Acid } \\ \left(\mathrm{H}_{2} \mathrm{SO}_{4}\right)\end{gathered} \longrightarrow \begin{gathered}\text { Precipitated Silica } \\ \left(\mathrm{SiO}_{2}\right)\end{gathered}+\begin{gathered}\text { Sodium Sulfate } \\ \left(\mathrm{Na}_{2} \mathrm{SO}_{4}\right)\end{gathered}+\begin{gathered}\text { Water } \\ \left(\mathrm{H}_{2} \mathrm{O}\right)\end{gathered}$}

Figure 2.14 Precipitated silica production - chemical reaction. ${ }^{[63]}$

As shown in Figure 2.15, in the beginning, small isolated particles are formed: primary particles. After a certain reaction time, the amount and size of the particles increases. Si-O-Si bonds are formed between the particles when their concentration reaches a sufficient level and they are close enough to each other: small clusters. The still ongoing process results in a continuous growth of the number and size of small clusters (smaller than $\pm 1 \mu \mathrm{m}$ ), until finally large clusters (larger than $\pm 1 \mu \mathrm{m}$ ) are formed. Large clusters can be destroyed by mechanical forces, because these are connected by van der Waals forces and hydrogen bonds. ${ }^{[63]}$

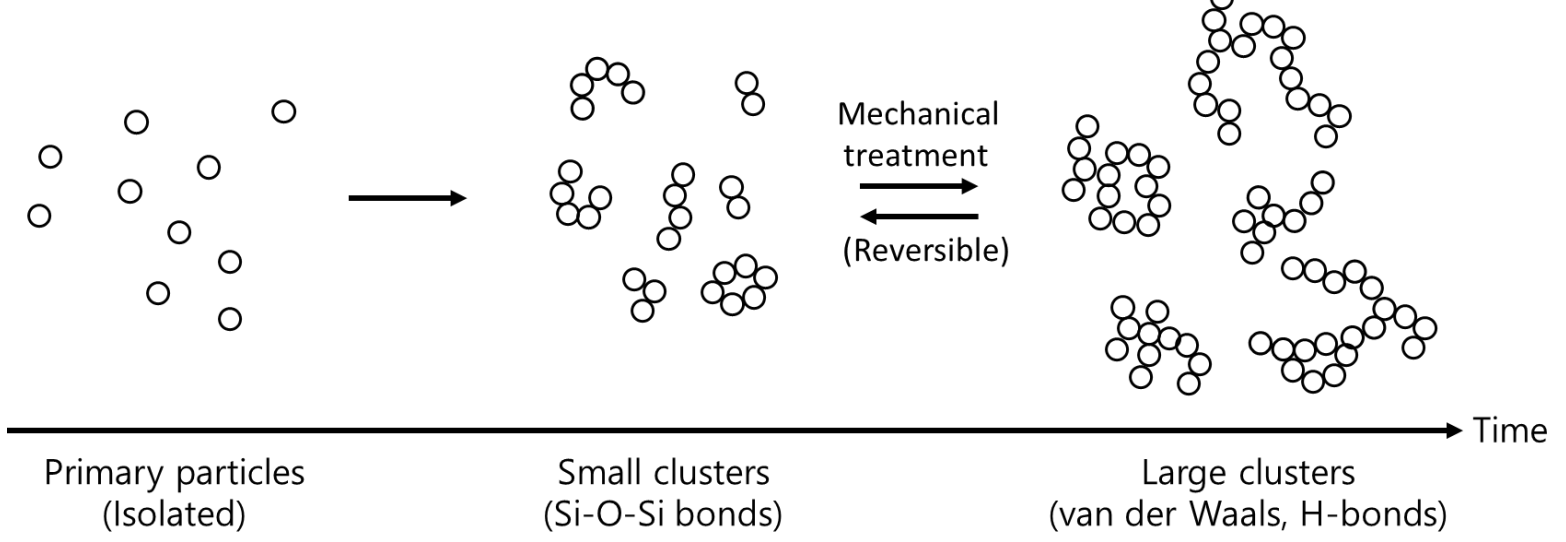

Figure 2.15 Particle formation during precipitation. ${ }^{[54]}$

After precipitation, a slurry of hydrated silica and salts is obtained. At the filtration stage, the salt concentration is reduced to $1 \sim 2 \%$ by washing with excess water in a filter-press. ${ }^{[64]}$ Electrical conductivity and solid contents of silica can be influenced within this stage. ${ }^{[63,65]}$ 
As the filter cake contains approximately $80 \%$ of water, a drying stage is required. During the drying stage, the water content is reduced to about 6\%. ${ }^{[64]}$ Several important properties of silica can be influenced within this stage: water content, surface area, structure, dispersibility, and particle size distribution. ${ }^{[63,65]}$ If it is necessary, the product passes through a milling and granulation stage in order to convert the product into a low-dust form for ease of handling. ${ }^{[54 . ~ 63-65]}$ Dustiness and visible dispersion, which is defined as the appearance of visible white particles, are mainly considered in order to select the appropriate physical form. ${ }^{[64]}$

\subsubsection{Silica properties}

In general, silica is classified into three different types: conventional, semi-dispersible, and highly dispersible silica. This classification is based on the basic properties and morphology of silica. Similar to carbon black, surface area and structure play an important role in reinforcement, but chemical characteristics such as surface activity, $\mathrm{pH}$ level, and water content can as well influence the properties of silica filled rubber. Thus studying the basic characteristics of silica is necessary in order to understand the dispersibility of silica.

\subsubsection{Primary particle and aggregate size of silica}

The primary particle size is the most important factor for prediction of rubber reinforcement: a high surface area means better reinforcement. The primary particle size can be measured by surface area. The size range of the particles with a reinforcing effect is $10^{\sim} 30 \mathrm{~nm}$, and this corresponds to a surface area of $125 \sim 250 \mathrm{~m}^{2} /$ g. $^{[64]}$

Brunauer-Emmett-Teller (BET) and cetyl-trimethyl-ammonium bromide (CTAB) adsorption methods are generally used for surface area measurements; ${ }^{[54,64]}$ both methods are using the same principle. The BET method uses nitrogen molecules: at very low temperatures, nitrogen is adsorbed onto the silica surface. Nitrogen molecules are small enough so that they are able to pass through the pores on the silica surface. ${ }^{[54]}$ The result is the measurement of the total surface area which is accessible to $N_{2}$.

The CTAB measurement uses cetyl-trimethyl-ammonium bromide molecules as the absorbed material. CTAB molecules are large, these molecules cannot enter into pores on the silica surface. Therefore, this method measures the external and geometrical surface of particles. ${ }^{[54]}$ The difference between BET and CTAB is demonstrated in Figure 2.16. 


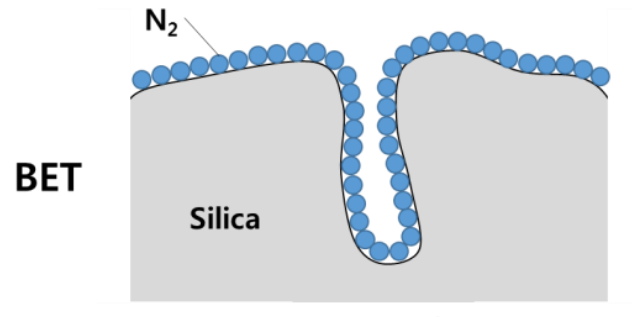

Porous surface

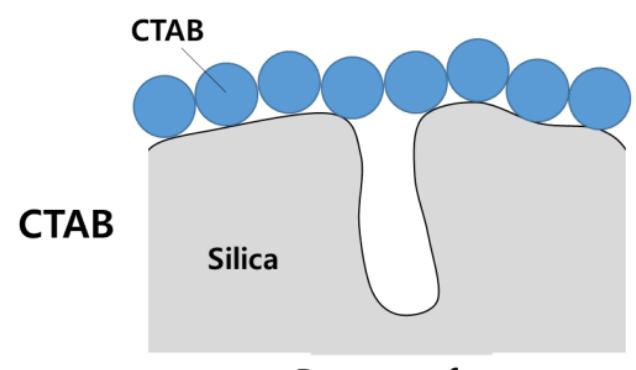

Porous surface

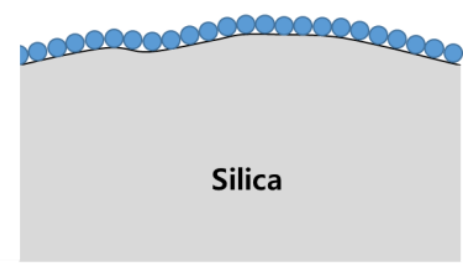

Nonporous surface

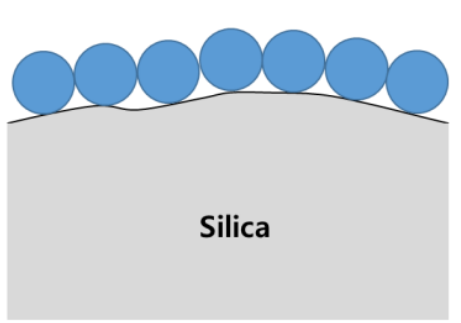

Nonporous surface

Figure 2.16 BET and CTAB measurements. ${ }^{[54]}$

Voet et al. ${ }^{[67]}$ reported that the surface area measured by CTAB showed good correlation with physical rubber properties, whereas BET did not. This shows that only the external surface is possible to interact with the large polymer molecules and that the pores are inactive.

\subsubsection{Structure of silica}

The structure of silica plays an important role for the physical properties of filled rubber, by changing the dispersion characteristics of silica. ${ }^{[45,63,68]}$ The structure can influence the degree of compactness in small clusters (smaller than $\pm 1 \mu \mathrm{m}$ ) which determines the inter-cluster and intra-cluster void volume. ${ }^{[54,63]}$ When HD silica, compared to conventional silica, undergoes very high shear forces, the initial voids remain for a longer period of time. Therefore, the polymer has enough time to penetrate into the voids during mixing. Ongoing mixing results in destruction of large silica clusters (larger than $\pm 1 \mu \mathrm{m}$ ) which include penetrated polymer. Therefore, a higher structure of silica indicates a better dispersion behavior. ${ }^{[54,63,68]}$ The structure of silica can be analyzed by evaluating the Oil Absorption Number (OAN) or DBP number. Oil absorption using dibutylphthalate (DBP) was generally used to predict the inter-cluster structure of silica: the amount of absorbed DBP gives an indication of the inter-cluster structure. ${ }^{[45,54,68]}$ Blume ${ }^{[68]}$ reported that the DBP number shows good correlation with the dispersion coefficient. However, DPB is no longer applied for silica structure prediction due to environmental and safety issues related to the oil; therefore paraffinic oil is nowadays recommended as an alternative to DPB. ${ }^{[69]}$ 
The initial structure can be estimated by the compressed volume structure test. The sample is subjected to a cylindrical glass chamber and compressed by a piston with constant pressure. The volume will be measured when there is no volume change anymore. Then the sample will be compressed again with increased pressure, and the more fragile structures will break with increasing pressure. A higher volume will be recorded for silica with a higher initial structure. A low structure silica will record a lower volume, which means it can be compacted with only small volume changes. These types of silica are difficult to disperse in the rubber matrix. ${ }^{[54,63]}$

\subsubsection{Surface chemistry of silica}

The surface of silica is covered with siloxane and silanol groups. Different silanol groups are formed during the precipitation stage of the silica manufacturing process, depending on the process parameters. ${ }^{[64]}$ The position of $-\mathrm{OH}$ with respect to a surface silicon is crucially important for rubber reinforcement. The reactivity and adsorption of polar molecules can be influenced by the distribution of hydroxyl moieties and the proximity between hydroxyl groups. ${ }^{[45,64]}$ Three different types of surface silanol groups are identified as shown in Figure 2.17. ${ }^{[45,54,64,65]}$

- Isolated: a lone silanol group, no close neighbor;

- Vicinal: two silanol groups on adjacent silicon atoms;

- Geminal: two silanols on the same silicon atom.

Geminal and isolated silanols are the most reactive. ${ }^{[54]}$ Blume et al. ${ }^{[70]}$ reported that during the hydrophobation process, all geminal and isolated silanol react.

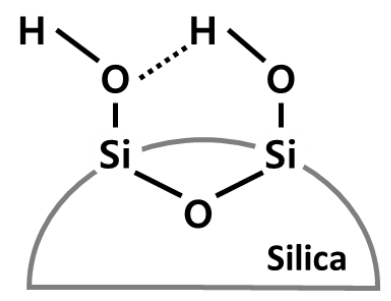

(a)

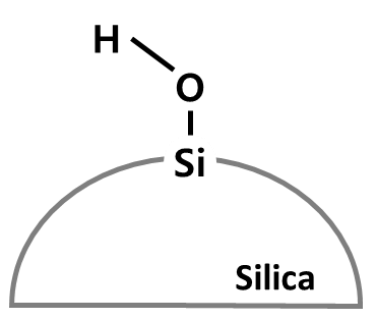

(b)

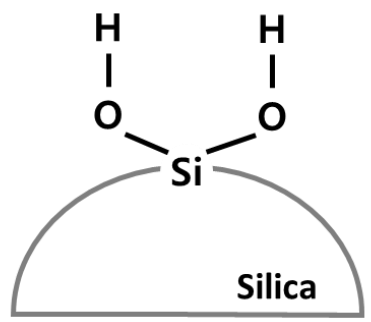

(c)

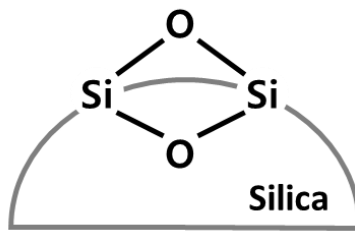

(d)

Figure 2.17 Different types of silanols on the silica surface;

(a): vicinal; (b): isolated; (c): geminal; (d): siloxane bridge. ${ }^{[54,64]}$ 


\subsubsection{Surface energy}

Due to the specific filler surface chemistry, the filler can be characterized by its surface energy $\gamma_{s}$, which can be expressed as follows: ${ }^{[41,71]}$

$$
\gamma_{s}=\gamma_{s}^{d}+\gamma_{s}^{s p}
$$

$\gamma_{s}{ }^{d}$ is the dispersive component based on van der Waals, London or nonspecific forces and $\gamma_{s}{ }^{\text {sp }}$ is the specific component based on polar forces, hydrogen bonding, acid-base interactions, etc. ${ }^{[71]}$ As mentioned previously, the silica surface is covered with silanols and siloxane bridges, which result in relatively high $\gamma_{s}^{\text {sp }}$ values compared to carbon black. However, silica forms a filler network more easily, indicating that the dispersion of silica in a non-polar elastomer matrix is much more difficult. ${ }^{[1]}$ By using a coupling agent, the surface energy of silica can be reduced. ${ }^{[72,73]}$ The closer the $\gamma_{s}$ of the filler (silica) to that of the rubber, the better the compatibility. The addition of ingredients with intermediate $\gamma_{s}$ can enhance compatibility like lubricants or process aids. ${ }^{[71]}$ The solubility parameters of common rubber ingredients are listed in Table 2.3.

Table 2.3 Solubility parameters of common rubber ingredients ${ }^{[7]}$

\begin{tabular}{cc}
\hline Polymer & Solubility parameter $\left[\mathrm{MPa}^{1 / 2}\right]$ \\
\hline NR, BR, IIR & $16-17$ \\
SBR & $17-18$ \\
Lubricants, process aids & $17-19$ \\
Carbon blacks & $24-30$ \\
Silicas & $28-36$ \\
\hline
\end{tabular}

As can be seen in Table 2.3, the solubility parameter difference between silica and polymer is greater than for carbon black, which means silica is more difficult to disperse in a rubber matrix. Recently, polymer chains are functionalized to reduce the solubility parameter difference between silica and polymer in order to improve the dispersion of silica in a rubber matrix. ${ }^{[46,75,76]}$ 


\subsubsection{Dispersibility of silica}

A higher degree of dispersion and distribution of fillers such as silica is a crucial factor for in-rubber properties. ${ }^{[77]}$ Not only the mixing conditions, but also the silica itself strongly influence the degree of dispersion in a rubber matrix. ${ }^{[77,78]}$ Measuring laser diffraction of a silica suspension after ultrasonic treatment can be an indication of silica dispersibility. ${ }^{[77,78]}$ When a sufficient amount of energy is applied to a silica suspension by ultrasonic treatment, clusters are destroyed to the initial structure. ${ }^{[63,68]}$ The results of this measurement for HD silica and conventional silica ${ }^{[63,68]}$ are shown in Figure 2.18.
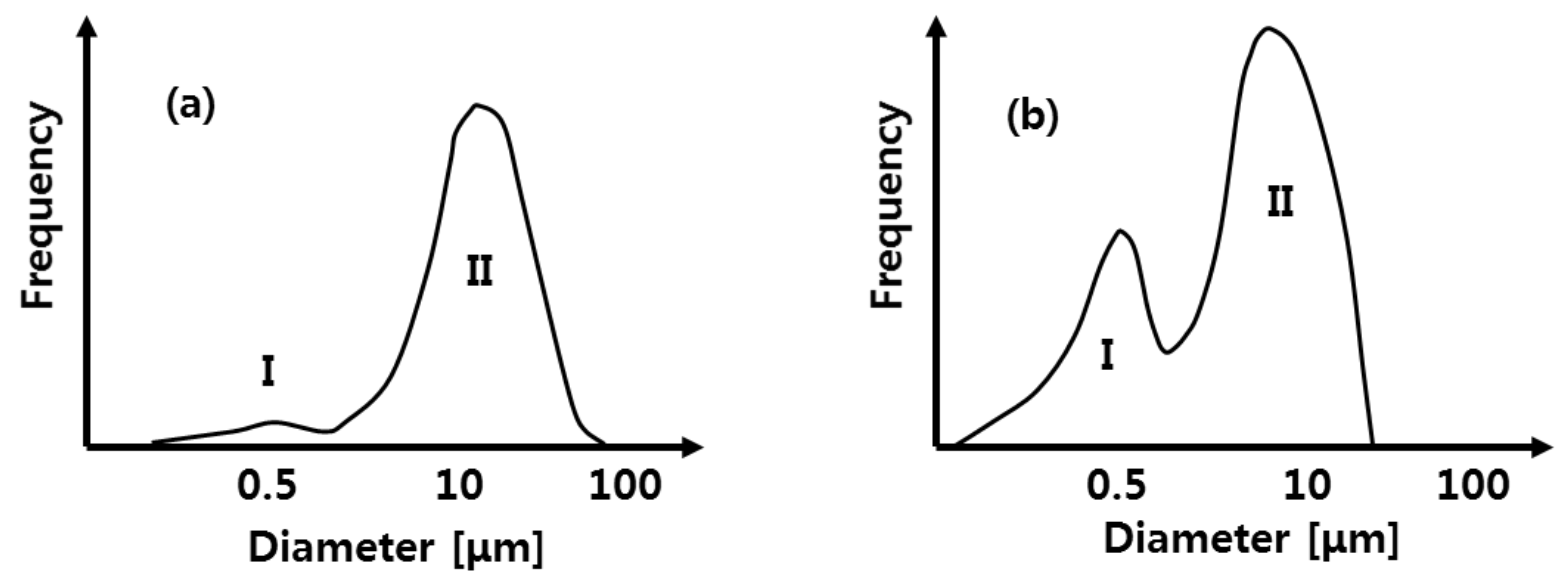

Figure 2.18 Particle size distribution; (a) conventional silica; (b) HD silica. ${ }^{[63,68]}$

The peak in the range of $0.5 \mu \mathrm{m}(\mathrm{I})$ indicates small clusters from decomposed larger clusters, and the one in the range of about $10 \mu \mathrm{m}$ (II) describes the initial structure with large clusters of silica, respectively. As can be seen in Figure 2.18, HD silica shows a relatively high amount of small clusters compared to conventional silica. It means that HD silica can be easily dispersed in a rubber matrix. ${ }^{[63]}$

\subsubsection{6 pH level of silica}

The $\mathrm{pH}$ level of the silica can influence the mechanical properties of silica filled rubber. In general, the $\mathrm{pH}$ level of precipitated silica lies within a range of 6.0 to 7.5. The effect of variation of the pH level of silica on processability and properties of rubber can be neglected within this range. ${ }^{[64]}$ However, if the $\mathrm{pH}$ level is extended to lower or higher values outside this range, the properties of silica filled rubber can be affected. Voet et al. ${ }^{[67]}$ reported, that for silica loadings above $40 \mathrm{phr}$, the $\mathrm{pH}$ level of the silica influences the adherence of filler and polymer. 
Hunsche et al. ${ }^{[72]}$ reported that with bis(triethoxysilylpropyl)tetrasufide (TESPT) as coupling agent, silica with a very low or very high $\mathrm{pH}$ level form a high amount of siloxane bonds on the surface. This means that the silanization reaction efficiency can be varied with the $\mathrm{pH}$ level of the silica. They assumed that an alkaline and an acid pH act as catalyst which results in an enhanced reaction between silica and coupling agent. This was clearly shown by their experiments. They obtained reaction rate constants by measuring evolved ethanol at $140^{\circ} \mathrm{C}$ as shown in Figure 2.19. The reaction speed was much faster when the silica has an acid or alkaline $\mathrm{pH}$ value. ${ }^{[72,73]}$ Hayichelaeh et al. ${ }^{[79]}$ compared the rate constant of the primary silanization reaction for silica filled natural rubber with and without amines (octadecylamine, OCT or diphenyl guanidine, DPG). They obtained a higher rate constant of the reaction when the compound contained an amine by forming basic conditions: the pKa value of DPG and OCT is 10.1 and 10.6, respectively.

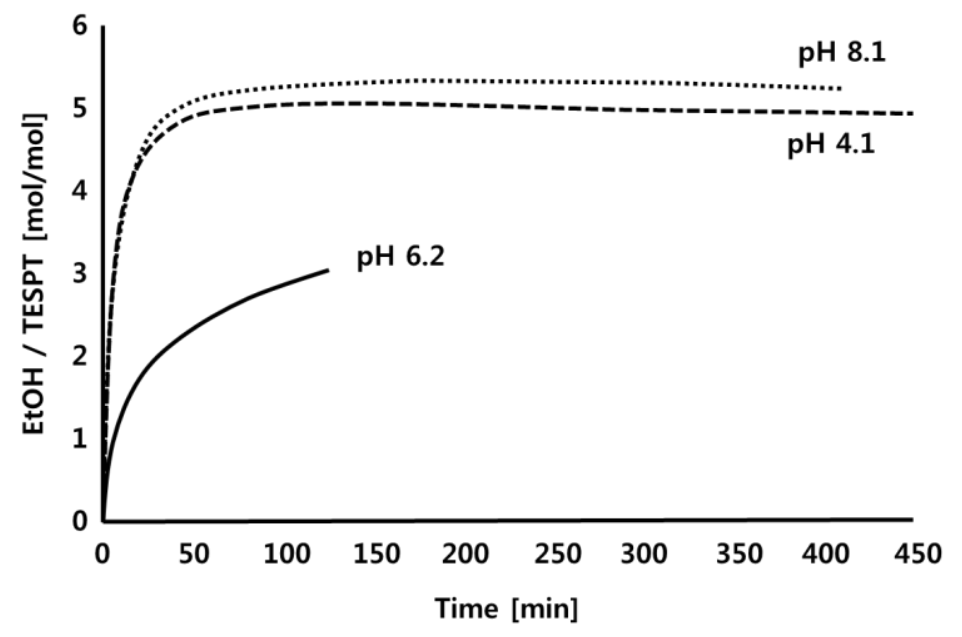

Figure 2.19 EtOH evolution at $140^{\circ} \mathrm{C}$ with three different $\mathrm{pH}$ values of silica. ${ }^{[73]}$

\subsubsection{Water content of silica}

The water content is also a characteristic property of silica. During silica manufacturing, sometimes water is added to adjust the concentration in the final product. ${ }^{[64]}$ The water content of silica can affect processability and properties of silica filled rubber. Lin et al. ${ }^{[80]}$ reported that during aging of a silica filled compound, the Mooney viscosity increment of a TESPT-containing compound was accelerated by humidity, while without TESPT, the compounds showed no change. However, the result of Schaal et al. ${ }^{\text {[81] }}$ showed an opposite trend. The main difference of the experiments between Lin et al. ${ }^{[80]}$ and Schaal et al. 
${ }^{[81]}$ was the range of moisture content. Schaal et al. ${ }^{[81]}$ compared undried (moisture contents 5 7wt\%) silica, while Lin studied the additional moisture effects during stock time. Less than $3 \%$ wt of moisture leads to a high viscosity of silica filled rubber. ${ }^{[54]}$

Kim and VanderKooi ${ }^{[82]}$ studied the moisture effect up to $50 \mathrm{wt} \%$ of a silica filled compound and observed a similar Mooney viscosity trend as Lin et al. ${ }^{[80]}$ They reported that the water content of silica affects the viscosity, curing behavior and mechanical properties. They also indicated that the mixing temperature profile can be affected by the moisture content of silica. They assumed the role of moisture as follows: ${ }^{[82]}$

- Acts as a lubricant and a protector of polymer chains during mechanical processing;

- Reduces polar bonding between silica agglomerates;

- Prevents pre-vulcanization;

- Accelerates crosslinking reactions;

- Improves the hydrolysis reaction of the coupling agent.

\subsection{Silane}

It is well known that silica shows strong filler-filler interactions between the particles, which result in poor dispersibility and processability of silica filled rubber. Many studies were done and are still ongoing for novel material development such as polymer functionalization and optimizing the dispersibility of silica in

order to overcome these problems. ${ }^{[77,78,83-85]}$ But still, using silane coupling agents is the best way to solve the problems. The silane coupling agent strongly affects the filler-filler and filler-polymer interaction, thus mechanical properties and processability of silica filled rubber can be enhanced. ${ }^{[47,86]}$ Therefore, studying the basics of silane coupling agents is necessary for a better understanding of silica reinforcement.

\subsubsection{Types of silane coupling agent}

Figure 2.20 shows three types of silanes, which are generally used for sulfur cured rubber compounds. 
Di- and poly sulfidic silanes

Mercaptosilanes

\section{Blocked mercaptosilanes}

$(\mathrm{RO})_{3} \mathrm{Si} \curvearrowright \mathrm{SSI}_{\mathrm{X}} \curvearrowright \mathrm{RO}_{3}$<smiles>O=[W][SiH2]CCCS</smiles>

Figure 2.20 Three types of silanes; $\mathrm{R}$ can be $-\mathrm{CH}_{3},-\mathrm{C}_{2} \mathrm{H}_{5}$, alkylpolyether; $x$ can be varied from 2 to 8;

PG corresponds to $\mathrm{C} \equiv \mathrm{N}$ and octanoyl. ${ }^{[54]}$

The most commonly used silane in the tire industry is bis(triethoxysilylpropyl)tetrasufide (TESPT). ${ }^{[54,72,87,88]}$ TESPT is composed of ethoxysilyl and poly-sulfide groups (sulfur rank: approximately 4). One part reacts with the hydroxyl groups on the silica surface, the other with the polymer. Due to the length of the poly-sulfidic moiety of TESPT, active sulfur can be released into the rubber matrix when it undergoes high temperature and shear conditions during mixing, which can cause processability problems. ${ }^{[72,89]}$

A shorter sulfur rank leads to better thermal stability. ${ }^{[54]}$ In case of bis(triethoxysilylpropyl)disulfide (TESPD), which has a di-sulfide group, better thermal stability is reached. As can be seen in Figure 2.21, TESPT has the most narrow optimum temperature frame. However, when TESPD is applied to silica filled rubber instead of TESPT, a higher amount of free sulfur is required to obtain the same level of mechanical properties. ${ }^{[90,91]}$ 


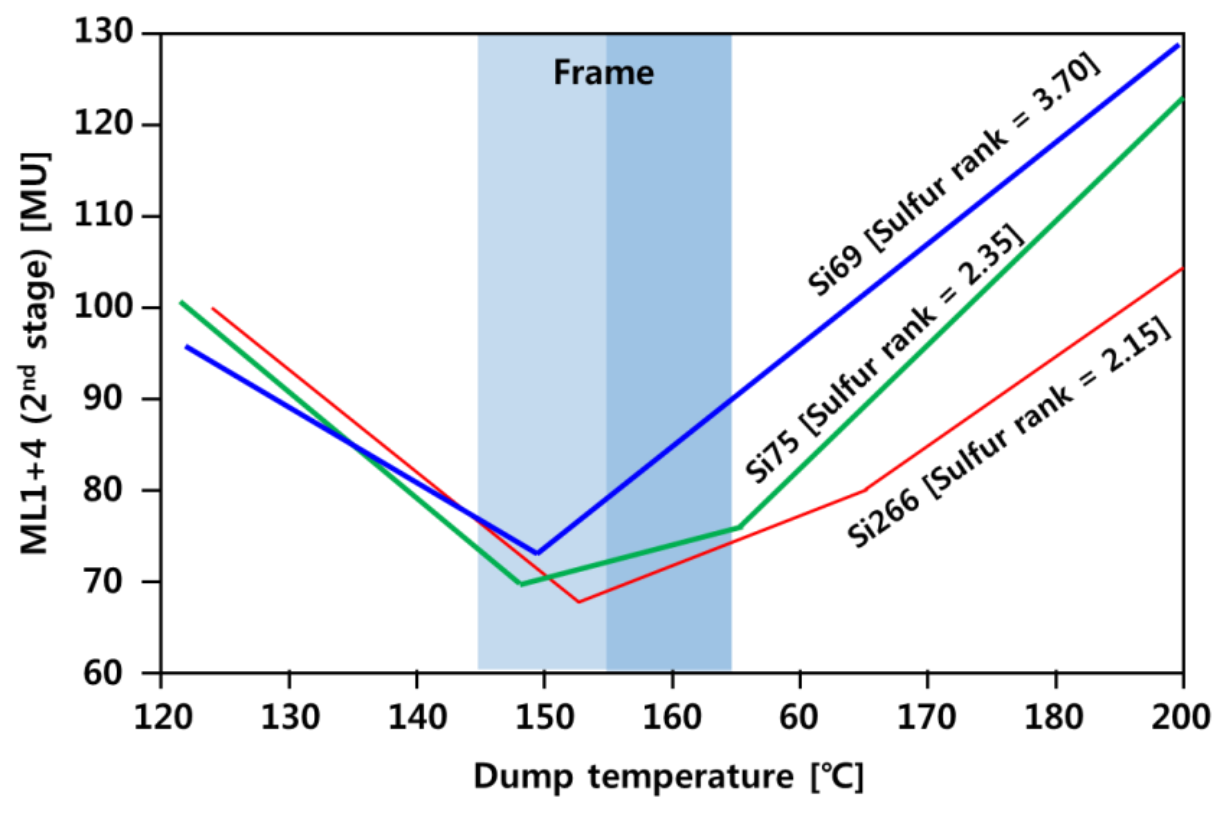

Figure 2.21 Thermal stability or scorch safety of the coupling agent represented by the compound viscosity (ML1+4); Si 69 = TESPT; Si 75 = TESPD; Si 266 = TESPD. ${ }^{[54,89]}$

Recently, a sterically hindered mercaptosilane (Si 363) and blocked mercaptosilane (NXT) received much attention from the tire industry. These silanes have more potential to enhance rolling resistance compared to the standard silane (TESPT). Si 363 has one ethoxy group $\left(\mathrm{C}_{2} \mathrm{H}_{5} \mathrm{O}-\right)$, two alkyl polyether groups $\left(\mathrm{CH}_{3}\left(\mathrm{CH}_{2}\right)_{12}\left(\mathrm{OCH}_{2} \mathrm{CH}_{2}\right)_{5} \mathrm{O}-\right)$ and a mercapto group (-RSH) on the silicon atom. The ethoxy and mercapto groups are responsible for silanization and coupling, respectively. ${ }^{[54,88]}$ The alkyl polyether groups can shield free silanol groups on the silica surface which leads to better hydrophobation of the silica as shown in Figure 2.22. ${ }^{[88]}$ 


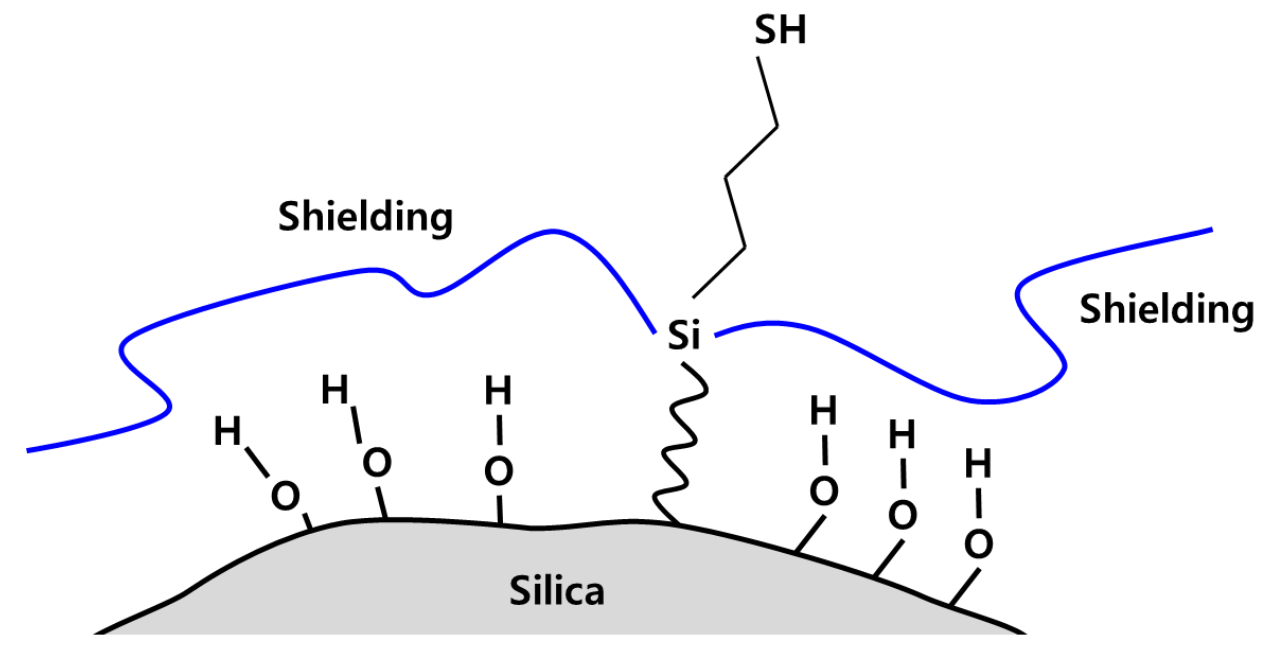

Figure 2.22 Silica hydrophobation by Si $363 .{ }^{[88]}$

Coupling yields of the free mercapto group is nearly $100 \%$, whereas the yield of poly- and di-sulfide moieties is approx. $50 \% .{ }^{[8,91]}$ However, silanes with mercapto groups often show poor processability such as high Mooney viscosity and scorch problems. ${ }^{[88]}$

\subsubsection{Silanization mechanism: Silica-Silane}

Silanization is a reaction between the silanol groups on the silica surface and the silane. ${ }^{[75]}$ As mentioned in the previous paragraph, silica can easily form a filler-filler network which results in a poor processability and reinforcing effect of silica filled rubber. Silanization leads to less polarity of the silica, therefore this reaction strongly affects the dispersion of silica. ${ }^{[14,92]}$ Thus, for the rubber industry, understanding the silanization reaction is crucially important. ${ }^{[54]}$

The silanization can be divided into a primary and a secondary reaction. The mechanism of the silanization reaction with TESPT is illustrated in Figure 2.23, as it is known from published literature, although other models are also proposed. ${ }^{[93]}$ 

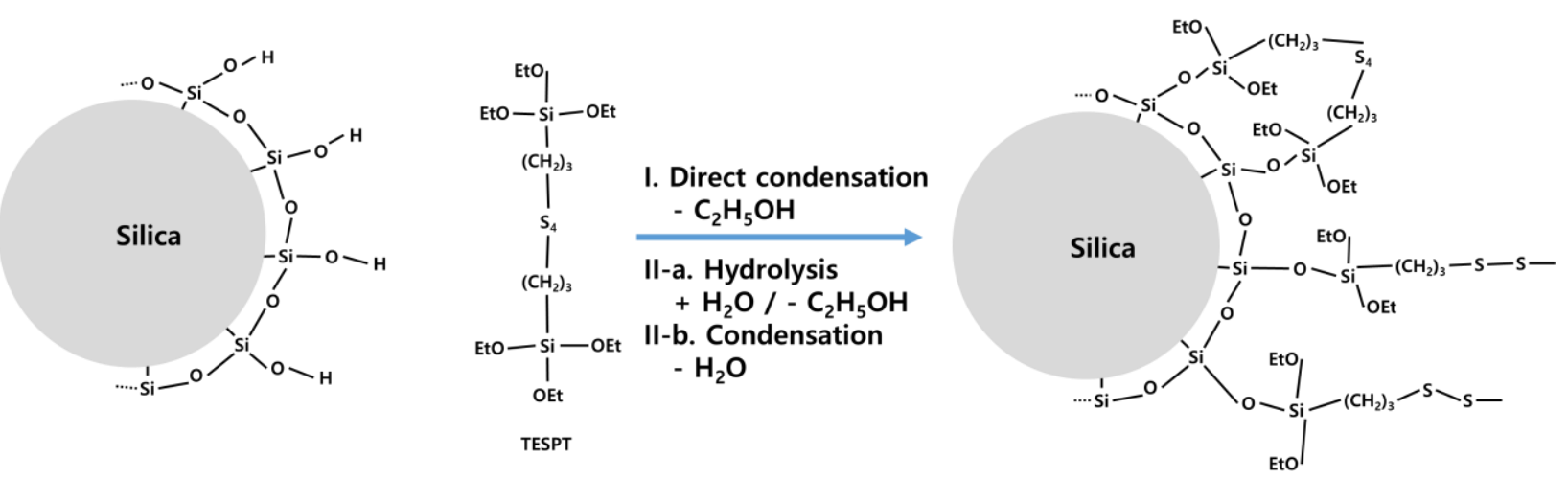

(a)
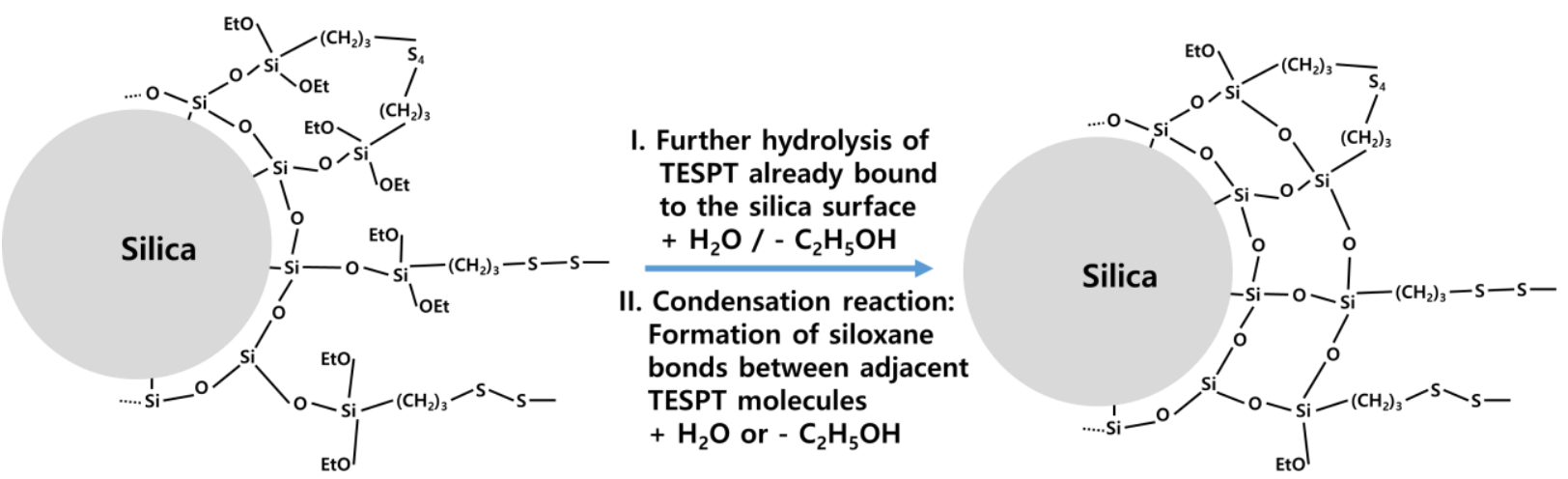

(b)

Figure 2.23 The silanization reaction mechanism; (a): primary reaction; (b): secondary reaction. ${ }^{\text {[72] }}$

First, silanol groups on silica surface and an alkoxy group of the silane react in the primary reaction. The reaction can occur in two ways: direct reaction or hydrolysis followed by a condensation reaction as described in Figure 2.23(a). ${ }^{[72,75]}$ The hydrolysis reaction is the rate-determining step for the silanization. As previously mentioned in Paragraph 2.3, the $\mathrm{pH}$ level of silica and the water content affect the rate of silanization. Also the mixing temperature can increase the reaction speed. ${ }^{[54,94]}$

The secondary reaction is a two stage process: hydrolysis of one or two ethoxy groups (Figure 2.23(b)-I), and a condensation reaction which generates water or ethanol (Figure 2.23(b)-II). ${ }^{[72,75]}$ This reaction also can be affected by the water content of silica and the mixing temperature. ${ }^{[72,75,94]}$ Compared to the rate of the primary reaction, the secondary reaction is rather slow. The rate constants of the primary and secondary reactions at elevated temperatures for TESPT are compared ${ }^{[94]}$ in Table 2.4. This result shows that the primary reaction is approx. 10 to 20 times faster than the secondary reaction and how the mixing temperature affects the rate of silanization. 
Table 2.4 Kinetic rate constants for the primary reaction $\left(k_{a}\right)$ and the secondary reaction $\left(k_{b}\right)^{[94]}$

\begin{tabular}{cccc}
\hline Temperature $\left[{ }^{\circ} \mathrm{C}\right]$ & 120 & 140 & 160 \\
\hline $\mathrm{k}_{\mathrm{a}}$ & 0.061 & 0.122 & 0.229 \\
$\mathrm{k}_{\mathrm{b}}$ & 0.005 & 0.008 & 0.012 \\
\hline
\end{tabular}

\subsubsection{Coupling mechanism: Silane-rubber}

The silane-rubber coupling reactions take place not only during vulcanization ${ }^{[95-98]}$ but already during the mixing process. ${ }^{[46,96-98]}$ In case of a sulfur bridged silane, at least two sulfur atoms in these silanes are required to enable a reaction with the rubber. Furthermore, the reactivity of the sulfur bridged silane increases with increasing sulfur chain length. ${ }^{[95,99]}$ Addition of free sulfur and accelerators plays an important role in the silane-rubber reaction. Without sulfur and accelerator, a coupling reaction cannot occur. The addition of sulfur and accelerators increases the speed and efficiency of the coupling reaction as shown in Figure 2.24. ${ }^{[91]}$

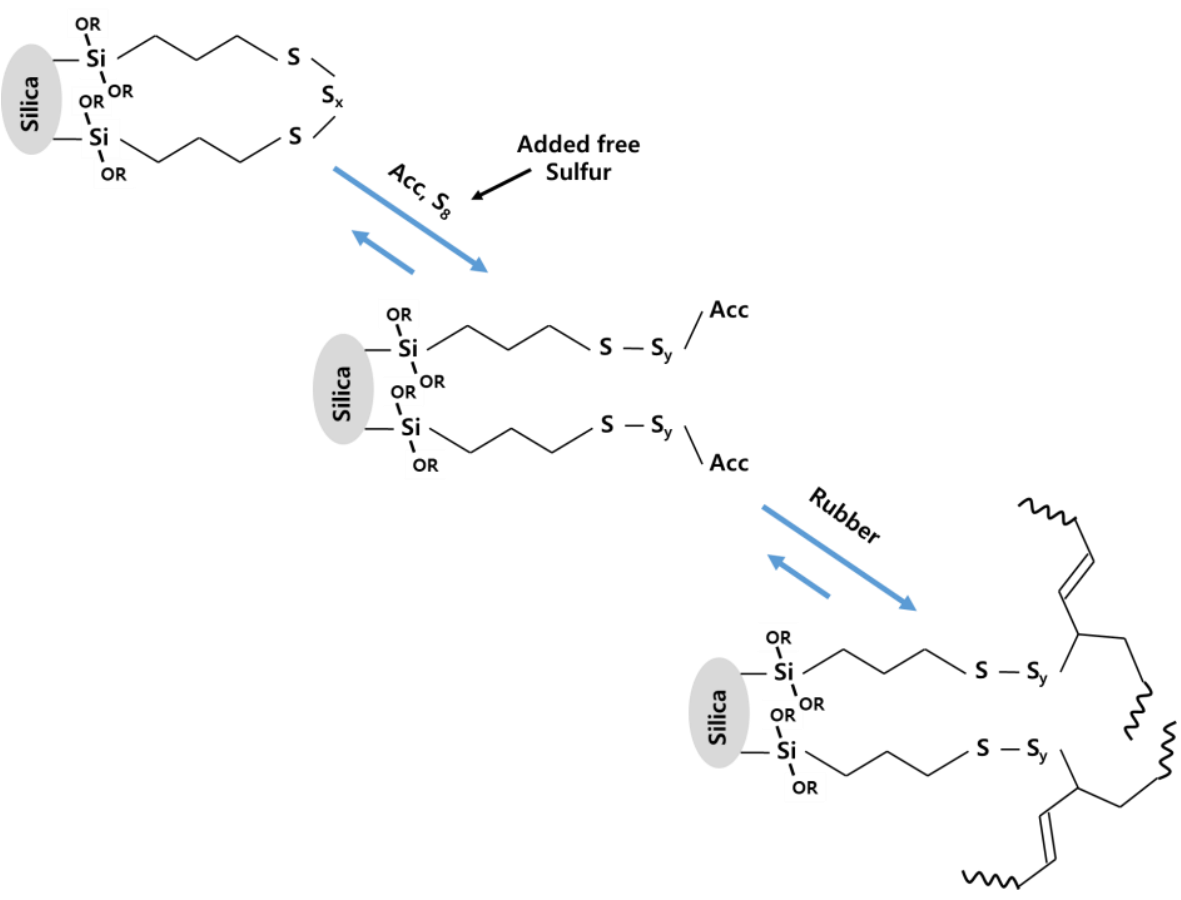

Figure 2.24 Coupling reaction to rubber (TESPT $(y=1.7)$, TESPD $(y=0)) .{ }^{[91]}$

Activation of the silane consumes free sulfur as shown in Figure 2.24. When a constant amount of free sulfur is introduced, silica-rubber coupling increases while the rubber matrix crosslink density decreases with increasing amount of TESPT (Figure 2.25 (a)). This result means that incorporation of sulfur by the 
silane indeed occurs. However, the total crosslink density increases (Figure 2.25 (b)) due to the fact, that TESPT can act as a sulfur donor as well and not only sulfur acceptor, as mentioned previously. ${ }^{[91]}$

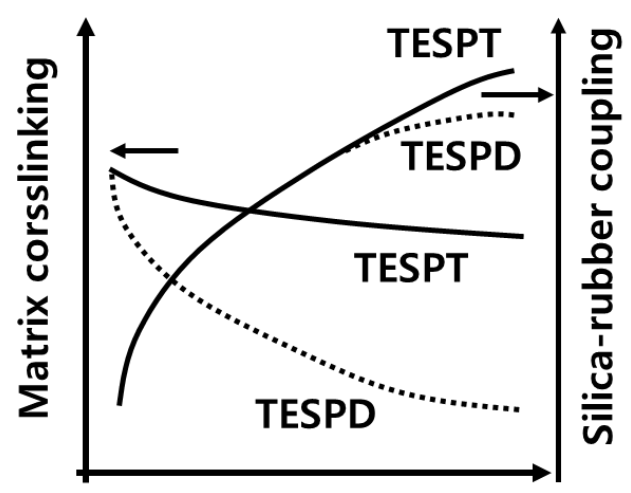

Amount of silane

(a)

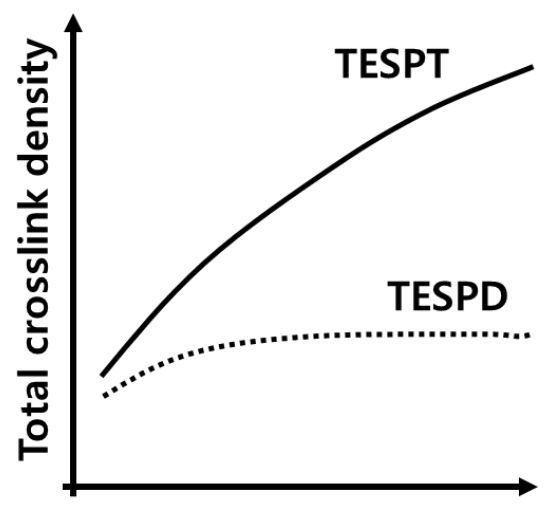

Amount of silane

(b)

Figure 2.25 Incorporation of sulfur by silane at a constant amount of sulfur. ${ }^{[1]}$

\subsubsection{Silane-rubber coupling reactivity of 1,2- and 1,4-double bonds in SBR or BR}

According to the work of Dogadkin et al., ${ }^{[100]}$ the 1,4-double bond structure in SBR or BR shows a higher reactivity for the sulfur crosslinking reaction during the vulcanization process. Marzocca et al. [101] obtained a higher crosslink density when the amount of cis-BR increased in a blend. However, the results of these studies are not valid for the coupling reaction between a sulfidic silane and butadiene units in SBR or BR.

Sato ${ }^{[102]}$ reported that the reactivity of the butadiene unit in SBR or BR towards sulfidic silane can differ according to their chemical structure. He compared the reactivity of several model olefins, which represent the vinyl-, cis- and trans- double bond structures in SBR or BR, toward sulfidic silanes, and found that the reactivity of butadiene units varies according to following order: vinyl > cis $>$ trans.

However, the reactivity of vinyl-groups of SBR or BR toward sulfidic silanes can again be changed by the presence of curing accelerators, such as $\mathrm{N}$-cyclohexylbenzothiazole-2-sulfenamide (CBS). Sato ${ }^{[102]}$ found that the presence of CBS makes the silane incorporating more sulfur in its structure rather than react with the model olefin: the filler-polymer coupling reaction between the vinyl-groups of SBR and silane will not occur much when the compound contains CBS. 


\subsection{Mixing}

Mixing is the first step in converting raw materials into a rubber compound. ${ }^{[103]}$ The goal in mixing is to provide a composition having a suitable processability with as high a consistency as possible. Since silica is used as a filler for tire tread compounds, mixing became a more crucial step of the tire manufacturing processes. The basic theory of mixing and previous work on silica mixing are discussed to understand the special aspects of silica mixing.

\subsubsection{Mixing mechanism}

Palmgren ${ }^{[104]}$ and Nakajima ${ }^{[105]}$ suggested a mixing model for a rubber compound in a batch mixer as shown in Figure 2.26. In order to obtain a well-mixed filled rubber compound, the size of the elastomer and filler domains should be reduced. Subdivision was described as the breaking up of large clusters of fillers into smaller units, suitable for the incorporation step. Subdivision is necessary not only for the incorporation into the elastomer matrix, but also for dispersion.

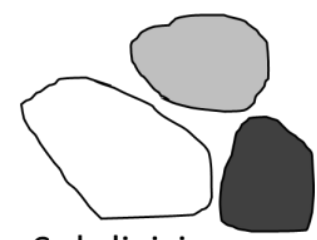

Subdivision

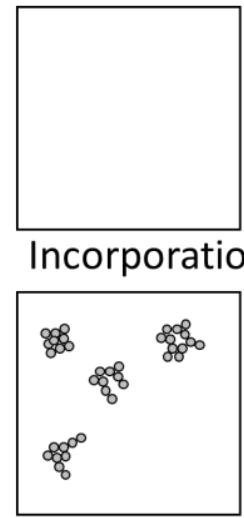

Dispersion

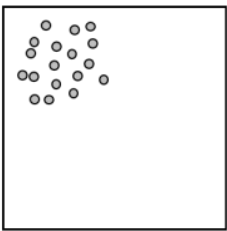

Simple (or distributive) mixing
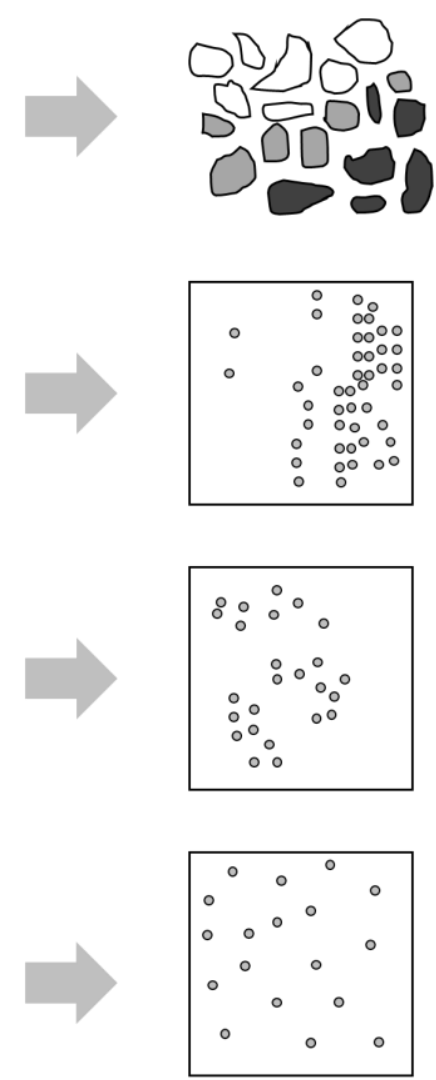

Figure 2.26 Rubber compound mixing stages. ${ }^{[104]}$ 
In the incorporation or wetting stage, ingredients are incorporated into the rubber matrix and form a coherent mass. The filler is absorbed into the rubber matrix, and polymer chains penetrate into filler voids. The total volume of the mixture becomes lower than the sum of the initial volumes of individual ingredients as shown in Figure 2.27. ${ }^{[37,106]}$

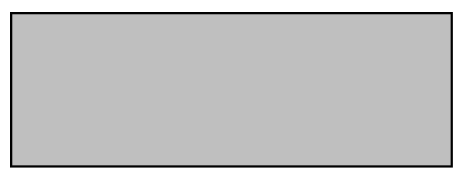

Rubber

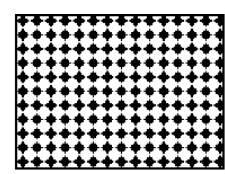

Filler

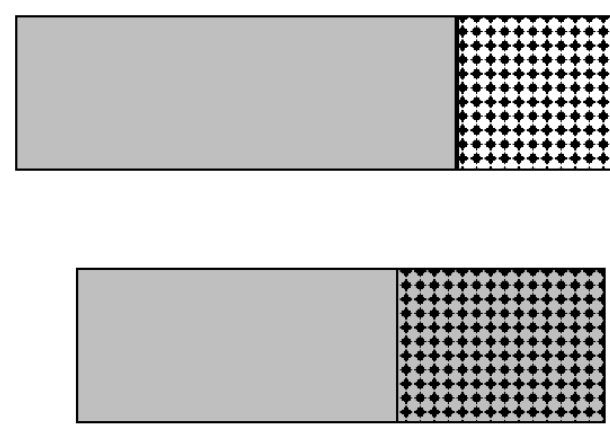

Incorporated filler

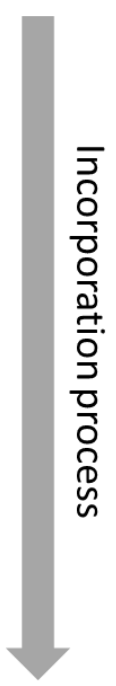

Figure 2.27 Total compound volume change after filler Incorporation process. ${ }^{[106]}$

The dispersion process should be considered separately from simple or distributive mixing. A more detailed diagram of dispersion and distribution is shown in Figure 2.28. ${ }^{[107]}$ The dispersion process involves the reduction of filler agglomerate size and changes the physical state of the fillers. Dispersion is dependent not only on mixing time but also on the shear stresses generated within the polymer.

Simple mixing or distributive mixing is the process of moving of the filler clusters from one point to another, without changing the shape and size of the particle to increase the homogeneity of the mixture. 

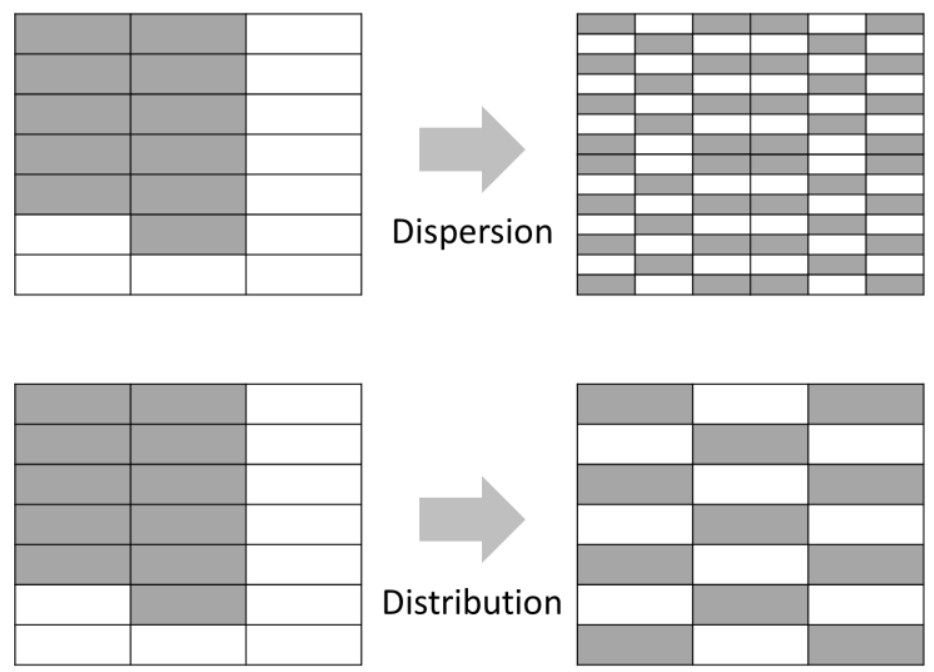

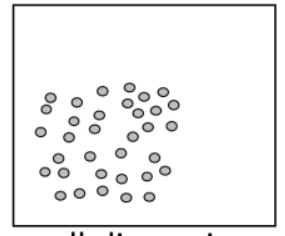

well dispersion Poor distribution

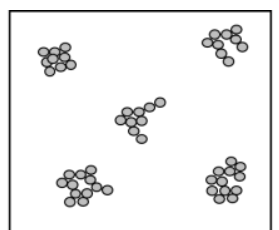

Poor dispersion well distribution

$$
\begin{aligned}
& \begin{array}{cccccc}
\circ & 0 & 0 & 0 & 0 \\
& 0 & 0 & \circ & 0 \\
\circ & 0 & 0 & 0 & 0
\end{array} \\
& \circ \circ \circ 000 \\
& \circ 0_{0}^{\circ} \circ \text { 。 }
\end{aligned}
$$

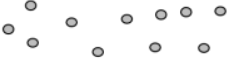

well dispersion well distribution

Figure 2.28 Dispersive and distributive mixing. ${ }^{[107]}$

The plasticization process is not illustrated in Figures 2.26 and 2.28 , but it as well takes place during the whole mixing process. This process involves change of the rheological properties of the rubber compound, such as viscosity reduction.

Besides plasticization, the dispersion of filler agglomerates also affects the viscosity by changing the effective volume fraction of the filler in the rubber compound. As mentioned in Paragraph 2.2.1, the volume fraction of the particles affects the viscosity of the fluid. ${ }^{[37]}$

$$
\varphi_{e}=\varphi+\varphi_{o r}(t)
$$

Where $\varphi_{e}$ is the effective volume fraction of the filler, $\varphi$ is the original volume fraction of the filler, $\varphi_{\text {or }}$ is the volume fraction of the occluded rubber and $t$ is mixing time. ${ }^{[37]}$

As shown in Figure 2.29 (a), at the beginning of mixing, filler clusters are not well dispersed and contain occluded rubber in filler voids. This entire filler cluster acts as a single filler particle and results in a higher effective volume fraction. Ideally, after all filler clusters are fragmented and all occluded rubber is released, $\varphi_{\text {or }}$ equals 0 , as shown in Figure 2.29 (b). The effective filler volume is decreased resulting in a lower viscosity. Accordingly, a poorly dispersed compound has high viscosity. ${ }^{[30,37]}$ 


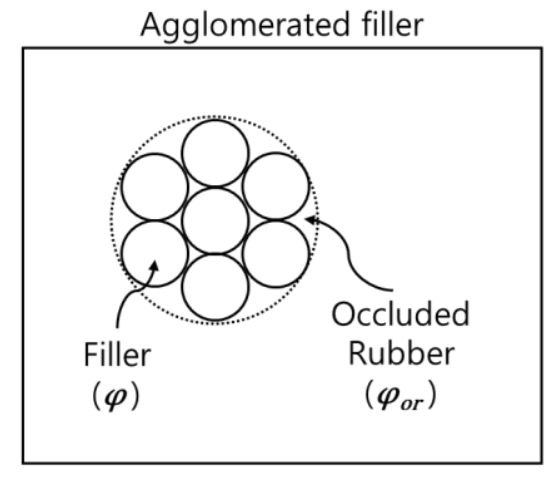

(a)

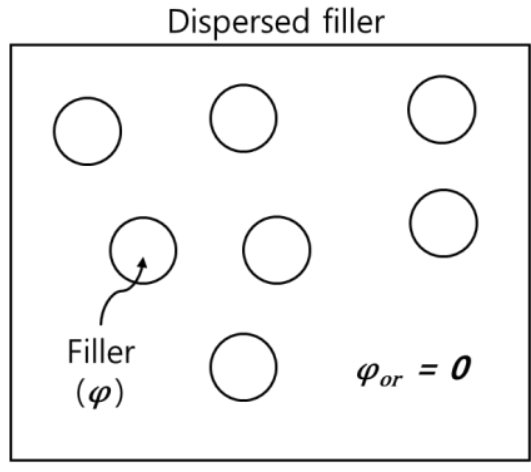

(b)

Figure 2.29 Occluded rubber volume fraction change by dispersion of the filler. ${ }^{[37]}$

\subsubsection{Dispersion morphology of filler in rubber}

It is common sense for rubber engineers that a higher degree of dispersion results in better mechanical properties. Dispersion plays the most important role for the viscoelastic properties of rubber compounds. ${ }^{[41,42]}$ However, there is a lower limit to the cluster size. ${ }^{[22]}$ Within the rubber matrix, the filler should remain in an appropriate form of small clusters in order to meet the demanding applications. Excessively deteriorated filler aggregates do not reinforce the elastomer. ${ }^{[40]}$

For a silica filler, the dynamic equilibrium between the different filler aggregation states during mixing is illustrated in Figure 2.30 .

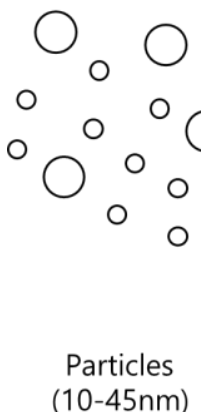

$(10-45 n m)$

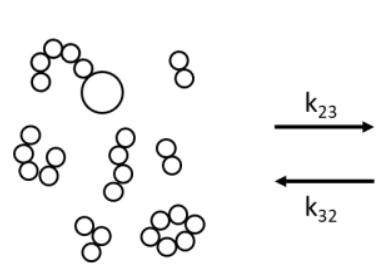

Small clusters (100-500nm)

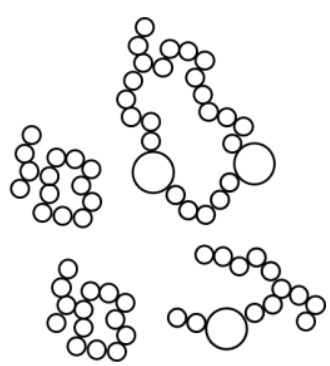

Large clusters $(1-40 \mu \mathrm{m})$

Figure 2.30 Filler aggregation equilibrium model. ${ }^{[96,108]}$

It is well known that mixing a silica compound to obtain a sufficient dispersion status is more difficult compared to a carbon black filled compound. The rate constants $k_{12}$ and $k_{23}$ for aggregation are far bigger than $k_{21}$ and $k_{32}$ for silica compared to carbon black, which means that silica can easily flocculate again. ${ }^{[96,108]}$ 


\subsubsection{Dispersion model}

Two basic mechanisms are proposed in literature to describe the dispersion process. The first mechanism is the agglomerate rupture model: ${ }^{[109]}$ Filler agglomerates cleave into two parts and further reduce their size by excess hydrodynamic forces to overcome the cohesive forces of the particles.

Another dispersion model was proposed by Shiga and Furuta ${ }^{[110]}$ via optical microscope observation: the onion peel model. ${ }^{[111,112]}$ Particularly for carbon black, they revealed that aggregates peel off from the surface of the agglomerates and form tails which consist of a large number of aggregates as illustrated in Figure 2.31. Collin and Peuverel-Diedier ${ }^{[113,114]}$ used a transparent counter-rotating shear cell which was capable of in-situ observation of carbon black dispersion induced by flow, and found two additional carbon black dispersion processes: debonding and collision. But they also observed that erosion and rupture were the main mechanisms of carbon black dispersion. Additionally, they reported that the kinetics of erosion depend on the size of the agglomerates. 


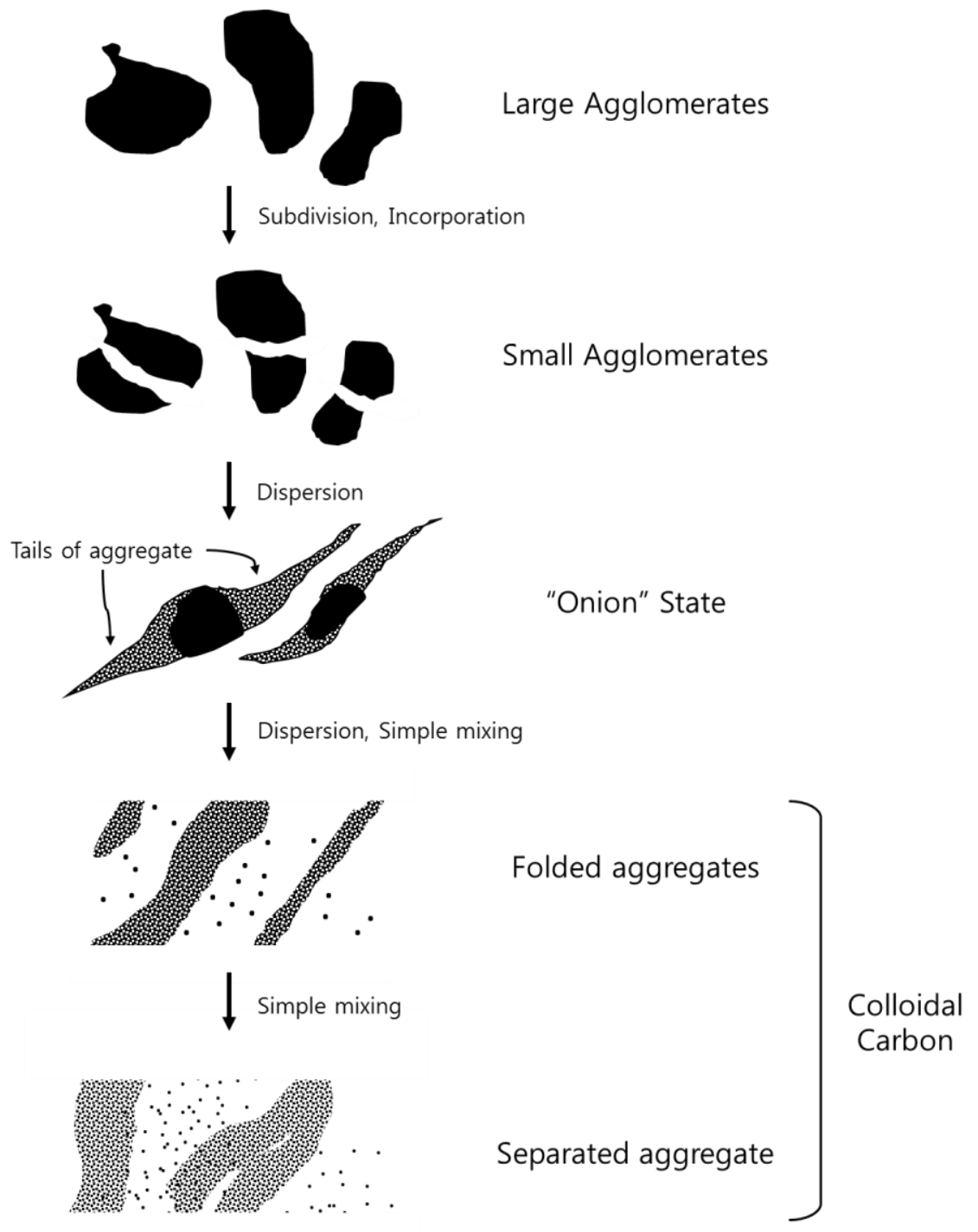

Figure 2.31 Onion peel model. ${ }^{[110]}$

\subsubsection{Mixing profile analysis}

Mixing a filled rubber compound is an energy-consuming process. In order to reach a certain level of rubber mastication, fragmentation of filler agglomerates and distribution of filler clusters require intense shear. ${ }^{[115]}$ For a given mixer, mixing temperature, instant and integrated power consumption, rotor speed, ram pressure and position over mixing time are possible to measure. By recording these parameters, the mixing process can be controlled and analyzed by the fingerprints, so called power curves. ${ }^{[92,107,115]}$ The power profile gives useful information of the four basic physical operations taking place during the mixing cycle: incorporation, dispersion, distribution and plasticization. Especially the instant power profile gives a good view of the different steps of the mixing process. ${ }^{[37,92,104,115,116]}$ Nakajima ${ }^{[116]}$ proposed a 
schematic mixing energy diagram. After dosing of filler, much more energy is consumed than the required amount of energy for rubber mastication as shown in Figure 2.32.

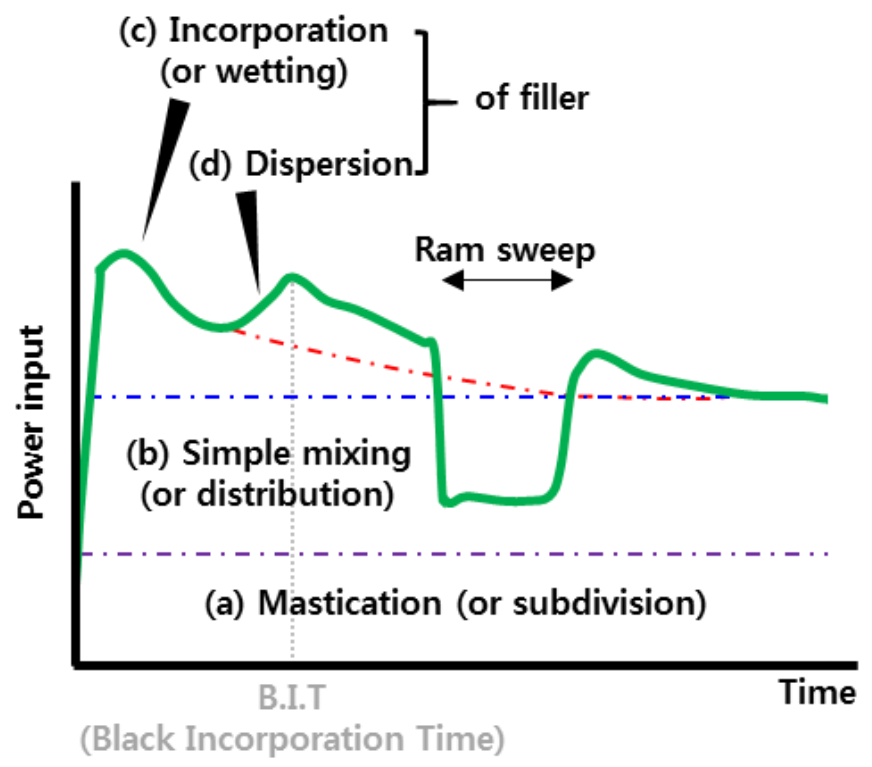

Figure 2.32 Energy absorption of a carbon black filled rubber compound during mixing. ${ }^{[115,116]}$

They explained the four different mixing energy contributions as follows:

- Mastication of rubber (a);

- Deformation-relaxation without rupturing rubber (b);

- Stretching and folding of rubber (c): filler incorporation;

- Breaking of filler agglomerates (d): filler dispersion.

According to his study, the type of charged rubber can affect the portion of (a) and (b), which means longer mixing time or more energy needed to incorporate and disperse the filler. ${ }^{[116]}$ However, the instant power curve gives only limited information. Recently mixers have been equipped with more sensors in order to obtain more knowledge on mixing. Especially the ram position combined with instant power input gives very useful information for determining the fill factor ${ }^{[107]}$ as shown in Figure 2.33 with the corresponding explanations shown in Table 2.5. 


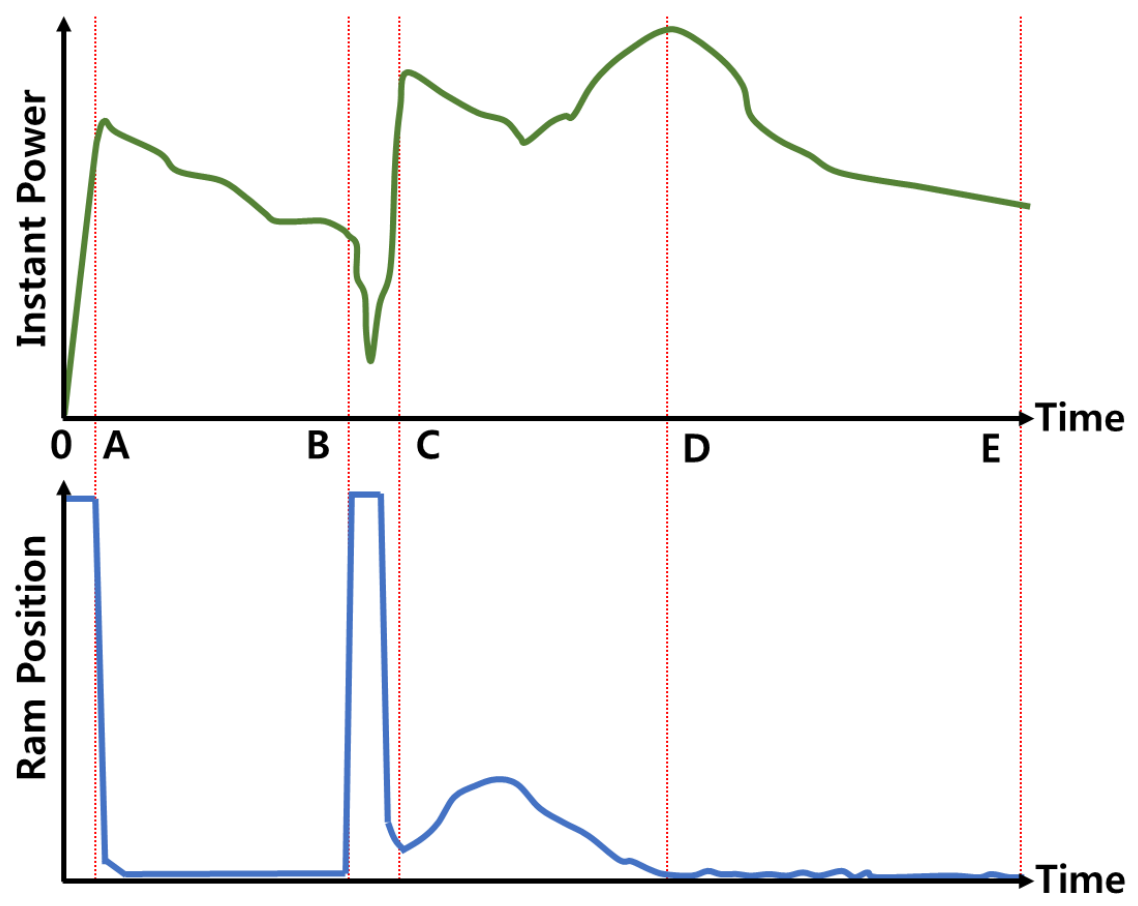

Figure 2.33 Ram position curve corresponding to instant power curve. ${ }^{[107]}$

Table 2.5 Description for each mixing sequence depicted in Figure $2.33^{[107]}$

\begin{tabular}{cc}
\hline Period & Details \\
\hline $0 \sim A$ & Rubber dosing \\
$A \sim B$ & Rubber mastication \\
$B \sim C$ & Filler dosing \\
$C \sim D$ & Black incorporation time (BIT) \\
$D \sim E$ & Homogenization \\
\hline
\end{tabular}

The ram reaches its end position after polymer dosing (A-B). After filler dosing, the ram reaches its lowest position (C) and goes up again. Because of the rotor movement, materials are transported to the area below the ram. Subsequently, the pressure increases until the material is able to lift the ram. After the ram reaches the maximum position, it goes down until its lowest position due to the total volume reduction: Figure 2.27. A longer BIT means an over-filled mixer and a short BIT means under-filled relative to the optimum. ${ }^{[107]}$ 


\subsubsection{Influencing parameters on the mixing process}

\subsubsection{Rotor geometry}

Many mixer manufacturers are developing new rotor designs in order to achieve better rubber mixing. Through the history of the internal mixer development, quality and productivity of the rubber compounding were improved by new rotor designs. ${ }^{[117]}$ Two types of rotors are widely used for tire compound mixing: tangential and intermeshing. Those rotors can be easily characterized by the working path of the rotor tip. ${ }^{[107]}$

\subsection{Tangential rotors}

Tangential rotors and the working path of the rotors are illustrated in Figure 2.34. From its name and as can be seen in Figure 2.34 (c), the rotor tips of the tangential mixer do not share their working path.

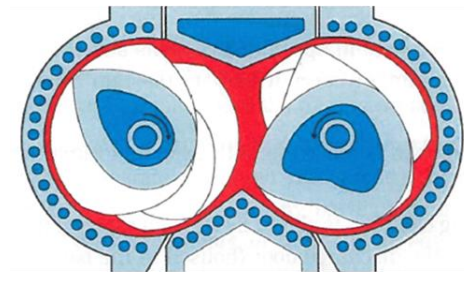

(a) Cross sectional view

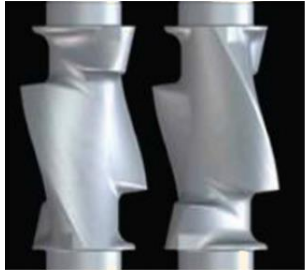

(b) Front view

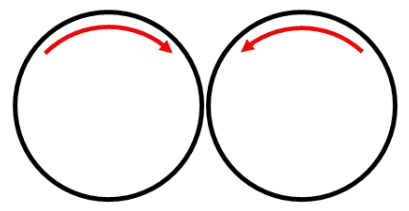

(c) Working path of the tangential rotor tips

Figure 2.34 Illustration of tangential rotor geometry. ${ }^{[107,118-120]}$

According to this characteristic, independent speed control of each rotor is possible. In practice, one rotor rotates $10 \%$ faster than the other: the friction ratio. However, previous experience has shown that even speed mixing improves productivity and reduces mixing energy. ${ }^{[107,121]}$ In case of even speed mixing, various rotor-to-rotor orientations are possible as shown in Figure 2.35. ${ }^{[119,121]}$

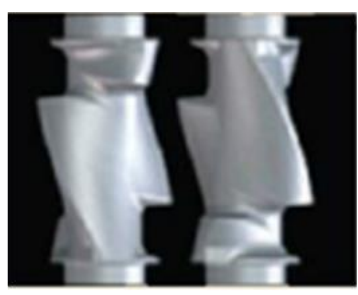

(a) $0-0$ orientation

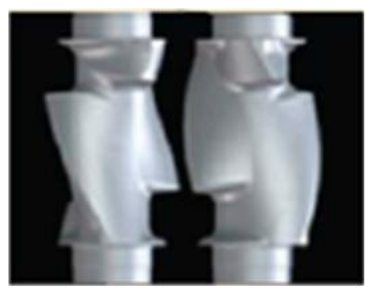

(b) $0-180$ orientation

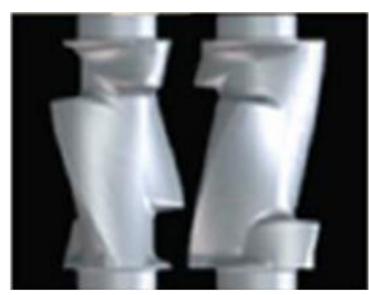

(c) $0-90$ orientation

Figure 2.35 The orientations of the tangential rotor. ${ }^{[119]}$ 
These different orientations of the rotors can result in enhanced distribution, dispersion and intake speed of the compounding ingredients. [119] Based on productivity and quality of the compounds, Donoian et al. ${ }^{[121]}$ reported that the optimum mixer performance can be obtained by $0-180$ orientation of the rotors.

The tangential rotors are designed to provide high shear mixing between the rotor tips and the chamber wall: no dispersive mixing can occur between the two rotors. There is a lot of empty space between the rotors which leads to a high fill factor. The actual useful volume is known as the range from $60 \%$ to $70 \%$. ${ }^{[107]}$

\subsection{Intermeshing rotors}

Intermeshing rotor and the working path of the rotors are illustrated in Figure 2.36. From its name and as can be seen in Figure 2.36 (c), the rotor tips of the intermeshing mixer share their working path. The rotors of the intermeshing mixer should rotate with even speed. Dispersive mixing occurs between the rotor tips and the chamber wall as well as between the two rotors, which leads to an additional mixing effect. ${ }^{[107,118]}$

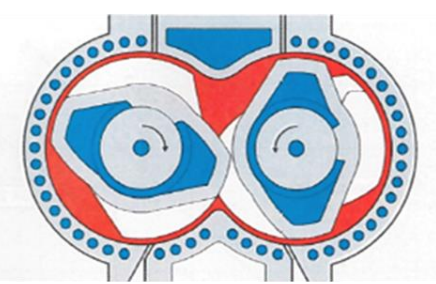

(a) Cross sectional view

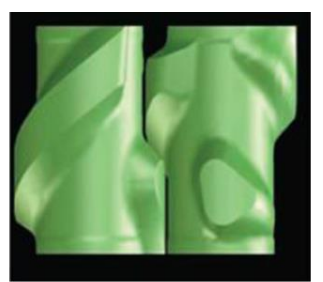

(b) Front view

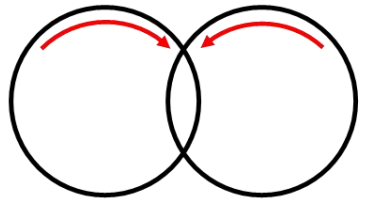

(c) Working path of the intermeshing rotor tip

Figure 2.36 Illustration of intermeshing rotor geometry. ${ }^{[107,118-120]}$

The intermeshing rotors are designed to provide effective shearing, enhanced cooling and aggressive movement of the material within the mixing chamber. ${ }^{[107]}$ The useful fill factor of the intermeshing mixer is approx. $5 \%$ lower than for a tangential mixer because of the small space between the rotors. ${ }^{[107,118]}$

\subsection{Technical comparison: tangential / intermeshing rotors}

The strength of the tangential mixer are good feeding and productivity. In contrast, the intermeshing mixer primarily offers better processability and quality of the compounds. ${ }^{[107,118]}$ The better mixing quality of intermeshing rotors can be explained by the additional mixing effect between the rotors. ${ }^{[107,118,119,122,123]}$ The relative mixing performances of the tangential vs. intermeshing rotors are compared as shown in Figure 2.37. 


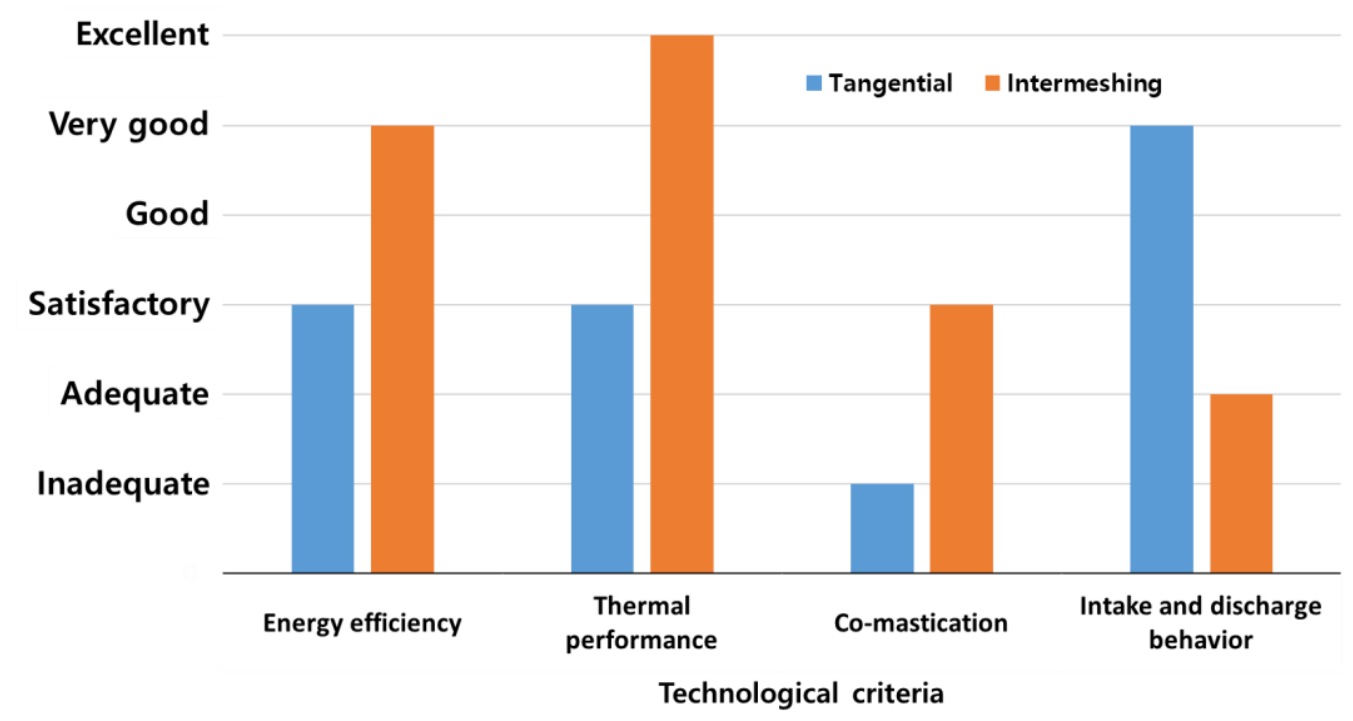

Figure 2.37 Technical comparison of tangential and intermeshing rotors. ${ }^{[107,118]}$

Kim and White ${ }^{[122]}$ have compared the effect of tangential and intermeshing rotors on the homogenization of rubber and filler, as well as an oil incorporation. They used a glass window equipped lab mixer and found that intermeshing rotors are more effective concerning these properties. Koolhiran and White ${ }^{[123]}$ reported that intermeshing rotors are more effective in distributive and dispersive mixing and filler incorporation. Within their study, smaller cluster sizes of the fillers and shorter filler incorporation times were observed when an intermeshing rotor was used. Additionally, the intermeshing rotors showed a lower viscosity of the compound than tangential rotors.

Another important technical advantage of the intermeshing rotors is the cooling efficiency and the temperature controllability. ${ }^{[107,118,119]}$ As can be seen in Figure 2.38 , intermeshing mixers have a larger surface area at the same empty volume of the mixer. 


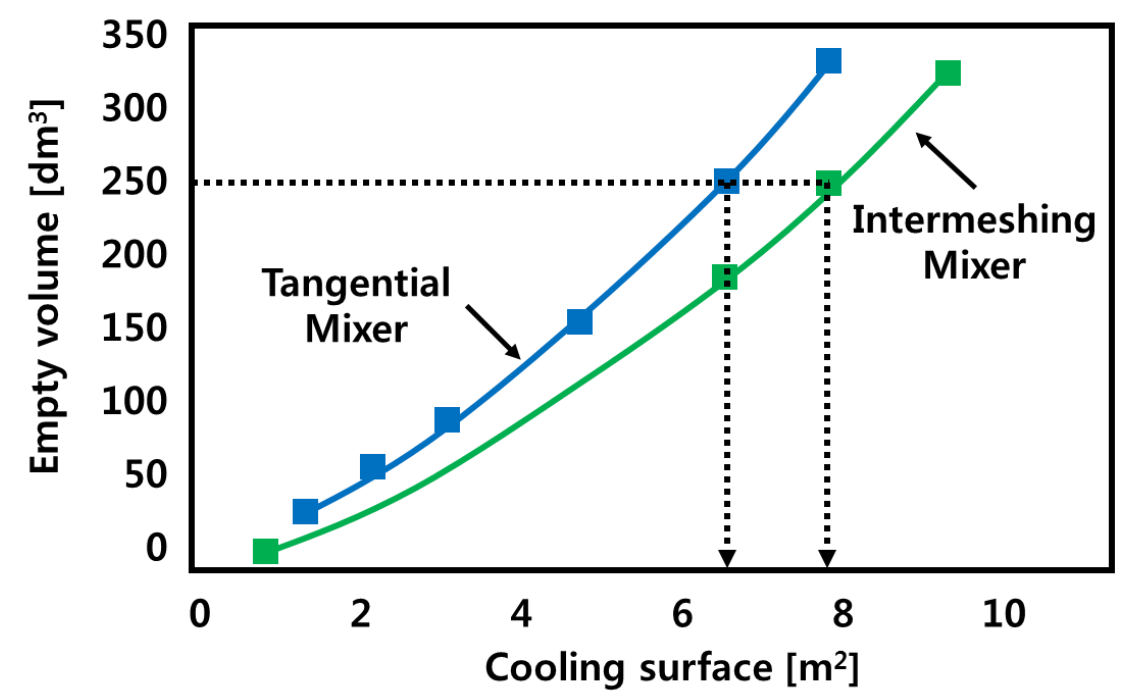

Figure 2.38 Empty volume vs. cooling surface of tangential and intermeshing mixers. ${ }^{[107,118]}$

The cooling performance of the internal mixer has become a crucial factor since silica was introduced as reinforcing filler. ${ }^{[107,118]}$ The mixer acts as a chemical reactor because the mixing of silica filled rubber involves a chemical reaction during the process. Therefore, better cooling and accurate temperature control is required. With the large cooling surface, intermeshing rotors have a better cooling efficiency and temperature controllability than tangential mixers. Dierkes ${ }^{[92]}$ compared the silanization efficiency of tangential and intermeshing rotors and found that intermeshing rotors give a better silanization efficiency with low energy consumption. Additionally, Dierkes and Noordermeer ${ }^{[124]}$ reported that intermeshing rotors can reduce the risk of pre-scorch because of their effective cooling performance.

\subsubsection{Fill factor}

The fill factor strongly affects the compound quality as well. ${ }^{[74,96,107,125]}$ Distributive and dispersive mixing is strongly related to the flow path (FP) of the material within the mixing chamber, and those paths are strongly affected by the fill factor. ${ }^{[107]}$ Figure 2.39 shows the material flow paths along the rotors within the mixing chamber. 


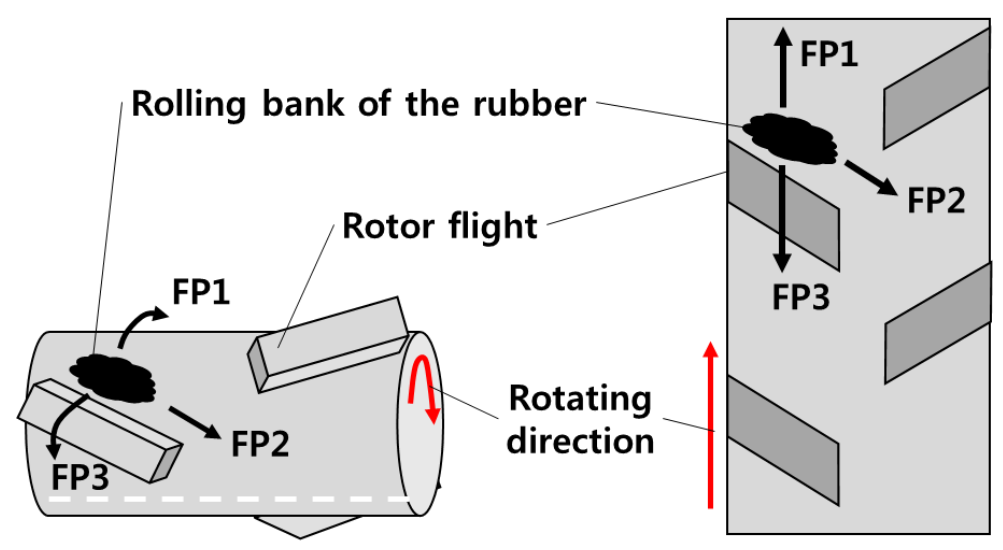

(a)

(b)

Figure 2.39 Flow path (FP) of the material along the rotor;

(a) in $3 \mathrm{D}^{[107]}$; (b) in 2D (cut along the dashed white line and unrolled rotor).

If the mixer is under-filled, the material tends to flow along the FP2 which results in mainly distributive mixing whereas dispersive mixing is poor. With increasing fill factor, the pressure along FP1 increases and blocks FP2. Consequently, a relatively high amount of material flows through the narrow gap between rotor tip and chamber wall (FP3) which results in intensive dispersive mixing. ${ }^{[107]}$ Over-filling of the mixing chamber also leads to poor dispersion quality. ${ }^{[92,107,125]}$ More material is captured in dead spots of the mixer and enters in the mixing process with delay. ${ }^{[92,107]}$ As a consequence, a better quality of the compound can be obtained with the optimal fill factor.

Beside dispersion quality, the fill factor influences the reaction efficiency of silica compounds. It is well known that the silanization reaction is an equilibrium reaction, thus removal of the by-product ethanol of the reaction is important for the silica compound quality. ${ }^{[124]}$ Dierkes ${ }^{\left[{ }^{[2]}\right.}$ reported that the fill factor is related to the fresh surface generation frequency of the compounds during mixing. Frequent generation of fresh surface will lead to easy release of ethanol and results in a better silanization reaction efficiency. ${ }^{[92]}$

However, estimation of the optimal fill factor has always been a problem for inexperienced mixing process engineers, because defining the optimal fill factor is typically based on experience and observation. ${ }^{[74]} \mathrm{A}$ lot of considerations should be take into account to define the optimum fill factor. Those are machine type, rotor geometry, compound formulation, viscosity and operating parameters. ${ }^{[74,92,119,126]}$ As can be seen in Figure 2.40, the optimal fill factor varies with rotor speed, ram pressure and rotor geometry. 


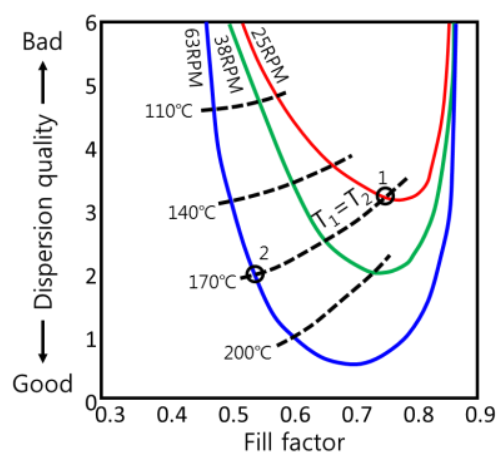

(a)

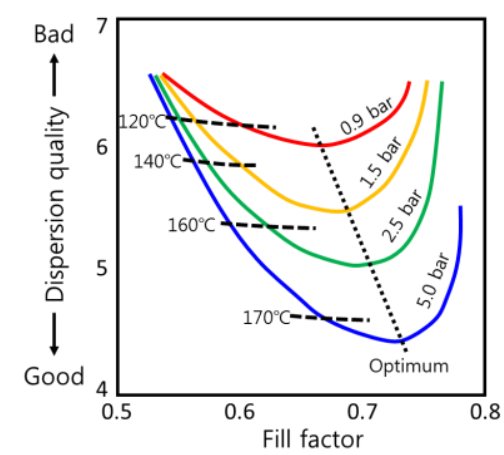

(b)

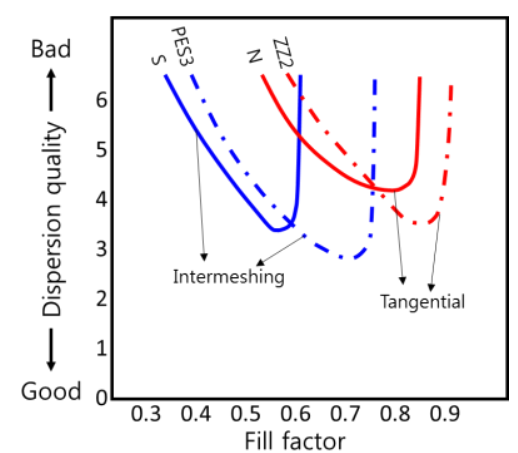

(c)

Figure 2.40 Optimum fill factors according to: (a) rotor speed; (b) ram pressure; (c) rotor geometry. ${ }^{\text {[126] }}$

\subsubsection{Mixing temperature control}

Especially in the field of silica compound mixing, temperature control is crucial because the silanization is a chemical reaction. Consistency of the compound properties improves with accurate mixing temperature control. ${ }^{[92,124,127]}$ Additionally, the efficiency of silanization is strongly affected by the temperature. ${ }^{[124]}$ The primary reaction of silanization as shown in Figure 2.23 (a), starts at a temperature around $130^{\circ} \mathrm{C}^{[92]}$, and the rate of silanization increases with increasing silanization temperature. ${ }^{[92,94]}$ Figure 2.41 shows the effect of temperature on silanization, represented by the Payne effect. As can be seen, the Payne effect decreases with increasing silanization temperature. This result indicates that the silanization efficiency improves with increasing temperature. ${ }^{[124]}$

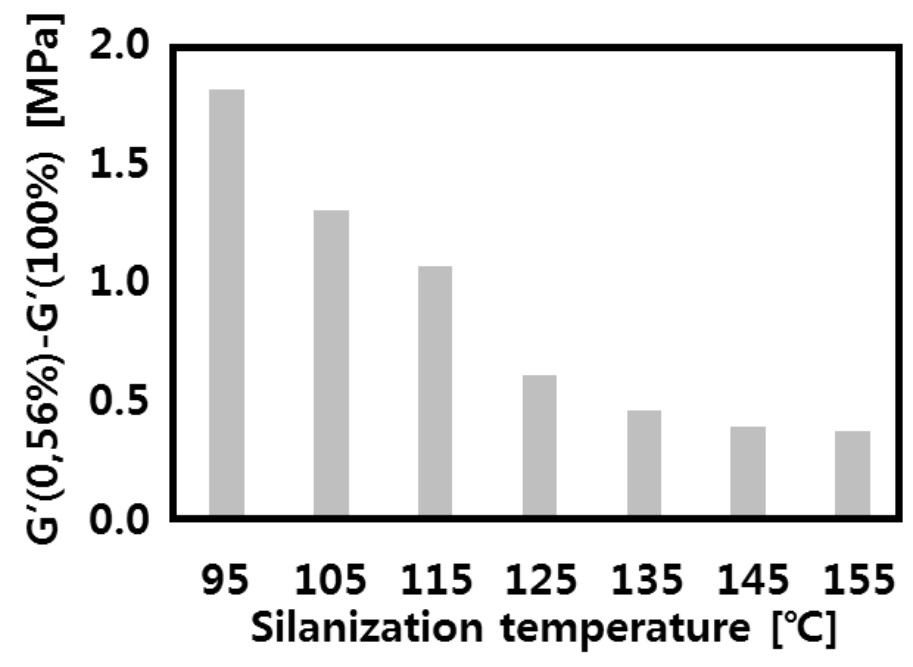

Figure 2.41 Temperature effect on silanization. ${ }^{[124]}$ 
However, an increasing temperature does not always result in good quality of a silica filled rubber compound. In case of TESPT as coupling agent, Reuvekamp et al. ${ }^{[127]}$ found that the storage modulus at $100 \%$ strain, which represents the rubber network effect, starts to increase at a temperature around $150^{\circ} \mathrm{C}$ : Figure 2.42.

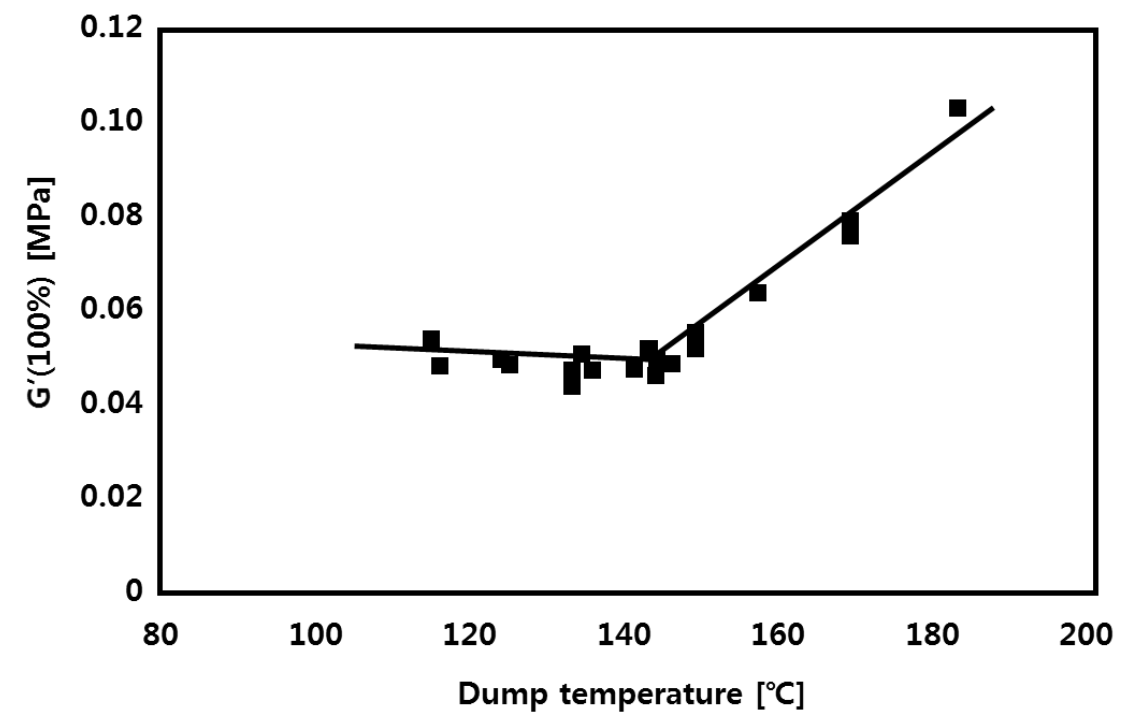

Figure 2.42 Temperature effect on $\mathrm{G}^{\prime}$ at $100 \%$ strain of a silica-silane (TESPT) compound. ${ }^{[27]}$

This is an indication of pre-scorch, which is caused by the reaction between TESPT and the rubber matrix ${ }^{[127]}$ or the formation of crosslinks by sulfur which is donated from TESPT. ${ }^{[128]}$ Consequently, higher mixing temperatures lead to pre-scorch. ${ }^{[54,89,127]}$ Depending on the thermal stability of the silane coupling agent, the maximum temperature can differ as explained in Paragraph 2.4.1 and Figure 2.21.

\subsubsection{Thermal boundaries and Temperature Control Unit (TCU) of the mixer}

The thermal boundary conditions of the mixer affect the mixing behavior and consequently influence the compound quality. ${ }^{[107]}$ As can be seen in Figures 2.43 and 2.44, the thermal boundary conditions strongly influence the polymer intake behavior, which is represented by ram seating time and the filler incorporation time. 


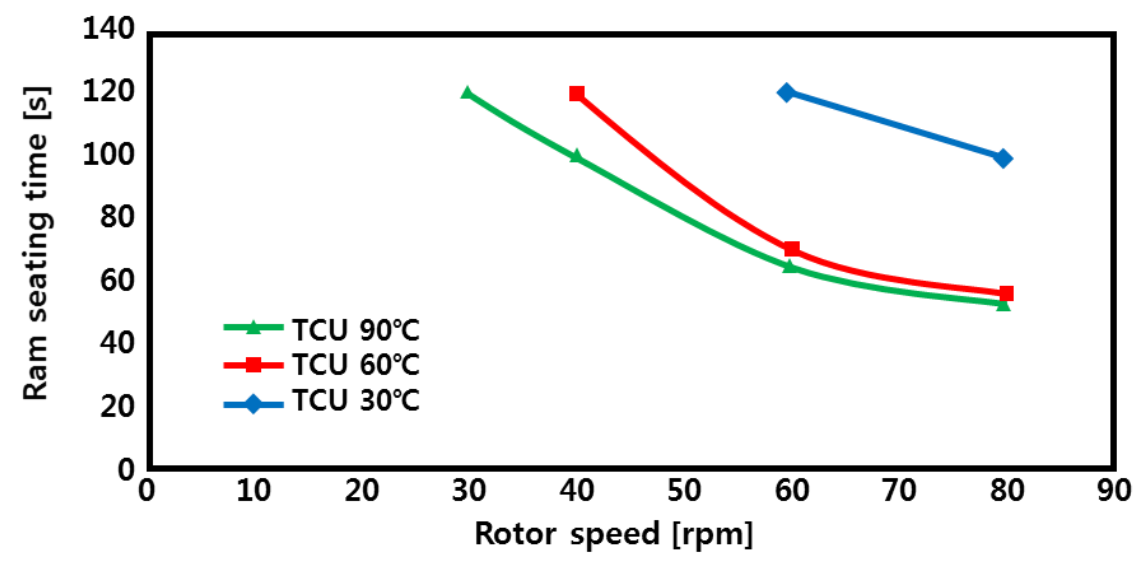

Figure 2.43 Change of ram seating time after polymer addition by different TCU settings. ${ }^{[107]}$

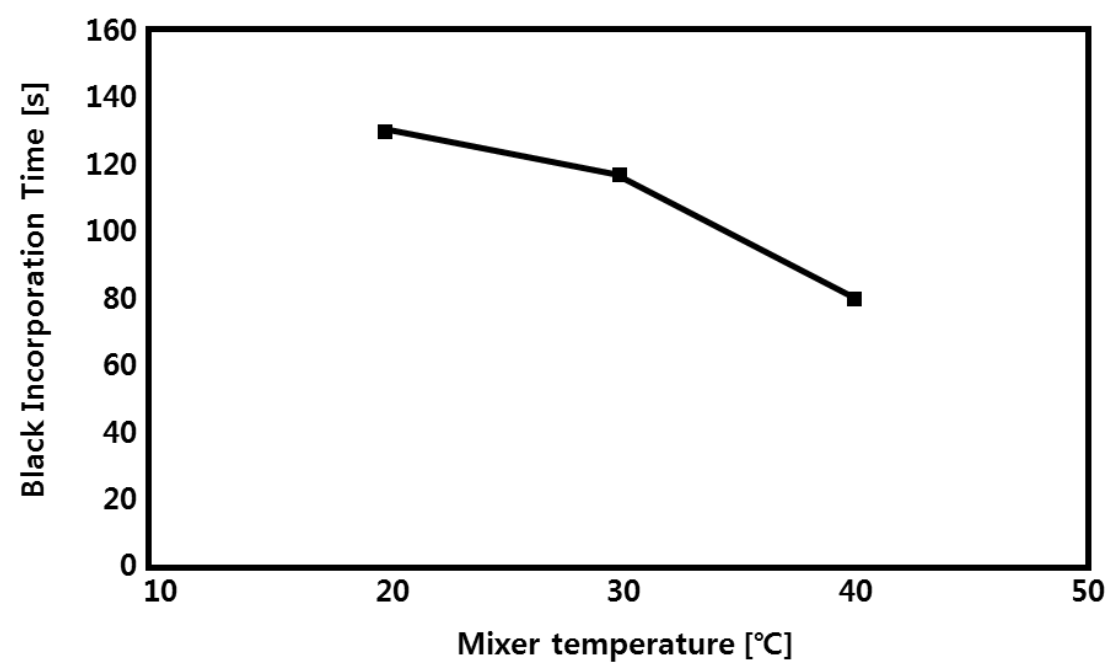

Figure 2.44 Black incorporation time variation of an EPDM compound mixed in a $45 \mathrm{~L}$ intermeshing mixer with different TCU settings. ${ }^{[107]}$

Additionally, the mechanical dispersive mixing can be affected by the viscosity of the compound: a higher viscosity of the compounds leads to better dispersive mixing. ${ }^{[54]}$ The viscosity $\mu$ of the compound depends on temperature $T$ as shown by the following equation: ${ }^{[129]}$

$$
\mu=A e^{E / R T}
$$

Where $R$ is the gas constant, $A$ is a coefficient dependent on the fluid, $E$ is an activation energy and T is the absolute temperature. 
Dierkes ${ }^{[91]}$ reported that the TCU temperature of the mixer also affects the silanization efficiency as shown in Figure 2.45. A lower TCU temperature results in a better silanization efficiency as represented by the Payne effect.

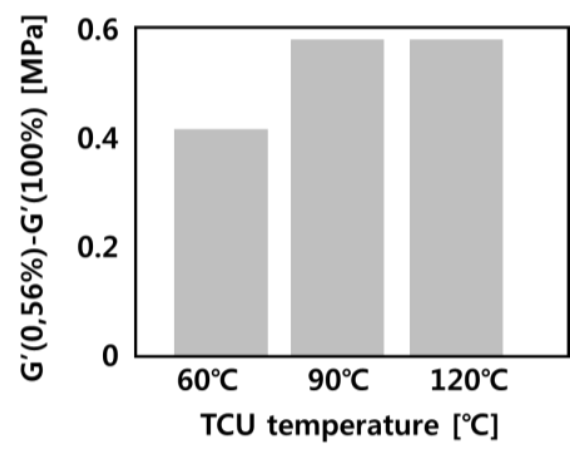

Figure 2.45 Effect of the mixer TCU temperature on the silanization efficiency as measured by the Payne effect. ${ }^{[91]}$

\subsubsection{Rotor speed}

The level of shear stress and mixing time strongly influences the filler dispersion quality: Figure 2.46. The shear stress applied to the compound is related to the rotor speed. ${ }^{[107]} \mathrm{A}$ higher rotor speed provides a higher level of shearing forces. However, a too high rotor speed induces a rapid increase of mixing temperature and may cause an unexpected processing problem: for example, pre-scorch of a silica-silane rubber compound. ${ }^{[127]}$ Therefore, a suitable rotor speed is required in order to achieve a good filler dispersion and processability within an adequate mixing time.

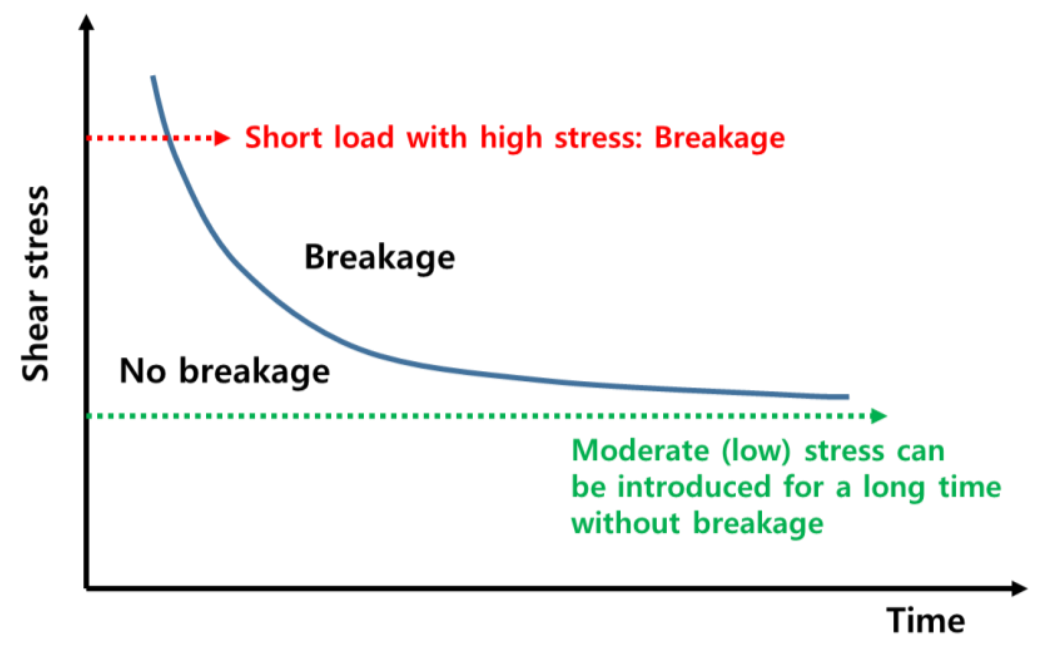

Figure 2.46 Filler particle size reduction as a function of applied shear force and time. ${ }^{[107]}$ 


\subsection{Motivation of the present project to avoid marching modulus}

It is already described in literature that the curing behavior of silica filled rubber compounds is affected by the mixing conditions ${ }^{[46,82]}$ and compound characteristics ${ }^{[33,130]}$, which are related to silica dispersion, the degree of silanization and the filler-polymer coupling reaction (Figure 2.47). This implies that these factors are the keys to reduce and finally avoid marching modulus.

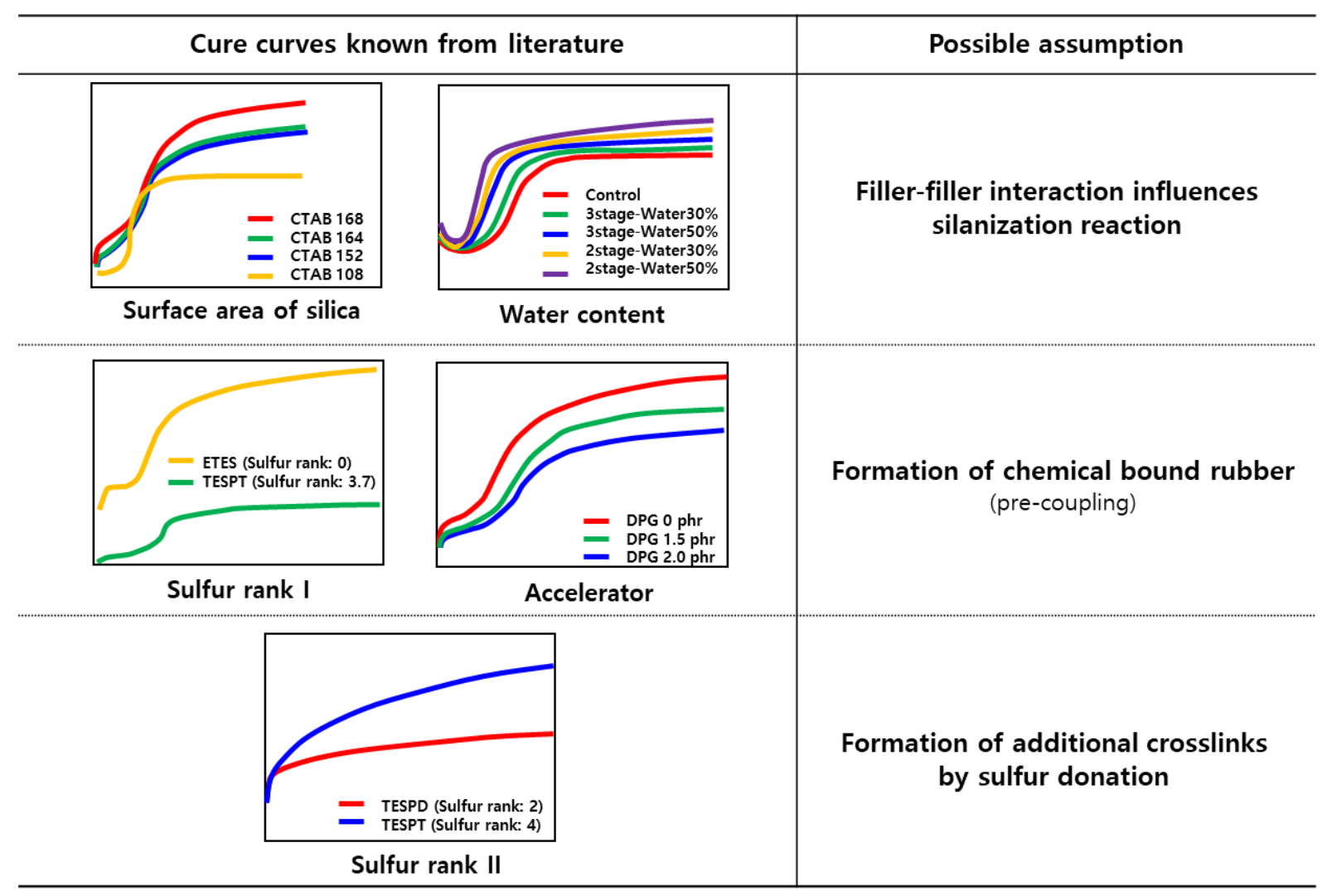

Figure 2.47 Influencing factors for marching modulus. ${ }^{[33,46,82,130]}$

The focus of this thesis is to provide further understanding of the origin of marching modulus of silicafilled rubber compounds. Mixing parameters and formulation characteristics, which are possible parameters for minimizing the marching modulus, will be explored.

Finally, based on these experiments, guidelines for a tailored mixing process and compound design will be provided in order to obtain a consistent quality of the compounds, enhanced productivity and better processability. 


\section{References}

[1] K. A. Grosch, Rubber Chem. Technol. 69, 495 (1996).

[2] D. E. Hall, J. C. Moreland, Rubber Chem. Technol. 74, 525 (2001).

[3] A. H. Tullo, Chem. Eng. News 87, 10 (2009).

[4] "Viscoelastic properties of polymer, 2nd edition", J. D. Ferry, Wiley-Interscience, New York (1970).

[5] "Polymer science \& technology, 2nd Edition", J. R. Fried, Prentice Hall Professional Technical Reference, Upper Saddle River (2003).

[6] R. R. Rahalkar, Rubber Chem. Technol. 62 (1989).

[7] "Deformation and fracture of high polymers", H. H. Kausch, J. A. Hassell, R. I. Jaffee, Springer, Boston (1973).

[8] "Science and technology of rubber", F. R. Eirich, Academic Press, New York (1978).

[9] "Rheology : Principle, Measurements and Applications", C. W. Macosko, VCH Publishers. Inc., New York (1994).

[10] S. Maghami, PhD Thesis: Silica-filled tire tread compounds, Univ. Twente, Enschede, the Netherlands (2016).

[11] Y. Saito, Kautsch. Gummi Kunstst. 38, 178 (1985).

[12] B. Duperray, J. L. Leblanc, Kautsch. Gummi Kunstst. 35, 298 (1982).

[13] H. H. Kausch, J. A. Hassell, R. I. Jaffee, Science 181, 961 (1973).

[14] M. J. Wang, Rubber Chem. Technol. 71, 520 (1998).

[15] K. H. Nordsiek, Kautsch. Gummi Kunstst. 38, 178 (1985).

[16] "Reinforcement of elastomers", G. Kraus, Ed. Interscience, New York (1965).

[17] D. W. Schaefer, T. Rieker, M. Agamalian, J. S. Lin, D. Fischer, S. Sukumaran, C. Chen, G. Beaucage, C. Herde, J. Iviee, J. Appl. Cryst. 33, 587 (2000).

[18] G. B. Ouyang, Kautsch. Gummi Kunstst. 59, 332 (2006).

[19] L. Bokobza, Macromol. Mater. Eng. 289, 607 (2004).

[20] J. Fröhlich, W. Niedermeier, H. D. Luginsland, Composites Part A 36, 449 (2005).

[21] A. Einstein, Ann. der Physik 19, 289 (1906).

[22] A. Einstein, Ann. der Physik 34, 591 (1911).

[23] E. Guth, O. Gold, Phys. Rev. 53, 322 (1938).

[24] E. Guth, J. Appl. Phys. 16, 20 (1945).

[25] H. M. Smallwood, J. Appl. Phys. 15, 758 (1944).

[26] G. Kraus, Adv. Polym. Sci. 8, 155 (1971). 
[27] E. M. Dannenberg, Rubber Chem. Technol. 48, 410 (1975).

[28] L. R. G. Treloar, Rep. Prog. Phys. 36, 755 (1973).

[29] "Science and technology of rubber 3rd edition", J. Mark, B. Erman, Academic Press, San Diego (2005).

[30] A. I. Medalia, Rubber Chem. Technol. 45, 1171 (1972).

[31] S. Wolff, M. J. Wang, E. H. Tan, Rubber Chem. Technol. 66, 163 (1993).

[32] M. J. Wang, S. Wolff, J. B. Donnet, Rubber Chem. Technol. 64, 714 (1991).

[33] J. Fröhlich, D. Luginsland, W. Nierdermeier, 157th meeting ACS Rub. Div., Dallas, Texas, Paper No. 9 (2000).

[34] P.P.A. Smit, Rubber Chem. Technol., 41, 1194 (1968).

[35] A. I. Medalia, J. Coll. Interf. Sci. 35, 115 (1970).

[36] G. Kraus, Rubber Chem. Technol. 44, 199 (1971).

[37] I. Pliskin, N. Tokita, J. Appl. Sci., 16, 473 (1972).

[38] J. O’Brien, E. Cashell, G. E. Wardell, V. J. McBrierty, Macromol. 9, 653 (1976).

[39] J. L. Leblanc, J. Appl. Polym. Sci. 78, 1541 (2000).

[40] J. L. Leblanc, Kautsch. Gummi Kunstst. 54, 327 (2001).

[41] J. L. Leblanc, Prog. Polym. Sci. 27, 627 (2002).

[42] L. L. Ban, W. M. Hess, and L. A. Papazian, Rubber Chem. Technol. 47, 858 (1974).

[43] Y. Fukahori, Rubber Chem. Technol. 76, 548 (2003).

[44] A. Kato, M. Nishioka, Y. Sato, E. Nagano, R. Yamashita, N. Kimura, T. Taguchi, Rubber Chem. Technol. 87, 471 (2014).

[45] M. P. Wagner, Rubber Chem. Technol. 49, 703 (1976).

[46] S. Mihara, PhD Thesis: Reactive processing of silica-reinforced tire rubber: new insight into the time- and temperature-dependence of silica rubber interaction, Univ. Twente, Enschede, the Netherlands (2009).

[47] L. L. Qu, G. Z. Yu, X. M. Xie, L. L. Wang, J. Li, Q. S. Zhao, Polym. Compos. 34, 1575 (2013).

[48] H. D. Luginsland, J. Fröhlich, A. Wehmeier, Rubber Chem. Technol. 75, 563 (2002).

[49] S. S. Choi, E. Ko, Polym. Test. 40, 170 (2014).

[50] J. M. Funt, Rubber Chem. Technol. 61, 842 (1988).

[51] A. R. Payne, R. E. Whittaker, Rubber Chem. Technol. 44, 440 (1971).

[52] A. R. Payne, J. Appl. Polym. Sci. 9, 2273 (1965).

[53] A. R. Payne, Rubber Chem. Technol. 39, 365 (1966). 
[54] "Rubber Compounding; Chemistry and Applications, 2nd Edition", B. Rodgers, CRC press, Boca Raton (2016).

[55] G. Kraus, J. Appl. Polym. Sci.: Appl. Polym. Symp. 39, 75 (1984).

[56] P. G. Maier, D. Göritz, Kautsch. Gummi Kunstst. 49, 18 (1996).

[57] G. Huber, T. A. Vilgis, Kautsch. Gummi Kunstst. 52, 102 (1999).

[58] G. Heinrich, M. Klüppel, Adv. Polym. Sci. 160, 1 (2002).

[59] "Reinforcement of Polymer Nano-Composites: Theory, Experiments and Applications", T. A. Vilgis, G. Heinrich, M. Klüppel, Cambridge University Press, Cambridge (2009).

[60] K. K. Jana, M. Patel, D. Rana, P. Maiti, Adv. Polym. Sci. 264, 15 (2014).

[61] "Rheology and Processing of Polymer Nanocomposites", S. Thomas, R. Muller, J. Abraham, John Wiley \& Sons, Inc., New Jersey (2016).

[62] M. Klüppel, G. Heinrich, Rubber Chem. Technol. 68, 623 (1995).

[63] A. Blume, S. Uhrlandt, 157th meeting ACS Rub. Div., Dallas, Texas, Paper No. 32 (2000).

[64] "Compounding Precipitated Silica in Elastomers: Theory and Practice", N. Hewitt, William Andrew Inc., Norwich (2007).

[65] T. A. Okel, 167th meeting ACS Rub. Div., San Antonio, Texas, Paper No. C (2005).

[66] "Rubber technologist's handbook", J. R. White, S. K. De, Rapra Technology Ltd., Shawbury (2001).

[67] A. Voet, A, J. C. Morawski, J. B. Donnet, Rubber Chem. Technol. 50, 342 (1977).

[68] A. Blume, Kautsch. Gummi Kunstst. 53, 338 (2000).

[69] ISO4556:2012, "Rubber compounding ingredients - carbon black - determination of oil absorption number (OAN) and oil absorption number of compressed sample (COAN)“, International Organization for Standardization (2012).

[70] A. Blume, M. Janik, J. P. Gallas, F. Thibault-Starzyk, A. Vimont, Kautsch. Gummi Kunstst. 61, 359 (2008).

[71] M. J. Wang, S. Wolff, J. B. Donnet, Rubber Chem. Technol. 64, 559 (1991).

[72] A. Hunsche, U. Görl, A. Müller, M. Knaack, T. Göbel, Kautsch. Gummi Kunstst. 50, 881 (1997).

[73] U. Goerl, A. Hunsche, A. Mueller, H. G. Koban, Rubber Chem. Technol. 70, 608 (1997).

[74] "The Mixing of Rubber", R. F. Grossman, Champman \& Hall, New York (1997).

[75] G. Heinrich, T. A. Vilgis, Kautsch. Gummi Kunstst. 61, 368 (2008).

[76] S. Maghami, W. K. Dierkes, J. W. M. Noordermeer, Rubber Chem. Technol. 89, 559 (2016).

[77] A. Blume, F. Grunert, A. Wehmeier, W. K. Dierkes, Kautsch. Gummi Kunstst. 71, 70 (2018).

[78] F. Grunert, A. Wehmeier, W. K. Dierkes, A. Blume, Kautsch. Gummi Kunstst. 72, 25 (2019). 
[79] C. Hayichelaeh, L. A. E. M. Reuvekamp, W. K. Dierkes, A. Blume, J. W. M. Noordermeer, K. Sahakaro, Rubber Chem. Technol. 91, 433 (2018).

[80] C. Lin. Jr., W. L. Hergenrother, A. S. Hilton, Rubber Chem. Technol. 75, 215 (2002).

[81] S. Schaal, A. Y. Coran, S. K. Mowdood, Rubber Chem. Technol. 73, 240 (2000).

[82] K. J. Kim, J. VanderKooi, Rubber Chem. Technol. 78, 84 (2005).

[83] B. Ahn, N. Park, D. Kim, W. Kim, Rubber Chem. Technol. 92, 364 (2019).

[84] M. V. Braum, M. A. M. Jacobi, Rubber Chem. Technol. 92, 431 (2019).

[85] P. Manoharan, M. G. Anagha, N. C. Das, K. Naskar, Rubber Chem. Technol. 91, 548 (2018).

[86] “Silane coupling agent", E. P. Plueddemann, Plenum Press, New York (1982).

[87] F. Vilmin, I. Bottero, A. Travert, N. Malicki, F. Gaboriaud, A. Trivella, F. Thibault-Starzyk, J. Phys. Chem. C 118, 4056 (2014).

[88] O. Klockmann, A. Hasse, Kautsch. Gummi Kunstst. 60, 82 (2007).

[89] H. D. Luginsland, A. Hasse, 157th meeting ACS Rub. Div., Dallas, Texas, Paper No. 34, (2000).

[90] R. W. Cruise, M. H. Hofstetter, L. M. Panzer, R. J. Pickwell, 150th meeting ACS Rub. Div., Louisville, Kentucky, Paper No. 75 (1996).

[91] A. Hasse, O. Klockmann, A. Wehmeier, H. D. Luginsland, Kautsch. Gummi Kunstst. 55, 236 (2002).

[92] W. K. Dierkes, PhD Thesis: Economic mixing of silica-rubber compounds, Univ. Twente, Enschede, the Netherlands (2005).

[93] A. Blume, J. Jin, A. Mahtabani, X. He, S. Kim, Z. Andrzejewska, International Rubber Conference 2019 (IRC2019), London, United Kingdom (2019).

[94] U. Görl, A. Hunsche, 150th meeting ACS Rub. Div., Louisville, Kentucky, Paper No. 76 (1996).

[95] U. Görl, J. Münzenberg, D. Luginsland, A. Müller, M. Wolfgang, Kautsch. Gummi Kunstst. 52, 588 (1999).

[96] S. Mihara, R. N. Datta, J. W. M. Noordermeer, Rubber Chem. Technol. 82, 524 (2009).

[97] W. Kaewsakul, K. Sahakaro, W. K. Dierkes, J. W. M. Noordermeer, Rubber Chem. Technol. 85, 277 (2012).

[98] W. Kaewsakul, K. Sahakaro, W. K. Dierkes, J. W. M. Noordermeer, Rubber Chem. Technol. 87, 291 (2014).

[99] H. D. Luginsland, Kautsch. Gummi Kunstst. 53, 10 (2000).

[100] B. A. Dogadkin, D. L. Fedyukin, V. E. Gul, Rubber Chem. Technol. 31, 756 (1958).

[101] A. J. Marzocca, S. Cerveny, J. M. Méndez, Polym. Int. 49, 216 (2000). 
[102] M. Sato, PhD Thesis: Reinforcing Mechanisms of Silica / Sulfide-Silane vs. Mercapto-Silane Filled Tire Tread Compounds, Univ. Twente, Enschede, the Netherlands (2018).

[103] P. van Raepenbusch, R. Pessina, D. Nichetti, 157th meeting ACS Rub. Div., Dallas, Texas, Paper No. 14 (2000).

[104] H. Palmgren, Rubber Chem. Technol. 48, 462 (1975).

[105] N. Nakajima, Rubber Chem. Technol. 55, 931 (1982).

[106] B. B. Boonstra, J. Appl. Polym. Sci. 11, 389 (1967).

[107] "Mixing of rubber compounds", A. Limper, Carl Hanser Verlag, Munich (2012).

[108] Y. Li, M. J. Wang, T. Zhang, F. Zhang, X. Fu, Rubber Chem. Technol. 67, 693 (1994).

[109] I. Manas-Zloczower, A. Nir, Z. Tadmor, Rubber Chem. Technol. 55, 1250 (1982).

[110] S. Shiga, M. Furuta, Nippon Gomu Kyokaishi 55, 491 (1982).

[111] H. H. Le, M. Tiwari, S. Ilisch, H. J. Radusch, Kautsch. Gummi Kunstst. 58,575 (2005).

[112] N. Nakajima, E. R. Harrell, Rubber Chem. Technol. 57, 153 (1984).

[113] V. Collin, E. Peuvrel-Disdier, Elastomery 9, 9 (2005).

[114] V. Collin, I. Boudimbou, E. Peuvrel-Disdier, J. Appl. Polym. Sci. 127, 2121 (2013).

[115] J. L. Leblanc, Prog. Trends Rheol. II, 32 (1988).

[116] N. Nakajima, Rubber Chem. Technol. 54, 266 (1981).

[117] N. Yamada, K. Takahara, K. Inoue, 157th meeting ACS Rub. Div., Dallas, Texas, Paper No. 4 (2000).

[118] D. Berkemeier, W. Haeder, M. Rinker, G. Heiss, 157th meeting ACS Rub. Div., Dallas, Texas, Paper No. 6 (2000).

[119] F. J. Borzenski, Q. Hartley, Rubber World 248, 18 (2013).

[120] N. Nortey, Rubber World 237, 23 (2008).

[121] G. S. Donoian, E. L. Canedo, L. N, Valsamis, Rubber Chem. Technol. 65, 792 (1992).

[122] P. S. Kim, J. L. White, Rubber Chem. Technol. 67, 880 (1994).

[123] C. Koolhiran, J. L. White, J. Appl. Polym. Sci. 78, 1551 (2000).

[124] W. Dierkes, J. W. M. Noordermeer, Kautsch. Gummi Kunstst. 56, 338 (2003).

[125] W. M. Wiedmann, H. M. Schmid, Rubber Chem. Technol. 55, 363 (1982).

[126] G. R. Cotten, Rubber Chem. Technol. 57, 118 (1984).

[127] L. A. E. M. Reuvekamp, J. W. ten Brinke, P. J. van Swaaij, J. W. M. Noordermeer, Rubber Chem. Technol. 75, 187 (2002).

[128] H. D. Luginsland, A. Hasse, 157th meeting ACS Rub. Div., Dallas, Texas, Paper No. 34 (2000). 
[129] "Engineering Principles of Plasticating Extrusion", Z. Tadmor, I. Klein, Van Nostrand Reinhold Co., New York (1970).

[130] J. W. ten Brinke, PhD Thesis: Silica Reinforced Tyre Rubbers: Mechanistic Aspects of the Role of Coupling Agents, Univ. Twente, Enschede, the Netherlands (2002). 


\section{Chapter 3 Investigation on the origin of marching modulus of silica filled tire tread compounds and its influence on the reinforcement}

Silica reinforced S-SBR/BR tire tread compounds often show characteristic vulcanization profiles that do not exhibit a distinct maximum in the cure curve, nor a plateau profile within acceptable time scales: marching modulus. In such a situation, it is difficult to determine the optimum curing time, and as a consequence the physical properties of the rubber compounds may vary. Previous studies stated that the curing behavior of silica filled rubber compounds is related to the degree of filler dispersion, the silanization and the filler-polymer coupling reaction, as well as the donation of free sulfur from the silane coupling agent (Paragraph 2.7 in Chapter 2).

Based on these work, several series of silica filled tire tread compounds were mixed in order to clarify the origin of marching modulus in Chapter 3.1. Various silane coupling agents with different sulfur ranks and functionalities were mixed for each mixing series at varied silanization temperatures in order to obtain; 1) different degrees of filler-polymer coupling; 2) different sulfur donation effects from the silanes; 3) different degrees of silanization. The Marching Modulus Intensity (MMI) of these compounds was monitored in two different deformation strains by measuring the vulcanization rheograms using a Rubber Process Analyzer. Additionally, the factors affecting the MMI were evaluated for the analysis of filler-filler interaction, Filler Flocculation Rate (FFR), filler-polymer Coupling Rate (CR), the amount of bound rubber, etc.

In Chapter 3.2, the mechanical properties alteration of the compounds having different MMI are detailed. The mechanical properties of the vulcanizates with three different curing times are investigated. The results were interpreted in terms of total crosslink density, filler-polymer interaction as well as fillerfiller interaction. 
Chapter 3 


\section{Chapter 3.1 Investigation of the origin of marching modulus of silica filled tire tread compounds}

\subsubsection{Introduction}

The polarity difference between silica fillers and rubber polymers induces major difficulties in the mixing process such as poor dispersion and high Mooney viscosities. Therefore, silane coupling agents, which act as compatibilizers between silica and polymer, have become an inevitable component in the formulation of a silica filled rubber compound. Using a silane coupling agent significantly improves the processability and reinforcement of silica filled rubber through the chemical reaction between the silica and the silane, commonly denoted as "silanization reaction". ${ }^{[1-3]}$

Many studies were done on the optimum rubber composition and mixing conditions for silica compounds, but commonly from a viewpoint of improving mixing and extrusion as well as the final vulcanizate properties. ${ }^{[4-10]}$ These studies are primarily focused on influences of mixer temperature settings, fill factor, number of mixing stages, removal of ethanol generated during the silica-coupling agent reaction, modifying the polymer, silica and silane, etc. ${ }^{[9-12]}$ Overall these studies indicate that the silanization reaction plays an important role for the dispersion and the bound rubber formation of a silica-filled rubber. ${ }^{[13-19]}$

The fact that this chemical reaction has to occur in a mechanical mixer which is not designed for this purpose causes an insufficient degree of silanization resulting in problems concerning vulcanization. The consequence of an incomplete silanization makes itself felt in a marching modulus during the vulcanization stage of such compounds. A marching modulus during vulcametry is a problem often observed in silica-filled S-SBR/BR tire tread compounds. A typical example is given in Figure 3.1, in which for one compound formulation but different mixing conditions a plateau torque or a marching modulus was obtained. This phenomenon makes it difficult to determine the optimum curing time, $t_{c, 90}$ or $t_{c, 95}$, from a rheogram. It is neither productive nor efficient to wait for a prolonged time in order to obtain a plateau in the vulcanization profile. Therefore, a marching modulus should be avoided or at least minimized. In order to overcome this problem, understanding of the physicochemical mechanisms underlying the marching modulus phenomena of silica filled rubber compounds is required. However, the mechanisms are not clearly defined yet. 


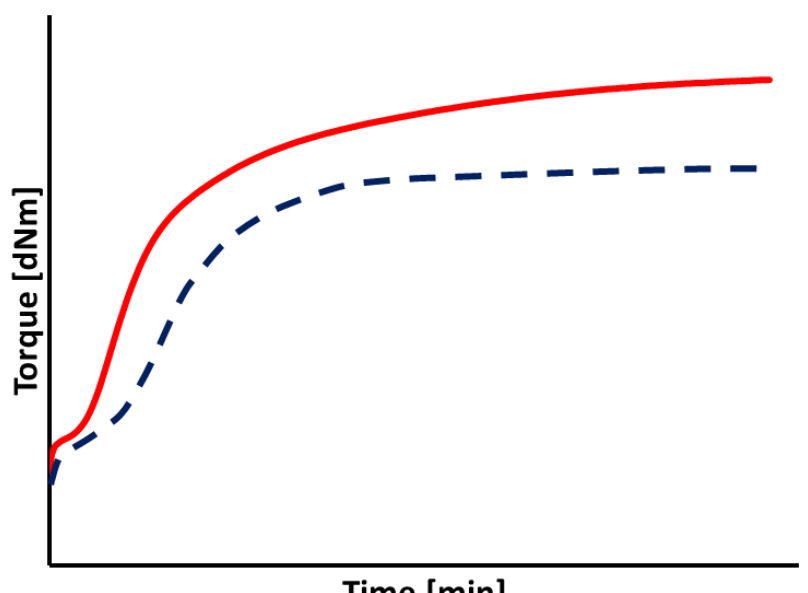

Time [min]

Figure 3.1 Typical rheograms of silica filled S-SBR/BR compounds mixed under different mixing

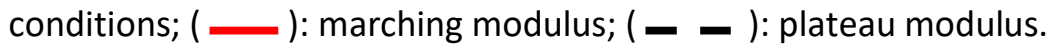

Other studies focused on obtaining the most optimal properties from the perspective of tire tread performance: Rolling Resistance, Wet traction and Wear resistance. ${ }^{[20-23]}$ Most of these studies are based on choosing either a constant curing time for all different samples in one study, or curing all the samples according to - for example - their calculated cure times.

Several authors state that the curing behavior of silica filled rubber compounds is related to the degree of filler dispersion, the silanization and the filler-polymer coupling reaction, as well as the donation of free sulfur from the silane coupling agent. ${ }^{[8,14,24,25]}$ Within the present study, these factors are analyzed in order to differentiate the effect of filler-filler and filler-polymer interaction as well as sulfur donation on the marching modulus. Therefore, compounds with various silane coupling agents are mixed at various silanization temperatures. Subsequently, the vulcanization behavior of these compounds is measured at small (approx. 7\%) and large (approx. 42\%) strain. The results will be interpreted in terms of the degrees of silanization reached.

\subsubsection{Experimental}

\subsubsection{Materials}

Different types of silane were used in the present study as given in Table 3.1. The silanes were chosen based on functionality and sulfur rank. With these silanes, all series of experiments were done based on a tire tread compound as shown in Table 3.2. Blends of oil-extended solution styrene-butadiene rubber (S-SBR, Mooney viscosity (ML1+4@100 $\mathrm{C}$ ) of 65 and composed of $75 \%$ butadiene with a $50 \%$ vinyl-content, 
and $25 \%$ styrene-content, extended with $37.5 \mathrm{phr}$ of TDAE oil) and high cis-1,4 polybutadiene rubber (BR, Mooney viscosity $\left(\mathrm{ML} 1+4 @ 100^{\circ} \mathrm{C}\right)$ of 44 and a cis-1,4 content of $96 \%$ ) were used in this study. Bis(triethoxysilylpropyl)tetrasulfide (TESPT) was used as the reference silane. The amount of TESPT applied in the formulations was adjusted according to Equation 3.1, based on the CTAB surface area of Zeosil 1165MP: $152 \mathrm{~m}^{2} / \mathrm{g}$ : [26]

$$
\operatorname{TESPT}(p h r)=5.3 \times 10^{-4} \times(C T A B)_{\text {silica }} \times(p h r)_{\text {silica }}
$$

Table 3.1 Silane coupling agents with their corresponding sulfur rank and functionality

\begin{tabular}{|c|c|c|c|c|c|}
\hline \multirow[b]{2}{*}{ Name of the silane } & & \multirow{2}{*}{$\begin{array}{l}\text { Sulfur } \\
\text { rank }\end{array}$} & \multicolumn{3}{|c|}{ Possible functions } \\
\hline & & & Silanization & Coupling & $\begin{array}{c}\text { Sulfur } \\
\text { donation }\end{array}$ \\
\hline $\begin{array}{l}\text { Bis(triethoxysilylpropyl)tetrasulfide } \\
(\text { EtO })_{3} \mathrm{Si}-\left(\mathrm{CH}_{2}\right)_{3}-\mathrm{S}_{3.85}-\left(\mathrm{CH}_{2}\right)_{3}-\mathrm{Si}(\mathrm{OEt})_{3}\end{array}$ & (TESPT) & 3 & $\bullet$ & $\bullet$ & $\bullet$ \\
\hline $\begin{array}{l}\text { Bis(triethoxysilylpropyl)disulfide } \\
(\mathrm{EtO})_{3} \mathrm{Si}-\left(\mathrm{CH}_{2}\right)_{3}-\mathrm{S}_{2.15}-\left(\mathrm{CH}_{2}\right)_{3}-\mathrm{Si}(\mathrm{OEt})_{3}\end{array}$ & (TESPD) & 2 & $\bullet$ & $\bullet$ & $x$ \\
\hline $\begin{array}{c}\text { 1,8-Bis(triethoxysilyl)octane } \\
(\mathrm{EtO})_{3} \mathrm{Si}-\left(\mathrm{CH}_{2}\right)_{8}-\mathrm{Si}(\mathrm{OEt})_{3}\end{array}$ & (TESO) & 0 & $\bullet$ & $x$ & $x$ \\
\hline $\begin{array}{l}\text { Propyltriethoxysilane } \\
(\text { EtO })_{3} \mathrm{Si}-\left(\mathrm{CH}_{2}\right)_{2}-\mathrm{CH}_{3}\end{array}$ & (PTEO) & 0 & $\bullet$ & $x$ & $x$ \\
\hline
\end{tabular}

$\bullet$ = yes

$x=$ no 
Table 3.2 Formulation of the compound

\begin{tabular}{|c|c|c|c|c|c|c|c|c|c|}
\hline $\begin{array}{l}\text { Mixing } \\
\text { stage }\end{array}$ & Ingredient & Product name & Company & $\begin{array}{l}\text { Ref. } \\
\text { [phr] }\end{array}$ & $\begin{array}{l}\text { Mix A } \\
{[p h r]}\end{array}$ & $\begin{array}{l}\text { Mix B } \\
\text { [phr] }\end{array}$ & $\begin{array}{l}\text { Mix C } \\
\text { [phr] }\end{array}$ & $\begin{array}{l}\text { Mix D } \\
\text { [phr] }\end{array}$ & $\begin{array}{l}\text { Mix E } \\
\text { [phr] }\end{array}$ \\
\hline \multirow{13}{*}{$\begin{array}{c}\text { Master } \\
\text { Batch }\end{array}$} & S-SBR ${ }^{*}$ & $\begin{array}{c}\text { Buna VSL5025- } \\
2 \mathrm{HM}\end{array}$ & Lanxess & 103.2 & 103.2 & 103.2 & 103.2 & 103.2 & 103.2 \\
\hline & BR & Buna CB24 & Lanxess & 25 & 25 & 25 & 25 & 25 & 25 \\
\hline & HD Silica & Zeosil 1165MP & Solvay & 80 & 80 & 80 & 80 & 80 & 80 \\
\hline & TESPT & Si69 & Evonik & 6.4 & - & - & - & - & - \\
\hline & TESPD & Si266 & Evonik & - & 5.7 & 5.7 & - & - & - \\
\hline & TESO & SIB1824.0 & Gelest & - & - & - & 5.2 & - & - \\
\hline & PTEO & VP Si203 & Evonik & - & - & - & - & 4.9 & - \\
\hline & TDAE Oil & VIVATEC 500 & $\begin{array}{l}\text { Hansen \& } \\
\text { Rosenthal }\end{array}$ & 7 & 7 & 7 & 7 & 7 & 7 \\
\hline & Stearic acid & - & Merck & 1 & 1 & 1 & 1 & 1 & 1 \\
\hline & Zinc oxide & - & Merck & 2 & 2 & 2 & 2 & 2 & 2 \\
\hline & DPG & Perkacit DPG & Flexsys & 2 & 2 & 2 & 2 & 2 & 2 \\
\hline & 6PPD & KUMANOX 13 & $\begin{array}{c}\text { Kumho } \\
\text { Petrochemical }\end{array}$ & 2 & 2 & 2 & 2 & 2 & 2 \\
\hline & Sulfur & $S$ & J. T Baker & - & - & 0.7 & - & - & - \\
\hline \multirow{3}{*}{ Final } & Sulfur & $S$ & J. T Baker & 0.7 & 1.4 & 0.7 & 1.4 & 1.4 & 1.4 \\
\hline & ZBEC & Vulkacit ZBEC & Lanxess & 0.2 & 0.2 & 0.2 & 0.2 & 0.2 & 0.2 \\
\hline & CBS & Santocure CBS & Flexsys & 2.2 & 2.2 & 2.2 & 2.2 & 2.2 & 2.2 \\
\hline
\end{tabular}

$※ 27.3$ wt $\%$ oil extended

As the molar masses of the various silanes used in this study differ from the one of TESPT, the quantities of the silanes were adjusted to be equimolar in terms of ethoxy groups in TESPT. Therefore, the amount of PTEO silane in Mix D was doubled. Additionally, the sulfur ranks of those silanes also differ from the one of TESPT; therefore the total amount of active sulfur was adjusted according to the amount of sulfur which can be released from TESPT. In case of Mix B, the extra amount of active sulfur was introduced in the first mixing step. 


\subsubsection{Mixing}

The compounds were mixed in two steps as shown in Table 3.3. The master batch stage was done by using a lab scale internal mixer (Brabender Plasticorder) with a $390 \mathrm{ml}$ chamber volume. The fill factor of the internal mixer was fixed to $75 \%$. The temperature of the mixer temperature control unit (TCU) was set at $50^{\circ} \mathrm{C}$. In order to avoid the first batch effect, one initial batch (B0) was mixed and discarded before the regular mixing starts. After $\mathrm{BO}$, the regular mixing was started when the mixing chamber reached $55^{\circ} \mathrm{C}$. The rotor speed was adjusted from 04:10 (minutes:seconds) onwards in order to reach and subsequently keep different silanization temperatures steady during a period of silanization of 150 seconds, as indicated in Figure 3.2. The silanization temperatures as indicated in Figure 3.2 are registered by the sensor embedded in the mixer wall and are 10 to $15^{\circ} \mathrm{C}$ lower than the actual discharge temperatures as measured with a hand-held thermometer. The silanization temperatures mentioned hereafter are the machine sensor values as depicted in Figure 3.2. After the first mixing step, the compounds were sheeted out immediately on a lab scale two-roll mill (Polymix 80T) in order to cool down the compounds and prevent further reaction. Three batches were mixed for each set of conditions in order to check the reproducibility. All batches were mixed with good reproducibility.

Table 3.3 Mixing procedure

\begin{tabular}{|c|c|c|c|}
\hline \multicolumn{2}{|c|}{ Masterbatch (first) step } & \multicolumn{2}{|c|}{ Final (second) step } \\
\hline \multicolumn{2}{|c|}{ Internal mixer } & \multicolumn{2}{|c|}{ Open mill } \\
\hline Action & time [mm:ss] & Action & time [mm:ss] \\
\hline Add polymer & $00: 00 \sim 00: 20$ & \multirow{2}{*}{ Add master batch } & \multirow{2}{*}{-} \\
\hline Mastication & $00: 20 \sim 01: 20$ & & \\
\hline $1 / 2$ Silica, silane & $01: 20 \sim 01: 40$ & \multirow{2}{*}{ Mixing } & \multirow{2}{*}{$00: 00 \sim 02: 00$} \\
\hline Mixing & $01: 40 \sim 02: 40$ & & \\
\hline $1 / 2$ Silica, other remains & $02: 40 \sim 03: 10$ & \multirow{2}{*}{ Add curatives } & \multirow{2}{*}{$02: 00 \sim 02: 30$} \\
\hline Mixing (Up to $120^{\sim} 170^{\circ} \mathrm{C}$ ) & 03:10 04:10 & & \\
\hline Ram sweep & $04: 10 \sim 04: 14$ & \multirow{2}{*}{ Mixing } & \multirow{2}{*}{$02: 30 \sim 09: 00$} \\
\hline Mixing (at target temp.) & $04: 14 \sim 06: 40$ & & \\
\hline Discharge and sheeting & - & Discharge & - \\
\hline
\end{tabular}


Ref. (TESPT)

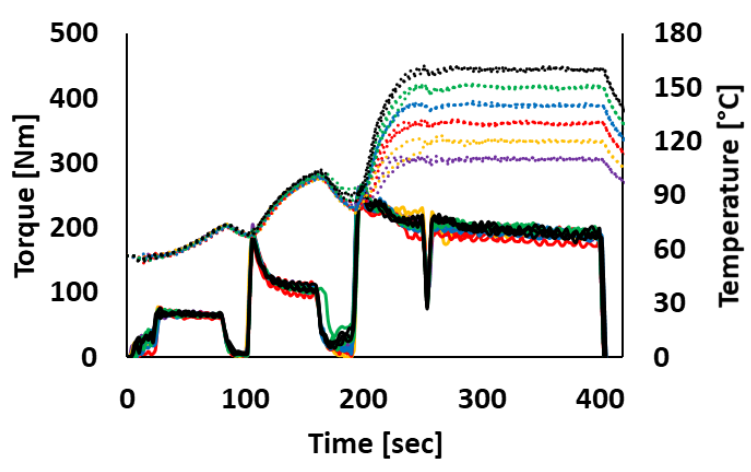

Mix B (TESPD+Sulfur)

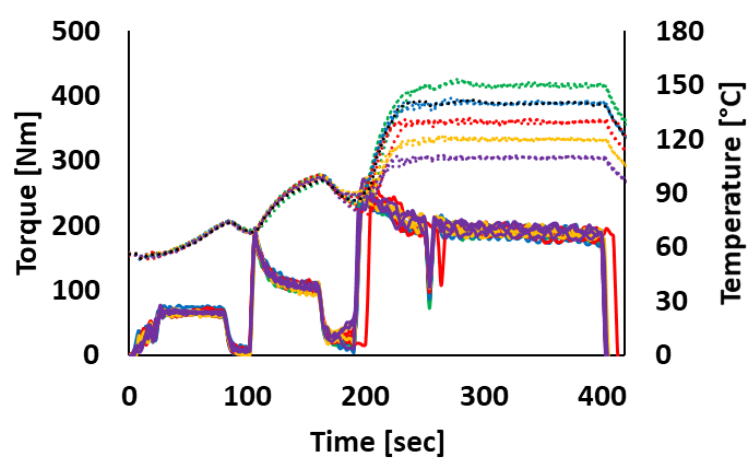

Mix D (PTEO)

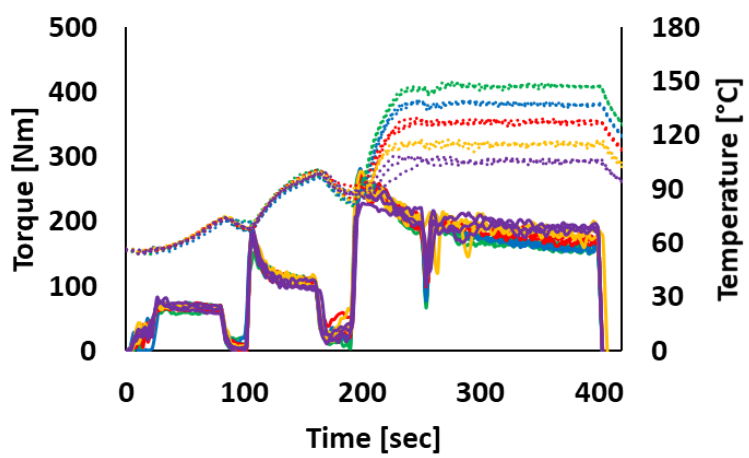

$\operatorname{Mix} A$ (TESPD)

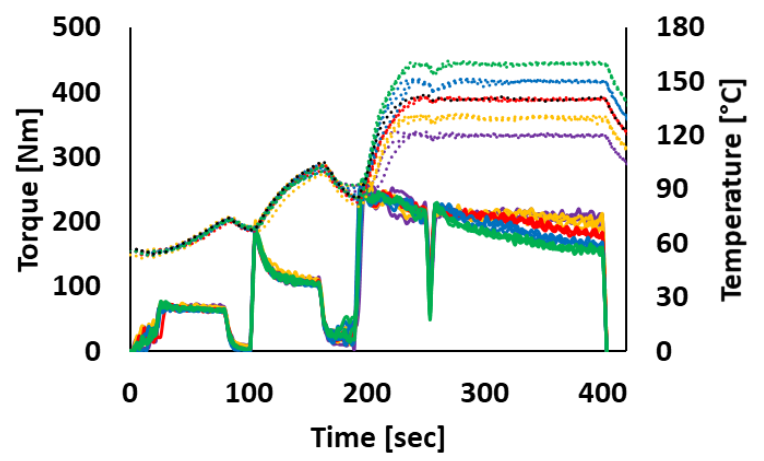

Mix C (TESO)

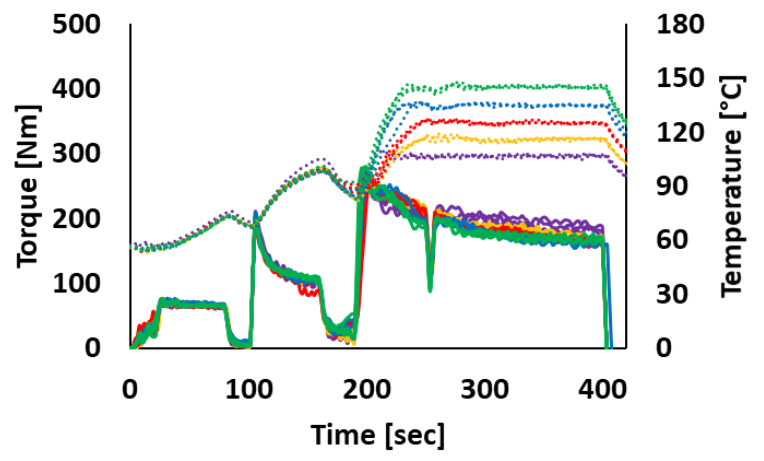

Mix E (No silane)

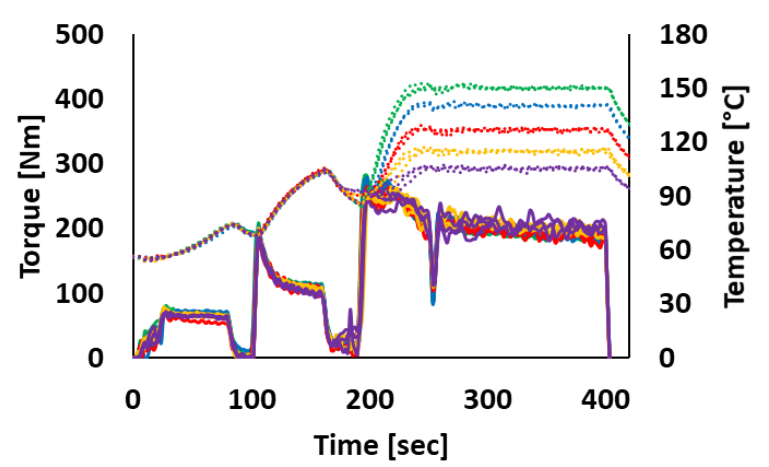

Figure 3.2 Mixing fingerprints of the compounds; ( solid line ): torque; (dotted line ): temperature;

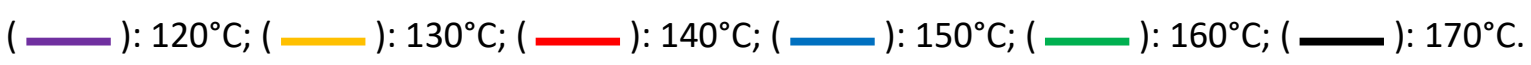

\subsubsection{Payne effect}

The Payne effect of silica-filled rubber compounds is generally used as an indicator of the degree of fillerfiller interaction. ${ }^{[13,27,28]}$ When a rubber is filled with a reinforcing filler, filler-filler interactions take place. Especially silica fillers have a lot of $-\mathrm{OH}$ groups on their surface and thus form a strong filler network in 
the rubber matrix via hydrogen bonding. ${ }^{[29]}$ In general, the storage modulus of such filled rubber compounds decreases with increasing strain amplitude due to the breakdown of the filler network.

In the present work, the storage shear moduli $\left(\mathrm{G}^{\prime}\right)$ of the uncured and cured rubber compounds were evaluated by using a Rubber Process Analyzer (RPA; RPA Elite, TA Instruments). The Payne effect of the uncured rubber was measured at a temperature of $100^{\circ} \mathrm{C}$, a frequency of $0.5 \mathrm{~Hz}$ and varying strains in the range of $0.56-200 \%$. The Payne effect of the cured rubber was measured at a temperature of $100^{\circ} \mathrm{C}$ after 30 minutes of curing at $160^{\circ} \mathrm{C}$, a frequency of $0.5 \mathrm{~Hz}$ and varying strains in the range of $0.56-120 \%$. The Payne effects were calculated from the difference in storage shear moduli at low strain $(0.56 \%)$ and high strain $(100 \%)$, i.e. $G^{\prime}(0.56 \%)$ - G'(100\%).

\subsubsection{Filler flocculation rate}

The Filler Flocculation Rate (FFR) of the uncured silica-reinforced S-SBR/BR compounds without curatives was studied by using the RPA mentioned above at $100^{\circ} \mathrm{C}$, a strain of $0.56 \%$ and test time of 14 minutes including 2 minutes of pre-heating time. The measurement temperature was selected according to a typical industrially employed extrusion temperature. The storage shear moduli were recorded at different measurement times. According to Mihara et al. ${ }^{[14]}$ it is possible to observe the flocculation of silica particles by monitoring the change of storage modulus $\left(G^{\prime}\right)$ at low strain under isothermal conditions. As shown in Figure 3.3, the results can best be fitted with Equations 3.2 or 3.3: ${ }^{[30,31]}$
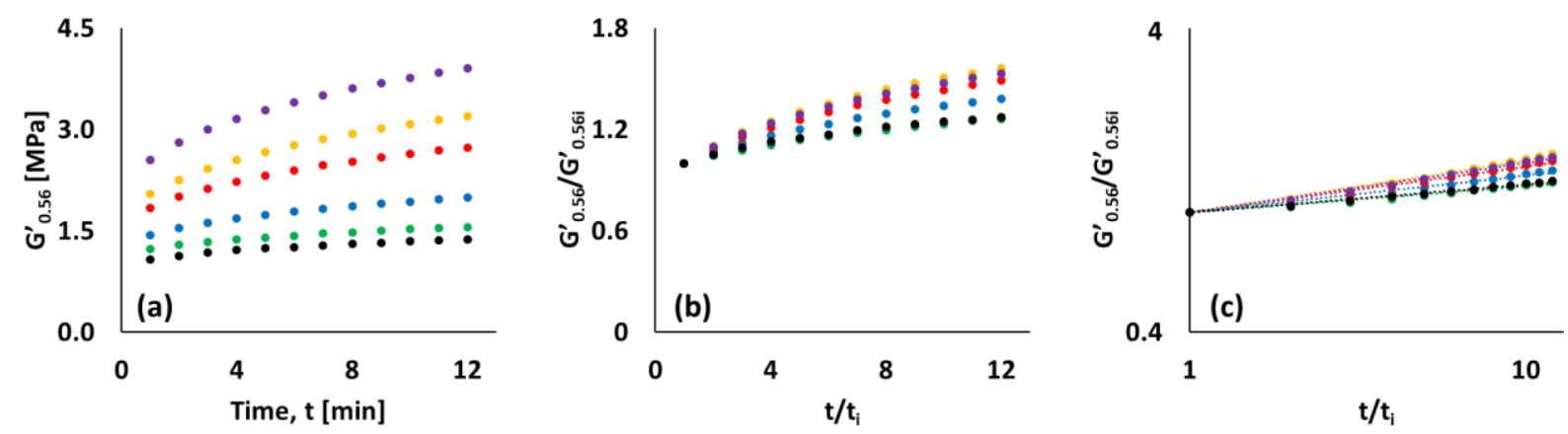

Figure 3.3 The procedure for FFR calculation; (a): raw data; (b): normalization; (c): linear fitting.

$$
G_{0.56}^{\prime} \sim t^{F F R}
$$




$$
F F R=\frac{d \log \left(G_{0.56}^{\prime}(t) / G_{0,56 i}^{\prime}\right)}{d \log \left(t / t_{i}\right)}
$$

Where FFR is the dimensionless flocculation rate, $\mathrm{G}_{0.56}^{\prime}(\mathrm{t})$ is the storage modulus at $0.56 \%$ strain at test time $t, G_{0.56 i}^{\prime}$ is the initial storage modulus at $t_{i}$, and $t_{i}$ is 1 minute after pre-heating.

\subsubsection{Filler-polymer coupling rate}

The filler-polymer Coupling Rate (CR) of the uncured silica-reinforced S-SBR/BR compounds without curatives was studied by using the RPA under the following conditions: $160^{\circ} \mathrm{C}, 1.677 \mathrm{~Hz}$ and 3 degrees ( $42 \%$ of strain) for 40 minutes. A large strain was applied for the CR measurement in order to break the filler-filler interaction. Therefore, only the filler-polymer interaction is taken into account in the CR.

The procedure for $\mathrm{CR}$ calculation is shown in Figure 3.4. After the torque levels at different times were recorded and normalized, CR was calculated following Equation 3.4, based on a same concept as Equation 3.2: ${ }^{[30,31]}$
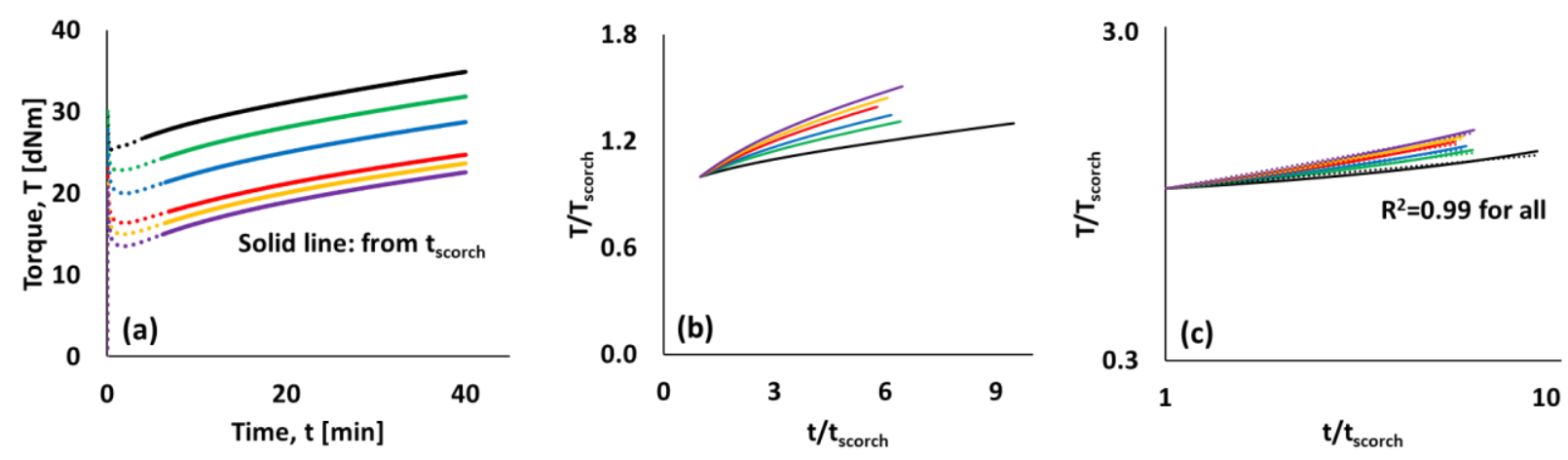

Figure 3.4 The procedure for CR calculation; (a): raw data curve; (b): normalization; (c): linear fitting.

$$
C R=\frac{d \log \left({ }^{T}(t) / T_{\text {scorch }}\right)}{d \log \left(t / t_{\text {scorch }}\right)}
$$

Where CR is the dimensionless filler-polymer coupling rate, $T(t)$ is the torque level at test time $t, T_{\text {scorch. }}$ is the torque level at $t_{\text {scorch., }}$ the time to incipient cure or scorch which corresponds to the time for the torque to increase by $1 \mathrm{dNm}: T_{\text {scorch }}=T_{\min }+1(\mathrm{dN} \cdot \mathrm{m}) . T_{\min }$ is the minimum torque level which is observed during the 
measurement.

Two examples are depicted in Figure 3.5. As can be seen in this figure, different torque profiles are recorded according to the filler-polymer coupling possibility of the silanes: Ref. compound (with TESPT) shows a clear torque increment along with measurement time, whereas Mix C (with TESO) shows a plateau profile, so that no $t_{\text {scorch }}$ can be indicated.
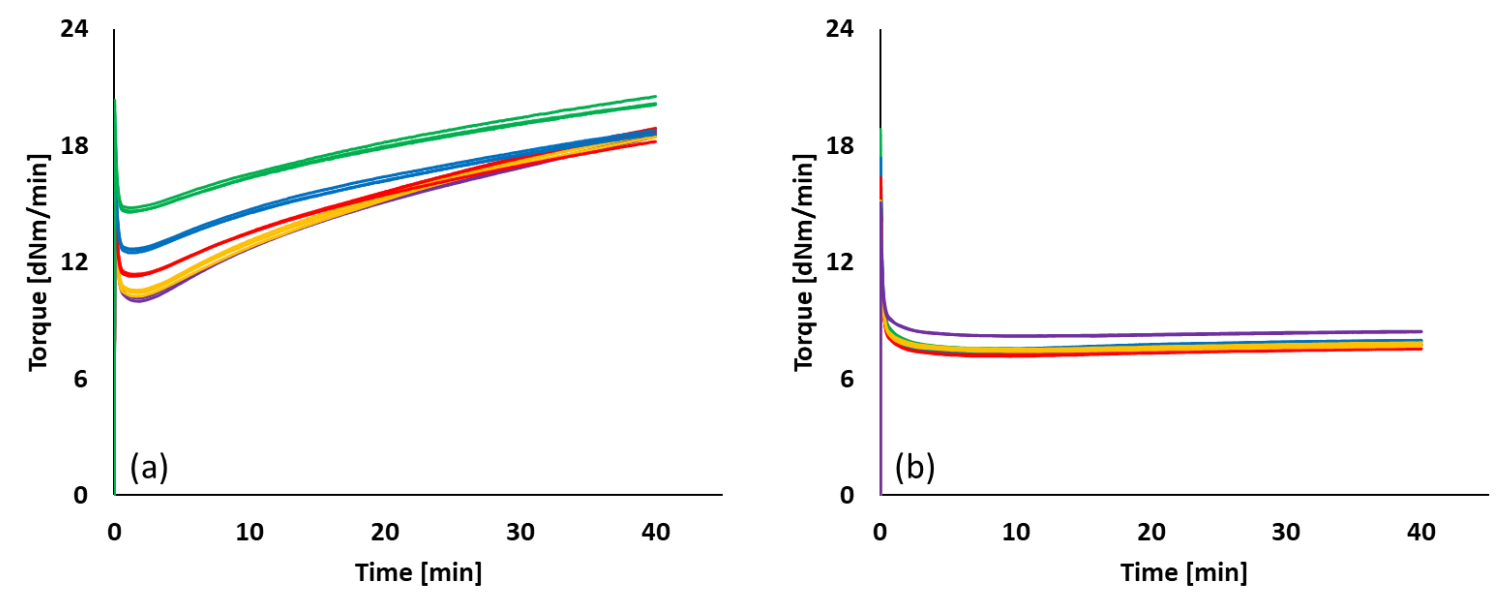

Figure 3.5 Examples of CR measurement; (a): Ref. compound (with TESPT); (b): Mix C (with TESO); $(\square): 120^{\circ} \mathrm{C} ;(\square): 130^{\circ} \mathrm{C} ;(\square): 140^{\circ} \mathrm{C} ;(\square): 150^{\circ} \mathrm{C} ;(\square): 160^{\circ} \mathrm{C}$.

\subsubsection{Cure characteristics and marching modulus intensity}

The rheograms were measured at $160^{\circ} \mathrm{C}$ for 40 minutes under two different strain conditions as follows:

- 0.5 degrees ( 7\% of strain) and a frequency of $1.667 \mathrm{~Hz}$ : ASTM D5289-95; [32]

- 3 degrees ( $\sim 2 \%$ of strain) and a frequency of $1.667 \mathrm{~Hz}$.

The Marching Modulus Intensity (MMI) was calculated from the rheograms by using Equation 3.5: ${ }^{[30,31]}$

$$
M M I=\frac{T_{40}-T_{20}}{40 \min -20 \min }
$$

Where $\mathrm{MMI}$ is the marching modulus intensity, $\mathrm{T}_{40}$ and $\mathrm{T}_{20}$ are the corresponding torques at 40 minutes and 20 minutes. 


\subsubsection{Vulcanization}

Vulcanization was done using a Wickert laboratory press (WLP1600). For the swelling ratio measurements, the compounds were vulcanized at $160^{\circ} \mathrm{C}$ at three different curing times: Calculated cure Time (CT), 20 minutes and 30 minutes. The compounds showed marching modulus behavior, therefore $\mathrm{CT}$ of the compound was calculated by using a differential curve of the rheogram according to Mihara's work, ${ }^{[33]}$ as shown in Figure 3.6. The time at the cross point of two tangential lines $A$ and $B$ of the differential curve plus $1 \mathrm{~min}$ was selected as CT.
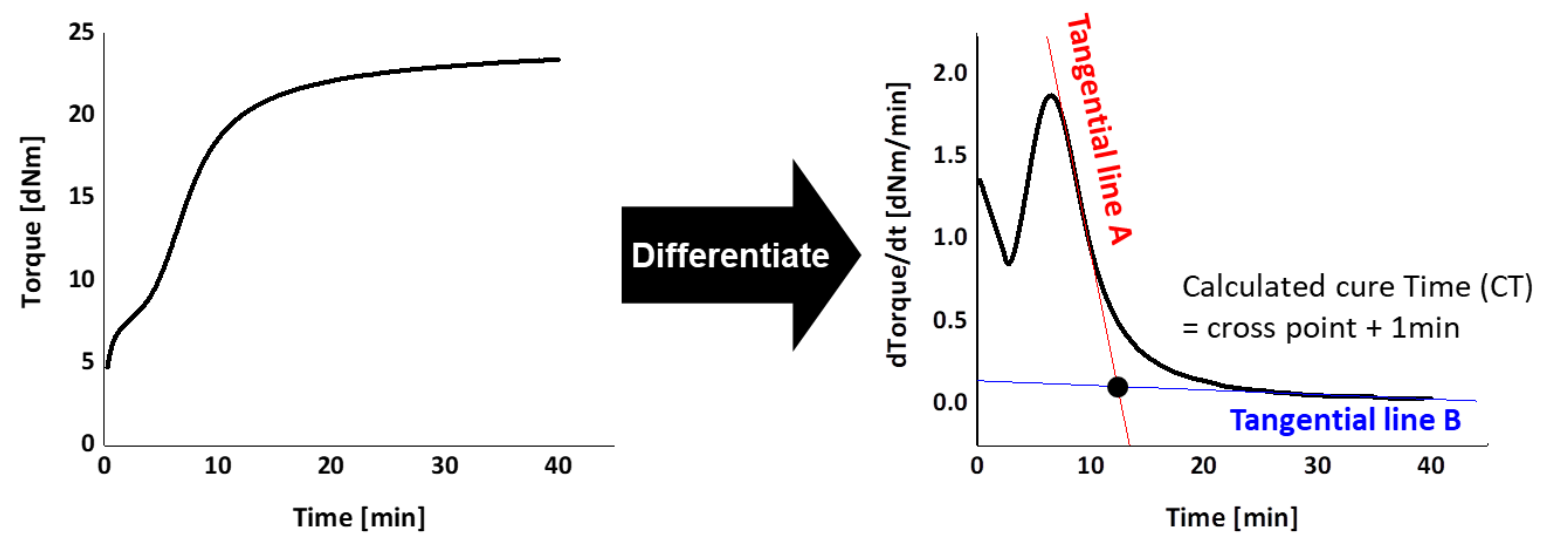

Figure 3.6 Cure time calculation for compounds showing marching modulus. ${ }^{[3]}$

\subsubsection{Total bound rubber content}

Approximately $0.2 \mathrm{~g}$ of the rubber compounds without curatives, as obtained from the first mixing step, were cut into small pieces and immersed in toluene at room temperature for 5 days, while the toluene was renewed every day. Thereafter, the samples were removed from the toluene, dried at $105^{\circ} \mathrm{C}$ for $24 \mathrm{~h}$ and weighed. The bound rubber content was calculated according to Equation 3.6: ${ }^{[34]}$

$$
\text { Bound rubber content }(\%)=\frac{W_{f g}-W\left(m_{f} /\left(m_{f}+m_{p}\right)\right)}{W\left(m_{p} /\left(m_{f}+m_{p}\right)\right)} \times 100
$$

Where $W_{f g}$ is the weight of filler plus gel, $W$ is the original weight of the specimen, and $m_{f}$ and $m_{p}$ are the weights of filler and polymer in the compound, respectively. 


\subsubsection{Chemically and physically bound rubber content}

The degree of filler-polymer coupling can be measured by the chemically bound rubber content. For this analysis, approximately $0.2 \mathrm{~g}$ of the rubber compounds without curatives, as obtained from the first mixing step, were cut into small pieces and immersed in toluene at room temperature for 5 days, this time under ammonia atmosphere in order to cleave physical linkages. The toluene was renewed every day. Then, the samples were removed from the toluene, dried at $105^{\circ} \mathrm{C}$ for $24 \mathrm{~h}$ and weighed. The chemically bound rubber content was calculated according to Equation 3.6. The physically bound rubber content was calculated by subtraction of the chemically bound rubber content from the total bound rubber content.

\subsubsection{Swelling ratio}

The swelling ratio was measured according to ASTM D471 ${ }^{[35]}$ in order to evaluate the crosslink density of the rubber vulcanizates indirectly. The samples cured at three different times having a dimension of approximately $25.0 \times 5.0 \times 2.0 \mathrm{~mm}^{3}$ were weighed and put into $50 \mathrm{ml}$ of toluene at room temperature for 7 days in order to obtain equilibrium state. The samples were then removed from the toluene, blotted to remove liquid from the surface, and weighed. The swelling ratio (Q) was calculated according to Equation 3.7. ${ }^{[36]}$

$$
Q(\%)=\frac{W_{1}-W_{0} / d_{2}}{W_{0} / d_{1}} \times 100
$$

Where $W_{0}$ is the weight of the specimen before swelling, $W_{1}$ is the weight of the specimen after swelling, $d_{1}$ is the density of blended polymer $(0.93 \mathrm{~g} / \mathrm{ml})$ and $d_{2}$ is the density of toluene $(0.87 \mathrm{~g} / \mathrm{ml})$.

\subsubsection{Results and discussion}

\subsubsection{Filler-Filler interaction (Payne effect)}

The Payne effects of the uncured compounds are plotted as a function of the silanization temperature for all mixing series, as shown in Figure 3.7. With increasing silanization temperature, a lower Payne effect was observed due to a higher degree of silanization for the silane-containing compounds. This trend was not expected for Mix E due to the absence of a silane coupling agent. So, all compounds in Mix E would show a similar level of Payne effect. The fact that still some decrease in Payne effect is seen for these compounds can be explained by the micro-dispersion mechanism of silica, as shown in Figure 3.8 . ${ }^{[13,37]}$ 


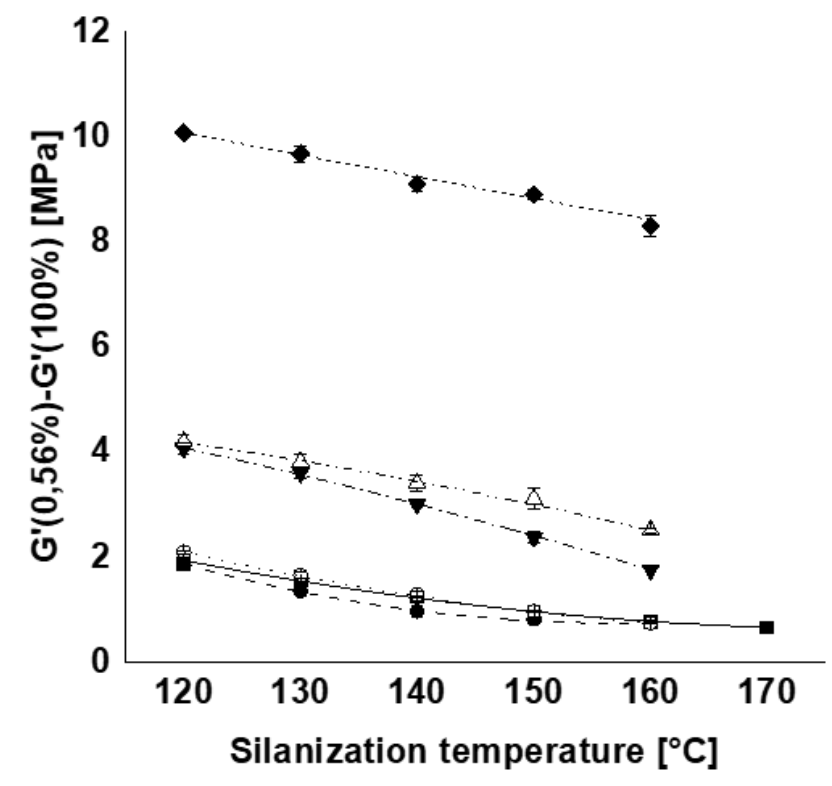

Figure 3.7 Payne effect as a function of silanization temperature;

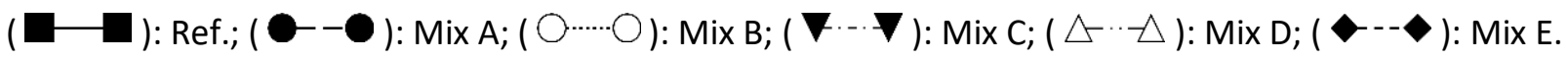

At higher temperatures the polymer mobility is enhanced, either by lower viscosity or possibly some polymer breakdown. This means that the polymer can more easily penetrate into the voids of the agglomerates. Consequently, aggregates are better separated by the polymer, resulting in a better microdispersion of silica. It is clear that the highest Payne effect is observed in Mix E, because no silanization is possible without any silane coupling agent. Mixes $C$ and $D$, which contain the silanes without sulfur moiety, show the second highest Payne effect. This effect can be explained by their bound rubber content which will be discussed here after.

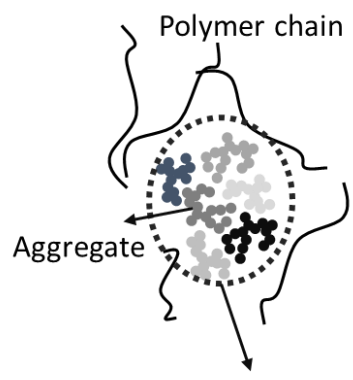

Agglomerate

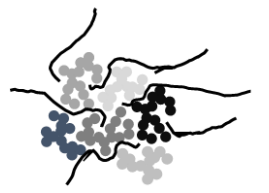

Polymer penetrates into the void

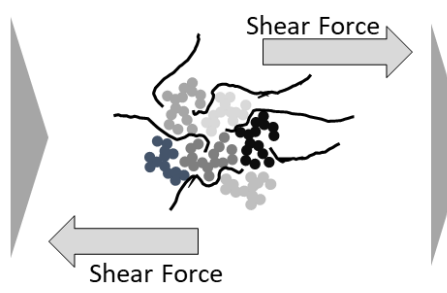

Shear Force

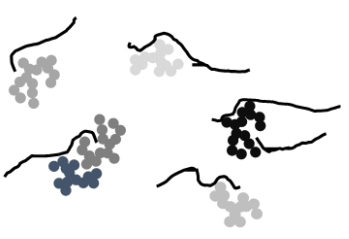

Separation of aggregates

Figure 3.8 Micro-dispersion mechanism of silica. ${ }^{[37]}$ 


\subsubsection{Bound rubber content}

Figure 3.9 shows the amount of bound rubber. In case of Ref. compound, Mix A and Mix B, the samples with TESPT, TESPD and TESPD with sulfur adjustment, a higher amount of chemically bound rubber was observed when the compounds were mixed at a higher silanization temperature. Mihara et al. ${ }^{[14]}$, and Choi and $\mathrm{Ko}^{[38]}$ reported that polymer-filler coupling via the silane occurs during mixing. Thus, a higher degree of polymer-filler coupling is established during mixing at a higher silanization temperature.
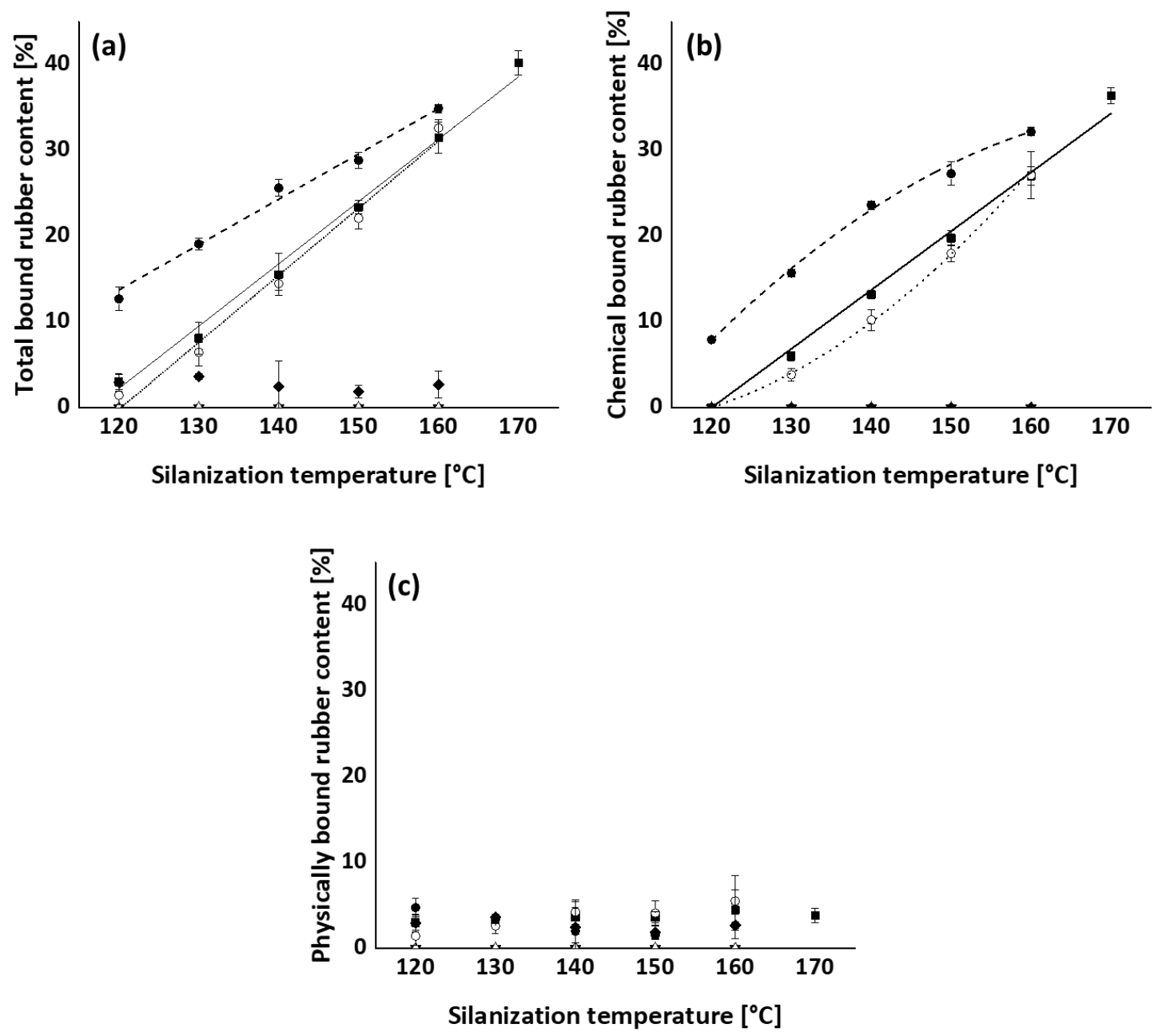

Figure 3.9 Bound rubber contents of samples with different types of coupling agents vs. silanization temperature; (a): total; (b): chemically; (c): physically; ( $\square$ ): Ref.; ( - - ): Mix A; ( $\cdots \cdots)$ ): Mix B; $(\nabla): \operatorname{Mix} C ;(\triangle): \operatorname{Mix} D ;(\bullet): \operatorname{Mix} E$.

No bound rubber at all was found for the samples with sulfur-free silanes (TESO, PTEO). However, approximately $2 \%$ of physically bound rubber was formed in Mix E even though there was no silane: 
Figure 3.9(c). To confirm this result, the storage moduli at the larger strains of the Payne effect measurement range: 60 to $120 \%$, of the cured compounds were compared as shown in Figure 3.10 . In that strain range, three mechanisms contribute to the storage modulus: the hydrodynamic effect of the presence of the fillers (same for all mixes), the polymer-polymer crosslinked network, and the fillerpolymer interaction. ${ }^{[4]}$ As can be seen in Figure 3.10, Mix E (no silane) shows an intermediate position between the compounds with sulfur bridged silanes (TESPT, TESPD) and no sulfur silanes (TESO, PTEO).

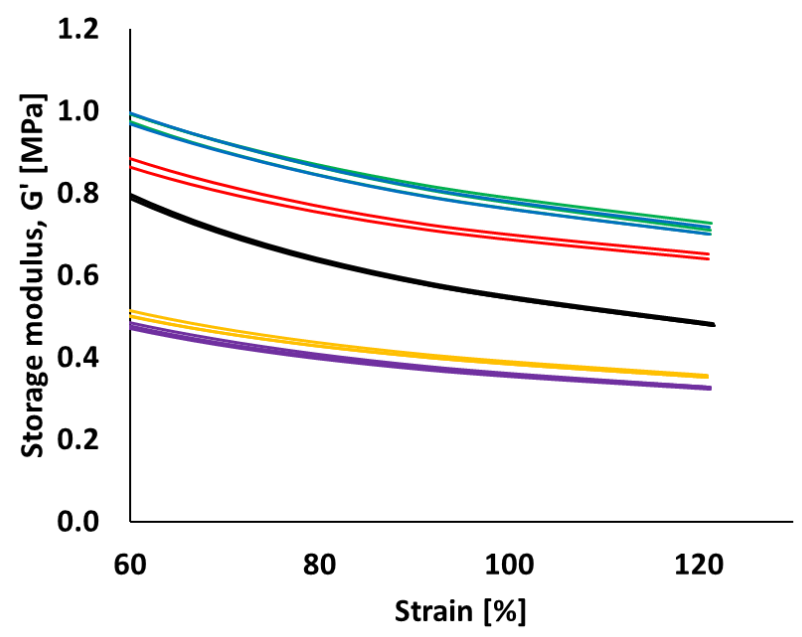

Figure 3.10 Payne effect of cured compounds in the large strain range;

( ): Ref.; ( $)$ ): Mix A; ( $)$ ):Mix B; ( $)$ ): Mix C; ( $)$ ):Mix D; ( $)$ ): Mix E; the lines of Ref. and Mix A are overlapping in this figure.

The amount of free sulfur of all mixing series was adjusted according to the Ref. compound. Therefore, the contribution induced by the polymer-polymer crosslinked network is assumed to be the same for all mixing series, except for Mix B, where the free sulfur is consumed for activation of TESPD. ${ }^{[39]}$ Thus, the possible contributions which lead to the result of Mix E in Figure 3.10 are the hydrodynamic effect and the filler-polymer network.

Due to the surface polarity characteristics of silica, a filler-filler network can easily be formed within the rubber matrix. Part of the rubber can be occluded within this filler-filler network and be immobilized. ${ }^{[13]}$ This occluded rubber surrounded by the filler structure is capable of causing a higher hydrodynamic effect. Additionally, due to the polarity of silica, the solvent toluene used for the bound rubber test cannot access the occluded rubber, as shown in Figure 3.11. As a result, a small amount of physically bound rubber remains. 


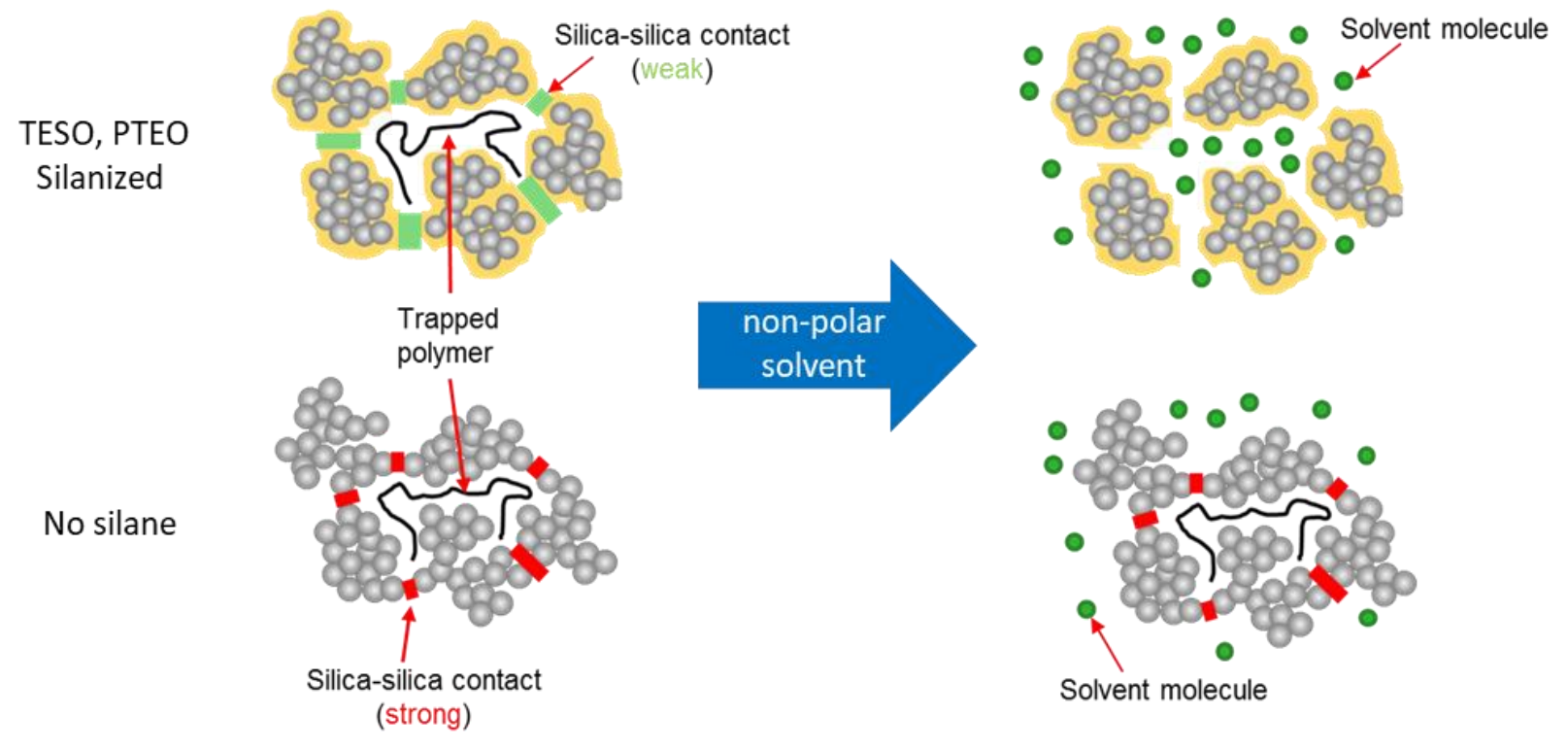

Figure 3.11 Trapped (occluded) polymer in a filler agglomerate with a non-polar solvent.

Stöckelhuber et al. ${ }^{[40]}$ evaluated wetting of the filler surface by polymers and the work of adhesion between rubber and filler. They compared those properties between surface modified and unmodified silica and found that hydrophobized silica shows good wetting properties while adhesion to the polymer was weak, and non-hydrophobized silica showed the opposite result. According to their result, it can be assumed that TESO and PTEO possibly result in good wettability but poor adhesion, which can be represented by physically bound rubber. However without any silane, the wettability is poor but the adhesion of polymer onto the silica surface could be better according to this theory.

\subsubsection{Silica filler flocculation rate}

The silica Filler Flocculation Rates (FFR) of all mixing series are shown in Figure 3.12. In Figure 3.12(a), the FFR values of Ref. compound, Mix A, and Mix B compounds are plotted as a function of the silanization temperature. As discussed in Paragraph 3.2.2, a higher amount of bound rubber, thus a higher degree of silanization is observed with the sulfur-bridged silanes (Ref. compound, Mix A, Mix B). As a result, a higher amount of bound rubber leads to lower filler-filler interaction and thus a reduced tendency for flocculation. No chemically bound rubber could be formed in Mix C (TESO), Mix D (PTEO) and Mix E (no silane). Due to that no clear tendency along with silanization temperature was found in these mixing series, except Mix C (TESO). The FFR slightly increased with rising silanization temperature in Mix C. A possible explanation is depicted in Figure 3.13, based on the special characteristic of TESO. This silane 
has a $\mathrm{C}_{8}$-chain between two silicon atoms in the molecule. This chain is more difficult to break than the $\mathrm{S}_{\mathrm{x}}$-bridge in TESPT. As a consequence, the effect of silica surface shielding by TESO is lower compared to other silanes, even though TESO has a longer alkyl chain in the structure. The FFR of all mixing series are plotted against total bound rubber content of these compounds in Figure 3.14. The FFR values start to decrease when the total bound rubber content exceeds approx. $15 \%$.
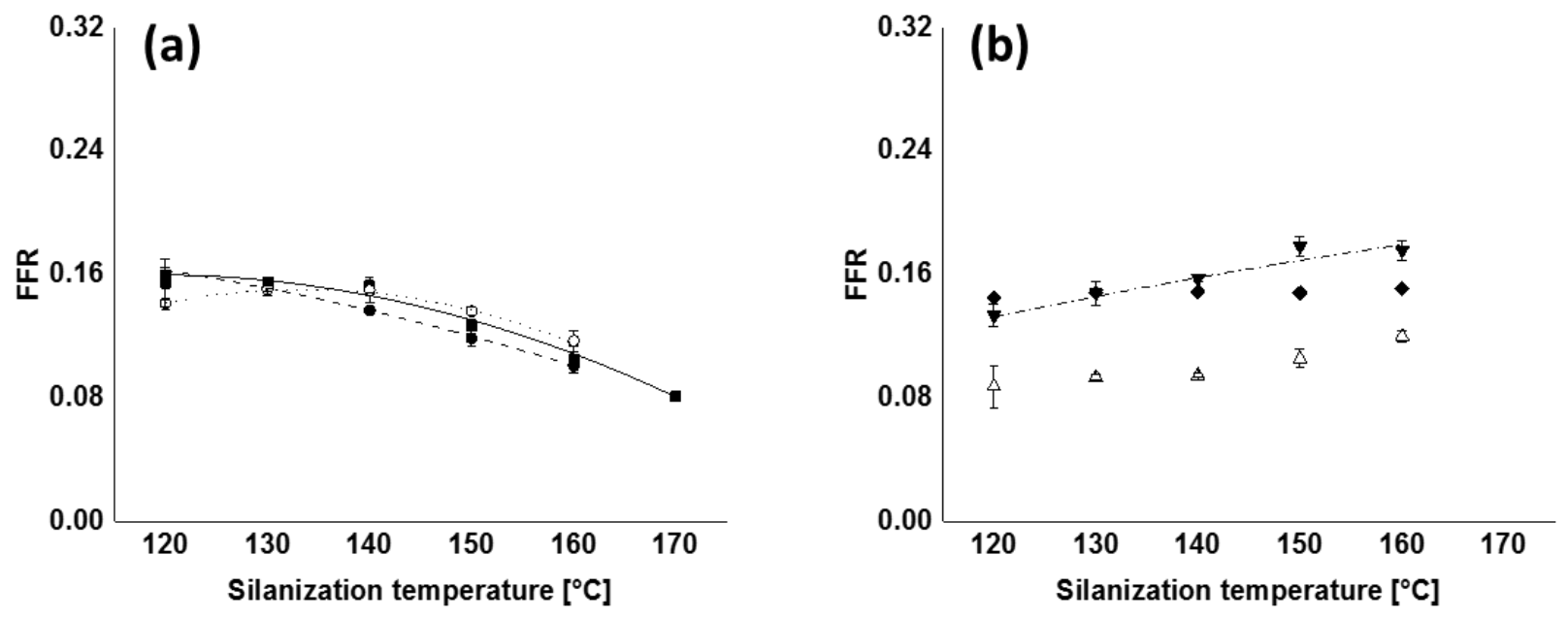

Figure 3.12 Silica flocculation rate (FFR) of samples with different types of coupling agents, vs. silanization temperature; (a): Ref. to Mix B; (b): Mix C to E; ( 1 ): Ref.; ( $(\bigcirc \cdots \cdots): \operatorname{Mix} B ;(\nabla \cdots): \operatorname{Mix} C ;(\triangle): \operatorname{Mix} D ;(\diamond): \operatorname{Mix} E$. 

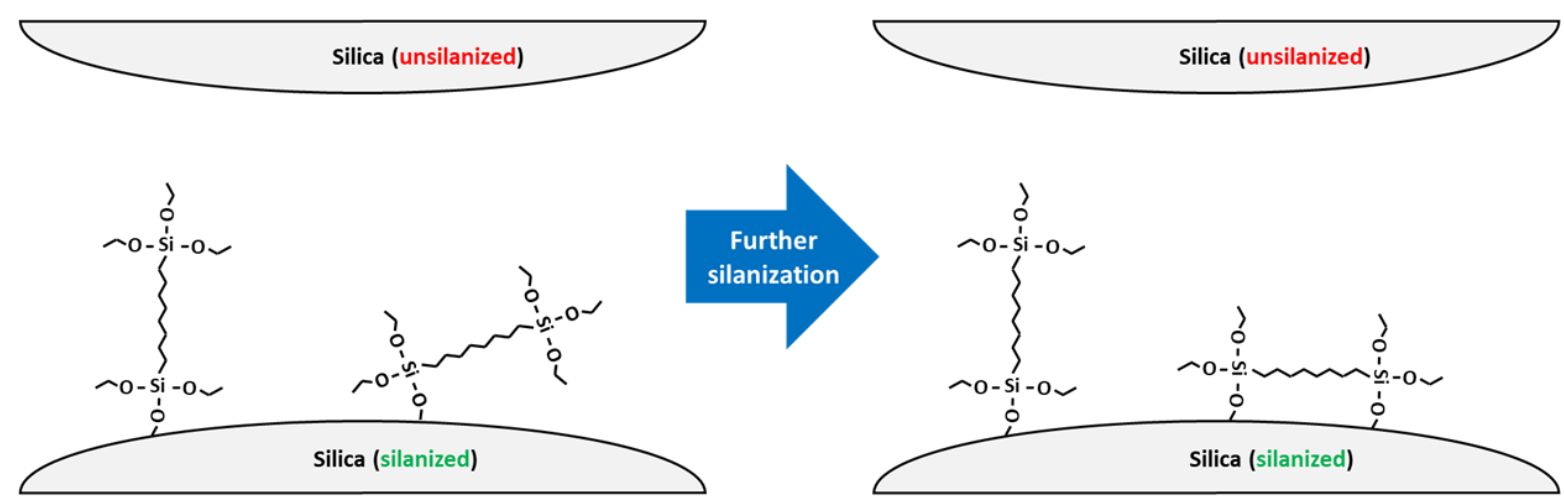

(a)
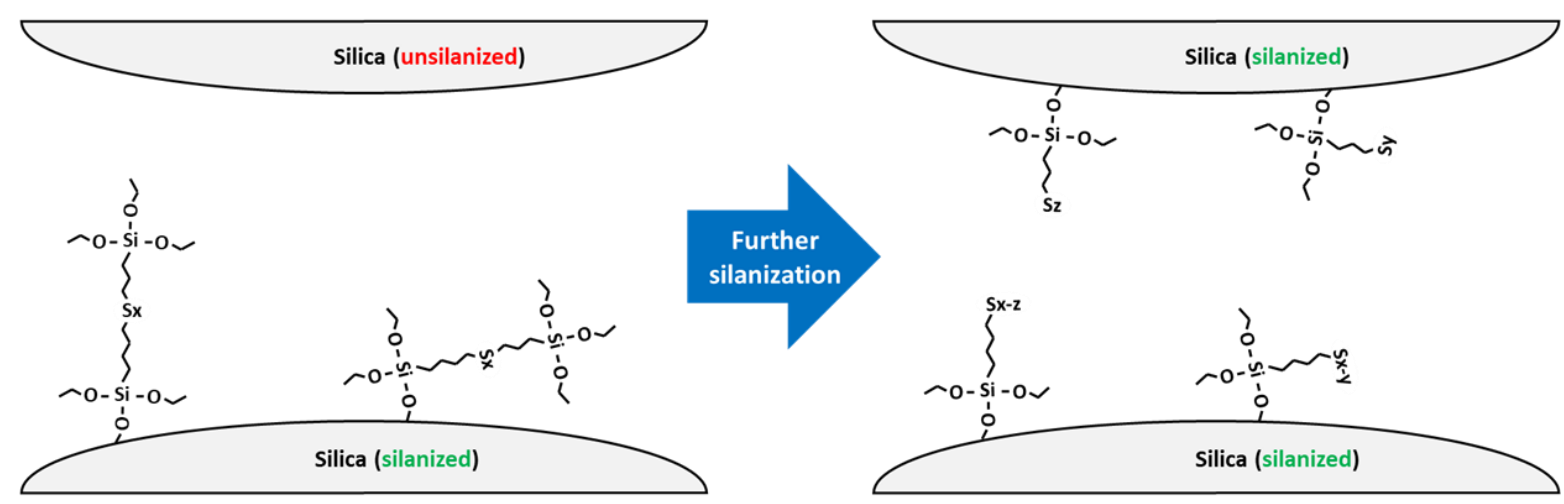

Further
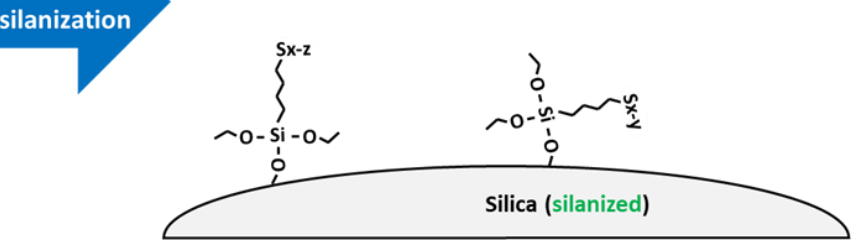

(b)

Figure 3.13 Silica surface coverage by silane molecules in case of using; (a): TESO; (b): TESPT.

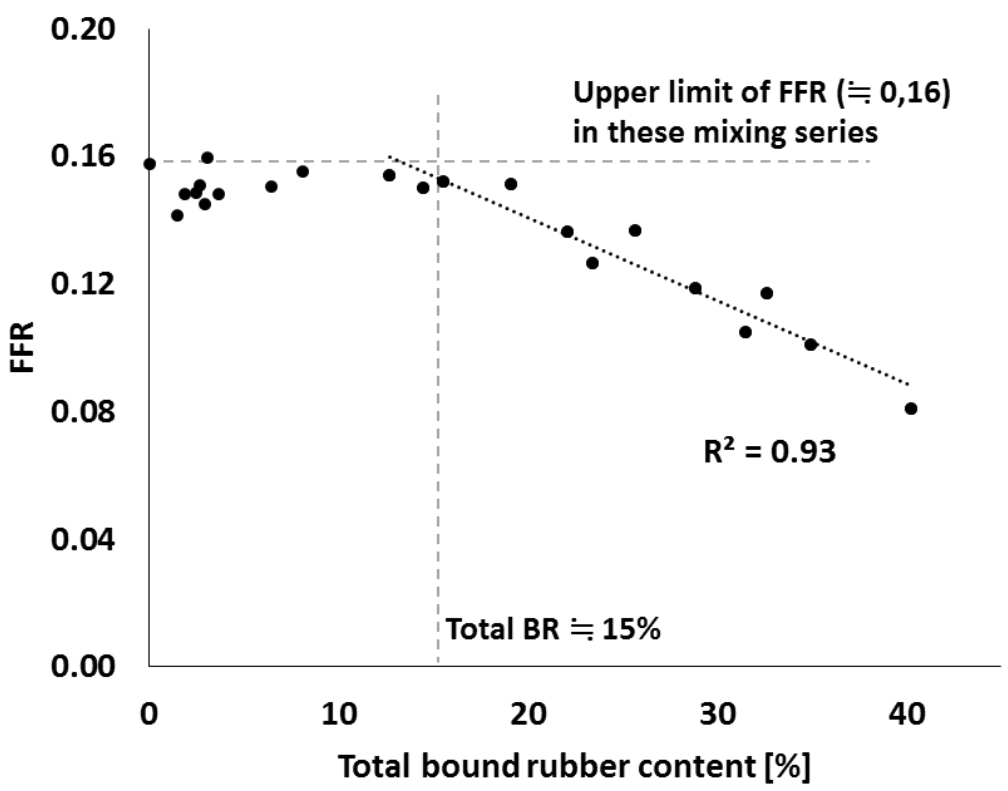

Figure 3.14 FFR versus total bound rubber content. 
The flocculation rate has an upper limit of 0.16 . For Mix E, the compound without silane, the highest flocculation rates are expected. As the values here do not exceed 0.16, it is logical to assume that the maximum FFR limit is around 0.16. Besides, the curves of the samples containing TESPT and TESPD in Figure 3.12(a) at low silanization temperatures also show this value.

\subsubsection{Filler-polymer coupling rate after mixing}

The filler-polymer Coupling Rates (CR) of all mixing series are shown in Figure 3.15. As expected, the CR is zero for Mixes C, D and E, (TESO, PTEO and no silane), as a coupling reaction was not possible. The CR of the Ref. compound (with TESPT) and Mix B (with TESPD and sulfur adjustment), plotted as a function of the silanization temperature, show a decreasing trend with increasing temperature. In contrast to this, Mix A shows a constant and slow CR (approx. 0.05) for the whole silanization temperature range. The trend of Mix A is clear when the sulfur rank of TESPD is taken into account: the sulfur bridge in TESPD consists of app. $90 \%$ of disulfide, which means that it is a rather pure material compared to a mixture of sulfur ranks in TESPT. ${ }^{[41,42]}$ The disulfidic bridge is not reactive enough to give coupling to the polymer without additional sulfur added, as in Mix B. Therefore, the filler-polymer coupling reaction rate of Mix A remains low and the same at all silanization temperatures.

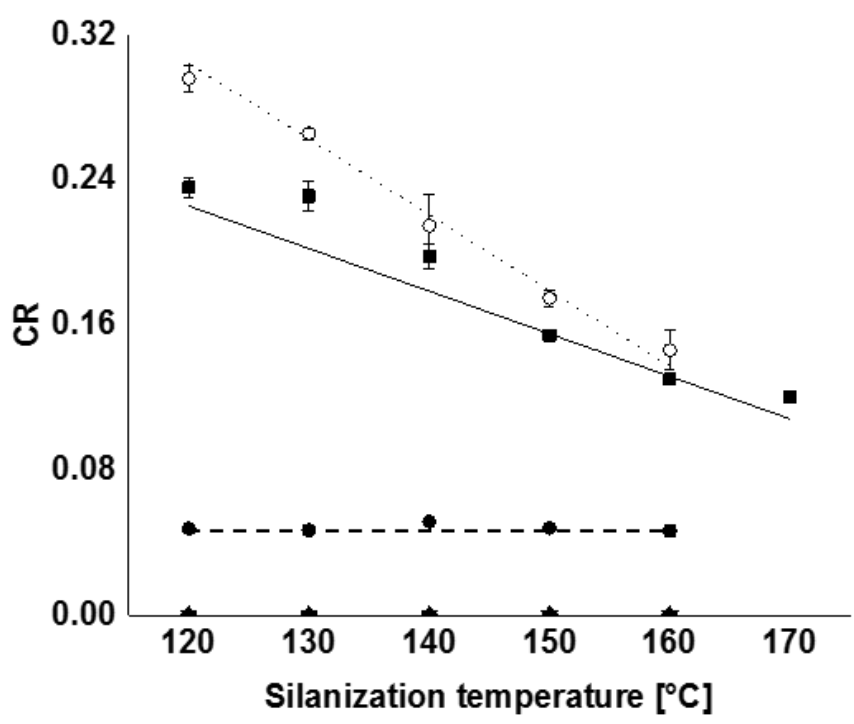

Figure 3.15 Filler-polymer coupling rate $(C R)$ of samples with different types of coupling agents vs. silanization temperature; ( 10 ): Ref.; ( - ): Mix A; $(\bigcirc \cdots \cdots)$ ): Mix B;

$(\nabla): \operatorname{Mix} C ;(\triangle): \operatorname{Mix} D ;(\bullet): \operatorname{Mix} E$. 
The results of the Ref. compound and Mix B clearly indicate that the presence of active sulfur enhances the speed and efficiency of the filler-polymer coupling reaction. At a higher silanization temperature, more active or free sulfur is available for filler-polymer bonding in this mixing step. As a result, the concentration of free sulfur will decrease when mixing is done at a higher temperature. Consequently, the $\mathrm{CR}$ will decrease due to the lowered concentration of active sulfur. Hasse et al. reported that activation of the silane consumes free sulfur. ${ }^{[39]}$ Additionally, they reported that the addition of sulfur increases the speed and efficiency of the filler-polymer coupling reaction. In case of the compound Ref., no free sulfur was added in the first mixing stage. However, TESPT is a mixture of silanes with various sulfur ranks from $S_{2}$ to $S_{10} \cdot{ }^{[42]}$ The longer sulfur bridges can donate active sulfur; this sulfur can in turn activate other silane molecules. ${ }^{[39,41]}$ This agrees well with the results shown here.

\subsubsection{Marching modulus intensity}

The Marching Modulus Intensities (MMI) measured at two different strains of all mixing series are plotted in Figure 3.16. 

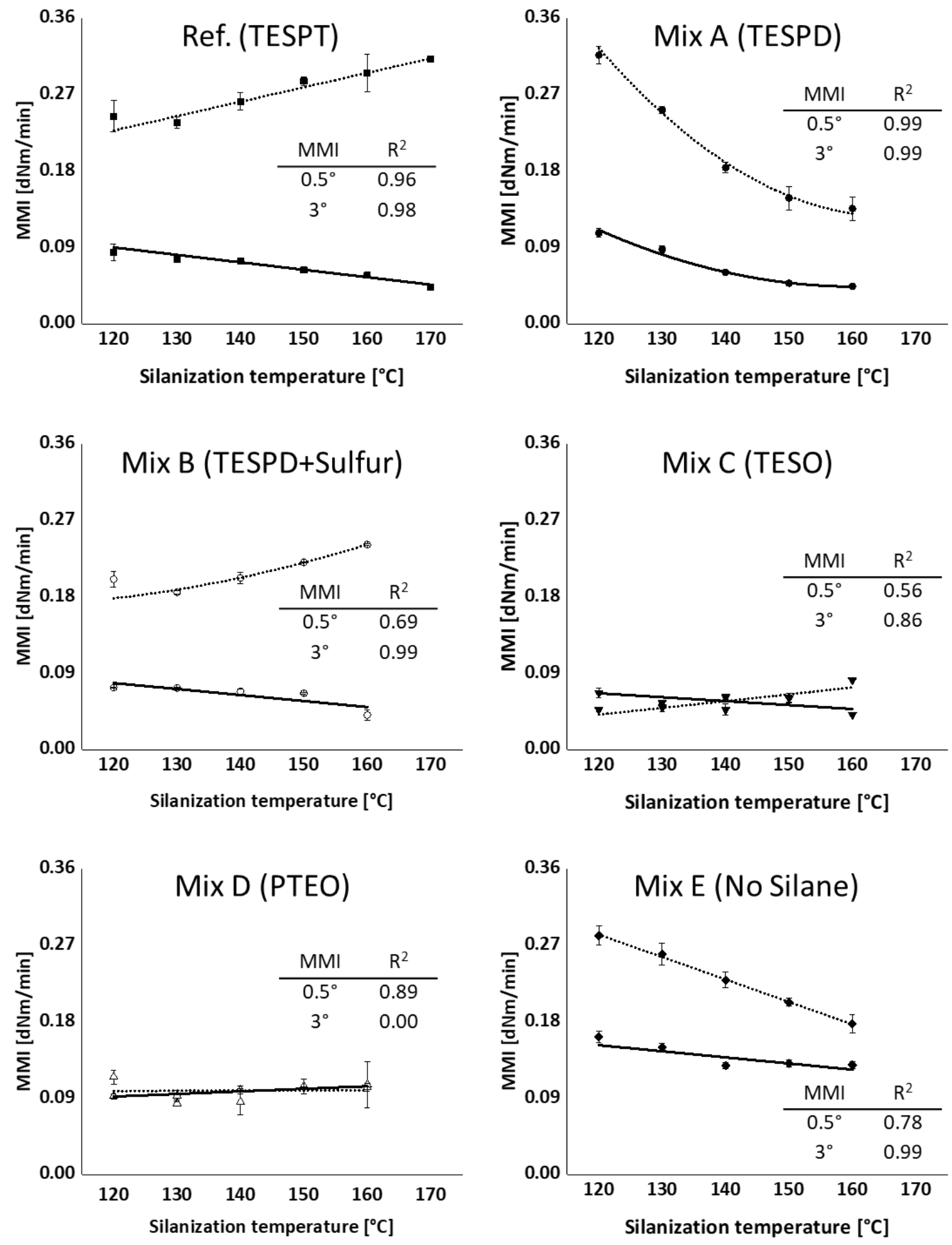

Figure 3.16 $\mathrm{MMI}$ of samples with different types of coupling agents vs. silanization temperature;

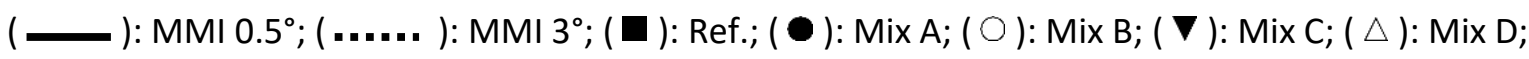
$(\bullet)$ : Mix E; fitting lines are based on polynomial or linear fit with maximum $\mathrm{R}^{2}$. 


\subsection{Marching modulus intensity at small (ASTM conditions, MMI $0.5^{\circ}$ ) and large strain $\left(\mathrm{MMI} 3^{\circ}\right)$}

Mix E has no silane which means that no reaction is possible between filler and polymer in this mixing series, and the highest MMI is observed in this mix. This indicates that the level of $0.5^{\circ}$ strain is particularly affected by filler-filler interaction. In addition, the MMI of $0.5^{\circ}$ of the Ref. compound (with TESPT) and Mix A (with TESPD) plotted as a function of the silanization temperature shows that increasing the latter lowers the $\mathrm{MMI} 0.5^{\circ}$. However, the trend of $\mathrm{MMI} 0.5^{\circ}$ cannot be fully explained with the intensity of fillerfiller interaction for all mixing series. Except for the Ref. compound and Mix A, MMI $0.5^{\circ}$ shows an almost constant level for the whole silanization temperature range for the other samples. In addition, it is interesting to note that Mix B does not follow the trend of Ref. compound and Mix A: the MMI $0.5^{\circ}$ is constant at first and only tends to decrease for the highest silanization temperature of $160^{\circ} \mathrm{C}$. This indicates that not only filler-filler interaction but also other factors affect the $\mathrm{MMI} 0.5^{\circ}$, as will be discussed later.

A different trend is observed in the $\mathrm{MMI}$ measurements done at a strain of $3^{\circ}$ or $42 \%$. Fröhlich and Luginsland ${ }^{[43]}$ reported that the breakage of the silica network starts at a higher strain amplitude, and the breakage takes place in a wider strain range than for a conventional filler such as carbon black. Their result agrees well with Figure 3.17. Therefore, it was expected that a large part of the filler-filler network will be broken when the filled rubber compound is subjected to large deformations, as shown in Figure 3.17; all storage moduli are the same at that level of strain. Consequently it may be stated that only filler-polymer and polymer-polymer interactions are active at the strain of $42 \%$.

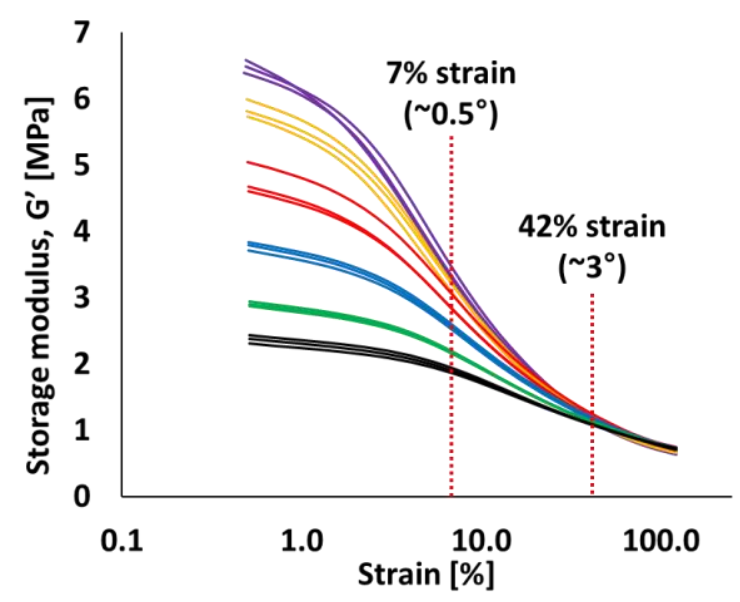

Figure 3.17 Examples of Payne effect curves: cured Ref. compound;

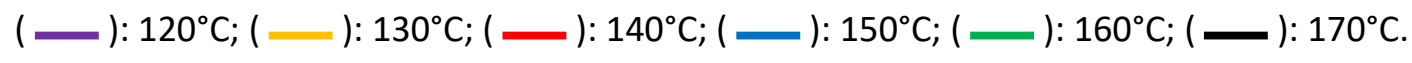


As already seen in Figure 3.9, no filler-polymer interaction was found in Mixes C and D. So only polymerpolymer interaction seems to exist for these mixes. As a result, these samples show an almost constant $\mathrm{MMI} 3^{\circ}$ for the whole silanization temperature range. Besides, the MMI values of these compounds do not depend on the measurement strain intensity: the levels of both MMI values are almost the same.

If, on the one hand, there is at least some filler-polymer interaction present, as a consequence of active or free sulfur in the compound because of release from the coupling agent TESPT (Ref.) or by sulfur added (Mix B), there is an increase of the $\mathrm{MMI} 3^{\circ}$ as a function of silanization temperature. On the other hand, a decreasing trend of the $\mathrm{MMI} 3^{\circ}$ along with increasing silanization temperature is observed for Mixes $\mathrm{A}$ and $\mathrm{E}$. These combined results demonstrate that the presence of free sulfur in the compound influences the polymer-filler as well as polymer-polymer interaction, while in the absence only polymer-polymer interaction plays a role.

As a rheogram according to ASTM standard D5289-95 commonly corresponds to MMI $0.5^{\circ}$, the free sulfur effect seen with the $\mathrm{MMI} 3^{\circ}$ is not of particular relevance but can be used as an evidence that MMI $0.5^{\circ}$ can be affected by the degree of filler-filler interaction. For the $\mathrm{MMI} 0.5^{\circ}$, a higher silanization temperature and consequently the degree of silanization has a positive effect on reducing the marching modulus irrespective of whether there is free sulfur present or not.

\subsection{Marching modulus intensity as a function of FFR and CR}

A correlation can be made between the MMI and FFR and CR as shown in Figures 3.18 and 3.19, respectively. As was seen in Figure 3.12(b), in case of Mix C and Mix E (TESO and no silane), the FFR values were at the maximum level of 0.16 . Therefore, no correlation could be made with both $\mathrm{MMI}$, because the FFR values were fixed while the MMI varied along with silanization temperature. Mix D (PTEO) showed a weak correlation $\left(R^{2}=0.82\right)$ between $\mathrm{MMI} 0.5^{\circ}$ and FFR, and is not depicted in Figure 3.18. Samples Ref., Mix $A$ and Mix $B$ show a good correlation of $M M I 0.5^{\circ}$ with FFR values: an $R^{2}$ higher than 0.9 . This indicates that silica flocculation is happening during the vulcanization process for all compounds, but mostly for the Ref. compound and mixes A and B. The degree of agglomeration has an influence on the viscosity of the rubber compounds. ${ }^{[44]}$ Thus, the viscosity will increase faster, the quicker the filler particles flocculate. Due to this, the shielding of the filler surface not only by a silane coupling agent but also by bound rubber will strongly affect the rheogram of a silica compound. No correlation was found between FFR and MMI $3^{\circ}$. 


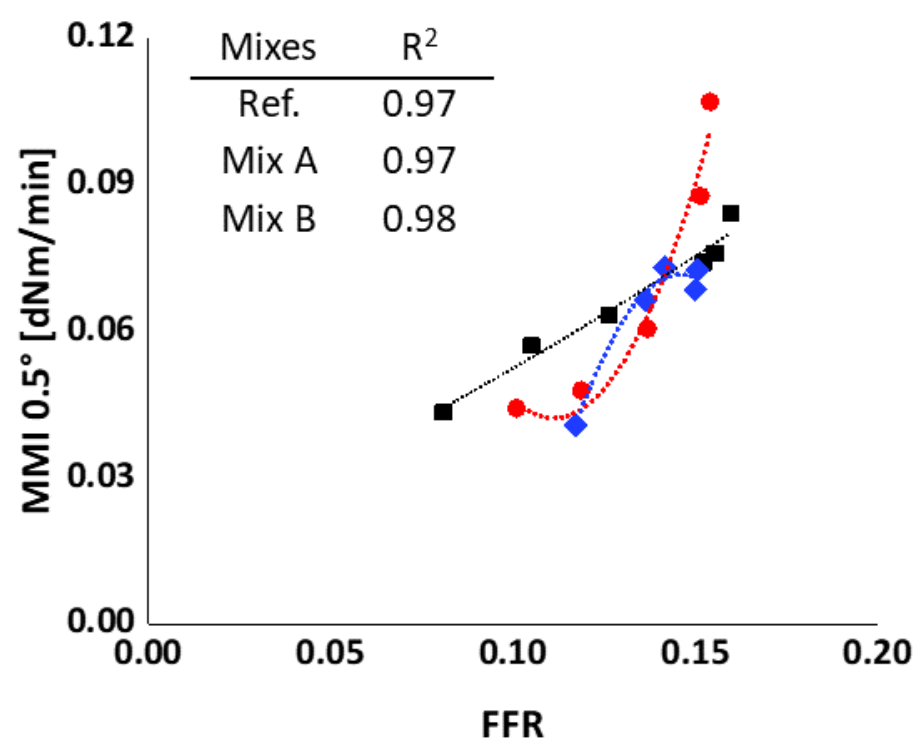

Figure 3.18 MMI 0.5 versus FFR; ( $\cdots \square$ ): Ref.; $(\bullet \cdots \cdots)$ ): Mix A; $(\diamond \cdots \cdots)$ ): Mix B; fitting lines are based on polynomial or linear fit with maximum $\mathrm{R}^{2}$.

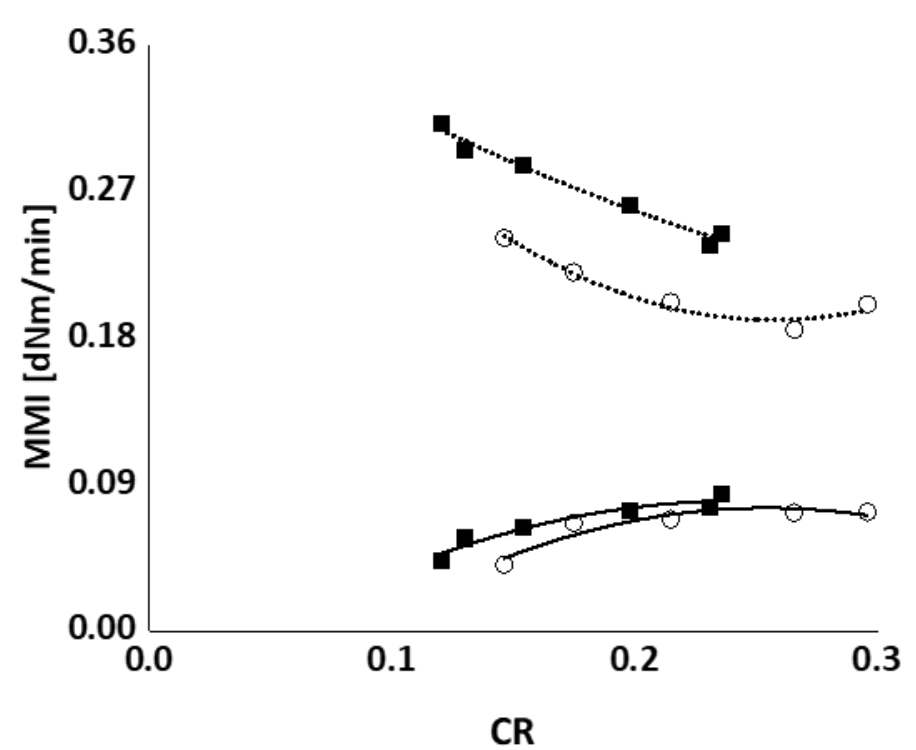

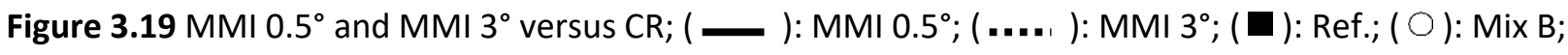
fitting lines are based on polynomial or linear fit with maximum $\mathrm{R}^{2}$.

$\mathrm{CR}$ is another important factor for the $\mathrm{MMI} 0.5^{\circ}$ and $\mathrm{MMI} 3^{\circ}$, when the compounds satisfy two conditions: Firstly, they should contain a silane with two functionalities: silanization and filler-polymer coupling. 
Secondly, additional active sulfur is required. Without active sulfur, the filler-polymer coupling reaction rate will be limited to a low level (Figure 3.15, Mix A with TESPD), and will not have a sizable effect on the MMI. When the filler-polymer interaction is established during the curing period, a fast filler-polymer reaction will lead to a quick increase of the torque level at the beginning of the measurement, overlying the torque increase due to the network formation.

However, the trends are depending on the intensity of the measurement strain in the rheometer. To further substantiate this, the rheograms of the Ref. mixing series at different silanization temperatures are shown in Figure 3.20 as examples. At small strains ( 7\%), a higher torque is measured for samples prepared at lower silanization temperatures, induced by more filler-filler interaction as a prevailing factor to the rheometer torque. However, at large strain ( $\sim 2 \%)$, the level of torque at 40 minutes is almost the same for all samples, except for the sample silanized at $170^{\circ} \mathrm{C}$, as the effect of filler-filler interaction is grossly absent at such a high deformation.
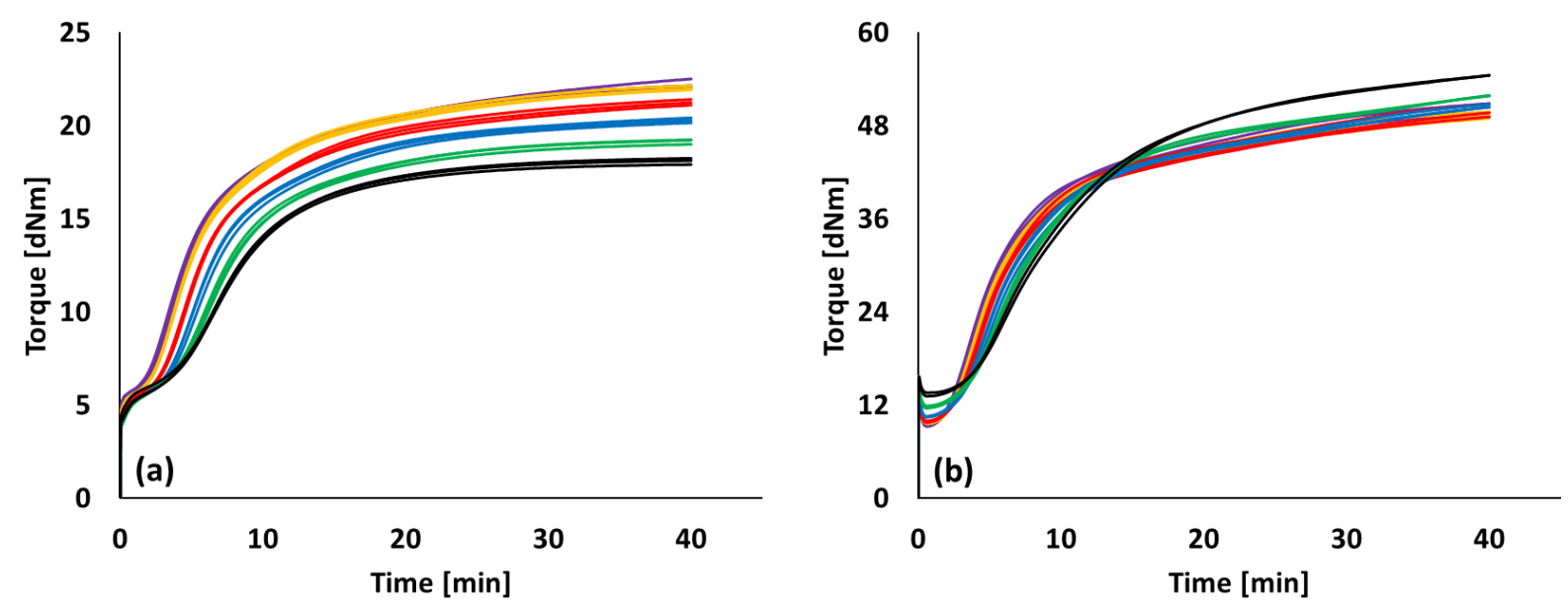

Figure 3.20 Rheograms of Ref. compound; (a): with $0.5^{\circ}$ of strain; (b): with $3^{\circ}$ of strain;

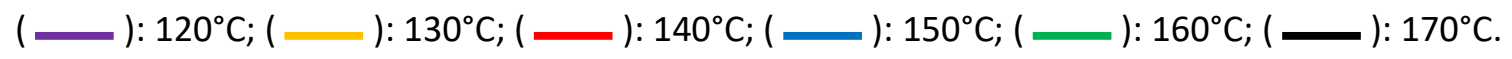

\subsection{The effect of crosslink density (swelling ratio) on marching modulus}

Marching modulus was always understood as a result of further formation of sulfur crosslinks. However, when the amount of sulfur in all mixes is taken into account (Table 3.2), it must be concluded that the crosslink density is not the major factor for the marching modulus phenomenon of the silica compounds. If the crosslink density would have been the major factor for marching modulus, then Mixes C (TESO), D (PTEO), and E (no silane) should have shown the same level of marching modulus intensity. TESO and 
PTEO cannot incorporate any sulfur into their chemical structure like TESPT or TESPD can. Therefore, the concentration of free sulfur was the same for all these mixes. However, these mixes did show major differences in marching modulus (Figure 3.16), which therefore must have another origin than increasing crosslink density: filler-filler interaction.

Besides, in case of Mix A (TESPD), all free sulfur for crosslinking was introduced in the final stage of mixing. The only variable in the Mix A series was the silanization temperature. A clear decreasing trend can be seen in $\mathrm{MMI} 0.5^{\circ}$ though along with silanization temperature: Figure 3.16. This result indicates again that other factors besides crosslink density influence the marching modulus phenomenon: degree of silanization, FFR, and CR.

However, in order to investigate the contribution of crosslink density on $\mathrm{MMI}$ level, swelling ratios $\left(\mathrm{Q}_{\mathrm{CT}}\right.$, $\mathrm{Q}_{20}$ and $\mathrm{Q}_{30}$ ) of all mixes were analyzed in Figure 3.21. The subscripts indicate the sample curing time: Calculated cure Time (CT), 20 minutes and 30 minutes, respectively. Comparing Figures 3.16 and 3.21 gives additional evidence that the crosslink density is not the major factor for the marching modulus phenomenon of the silica compounds. As can be seen in Figure 3.21, the compounds with sulfidic silane (Ref. compound, Mixes A and B) - which initiate filler-polymer coupling - show lower $Q$ values compared to the compounds with non-sulfidic silanes (Mixes C, D) or the silane free compound (Mix E). In other words, Mixes C, D and E have less crosslinks, because a higher $Q$ value indicates a lower crosslink density. However, the highest $\mathrm{MMI} 0.5^{\circ}$ was observed in Mix $E$, and Mixes $C$ and $D$ does not show same level of MMI 0.5 (Figure 3.16): Mixes C, D are silanized the least. Moreover, Ref. compound showed a decreasing trend in $\mathrm{MMI} 0.5^{\circ}$ along with silanization temperature (Figure 3.16); however, $\mathrm{Q}$ values of this series were almost constant (Figure 3.21). Additionally, the $Q_{C T}, Q_{20}$ and $Q_{30}$ values of Ref. compound do not show a significant difference. Overall, these results again clearly indicate that the crosslink density is not the major factor for the marching modulus phenomenon of the silica compounds. 

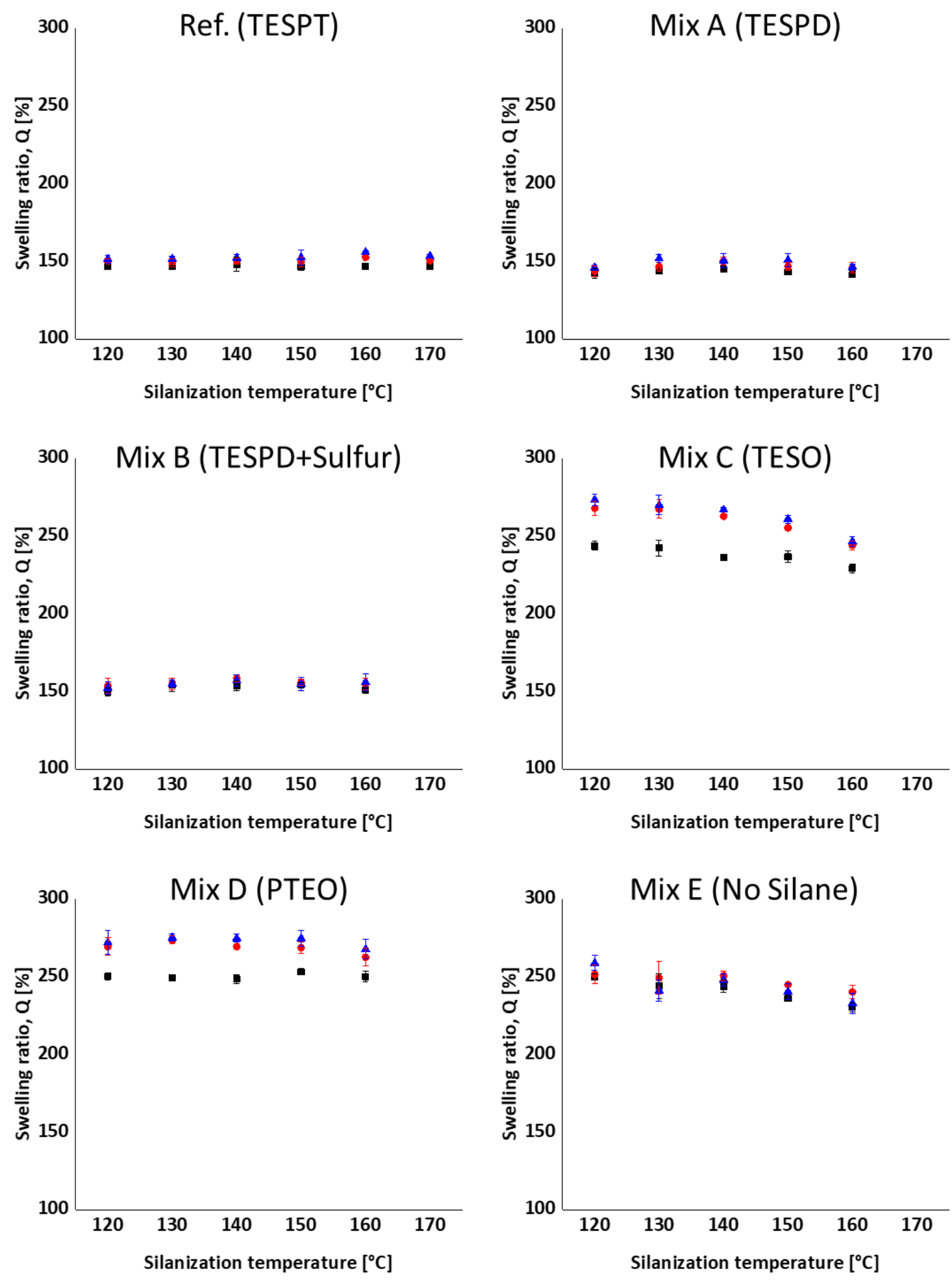

Figure 3.21 Swelling ratio of all Mixes; $(\boldsymbol{\square})$ : $\mathrm{Q}_{\mathrm{cT}} ;(\boldsymbol{O}): \mathrm{Q}_{20} ;(\boldsymbol{\Delta}): \mathrm{Q}_{30}$. 


\subsubsection{Conclusions}

The focus of this study was to elucidate the causes of marching modulus under ASTM conditions in a rheometer during curing of silica filled S-SBR/BR rubber compounds. Three factors: the intensity of fillerfiller interaction, the silica flocculation rate and the filler-polymer coupling rate turned out to be the major influencing factors on the marching modulus phenomenon of silica compounds, in order of decreasing importance. All three are the consequence of proper silanization of the silica during mixing as shown in Figure 3.22. When silica is not properly silanized, the curing process cannot repair that. Unexpectedly, the total crosslink density was not a major influencing factor for the marching modulus phenomenon.

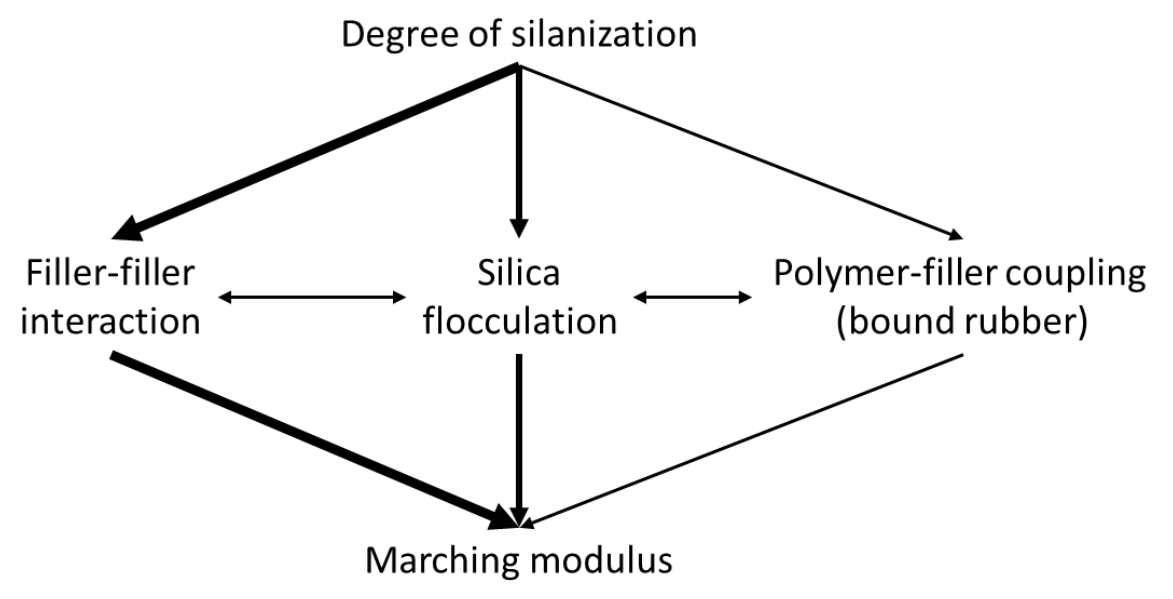

Figure 3.22 Silanization as a key factor for marching modulus for silica filled rubber compounds.

While a silica-filled rubber compound undergoes vulcanization, silica flocculation occurs at the same time; the more, the lower the degree of silanization. There is a lower limit of total bound rubber of $15 \%$, below which the flocculation is at its upper level and does not show a trend anymore with marching modulus. The polymer-filler coupling rate during the curing process contributes as well to the marching modulus intensity. When the compound shows a fast coupling rate, the compound becomes quickly stiffer and a higher torque is measured in the rheometer. As a consequence, the fast increase of the torque level also limits flocculation. However, the coupling rate is only an effective factor in the presence of active sulfur. The addition of free sulfur or its release from TESPT speeds up the filler-polymer coupling reaction, because the activation of the silane consumes free sulfur. Additionally, the reactivity of a sulfur-bridged silane increases with increasing sulfur moiety length beyond $S_{2}$.

All in all, both filler flocculation and polymer-filler coupling are strongly influenced by the mixing conditions, in particular the silanization. Mixing time and temperature are the crucial factors. 


\section{References}

[1] U. Goerl, A. Hunsche, A. Mueller, H. G. Koban, Rubber Chem. Technol. 70, 608 (1997).

[2] U. Görl, A. Hunsche, 150th meeting Rub. Div. ACS Louisville, paper no. 76 , Kentucky (1996).

[3] G. Heinrich, T. A. Vilgis, Kautsch. Gummi Kunstst. 61, 368 (2008).

[4] M-J. Wang, Rubber Chem. Technol. 71, 520 (1998).

[5] C. Jr. Lin, W. L. Hergenrother, A. S. Hilton, Rubber Chem. Technol. 75, 215 (2002).

[6] L. A. E. M. Reuvekamp, J. W. ten Brinke, P. J. van Swaaij, J. W. M. Noordermeer, Rubber Chem. Technol. 75, 187 (2002).

[7] L. Ladouce-Stelandre, Y. Bomal, L. Flandin, D. Labarre, Rubber Chem. Technol. 76, 145 (2003).

[8] K-J. Kim, J. VanderKooi, Rubber Chem. Technol. 78, 84 (2005).

[9] W. K. Dierkes, J. W. M. Noordermeer, M. Rinker, K.-U. Kelting, C. Van de Pol, Kautsch. Gummi Kunstst. 56, 338 (2003).

[10] P. Manoharan, M. G. Anagha, N. C. Das, K. Naskar, Rubber Chem. Technol. 91, 548 (2018).

[11] Y. Li, M. J. Wang, T. Zhang, F. Zhang, X. Fu, Rubber Chem. Technol. 64, 693 (1994).

[12] S. S. Sarkawi, W. K. Dierkes, J. W. M. Noordermeer, Rubber Chem. Technol. 87, 103 (2014).

[13] H-D. Luginsland, J. Fröhlich, A. Wehmeier, Rubber Chem. Technol. 75, 563 (2002).

[14] S. Mihara, R. N. Datta, J. W. M. Noordermeer, Rubber Chem. Technol. 82, 524 (2009).

[15] W. Kaewsakul, K. Sahakaro, W. K. Dierkes, J. W. M. Noordermeer, Rubber Chem. Technol. 85, 277 (2012).

[16] W. Kaewsakul, K. Sahakaro, W. K. Dierkes, J. W. M. Noordermeer, Rubber Chem. Technol. 87, 291 (2014).

[17] S. S. Sarkawi, W. K. Dierkes, J. W. M. Noordermeer, Rubber Chem. Technol. 88, 359 (2015).

[18] C. Hayichelaeh, L. A. E. M. Reuvekamp, W. K. Dierkes, A. Blume, J. W. M. Noordermeer, K. Sahakaro, Rubber Chem. Technol. 91, 433 (2018).

[19] M. V. Braum, M. A. M. Jacobi, Rubber Chem. Technol. 92, 431 (2019).

[20] J. M. Collins, W. L. Jackson, P. S. Oubridge, Rubber Chem. Technol. 38, 400 (1965).

[21] W. H. Waddell, Rubber Chem. Technol. 69, 377 (1996).

[22] W. H. Waddell, R. C. Napier, R. R. Poulter, Kautsch. Gummi Kunstst. 54, 181 (2001).

[23] S. Futamura, Rubber Chem. Technol. 64, 57 (1991).

[24] H.-D. Luginsland, A. Hasse, 157th meeting Rub. Div. ACS Dallas, Texas, paper 34 (2000).

[25] J. W. ten Brinke, P. J. van Swaaij, L. A. E. M. Reuvekamp, J. W. M. Noordermeer, Rubber Chem. Technol. 76, 12 (2003). 
[26] L. Guy, S. Daudey, P Cochet, Y. Bomal, Kautsch. Gummi Kunstst. 63, 383 (2009).

[27] A. R. Payne, Rubber Chem. Technol. 39, 365 (1966).

[28] A. R. Payne, R. E. Whittaker, Rubber Chem. Technol. 44, 440 (1971).

[29] J. H. Bachmann, J. W. Sellers, M. P. Wagner, R. F. Wolf, Rubber Chem. Technol. 32, 1286 (1959).

[30] J. Jin, J. W. M. Noordermeer, W. K. Dierkes, A. Blume, poster 28 at the Deutsche Kautschuk-Tagung, Nuremberg, Germany (2018).

[31] J. Jin, J. W. M. Noordermeer, W. K. Dierkes, A. Blume, Rubber Chem. Technol. in press (2019). https://doi.org/10.5254/rct.19.80453

[32] ASTM Standard D5289-95, "Standard Test Method for Rubber Property-Vulcanization Using Rotorless Cure Meters", ASTM International (2001).

[33] S. Mihara, PhD Thesis: Reactive processing of silica-reinforced tire rubber: new insight into the time- and temperature-dependence of silica rubber interaction, Univ. Twente, Enschede, the Netherlands (2009).

[34] S. Wolff, M. J. Wang, E-H. Tan, Rubber Chem. Technol. 66, 163 (1993).

[35] ASTM D471-06, "Standard Test Method for Rubber Property-Effect of Liquids", ASTM International (2006).

[36] S-H, Lim, S. Lee, N. Lee, B. K. Ahn, N. Park, W. Kim, Elastomers and Composites 51, 81 (2016).

[37] A. Blume, S. Uhrlandt, 157th meeting Rub. Div. ACS, Dallas, Texas, paper 32 (2000); Kautsch. Gummi Kunstst. 54, 520 (2001).

[38] S-S. Choi, E. Ko, Polymer Testing 40, 170 (2014).

[39] A. Hasse, O. Klockmann, A. Wehmeier, H.-D. Luginsland, Kautsch. Gummi Kunstst. 55, 236 (2002).

[40] K. W. Stöckelhuber, A. S. Svistkov, A. G. Pelevin, G. Heinrich, Macromolecules 44, 4366 (2011).

[41] "Rubber compounding: Chemistry and applications, 2nd Edition", B. Rodgers, CRC Press, New York (2016).

[42] H.-D. Luginsland, Kautsch. Gummi Kunstst. 53, 10 (2000).

[43] J. Fröhlich, H. D. Luginsland, oral presentation at the Kautschuk-Herbst-Kolloquium DIK, Hannover, Germany (2000).

[44] N. Tokita, I. Pliskin, Rubber Chem. Technol. 46, 1166 (1973). 
Chapter 3.1 


\section{Chapter 3.2 The effect of marching modulus phenomenon on the mechanical properties alteration of silica filled rubber}

\subsubsection{Introduction}

This chapter deals with the effect of marching modulus on the consistency as well as the level of the mechanical properties and reinforcement of silica filled rubber. The mechanical properties, such as the Moduli at strains of 100\% (M100) and 200\% (M200), reinforcing index (M200/M100), Tensile Strength (TS) and Elongation at break (Eb) of the rubber vulcanizates in chapter 3.1 are evaluated. In order to observe the effect of $\mathrm{MMI}$ on the consistency of the mechanical properties, the vulcanizates are prepared with three different curing times: Calculated cure Time (CT, see Figure 3.6), 20 minutes and 30 minutes. The property alteration is analyzed along with the curing time as well as with the silanization temperatures. Additionally, the influencing factors affecting the properties are investigated.

\subsubsection{Experimental}

\subsubsection{Tensile properties}

According to ISO 37, ${ }^{[1]}$ type 2 dumbbell test specimens of all Mixes of Chapter 3.1 were prepared using three different curing times: Calculated cure Time (CT), 20 minutes and 30 minutes. The tensile properties (i.e., modulus at different strains, tensile strength, and elongation at break) were tested with a Zwick tensile tester model Z1.0/TH1S (Zwick Roell Group, Ulm, Germany) at a cross-head speed of $500 \mathrm{~mm} / \mathrm{min}$ according to ISO 37. ${ }^{[1]}$ The moduli at $300 \%$ strain (M300) was not taken into account in this work because some of the samples were broken before they reached $300 \%$ strain.

\subsubsection{Mooney-Rivlin plot}

Limited polymer chain extensibility, evaluated by the Mooney-Rivlin equation, gives additional information on the filler-polymer interaction of vulcanizates. ${ }^{[2-7]}$ The Mooney-Rivlin equation is shown in Equation 3.8: ${ }^{[4]}$

$$
\sigma^{*}=\frac{\sigma}{\lambda-\lambda^{-2}}=2 C_{1}+2 C_{2} \lambda^{-1}
$$

Where $\sigma$ is the stress and $C_{1}, C_{2}$ are constants independent of the extension ratio $(\lambda)$. The reduced stress 
$\left(\sigma^{*}\right)$ decreases until a flat region with a decline of the reciprocal of the extension ratio $\left(\lambda^{-1}\right)$, and then rises again. The $\lambda^{-1}$ value at the upturn point implies finite extensibility of polymer chains while stretching. ${ }^{[3]}$ In a better reinforcement (i.e., higher degree of filler-polymer coupling), the length of bridging polymer chains between adjacent filler particles become short and restricted, thus contributes to the modulus. Therefore, the $\lambda^{-1}$ value at the upturn point can be used as an indicator for the intensity of filler-polymer interaction as well as the crosslink network. By using mathematical software, the $\lambda^{-1}$ value where the slope of the tangential line of the Mooney-Rivlin plot becomes zero was selected as the $\lambda^{-1}$ value at the upturn point.

\subsubsection{Results and discussion}

\subsubsection{The relation between $\mathrm{MMI} 0.5^{\circ}$ and tensile properties alteration}

The Moduli at strains of 100\% (M100) and 200\% (M200), reinforcing index (M200/M100), Tensile Strength (TS) and Elongation at break (Eb) of the compounds are shown in Figures 3.23 to 3.27. Surprisingly, all the compounds within each Mix show only in a few cases a significant tensile property difference along with the cure time whether they have higher marching modulus intensity or not. Mix E which showed the highest $\mathrm{MMI} 0.5^{\circ}$ compared to the other mixes, shows almost constant tensile properties regardless of curing time. Mix A showed a different level of $\mathrm{MMI} 0.5^{\circ}$ but the tensile properties are almost constant for the whole range of silanization temperature and curing time. Mixes $C$ and $D$ show almost constant tensile properties regardless of curing time as well as the silanization temperature, even though their $\mathrm{MMI} 0.5^{\circ}$ values were not zero. Additionally, the maximum deviation with curing time in M100 is found for the sample silanized at $160^{\circ} \mathrm{C}$ in Mix B - but less than $1 \mathrm{MPa}$ - however when the $\mathrm{MMI} 0.5^{\circ}$ of the sample is taken into account, it is clear that $\mathrm{MMI} 0.5^{\circ}$ gives no information on tensile property differences along curing time: within Mix B, the lowest MMI $0.5^{\circ}$ was obtained for the sample silanized at $160^{\circ} \mathrm{C}$. 
Ref. (TESPT)

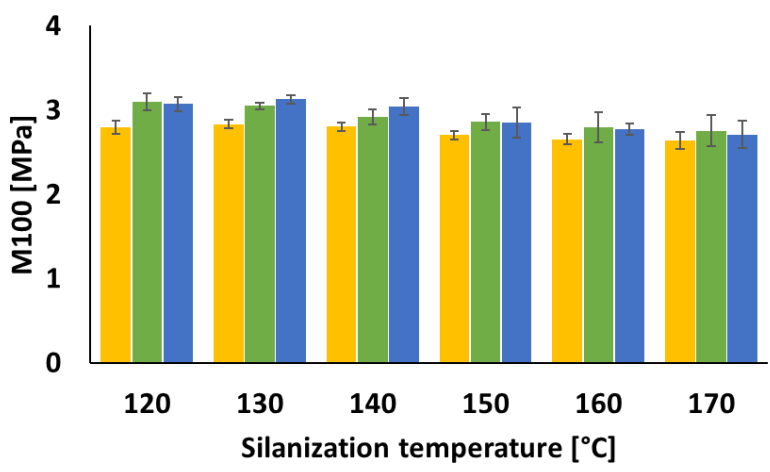

Mix B (TESPD+Sulfur)

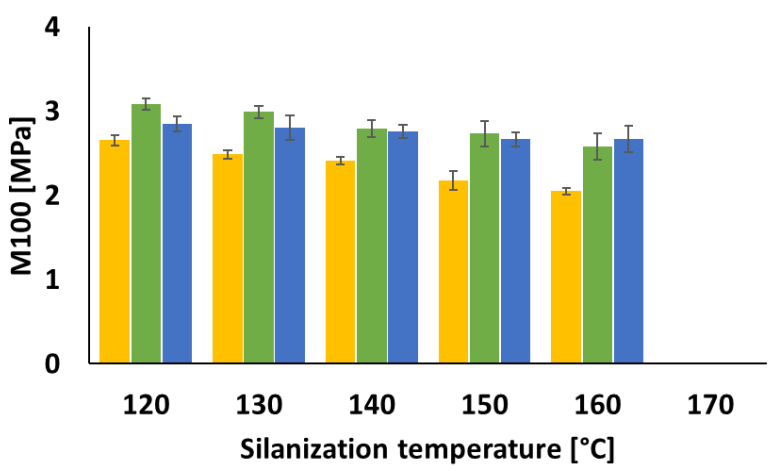

Mix D (PTEO)

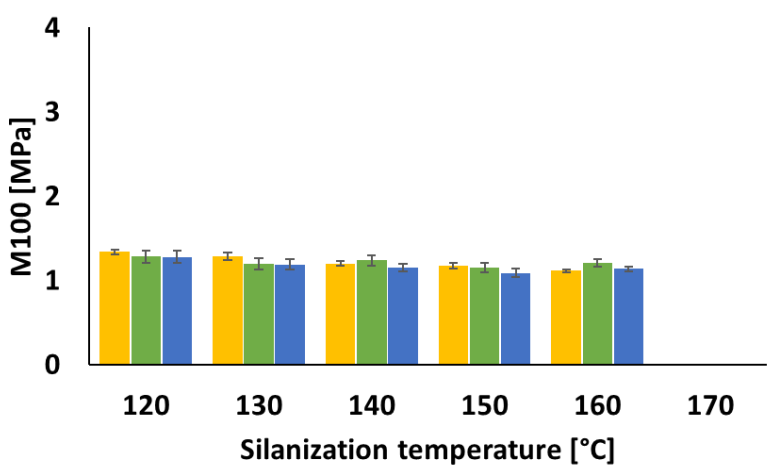

Mix A (TESPD)

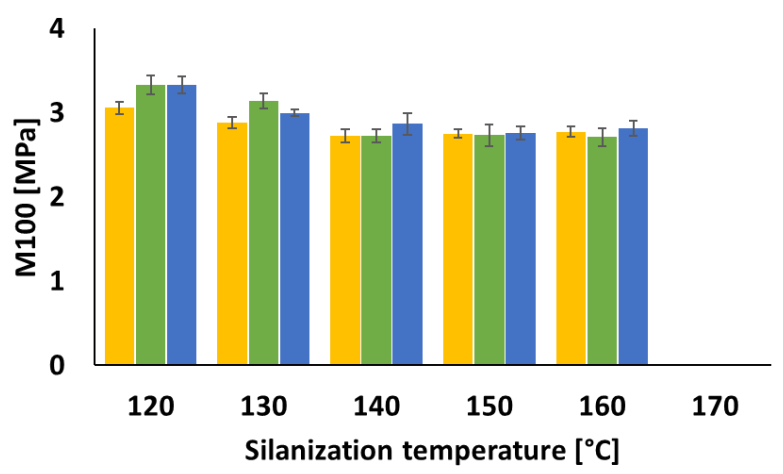

Mix C (TESO)

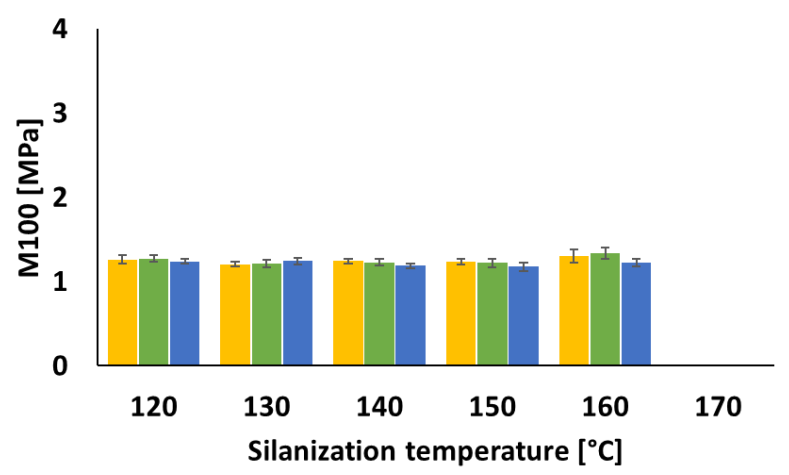

Mix E (No silane)

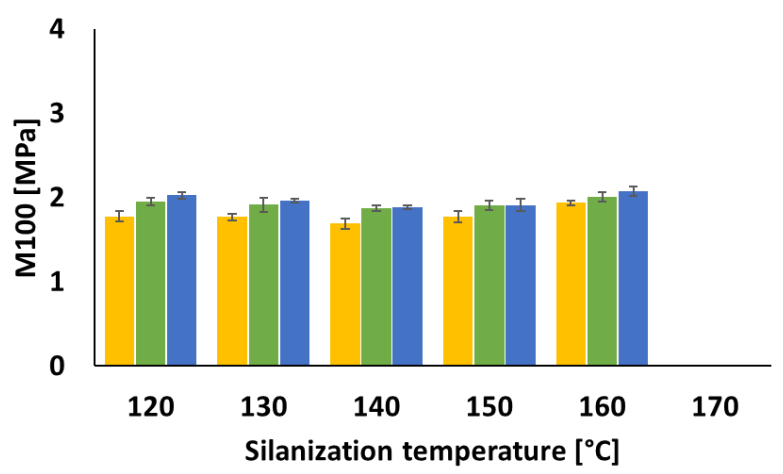

Figure 3.23 M100 with three different curing times; ( $\square$ ): CT; ( $\square$ ): 20 min; ( $\square$ ): 30 min. 
Ref. (TESPT)

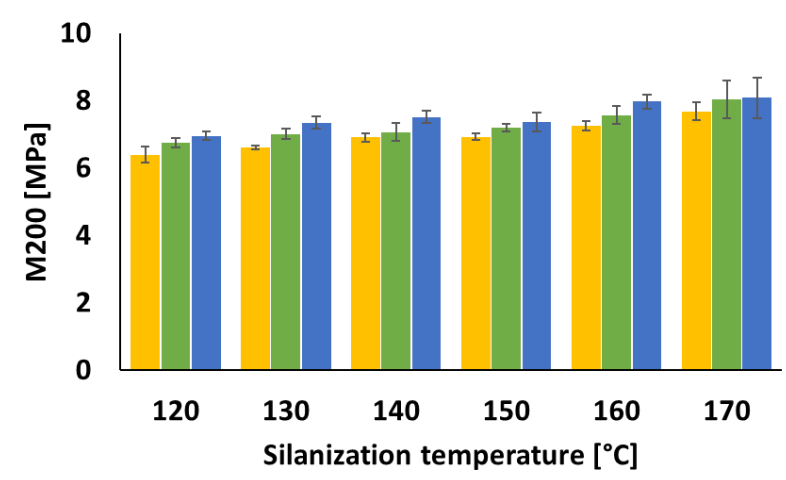

Mix B (TESPD+Sulfur)

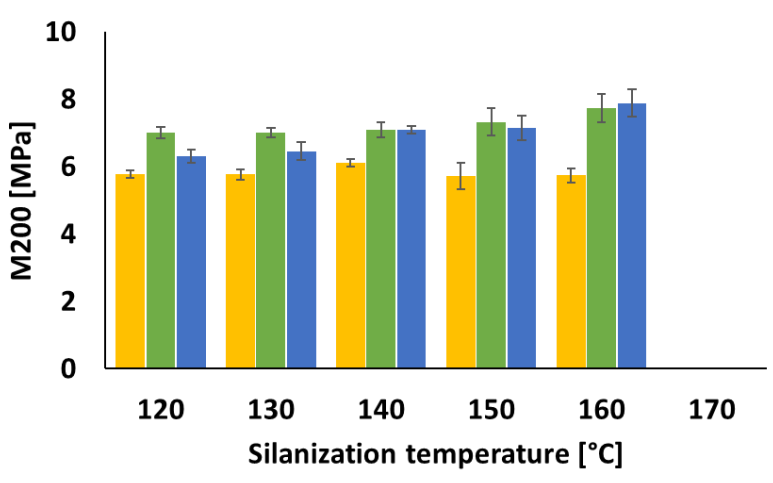

Mix D (PTEO)

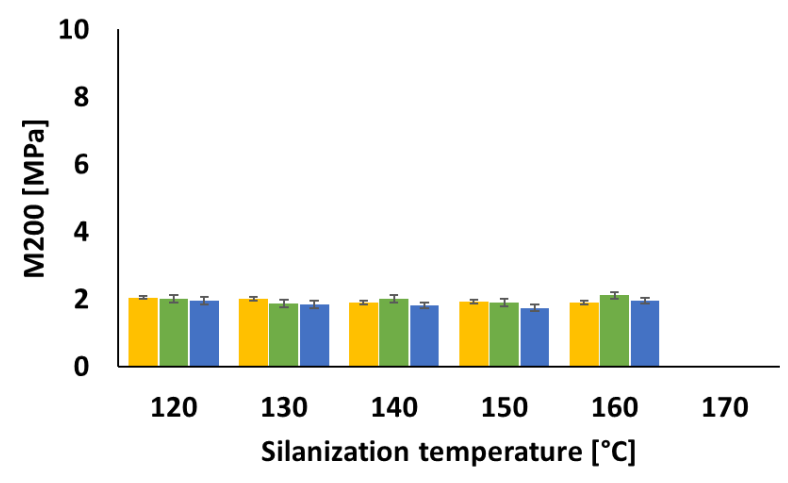

Mix A (TESPD)

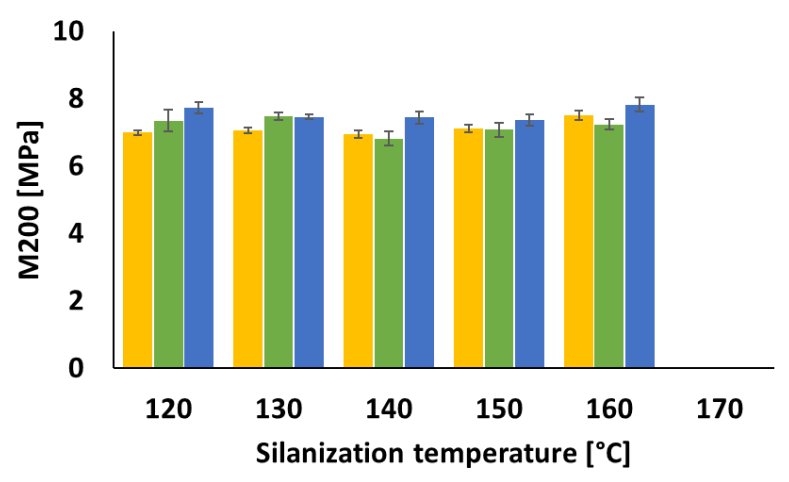

Mix C (TESO)

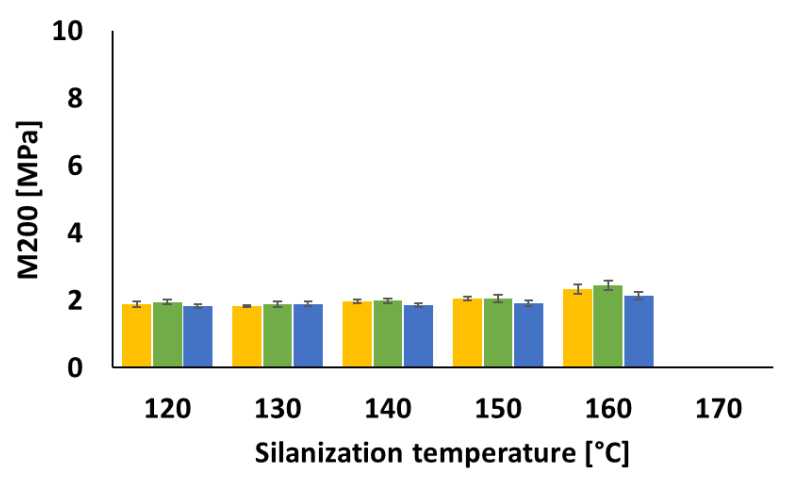

Mix E (No silane)

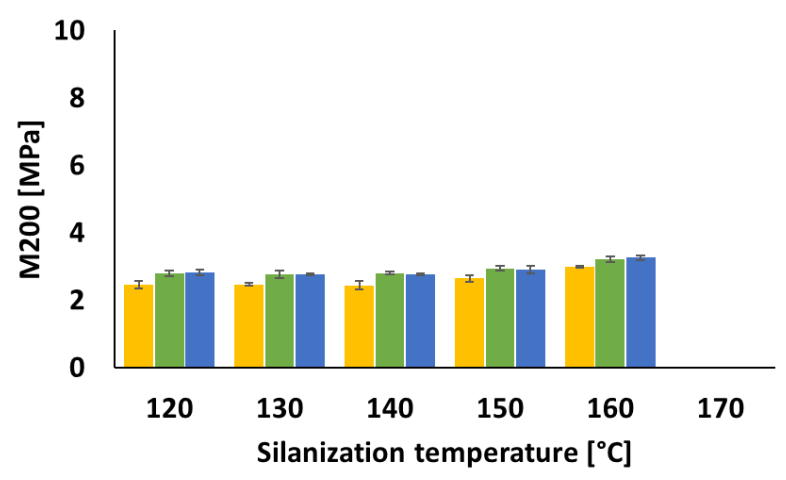

Figure 3.24 M200 with three different curing times; ( $\square$ ): CT; ( $\square$ ): 20 min; ( $\square$ ): 30 min. 
Ref. (TESPT)

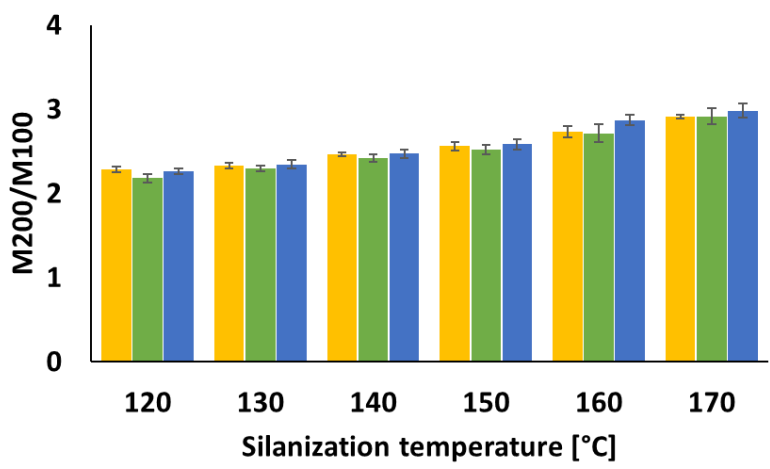

Mix B (TESPD+Sulfur)

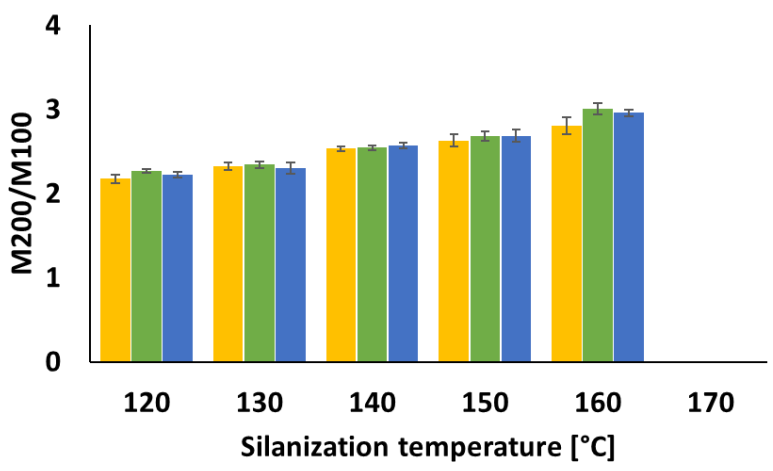

Mix D (PTEO)

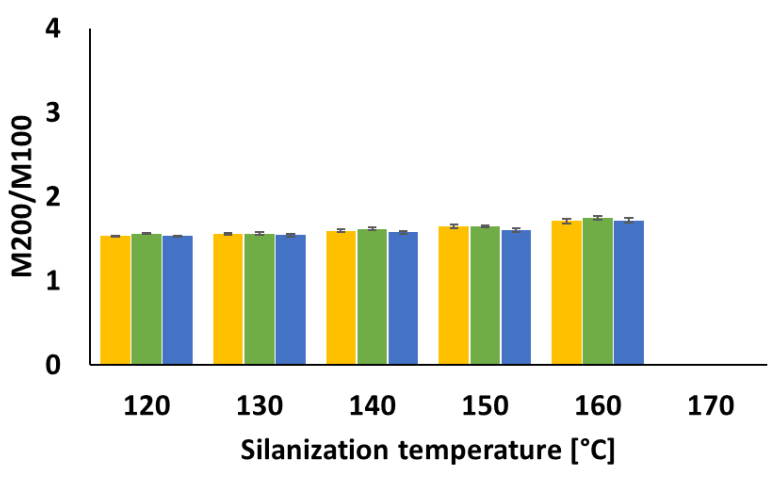

Mix A (TESPD)

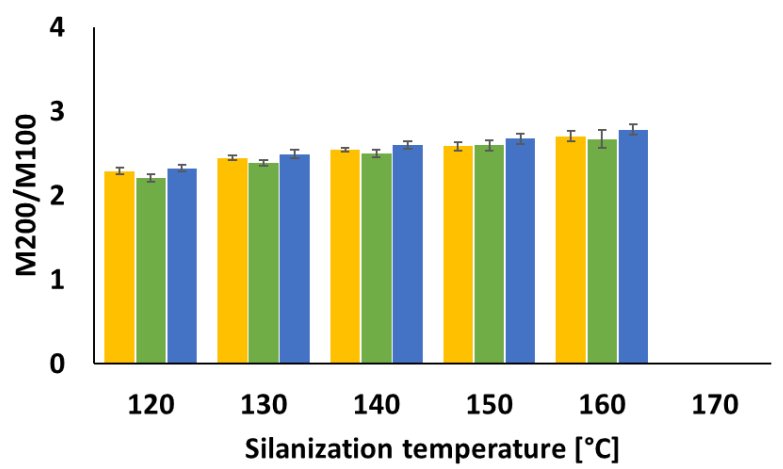

Mix C (TESO)

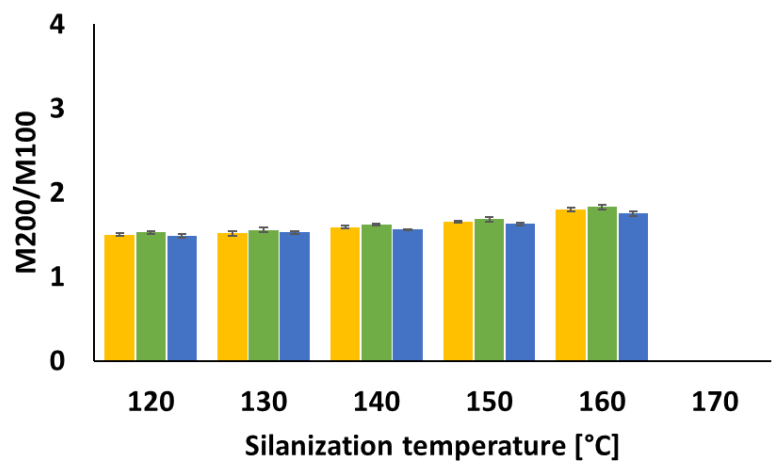

Mix B (No silane)

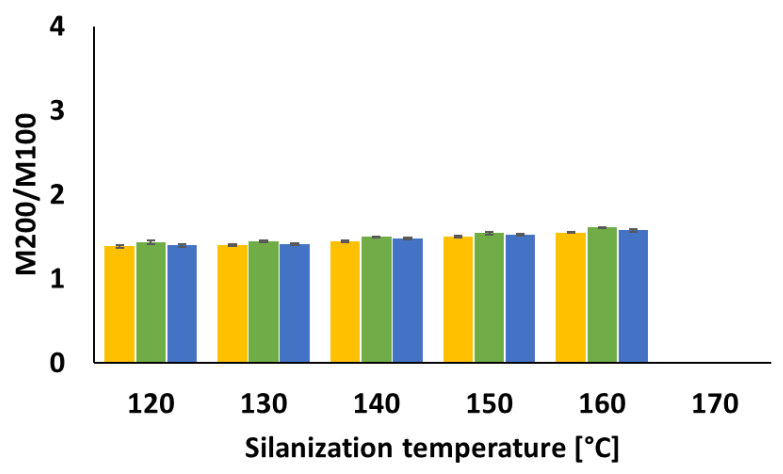

Figure 3.25 Reinforcement index (M200/M100) with three different curing times;

$(\square): \mathrm{CT} ;(\square): 20 \mathrm{~min} ;(\square): 30 \mathrm{~min}$. 
Ref. (TESPT)

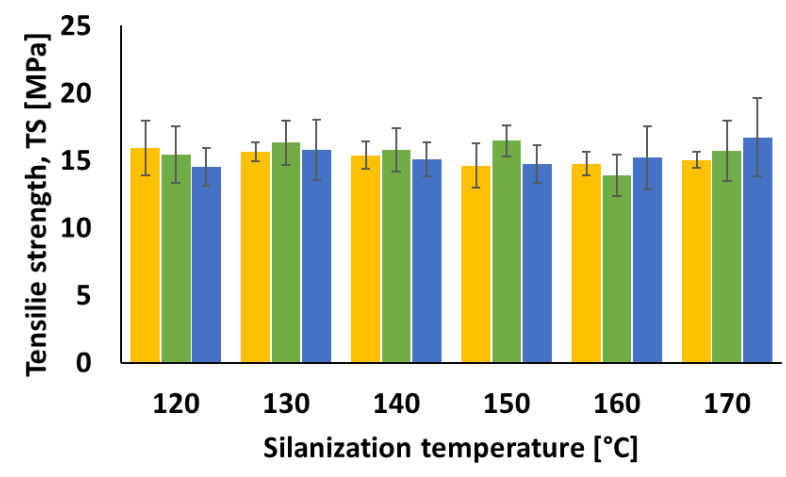

Mix B (TESPD+Sulfur)

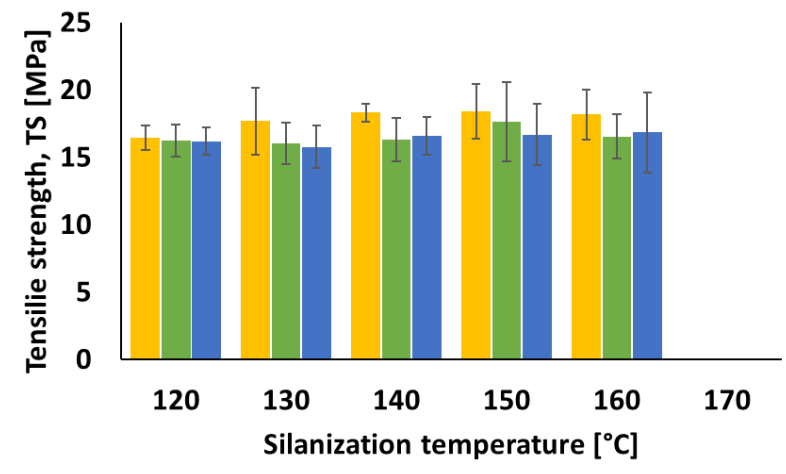

Mix D (PTEO)

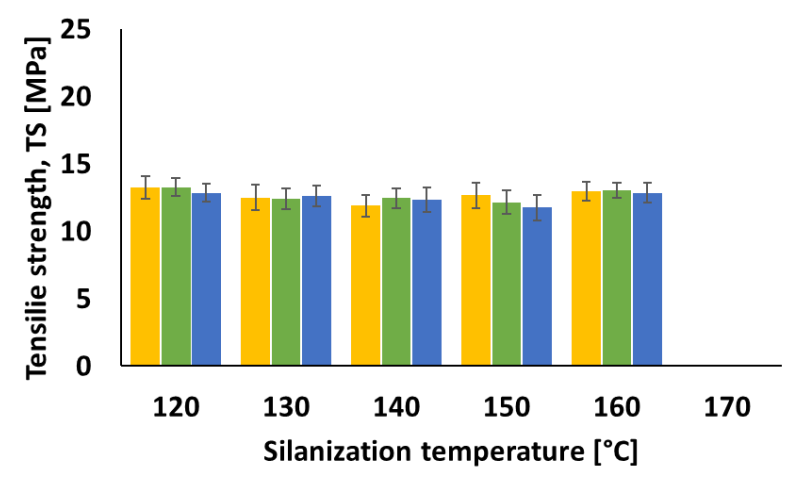

Mix A (TESPD)

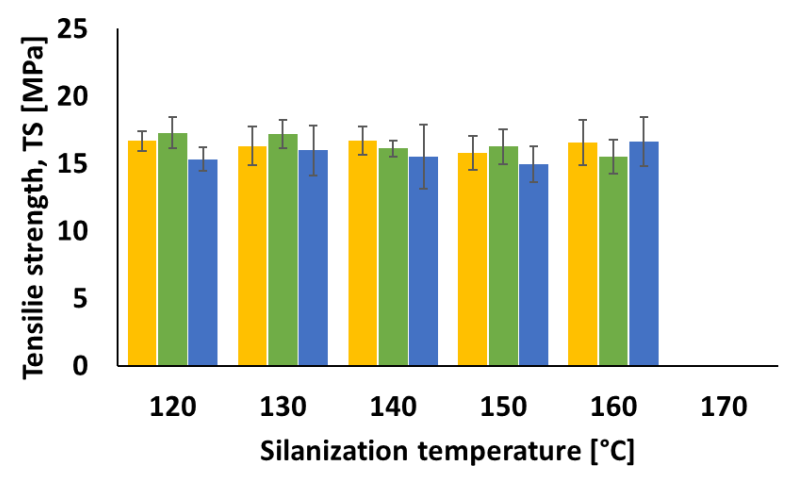

Mix C (TESO)

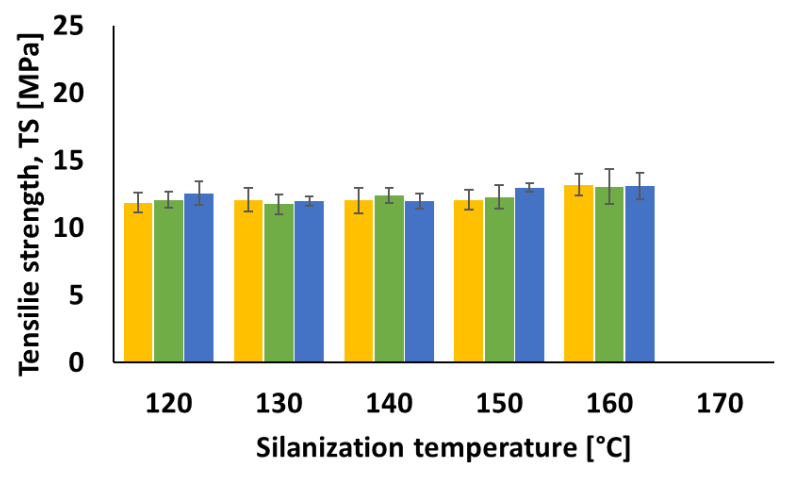

Mix E (No silane)

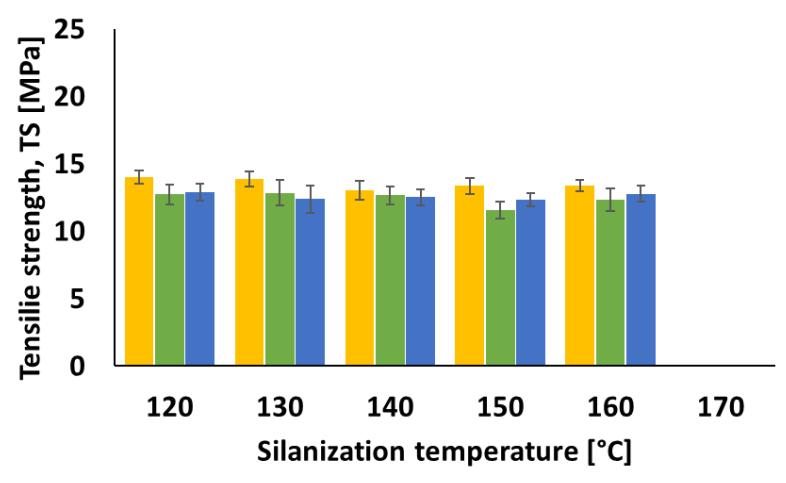

Figure 3.26 Tensile strength (TS) with three different curing times;

$(\square)$ ): CT; ( $\square$ ): $20 \mathrm{~min}$; ( $\square$ ): $30 \mathrm{~min}$. 
Ref. (TESPT)

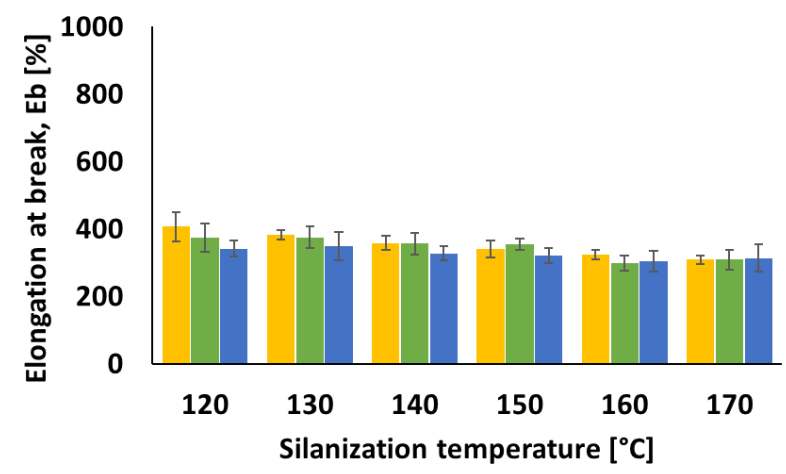

Mix B (TESPD+Sulfur)

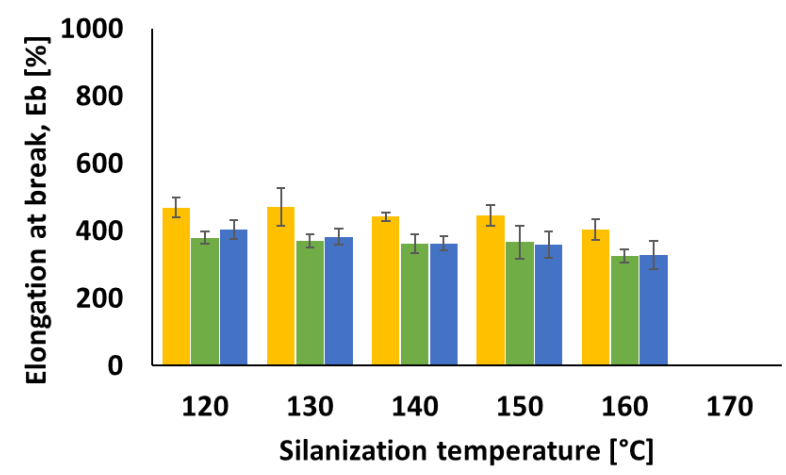

Mix D (PTEO)

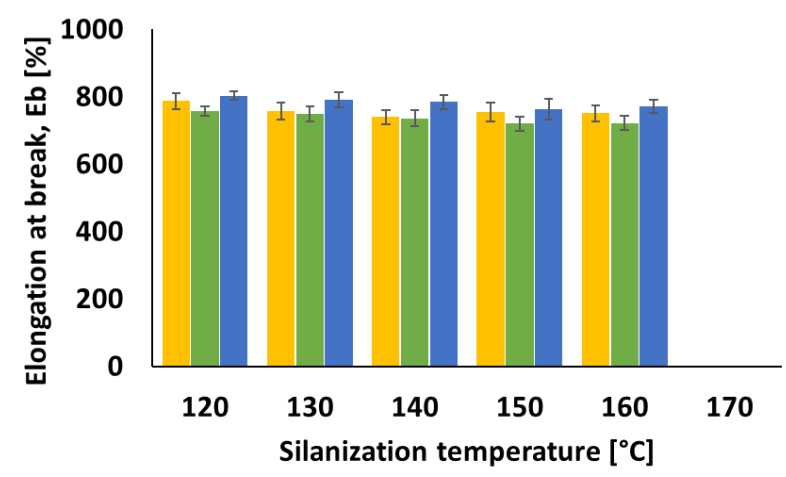

Mix A (TESPD)

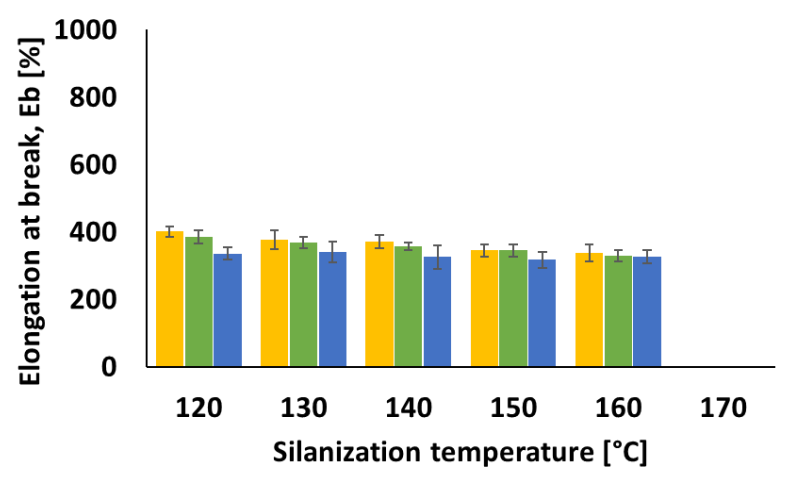

Mix C (TESO)

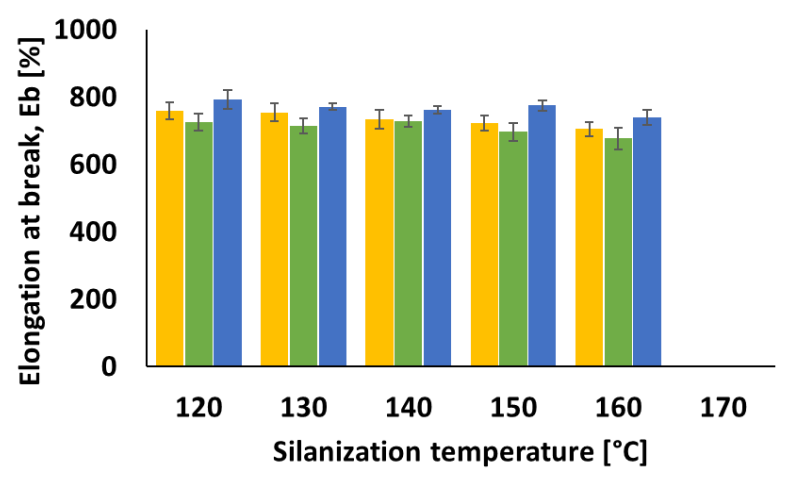

Mix E (NS)

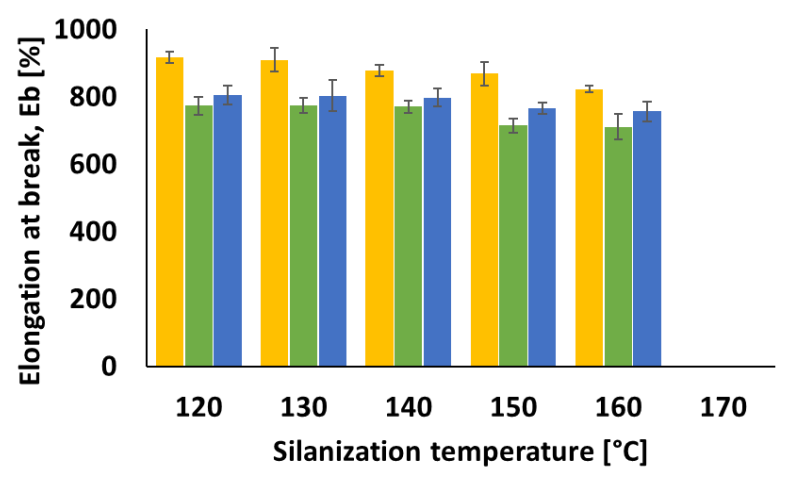

Figure 3.27 Elongation at break (Eb) with three different curing times;

$(\square): \mathrm{CT} ;(\square): 20 \mathrm{~min} ;(\square): 30 \mathrm{~min}$. 


\subsubsection{Influencing factors for the tensile properties of silica filled compounds}

Beside the relation between $\mathrm{MMI} 0.5^{\circ}$ and tensile properties, Figures 3.23 to 3.27 still show some interesting trends of the properties (except TS and Eb). The factors for the trends are analyzed in the following paragraphs in terms of total crosslink density, the degree of filler-polymer interaction and fillerfiller interaction.

\subsection{Crosslink density and overall tensile properties}

According to Figures 3.23 to 3.27 , a clear property level difference was observed between the mixes having a sulfidic silane (Ref. compound, mixes A and B) and those without (mixes C, D and E). Therefore, the overall tensile properties were first correlated with the swelling ratio. Dijkhuis et al. ${ }^{[8]}$ reported that tensile properties strongly depend on crosslink density, but independent of vulcanization system or length distribution of the crosslinks. Additionally, many studies confirmed that filler-polymer interaction results in high crosslink density and can be evaluated by equilibrium swelling. ${ }^{[3,9,10]}$ As can be seen in Figure 3.28, the tensile property levels can roughly be separated into two zones according to the major function of the silane: filler-polymer coupling. Even though the same amount of free sulfur was introduced for all mixes, sulfur-free silane and no-silane compounds show lower mechanical strengths (M100, M200, TS) and crosslink density (a higher swelling ratio (app. 250\%) and higher Eb values (app. 800\%)). These results indicate that not only the sulfur crosslinking but also the filler-polymer coupling via silane gives a major contribution for total crosslink density, and as a consequence enhanced mechanical strength of silica filled rubber compounds. 

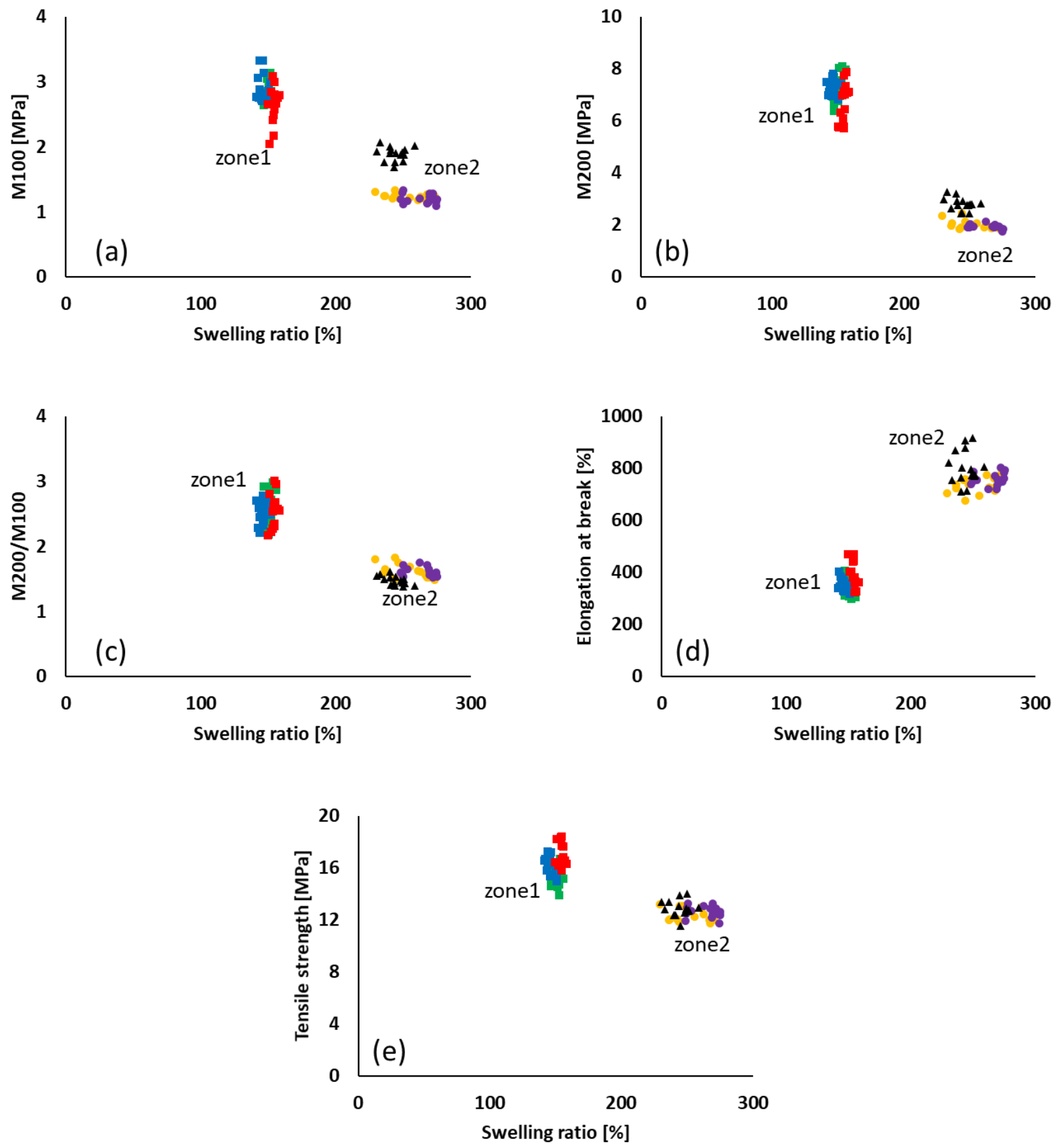

Figure 3.28 Tensile properties vs. swelling ratio of all mixes distinguished by the shape and the color of the data points; ( $\boldsymbol{\square}$ ): sulfidic silane; ( $)$ ): sulfur-free silane; ( $\boldsymbol{\Delta}$ ): no silane; ( $\square$ ): Ref. compound; ( $\square$ ): Mix A; ( ): Mix B; ( ): Mix C; ( $\square$ ): Mix D; ( $)$ ): Mix E; (a): M100; (b): M200; (c): reinforcing index; (d): tensile strength; (e): Elongation at break. 


\subsubsection{Filler-polymer interaction and moduli at $200 \%$ of strain (M200)}

As expected from Figure 3.28, the compounds with sulfidic silanes (Ref. compound, Mixes A, B) show a higher $\lambda^{-1}$ upturn point compared to the compounds with a sulfur-free silane or no silane (Mixes C, D and E): Figure 3.29.

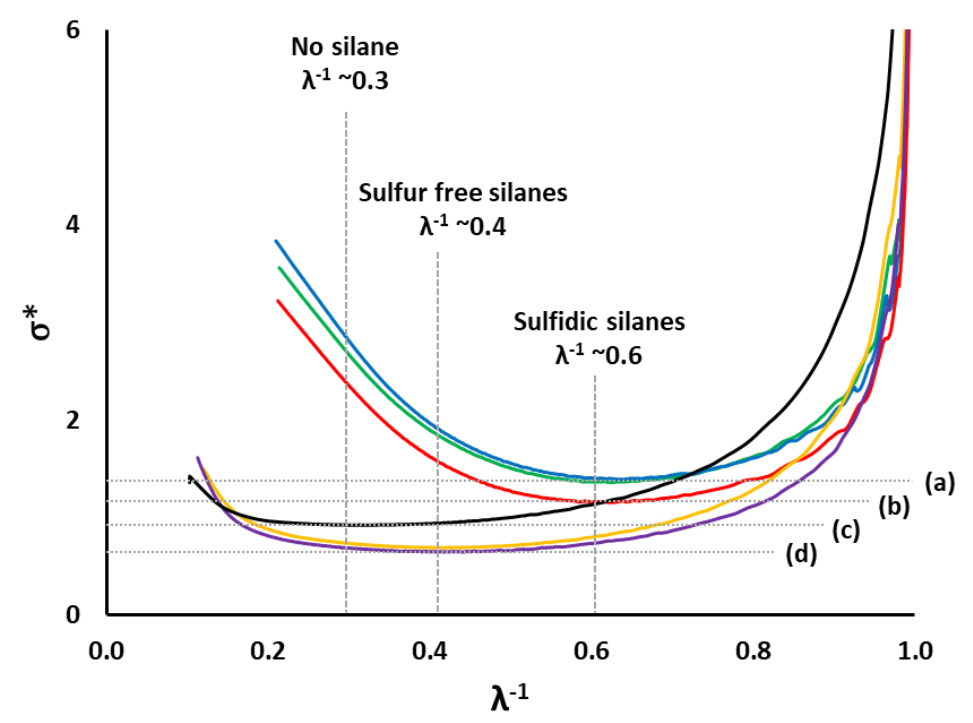

Figure 3.29 Mooney-Rivlin plots for the samples silanized at $150^{\circ} \mathrm{C}$ and vulcanized with calculated cure time of all mixes and their tangential lines where the slope becomes zero; (a): Ref. compound and Mix A;

(b): Mix B; (c): Mix E; (d): Mixes C and D; ( — ): Ref. Compound; ( — ): Mix A; ( — ): Mix B;

$$
(-) \text { : Mix C; ( ) ):Mix D; ( ) ): Mix E. }
$$

The upturn points of the compounds containing a sulfidic silane (Ref. compound, Mixes A and B) are shown in Figure 3.30. The upturn point of Ref. Compound (TESPT) gradually increased with higher silanization temperature: Figure 3.30(a). This result implies that the degree of filler-polymer coupling strongly influences the reinforcement of the rubber compound. Due to the fact that the presence of free sulfur affects the reactivity as well as thermal stability of TESPD, Mix B (TESPD with sulfur adjustment) shows a similar trend to the Ref. compound with 20 minutes of curing time (Figure 3.30(c)) whereas Mix A (TESPD) shows almost the same upturn point values for the whole range of silanization temperature and curing time (Figure 3.30(b)). The upturn points of the compounds with sulfur-free silane (Mixes C and D) or no silane (Mix E) showed constant values regardless of the silanization temperature and curing time (0.4 and 0.3 , respectively), and are therefore not depicted in the figure. 

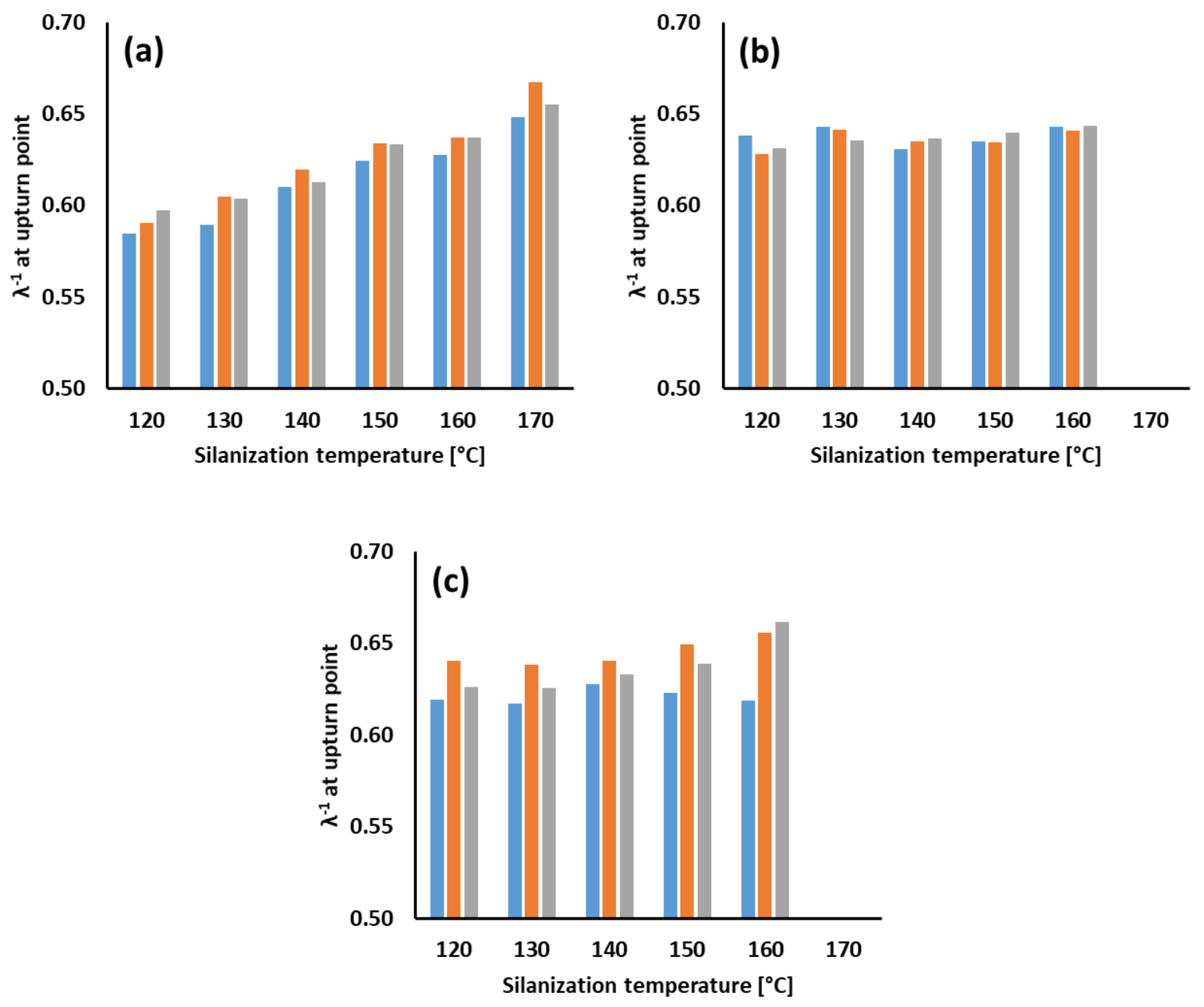

Figure 3.30 $\lambda^{-1}$ at upturn point vs. silanization temperature; (a): Ref. compound; (b): Mix A; (c): Mix B;

$$
\text { ( } \square): C T \text {; ( } \square): 20 \mathrm{~min} ;(\square) \text { ): } 30 \mathrm{~min} \text {. }
$$

The upturn point values show the best correlation with M200 properties as shown in Figure 3.31. For Mix A, both the upturn point and M200 values were almost the same for the whole range of silanization temperature as well as the curing time. Therefore, all the data points of Mix A were clustered at a certain point. 

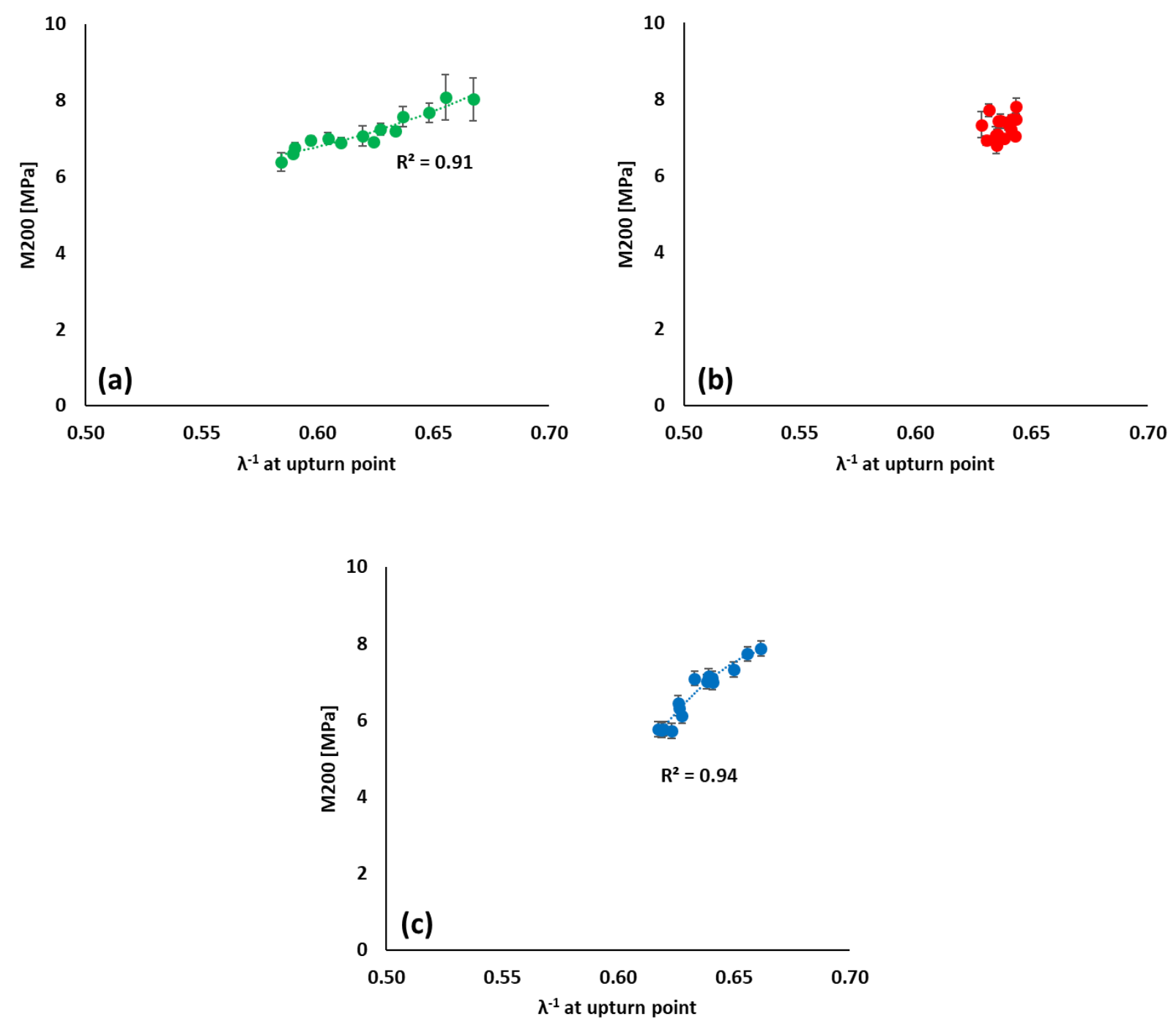

Figure 3.31 M200 vs. upturn point; (a): Ref. compound; (b): Mix A; (c): Mix B.

\subsection{Filler-filler interaction and moduli at $100 \%$ of strain (M100)}

As was seen in Figure 3.28(a), total crosslink density first gives a major contribution to the level of M100. However, the tendency observed in total crosslink density (Figure 3.21) as well as filler-polymer interaction of Ref. Compound and Mixes A and B (Figure 30) cannot explain their M100 trends along with the silanization temperatures. The reason is that the M100 levels of Ref. compound and Mix B slightly decrease along with silanization temperature while total crosslink density remains constant (Figure 3.21), and the filler-polymer interaction increases (Figure 3.30(a) and (c)). In the case of Mix A, a constant total crosslink density (Figure 3.21) as well as filler-polymer interaction (Figure 3.30(b)) regardless of the silanization temperature were observed, however higher M100 values were obtained when the 
compound was silanized at a lower temperature $\left(120^{\circ} \mathrm{C}\right)$ : but levels off starting from $140^{\circ} \mathrm{C}$.

The M100 trends for the mixes with sulfidic silanes are possible to be explained with filler-filler interaction based on Figure 3.10: the reduction of the storage modulus $\left(G^{\prime}\right)$ still occurs due to a strong filler-filler interaction of the silica. As a result, for the M100 of mixes with sulfidic silanes plotted as a function of filler-filler interaction as shown in Figure 3.32: higher filler-filler interaction increases the M100 values. No correlation is found between M100 and the Payne effect for the Mixes with the sulfur-free silanes (Mixes C, D) or no silane at all (Mix E). This result implies that in cases where there is filler-polymer interaction, the polymer chain extensibility and mobility can be influenced by the filler network and as consequence results in a slight difference in modulus.
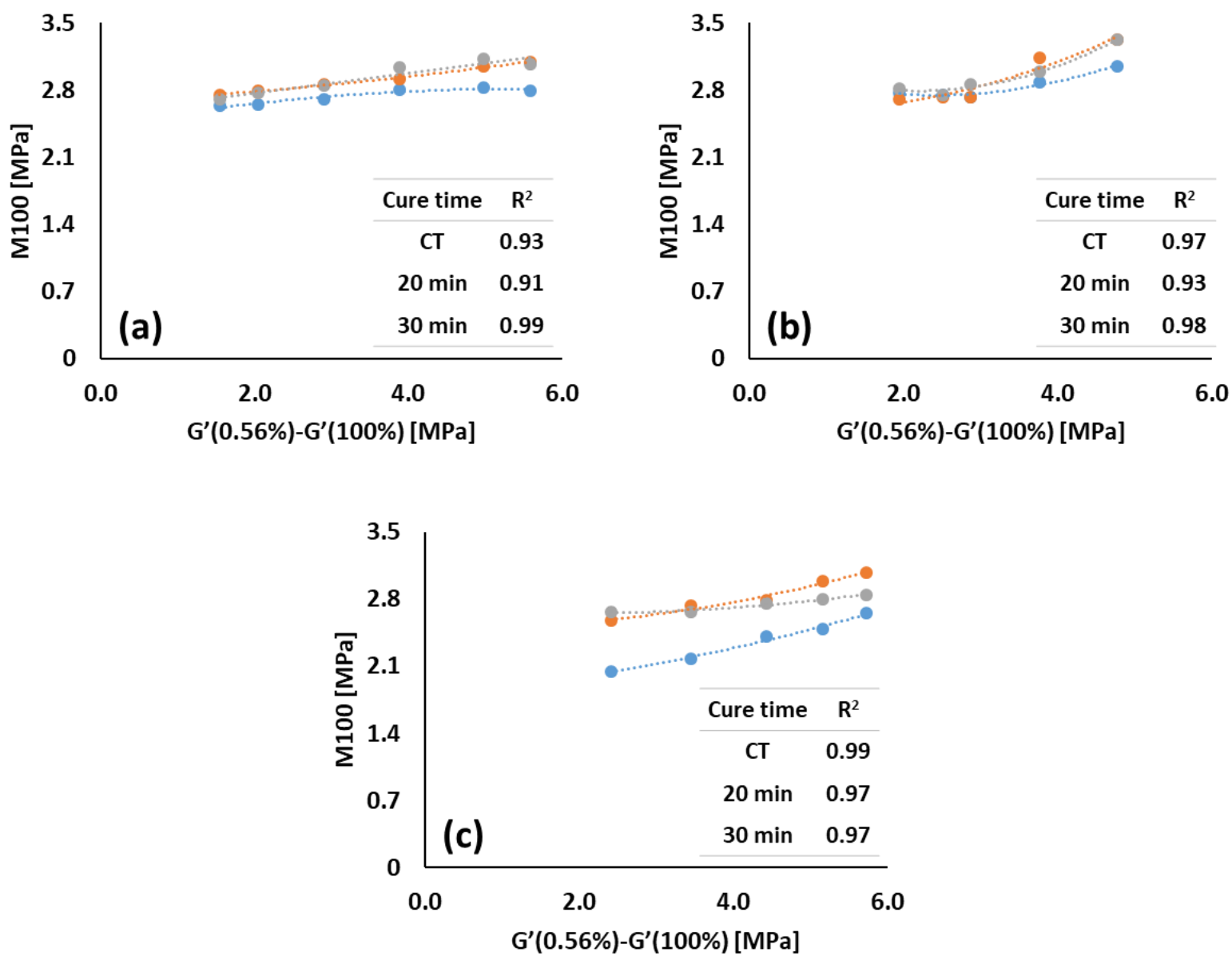

Figure 3.32 M100 vs Payne effect; (a): Ref. Compound; (b): Mix A; (c): Mix B; ( ): CT; ( ): 20 min;

( ): 30 min; fitting lines are based on polynomial or linear fit with maximum $\mathrm{R}^{2}$. 


\subsection{The effect of silane and the dispersion of silica on rubber reinforcement}

As expected from the compound formulation and already seen in Figure 3.25, the mixes with silane, which establishes a silica-polymer coupling, show a higher reinforcement index. Luginsland et al. ${ }^{[11]}$ reported that the capability of filler-polymer coupling is crucial for rubber reinforcing by silica. They obtained poor mechanical strength - i.e. moduli at $300 \%$ of strain - for the compound with a silane which could not form crosslinks between silica and polymer.

Beside the effect of filler-polymer coupling, another interesting point can be found in Figure 3.33. In this figure, the reinforcing indices (M200/M100) of all compounds are plotted as a function of the Payne effect regardless of the functions of the silanes or the curing times. It can be explained with the degree of microdispersion which can be indirectly deduced from the Payne effect results. Many researchers already have confirmed that the degree of micro-dispersion of the filler is crucial in order to achieve the best reinforcement of rubber. ${ }^{[11,12]}$ The results in Figure 3.33 also indicate that the degree of micro-dispersion of filler has an impact on rubber reinforcement. Once the silica is poorly silanized or dispersed during mixing, the rubber reinforcement effect by the filler cannot be repaired even though the vulcanizates are properly cured. Further discussion will be held later on in Chapter 7. 

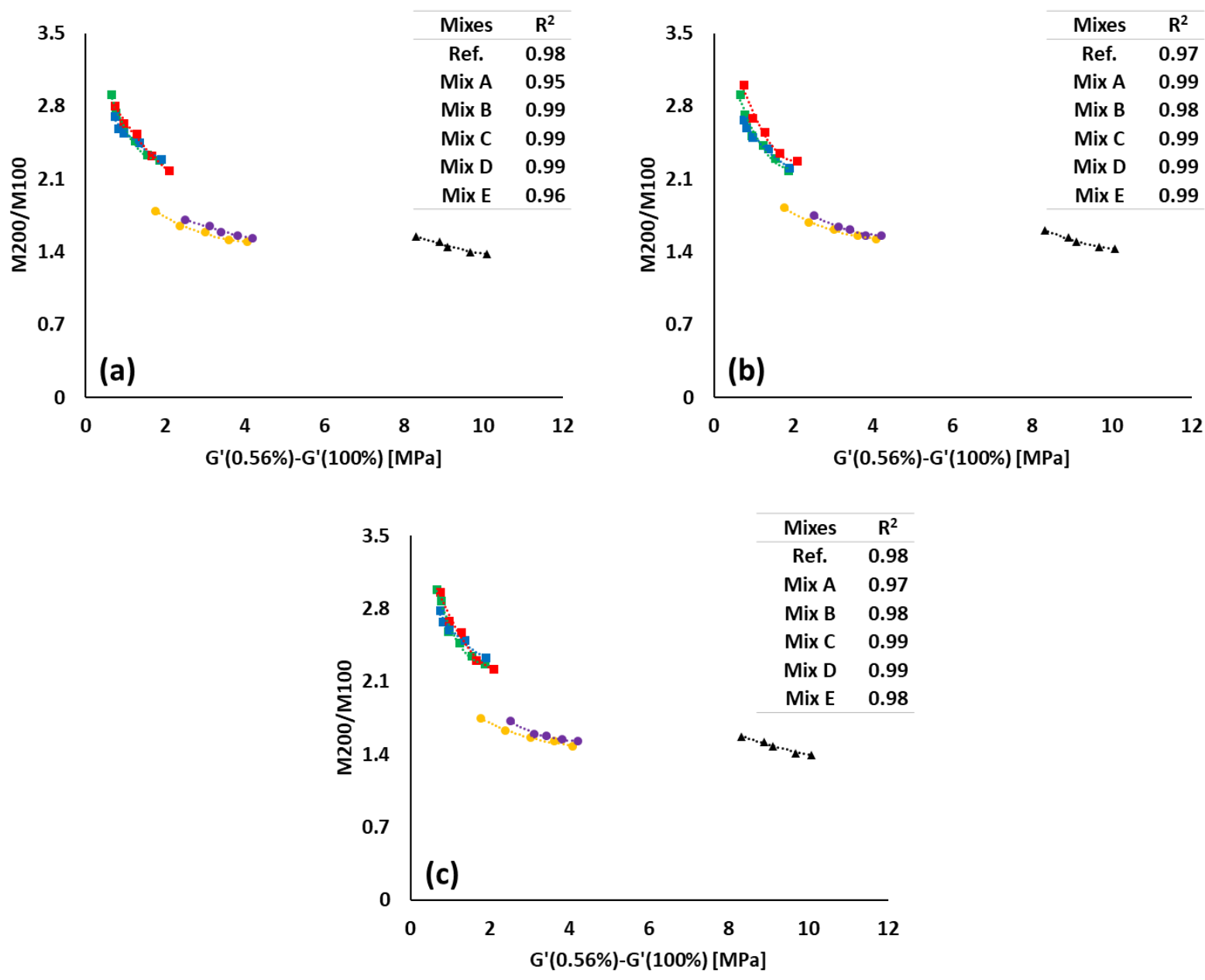

Figure 3.33 Reinforcing index vs. Payne effect of all mixes with different curing times distinguished by the shape and the color of the data points; $(\boldsymbol{\square})$ : sulfidic silanes; $(\boldsymbol{O})$ : sulfur-free silanes; $(\boldsymbol{\Delta})$ : no silane;

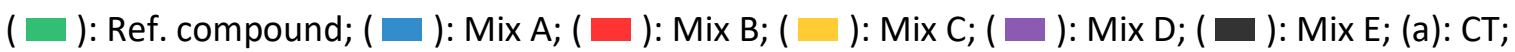

(b): $20 \mathrm{~min}$; (c): $30 \mathrm{~min}$; fitting lines are based on polynomial or linear fit with maximum $\mathrm{R}^{2}$.

\subsubsection{Conclusions}

The focus of this study was to observe the alteration of the mechanical properties, such as tensile properties, for the compounds showing marching modulus phenomenon. Before the study, a large alteration of tensile properties was expected for the compounds having a higher marching modulus intensity (MMI). However, it is turned out that the $\mathrm{MMI}$ does not give any information about mechanical property variation along with the vulcanization time.

The level of tensile properties of silica filled rubbers were first (and roughly) determined by the total crosslink density which includes not only polymer-polymer crosslinks by free sulfur but also filler-polymer 
coupling established by silane. This means that the filler-polymer coupling has a large impact on rubber reinforcement. The higher amount of filler-polymer coupling results in a better reinforcement of silica filled rubber. Even though the silica is well silanized, the reinforcing effect by the silica can be poor with absence of filler-polymer coupling via silane.

The degree of micro-dispersion turned out to be another key factor for rubber reinforcement. Even though a similar quantity of total crosslinks are formed during vulcanization process, the final reinforcing effect can be lower when the silica is not well dispersed in the rubber matrix.

To wrap it up in a nutshell, using an appropriate silane coupling agent as well as optimizing the mixing conditions is crucial in order to achieve the best reinforcement of silica filled rubber.

\section{References}

[1] ISO 37:2011, "Rubber, vulcanized or thermoplastic - Determination of tensile stress-strain properties", International Organization for Standardization (2011).

[2] C. Hayichelaeh, L. A. E. M. Reuvekamp, W. K. Dierkes, A. Blume, J. W. M. Noordermeer, K. Sahakaro, Rubber Chem. Technol. 91, 433 (2018).

[3] L. Bokobza, Macromol. Mater. Eng. 289, 607 (2004).

[4] "The Physics of Rubber Elasticity, 3rd Edition" L. R. G. Treloar, Oxford University Press, New York (1975).

[5] Y. Zhan, J. Wu, H. Xia, N. Yan, G. Fei, and G. Yuan, Macromol. Mater. Eng. 296, 590 (2011).

[6] B. Zhong, Z. Jia, D. Hu, Y. Luo, and D. Jia, Composites Part A 78, 303 (2015).

[7] Z. Tang, J. Huang, X. Wu, B. Guo, L. Zhang, and F. Liu, Ind. Eng. Chem. Res. 54, 10747 (2015).

[8] K. A. J. Dijkhuis, J. W. M. Noordermeer, W. K. Dierkes, European Polymer Journal 45, 3302 (2009).

[9] M. L. Kralevich, J. L. Koenig, Rubber Chem. Technol. 71, 300 (1998).

[10] L. Bokobza, O. Rapoport, J. Appl. Polym. Sci. 85, 2301 (2002).

[11] H-D. Luginsland, J. Fröhlich, A. Wehmeier, Rubber Chem. Technol. 75, 563 (2002).

[12] S. Mihara, R. N. Datta, J. W. M. Noordermeer, Rubber Chem. Technol. 82, 524 (2009). 


\section{Chapter 4 The effect of mixing parameters on marching modulus of silica filled tire tread compounds}

The degree of silanization turned out to be a key factor for marching modulus of silica filled S-SBR/BR tire tread compounds. This implies that the intensity of marching modulus of a silica filled compound can be reduced by controlling the mixing parameters for a better silanization.

The silanization reaction has to take place during the different mixing cycle(s), therefore many studies were performed in order to tailor the mixing parameters. However, this is commonly done with the objective to improve the mixing or extrusion performance of the compounds, and the curing or final vulcanizate properties.

In this chapter, the mixing parameters, which are known as contributors to the degree of silanization, are evaluated in terms of their effect on marching modulus. The following parameters are taken into consideration:

4.1 The effect of silanization temperature and time on marching modulus;

4.2 The effect of zinc oxide concentration in the early and later mixing stage on marching modulus; 4.3 The effect of the 1,3-diphenylguanidine concentration the early and later mixing stage on marching modulus

The correlation between these mixing parameters and their effect on the factors, which have a strong relation with the Marching Modulus Intensity (MMI), are investigated: the amount of bound rubber, Filler Flocculation Rate (FFR), and filler-polymer Coupling Rate (CR). The results are interpreted in view of a correlation between these factors and their effect on the Marching Modulus Intensity (MMI).

Additionally, a series of model compound studies - Chapter 4.4 - is excuted in order to explain the results of Chapters 4.2 and 4.3. Based on the outcome, a new role of 1,3-diphenylguanidine (DPG) is defined: DPG can react with bis-(triethoxysilylpropyl)tetrasulfide (TESPT) and is thus consumed during the mixing process. Finally, the chemical structure of DPG-TESPT complex is suggested. 


\section{Chapter 4.1 The effect of silanization temperature and time on marching modulus}

\subsubsection{Introduction}

In this chapter, the effects of mixer silanization temperature and time - pronounced parameters for the degree of silanization - are evaluated and compared. Dierkes et al. ${ }^{[1]}$ and Reuvekamp et al. ${ }^{[2]}$ reported that the mixing temperature and time is crucial in mixing silica and rubber with bis(triethoxysilylpropyl)tetrasulfide (TESPT) as coupling agent in order to achieve a proper degree of silanization. Additionally, Mihara et al. ${ }^{[3]}$ and Jin et al. ${ }^{[4]}$ reported that the silanization temperature strongly affects the degree of silanization and the formation of chemically bound rubber. If the temperature is too low, this results in a higher degree of remaining filler-filler interaction and consequently in silica re-agglomeration. Since the degree of silanization has a great impact on the intensity of marching modulus by affecting silica flocculation as well as filler-polymer coupling, an alteration of the Marching Modulus Intensity (MMI) is expected by changing these mixing parameters.

First, the compounds with TESPT are mixed at various silanization temperatures and times. Subsequently, the factors that largely contribute to the vulcanization behavior are evaluated based on the results in Chapter 3.1: the degree of silanization, the amount of bound rubber, the Filler Flocculation Rate (FFR) and the filler-polymer Coupling Rate (CR). These acquired factors are then correlated with the MMIs measured at small (approx. 7\%) and large (approx. 42\%) strain.

\subsubsection{Experimental}

\subsubsection{Materials and mixing}

All series of experiments were done based on a tire tread compound as shown in Table 4.1. The compounds were mixed in two steps as shown in Tables 4.2 and 4.3. The master batch stage was done by using a lab scale internal mixer (Brabender Plasticorder) with a chamber volume of $390 \mathrm{ml}$. The fill factor of the internal mixer was fixed at $63 \%$. The temperature of the mixer Temperature Control Unit (TCU) was set at $50^{\circ} \mathrm{C}$, except for the silanization temperature mixing test at $170^{\circ} \mathrm{C}: 70^{\circ} \mathrm{C}$ of $\mathrm{TCU}$ temperature was applied for this test to reach $170^{\circ} \mathrm{C}$. In order to avoid the "first batch effect", one initial batch was mixed and discarded before the regular mixing started. This was started when the mixing chamber had reached $55^{\circ} \mathrm{C}$. The mixing fingerprints are depicted in Figures 4.1 and 4.2. The silanization temperatures as indicated in Figures 4.1 and 4.2 are registered by a sensor embedded in the mixer wall and are almost the 
same compared to the actual discharge temperatures as measured with a hand-held thermometer: the deviation between these two temperatures was 2 to 3 degrees Centigrade. In case of Mixing procedure A, the rotor speed was adjusted from 04:10 ( $\mathrm{min}: \mathrm{sec}$ ) onwards in order to reach and subsequently keep different silanization temperatures steady during a period of silanization of 150 seconds. The same temperature control technique was used for Mixing procedure $B$, but here the silanization temperature was fixed at $150^{\circ} \mathrm{C}$ and the silanization period varied. After the first mixing step, the compounds were sheeted out immediately on a lab scale two-roll mill (Polymix $80 \mathrm{~T}$ ) with $40^{\circ} \mathrm{C}$ of TCU setting in order to cool down the compounds and prevent further reaction. Three batches were mixed for each set of conditions in order to check the reproducibility. All batches were mixed with good reproducibility.

Table 4.1 Formulation of the compound

\begin{tabular}{|c|c|c|c|c|}
\hline Mixing stage & Ingredient & Product name & Company & Content [phr] \\
\hline \multirow{8}{*}{ Master Batch } & S-SBR* & Buna VSL5025-2HM & Lanxess & 110 \\
\hline & BR & Buna CB24 & Lanxess & 20 \\
\hline & HD Silica & $\begin{array}{l}\text { ULTRASIL }{ }^{\circledR} 7005 \\
\left.\text { (CTAB: } 177 \mathrm{~m}^{2} / \mathrm{g}\right)\end{array}$ & Evonik & 90 \\
\hline & Silane (TESPT) & Si69 & Evonik & 8.05 \\
\hline & TDAE Oil & VIVATEC 500 & Hansen \& Rosenthal & 5 \\
\hline & Stearic acid & Stearic acid & Merck & 1 \\
\hline & Zinc Oxide & ZnO & Merck & 2 \\
\hline & DPG & Perkacit DPG & Flexsys & 1.5 \\
\hline \multirow{3}{*}{ Final } & Sulfur & $S$ & J. T Baker & 0.7 \\
\hline & ZBEC & Vulkacit ZBEC & Lanxess & 0.2 \\
\hline & CBS & Santocure CBS & Flexsys & 2.2 \\
\hline
\end{tabular}

*27.3 wt\% TDAE oil extended 
Table 4.2 Master batch mixing procedures

\begin{tabular}{|c|c|c|c|}
\hline \multicolumn{2}{|c|}{ Mixing procedure $A$} & \multicolumn{2}{|l|}{ Mixing procedure $B$} \\
\hline Action & time $[\mathrm{mm}: \mathrm{ss}]$ & Action & time $[\mathrm{mm}: \mathrm{ss}]$ \\
\hline Add polymer & $00: 00 \sim 00: 20$ & Add polymer & $00: 00 \sim 00: 20$ \\
\hline Mastication & $00: 20 \sim 01: 20$ & Mastication & $00: 20 \sim 01: 20$ \\
\hline $1 / 2$ Silica, silane & $01: 20 \sim 01: 40$ & $1 / 2$ Silica, silane & $01: 20 \sim 01: 40$ \\
\hline Mixing & $01: 40 \sim 02: 40$ & Mixing & $01: 40 \sim 02: 40$ \\
\hline $1 / 2$ Silica, remaining ingredients & $02: 40 \sim 03: 10$ & $1 / 2$ Silica, remaining ingredients & $02: 40 \sim 03: 10$ \\
\hline $\begin{array}{l}\text { Mixing till target temperature: } \\
120,130, \ldots ., 170^{\circ} \mathrm{C}\end{array}$ & 03:10 04:10 & Mixing till $150^{\circ} \mathrm{C}$ & 03:10 04:10 \\
\hline Ram sweep & $04: 10 \sim 04: 14$ & Ram sweep & $04: 10 \sim 04: 14$ \\
\hline Mixing (at target temperature) & $04: 14 \sim 06: 40$ & $\begin{array}{c}\text { Mixing for various time laps: } \\
0,50,100, \ldots ., 250 \mathrm{sec}\end{array}$ & $04: 14 \sim 08: 20$ \\
\hline Discharge and sheeting & - & Discharge and sheeting & - \\
\hline
\end{tabular}

Table 4.3 Final mixing procedure on mill

\begin{tabular}{c|c}
\hline \multicolumn{2}{c}{ Mixing procedures A and B } \\
\hline Action & time [mm:ss] \\
\hline Add master batch & $00: 00 \sim 02: 00$ \\
\hline Mixing & $02: 00 \sim 02: 30$ \\
\hline Add curatives & $02: 30 \sim 09: 00$ \\
\hline Mixing & - \\
\hline Discharge & \\
\hline
\end{tabular}




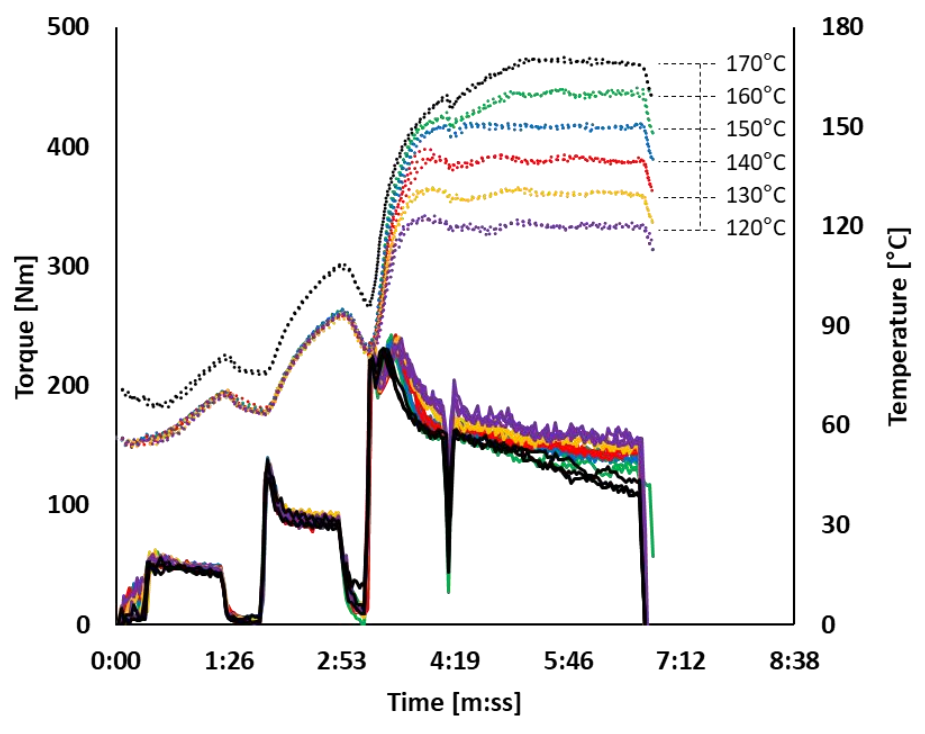

Figure 4.1 Fingerprints of Mixing procedure A; ( solid line ): Torque; ( dotted line ): temperature; $(\square): 120^{\circ} \mathrm{C} ;(\square): 130^{\circ} \mathrm{C} ;(\square): 140^{\circ} \mathrm{C} ;(\square): 150^{\circ} \mathrm{C} ;(\square): 160^{\circ} \mathrm{C} ;(\square): 170^{\circ} \mathrm{C}$

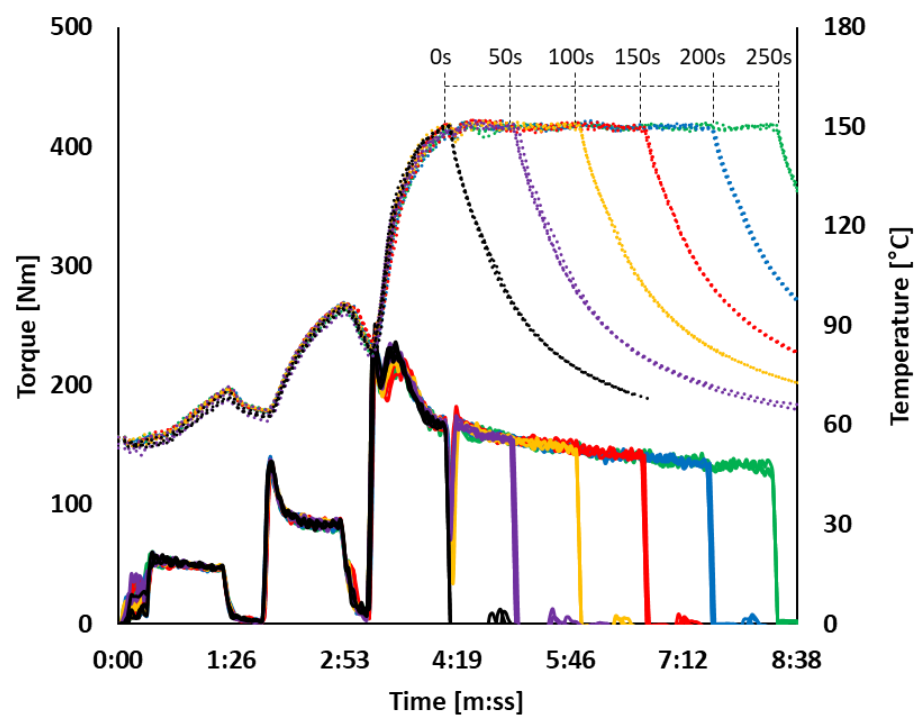

Figure 4.2 Fingerprints of Mixing procedure B; ( solid line ): Torque; ( dotted line ): temperature;

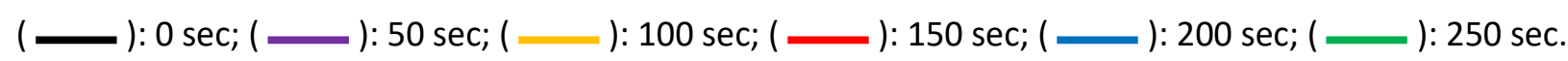

\subsubsection{Property analysis and testing}

Payne effect, Filler Flocculation Rate (FFR), filler-polymer Coupling Rate (CR), cure characteristics and Marching Modulus Intensity (MMI), resp. bound rubber content of the compounds were investigated according to the methods as described in Chapter 3.1. 


\subsubsection{Results and discussion}

\subsubsection{Filler-Filler interaction (Payne effect) as a function of silanization temperature and time}

The Payne effects of the uncured compounds are plotted as a function of the silanization temperature and time for both mixing procedures, as shown in Figure 4.3. With increasing silanization temperature and time, a lower Payne effect value is observed due to a higher degree of silanization.
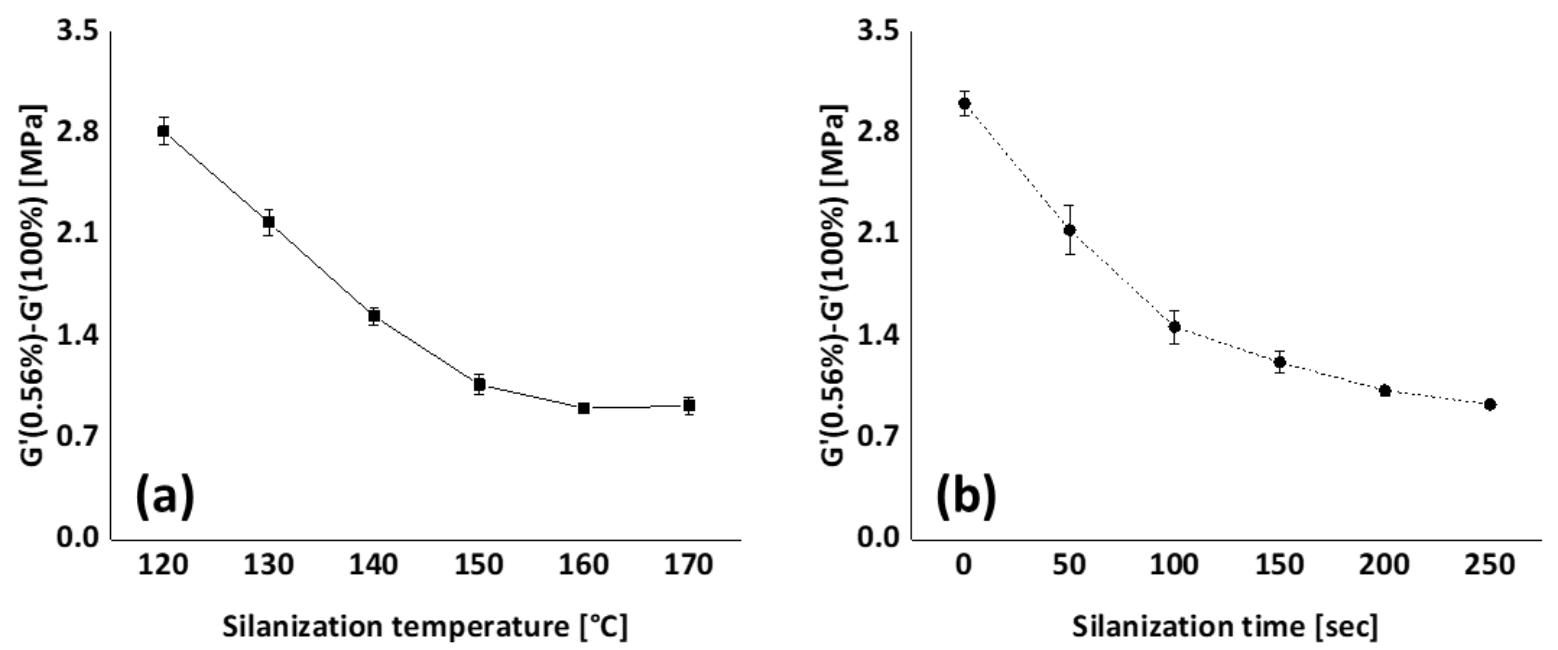

Figure 4.3 Payne effect as a function of: (a): silanization temperature; (b): silanization time.

As was already stated in Chapter 3.1, the mixing temperature greatly affects the degree of silanization. Besides silanization temperature, the silanization time is also a major factor that enhances the degree of silanization. Even though the mixing temperature of all compounds in Mixing procedure $\mathrm{B}$ reached $150^{\circ} \mathrm{C}$, a higher Payne effect value is observed when a relatively short or no silanization time was applied. The compound mixed according to procedure B without silanization (silanization time zero) shows even a $7 \%$ higher Payne effect compared to the compound which was mixed at $120^{\circ} \mathrm{C}$ according to Mixing procedure A. These results indeed show that not only the silanization temperature, but also the silanization time is crucial for the degree of silanization. The trends starts to level off when the silanization temperature and time reach $160^{\circ} \mathrm{C}$ and 200 seconds, respectively. Therefore, a higher temperature than $160^{\circ} \mathrm{C}$ and mixing duration longer than 200 seconds have little effect on reducing filler-filler interaction any further. 


\subsubsection{Bound rubber contents as a function of silanization temperature and time}

Figure 4.4 shows the amounts of bound rubber as a function of silanization temperature (Mixing procedure A) and silanization time (Mixing procedure B). A higher amount of chemically bound rubber is observed when the compounds were mixed at a higher silanization temperature and for a longer silanization time. It is well known that during mixing already some polymer-filler coupling via the silane may occur, in the worst case resulting in pre-scorch. Thus, a higher degree of polymer-filler coupling is already established during mixing at a higher silanization temperature or with a longer silanization time. For Mixing procedure $A$, the amount of chemically bound rubber gradually increased along with silanization temperature, and a maximum value ( $40 \%)$ was obtained at the highest silanization temperature employed: $170^{\circ} \mathrm{C}$. The maximum content of chemically bound rubber ( 35\%) for Mixing procedure B was observed after 200 seconds of silanization time, and leveled off from this point onwards. This indicates that the silanization temperature is more effective for bound rubber formation than the silanization time. The physically bound rubber does not change irrespective of the silanization conditions in either mixing procedure.
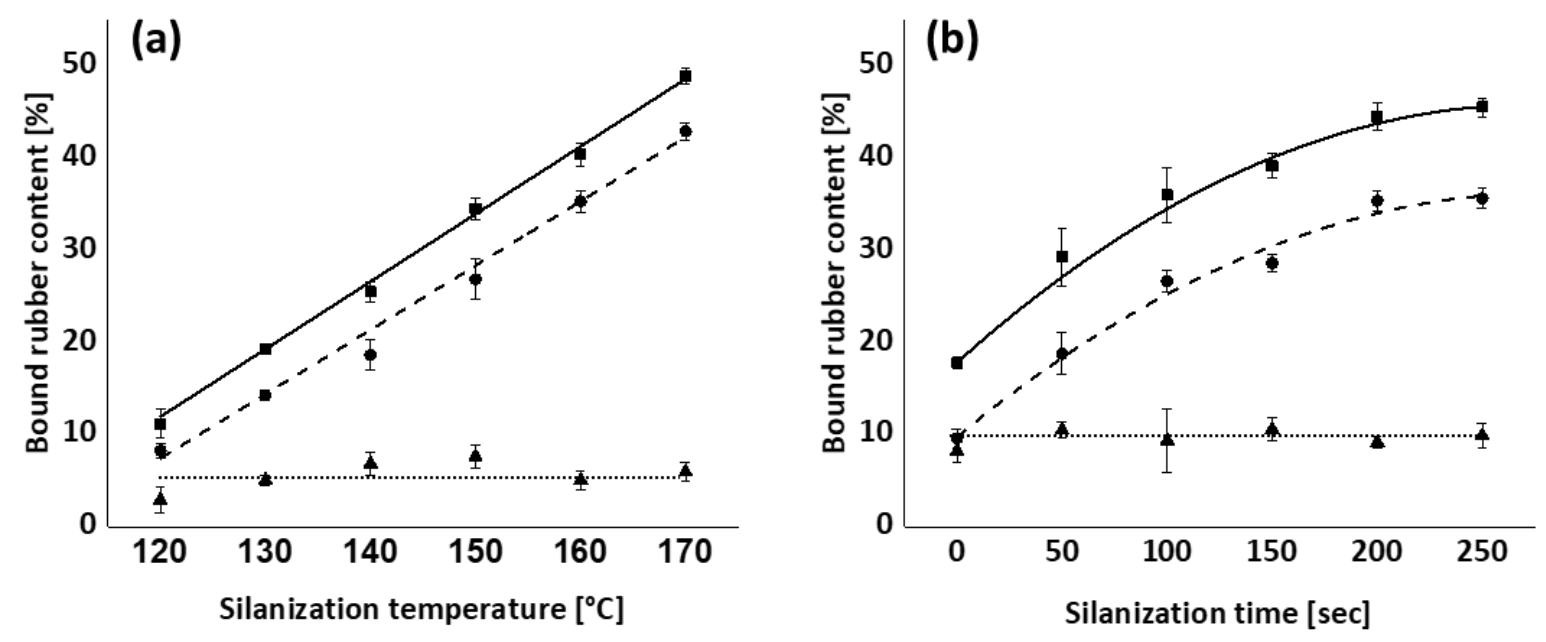

Figure 4.4 Bound rubber content as a function of: (a): silanization temperature; (b): silanization time; $(\mathbf{D})$ : total bound rubber; $(\mathbf{-})$ ): chemically bound rubber; $(\mathbf{\Delta})$ : physically bound rubber.

All the Payne effect values of Mixing procedures $A$ and $B$ and their chemically bound rubber contents show a good correlation, as shown in Figure 4.5: an $\mathrm{R}^{2}$ value higher than 0.9. This indicates that the amount of chemically bound rubber strongly influences the reduction of filler-filler interaction. Another interesting point is seen in this figure: as was already stated in Figure 4.3 , from $160^{\circ} \mathrm{C}$ onwards in Mixing procedure 
A or after 200 seconds in Mixing procedure B, the Payne effect levels off, and at these points both Mixing procedures reach the same chemically bound rubber content: app. 35\%, see Figure 4.4 . This indicates that a further reduction of filler-filler interaction with increasing chemically bound rubber content does not occur beyond $35 \%$.

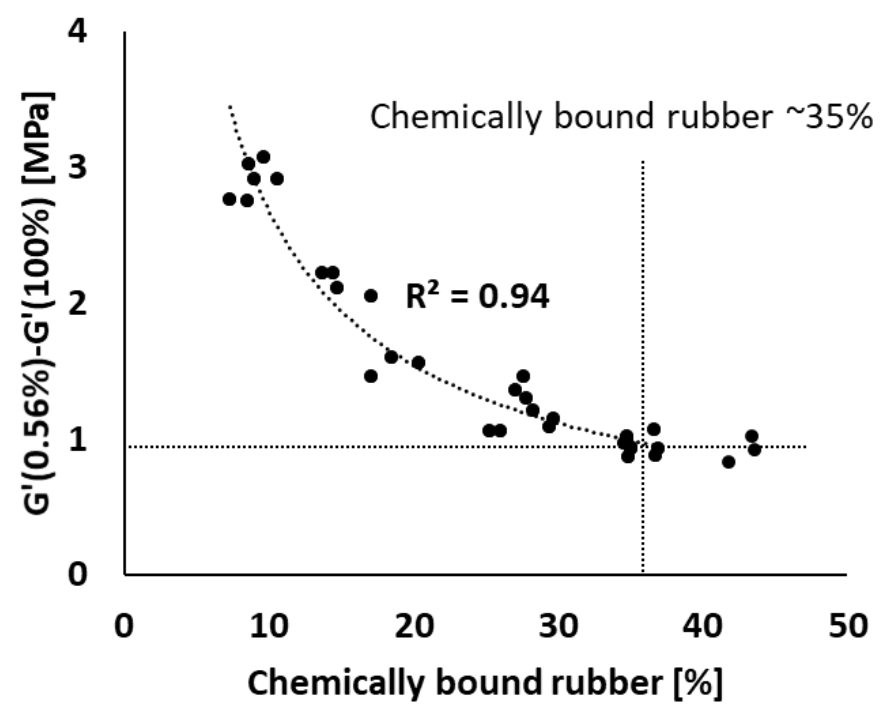

Figure 4.5 Payne effect vs. chemically bound rubber content; fitting line is based on polynomial fit with maximum $\mathrm{R}^{2}$.

\subsubsection{Silica filler flocculation rate}

The silica Filler Flocculation Rates (FFR) are shown in Figure 4.6. The FFR values are plotted as a function of silanization temperature and time. A higher amount of bound rubber leads to lower filler-filler interaction and thus a reduced tendency for flocculation. However, the maximum difference of FFR for Mixing procedure B is smaller than for Mixing procedure A due to a lower amount of total and chemically bound rubber.

Jin et al. ${ }^{[4]}$ reported that FFR has an upper limit. However, in the present work, Mixing procedure A shows two plateau regions: one for a silanization temperature lower than $130^{\circ} \mathrm{C}$ and one for temperatures higher than $160^{\circ} \mathrm{C}$. This indicates that the FFR has an upper and lower limit: in this compound, an upper limit of 0.17 and a lower limit of 0.09 was found. In case of Mixing procedure B, no upper and lower limits are observed because the range of FFR values was within the above mentioned limits of FFR. 

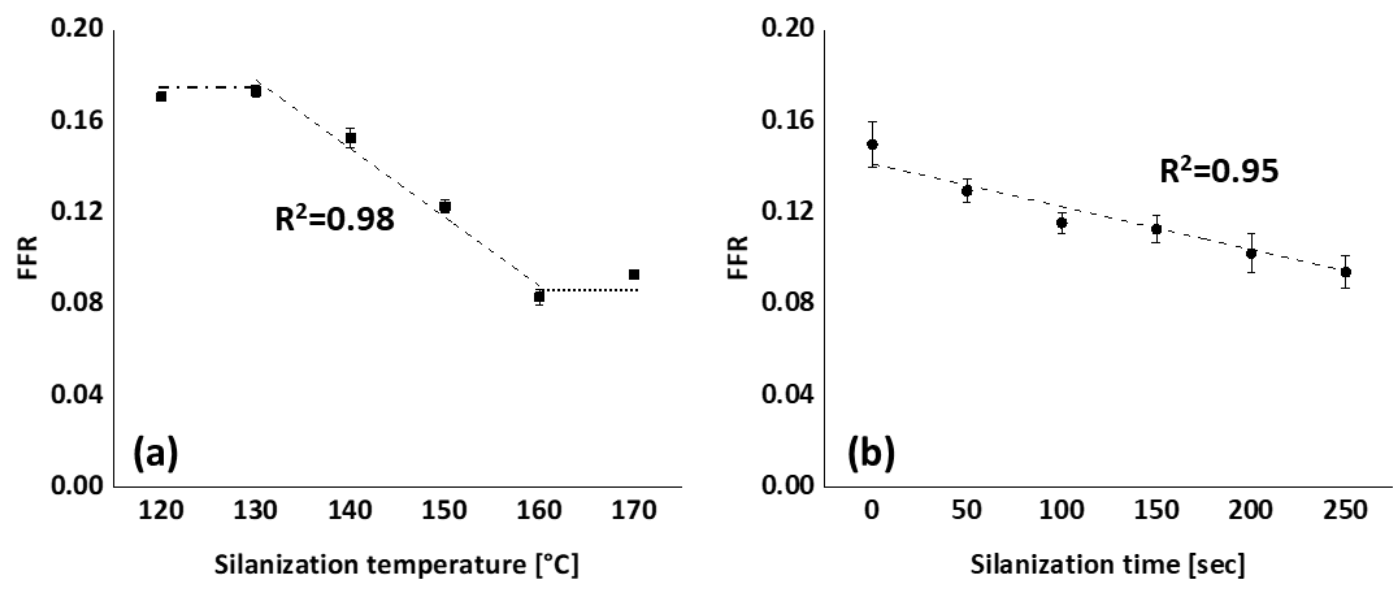

Figure 4.6 FFR as a function of: (a): silanization temperature; (b): silanization time; ( $\mathbf{c}$ ): silanization temperature or time range where FFR shows good correlation with those parameters; ( - . - ): upper plateau; ( ..... ) : lower plateau; fitting lines for (a) and (b) are based on linear fit with maximum $\mathrm{R}^{2}$.

FFR values of all mixing series are plotted against total and chemically bound rubber content of these compounds in Figure 4.7. The FFR values start to decrease when the total and chemically bound rubber contents exceed approximately $20 \%$ and $15 \%$, respectively. No correlation was observed between FFR and physically bound rubber. This means that it is only the chemically bound rubber that strongly influences the silica flocculation behavior, and thus the degree of silanization is the key for FFR reduction.
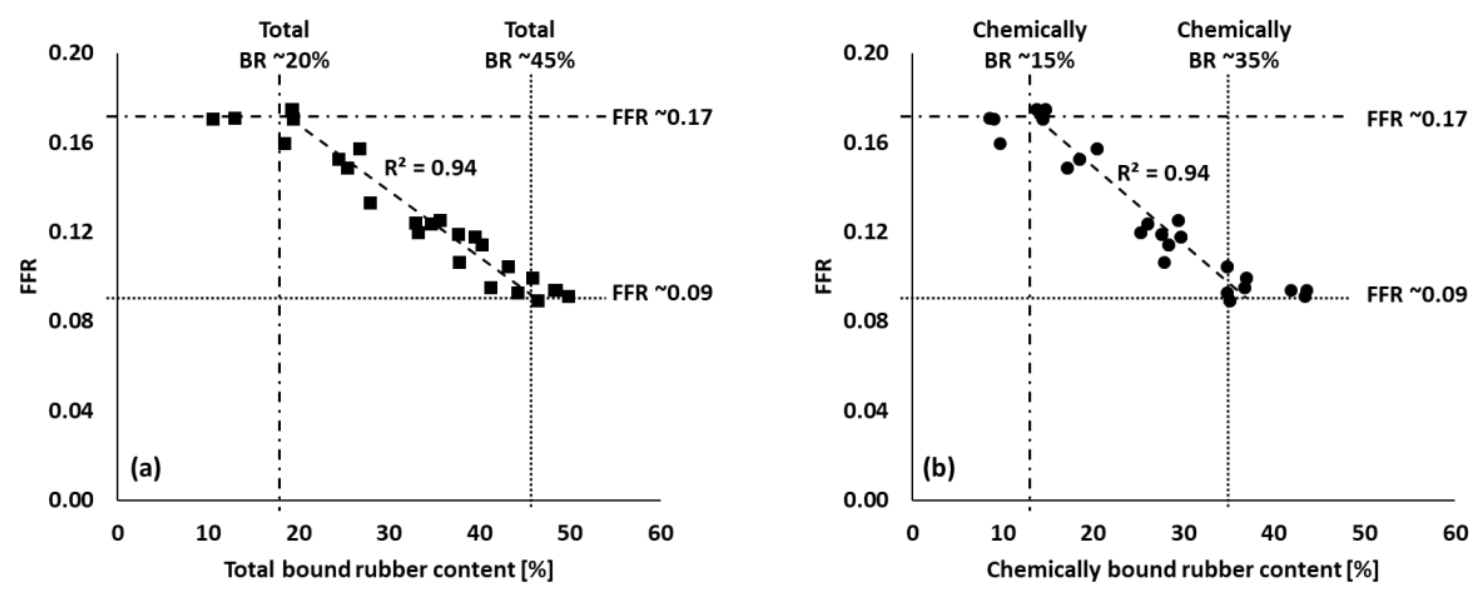

Figure 4.7 FFR versus bound rubber content; (a): FFR vs. total bound rubber; (b): FFR vs. chemically bound rubber; ( - a ): silanization temperature or time range where FFR shows good correlation with those parameters; ( _ . ): upper limit; ( ... ): lower limit; fitting lines are based on linear fit with maximum $\mathrm{R}^{2}$. 


\subsubsection{Filler-polymer coupling rate after mixing}

The filler-polymer Coupling Rates (CR) of all mixing series are shown in Figure 4.8. The CR of the compounds plotted as a function of the silanization temperature and time show a decreasing trend with temperature and time. Similar to the FFR results, the difference of CR for Mixing procedure $B$ is smaller than for Mixing procedure A: silanization temperature has more effect on the filler-polymer coupling reaction than silanization time.
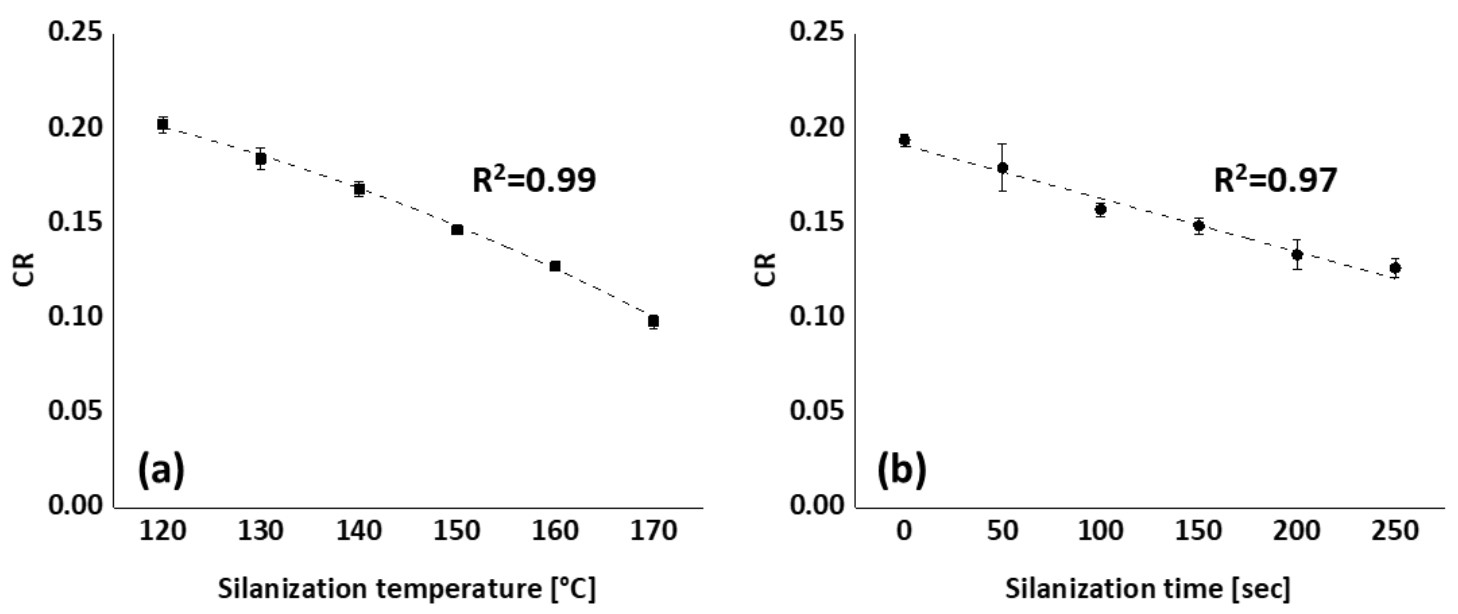

Figure 4.8 CR as a function of: (a): silanization temperature; (b): silanization time; fitting lines for (a) and (b) are based on polynomial and linear fit with maximum $\mathrm{R}^{2}$, respectively.

The CR of all mixing series are plotted as a function of total and chemically bound rubber contents of these compounds in Figure 4.9: Both types of bound rubber show a good correlation with CR. As was already reported, at a higher silanization temperature during mixing, the coupling agent TESPT will release more active or free sulfur and thus induce filler-polymer bonding. ${ }^{[4,5]}$ As a result, the concentration of free sulfur as well as the amount of leftover silane decreases when mixing is done at a higher temperature. Consequently, the CR will decrease due to the lowered concentration of active sulfur and remaining free silane. 

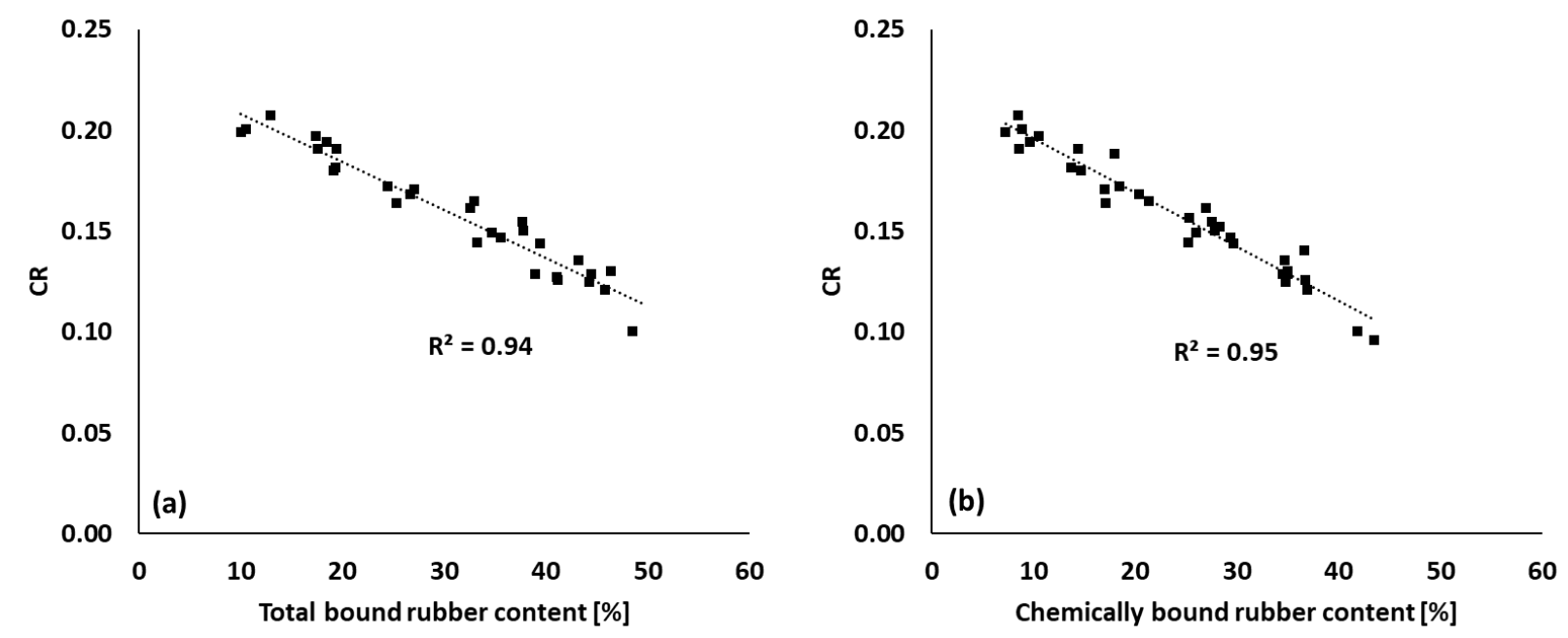

Figure 4.9 CR versus bound rubber content; (a): CR vs. total bound rubber; (b): CR vs. chemically bound rubber; fitting lines for (a) and (b) are based on linear fit with maximum $\mathrm{R}^{2}$.

\subsubsection{Marching modulus intensity}

The rheograms of the compounds mixed according to procedure A and B, and their Marching Modulus Intensities (MMI) measured at two different strains are plotted in Figures $4.10-4.12$, respectively.
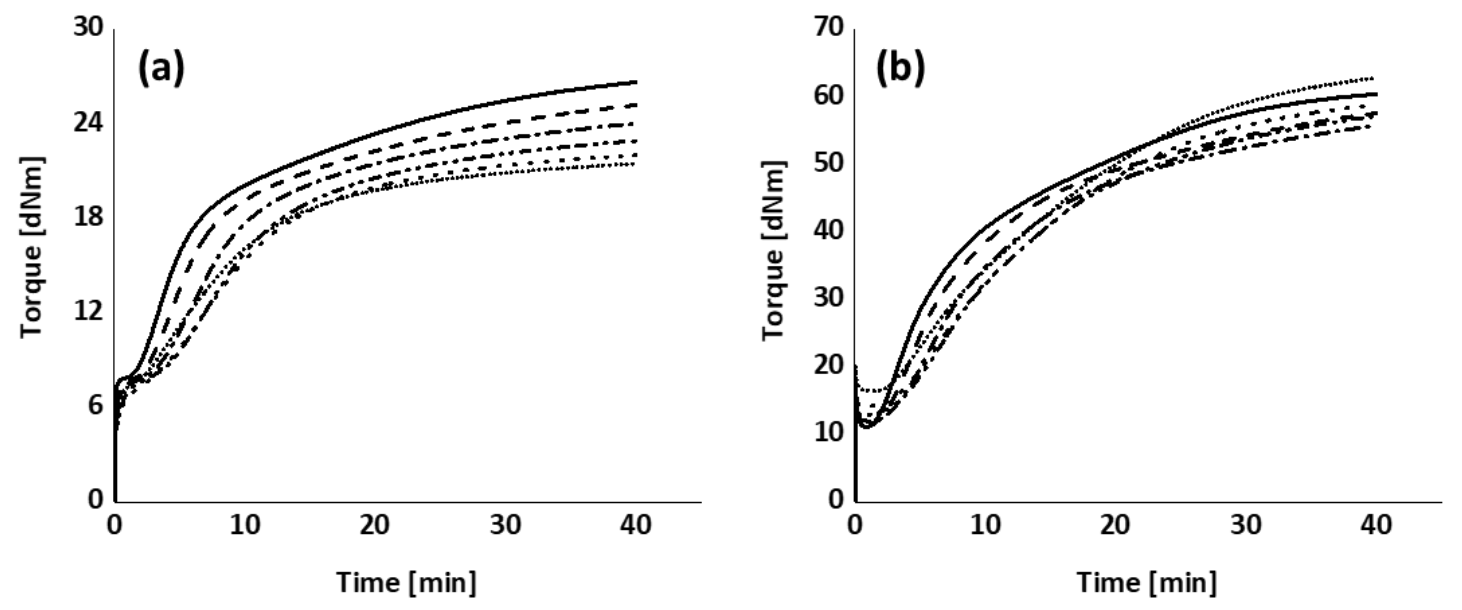

Figure 4.10 Rheograms of Mixing procedure A; (a): $0.5^{\circ}$ ( 7\% of strain); (b): $3^{\circ}$ ( $42 \%$ of strain);

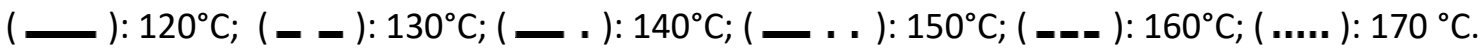



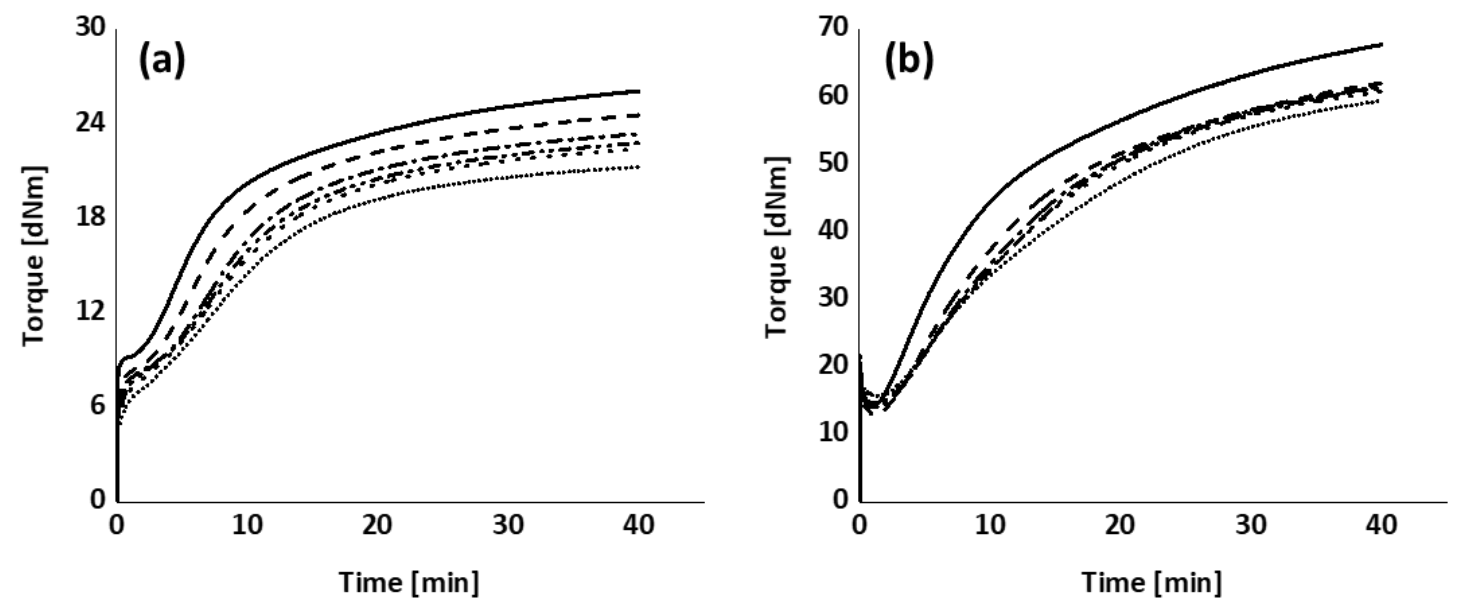

Figure 4.11 Rheograms of Mixing procedure B; (a): $0.5^{\circ}$ ( 7\% of strain); (b): $3^{\circ}$ ( $42 \%$ of strain); (_ ): $0 \mathrm{sec}$; ( _ _ ): $50 \mathrm{sec}$ ( _ . ): $100 \mathrm{sec}$ ( _ . . ): $150 \mathrm{sec}$ ( _. _ ): $200 \mathrm{sec}$ ( ..... ): $250 \mathrm{sec}$.
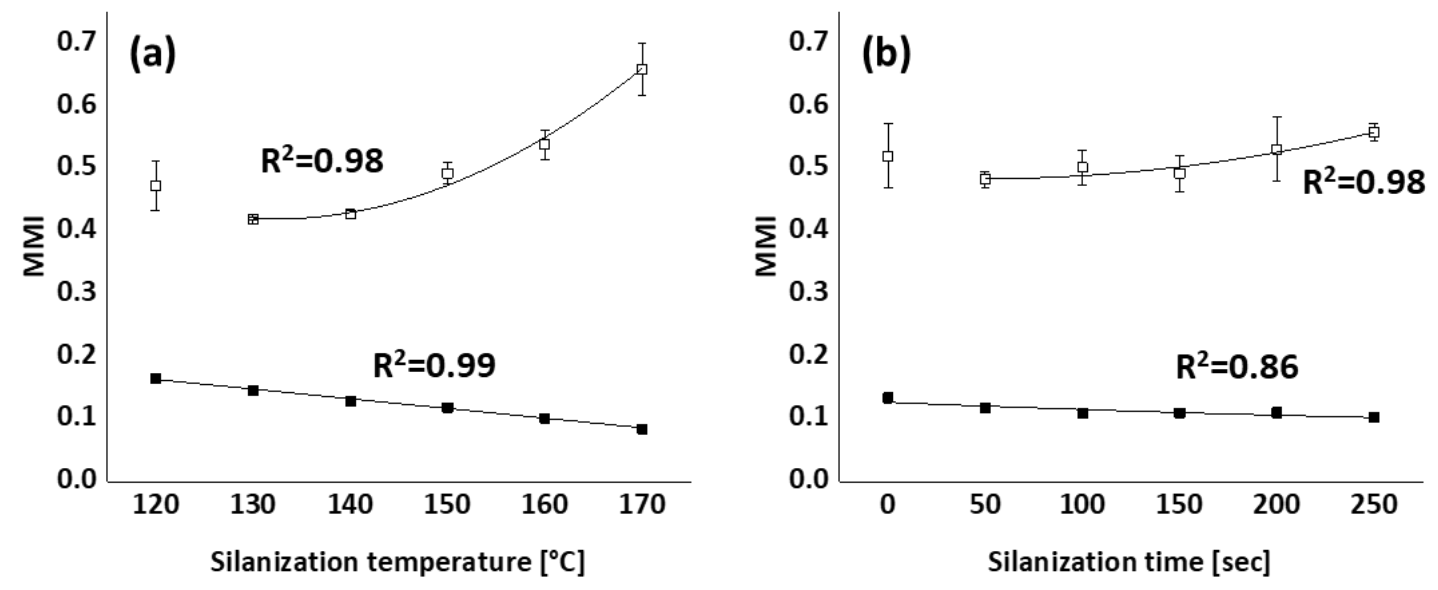

Figure 4.12 MMI as a function of silanization temperature and time; (a): MMI vs. silanization temperatures; (b): MMI vs. silanization time; ( $\square$ ): MMI 0.5 ; $\square-\square$ ): MMI $3^{\circ}$; fitting lines for each $\mathrm{MMI}$ in (a) and (b) are based on linear or polynomial fit with maximum $\mathrm{R}^{2}$.

\subsection{Marching modulus intensity at small (ASTM conditions, MMI $0.5^{\circ}$ ) and large strain $\left(\mathrm{MMI}^{\circ}\right)$}

As can be seen in Figure 4.12 , the $\mathrm{MMI} 0.5^{\circ}$ decreases with silanization temperature and time, with silanization time showing a slightly lower correlation coefficient. The maximum difference of $\mathrm{MMI} 0.5^{\circ}$ of the compounds mixed according to procedure B is about $40 \%$ of the difference found for compounds with Mixing procedure $\mathrm{A}$, and the $\mathrm{MMI}$ values of the former in the range of 50 seconds to 200 seconds only 
marginally decrease. The $\mathrm{MMI}$ at $3^{\circ}$ plotted as a function of both, silanization temperature and time, in Figure 4.12 show good correlations, except for the compounds mixed at a very low silanization temperature of $120^{\circ} \mathrm{C}$ or silanization time of 0 seconds. These exceptional MMI $3^{\circ}$ values can be explained by the intensity of filler-filler interaction still present at the early stage of silanization, as seen in Figure 4.3 with the high Payne effects.

Different from the trend of $\mathrm{MMI} 0.5^{\circ}$, the $\mathrm{MMI} 3^{\circ}$ increases with silanization temperature and time: Figure 4.12. The reason for this opposite trend is the decreasing vulcanization speed as observed in Figures 4.10(b) and 4.11(b) as well as shown in Table 4.4: where $T_{\min }$ is the values of the minimum torque, $T_{40}$ is the torque recorded at 40 minutes of measurement time, $\Delta T$ is the value difference between $T_{40}$ and $T_{\min }$ and maximum cure rate is the steepest slope observed in the rheogram. Even though all the mixes reach approximately the same end-level - with exception of the curves at $120^{\circ} \mathrm{C}$ and $170^{\circ} \mathrm{C}$ or for 0 seconds of silanization time - the curing rate becomes slower when the compounds are silanized at a higher temperature or with a long time.

Table 4.4 $T_{\min }, T_{40}, \Delta T$, maximum cure rate values obtained from Figures $4.10(\mathrm{~b})$ and $4.11(\mathrm{~b})$ Mixing procedure $A$

\begin{tabular}{c|cccccc}
\hline Silanization temperature $\left[{ }^{\circ} \mathrm{C}\right]$ & 120 & 130 & 140 & 150 & 160 & 170 \\
\hline $\mathrm{T}_{\min }[\mathrm{dNm}]$ & 11.2 & 11.2 & 11.8 & 12.8 & 14.1 & 16.5 \\
$\mathrm{~T}_{40}[\mathrm{dNm}]$ & 61.0 & 57.6 & 56.4 & 57.1 & 58.9 & 62.8 \\
$\Delta \mathrm{T}\left(\mathrm{T}_{40}-\mathrm{T}_{\min }\right)[\mathrm{dNm}]$ & 49.9 & 46.3 & 44.6 & 44.3 & 44.7 & 46.3 \\
Max. cure rate $[\mathrm{dNm} / \mathrm{min}]$ & 5.5 & 4.8 & 3.5 & 2.8 & 2.7 & 2.5 \\
\hline
\end{tabular}

Mixing procedure $B$

\begin{tabular}{c|cccccc}
\hline Silanization time $[\mathrm{sec}]$ & 0 & 50 & 100 & 150 & 200 & 250 \\
\hline $\mathrm{T}_{\min }[\mathrm{dNm}]$ & 13.5 & 13.0 & 14.2 & 14.7 & 15.8 & 15.7 \\
$\mathrm{~T}_{40}[\mathrm{dNm}]$ & 69.0 & 62.1 & 61.6 & 61.4 & 60.8 & 59.4 \\
$\Delta \mathrm{T}\left(\mathrm{T}_{40} \mathrm{~T}_{\min }\right)[\mathrm{dNm}]$ & 55.5 & 49.1 & 47.4 & 46.7 & 45.0 & 43.7 \\
Max. cure rate $[\mathrm{dNm} / \mathrm{min}]$ & 4.3 & 3.5 & 3.2 & 2.8 & 2.7 & 2.7 \\
\hline
\end{tabular}

\subsection{Marching modulus intensity as a function of FFR and CR}

A correlation was found between the MMI and FFR and CR respectively as shown in Figures 4.13 and 4.14. Within the range of lower and upper limit of FFR, both Mixing procedures A and B show good correlations 
with silanization temperature and time: $R^{2}=0.96$ and 0.91 , respectively: Figures $4.13(a)$. Since the influence of filler-filler interaction becomes less for $\mathrm{MMI} 3^{\circ}$ due to the high deformation, no correlation was found between FFR and MMI $3^{\circ}$ : Figure 4.13(b).
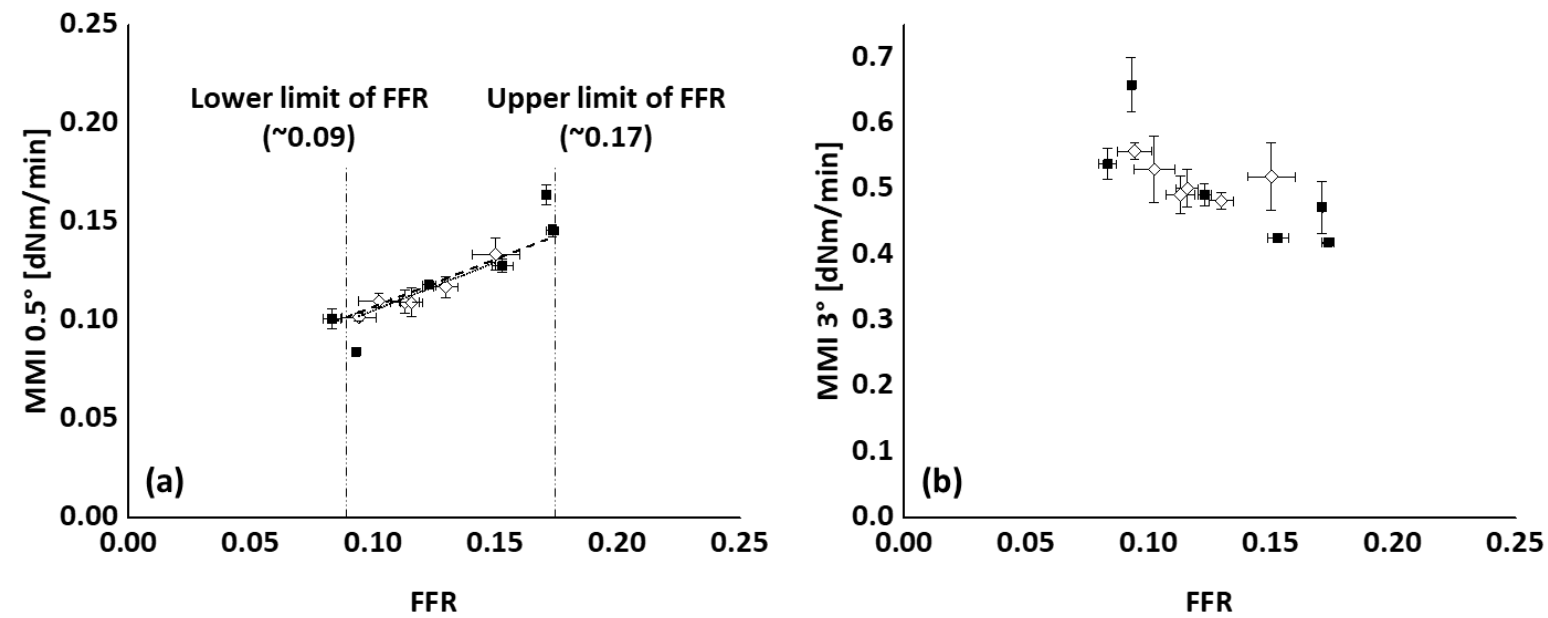

Figure 4.13 $\mathrm{MMI} 0.5^{\circ}$ versus FFR; (a): $\mathrm{MMI} 0.5^{\circ}$; (b): $\mathrm{MMI} 3^{\circ}$;

$(\boldsymbol{\square}-\mathbf{\square}$ or $\mathbf{\square})$ : Mixing procedure $\mathrm{A} ;(\diamond \cdots \diamond$ or $\diamond)$ : Mixing procedure B.
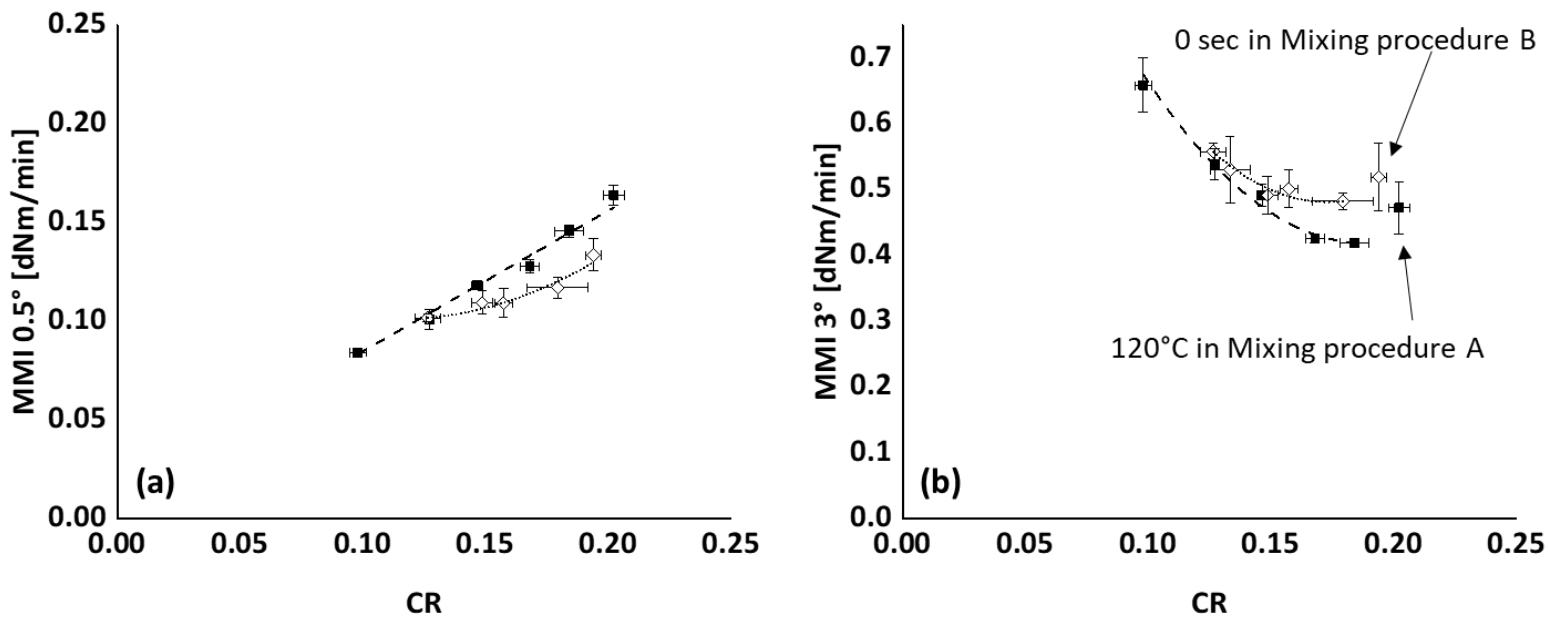

Figure 4.14 $\mathrm{MMI}$ versus $\mathrm{CR}$; (a): $\mathrm{MMI} 0.5^{\circ}$; (b): $\mathrm{MMI} 3^{\circ}$;

$(\boldsymbol{\square}-\mathbf{\square})$ : Mixing procedure $\mathrm{A} ;(\diamond \cdots . . \vee)$ : Mixing procedure B.

Not only the FFR, but also the CR is important for the $\mathrm{MMI} 0.5^{\circ}$ and $\mathrm{MMI} 3^{\circ}$. In Figure 4.14 , both MMIs are plotted as a function of CR. A higher silanization temperature and longer silanization time lead to a higher amount of bound rubber and indicate a lower concentration of free silane and released free sulfur from 
TESPT. As a consequence, a slower and lower degree of the coupling reaction would occur during the vulcanization process. Finally, a lower torque level and corresponding flat plateau of the rheograms is obtained when the rheometer runs at small strains, approx. $7 \%\left(\sim 0.5^{\circ}\right)$. In case of large strain: approx. $42 \%\left(\sim 3^{\circ}\right)$, a rapid rise of the rheometer torque can be seen in the compound, which was mixed at a lower silanization temperature or a shorter silanization time, due to a faster filler-polymer coupling reaction: a relatively large amount of free silane and free sulfur remains within the compound. These results again show that the shielding of the filler surface not only by a silane coupling agent but also by bound rubber does strongly affect the rheometer torque.

\subsubsection{Conclusions}

The focus of this study was to investigate mixing parameters that can reduce the marching modulus during curing of silica filled S-SBR/BR rubber compounds. The silanization temperature and time strongly influence the marching modulus phenomenon. A higher temperature and a longer time for the silanization lead to a better degree of silanization, where temperature has the largest effect.

The amount of chemically bound rubber of a silica compound has an intricate influence on the filler-filler interaction as well as on the silica flocculation rate and the filler-polymer coupling rate, all strongly related to the marching modulus. The chemically bound rubber suppresses the flocculation behavior of the silica in rubber during the vulcanization process. However, a very low or even very high amount of chemically bound rubber no longer affects the silica flocculation rate: FFR has an upper and a lower limit. The consequence of a higher amount of chemically bound rubber is a lower concentration of free silane which may enhance the filler-polymer coupling reaction during vulcanization. Therefore, as the amount of bound rubber increases, a slower filler-polymer coupling rate is obtained during vulcanization and results in a reduction of the marching modulus phenomenon of silica compounds.

\section{References}

[1] W. K. Dierkes, J. W. M. Noordermeer, M. Rinker, K.-U. Kelting, C. Van de Pol, Kautsch. Gummi Kunstst. 56, 338 (2003).

[2] L. A. E. M. Reuvekamp, J. W. ten Brinke, P. J. van Swaaij, J. W. M. Noordermeer, Rubber Chem. Technol. 75, 187 (2002).

[3] S. Mihara, R. N. Datta, J. W. M. Noordermeer, Rubber Chem. Technol. 82, 524 (2009).

[4] J. Jin, J. W. M. Noordermeer, W. K. Dierkes, A. Blume, Rubber Chem. Technol. in press (2019).

[5] A. Hasse, O. Klockmann, A. Wehmeier, H.-D. Luginsland, Kautsch. Gummi Kunstst. 55, 236 (2002). 


\section{Chapter 4.2 The effect of zinc oxide concentration in the early and later mixing stage on marching modulus}

\subsubsection{Introduction}

In the rubber industry, zinc oxide (ZnO) was initially used as a reinforcing filler. In the early 1920s, it was discovered that $\mathrm{ZnO}$ is capable of accelerating the sulfur-based crosslinking reaction of rubber, which is commonly denoted as vulcanization. ${ }^{[1]}$ When $\mathrm{ZnO}$ is combined with stearic acid, shorter curing times and enhanced vulcanizate properties can be obtained. Fatty acids, such as stearic acid, enhance the solubility of zinc ions and helps to form the zinc-accelerator complex, thus catalyze the vulcanization process. ${ }^{[2]}$ In special cases, magnesium oxide $(\mathrm{MgO})$, lead oxide $(\mathrm{PbO})$ and calcium hydroxide $\left(\mathrm{Ca}(\mathrm{OH})_{2}\right)$ are used instead of $\mathrm{ZnO}$; however, $\mathrm{ZnO}$ is known as the best activator for sulfur vulcanization. ${ }^{[1,3]}$ Therefore, $\mathrm{ZnO}$ is still widely used as an essential ingredient for rubber compounding in the tire and other rubber industries. However, in case of silica filled compounds, it is proposed that $\mathrm{ZnO}$ can inhibit the silanization reaction ${ }^{[4-6]}$ due to its alkalinity: Acidic hydroxyl $(-\mathrm{OH})$ groups on the silica surface can lead to physisorption of the basic ZnO molecules. Magami et al. ${ }^{[3]}$ observed a higher Payne effect value for a silica

filled functionalized S-SBR compound, when $\mathrm{ZnO}$ is introduced in an earlier mixing stage. Blume ${ }^{[7]}$ found in a series of model reactions with $\mathrm{ZnO}$, stearic acid and several types of silane coupling agents, that the silanization reaction rate at the very beginning was delayed in the presence of $\mathrm{ZnO}$, and even when $\mathrm{ZnO}$ was combined with stearic acid. Overall, those studies indicate that the presence of $\mathrm{ZnO}$ in a rubber matrix has a negative effect on the degree of silanization.

In Chapters 3.1 and 4.1, it is concluded that the degree of silanization is a key factor for the marching modulus of silica filled tire tread compounds. Based on these precedent studies, the concentration of $\mathrm{ZnO}$ in the MasterBatch (MB) and final mixing step was considered as one main variable for this study on the efficiency of the silanization and the correlation with the marching modulus. A series of compounds was prepared varying the concentration of $\mathrm{ZnO}$ in the $\mathrm{MB}$ and the final mixing stage, with the final compound formulation kept the same. Subsequently, the vulcanization behavior of these compounds was measured at small (approx. 7\%) and large (approx. 42\%) strain. The results are interpreted in terms of degree of silanization, as well as the Filler Flocculation Rate (FFR), filler-polymer Coupling Rate (CR) and the amount of bound rubber. 


\subsubsection{Experimental}

\subsubsection{Materials and Mixing}

All series of experiments were done based on a tire tread compound as shown in Table 4.1 in Chapter 4.1. The acronyms of the compounds and their $\mathrm{ZnO}$ concentrations in the MasterBatch (MB) and the final mixing steps are shown in Table 4.5. The compounds were mixed in two steps as shown in Table 4.6. The masterbatch stage was done using a lab scale internal mixer (Brabender Plasticorder) with a chamber volume of $390 \mathrm{ml}$. The fill factor of the internal mixer, TCU settings, starting temperature of mixing, silanization temperature control, the compound cooling after the MB mixing and the number of batches per each set of conditions were the same as described in Chapter 4.1. The compounds were mixed with good reproducibility as judged by the mixing fingerprints depicted in Figure 4.15.

Table 4.5 Formulation of the compound: $\mathrm{ZnO}$ concentration

\begin{tabular}{c|ccccc}
\hline Acronyms & Z00 [phr] & Z05 [phr] & Z10 [phr] & Z15 [phr] & Z20 [phr] \\
\hline MasterBatch (MB) & 0.0 & 0.5 & 1.0 & 1.5 & 2.0 \\
Final & 2.0 & 1.5 & 1.0 & 0.5 & 0.0 \\
\hline
\end{tabular}

Table 4.6 Mixing procedures

\begin{tabular}{|c|c|c|c|}
\hline \multicolumn{2}{|c|}{ MasterBatch (MB) step } & \multicolumn{2}{|c|}{ Final step } \\
\hline \multicolumn{2}{|c|}{ Internal mixer } & \multicolumn{2}{|c|}{ Open mill } \\
\hline Action & time [mm:ss] & Action & time $[\mathrm{mm}: \mathrm{ss}]$ \\
\hline Add polymer & $00: 00 \sim 00: 20$ & \multirow{2}{*}{ Add masterbatch } & \multirow{2}{*}{-} \\
\hline Mastication & $00: 20 \sim 01: 20$ & & \\
\hline $1 / 2$ Silica, silane & 01:20 01:40 & \multirow{2}{*}{ Mixing } & \multirow{2}{*}{ 02:00 } \\
\hline Mixing & $01: 40 \sim 02: 40$ & & \\
\hline $\begin{array}{l}1 / 2 \text { Silica, ZnO (except for Z00), } \\
\text { remaining ingredients }\end{array}$ & 02:40 03:10 & \multirow{2}{*}{$\begin{array}{l}\text { Add curatives, } \\
\text { remaining ZnO } \\
\text { (except for Z20) }\end{array}$} & \multirow{2}{*}{$02: 30$} \\
\hline Mixing till $150^{\circ} \mathrm{C}$ & 03:10 04:10 & & \\
\hline Ram sweep & 04:10 04:14 & \multirow{2}{*}{ Mixing } & \multirow{2}{*}{ 09:00 } \\
\hline Mixing (at $150^{\circ} \mathrm{C}$ ) & $04: 14 \sim 06: 40$ & & \\
\hline Discharge and sheeting & - & Discharge & - \\
\hline
\end{tabular}




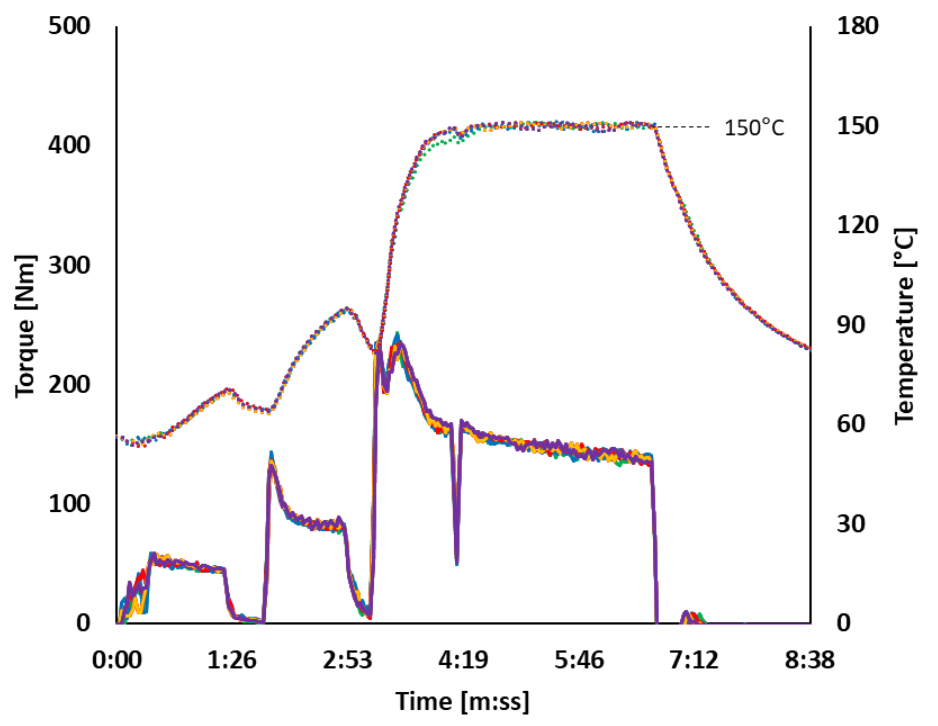

Figure 4.15 Mixing fingerprints of Mixing procedure A; ( solid line ): torque; ( dotted line ): temperature;
( $):$ ZOO;
): ZO5 ; (
): Z10;
): Z15; (
): Z20 .

\subsubsection{Property analysis and testing}

Payne effect, Filler Flocculation Rate (FFR), filler-polymer Coupling Rate (CR), cure characteristic and Marching Modulus Intensity (MMI), bound rubber content of the compounds were investigated according to the methods as described in Chapter 3.1.

\subsubsection{Results and discussion}

\subsubsection{Filler-filler interaction (Payne effect) as a function of $\mathrm{ZnO}$ concentration in the MB}

The Payne effect values of the uncured compounds are plotted as a function of $\mathrm{ZnO}$ concentration in the $\mathrm{MB}$ stage: Figure 4.16. An increment of the Payne effect value is observed with increasing $\mathrm{ZnO}$ concentration in the $\mathrm{MB}$ stage until the $\mathrm{ZnO}$ concentration reaches $1.5 \mathrm{phr}$. This result can be explained with the negative role of $\mathrm{ZnO}$ in silica filled rubber as explained in 4.2.1. However, the effect of $\mathrm{ZnO}$ on filler-filler interaction (Payne effect) in this study is much smaller that the effect of silanization time and temperature as discussed in Chapter 4.1: Figure 4.3. 


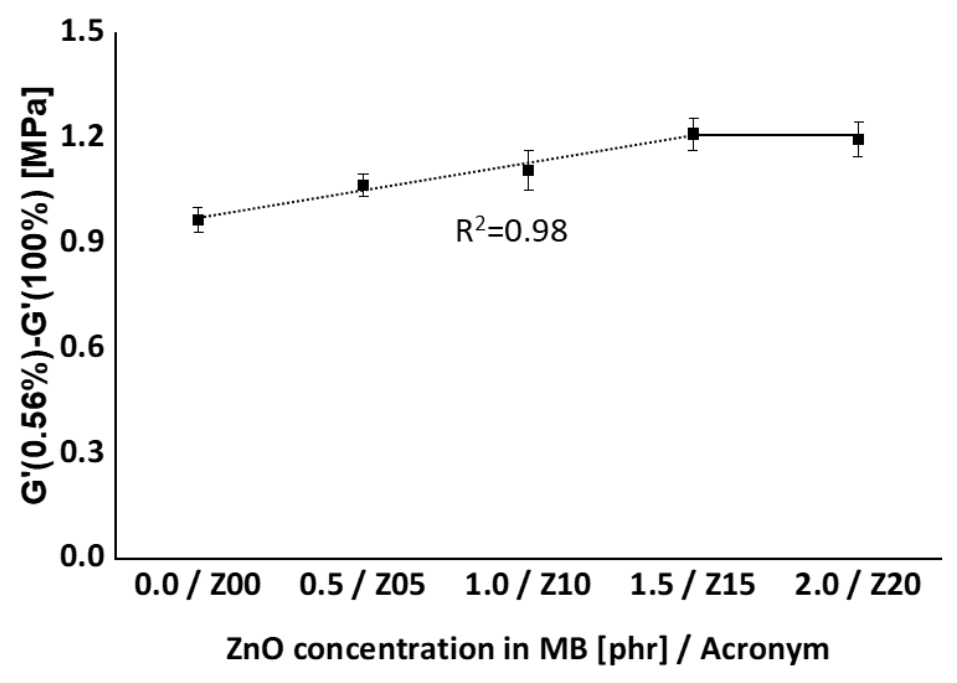

Figure 4.16 Payne effect as a function of $\mathrm{ZnO}$ concentration in the $\mathrm{MB} ;$ ( . ... ) : where the Payne effect shows good correlation with ZnO concetration in MB; ( $)$ ): where the Payne effect shows plateau; the fitting line is based on linear fit with maximum $\mathrm{R}^{2}$.

Reuvekamp et al. ${ }^{[6]}$ reported that presence of $\mathrm{ZnO}$ increases the risk of pre-scorch due to its catalytic effect on sulfur release from bis-(triethoxysilylpropyl)tetrasulfide (TESPT). They observed an increase of $\mathrm{G}^{\prime}(100 \%)$ for the compound with $\mathrm{ZnO}$ added in an early mixing stage, when the dump temperature exceeded $150^{\circ} \mathrm{C}$ : Figure $4.17(\mathrm{a})$. However, in the study described in this chapter, the silanization temperature did not exceed $150^{\circ} \mathrm{C}$, thus below the critical temperature of pre-scorch activated by the presence of $\mathrm{ZnO}$. Therefore, a variation of $\mathrm{G}^{\prime}(100 \%)$ along with $\mathrm{ZnO}$ concentration in the MB can hardly be seen: Figure 4.17(b). The effect of $\mathrm{ZnO}$ and stearic acid on sulfur release from TESPT will be further investigated in a model compound study, which is the topic of Chapter 4.4. 

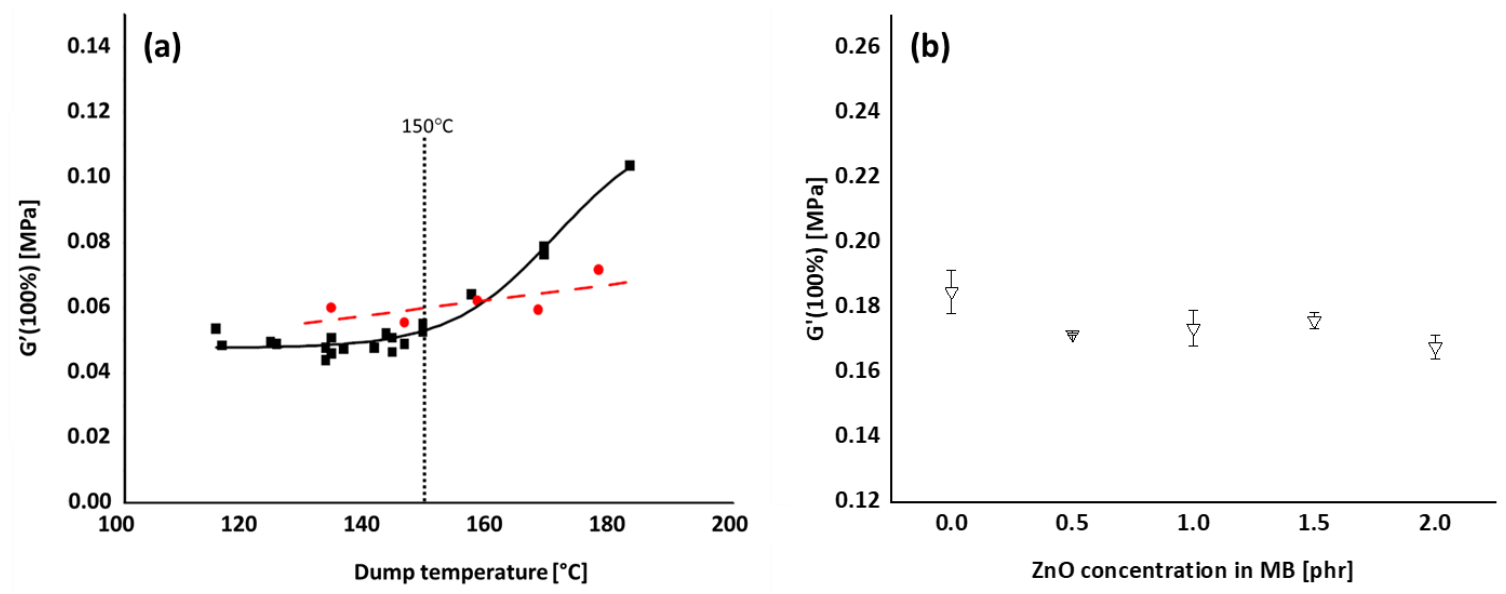

Figure $4.17 \mathrm{G}^{\prime}$ at $100 \%$ strain as indication of filler-polymer interaction; (a): dump temperature and presence of $\mathrm{ZnO}$ in $\mathrm{MB}^{[6]}$ : ( $\left.\mathbf{\square}\right)$ : with $\mathrm{ZnO}$; $(\mathrm{O})$ : without $\mathrm{ZnO}$; (b): $\mathrm{G}^{\prime}(100 \%)$ vs $\mathrm{ZnO}$ concentration in $\mathrm{MB}$.

\subsubsection{Bound rubber contents as a function of $\mathrm{ZnO}$ concentration in $\mathrm{MB}$}

As was discussed in Chapters 3.1 and 4.1, the Payne effect is strongly influenced by the amount of chemically bound rubber. Besides, $\mathrm{G}^{\prime}(100 \%)$ can be used as an indicator for pre-scorch. ${ }^{[6]}$ As expected from the small changes in Payne effect and the $G^{\prime}(100 \%)$ values in Figures 4.16 and $4.17(\mathrm{~b})$ respectively, the total and chemically bound rubber content within these mixing series should be rather constant as a function of $\mathrm{ZnO}$ concentration in the $\mathrm{MB}$ except for Z00: Figure 4.18. Significant decrements in total and chemically bound rubber are only observed for the data points between Z00 and Z05; the values of the others compounds only slightly decreased with increasing amount of $\mathrm{ZnO}$ in the MB.

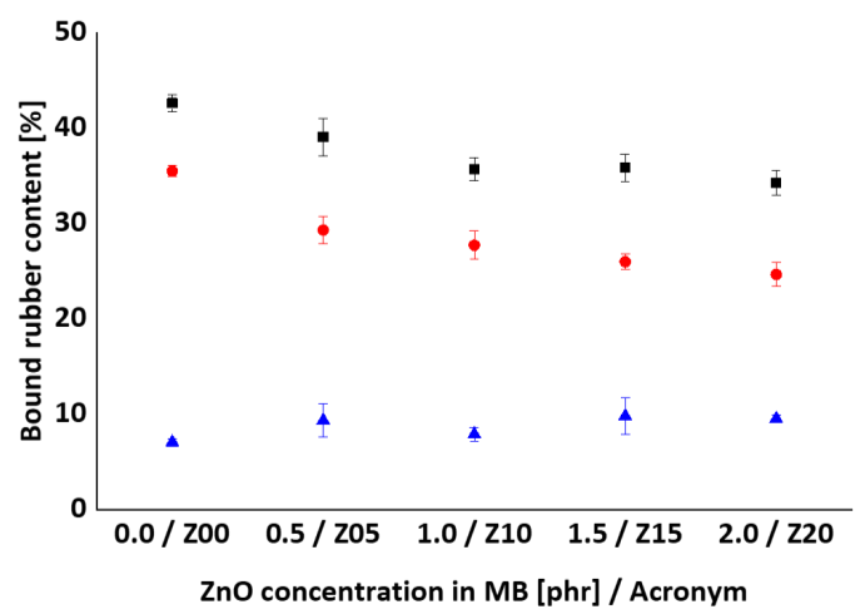

Figure 4.18 Bound rubber content as a function of $\mathrm{ZnO}$ concentration in the $\mathrm{MB}$;

$(\boldsymbol{\square})$ : total bound rubber; $(\boldsymbol{O})$ : chemically bound rubber; $(\boldsymbol{\Delta})$ : physically bound rubber. 
Taking into account the small differences of the bound rubber and the Payne effect values - except for ZOO - it can be stated that the presence of zinc stearate interferes with the silanization reaction, as depicted in Figure 4.19. The Payne effect does show a trend, but compared to the results of Chapter 4.1 (Figure 4.4), the value difference between the data points of $Z 05$ to $Z 20$ in the figure is rather small due to a higher $\mathrm{ZnO}$ concentration. $\mathrm{ZnO}$ itself cannot cover the silica surface; this becomes only possible when it forms zinc stearate. If $\mathrm{ZnO}$ itself would cover the silica surface, $\mathrm{Z20}$ should show a higher Payne effect level and lower amount of total and chemically bound rubber compared to the values of Z15. However, the Payne effect shows a rather constant value (Figure 4.16) compared to earlier trends (see Figure 4.4) and a small difference in the bound rubber values, see Figure 4.18.

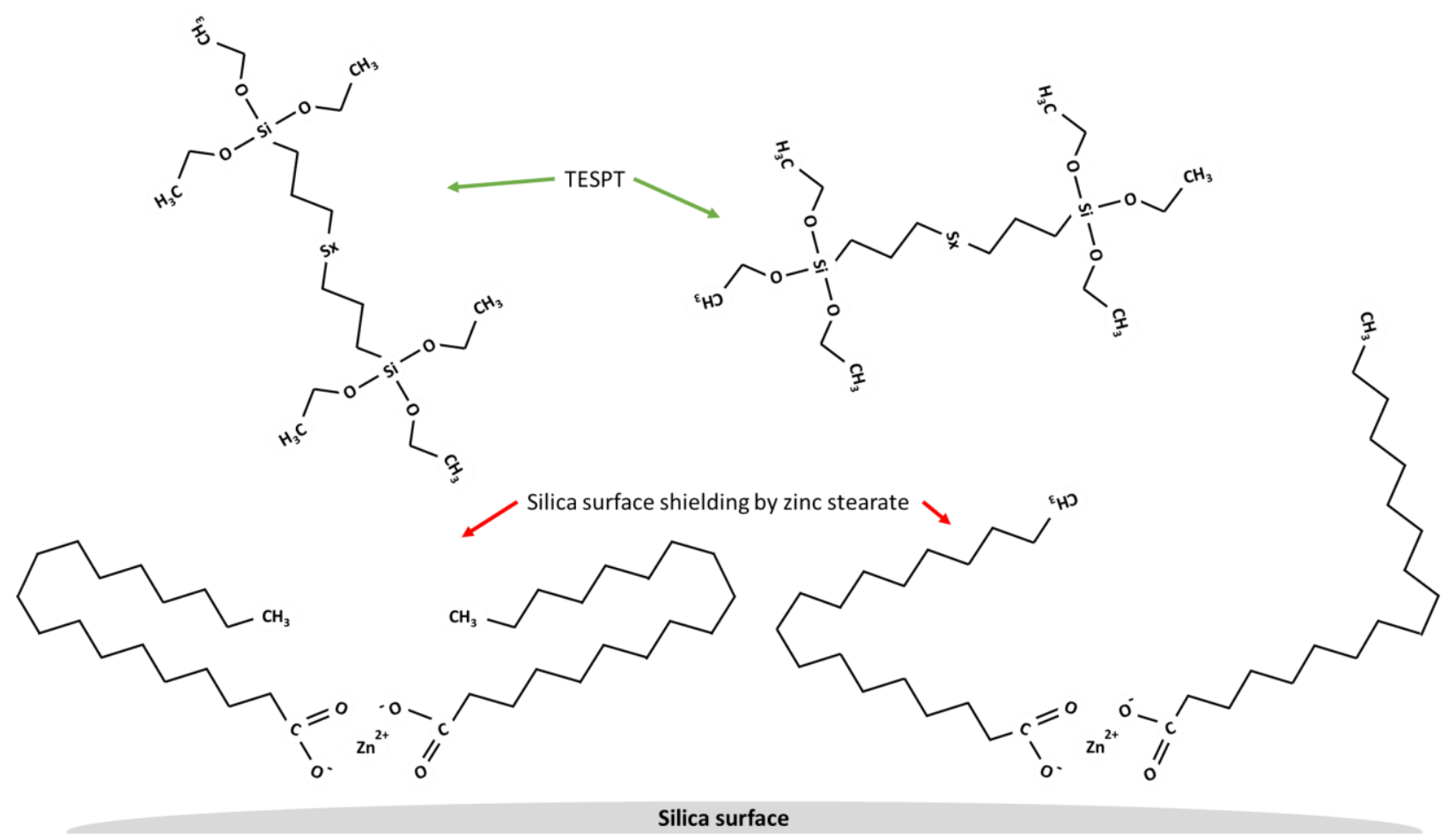

Figure 4.19 Possible mechanism of shielding of the silica surface and blocking for silane by zinc stearate.

In this sense, zinc stearate can have a positive effect on lowering the filler-filler interaction due to its long hydrocarbon side chains ( $2 \mathrm{C}_{18}$ groups). It is for this reason, that the zinc soap and the fatty acid are the most popular processing agents for this type of compounds. ${ }^{[9,10]}$ However, when zinc stearate blocks the access of silane molecules, it will result in a lower amount of chemically bound rubber - which has a significant effect on reducing filler-filler interaction - as a consequence of insufficient silanization. The effect of chemically bound rubber on reducing the Payne effect level is already confirmed in Chapters 3.1 
and 4.1 and can also be seen in this work. In Figure 4.20, the Payne effect is plotted as a function of chemically bound rubber, and it confirms the trend observed in Chapters 3.1 and 4.1: a higher content of chemically bound rubber leads to a lower Payne effect.

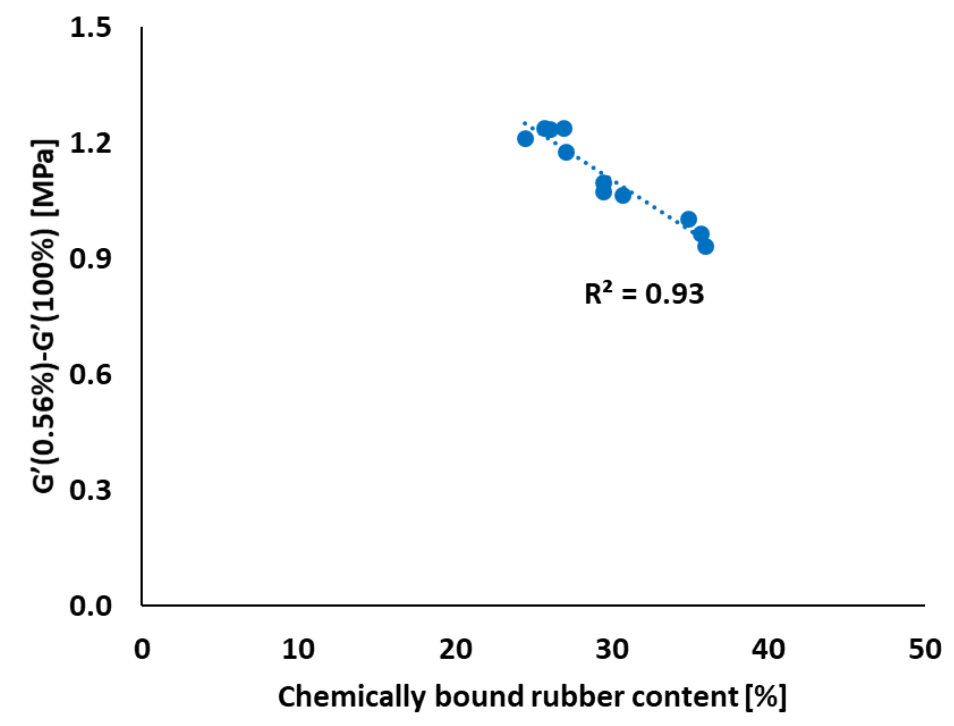

Figure 4.20 Payne effect vs. chemically bound rubber content.

\subsubsection{Silica filler flocculation rate}

The silica Filler Flocculation Rate (FFR) is shown in Figure 4.21. No clear trend is observed along with the $\mathrm{ZnO}$ concentration in the $\mathrm{MB}$, but a slight difference in the level of FFR between the compounds with and without $\mathrm{ZnO}$ is observed. The result firstly indicates that FFR is influenced by the presence of $\mathrm{ZnO}$ in the $\mathrm{MB}$, but is independent of the concentration. As was seen in Figure 4.19 showing the complex formed from $\mathrm{ZnO}$ and stearic acid, this results in blocking the silica surface for a reaction with silane. Subsequently, less bound rubber compared to the compound without $\mathrm{ZnO}$ in the $\mathrm{MB}$ will be formed. As a consequence, a higher FFR value will be obtained. 


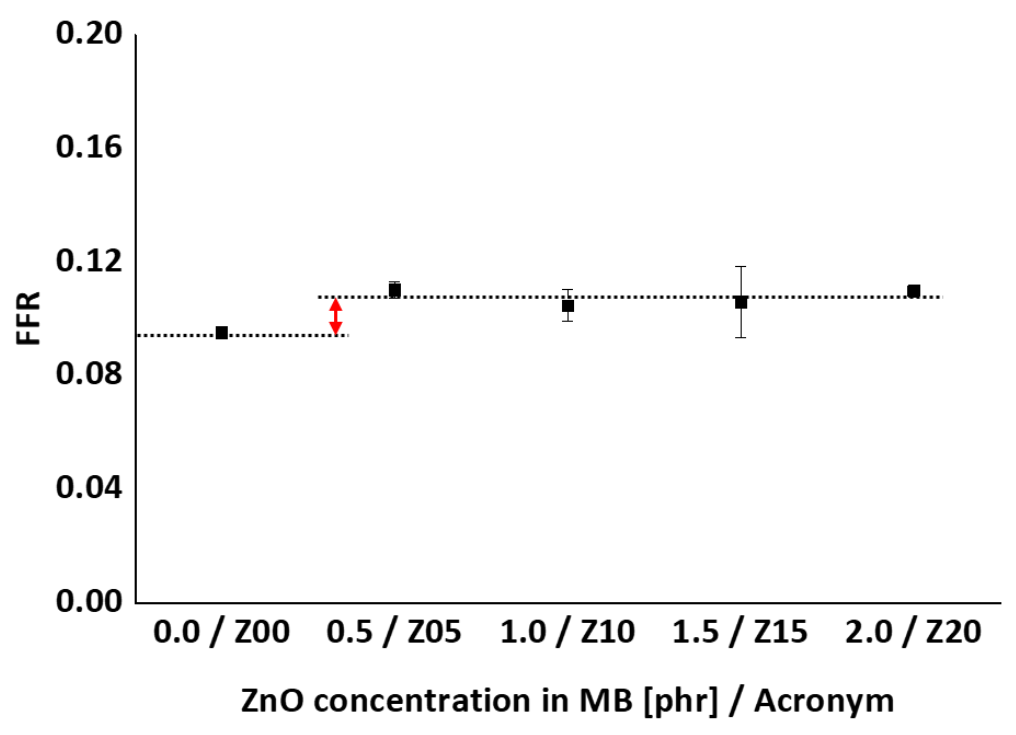

Figure 4.21 FFR vs. ZnO concentration in MB.

$\mathrm{ZnO}$ addition in the final mixing stage (Z00) has an advantage over the other compounds (Z05 to Z20, with $\mathrm{ZnO}$ in the MB stage): the filler flocculation rate is lower in the case of Z00. The FFR measured here (0.09) is comparable to the rate found in Chapter 4.1 for the compounds silanized at $160^{\circ} \mathrm{C}$ for 150 seconds (with Mixing procedure A) or at $150^{\circ} \mathrm{C}$ for 250 seconds (with Mixing procedure B): Figure 4.6. The other compounds ( $\mathrm{Z05}$ to $\mathrm{Z20}$, with $\mathrm{ZnO}$ ) gave similar FFR values as the compound silanized at $150^{\circ} \mathrm{C}$ for 150 seconds in both mixing procedures ( 0.11) as shown in Chapter 4.1: Figure 4.6. This result indicates that $\mathrm{ZnO}$ addition in later mixing stage will provide a lower FFR value, as does mixing at a higher temperature or for a longer silanization time: it results in a better compound quality and enhanced processability.

\subsubsection{Filler-polymer coupling rate after mixing}

The filler-polymer Coupling Rate $(C R)$ is strongly related to the concentration of the remaining silane after mixing, as well as to the presence of active sulfur (additionally introduced or released from the silane). ${ }^{[8]}$ As seen in Figures 4.17(b) and 4.18, it can easily be noticed that the amount of silane as well as the free sulfur released from TESPT after the MB phase are almost the same for all compounds in this work. This can be seen in Figure 4.17(b) in which similar levels of $G^{\prime}(100 \%)$ - an indication of pre-scorch - were obtained regardless of the $\mathrm{ZnO}$ concentration in the MB. Additionally, the amount of chemically bound rubber of the compounds - an indication of filler-polymer coupling during mixing, Figure 4.18 - did not differ much except for Z00. As a consequence, the value of CR is rather constant as shown in Figure 4.22. 


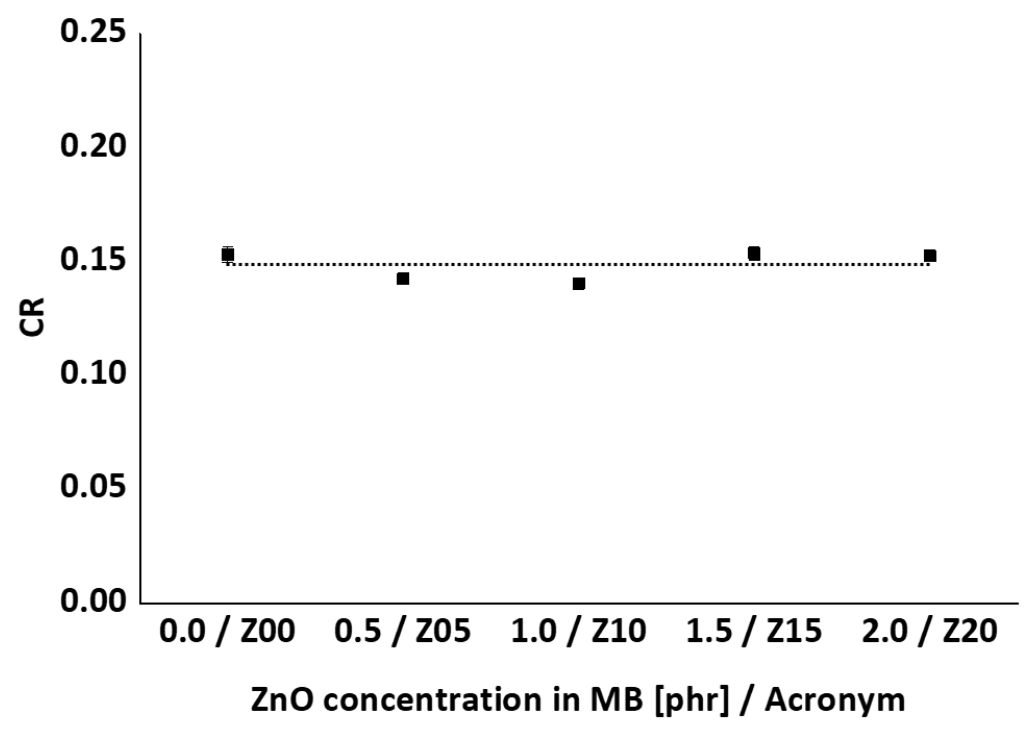

Figure 4.22 CR vs. ZnO concentration in $\mathrm{MB}$; the error bars are smaller than the symbol.

\subsubsection{Presence of $\mathrm{ZnO}$ as an influencing factor for marching modulus intensity}

The rheograms and their Marching Modulus Intensities (MMI) measured at two different strains for the compounds with different $\mathrm{ZnO}$ concentrations in the MB stage are plotted in Figures 4.23 and 4.24, respectively. No clear tendency neither level difference in the MMI values are observed for the rheograms measured at $3^{\circ}$ of strain ( $42 \%$ ): Figure $4.24(b)$.
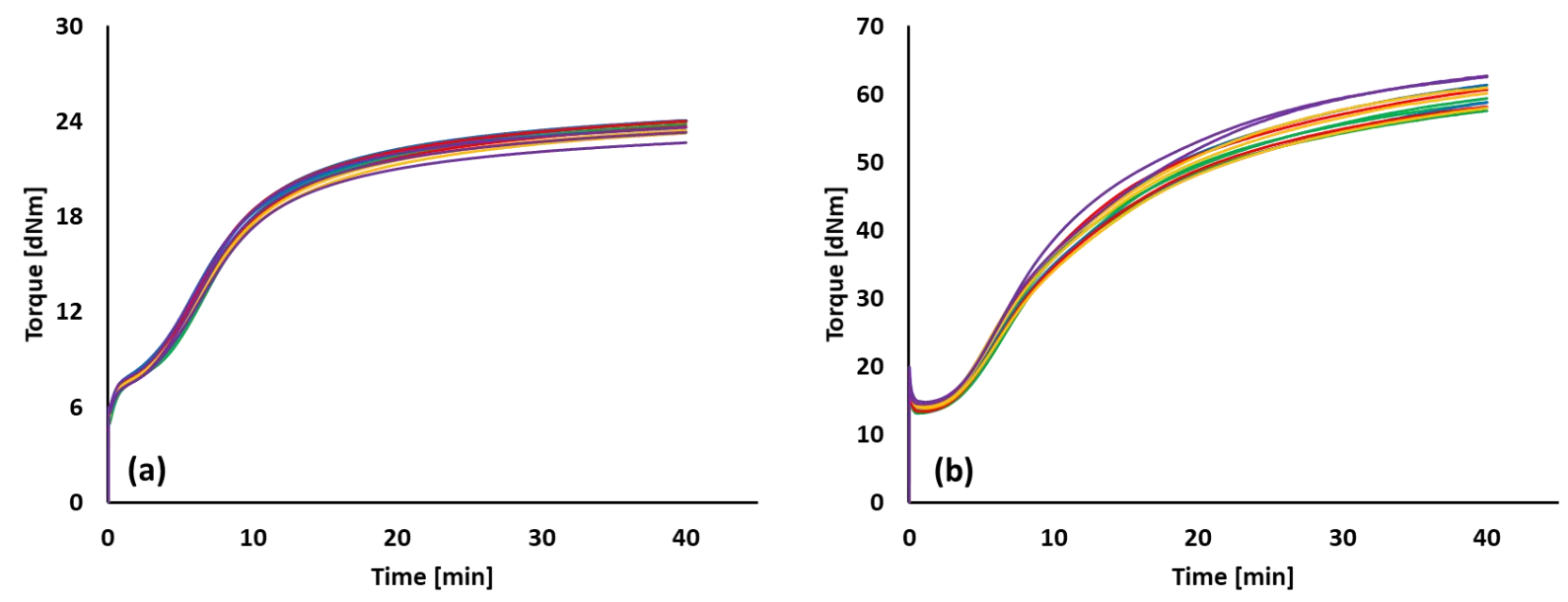

Figure 4.23 Rheograms of the compound; (a): $0.5^{\circ}$ ( 7\% of strain); (b): $3^{\circ}$ ( $42 \%$ of strain);

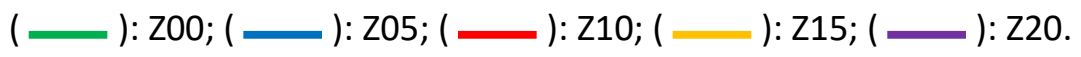



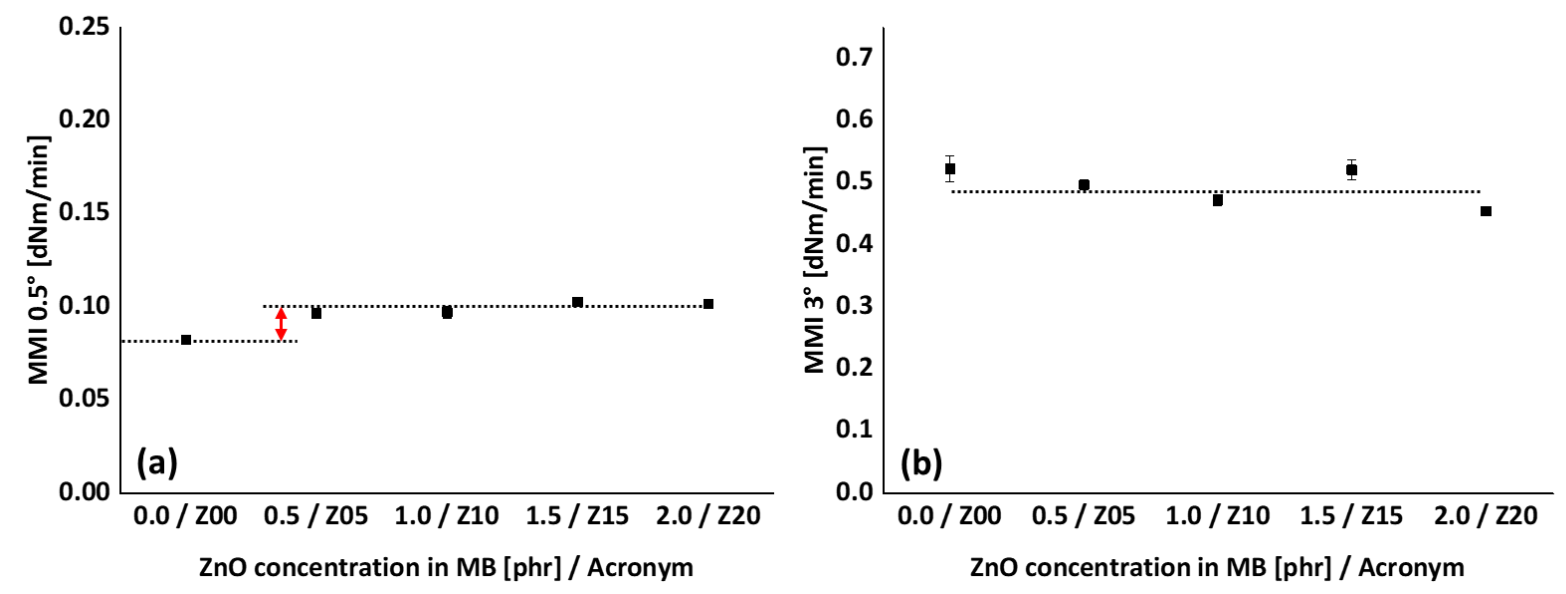

Figure 4.24 $\mathrm{MMI}$ vs. $\mathrm{ZnO}$ concentration in the $\mathrm{MB}$; (a): $\mathrm{MMI} 0.5^{\circ}$; (b): $\mathrm{MMI} 3^{\circ}$; in some cases, the range of error bars are smaller the data points thus not visible.

The rheograms measured at $0.5^{\circ}$ ( 7\%) of strain: Figure 4.24(a), do not show a significant difference in MMI neither. However, it can be seen in Figure 4.24(a), that the MMI value of Z00 (without $\mathrm{ZnO}$ ) is on a different level than the MMI values of the other compounds with $\mathrm{ZnO}$ added in the earlier mixing step. As already stated in Chapters 3.1 and 4.1, FFR and CR strongly influence the MMI $0.5^{\circ}$. In this part of the study, the CR values for all compounds are comparable, see Figure 4.22, an influence on $\mathrm{MMI} 0.5^{\circ}$ therefore cannot be identified. However, as was seen in Figure 4.21, the presence of $\mathrm{ZnO}$ in the MB does influence the FFR. As a consequence, the $\mathrm{MMI} 0.5^{\circ}$ shows a difference between $\mathrm{ZOO}$ (without $\mathrm{ZnO}$ ) and all other compounds with $\mathrm{ZnO}$ added in the earlier mixing stage: the data points of $\mathrm{Z} 05$ to $\mathrm{Z20}$ are clustered, while the compound without $\mathrm{ZnO}$ in the $\mathrm{MB}$ is on a different level: Figure 4.25. This result again confirms that the MMI $0.5^{\circ}$ is a function of FFR. 


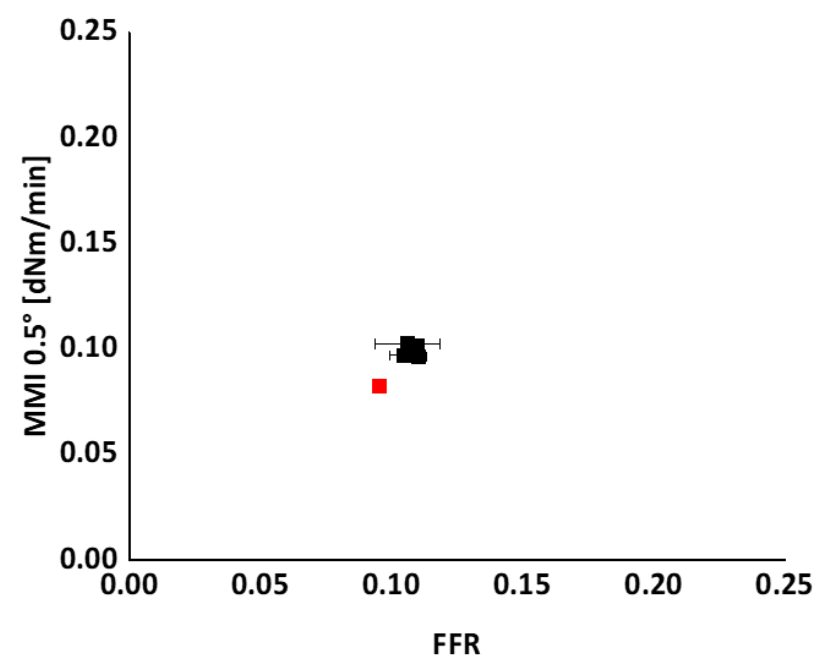

Figure 4.25 $\mathrm{MMI} 0.5^{\circ}$ versus FFR; $(\square)$ : $\mathrm{MB}$ mixed without $\mathrm{ZnO}$; $(\boldsymbol{\square})$ : ZnO present in $\mathrm{MB}$.

Similar to the FFR results, the MMI $0.5^{\circ}$ of $Z 00$ (without $\mathrm{ZnO}$ in the $\mathrm{MB}$ ) shows the same value ( 0.08 ) as the compounds silanized at $160^{\circ} \mathrm{C}$ according to Mixing procedure A or for 200 seconds according to Mixing procedure B, seen in Chapter 4.1. The other compounds show the same MMI value ( 0.11) which was obtained for the compounds silanized at $150^{\circ} \mathrm{C}$ for 150 seconds in both mixing procedures in Chapter 4.1. Adding $\mathrm{ZnO}$ in a later mixing step lowers the FFR, thus the $\mathrm{MMI} 0.5^{\circ}$ level is reduced as well: a better compound quality and enhanced processability can be achieved by a lower FFR and MMI value.

\subsubsection{Interaction between zinc stearate and the silica surface}

Taking into account the low degree of variation of the Payne effect and bound rubber, it can be stated that the interaction between zinc stearate and the silica surface is not very strong. Therefore, the zinc complex can leave the surface easily and can be replaced by silane. If this would not be the case, the results of the Payne effect and bound rubber content would show larger value differences between data points of the samples as was seen in Figures 4.3 and 4.4. In accordance with this result, Blume ${ }^{[7]}$ obtained the same yield of the silica-silane reaction, independent if $\mathrm{ZnO}$ or stearic acid or both are present in the system. Additional evidence for the weak interaction of silica-zinc stearate can be found by acquiring the calculated cure times using the method described in Paragraph 3.1.2.7 and the maximum cure rate (the steepest slope in the rheogram) from Figure 4.23(a). The values of the calculated cure time and maximum cure rate are listed in Table 4.7. 
Table 4.7 Calculated cure times and maximum cure rates acquired from Figure 4.23(a)

\begin{tabular}{cccc}
\hline Acronym & $\begin{array}{c}\text { ZnO concentration in MB } \\
{[\mathrm{phr}]}\end{array}$ & Calculated cure time [min] & $\begin{array}{c}\text { Max. cure rate } \\
{[\mathrm{dNm} / \mathrm{min}]}\end{array}$ \\
\hline Z00 & 0.0 & 13.9 & 1.6 \\
Z05 & 0.5 & 13.7 & 1.7 \\
Z10 & 1.0 & 13.7 & 1.7 \\
Z15 & 1.5 & 13.6 & 1.7 \\
Z20 & 2.0 & 13.7 & 1.6 \\
\hline
\end{tabular}

If the interaction between zinc stearate and silica would be strong, it would inhibit the crosslinking reaction and thus a longer calculated cure time and a slower maximum cure rate would be obtained with increasing $\mathrm{ZnO}$ concentration in the $\mathrm{MB}$ : the adsorbed zinc stearate hardly participates in the vulcanization reaction. However, as can be seen in Table 4.7, the calculated cure time and maximum cure rate were the same for all $\mathrm{ZnO}$ concentrations in the $\mathrm{MB}$, which means they can easily leave the silica surface.

\subsubsection{Conclusions}

The focus of this part of the study are the mixing protocol that can reduce the marching modulus during curing of silica filled S-SBR/BR rubber compounds. This study showed that the presence of $\mathrm{ZnO}-$ to be exact, zinc stearate - influences the marching modulus behavior of silica filled rubber, but its concentration in the MB has no effect at all. It is the presence or absence of $\mathrm{ZnO}$ in the MB that makes the difference. Additionally, a possible mechanism of blocking the silica surface for access of the silane is suggested based on the results of this study: Figure 4.19.

$\mathrm{ZnO}$ influences the FFR, when it is introduced in an early mixing stage, as was already found as a major influencing factor for the marching modulus phenomenon. When $\mathrm{ZnO}$ reacts with stearic acid to form zinc stearate, the two long hydrocarbon groups - having each 18 carbon atoms - are capable of blocking the access to the silica surface for the silane. Zinc stearate might provide a positive effect on reducing fillerfiller interaction; however, as soon as $\mathrm{ZnO}$ is added in the early stage the amount of bound rubber is reduced (Figure 4.18).

All in all, the effect of $\mathrm{ZnO}$ addition in the final mixing stage on the MMI reduction was not as significant as mixing at a higher silanization temperature or for a longer time. However, it will give a better quality of the compound in terms of filler-filler interaction, FFR, and bound rubber content. 


\section{References}

[1] G. Heideman, R. N. Datta, J. W. M. Noordermeer, Rubber Chem. Technol. 77, 512 (2004).

[2] "Elastomer Technology Handbook", M. R. Kresja, J. L. Koening, CRC Press, New Jersey (1993).

[3] S Maghami, W. K. Dierkes, J. W. M. Noordermeer, Rubber Chem. Technol. 89, 559 (2016).

[4] S. H. Laning, M.P. Wagner and J.W. Sellers, J. Appl. Polymer Sci. 2, 225 (1959).

[5] F. Thurn, S. Wolff, Kautsch. Gummi Kunstst., 28, 733 (1975).

[6] L. A. E. M. Reuvekamp, S. C. Debnath, J. W. ten Brinke, P. J. van Swaaij, J. W. M. Noordermeer, Rubber Chem. Technol. 77, 34 (2004).

[7] A. Blume, 180th meeting Rub. Div. ACS Cleveland, Ohio, Paper No. 22 (2011).

[8] J. J. Jin, J. W. M. Noordermeer, W. K. Dierkes, A. Blume, Rubber Chem. Technol. in press (2019). https://doi.org/10.5254/rct.19.80453

[9] J. van der Kooi, 150th meeting Rub. Div. ACS Louisville, Kentucky, Paper No. 2 (1996).

[10] K. -J. Kim, J. van der Kooi, 162nd meeting ACS Rub. Div., Pittsburgh, Pennsylvania, Paper No. 70 (2002). 
Chapter 4.2 


\section{Chapter 4.3 The effect of 1,3-diphenylguanidine concentration in the earlier and later mixing stages on marching modulus}

\subsubsection{Introduction}

1,3-diphenylguanidine (DPG) is widely used as an essential ingredient in silica filled tire rubber compounding, mainly as a secondary accelerator of the vulcanization reaction. ${ }^{[1,2]}$ But it has additional known functions: First, DPG reduces the polarity of silica by being adsorbed onto the silica surface; ${ }^{[3]}$ and second, DPG is capable of accelerating the silanization reaction. ${ }^{[2,4]}$ Many studies were done, which support these roles of DPG in silica filled rubber compounds.

The silica surface can lead to physisorption of the basic DPG molecules due to its own acidity; DPG is a basic substance with a pH level of 10.1. ${ }^{[5,6]}$ Zaborski $^{[5]}$ reported that 1 gram of silica with a specific surface area of $160 \mathrm{~m}^{2} / \mathrm{g}$ is capable of adsorbing $2.9 \times 10^{20}$ molecules of DPG. Moreover, Zaborski and Donnet ${ }^{[3]}$ found that desorption of DPG from the silica surface and crosslink formation of polymers occur simultaneously during the vulcanization process, where the latter is faster. Consequently the silica surface tends to adsorb DPG rather than to release it. Lim et al. ${ }^{[7]}$ identified a slower vulcanization rate when most of DPG is added in an early mixing stage of a silica filled compound. They reported that the slower vulcanization rate is induced by the adsorption of DPG onto silica surface. Goerl et al. ${ }^{[8]}$ reported that the silanization reaction efficiency can be varied with the $\mathrm{pH}$ level, either acidic or basic. Thus, an enhanced reaction between silica and a coupling agent is possible in presence of DPG. Kaewsakul et al. ${ }^{[9]}$ studied the effect of the amount of DPG in silica filled rubber compounds and found that the amount of chemically bound rubber increased with higher DPG concentration due to a better silanization efficiency.

Based on those earlier studies, the concentration of DPG in the MasterBatch (MB) and final mixing step was considered as a main variable for this part of the study. A series of compounds is prepared varying the concentration of DPG in the MB and the last mixing stage, but the final compound formulation was kept the same. Subsequently, the factors which predominantly contribute to the vulcanization behavior are evaluated based on the results in Chapters 3.1 and 4.1: the degree of silanization, the amount of bound rubber, the Filler Flocculation Rate (FFR) and the filler-polymer Coupling Rate (CR). These factors are then correlated with the Marching Modulus Intensities (MMIs) measured at small (approx. 7\%) and large (approx. 42\%) strains. Additional evaluations such as crosslink density, vulcanization efficiency as 
represented by the calculated cure time, and maximum vulcanization rate are done in order to elucidate the special curing behavior observed in this study.

\subsubsection{Experimental}

\subsubsection{Materials and mixing}

All series of experiments were done based on a tire tread compound as shown in Table 4.1 in Chapter 4.1. The acronyms of the compounds correspond to their DPG concentrations in the MB: Table 4.8.

Table 4.8 Formulation of the compound

\begin{tabular}{c|c|cccc}
\hline \multicolumn{2}{c|}{ Acronym } & D00 & D05 & D10 & D15 \\
\hline $\begin{array}{c}\text { DPG concentration } \\
{[\text { phr }]}\end{array}$ & MasterBatch (MB) & 0.0 & 0.5 & 1.0 & 1.5 \\
\hline & Final step & 1.5 & 1.0 & 0.5 & 0.0 \\
\hline
\end{tabular}

The compounds were mixed in two steps as shown in Table 4.9. The masterbatch stage was done by using a lab scale internal mixer (Brabender Plasticorder) with $390 \mathrm{ml}$ of chamber volume. The fill factor of the internal mixer, TCU settings, starting temperature of mixing, silanization temperature control, the compound cooling after the MB mixing and the number of mixings per each set of conditions were the same as described in Chapter 4.1. The compounds were mixed with good reproducibility as judged by the mixing fingerprints, depicted in Figure 4.26. 
Table 4.9 Mixing procedures

\begin{tabular}{|c|c|c|c|}
\hline \multicolumn{2}{|c|}{ MasterBatch (MB) step } & \multicolumn{2}{|c|}{ Final step } \\
\hline \multicolumn{2}{|c|}{ Internal mixer } & \multicolumn{2}{|c|}{ Open mill } \\
\hline Action & time [mm:ss] & Action & time $[\mathrm{mm}: \mathrm{ss}]$ \\
\hline Add polymer & $00: 00 \sim 00: 20$ & \multirow{2}{*}{ Add masterbatch } & \multirow{2}{*}{-} \\
\hline Mastication & $00: 20 \sim 01: 20$ & & \\
\hline $1 / 2$ Silica, silane & $01: 20 \sim 01: 40$ & \multirow{2}{*}{ Mixing } & \multirow{2}{*}{ 02:00 } \\
\hline Mixing & $01: 40 \sim 02: 40$ & & \\
\hline $\begin{array}{l}1 / 2 \text { Silica, DPG (except for D00), } \\
\text { remaining ingredients }\end{array}$ & $02: 40 \sim 03: 10$ & \multirow{2}{*}{$\begin{array}{l}\text { Add curatives, } \\
\text { remaining DPG } \\
\text { (except for D15) }\end{array}$} & \multirow{2}{*}{$02: 30$} \\
\hline Mixing till $150^{\circ} \mathrm{C}$ & 03:10 04:10 & & \\
\hline Ram sweep & $04: 10 \sim 04: 14$ & \multirow{2}{*}{ Mixing } & \multirow{2}{*}{ 09:00 } \\
\hline Mixing (at $150^{\circ} \mathrm{C}$ ) & $04: 14 \sim 06: 40$ & & \\
\hline Discharge and sheeting & - & Discharge & - \\
\hline
\end{tabular}

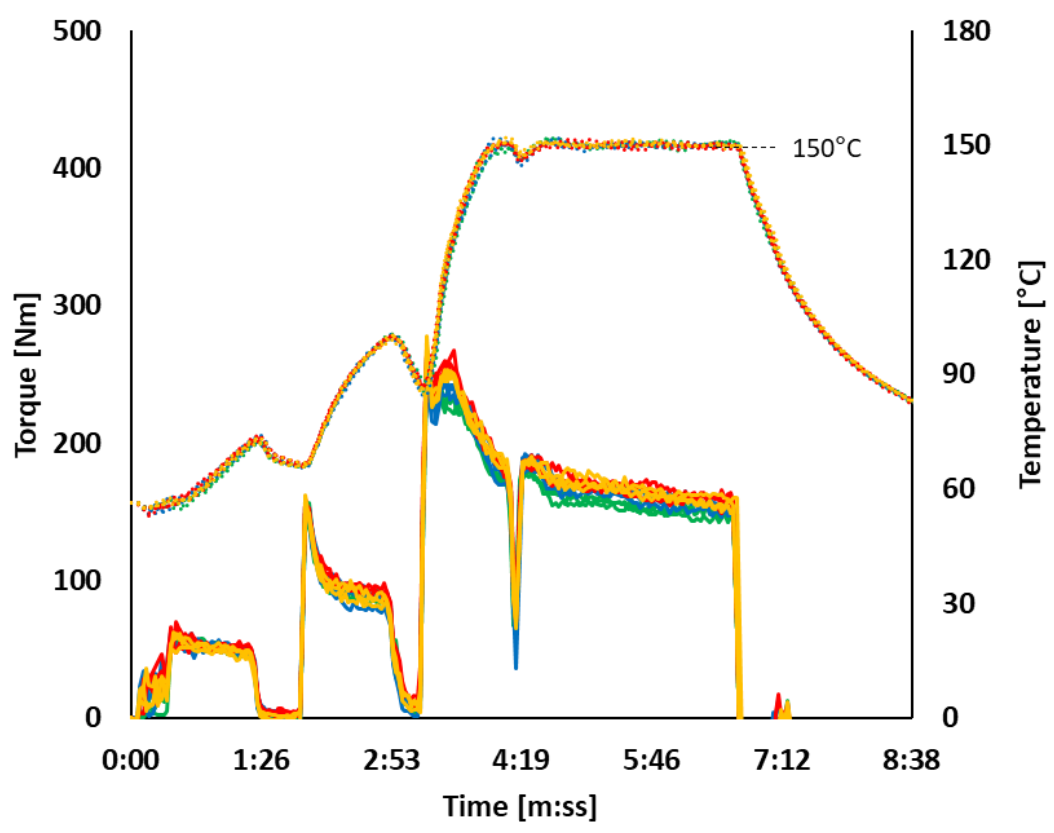

Figure 4.26 Mixing fingerprints; ( solid line ): Torque; ( dotted line ): temperature;

$$
\text { ( }) \text { : D00; ( }) \text { : D05; ( }) \text { : D10; ( }) \text { : D15. }
$$




\subsubsection{Property analysis and testing}

Payne effect, Filler Flocculation Rate (FFR), filler-polymer Coupling Rate (CR), cure characteristics and Marching Modulus Intensity (MMI) were investigated using a Rubber Process Analyzer (RPA; RPA2000, Alpha Technologies) according to the methods described in Chapter 3.1. The evaluation of the other properties such as bound rubber content, vulcanization, tensile properties, and swelling ratio (Q) of the compounds followed the methods described in Chapter 3.2.

\subsubsection{Results and discussion}

\subsubsection{Filler-Filler interaction (Payne effect) as a function of DPG concentration in $\mathrm{MB}$}

The Payne effect values of the uncured compound are plotted as a function of DPG concentration in the MB stage: Figure 4.27. A decreasing trend of the Payne effect values is observed with increasing DPG concentration. This result can be explained two ways: boosting of the silanization reaction induced by the presence of DPG, or absorption of DPG on the silica surface. Both phenomena will reduce the filler-filler interaction.

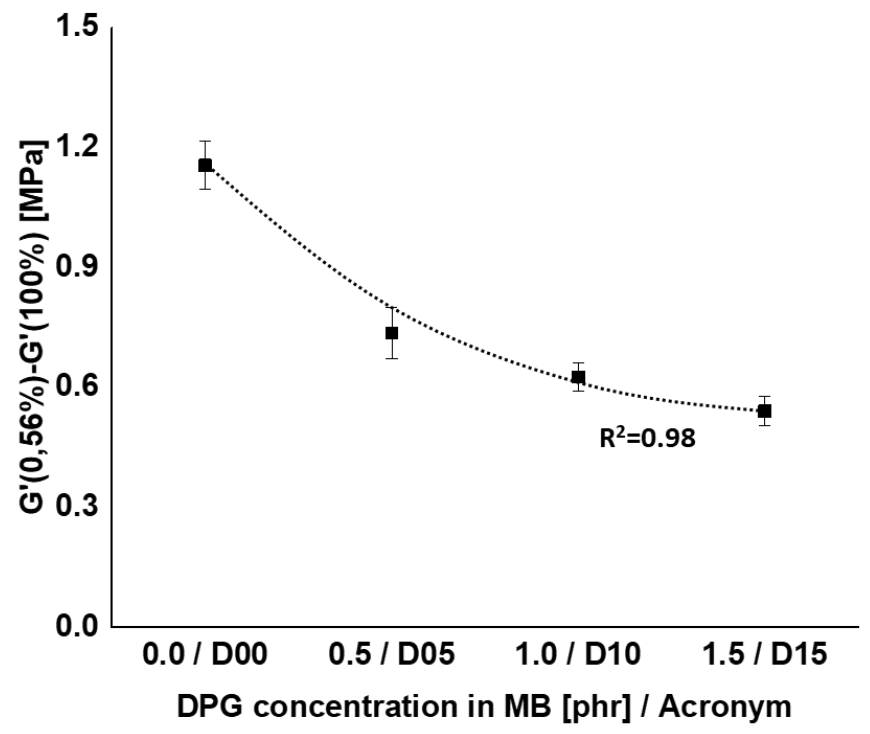

Figure 4.27 Payne effect as a function of DPG concentration in MB; fitting line is based on polynomial fit with maximum $\mathrm{R}^{2}$.

\subsubsection{Bound rubber content as a function of DPG concentration in the MB}

Total and chemically bound rubber contents are plotted as functions of DPG concentration in the MB: 
Figure 4.28. The amount of chemically bound rubber as well as total bound rubber increase along with the DPG concentration in the MB. The amounts of total and chemically bound rubber versus the DPG concentration in the MB indicate that DPG supports the formation of chemically bound rubber and thus leads to reduced filler-filler interaction. It speeds up the silanization reaction, thus resulting in a higher degree of silanization. More chemically bound rubber does form when DPG is present, so the reaction sites on the silica surface are more shielded by DPG molecules but not blocked for the access of silane, as is the case for zinc stearate: see Figure 4.19. Additionally, according to the bound rubber model proposed by Ko and Choi ${ }^{[10]}$, silanization should first occur before DPG adsorption in order to form chemically bound rubber: Figure 2.12(c).

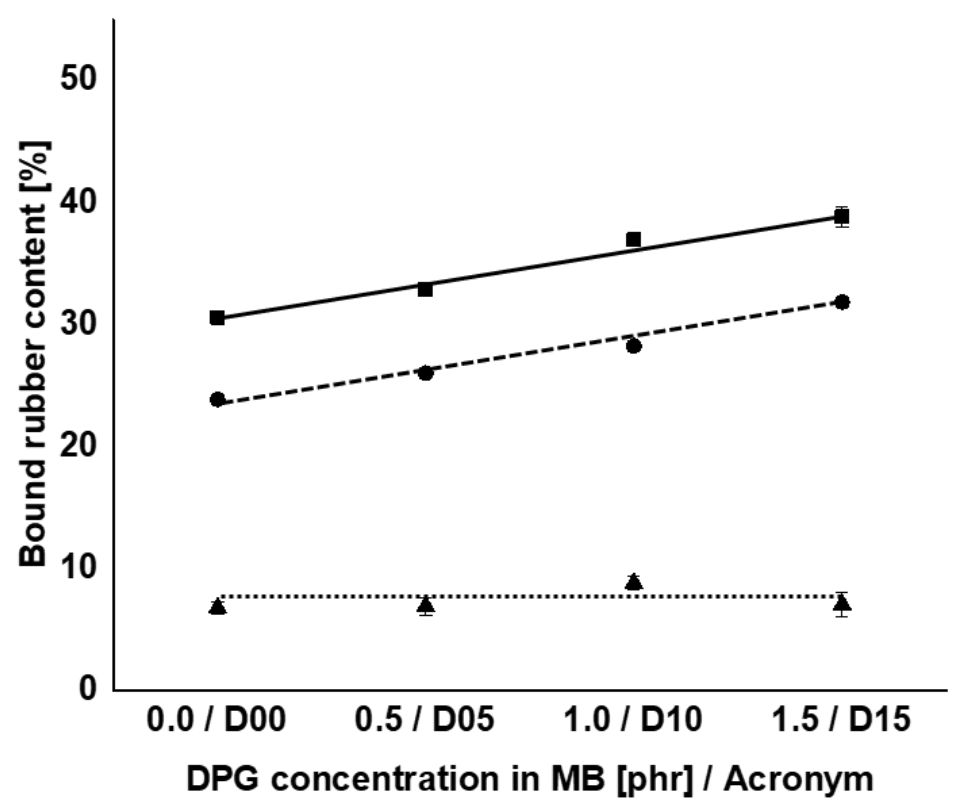

Figure 4.28 Bound rubber contents as function of DPG concentration in the MB;

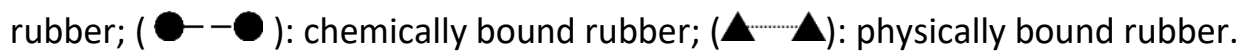

\subsubsection{Silica filler flocculation rate}

The silica Filler Flocculation Rate (FFR) is shown in Figure 4.29. As already confirmed in Chapter 4.1, the amounts of chemically as well as total bound rubber give a large contribution to the reduction of FFR. However, the overall difference in bound rubber content within this mixing series was not large: 6\%, as shown in Figure 4.28. Therefore, comparable FFR values are obtained for all compounds except for D15, which was mixed with the full amount of DPG in the MB and showed the highest amount of bound rubber content. 


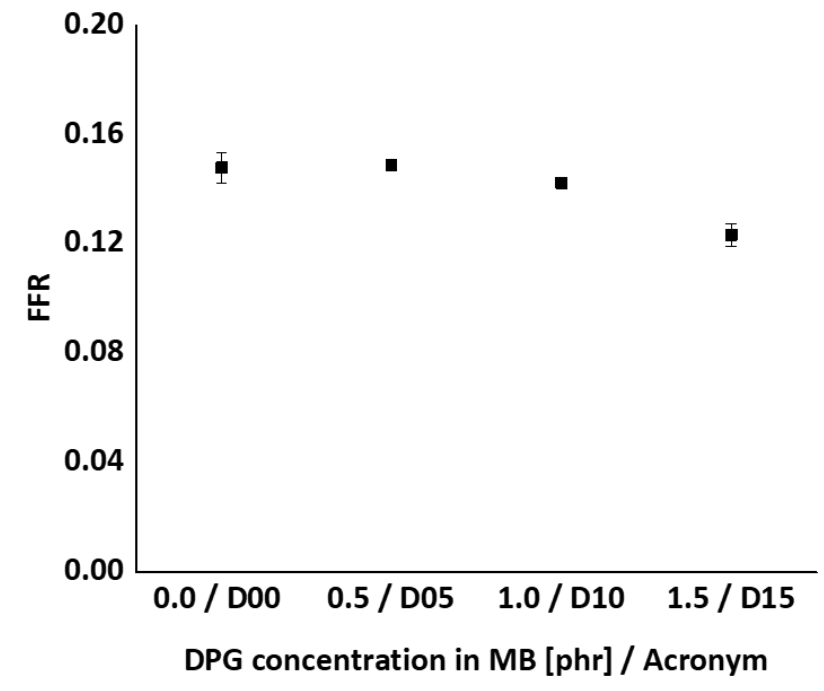

Figure 4.29 FFR versus DPG concentration in MB.

\subsubsection{Filler-polymer coupling rate after mixing}

As can be seen in Figure 4.30, the filler-polymer Coupling Rate (CR) values showed a difference between D00 (mixed without DPG in the MB) and all other compounds. This result indicates that the presence of DPG in the MB - independent from the concentration - promotes not only the silanization, but also the filler-polymer coupling reaction via the silane coupling agent (TESPT). This function of DPG will be further discussed in Chapter 4.4.

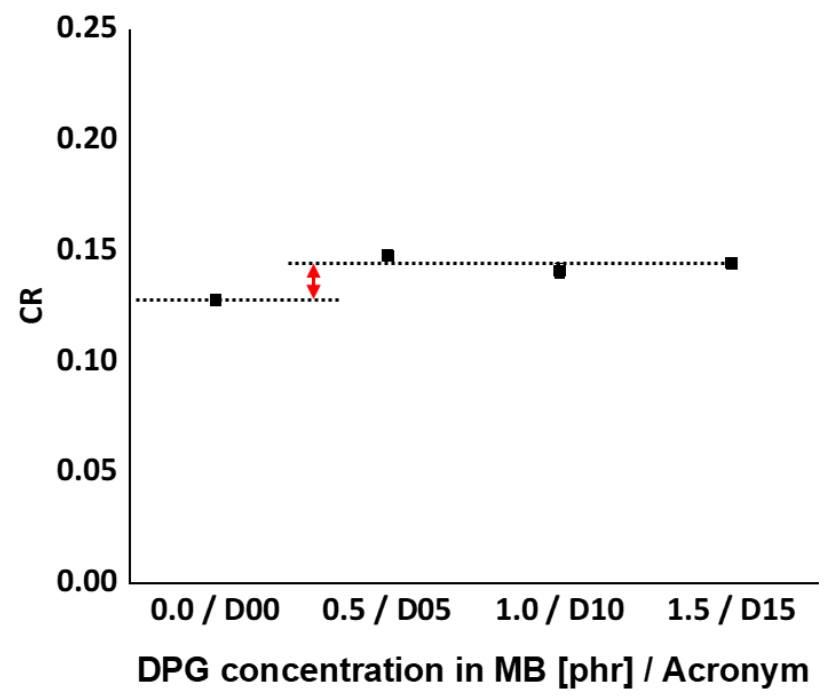

Figure 4.30 CR versus DPG concentration in $\mathrm{MB}$; the error bars are hidden by the data points; the error bars are smaller than the symbol. 


\subsubsection{The effect of DPG concentration in the MB on vulcanization behavior}

\subsection{Marching modulus intensity}

The rheograms, from which the Marching Modulus Intensities (MMI) are calculated and measured at two different strains of the compounds, are plotted in Figures 4.31 and 4.32, respectively. $\mathrm{MMI} 0.5^{\circ}$ values were almost constant (Figure 4.32(a)) and neither FFR nor CR show a clear correlation with MMI $0.5^{\circ}$. This result indicates that the DPG concentration is not an influencing factor for $\mathrm{MMI} 0.5^{\circ}$.

In contrast to this, in general it was observed that the $\mathrm{MMI} 3^{\circ}$ decreases when the $\mathrm{CR}$ values increase due to a slower vulcanization speed as explained in Paragraph 4.1.3.5.2. DPG is capable of accelerating fillerpolymer coupling (Figure 4.30); therefore, higher MMI $3^{\circ}$ values are expected for the compounds mixed with DPG in the MB (D05, D10 and D15). However, the MMI $3^{\circ}$ values are gradually reduced along with DPG concentration in the MB: Figure 4.32(b) This result indicates that there must be a different mechanism to explain this result. Further discussion will be done in Chapter 4.4.
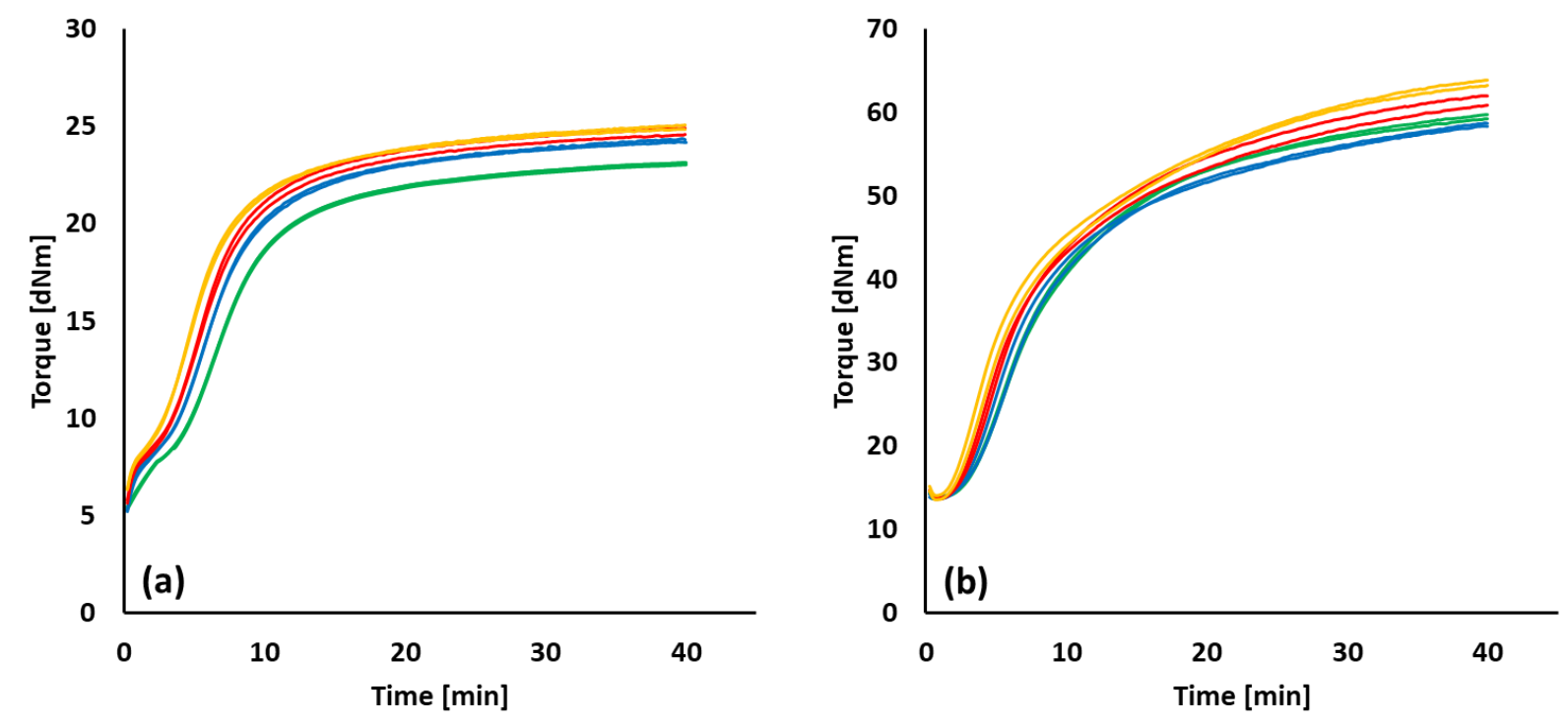

Figure 4.31 Rheograms of the compounds; (a): $0.5^{\circ}$ ( 7\% of strain); (b): $3^{\circ}$ ( $42 \%$ of strain);

( $)$ ): D00; ( $)$ ): D05; ( $)$ ): D10; ( $)$ ): D15. 

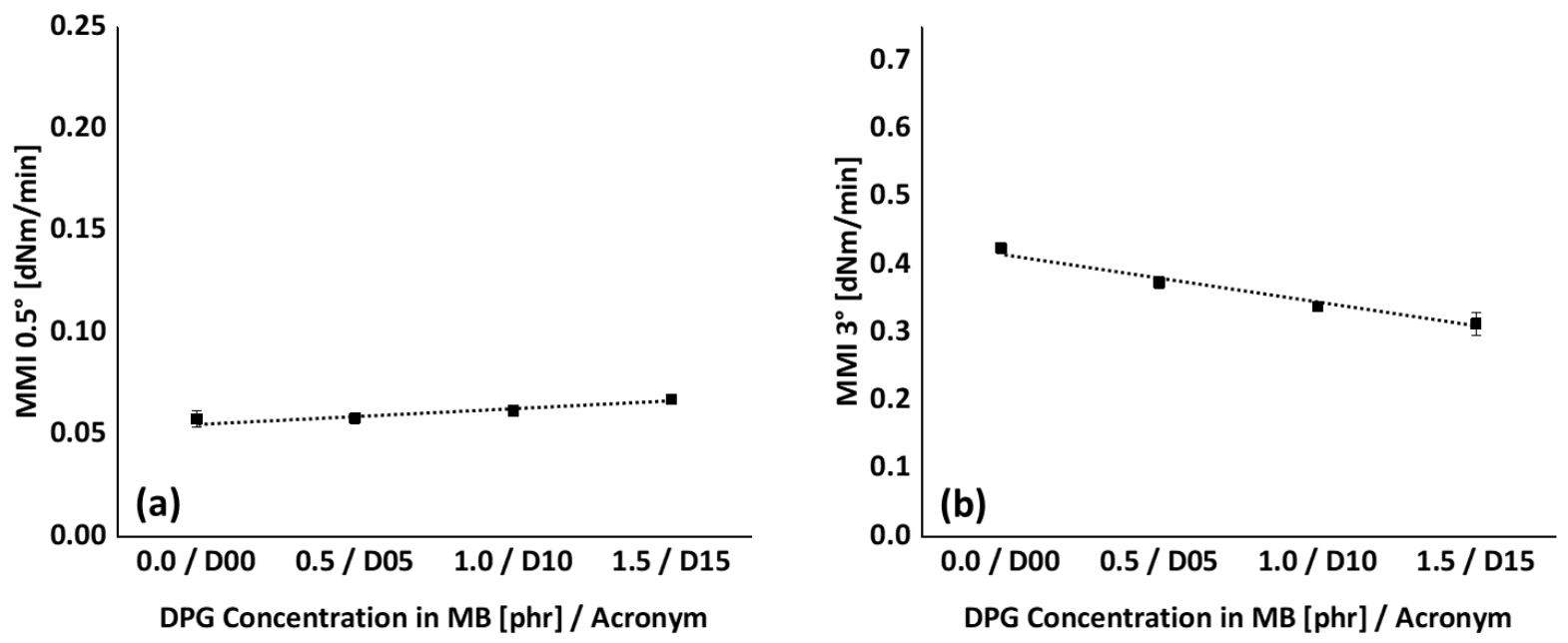

Figure 4.32 MMI vs. DPG concentration in $\mathrm{MB}$; (a): $\mathrm{MMI} 0.5^{\circ}$; (b): $\mathrm{MMI} 3^{\circ}$; fitting lines are based on linear fit with maximum $\mathrm{R}^{2}$.

\subsection{Calculated cure time and maximum cure rate as a function of DPG concentration in MB}

Besides variation of the $\mathrm{MMI}$, a change in the curing behavior is observed, even though the silanization temperature and final amount of DPG were constant for all compounds. From Figure 4.31(a), the calculated curing time $\left(t_{C T}\right)$ and maximum cure rate were acquired according to the method as stated in Chapters 3.1 and 4.1 - Paragraphs 3.1.2.7 and 4.1.3.5.1 - and depicted in Figure 4.33. The $t_{C T}$ and the maximum cure rate nicely correlate with the DPG concentration in the $M B: R^{2}=0.98$ for both. 

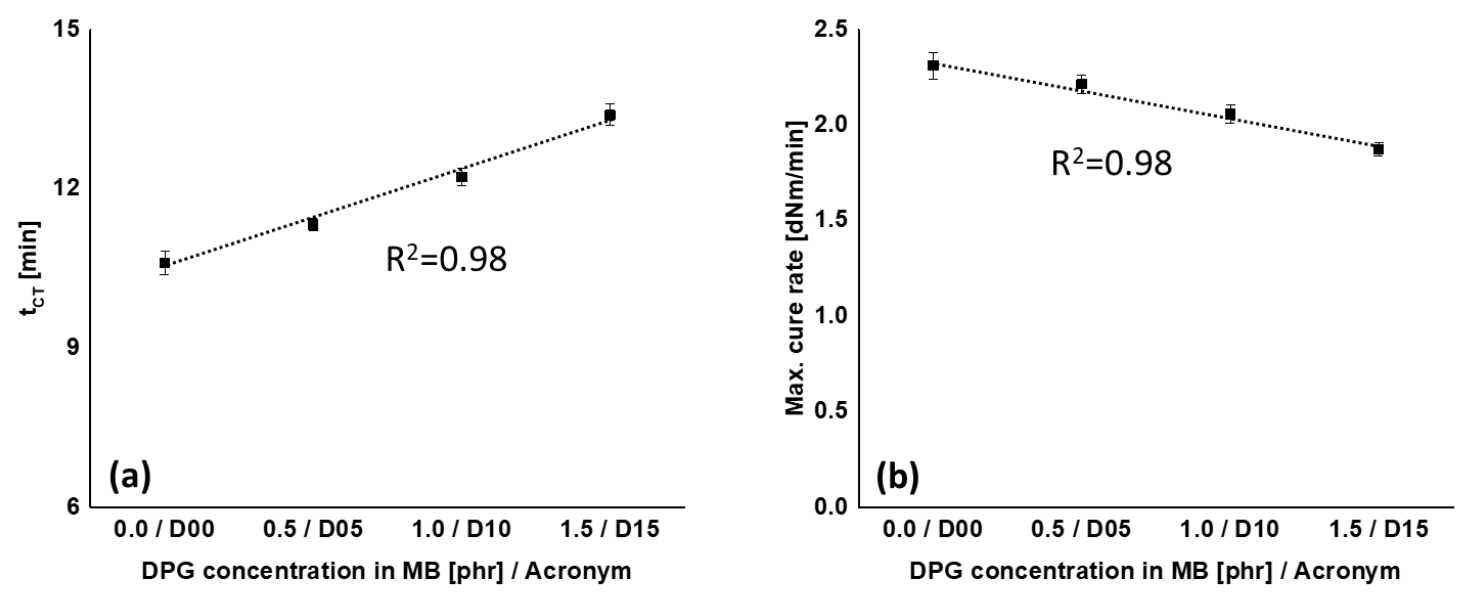

Figure 4.33 Calculated cure time $\left(\mathrm{t}_{\mathrm{C} T}\right)$ and maximum cure rate as a function of DPG concentration in the $M B ;(a): t_{c T}$ vs. DPG concentration in the MB; (b): max. cure rate vs. DPG concentration in the MB; fitting lines are based on linear fit with maximum $\mathrm{R}^{2}$.

As can be seen in this figure, delayed $t_{\text {Ст }}$ and reduced maximum curing speed are obtained when the DPG concentration in the MB increases. These results can be explained with the adsorption of DPG onto the silica surface. DPG added in the early mixing stage results in a higher degree of adsorption of this additive onto the silica surface, as a consequence the adsorbed species would hardly participate in the vulcanization process. ${ }^{[7]}$ However, this explanation is in conflict with the results of chemically bound rubber and CR (Figures 4.28 and 4.30): the high degree of silanization as well as a large amount of chemically bound rubber obtained there, even when DPG molecules would occupy the silanization sites on the silica surface by adsorption. Therefore, the conclusion must be drawn that there are additional functions of DPG which can harmonize the conflicting results. This will be further evaluated and discussed in Chapter 4.4.

\subsubsection{Crosslink density as a function of DPG concentration in the MB}

When the amount of DPG captured on the silica surface increases, and this part of DPG cannot participate in the vulcanization, then the crosslink density of the compound would be lower. In Figure 4.34, the crosslink density of the vulcanizates with three different curing times (calculated cure time $\left(t_{C T}\right.$, see Figure 3.6), 20minutes and 30minutes) are compared indirectly by measuring the swelling ratios $\left(Q_{C T}, Q_{20}\right.$ and $\left.\mathrm{Q}_{30}\right)$ and the difference between the swelling ratios $\left(\Delta \mathrm{Q}_{20}, \Delta \mathrm{Q}_{30}\right)$. The subscripts $(C T, 20,30)$ for the swelling ratios correspond to the curing times and the subscript CT means Calculated cure Time; $\Delta \mathrm{Q}_{20}, \Delta \mathrm{Q}_{30}$ are defined in Equations 4.1 and 4.2, respectively: 


$$
\begin{aligned}
& \Delta Q_{20}=Q_{C T}-Q_{20} \\
& \Delta Q_{30}=Q_{C T}-Q_{30}
\end{aligned}
$$
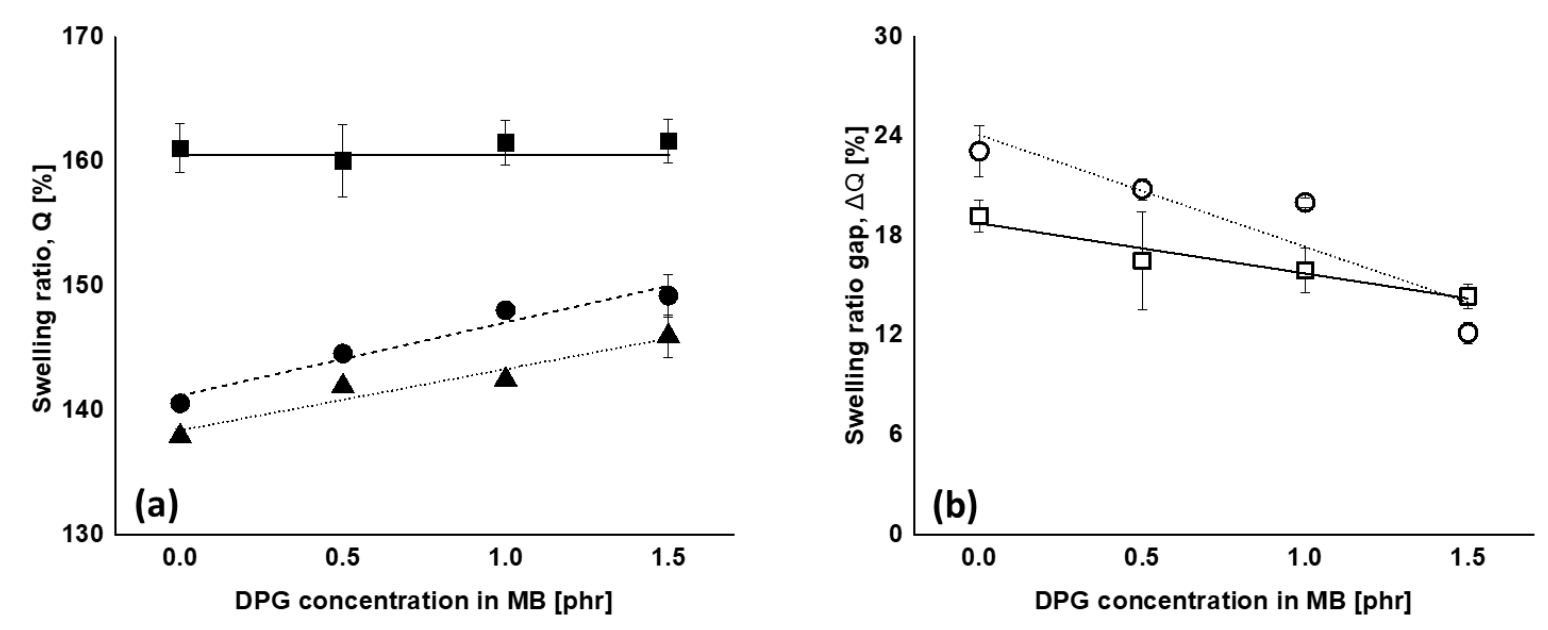

Figure 4.34 Swelling ratios and their difference as a function of the curing time and DPG concentration in the MB; (a): swelling ratios vs. DPG concentration in the MB; (b): swelling ratio difference vs. DPG concentration in the MB; $(\square)$ : $\mathrm{Q}_{c T} ;(--)$ ): $\mathrm{Q}_{20} ;(\boldsymbol{\Delta} \cdots \cdots)$ : $\mathrm{Q}_{30} ;(\square-\square): \Delta \mathrm{Q}_{20} ;(\bigcirc \cdots \cdots): \Delta \mathrm{Q}_{30}$.

The crosslink densities are similar when the compounds were cured according to their $\mathrm{t}_{\mathrm{CT}}$ : a constant $\mathrm{Q}_{\mathrm{CT}}$ level was obtained. The crosslink densities of the compounds increase with increasing DPG concentration in the $M B$, but to a different extent when a longer curing time is applied: larger $\Delta Q_{20}$ and $\Delta Q_{30}$ values are obtained when the compound had a lower DPG concentration in the MB. This result can also be explained by the adsorption of DPG onto the silica surface, but still contradicts the results of chemically bound rubber and $\mathrm{CR}$ as was stated in Paragraph 4.3.3.5.2.

Another interesting point can be seen when these results are compared to the MMI $0.5^{\circ}$ values: Similar levels of $\mathrm{MMI} 0.5^{\circ}$ are obtained (Figure 4.31), even though the crosslink densities of the samples are changing along with vulcanization time. This result again leads to the conclusion that the crosslink density is not the major factor for the marching modulus phenomenon of silica compounds, as was already discussed in Chapter 3.1.

\subsubsection{Tensile properties as a function of crosslink density}

The moduli at different strains (100\% (M100), 200\% (M200)) as well as Tensile Strength (TS) and 
Elongation at break (Eb) of the rubber vulcanizates are shown in Figure 4.35 . The modulus at $300 \%$ strain (M300) was not taken into account, as some of the samples were broken before they reached $300 \%$ strain.
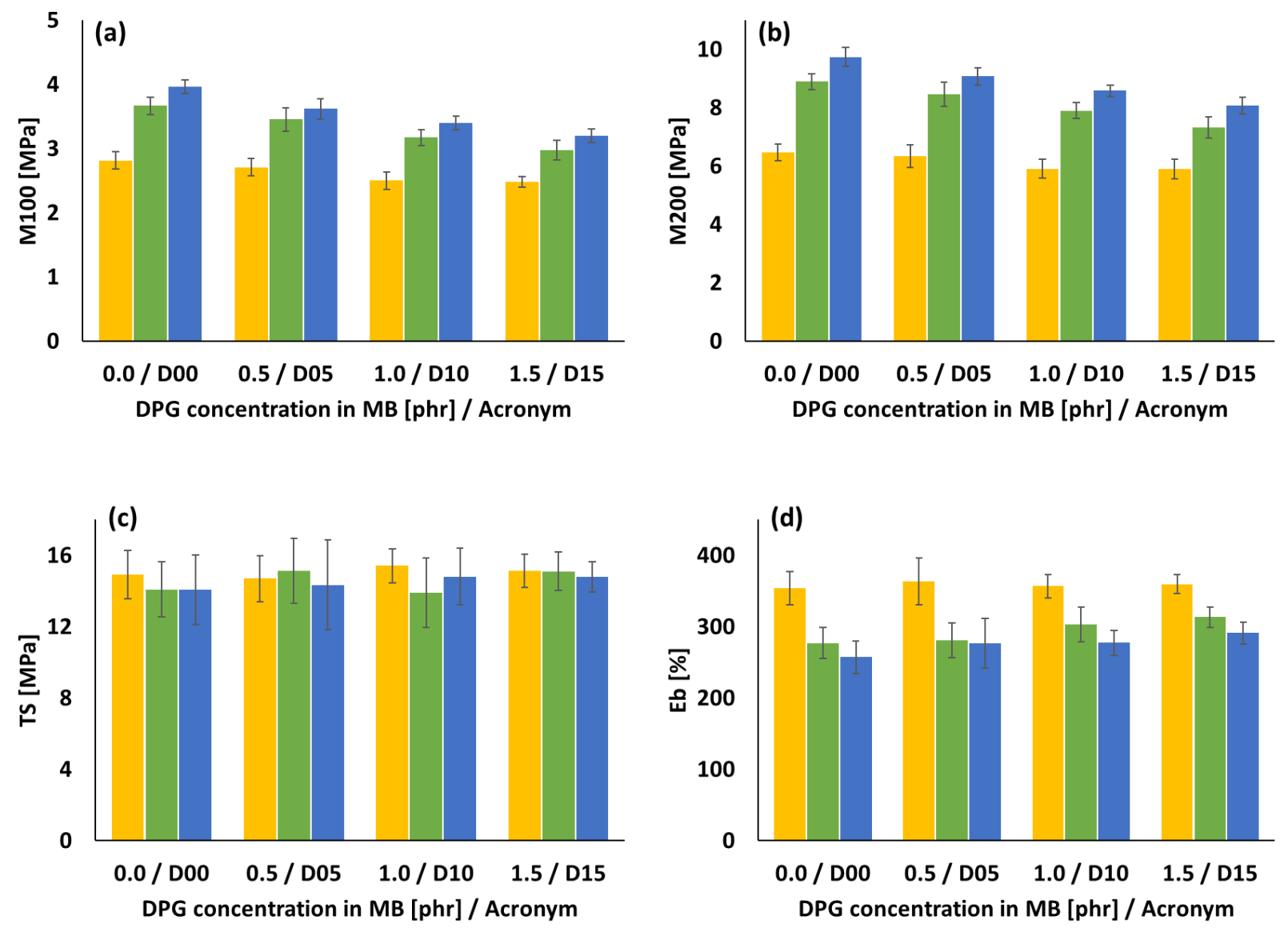

Figure 4.35 Tensile properties corresponding to the DPG concentration in the MB and to the curing times; (a): M100; (b): M200; (c): TS; (d): Eb; ( $\square$ ): $t_{c T} ;(\square)$ ): 20 min; ( $\square$ ): 30 min.

As expected from Figure 4.34, the same levels of tensile properties were obtained when the compounds were cured for their optimum cure time, $\mathrm{t}_{\mathrm{CT}}$. However, the property differences between the samples cured for $t_{C T}, 20$ minutes and 30 minutes became smaller when the DPG concentration in the MB increased, except for TS: TS was almost the same and not affected by the DPG concentration in the MB nor the curing time. M100, M200 and Eb show a correlation with the swelling ratio values, as shown in Figure 4.36. This result indicates that adding DPG in the later mixing stage increases the amount of free DPG, which can participate in the vulcanization process of the rubber matrix, even though the overall concentration in the compound is the same. But still, this phenomenon is in contrast with the result of chemically bound rubber and CR. The reason will again be further discussed in Chapter 4.4. 

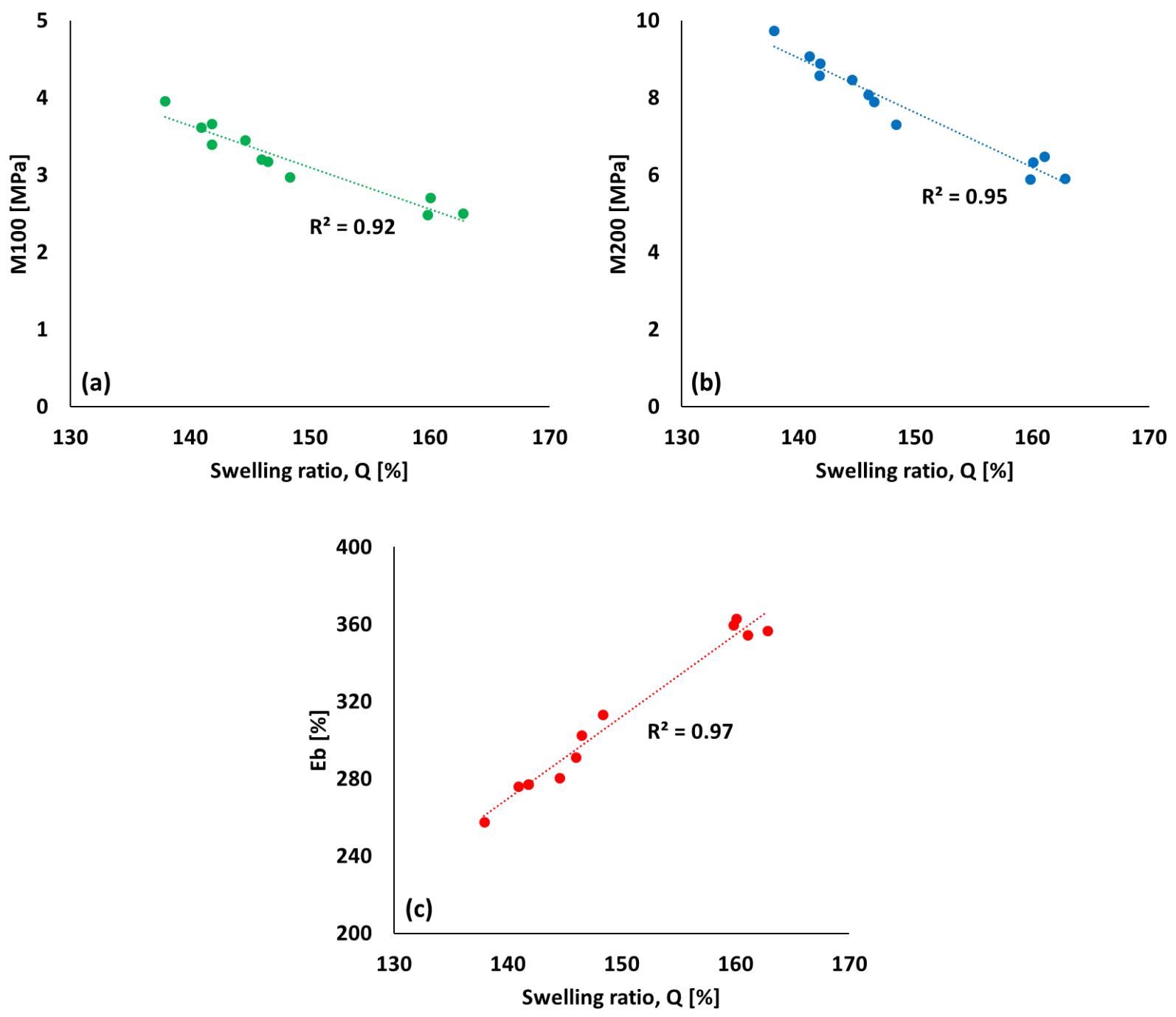

Figure 4.36 Tensile properties versus swelling ratio; (a): M100 vs. Q; (b): M200 vs. Q; (c): Eb vs. Q.

\subsubsection{Conclusions}

The focus of this study was to investigate the mixing parameters that can reduce the marching modulus during curing of silica filled S-SBR/BR rubber compounds. The outcome of this part is that neither the presence nor concentration of DPG in the early mixing stages affect the marching modulus behavior of silica filled rubber.

However, regarding the marching modulus phenomenon the concentration of DPG in the first or final mixing stage does influence the efficiency of the silanization as well as the filler-polymer coupling reaction via the silane coupling agent. Adding DPG in an early mixing stage results in a higher amount of chemically bound rubber due to a boosting effect of the silanization and filler-polymer coupling. 
The vulcanization efficiency is also strongly influenced by the concentration of DPG in the earlier or later mixing steps. The amount of free DPG is reduced when it is introduced in an earlier mixing stage and results in delayed vulcanization and lower crosslink density.

However, these two phenomena cannot be explained with the known functions of DPG, as was stated in Paragraph 4.3.1, due to the following reasons:

- If DPG would mainly boost the silanization reaction, an enhanced degree of silanization as well as a higher amount of chemically bound rubber should be seen. Besides, a similar level of crosslink density as well as vulcanization efficiency ( $t_{C T}$, max. cure rate) should be obtained regardless of the DPG concentration in earlier or later mixing steps. The reason is that the final concentration of DPG in the vulcanization step is the same.

- If DPG blocks the access of silane molecules by absorption onto the silica surface, the vulcanization efficiency and crosslink density would be lowered as the amount of absorbed DPG increases. As a consequence, a lower degree of silanization as well as a lower amount of chemically bound rubber could be expected. However, the Payne effect and bound rubber results (Figures 4.27 and 4.28) show opposite trends.

Taking these conflicting results into account, it is now clear that there must be another function of DPG, which will explain all the contradicting results: further evaluation and discussion will be done in Chapter 4.4.

Based on this study, it can be recommended to add all DPG in the early mixing stage. This will give a better quality and processability of the silica-filled rubber: lower filler-filler interaction, slow FFR, and a relatively consistent crosslink density along with curing time, though it does not influence the marching modulus.

\section{References}

[1] S. Mihara, PhD Thesis: Reactive processing of silica-reinforced tire rubber: new insight into the time- and temperature-dependence of silica rubber interaction, Univ. Twente, Enschede, the Netherlands (2009).

[2] S. Mihara, R. N. Datta, A. G. Talma, and J. W. M. Noordermeer, U.S. Patent 7923493 B2 (to The Yokohama Rubber Co., Ltd.), April 12 (2011).

[3] M. Zaboski and J. B. Donnet, Macromol. Symp. 194, 87 (2003).

[4] K. J. Kim, and J. van der Kooi, Rubber Chem. Technol. 65, 1016 (1992). 
[5] J. Ramier, L. Chazeau, C. Gauthier, L. Guy, and M. N. Bouchereau, Rubber Chem. Technol. 80, 183 (2008).

[6] C. Hayichelaeh, L. A. E.M. Reuvekamp, W. K. Dierkes, A. Blume, J. W. M. Noordermeer, K. Sahakaro, Rubber Chem. Technol. 90, 651 (2017).

[7] S-H, Lim, S. Lee, N. Lee, B. K. Ahn, N. Park, W. Kim, Elastomers and Composites 51, 81 (2016).

[8] U. Goerl, A. Hunsche, A. Mueller, H. G. Koban, Rubber Chem. Technol. 70, 608 (1997).

[9] W. Kaewsakul, K. Sahakaro, W. K. Dierkes, J. W. M. Noordermeer, Rubber Chem. Technol. 86, 313 (2013).

[10] S-S. Choi, E. Ko, Polymer Testing 40, 170 (2014). 


\section{Chapter 4.4 Model compound study: effect of $\mathrm{ZnO}$, stearic acid and DPG on the silane chemistry}

\subsubsection{Introduction}

In Chapters 4.2 and 4.3, the effect of the zinc oxide ( $\mathrm{ZnO})$ and 1,3-diphenylguanidine (DPG) concentration in the master batch and final mixing steps on silanization, as well as the filler-polymer coupling was evaluated through series of mixing trials. However, the outcomes of these chapters could not completely be explained with their well-known functions of these compounding ingredients. The remaining questions from these two chapters are:

- Does ZnO or zinc stearate facilitate release of free sulfur from the TESPT molecule? (Chapter 4.2)

- What is (are) the missing piece(s) of the puzzle which can connect the conflicting functions of DPG stated in Paragraph 4.3.4? (Chapter 4.3)

Especially for the second question, Mihara ${ }^{[1]}$ proposed that DPG helps to release sulfur from the silane; it reacts directly with the sulfur. This can explain the change in filler-polymer coupling rate, vulcanization rate and the amount of bound rubber along with the concentration of DPG in the early mixing stage (Paragraphs 4.3.3.2, 4.3.3.4 and 4.3.3.5.2 in Chapter 4.3). However, so far little attention was paid to study more this potential role of DPG in depth.

Therefore, the present chapter focuses on a model compound study in order to find answers to and proof for the questions above. The possible reactions between TESPT and ingredients such as ZnO (Z), Stearic acid (Sa) and DPG (D) are evaluated. The ingredients are added alone or in combination to ampoules containing a $\mathrm{n}$-decane-TESPT solution and dipped into a hot silicon oil-bath to react with TESPT. Subsequently, the reactants and reaction products are analyzed by appearance as well as by using High Pressure Liquid Chromatography (HPLC), Liquid Chromatography Mass Spectrometry (LC-MS) and ${ }^{13} \mathrm{C}$ Nuclear Magnetic Resonance $\left({ }^{13} \mathrm{C}-\mathrm{NMR}\right)$.

\subsubsection{Experimental}

\subsubsection{Sample preparation}

The formulations of the model compounds are given in Table 4.10. The experimental procedure is shown in Figure 4.37. A large quantity of bulk solution consisting of $n$-decane and TESPT was first prepared in 
order to minimize the experimental error. Additionally, three ampoules per each set of conditions were prepared to get information on repeatability.

Table 4.10 Formulation of the model compounds

\begin{tabular}{|c|c|c|c|c|c|c|c|c|c|c|c|c|}
\hline \multirow{2}{*}{$\frac{\text { Ingredients }}{\text { n-Decane }}$} & \multirow{2}{*}{$\frac{\text { Supplier }}{\text { Sigma-Aldrich }}$} & \multirow{2}{*}{$\frac{\text { Amount }^{\#}}{100}$} & \multicolumn{6}{|c|}{ Series1 } & \multicolumn{4}{|c|}{ Series2 } \\
\hline & & & $\bullet$ & $\bullet$ & $\bullet$ & $\bullet$ & $\bullet$ & $\bullet$ & $\bullet$ & $\bullet$ & $\bullet$ & $\bullet$ \\
\hline TESPT & Evonik & 8 & $x$ & $\bullet$ & $\bullet$ & $\bullet$ & $\bullet$ & $\bullet$ & $\bullet$ & $\bullet$ & $\bullet$ & $\bullet$ \\
\hline Zinc oxide & Merck & 2 & $x$ & $x$ & $x$ & $\bullet$ & $x$ & $x$ & $\bullet$ & $\bullet$ & $x$ & $\bullet$ \\
\hline Stearic acid & Merck & 2 & $x$ & $x$ & $x$ & $x$ & $\bullet$ & $x$ & $\bullet$ & $x$ & $\bullet$ & $\bullet$ \\
\hline DPG & Flexsys & 2 & $\bullet$ & $x$ & $x$ & $x$ & $x$ & $\bullet$ & $x$ & $\bullet$ & $\bullet$ & $\bullet$ \\
\hline \multicolumn{3}{|c|}{ Heating for 1hr @140 ${ }^{\circ} \mathrm{C}$} & $\bullet$ & $x$ & $\bullet$ & $\bullet$ & $\bullet$ & $\bullet$ & $\bullet$ & $\bullet$ & $\bullet$ & $\bullet$ \\
\hline \multicolumn{3}{|c|}{ Acronyms } & RO & $\mathrm{R} 1$ & $\mathrm{R} 2$ & Z & Sa & $D$ & ZSa & ZD & $\mathrm{SaD}$ & ZSaD \\
\hline
\end{tabular}

x: not added or not applied; $\bullet$ : added or applied

\#The amount of ingredients was calculated based on parts per hundred n-decane.

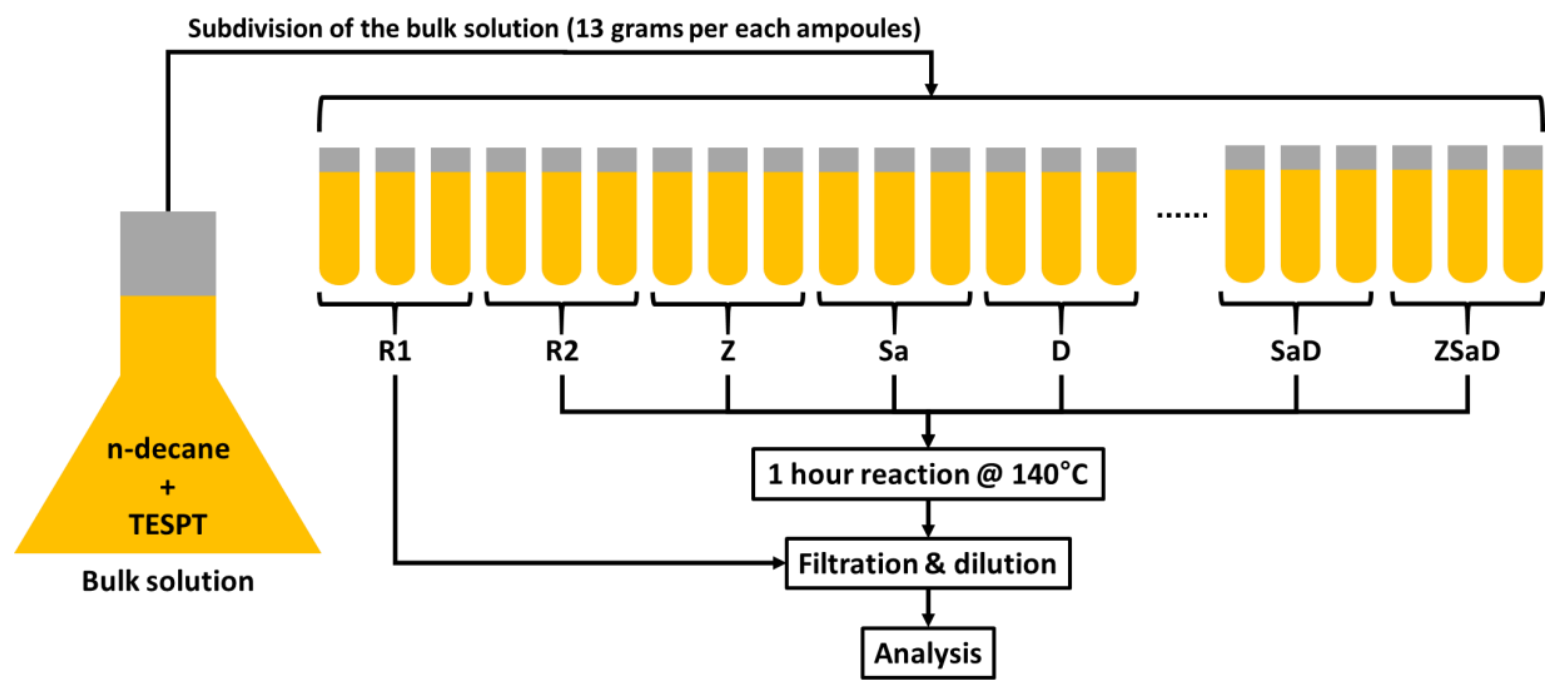

Figure 4.37 Experimental procedure.

The ampoules were flushed with $\mathrm{N}_{2}$ gas before and after material addition in order to prevent side reactions induced by $\mathrm{O}_{2}$ or $\mathrm{H}_{2} \mathrm{O}$ in the air. After this step, the ampoules were sealed. Subsequently, the ampoules were dipped into an oil-bath and stirred for one hour at $140^{\circ} \mathrm{C}$ except for sample R1. The 
reaction temperature was selected according to the thermal stability of TESPT shown in Figure 2.21. After the reaction, the ampoules were dipped in an ice/water bath immediately in order to stop further reaction.

\subsubsection{Appearance comparison}

As it is difficult to dissolve sulfur in a non-polar organic solvent such as n-decane, precipitated sulfur is expected to be visually detectable when the ingredients affect the release of sulfur from the TESPT molecule during the reaction. The colors of the mixtures before and after the reaction were compared simply by taking and comparing pictures.

\subsubsection{The amount and average sulfur rank of TESPT after the reaction}

The amount and average sulfur rank of TESPT before and after the reaction were monitored by High Pressure Liquid Chromatography (HPLC, Varian ProStar Model 500, Varian Analytical Instruments). The cooled ampoules were opened and the reaction mixture was taken and filtered using a $45 \mu \mathrm{m}$ porous filter. $75 \mu \mathrm{l}$ of diethyleneglycol-monobutylether was added to $75 \mu \mathrm{l}$ of the filtered reaction mixture as a compatibilizer for $n$-decane and acetonitrile. Then, these sample were diluted with $1.5 \mathrm{ml}$ of acetonitrile and injected into to HPLC device. An example-chromatogram is shown in Figure 4.38. The positions of the peaks, which are related to the number of sulfur atoms in TESPT (the sulfur-rank), are determined by comparing with the chromatogram of TESPD which contains $90 \%$ of disulfidic bridges.

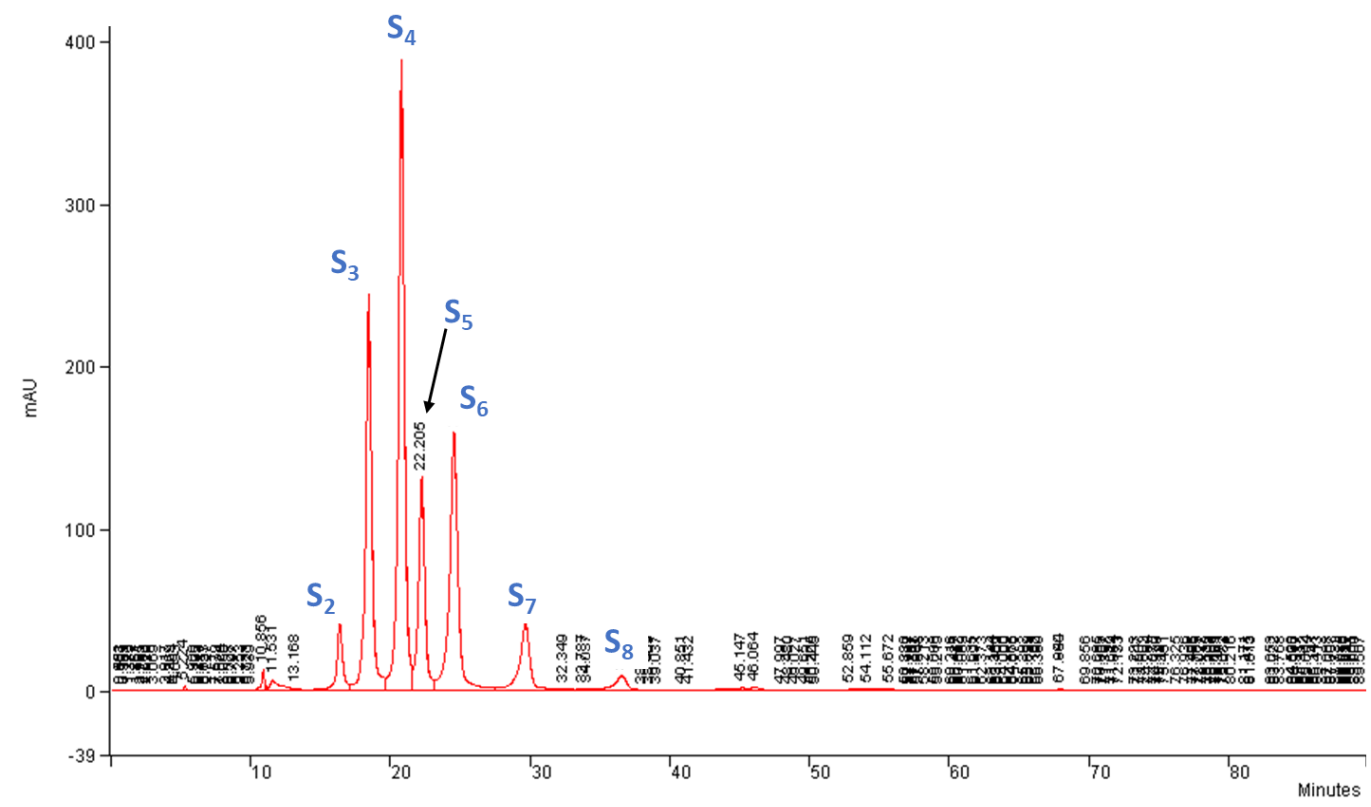

Figure 4.38 HPLC chromatogram of sample R1. 


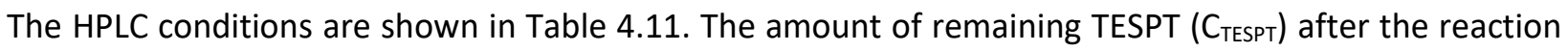
was calculated by using Equation 4.3 obtained from a calibration curve (Figure 4.39) prepared before the experiments. Using Equation 4.3, C C TESPT was calculated in terms of parts per hundred n-decane.

$$
C_{\text {TESPT }}[\text { parts per hundred } \mathrm{n}-\text { decane }]=\frac{\text { Total peak area }}{2.36 \times 10^{7}}
$$

Where the total peak area corresponds to the sum of the areas under the peaks of $S_{2}$ to $S_{8}$.

Table 4.11 HPLC conditions

\begin{tabular}{cc}
\hline Column & Valco Microsorb $300-5 \mathrm{C} 18$ \\
Length of the column & $250 \mathrm{~mm}$ \\
Internal diameter of the column & $4.6 \mathrm{~mm}$ \\
Mobile phase & Acetonitrile : water $=97: 3$ \\
Flow rate & $0.3 \mathrm{ml} / \mathrm{min}$ \\
Temperature & $23^{\circ} \mathrm{C}$ \\
Detector & UV (DAD) \\
Wavelength & $254 \mathrm{~nm}(200 \mathrm{to} 700 \mathrm{~nm})$ \\
Injection volume & $0.5 \mathrm{ml}$ \\
\hline
\end{tabular}

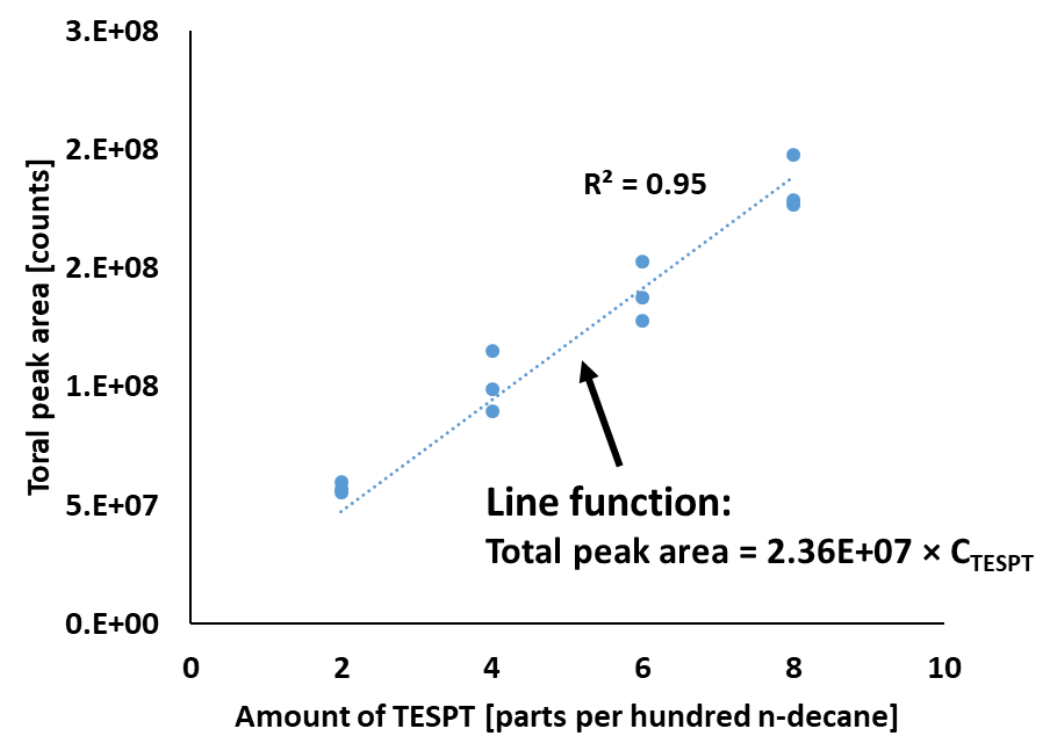

Figure 4.39 Calibration curve for calculating the amount of TESPT. 
The average sulfur ranks of TESPT before and after the reaction were calculated by using Equation 4.4. It is well known that TESPT is a mixture of silanes with sulfur ranks from $S_{1}$ to $S_{10 .}{ }^{[2]}$ However, the peaks of $S_{1}, S_{9}$ and $S_{10}$ were not identified in this work: Figure 4.38. Therefore, only $S_{2}$ to $S_{8}$ were taken into account for the amount of remaining TESPT after the reaction and for the average sulfur rank calculation.

$$
A V G \text { sulfur rank }=\sum_{n=2}^{8} n\left(\frac{A_{n}}{A_{T}}\right)
$$

Where $n$ is the sulfur rank, $A_{n}$ is the peak area corresponding to sulfur rank $n$, and $A_{T}$ is the total peak area.

\subsubsection{Analysis of the reaction products}

\subsection{Liquid Chromatography-Mass Spectrometry (LC-MS)}

High Pressure Liquid Chromatography (LC, Ultramate 3000, ThermoFisher SCIENTIFIC) coupled to Mass Spectrometry (MS, amaZon SL, Bruker) was used for defining the chemical structure of the DPG-TESPT reaction products. The LC-MS conditions are shown in Table 4.12.

Table 4.12 HPLC and MS conditions

\begin{tabular}{c|cc}
\hline Column & Kintex ${ }^{\circledR} 5 \mu \mathrm{m} \mathrm{C18} 100 \AA$ \\
Length of the column & $150 \mathrm{~mm}$ \\
Internal diameter of the column & $2.1 \mathrm{~mm}$ \\
MPLC conditions & Acetonitrile : water $=97: 3$ \\
& Flow rate & $0.2 \mathrm{ml} / \mathrm{min}$ \\
& Temperature & $30^{\circ} \mathrm{C}$ \\
Detector & UV (DAD) \\
WS ionization conditions in ESI & Injection volume & $10 \mu \mathrm{l}$ \\
\cline { 2 - 2 } & Flow rate & $230 \mathrm{~nm}(200 \mathrm{to} 700 \mathrm{~nm})$ \\
& Nebuliser gas pressure & $103 \mathrm{kPa}(15 \mathrm{psi})$ \\
& Dry gas flow & $8 \mathrm{l} / \mathrm{min}$ \\
& Dry gas temperature & $200^{\circ} \mathrm{C}$ \\
\hline
\end{tabular}


The flow rate of the mobile phase was set at $0.2 \mathrm{ml} / \mathrm{min}$ in order to optimize the separation and MS ionization. The ingredients were ionized using the Electrospray lonization (ESI) technique. The ions were identified according to their mass to charge ratio $(\mathrm{m} / \mathrm{z})$ encountered with a proton, sodium, potassium or ammonium adduct. ${ }^{[3,4]}$ For example, TESPT (R1 sample) showed a mass to charge ratio as molecular weight of TESPT + sodium as shown in Figure 4.40 and Table 4.13. The extracted ion spectrum, which does not give clear information of reaction products, was further analyzed using the MS-MS technique. With this technique it is possible to obtain additional extracted ion spectra from the selected extracted ion spectra by further ionization and fragmentation.
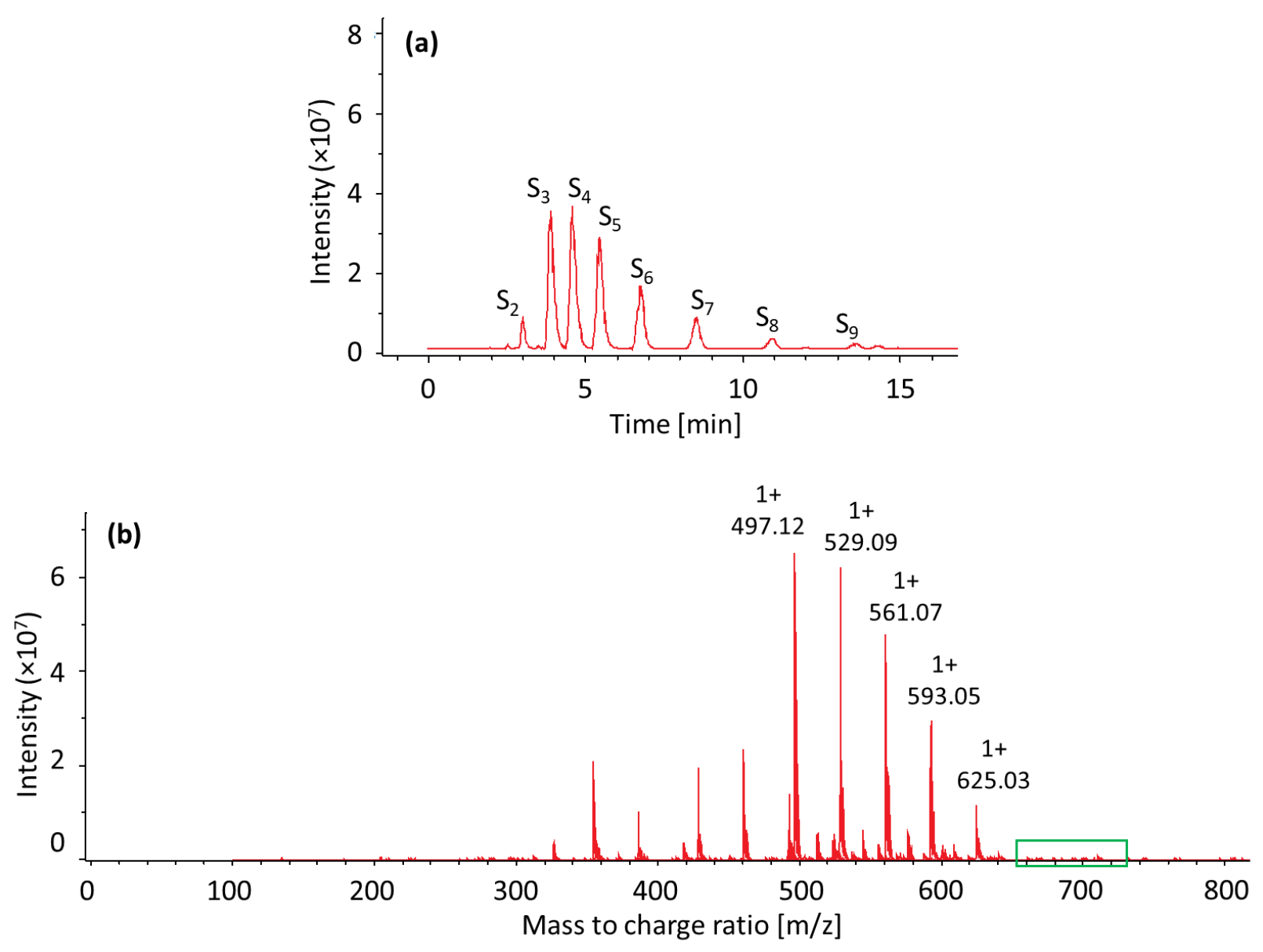

Figure 4.40 LC-MS spectra of R1 sample; (a): mass spectra; (b): extracted ion spectrum from (a);

$\left(\square\right.$ ): $\mathrm{m} / \mathrm{z}$ range, in which the extracted ion spectra of $S_{7}, S_{8}$ and $S_{9}$ sulfidic silane appears with very low intensity $\left(\times 10^{6}\right)$. 
Table 4.13 Molecular weight obtained from the extracted ion spectrum

\begin{tabular}{|c|c|c|c|}
\hline \multirow{2}{*}{ Structure of the silane } & \multirow{2}{*}{$\begin{array}{l}\text { Number of } \\
\text { sulfur, X }\end{array}$} & \multicolumn{2}{|c|}{ Molecular weight (Mw, g/mol) } \\
\hline & & $\begin{array}{c}\text { Calculated Mw according to } \\
\text { sulfur rank }\end{array}$ & $\begin{array}{l}\text { Detected Mw by LC-MS } \\
\text { with sodium adduct }\end{array}$ \\
\hline & 2 & 474 & 497 \\
\hline $\begin{array}{c}\text { OEt } \\
\text { I }\end{array}$ & 3 & 506 & 529 \\
\hline $\begin{array}{c}\text { EtO }-\mathrm{Si}-\mathrm{OEt} \\
\mathrm{I} \\
\left(\mathrm{CH}_{2}\right)_{3}\end{array}$ & 4 & 538 & 561 \\
\hline $\begin{array}{l}1 \\
x\end{array}$ & 5 & 571 & 593 \\
\hline$\left(\mathrm{CH}_{2}\right)_{3}$ & 6 & 603 & 625 \\
\hline $\mathrm{EtO}-\stackrel{\mathbf{I}}{\mathrm{Si}}-\mathrm{OEt}$ & 7 & 635 & \#657 \\
\hline $\begin{array}{c}\text { I } \\
\text { OEt }\end{array}$ & 8 & 667 & \#689 \\
\hline & 9 & 699 & $\# 721$ \\
\hline
\end{tabular}

\#Compared to the silane having $S_{2}$ to $S_{6}$ sulfide, the intensities of these peaks in the extracted ion spectra were very low $\left(\times 10^{6}\right)$, therefore not visible in Figure 4.40 .

\subsubsection{2 ${ }^{13} \mathrm{C}$-Nuclear Magnetic Resonance $\left({ }^{13} \mathrm{C}-\mathrm{NMR}\right)$}

In order to elucidate the chemical structure of the DPG-TESPT complex, a direct reaction between DPG and TESPT without $n$-decane was done and analyzed by ${ }^{13} \mathrm{C}$-Nuclear Magnetic Resonance (NMR, Ascend $400 \mathrm{MHz}$, Bruker) spectroscopy. DPG and TESPT were added to the ampoules flushed with $\mathrm{N}_{2}$ gas and dipped into the hot oil-bath and stirred for one hour at $140^{\circ} \mathrm{C}$. Subsequently, the sample was taken out from the ampoule and dissolved in deuterated chloroform $\left(\mathrm{CDCl}_{3}\right)$ for the analysis. Additionally, untreated and heated $\left(140^{\circ} \mathrm{C}\right.$ for $\left.1 \mathrm{hr}\right) \mathrm{DPG}$ and TESPT were also analyzed with ${ }^{13} \mathrm{C}-\mathrm{NMR}$ as references. The chemical structure of the DPG-TESPT reaction product was characterized by using software $(A C D / S p e c t r u s$ Processor 2019.1.2, Advanced Chemistry Development, Inc.).

\subsubsection{Results and discussion}

\subsubsection{Appearance comparison: before and after reaction}

As can be seen in Figure 4.41, a significant color change of the mixtures is observed for the ampoules containing DPG (D, ZD, SaD and ZSaD); sample RO shows that this is not caused by the presence of DPG as such (see Figure 4.41(a)). The color change of these samples can be explained by the dissolution of sulfur released from TESPT - in the solvent. 


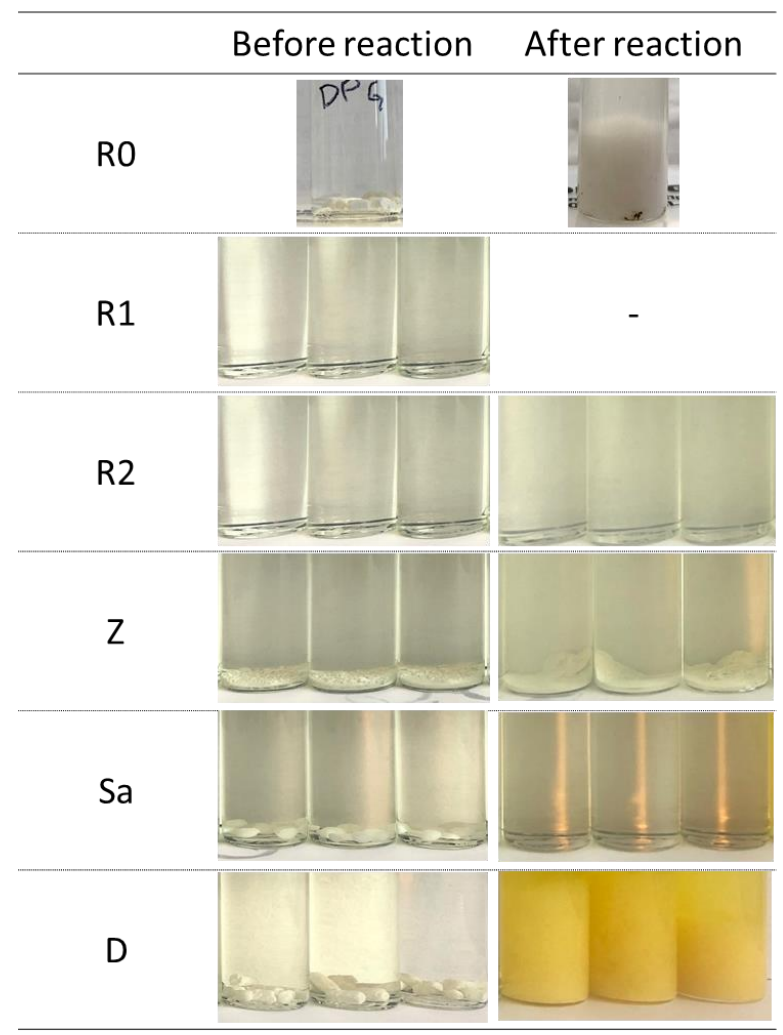

(a)

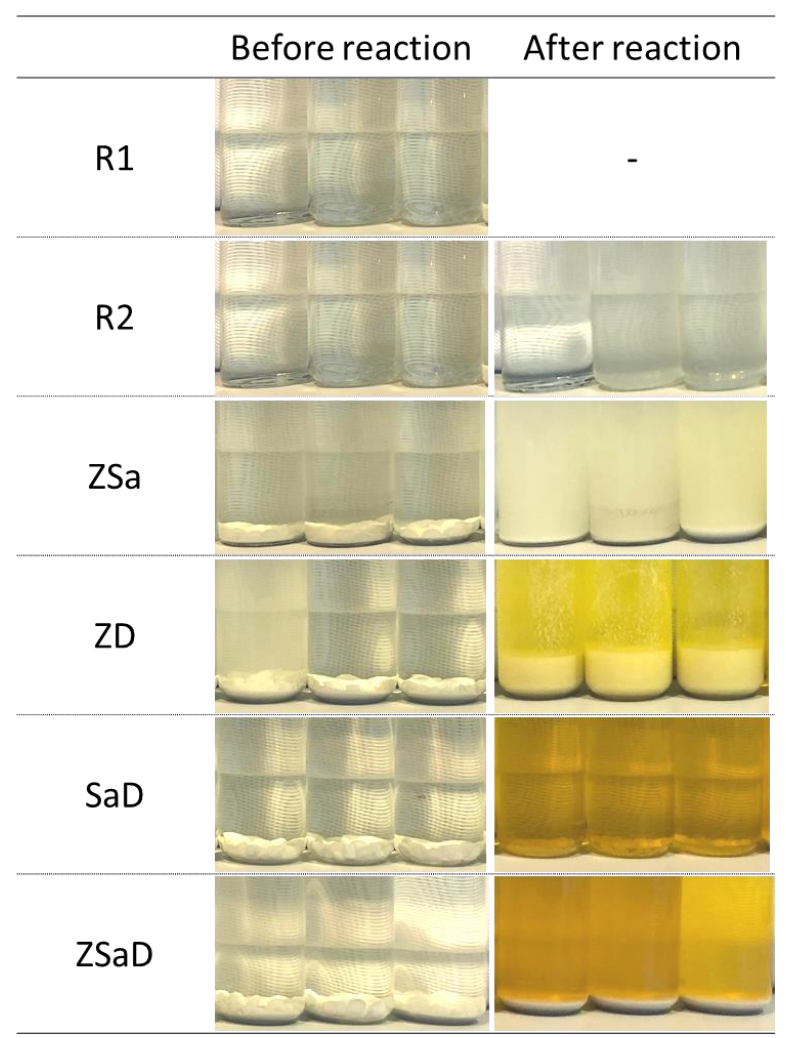

(b)

Figure 4.41 Color change of the mixtures before and after the reaction; (a): Series 1; (b): Series 2; the line in (b) is due to the background and not a sign of phase separation.

An unusual color could be observed - yellow, orange and even green - when sulfur dissolves in an amine solvent or stemming from sulfur radicals. ${ }^{[5,6]}$ In the present test series, an amine-containing solvent can be formed when DPG is transformed or fragmented and dissolved in $\mathrm{n}$-decane: Hummel et al. ${ }^{[7]}$ reported that the chemical structure of DPG can be transformed or fragmented when heat is applied. In addition, it is interesting to note that stearic acid as well as DPG disappeared by forming a salt which precipitates in $\mathrm{SaD}$ (stearic acid+DPG) and ZSaD (ZnO+stearic acid+DPG) after the reaction, whereas a lot of precipitated DPG is observed in Samples D (DPG only) and ZD (ZnO+DPG). This indicates that a higher amount of DPG as such or in its transformed or fragmented state can be dissolved in $\mathrm{n}$-decane under these circumstances. And more sulfur can be dissolved when DPG is combined with stearic acid ( $\mathrm{SaD}$ and ZSaD). As a result, the color of these samples (D, ZD, SaD and ZSaD) is deep yellow or orange. The other samples without DPG (including R2) show almost no appearance change: no precipitated sulfur neither color change is observed. This means that $\mathrm{ZnO}$ as well as stearic acid do not affect the release of sulfur from TESPT molecules. 


\subsubsection{Total amount, average sulfur rank and sulfur rank distribution of TESPT}

The total amount and the average sulfur rank of TESPT after the reaction is shown in Figure 4.42. As can be seen in Figure 4.42(a), ZnO and stearic acid do not have much impact on the amount of TESPT ( $\mathrm{Z}$ and Sa vs. R2). In particular ZnO shows almost no effect on the amount of TESPT, even when it is combined with stearic acid (ZSa). Therefore, it can be stated that $\mathrm{ZnO}$ or zinc stearate do not affect the release of sulfur from the TESPT molecule.

A significant reduction in the amount of TESPT can be seen in Figure 4.42(a) for sample ZD (ZnO+DPG). However, when the value is compared with sample $D$, it can be easily noticed that the decrement in the amount of TESPT is mainly induced by DPG. The mixtures containing DPG show a significant reduction in the amount of TESPT: compared to R1 and R2, the reduction is app. $20 \%$ and $10 \%$, respectively. The largest reduction in the amount of TESPT is observed when DPG is combined with stearic acid (SaD and ZSaD). These results indicate that the acidic component helps the reaction between TESPT and DPG, however further evaluation is required in order to clarify the role of the acidic substance on the reaction between TESPT and DPG.

Figure 4.42(b) shows as well, that a shorter sulfide chain is found in the samples containing DPG and stearic acid. The shortest sulfur moiety is found for the samples SaD (stearic acid+DPG) and ZSaD (ZnO+stearic acid+DPG). This result again indicates that the reaction between TESPT and DPG is enhanced when an acidic substance like stearic acid is present.
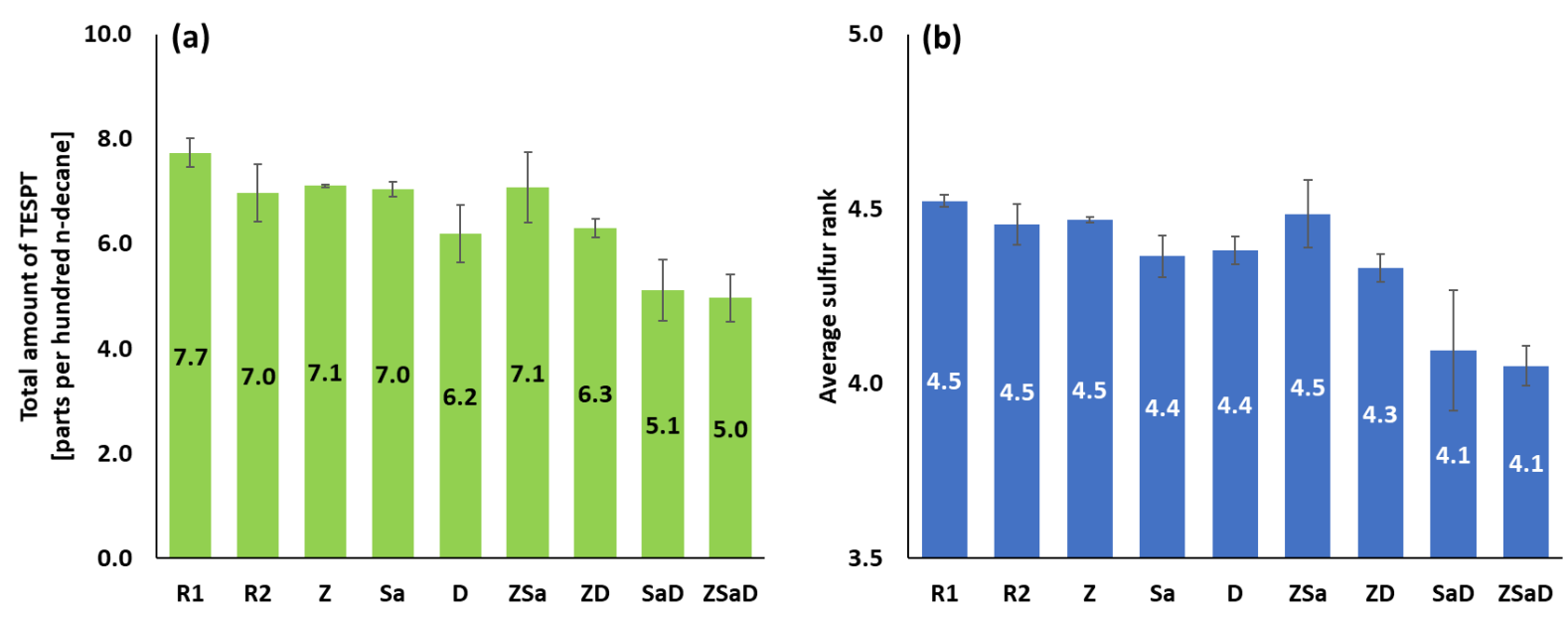

Figure 4.42 Total amount and the average sulfur rank of TESPT in the mixture after the reaction;

(a): total amount of TESPT; (b): average sulfur rank. 
The average sulfur rank distribution index of the mixtures after the reaction are shown in Figure 4.43. The amount of each sulfide in the R1 sample was set as $100 \%$. Regardless of the ingredients, the longest sulfides showed the largest reduction, see e.g. the grey bars. The sulfur rank distribution of TESPT is changed already by the heating process due to its thermal stability, as seen for sample R1 versus R2. ${ }^{[2,8,9]}$ However, not only the thermal stability of the sulfides, but also the presence of DPG has a large impact on the breaking pattern of the sulfidic bridges. The samples containing DPG (D, ZD, SaD and ZSaD) show the largest decrement in the amount of the longer polysulfidic silanes. And this phenomenon is enhanced when DPG is combined with stearic acid: samples SaD and ZSaD show the lowest percentages of the longer polysulfidic silanes.

A significant increment in the number of short sulfidic species, such as di- and tri-sulfides, is observed for the samples containing stearic acid ( $\mathrm{Sa}, \mathrm{ZSa}, \mathrm{SaD}$ and $\mathrm{ZSaD}$ ), whereas $\mathrm{Z}, \mathrm{D}$ and ZD show almost similar values compared to R2. This result indicates that stearic acid assists to bind the broken silane molecules after they release the sulfur moiety.

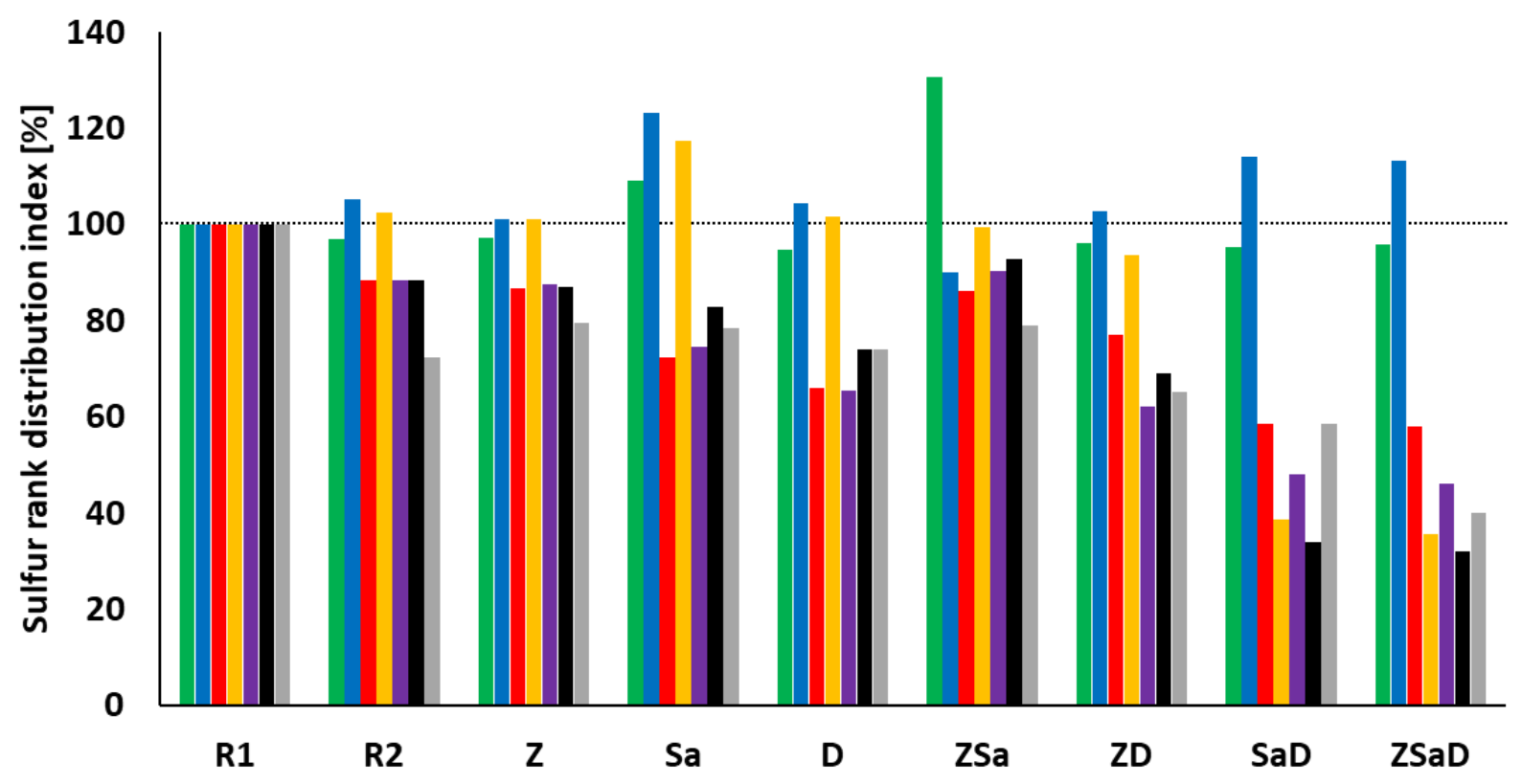

Figure 4.43 Sulfur rank distribution;

$(\square): S_{2} ;(\square): S_{3} ;(\square): S_{4} ;(\square): S_{5} ;(\square): S_{6} ;(\square): S_{7} ;(\square): S_{8}$. 


\subsubsection{Analysis of the reaction products}

\subsection{LC-MS analysis}

As can be seen in Figure 4.44, the mass spectra of the samples containing DPG (D, SaD and ZSaD) show a new peak with a higher intensity $\left(\times 10^{9}\right)$ at the very beginning of the measurement. Sample ZD could not be evaluated as the sample was broken during preparation, and due to time constraints this measurement could not be repeated.

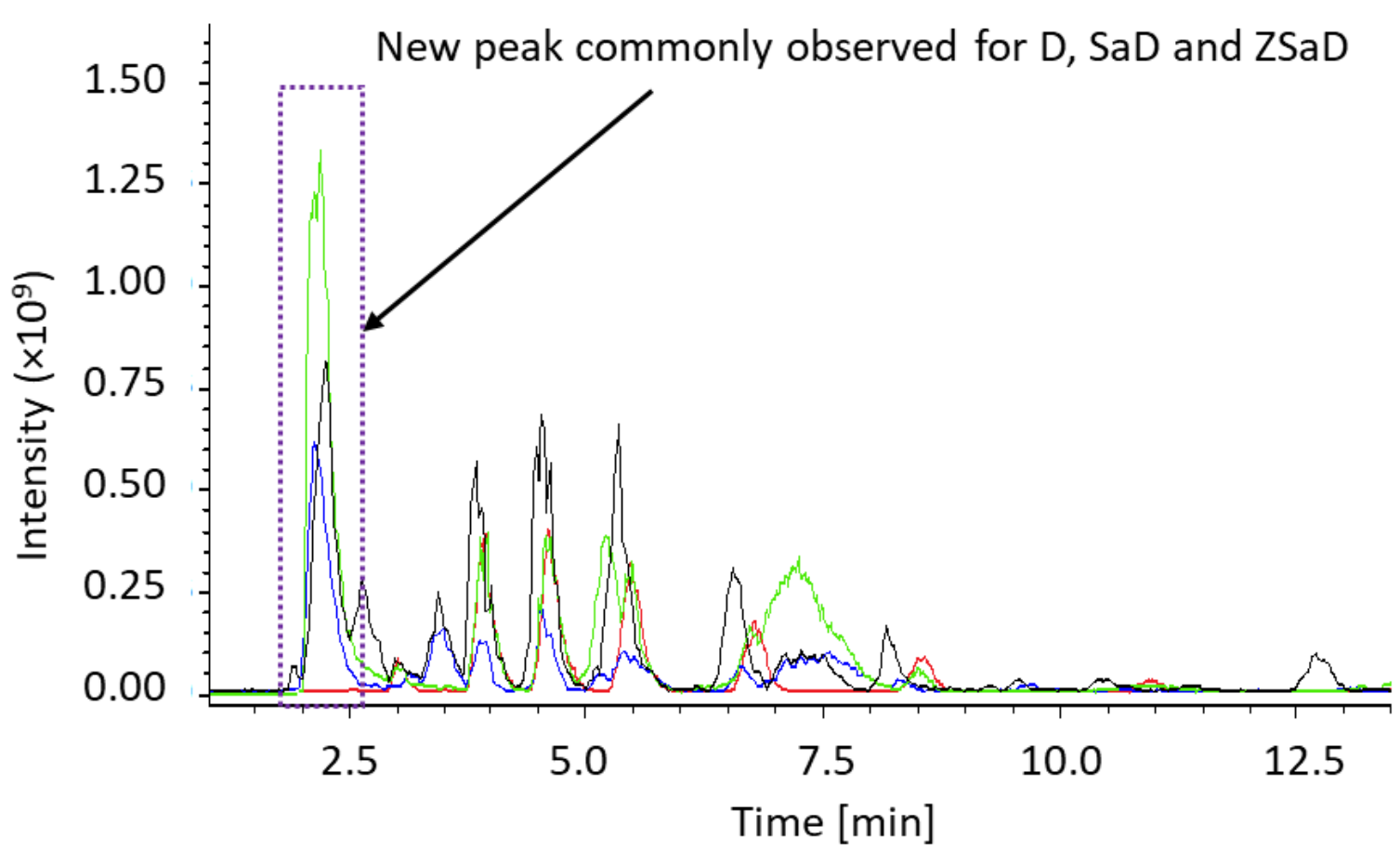

Figure 4.44 Mass spectra; (_ ): R2; ( $)$ ): D; ( $)$ ): SaD; ( $)$ ): ZSaD.

The extracted ion spectra corresponding to the new peak observed for $\mathrm{D}, \mathrm{SaD}$ and $\mathrm{ZSaD}$, are depicted in Figure 4.45. These samples all show extracted ion spectra peaks at $211 \mathrm{~m} / \mathrm{z}, 406 \mathrm{~m} / \mathrm{z}$ and $431 \mathrm{~m} / \mathrm{z}$ with high intensity $\left(\times 10^{7}\right)$. The origin of the peak at $211 \mathrm{~m} / \mathrm{z}$ is obvious, because the value corresponds to the molecular weight of DPG. The peak at $431 \mathrm{~m} / \mathrm{z}$ is most probably be the reaction product similar to the model structure of Mihara (Figure 4.46(a)) with a mono-sulfidic moiety: Figure 4.46(b). However, the mass spectrum of $406 \mathrm{~m} / \mathrm{z}$ is so far unknown. 

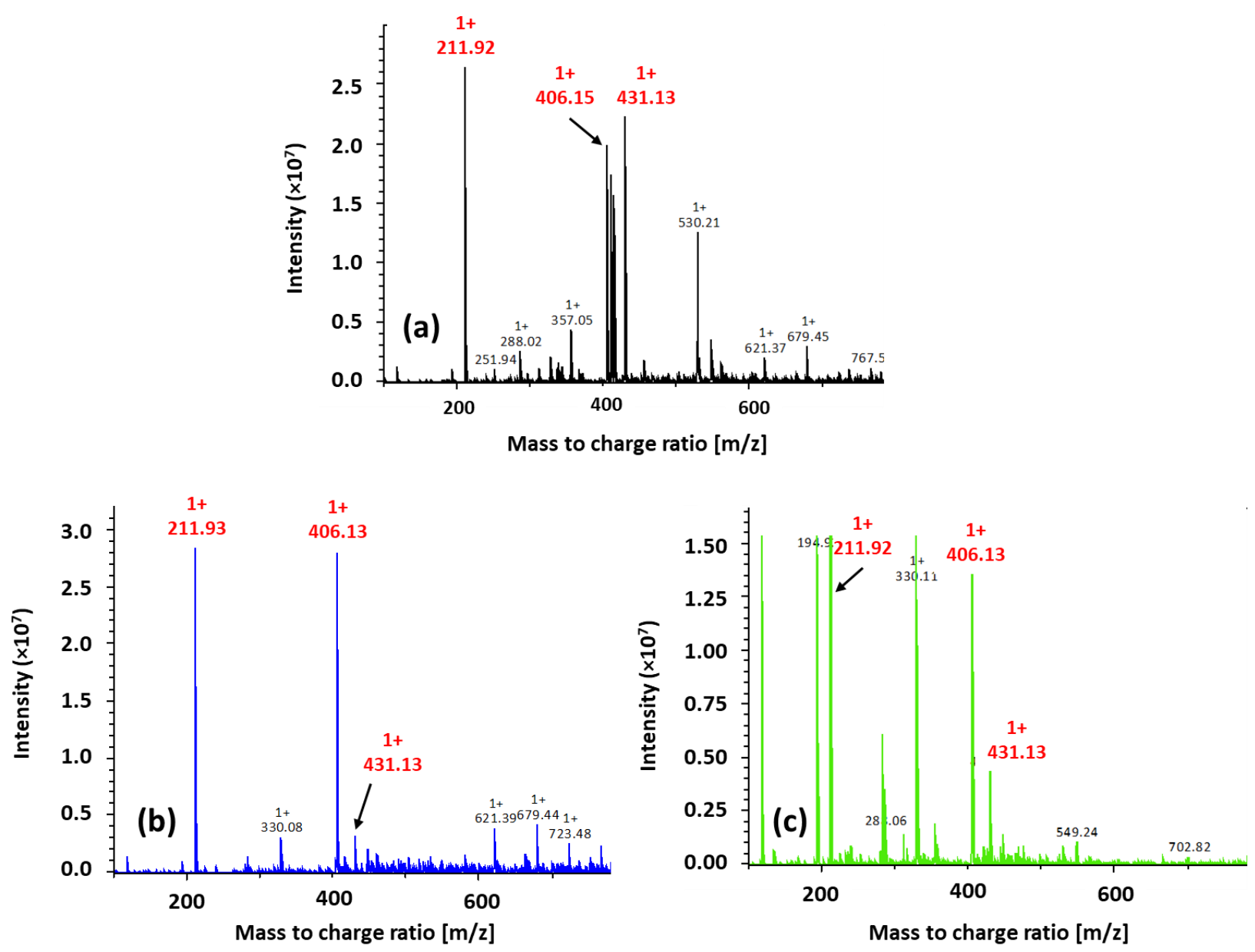

Figure 4.45 The extracted ion spectra of the new peaks in Figure 4.44; (a): D; (b): SaD; (c): ZSaD.

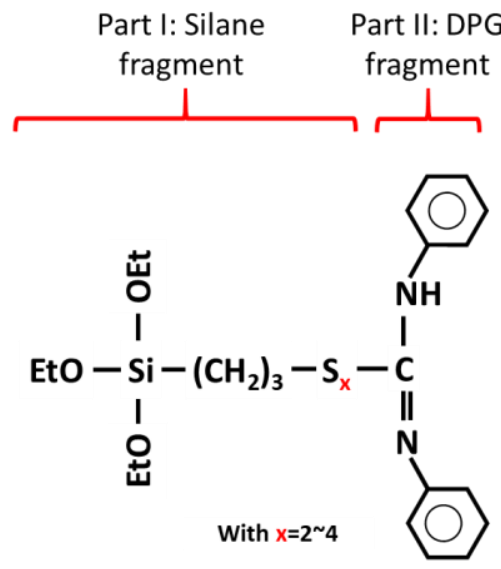

(a)

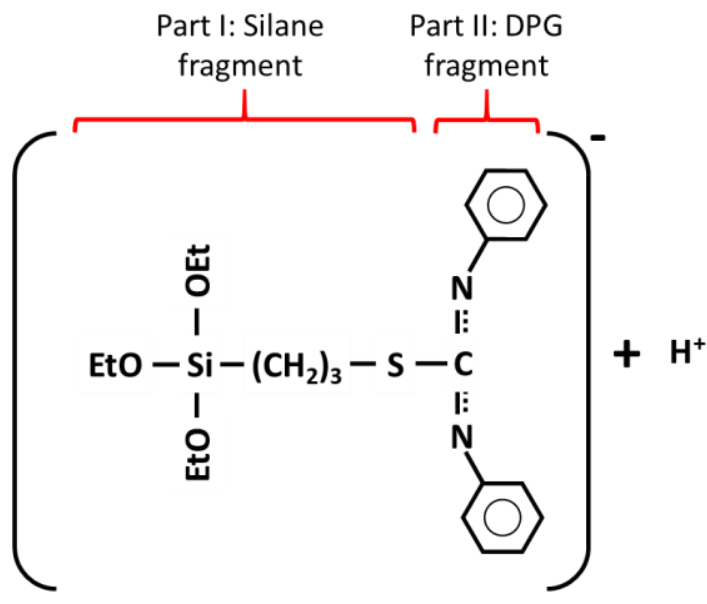

(b)

Figure 4.46 Chemical structure models of DPG-TESPT complex;

(a) Mihara's model ${ }^{[1]}$; (b): suggested model in the present work with mono-sulfide moiety. 
The reaction product showing $406 \mathrm{~m} / \mathrm{z}$ in Figure 4.45 was further analyzed by using MS-MS; the results are shown in Figure 4.47. The chemicals were further ionized and fragmented, therefore additional extracted ion spectra were obtained. Based on the values obtained in Figure 4.47 and the work of Hummel et al. ${ }^{[7]}$, it turned out that $406 \mathrm{~m} / \mathrm{z}$ corresponds to transformed or fragmented structures of DPG: Figure 4.48. Especially, the DPG fragment corresponding to $194 \mathrm{~m} / \mathrm{z}$ in Figure 4.48 shows a similar structure as Part II depicted in Figure 4.46.

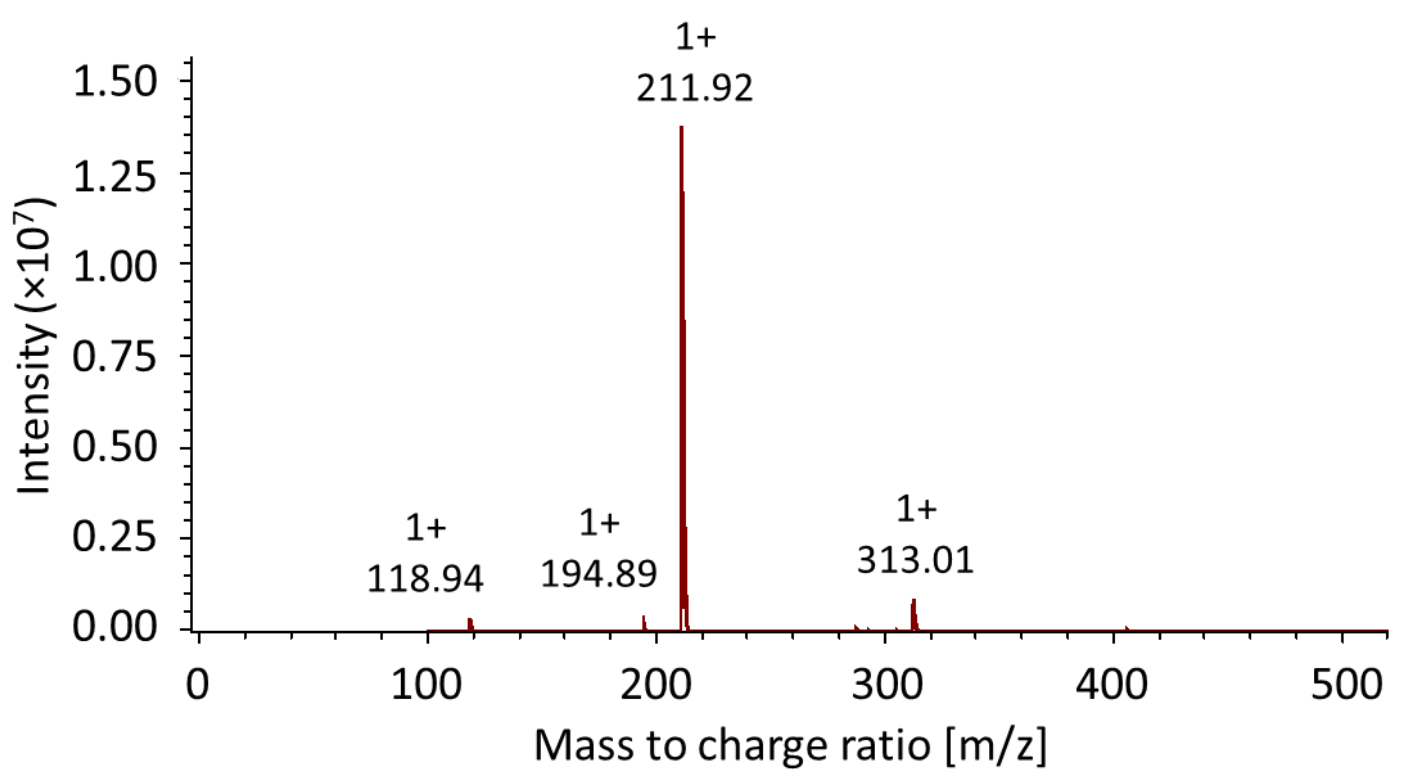

Figure 4.47 MS-MS spectra corresponding to the reaction product showing $406 \mathrm{~m} / \mathrm{z}$ in Figure 4.45 .

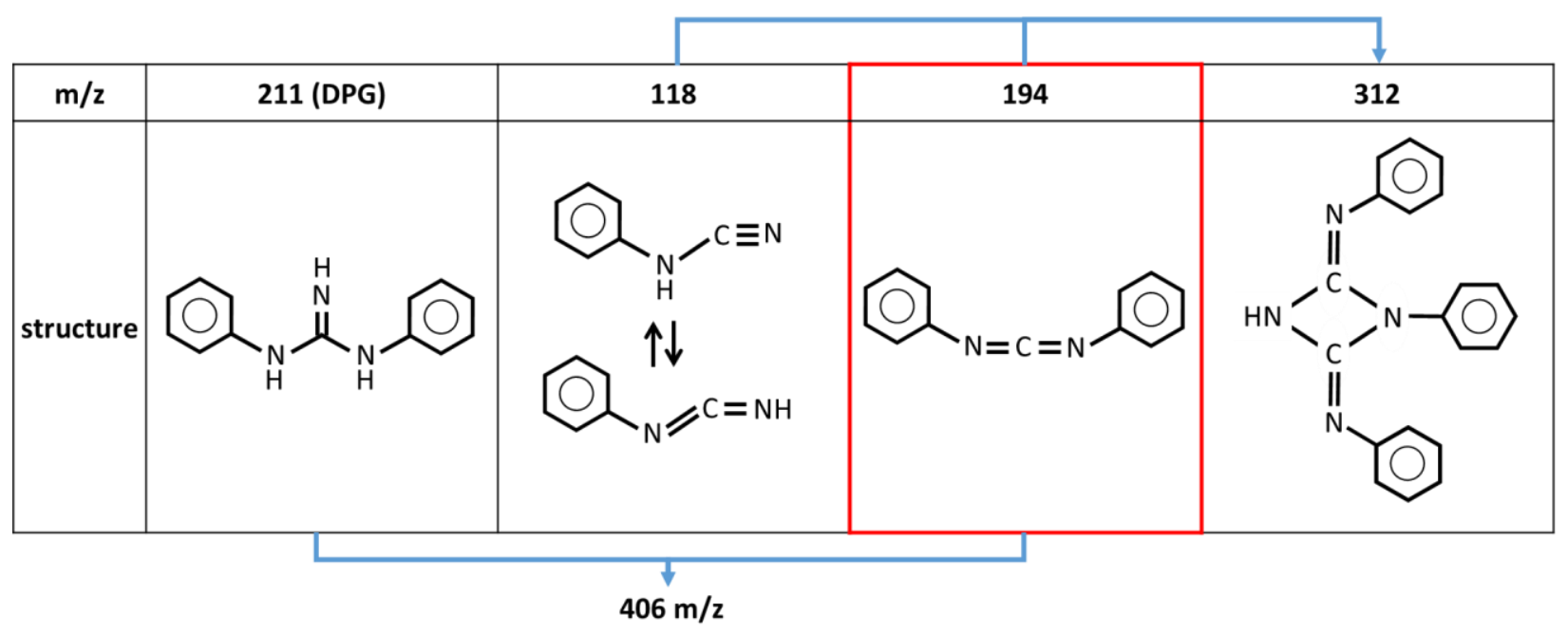

Figure 4.48 Transformed or fragmented structures of DPG by heat from literature. ${ }^{[7]}$ 


\subsubsection{2 ${ }^{13} \mathrm{C}-\mathrm{NMR}$ analysis}

The ${ }^{13}$ C-NMR spectrum of heated TESPT and untreated DPG are shown in Figures 4.49 and 4.50, respectively, and their chemical shifts are listed in Tables 4.14 and 4.15. Untreated TESPT showed the same spectra as shown in Figure 4.49 with different intensities, and is therefore not depicted.

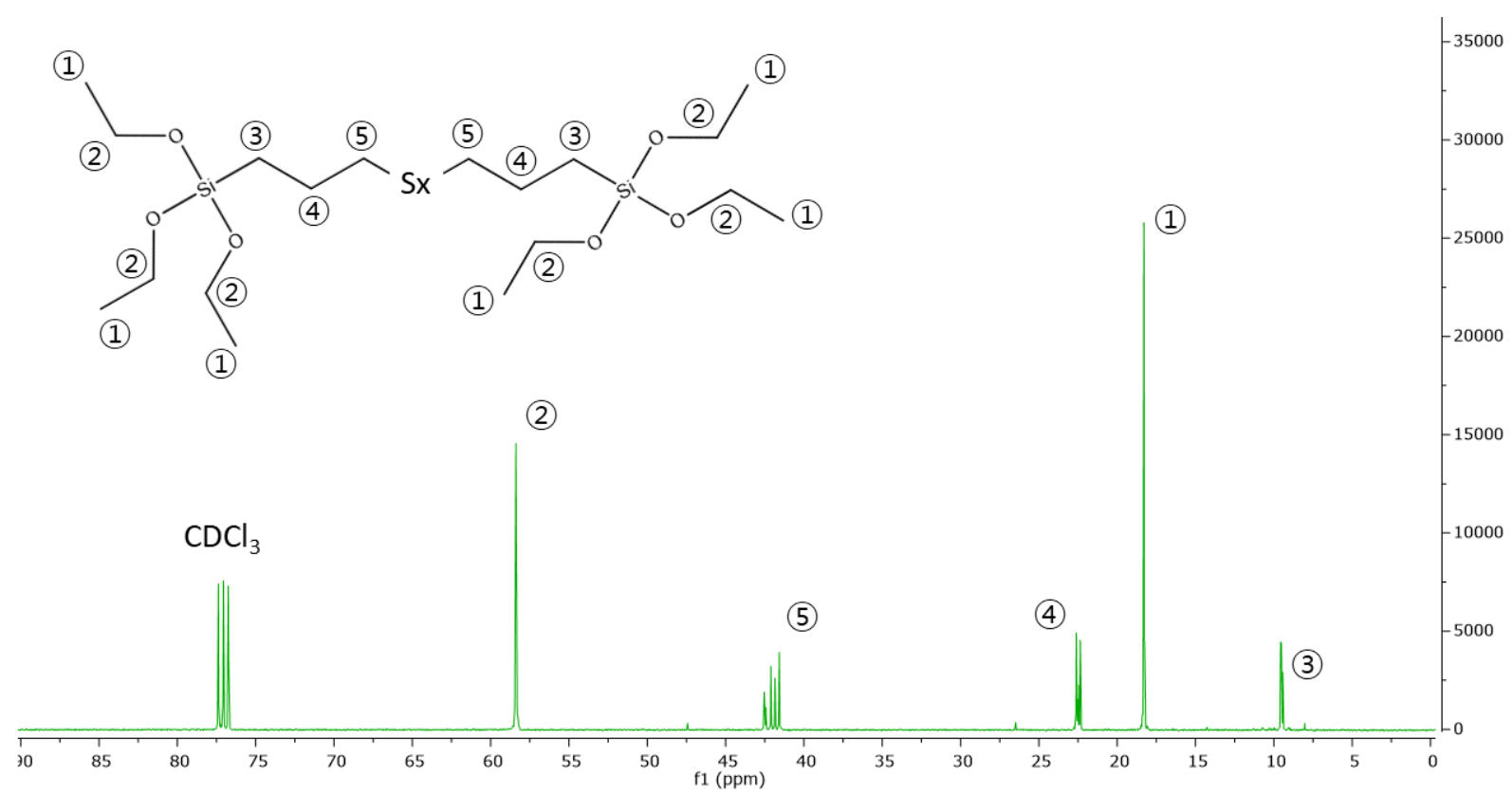

Figure $4.49{ }^{13} \mathrm{C}$-NMR spectrum of heated TESPT.

Table 4.14 Chemical shift of TESPT

\begin{tabular}{c|c}
\hline Peak & Chemical shift [ppm] \\
\hline (1) & 18.29 \\
(2) & 58.39 \\
(3) & 9.50 \\
(4) & 22.46 \\
(5) & 42.10 \\
\hline
\end{tabular}




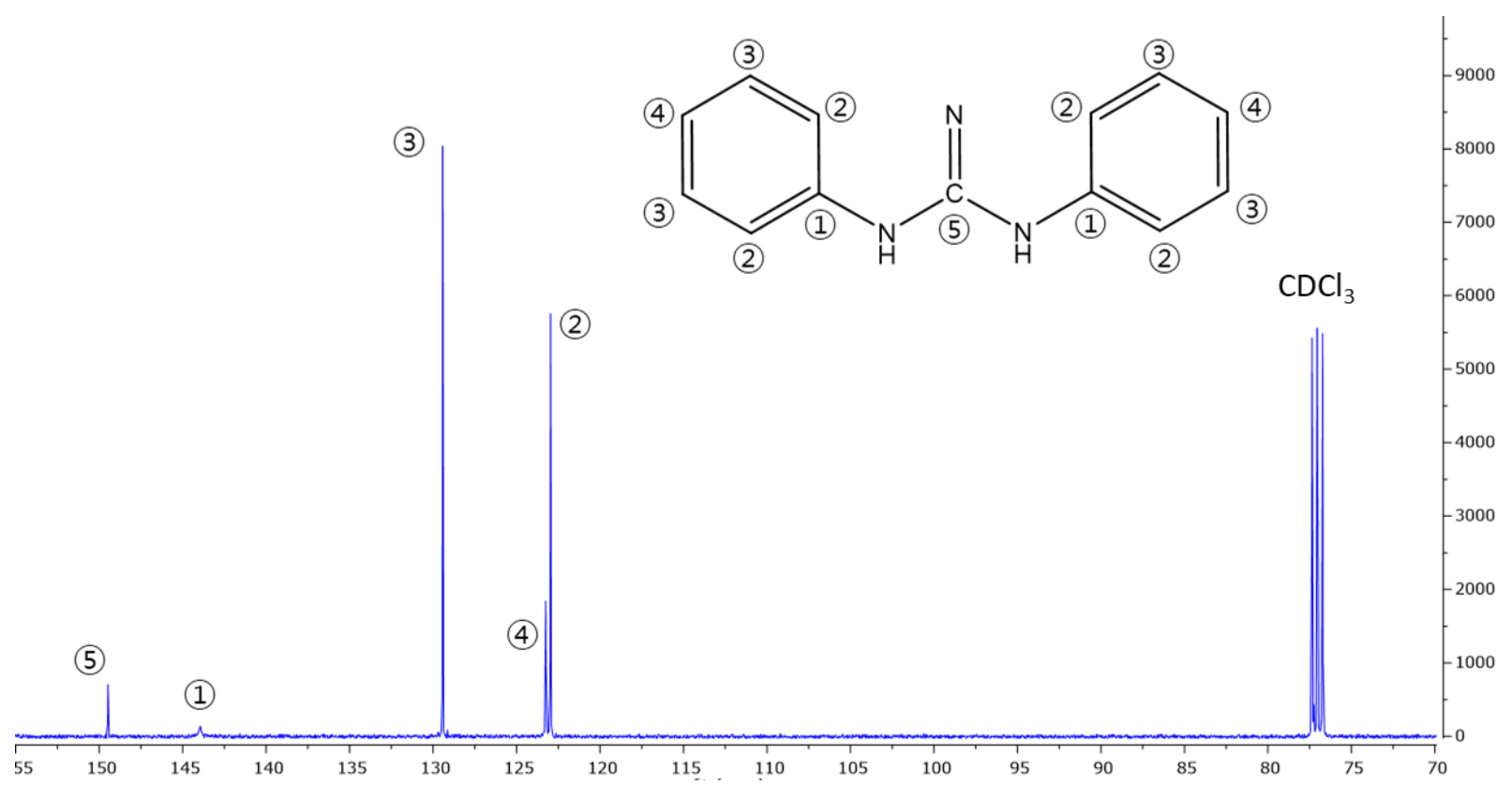

Figure $4.50{ }^{13} \mathrm{C}-\mathrm{NMR}$ spectrum of DPG (untreated).

Table 4.15 Chemical shift of DPG

\begin{tabular}{c|c}
\hline Peak & Chemical shift [ppm] \\
\hline (1) & 143.96 \\
(2) & 122.96 \\
(3) & 129.42 \\
(4) & 123.25 \\
(5) & 149.47 \\
\hline
\end{tabular}

The NMR spectra of unheated/heated DPG are compared (Figure 4.51) and the chemical shift values are listed in Table 4.16. As can be seen in Figure 4.51, heated DPG shows seven peaks in total. Five of them are the same as in the spectra of untreated DPG: peaks a-e in Figure 4.51 represent the same structural units as peaks 1-5 in Figure 4.50. Two additional new peaks ( $\mathrm{f}$ and $\mathrm{g}$ ) are observed and remained unknown. The new peaks are assumed to be the spectra of DPG fragment(s) due to the fact that there were no other ingredients present while DPG was heated. 


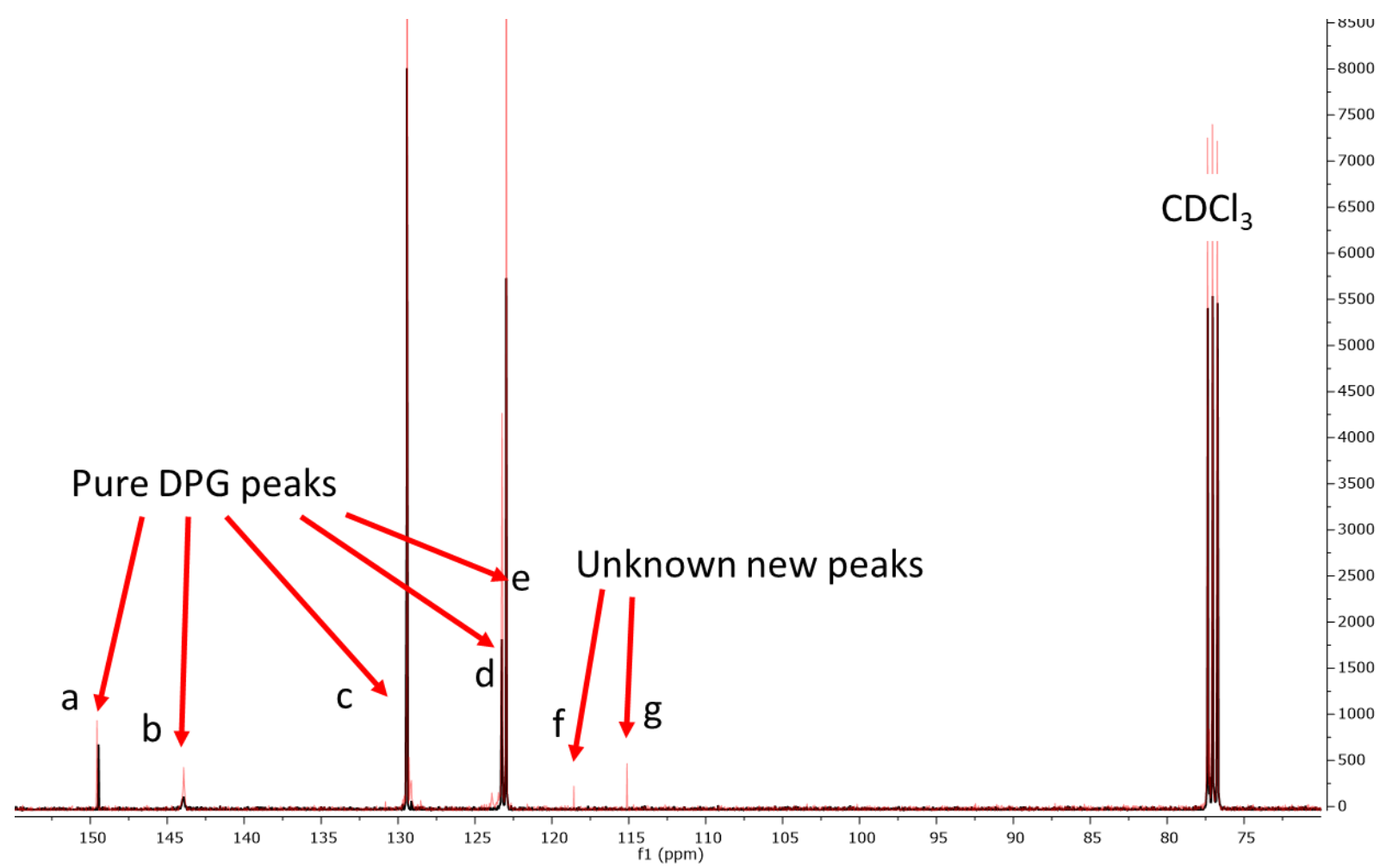

Figure $4.51{ }^{13} \mathrm{C}-\mathrm{NMR}$ spectrum of DPG; (_ ) : untreated; ( $(\longrightarrow$ ): heated DPG.

Table 4.16 Chemical shift of the peaks

\begin{tabular}{c|c|c}
\hline Peak & Chemical shift [ppm] & Division \\
\hline a & 149.47 & \\
b & 143.96 & known peaks \\
c & 129.42 & \\
d & 123.25 & \\
e & 122.96 & \\
\hline f & 118.57 & (from DPG; see Figure 4.50) \\
g & 115.11 & (DPG fragments) \\
\hline
\end{tabular}

It was assumed that, in the case that DPG and TESPT would not react mutually, the sample would show the same NMR spectra as a simple mixture of heated DPG and TESPT. Therefore, the stacked NMR spectra of heated DPG and TESPT was used as background for elucidating the DPG-TESPT reaction: Figure 4.52. 


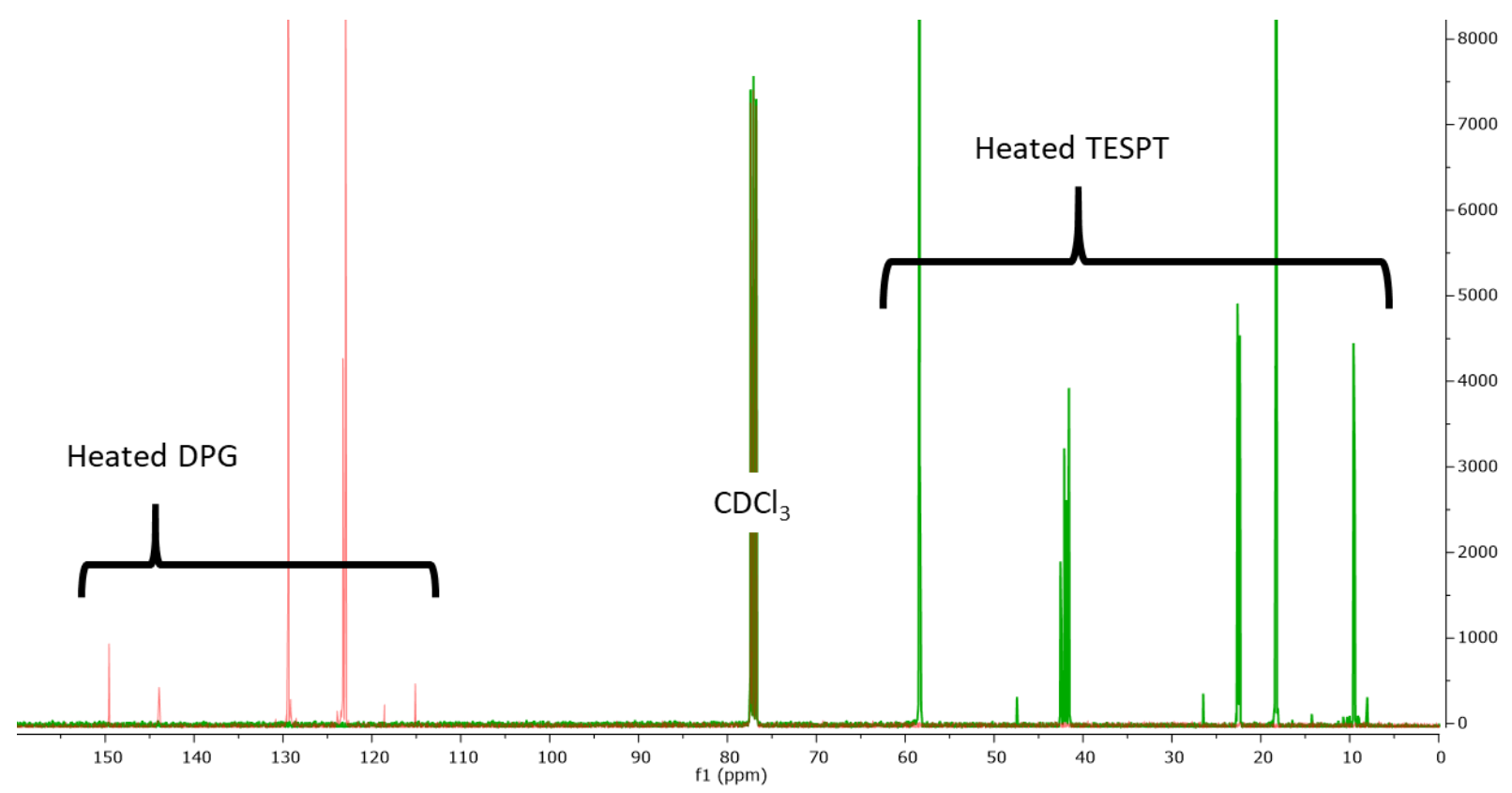

Figure 4.52 Background ${ }^{13} \mathrm{C}-\mathrm{NMR}$ spectrum for DPG-TESPT reaction elaboration;

( $)$ : heated TESPT; (

The spectra of the real DPG-TESPT reactant is compared with Figure 4.52 as shown in Figure 4.53. As can be seen in this figure, five new peaks $(v-z)$ are observed. These peaks clearly indicate that DPG and TESPT reacted directly and formed a complex. The predicted chemical structure of the DPG-TESPT reaction is depicted in Figure 4.54, and the chemical shift values are listed in Table 4.17. These results confirm the predicted DPG-TESPT complex structure, as well as Mihara's model.

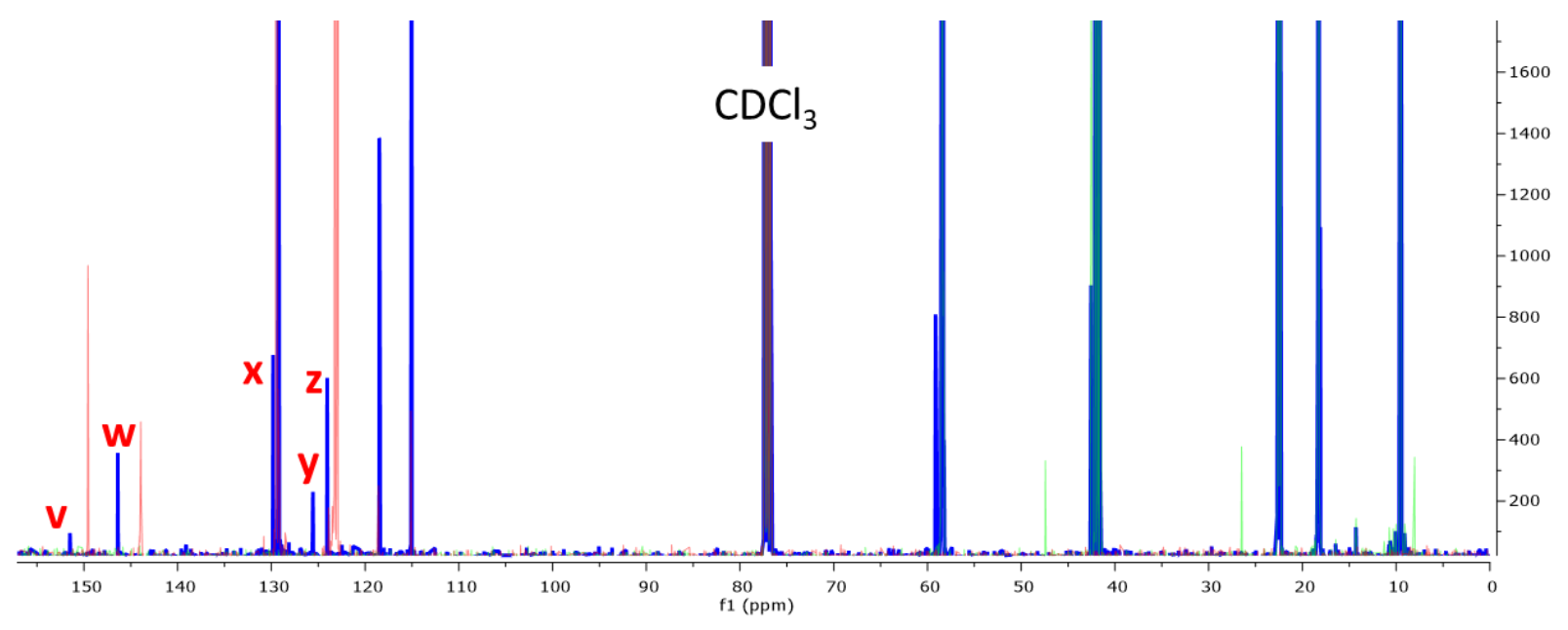

Figure $4.53{ }^{13} \mathrm{C}$-NMR spectrum of DPG-TESPT reaction product with background;

( _ ): heated TESPT; ( _ ): heated DPG; (_ ) ): DPG-TESPT reaction product. 


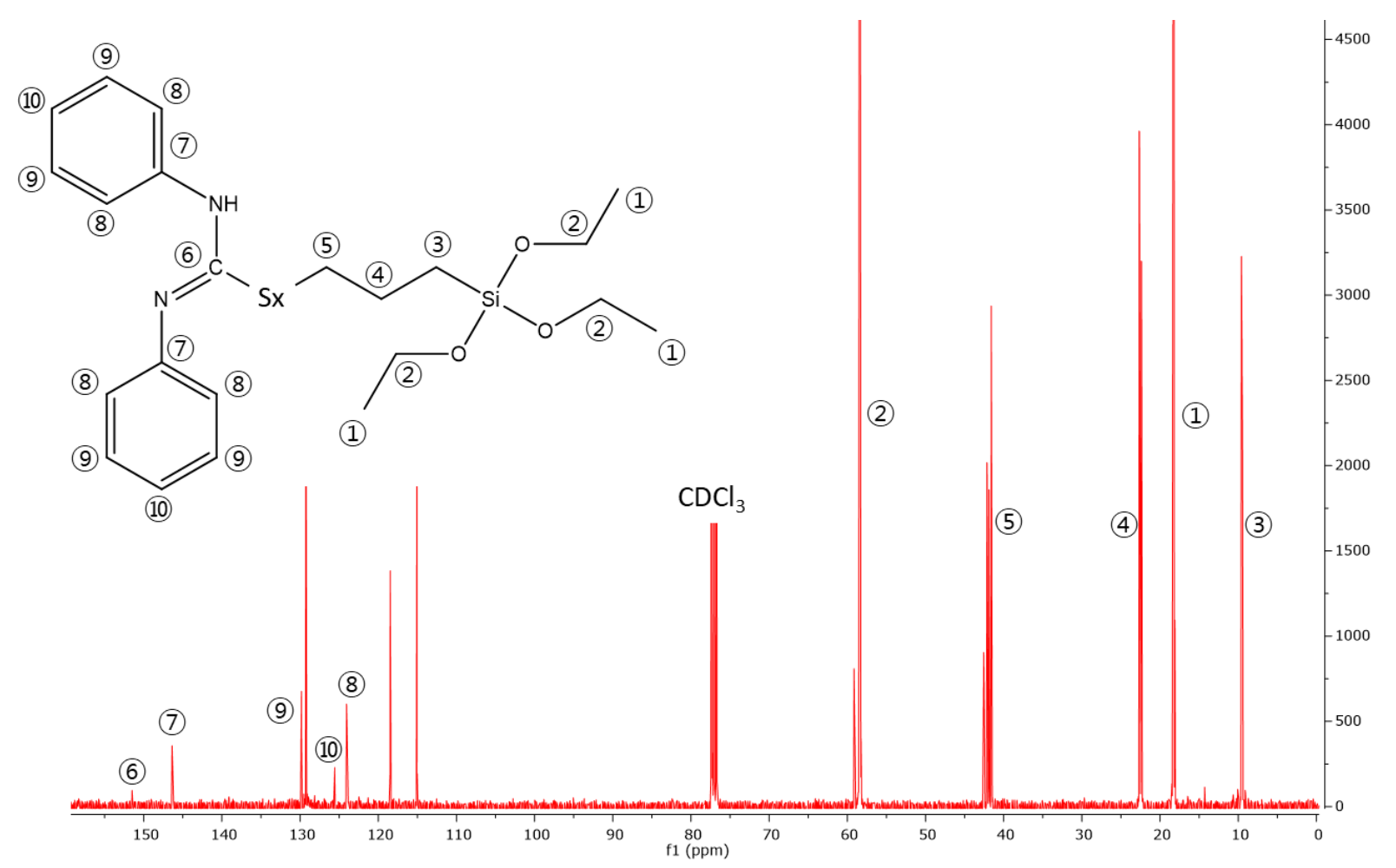

Figure $4.54{ }^{13} \mathrm{C}-\mathrm{NMR}$ spectrum of TESPT-DPG reaction product and its structure.

Table 4.17 Chemical shifts of the TESPT-DPG reaction product

\begin{tabular}{c|c}
\hline \multicolumn{1}{c|}{ Peak } & Chemical shift [ppm] \\
\hline (1) & 18.31 \\
(2) & 58.41 \\
(3) & 9.52 \\
(4) & 22.48 \\
(5) & 42.03 \\
(6) & 151.49 \\
(7) & 146.38 \\
(8) & 124.05 \\
(9) & 129.81 \\
(10) & 125.57 \\
\hline
\end{tabular}




\subsubsection{Conclusions}

The focus of this study was to investigate the secondary roles of the ingredients $\mathrm{ZnO}$, stearic acid and DPG, to elucidate their impact on the sulfidic silane in order the answer the questions in Paragraph 4.4.1.

$\mathrm{ZnO}(\mathrm{Z})$ and stearic acid ( $\mathrm{Sa}$ ) had no effect on TESPT. Compared to the reference R2, the samples containing ZnO (with/without stearic acid) showed no significant change in appearance, amount, average sulfidic bridge length and the sulfur length distribution of TESPT.

Another functionality of DPG, which was proposed by Mihara ${ }^{[1]}$, was confirmed in this study: DPG reacts directly with TESPT. ${ }^{[1]} \mathrm{A}$ first indication for such a reaction was observed in the change in appearance before and after the reaction. The samples containing DPG changed their color to deep yellow. A reduced amount of TESPT and a higher percentage of short sulfidic bridge molecules were detected in the samples containing DPG: A lot of long sulfidic species of TESPT turned into short sulfides. Additionally, the results of LC-MS and ${ }^{13} \mathrm{C}-N M R$ strongly supported the reaction between DPG and TESPT. LC-MS allowed to identify fragments and transforms of DPG. Especially, one of the DPG fragments (diphenylcarbodiimide), which shows $194 \mathrm{~m} / \mathrm{z}$ in the mass spectrum in LC-MS, seems to react with TESPT after splitting the molecule into two parts. The mass spectrum of the samples containing DPG showed a peak at $431 \mathrm{~m} / \mathrm{z}$, which confirmed the predicted DPG-TESPT complex. The proposed chemical structure of the DPG-TESPT complex was also confirmed based on the results of ${ }^{13} \mathrm{C}-\mathrm{NMR}$.

These results indicate that DPG can react with TESPT and both are thus partly consumed during the mixing process. Through this interpretation, a slower vulcanization rate, lower crosslink density, and a larger amount of bound rubber with increasing DPG concentration in the masterbatch mixing step in Chapter 4.3 can thus be explained as shown in Table 4.18.

Table 4.18 The effect of a higher concentration of DPG in the early mixing step

\begin{tabular}{|c|c|c|}
\hline $\begin{array}{l}\text { Increasing DPG concentration } \\
\text { in the early mixing step }\end{array}$ & Trend & Possible explanation \\
\hline $\begin{array}{l}\text { Filler-filer interaction } \\
\text { (Payne effect) }\end{array}$ & & $\begin{array}{l}\text { - absorption onto silica surface } \\
\text { - enhancing the silanization reaction } \\
\text { - formation of higher amount of bound rubber }\end{array}$ \\
\hline $\begin{array}{l}\text { Chemically bound rubber, } \\
\text { Filler-polymer coupling rate }\end{array}$ & & $\begin{array}{l}\text { - enhancing the silanization reaction } \\
\text { - TESPT-DPG reaction (sulfur release from TESPT) }\end{array}$ \\
\hline $\begin{array}{l}\text { Cure rate, } \\
\text { Crosslink density (with increasing cure time) }\end{array}$ & & $\begin{array}{l}\text { - absorption onto silica surface } \\
\text { - TESPT-DPG reaction (consumed during mixing) }\end{array}$ \\
\hline
\end{tabular}




\section{References}

[1] S. Mihara, PhD Thesis: Reactive processing of silica-reinforced tire rubber: new insight into the time- and temperature-dependence of silica rubber interaction, Univ. Twente: Enschede, the Netherlands (2009).

[2] H. D. Luginsland, Kautsch. Gummi Kunstst. 53, 10 (2000).

[3] A. Kruve, K. Kaupmees, J. Am. Soc. Mass Spectrom. 28, 887 (2017).

[4] N. B. Cech, C. G. Enke, Mass Spectrom. Rev. 20, 362 (2001).

[5] R. E. Davis, H. F. Nakshbendi, J. Am. Chem. Soc. 84, 2085 (1962).

[6] B. Eckert, R. Steudel, Top Curr. Chem. 231, 31 (2003).

[7] D. O. Hummel, G. Czybulka, H-J. Dussel, Macromol. Chem., Rapid Commun. 3, 335 (1982).

[8] A. Hasse, O. Klockmann, A. Wehmeier, H.-D. Luginsland, Kautsch. Gummi Kunstst. 55, 236 (2002).

[9] U. Görl, J. Munzenburg, H.D Luginsland, A. Muller, Kautsch. Gummi Kunstst., 52, 588 (1999). 


\section{Chapter 5 The effect of polymer and silica characteristics on marching modulus of silica filled tire tread compounds}

The technical and chemical characteristics of the raw materials are recognized as crucial factors for the degree of filler dispersion as well as bound rubber formation. Within this chapter, the effect of polymer variation and silica characteristics on marching modulus are studied.

In earlier studies, the chemical structure of the silane was found to strongly influence the intensity of the filler-filler interaction, the amount of bound rubber as well as the filler-polymer coupling rate for silica compounds: Figures 3.7, 3.9 and 3.15 in Chapter 3.1. Besides, the polymer type such as SBR and BR, and their physical affinity towards silica influence the degree of silica dispersion. Additionally, the chemical configuration of the double bonds of the butadiene unit in SBR and BR can affect the degree of fillerpolymer coupling due to their reactivity as theoretically elaborated in Chapter 2, Paragraph 2.4.3.1. Silica itself has large impact on its dispersion quality in a rubber matrix due to the dispersibility induced by the physical characteristics. The development of Highly-Dispersible (HD) silica is a good example, indicating the importance of the properties of the filler, as explained in Paragraph 2.3.2.5 in Chapter 2.

In this chapter, the effect of the polymer and silica characteristics on marching modulus of a silica filled tire tread compound are investigated as follows:

5.1 The effect of the SBR/BR blend ratio;

5.2 The effect the dispersibility of silica.

In these chapters, compounds were mixed with different polymer blending ratios (Chapter 5.1) and using two silicas having different dispersibility (Chapter 5.2). Subsequently, the degree of silica dispersion, the amount of bound rubber, Filler Flocculation Rate (FFR), and filler-polymer Coupling Rate (CR) of the compounds are evaluated. Finally, those factors are correlated with the curing behavior of the compounds. The outcome of these chapters are interpreted in terms of the technical characteristics of polymers and silicas. 


\section{Chapter 5.1 The effect of the SBR/BR blend ratio on marching modulus}

\subsubsection{Introduction}

In tire tread compounding, two or more different types of polymers are in general blended in order to achieve appropriate rubber properties as well as tire performance. The elastomer blending technique can result in dissimilar benefits for a tire tread. As an example, styrene-butadiene rubber (SBR) and polybutadiene rubber (BR) are often blended for winter tire applications. ${ }^{[1,2]} A$ high styrene content of SBR leads to better wet skid and traction properties ${ }^{[3]}$, however, also results in a higher glass transition temperature $\left(\mathrm{T}_{\mathrm{g}}\right)$ which on its turn in particular influences the low temperature performance of a tire. ${ }^{[1]}$ $B R$ has a low glass transition temperature, thus the $T_{g}$ of a tire tread compound can be controlled by changing the ratio of SBR to BR in the blend.

Due to thermodynamic immiscibility, elastomer blends are heterogeneous and show a certain phase morphology according to the level of and miscibility of the blended species. ${ }^{[1,4-6]}$ Further, each type of elastomer has its own specific affinity to reinforcing fillers. ${ }^{[4,7]}$ Wang and Wolff ${ }^{[8]}$ compared the affinity of model chemical probes representing elastomers and found, that the degree of interaction of rubber with silica varies in the following order: NBR $>$ SBR $>$ NR $>$ BR $>$ EPDM $>$ IIR. Le et al. ${ }^{[7,9,10]}$ reported that the nature of the rubber-filler interactions strongly affect the filler surface wetting behavior. In return, this will also have an influence on the flocculation behavior. Stöckelhuber ${ }^{[11]}$ reported that flocculation can be reduced by a higher compatibility between rubber and filler surface. These studies imply that the characteristics of the materials such as the chemistry of the polymer and the filler surface, are recognized as crucial factors for filler dispersion and network. ${ }^{[12-16]}$

In the present chapter, the effect of incompatible SBR/BR blends with different polymer ratios on factors affecting the marching modulus of silica filled compounds are evaluated. These factors are correlated with the vulcanization characteristics of these compounds as measured at small (approx. 7\%) and large (approx. 42\%) strain. According to literature, the chemical structure of the polymers influences their interaction with the silane as well as the curatives. ${ }^{[17]}$ Therefore, the results are interpreted in terms of the degree of silanization, but also concerning the chemical structure of SBR and BR and their respective reactivities. 


\subsubsection{Experimental}

\subsubsection{Materials}

Blends of the flowing polymers were used in this study:

- $\quad$ Oil-extended solution styrene-butadiene rubber (S-SBR) with a Mooney viscosity $\left(\mathrm{ML} 1+4 @ 100^{\circ} \mathrm{C}\right)$ of 65 and composed of $75 \mathrm{wt} \%$ butadiene with a $50 \%$ vinyl-content, and $25 \mathrm{wt} \%$ styrene-content, extended with $37.5 \mathrm{phr}$ of TDAE oil,

- High cis-1,4 polybutadiene rubber (BR) with a Mooney viscosity (ML1+4@100 ${ }^{\circ} \mathrm{C}$ ) of 44 and a cis1,4 content of $96 \%$.

With these polymers, all series of experiments were done based on a tire tread compound as shown in

Table 5.1. The amount of TESPT applied in the formulations was calculated according to Equation 3.1.

Table 5.1 Compound formulation

\begin{tabular}{|c|c|c|c|c|c|c|c|c|c|c|}
\hline $\begin{array}{l}\text { Mixing } \\
\text { stage }\end{array}$ & Ingredient & Product name & Company & $\begin{array}{l}\text { SBR100 } \\
\text { (phr) }\end{array}$ & $\begin{array}{c}\text { SBR80 } \\
\text { (phr) }\end{array}$ & $\begin{array}{c}\text { SBR60 } \\
\text { (phr) }\end{array}$ & $\begin{array}{c}\text { SBR50 } \\
\text { (phr) }\end{array}$ & $\begin{array}{c}\text { SBR40 } \\
\text { (phr) }\end{array}$ & $\begin{array}{c}\text { SBR20 } \\
\text { (phr) }\end{array}$ & $\begin{array}{l}\text { SBRO } \\
\text { (phr) }\end{array}$ \\
\hline \multirow{4}{*}{$\begin{array}{l}\text { Pre- } \\
\text { blending }\end{array}$} & S-SBR* & $\begin{array}{c}\text { Buna VSL5025- } \\
2 \mathrm{HM}\end{array}$ & Arlanxeo & 137.6 & 110.0 & 82.5 & 68.8 & 55.0 & 27.5 & 0.0 \\
\hline & $\mathrm{BR}$ & Buna CB24 & Arlanxeo & 0.0 & 20.0 & 40.0 & 50.0 & 60.0 & 80.0 & 100.0 \\
\hline & HD Silica & $\begin{array}{l}\text { ULTRASIL }^{\circledR} 7005 \\
\text { (CTAB: } 177 \mathrm{~m}^{2} / \mathrm{g} \text { ) }\end{array}$ & Evonik & 50 & $\rightarrow$ & $\rightarrow$ & $\rightarrow$ & $\rightarrow$ & $\rightarrow$ & $\rightarrow$ \\
\hline & TDAE Oil & VIVATEC 500 & $\begin{array}{l}\text { Hansen \& } \\
\text { Rosenthal }\end{array}$ & 0.0 & 7.6 & 15.1 & 18.8 & 22.6 & 30.1 & 37.6 \\
\hline \multirow{6}{*}{$\begin{array}{l}\text { Master } \\
\text { batch }\end{array}$} & Pre-blendings & - & - & 187.6 & $\rightarrow$ & $\rightarrow$ & $\rightarrow$ & $\rightarrow$ & $\rightarrow$ & $\rightarrow$ \\
\hline & HD Silica & $\begin{array}{l}\text { ULTRASIL }^{\circledR} 7005 \\
\left.\text { (CTAB: } 177 \mathrm{~m}^{2} / \mathrm{g}\right)\end{array}$ & Evonik & 50 & $\rightarrow$ & $\rightarrow$ & $\rightarrow$ & $\rightarrow$ & $\rightarrow$ & $\rightarrow$ \\
\hline & Silane (TESPT) & Si 69 & Evonik & 8.4 & $\rightarrow$ & $\rightarrow$ & $\rightarrow$ & $\rightarrow$ & $\rightarrow$ & $\rightarrow$ \\
\hline & Stearic acid & Stearic acid & Merck & 2 & $\rightarrow$ & $\rightarrow$ & $\rightarrow$ & $\rightarrow$ & $\rightarrow$ & $\rightarrow$ \\
\hline & Zinc Oxide & $\mathrm{ZnO}$ & Merck & 2 & $\rightarrow$ & $\rightarrow$ & $\rightarrow$ & $\rightarrow$ & $\rightarrow$ & $\rightarrow$ \\
\hline & DPG & Perkacit DPG & Flexsys & 1.5 & $\rightarrow$ & $\rightarrow$ & $\rightarrow$ & $\rightarrow$ & $\rightarrow$ & $\rightarrow$ \\
\hline \multirow{4}{*}{ Final } & Masterbatches & - & - & 251.5 & $\rightarrow$ & $\rightarrow$ & $\rightarrow$ & $\rightarrow$ & $\rightarrow$ & $\rightarrow$ \\
\hline & Sulfur & $S$ & J. T Baker & 0.7 & $\rightarrow$ & $\rightarrow$ & $\rightarrow$ & $\rightarrow$ & $\rightarrow$ & $\rightarrow$ \\
\hline & ZBEC & Vulkacit ZBEC & Lanxess & 0.2 & $\rightarrow$ & $\rightarrow$ & $\rightarrow$ & $\rightarrow$ & $\rightarrow$ & $\rightarrow$ \\
\hline & CBS & Santocure CBS & Flexsys & 2.2 & $\rightarrow$ & $\rightarrow$ & $\rightarrow$ & $\rightarrow$ & $\rightarrow$ & $\rightarrow$ \\
\hline
\end{tabular}

*27.3 wt\% TDAE oil extended 
The S-SBR used in this work contained $27.3 \mathrm{wt} \%$ of TDAE oil whereas the BR did not contain any oil. Therefore, except for SBR100, the amount of TDAE oil for all the blends was adjusted and introduced in the pre-blending step. $50 \mathrm{phr}$ of silica - without TESPT - was introduced at the same time with TDAE oil in order to avoid slippage of the compound in the mixing chamber as well as to provide a similar amount of mixing energy for all samples.

\subsubsection{Mixing}

As shown in Figure 5.1, the compounds were mixed in four steps: pre-blending, batch fusion, masterbatch and final steps. The pre-blending and masterbatch steps were done by using a lab scale internal mixer (Brabender Plasticorder) with $390 \mathrm{ml}$ of chamber volume, and the other steps took place on a lab scale two-roll mill (Polymix 80T). The details of each mixing step are shown in Tables 5.2 and 5.3. Before the masterbatch step, two batches of pre-blending having the same formulation were combined in order to minimize unintended variations from the pre-blending step.

The fill factor for the pre-blending and masterbatch steps were fixed to $75 \%$ and $65 \%$, respectively, limited by the maximum mechanical load capacity of the mixer. The temperature of the mixer temperature control unit (TCU) was set at $50^{\circ} \mathrm{C}$ for the pre-blending and masterbatch steps. In order to avoid the "first batch effect", one initial batch was mixed and discarded before the regular mixing started for both steps. In case of the masterbatch step, the rotor speed was adjusted from 04:10 ( $\mathrm{min}: \mathrm{sec}$ ) onwards in order to reach and subsequently keep $150^{\circ} \mathrm{C}$ steady chamber temperature during a period of 150 seconds for silanization. After the mixing steps done in the internal mixer, the compounds were sheeted out immediately on the two-roll mill in order to cool them down and prevent further silanization as well as filler-polymer coupling reaction, in particular the masterbatch compounds. Two batches were mixed for each set of conditions in order to check the reproducibility. All batches were mixed with good reproducibility as judged by the mixing finger prints: Figure 5.2. 


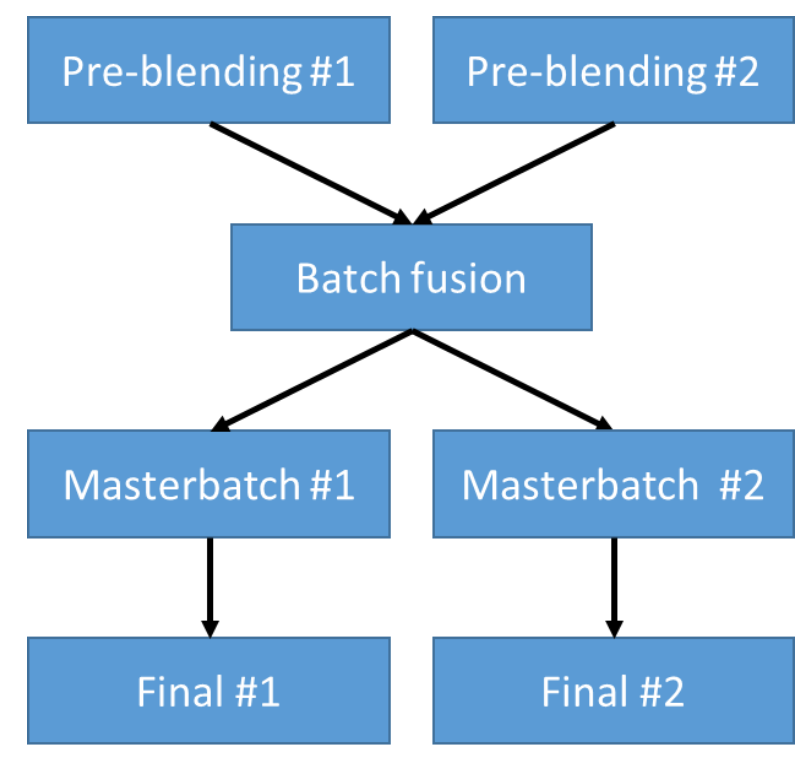

Figure 5.1 Mixing process diagram.

Table 5.2 Pre-blending and batch fusion mixing procedures

\begin{tabular}{|c|c|c|c|}
\hline \multicolumn{2}{|c|}{ Pre-blending } & \multicolumn{2}{|l|}{ Batch fusion } \\
\hline \multicolumn{2}{|c|}{ Internal mixer } & \multicolumn{2}{|l|}{ Open mill } \\
\hline Action & time [mm:ss] & Action & time [mm:ss] \\
\hline Add polymer & $00: 00 \sim 00: 20$ & $\begin{array}{l}\text { Add pre-blends } \\
\text { having the same formulation }\end{array}$ & $00: 00 \sim 01: 00$ \\
\hline Mastication & $00: 20 \sim 01: 20$ & Milling & $01: 00 \sim 06: 00$ \\
\hline Silica (50 phr), TDAE oil & $01: 20 \sim 01: 40$ & $\begin{array}{l}\text { Discharge and separation } \\
\text { (by weight) }\end{array}$ & - \\
\hline Mixing & $01: 40 \sim 02: 40$ & & \\
\hline Ram sweep & $02: 40 \sim 03: 10$ & & \\
\hline Mixing & 03:10 04:10 & & \\
\hline Discharge and sheeting & - & & \\
\hline
\end{tabular}


Table 5.3 Masterbatch and final mixing procedure

\begin{tabular}{|c|c|c|c|}
\hline \multicolumn{2}{|c|}{ Masterbatch } & \multicolumn{2}{|c|}{ Final } \\
\hline \multicolumn{2}{|c|}{ Internal mixer } & \multicolumn{2}{|c|}{ Open mill } \\
\hline Action & time $[\mathrm{mm}: \mathrm{ss}]$ & Action & time [mm:ss] \\
\hline Add fused batch & $00: 00 \sim 00: 20$ & Add masterbatch & - \\
\hline Mixing & $00: 20 \sim 01: 20$ & Mixing & $00: 00 \sim 02: 00$ \\
\hline $\begin{array}{l}\text { Silica (50 phr), silane, } \\
\text { remaining ingredients }\end{array}$ & 01:20 01:40 & Add curatives & $02: 00 \sim 02: 30$ \\
\hline Mixing & $01: 40 \sim 02: 40$ & Mixing & $02: 30 \sim 09: 00$ \\
\hline Ram sweep & $02: 40 \sim 03: 10$ & Discharge & - \\
\hline Mixing till $150^{\circ} \mathrm{C}$ & 03:10 04:10 & & \\
\hline Ram sweep & $04: 10 \sim 04: 14$ & & \\
\hline Mixing at $150^{\circ} \mathrm{C}$ & $04: 14 \sim 06: 40$ & & \\
\hline Discharge and sheeting & - & & - \\
\hline
\end{tabular}
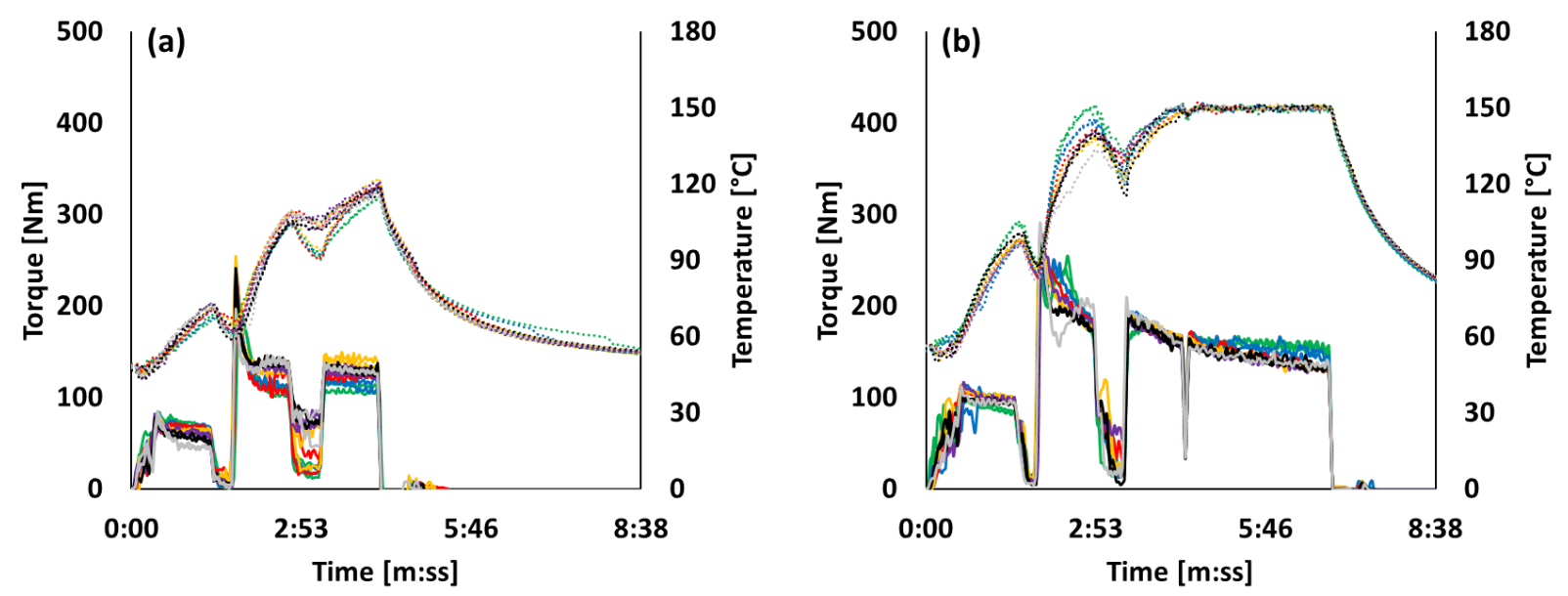

Figure 5.2 Mixing fingerprints; (a): pre-blending; (b): masterbatch; ( solid lines ): Torque;

(dotted lines ): temperature; (_ ) ): SBR100; (_ ): SBR80; (_ ) SBR60; ( ( ) ): SBR40; ( $)$ ): SBR20; ( $)$ ): SBR00.

The final mixing stage was done by using a lab scale two-roll mill (Polymix 80T). All ingredients to vulcanize the compounds, alternatively called curatives (sulfur and curing accelerators zinc 
dibenzyldithiocarbamate (ZBEC) and N-cyclohexyl-2-benzothiazolesulphenamide (CBS)), were added in this step.

\subsubsection{Property analysis and testing}

Payne effect, Filler Flocculation Rate (FFR), filler-polymer Coupling Rate (CR), cure characteristic as well as the Marching Modulus Intensity (MMI), and bound rubber content of the compounds were investigated according to the methods as described in Chapter 3.1.

The samples were vulcanized at two different cure times for solvent swelling and Mooney-Rivlin plot in order to be able to compare the overall crosslink density depending on the choice of the curing time of the compounds: calculated cure time (see Figure 3.6) and a fixed time of 30 minutes.

\subsubsection{Results and discussion}

\subsubsection{Filler-filler interaction (Payne effect) as a function of SBR content}

The Payne effect values of the uncured compounds of the pre-blend and masterbatch are plotted as a function of SBR content in Figure 5.3.
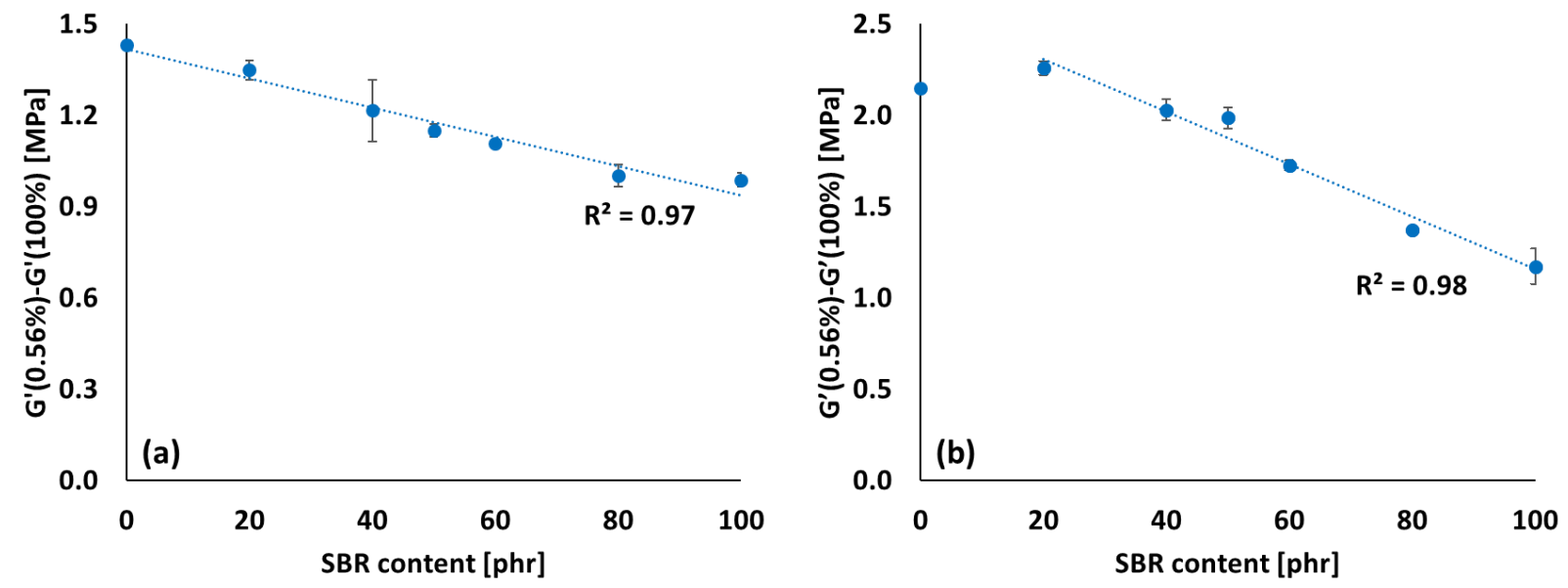

Figure 5.3 Payne effect as a function of SBR content; (a): Pre-blending; (b): masterbatch; fitting lines are based on linear fit with maximum $R^{2}$.

Pre-blended compounds - containing 50 phr of silica - show a decreasing Payne effect value with increasing SBR content, even though there was no silane present at all: Figure 5.3(a). This trend indicates that the affinity or surface wetting behavior of SBR toward silica is better than BR, as also reported by 
Le et al. ${ }^{[7,9,10]}$ and Wang and Wolff ${ }^{[8]}$. Masterbatch compounds - containing $100 \mathrm{phr}$ of silica and $8.4 \mathrm{phr}$ of silane - also show a lower Payne effect value with increasing SBR content except for SBRO0, which contains only BR. This trend can be explained with the affinity or surface wetting behavior of SBR; however, it is better interpreted with bound rubber content which will be discussed lateron.

\subsubsection{Bound rubber content as a function of SBR content}

Figure 5.4 shows the amount of bound rubber of masterbatch compounds as a function of SBR content. Except for SBROO ( 0 phr of SBR, full BR), a higher amount of total and chemically bound rubber is observed when the SBR content increases.

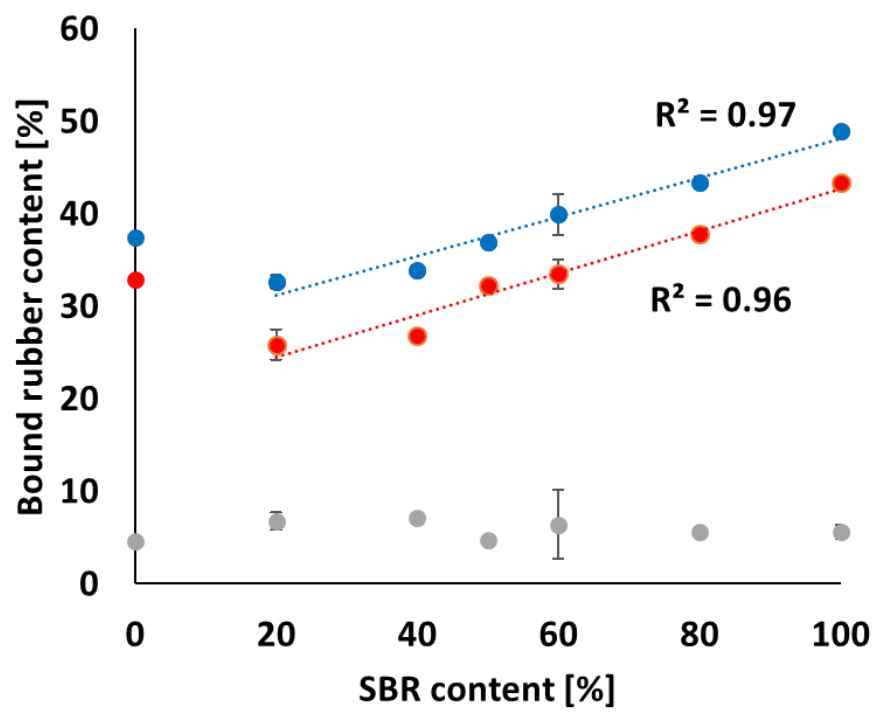

Figure 5.4 Bound rubber content as a function of $\operatorname{SBR}$ content; $(\bullet)$ : total bound rubber; $(\bullet)$ : chemically bound rubber; $(\bullet)$ : physically bound rubber; fitting lines are based on linear fit with maximum $\mathrm{R}^{2}$.

This result can be explained with the high reactivity of vinyl groups in S-SBR and thiol radicals generated from the silane (TESPT). Sato ${ }^{[17]}$ compared the reactivity between sulfidic silane and model olefins representing the vinyl group in SBR and cis as well as trans double bonds in SBR and BR - and found that the vinyl group is the most reactive moiety. Additionally, he analyzed the chemical structure of the reaction product by using ${ }^{1} \mathrm{H}$ - and ${ }^{13} \mathrm{C}-\mathrm{NMR}$ and found that the reaction between sulfidic silane and the vinyl group follows a radical and not an ionic pathway: Figure 5.5. The reaction between a double bond (ene) and a thiol radical - thiol-ene click reaction - is well known for its rapid rate and high efficiency of the reaction. ${ }^{[18-21]}$ An additional factor may be the higher Mooney viscosity (ML1+4@100 $\mathrm{C}$ ) of 62 for S- 
SBR versus 44 for BR, which results in a gradual increase of the viscosity of the blends with increasing SSBR content. This leads to higher shear forces during mixing, which helps to generate more fresh surface of silica (more reaction sites), and may result in a higher bound rubber level.

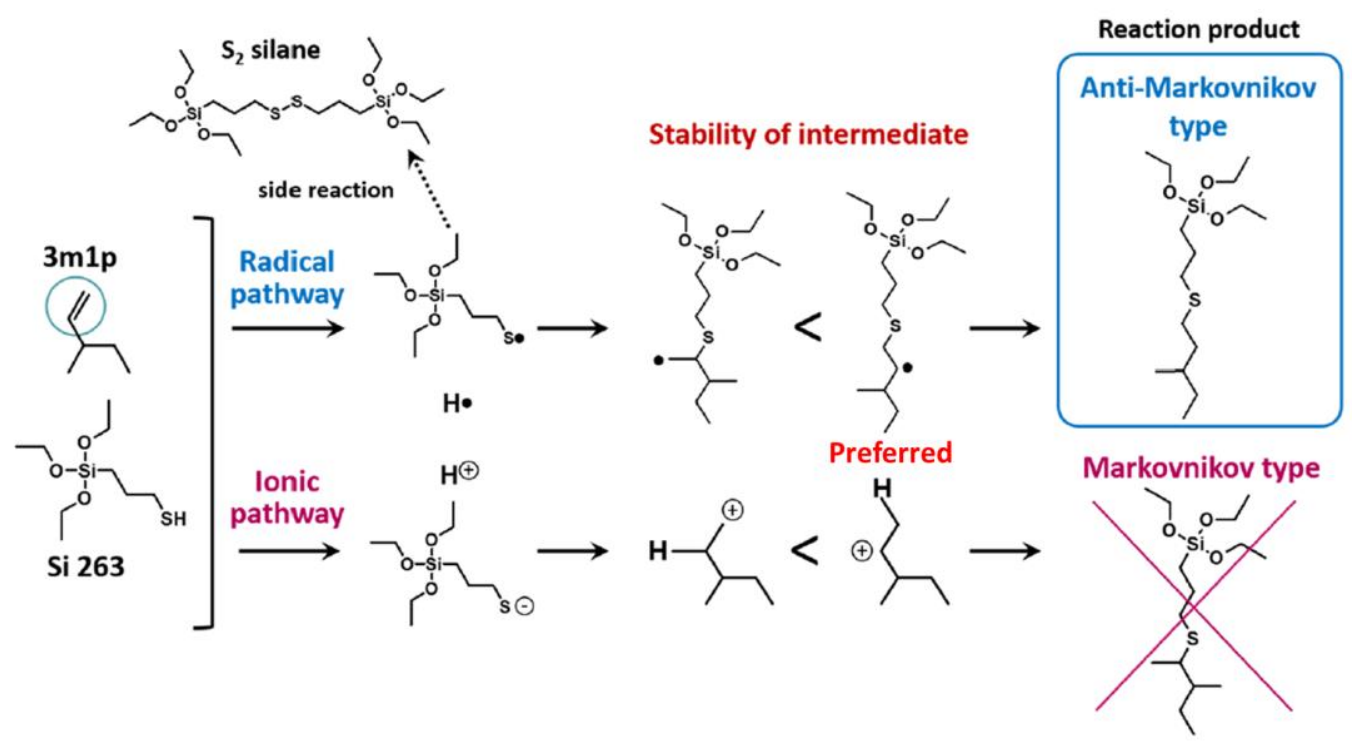

Figure 5.5 Possible mechanism of the reaction between the model olefin representing vinyl groups of SBR (3-methyl-1-pentene, 3m1p) and a sulfidic silane ((3-mercaptopropyl)triethoxysilane, Si $\left.263^{\mathrm{TM}}\right)^{{ }^{[17]}}$

As was seen in Figures 5.3(b) and 5.4, the Payne effect and bound rubber content values of SBROO (0 phr of SBR, full BR) did not follow the trend. However, all Payne effect values of the masterbatch compounds and their chemically bound rubber contents show a fair correlation, as shown in Figure 5.6: an coefficient of determination $\left(R^{2}\right)$ higher than 0.9 . This result indicates that the amount of chemically bound rubber is more effective in reducing filler-filler interaction than the physical affinity of the polymer towards the silica filler. 


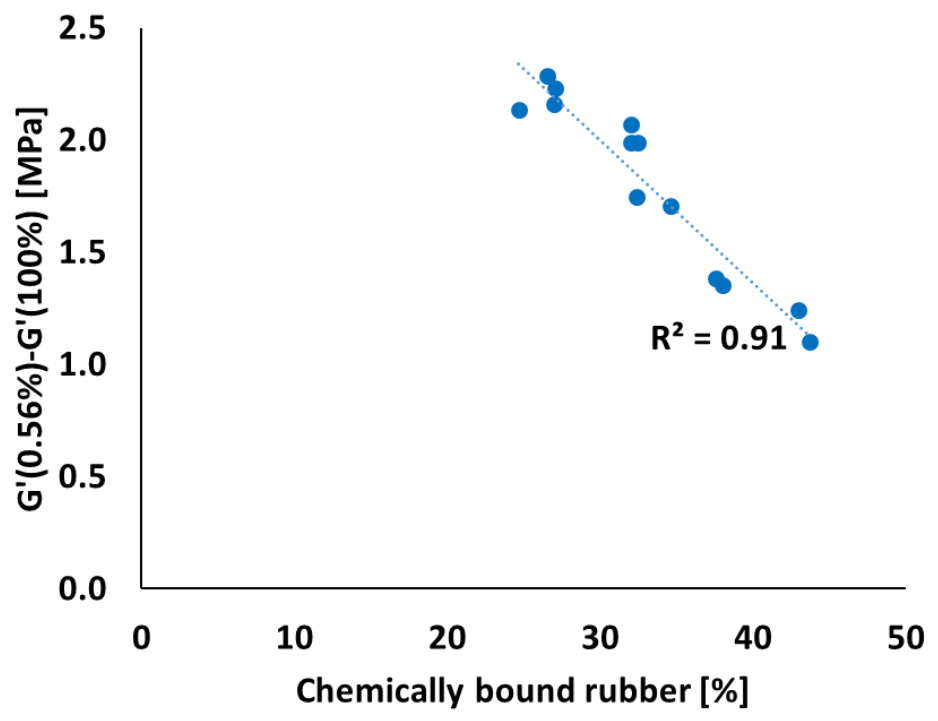

Figure 5.6 Payne effect vs. chemically bound rubber content;

fitting lines are based on linear fit with maximum $R^{2}$.

\subsubsection{Silica filler flocculation rate}

The silica Filler Flocculation Rates (FFR) are shown in Figure 5.7. The FFR values first increase along with the SBR content up to SBR50 (50 phr of SBR content), and then decrease.

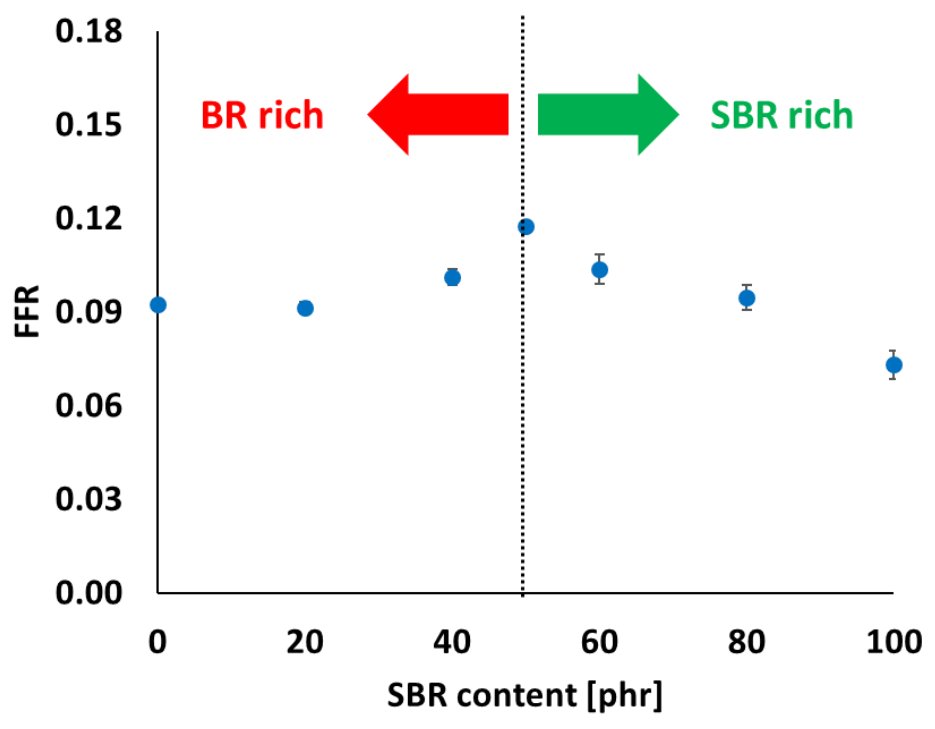

Figure 5.7 FFR vs. SBR content.

These results can be explained by a filler re-localization process. ${ }^{[7,22-25]}$ Reinforcing fillers such as silica can 
be localized in one blend phase or at the interphase between the two components. ${ }^{[22,23]}$ Le et al. ${ }^{[7]}$ reported that filler transfer occurs from a less preferred blend phase into which silica was introduced by the mixing process to the more favorable one. A model of filler localization for silica filled compounds with $100 \%$ SBR, SBR rich, BR rich and 100\% BR based on the results of this work is suggested in Figure 5.8 .

(b)

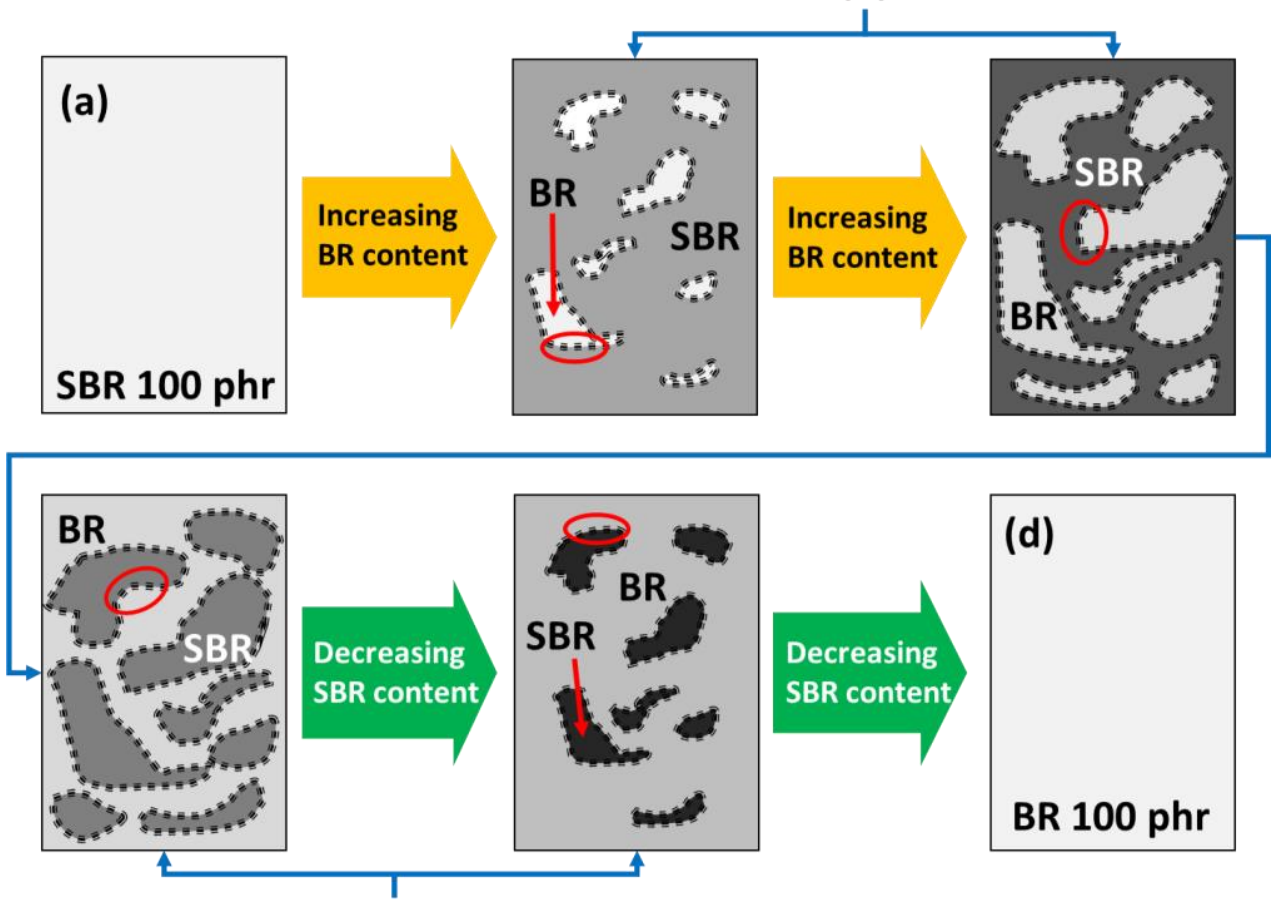

(c)

Figure 5.8 Filler localization model; (a): SBR 100\%; (b): SBR rich; (c): BR rich; (d): BR 100\%; $(\bigcirc$ ): filler localized at the interphase of SBR and BR; the population of silica is expressed by the color; darker color represents higher population of silica.

In the extreme cases (with 100 phr SBR or BR, Figures 5.8(a), (d)), silica has no alternative than the matrix of the respective polymer, therefore the silica clusters will be first distributed more homogeneously during mixing compared to a matrix with SBR or BR rich regions. When these compounds undergo the FFR measurements, the clusters will start to flocculate without any other effects induced by the immiscibility ${ }^{[1,4-6]}$ of SBR and BR. Due to the fact that the physical affinity of silica towards BR is lower compared to SBR, ${ }^{[8]}$ silica would flocculate slightly faster for the compound with $100 \%$ of BR: Figure 5.7 . In the case of the SBR rich region (Figure 5.8(b)), the silica localized in the BR phase or at the interphase of BR and SBR tends to migrate to the SBR phase, the preferred one for silica in this system. Increasing the BR content enhances the migration of silica to SBR, as a higher percentage of the total amount of silica is 
present in the BR phase or at the interphase of BR and SBR due to the higher amount of BR compared to SBR. Additionally, a smaller SBR volume (dark area in Figure 5.8(b)) induced by increasing BR content would result in a shorter distance between silica clusters in this phase. As a consequence, silica flocculation will be enhanced.

The same phenomenon will take place for the BR rich region as well (Figure 5.8(c)). In the BR rich region, the migration of the silica from the BR phase or the BR/SBR interphase into the SBR phase is blocked, when the silica incorporation capacity of the SBR phase reaches its limit. The absolute maximum silica incorporation capacity of the SBR phase decreases as the SBR content in the BR rich region becomes lower. As a consequence, the flocculation of silica mainly occurs in the BR phase or at the SBR/BR interphase. FFR values of the compounds are plotted against total and chemically bound rubber content in Figure 5.9. In Chapters 3.1 and 4.1, it turned out that the amount of total and chemically bound rubber are the key factors for FFR reduction. However, these bound rubber contents are not effective in the BR rich compounds. This result indicates that not only the amount of bound rubber, but also the chemical characteristics of rubber and silica - affinity or wettability - influence the silica flocculation behavior.
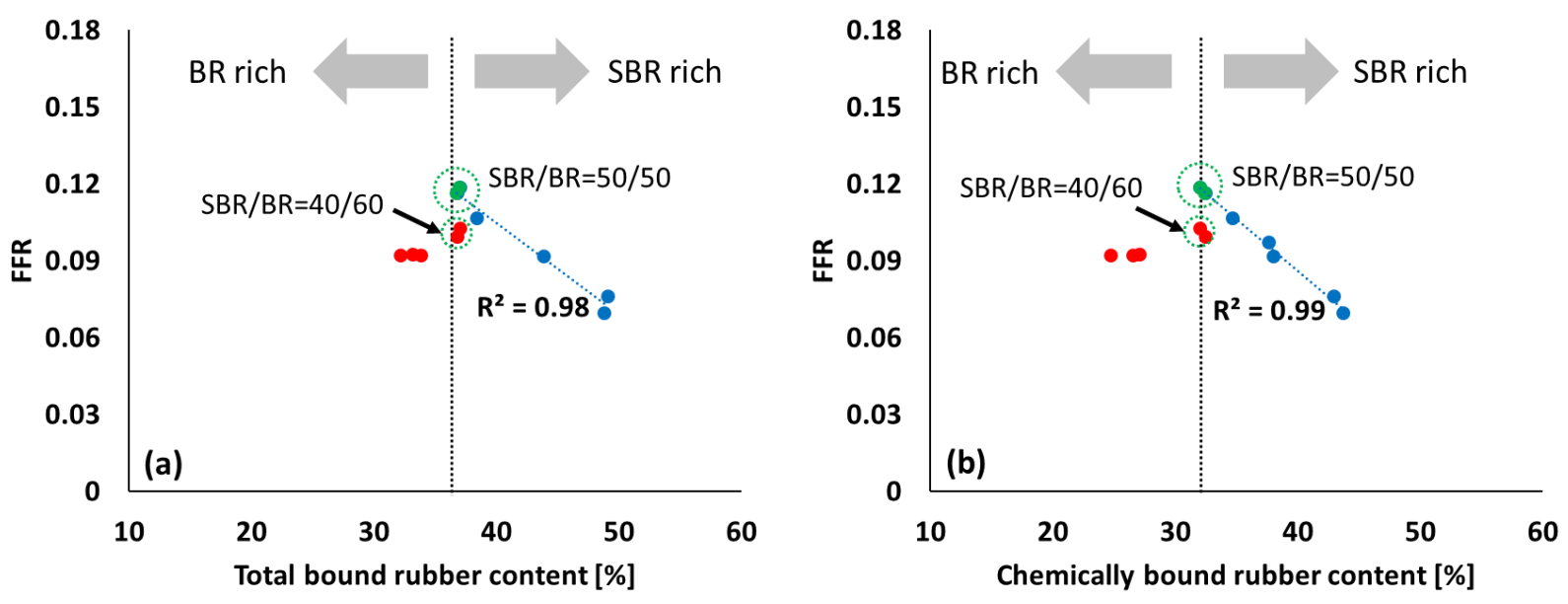

Figure 5.9 FFR versus bound rubber content; (a): FFR vs. total bound rubber;

(b): FFR vs. chemically bound rubber; $(\bullet)$ : SBR rich; $(\bullet)$ : BR rich; $(\bullet)$ : SBR/BR=50/50;

fitting lines are based on linear fit with maximum $R^{2}$.

\subsubsection{Filler-polymer coupling rate after mixing}

The filler-polymer Coupling Rates (CR) are shown in Figure 5.10. The CR of the compounds plotted as a function of SBR content show an increasing trend with SBR content. As already stated in Paragraph 5.1.3.2, 
increasing the amount of SBR in the compound formulation leads to a higher concentration of vinyl groups and thus to an enhancement of the thiol-ene click reaction.

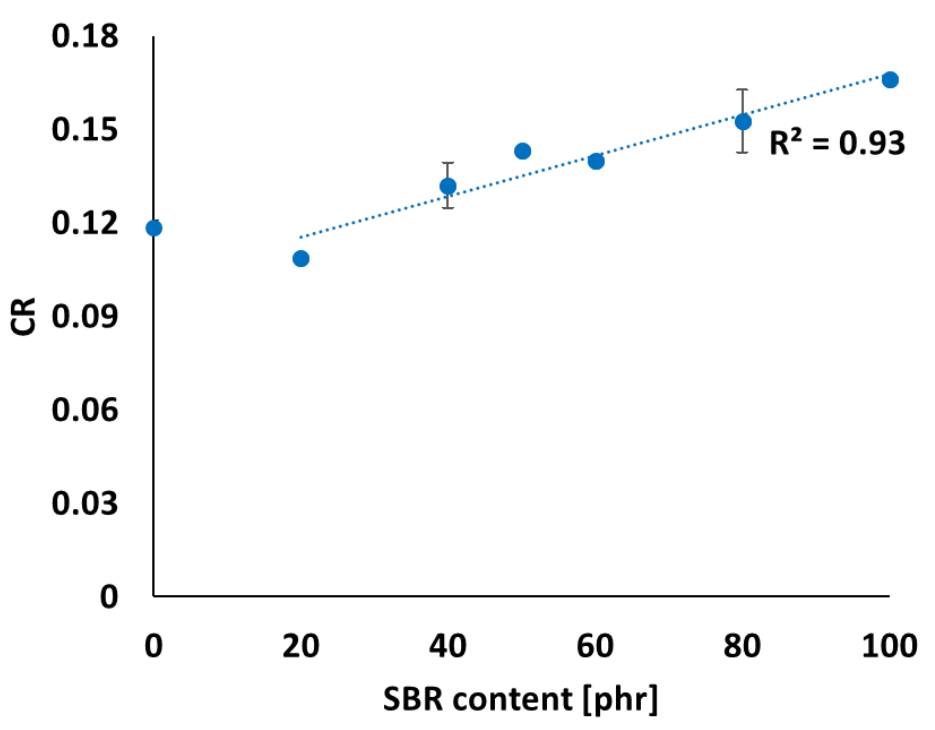

Figure 5.10 CR as a function of SBR content; fitting lines are based on linear fit with maximum $\mathrm{R}^{2}$.

In Chapters 3.1 and 4.1, CR usually showed a negative correlation with the amount of chemically bound rubber: for low vinyl content in the compound. However, in the present study, higher CR values were obtained for the compounds having a high percentage of total and chemically bound rubber: positive correlation, Figure 5.11. This result is another indication for the thiol-ene click reaction between the vinyl groups of SBR and the sulfur radicals generated from TESPT: when more vinyl groups are present, enhanced filler-polymer coupling via the sulfidic silane is occurring. 

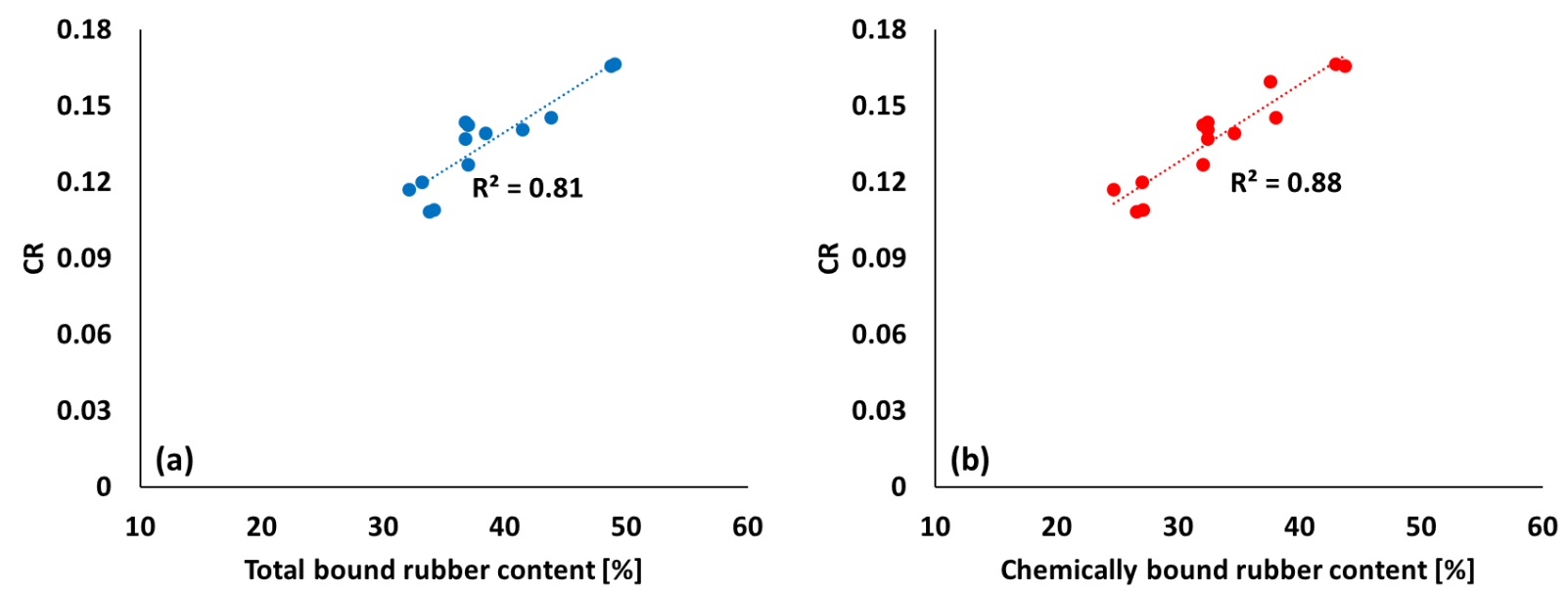

Figure 5.11 CR as a function of total and chemically bound rubber; (a): CR vs. total bound rubber;

(b): CR vs. chemically bound rubber; fitting lines are based on linear fit with maximum $\mathrm{R}^{2}$.

\subsubsection{Marching modulus intensity}

The rheograms of the compounds and their Marching Modulus Intensities (MMI) measured at two different strains are plotted in Figures 5.12 and 5.13, respectively.
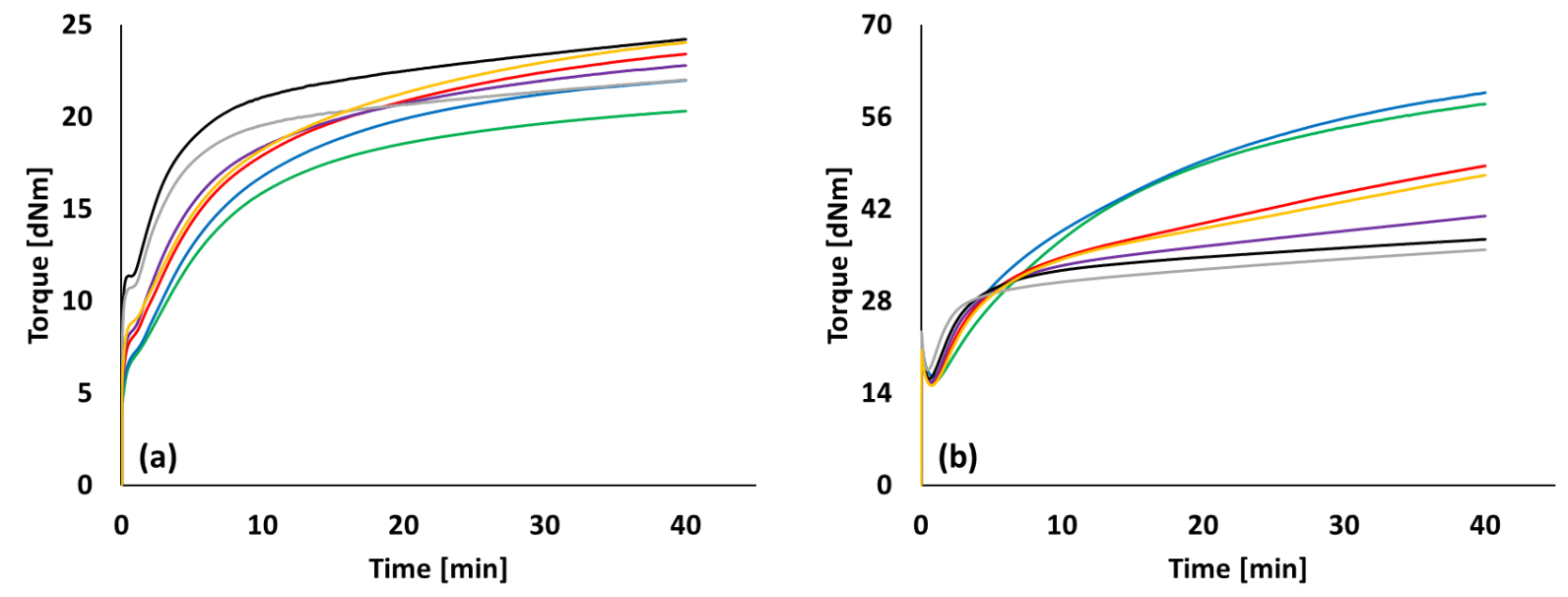

Figure 5.12 Rheograms of the compounds; (a): $0.5^{\circ}$ ( 7\% of strain); (b): $3^{\circ}$ ( $42 \%$ of strain);

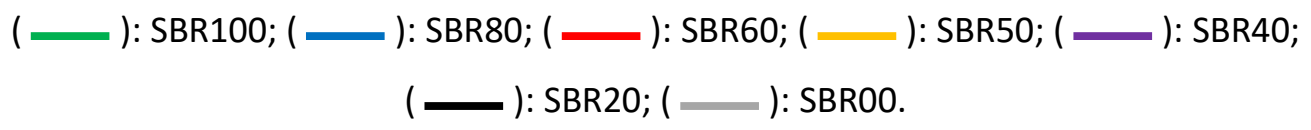



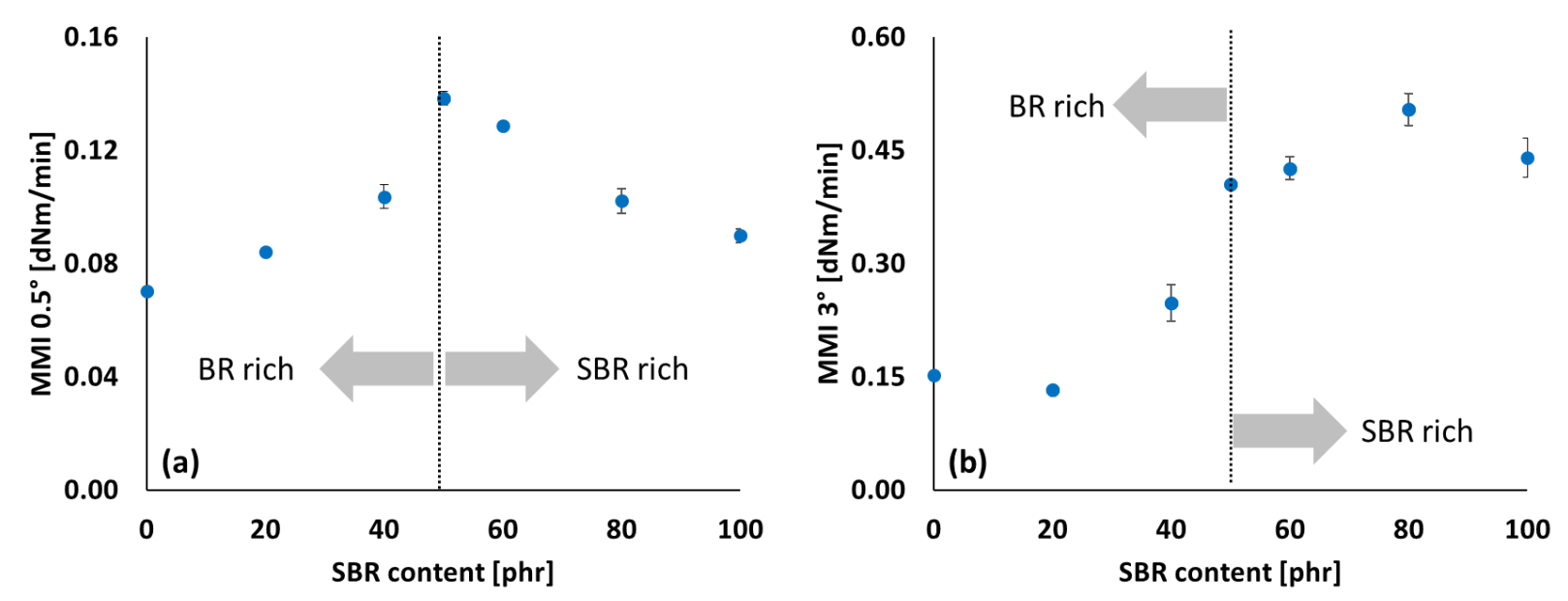

Figure 5.13 MMI vs. SBR content; (a): $\mathrm{MMI} 0.5^{\circ}$; (b): $\mathrm{MMI} 3^{\circ}$.

\subsection{Marching modulus intensity at small strain (ASTM conditions, MMI $0.5^{\circ}$ ) as a function of FFR}

As can be seen in Figure 5.13(a), the MMI $0.5^{\circ}$ values corresponding to the SBR contents show a similar trend as the FFR trend. Taken from Figures 5.7 and 5.13(a), the MMI 0.5 values of the BR rich and SBR rich regions are plotted as functions of FFR and depicted in Figure 5.14. Both polymer regions show a positive correlation, the same as the results of Chapters 3.1 and 4.1. These results indicate that the silica filler flocculation behavior in the rubber matrix is still the strongly influencing factor for $\mathrm{MMI} 0.5^{\circ}$. However, the driving force of silica flocculation in this case should be distinguished from that of the compounds in Chapters 3.1 and 4.1: the amount of chemically bound rubber. The reason is that in Chapter 3.1 and 4.1, the compound formulation was fixed and the only variable was the mixing condition. In the present case, FFR is affected not only by the amount of chemically bound rubber but also by the relocalization or migration of silica between the rubber phases induced by the different polymer blending ratios. 

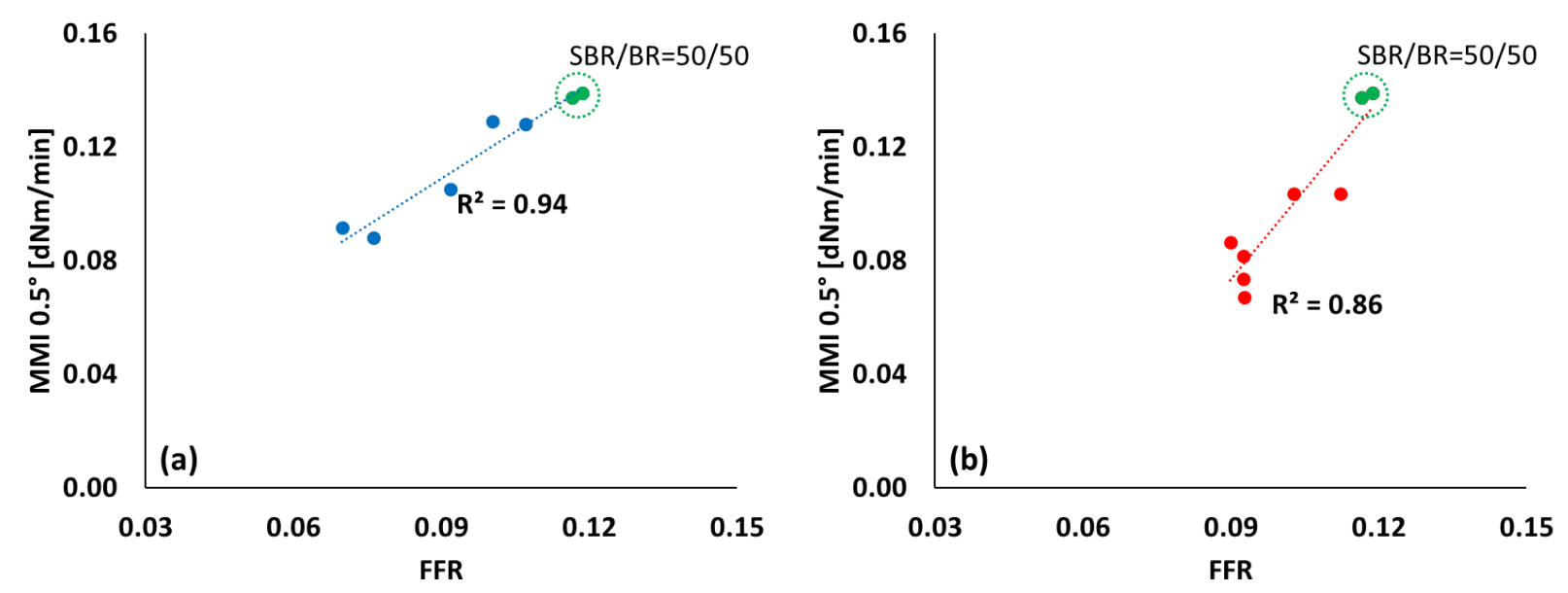

Figure 5.14 $\mathrm{MMI} 0.5^{\circ}$ as a function of FFR; (a): SBR rich; (b): BR rich; $(\bullet$ ): SBR/BR=50/50;

fitting lines are based on linear fit with maximum $\mathrm{R}^{2}$.

Different from the previous results in Chapters 3.1 (Figure 3.19) and 4.1 (Figure 4.14(a)), the CR does not affect the MMI $0.5^{\circ}$ : Figure 5.15. In order to explain this phenomenon, the mechanism of the reaction of filler-polymer coupling via the silane coupling agent and sulfur curing as well as the formulations of the masterbatch and final compounds need to be taken into account.

$\mathrm{CR}$ was measured on the masterbatch, which means that there were no accelerators present such as $\mathrm{N}$ cyclohexylbenzothiazole-2-sulfenamide (CBS) and zinc dibenzyldithiocarbamate (ZBEC). In the case of the masterbatch compound, therefore, higher values of CR were obtained with increasing the SBR content due to the increasing vinyl groups concentration: Figure 5.10.

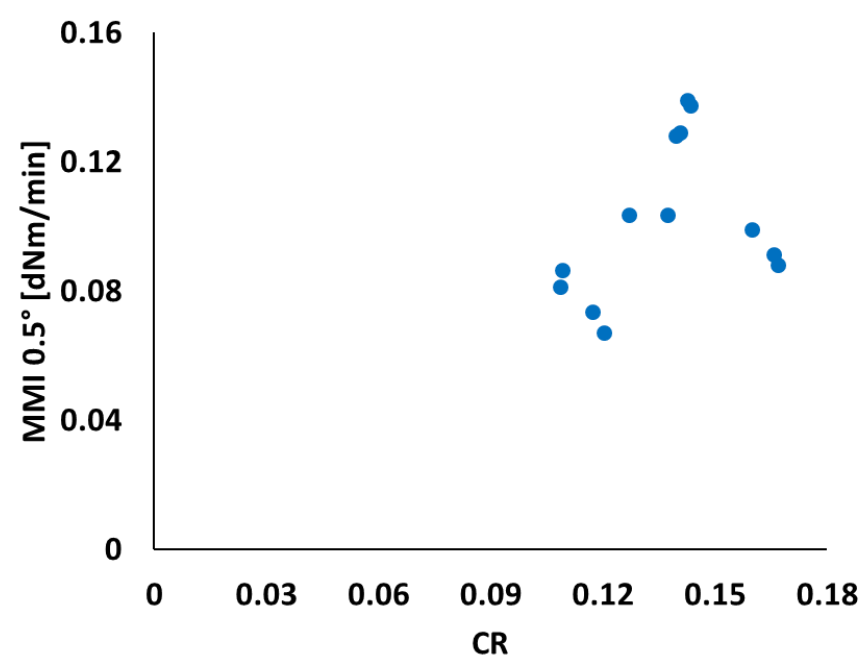

Figure 5.15 $\mathrm{MMI} 0.5^{\circ}$ vs. CR. 
However, the final compounds do contain accelerators (CBS and ZBEC). Sato ${ }^{[17]}$ compared the reaction between a model olefin - representing the vinyl groups of SBR - and a sulfidic silane with and without CBS. He found that the model olefin and the sulfidic silane do not react in the presence of CBS: the silane tends to incorporate more sulfur into its structure rather than reacting with the model olefin. Sato's results indicate that filler-polymer coupling by vinyl groups of the SBR will not occur to a large extent during curing of the final compound containing CBS.

Klockmann et al. ${ }^{[26]}$ stated that, once the silane is coupled to the silica, the silane can be stabilized and react further with the polymer after forming a silica-silane-CBS intermediate: Figure 5.16.
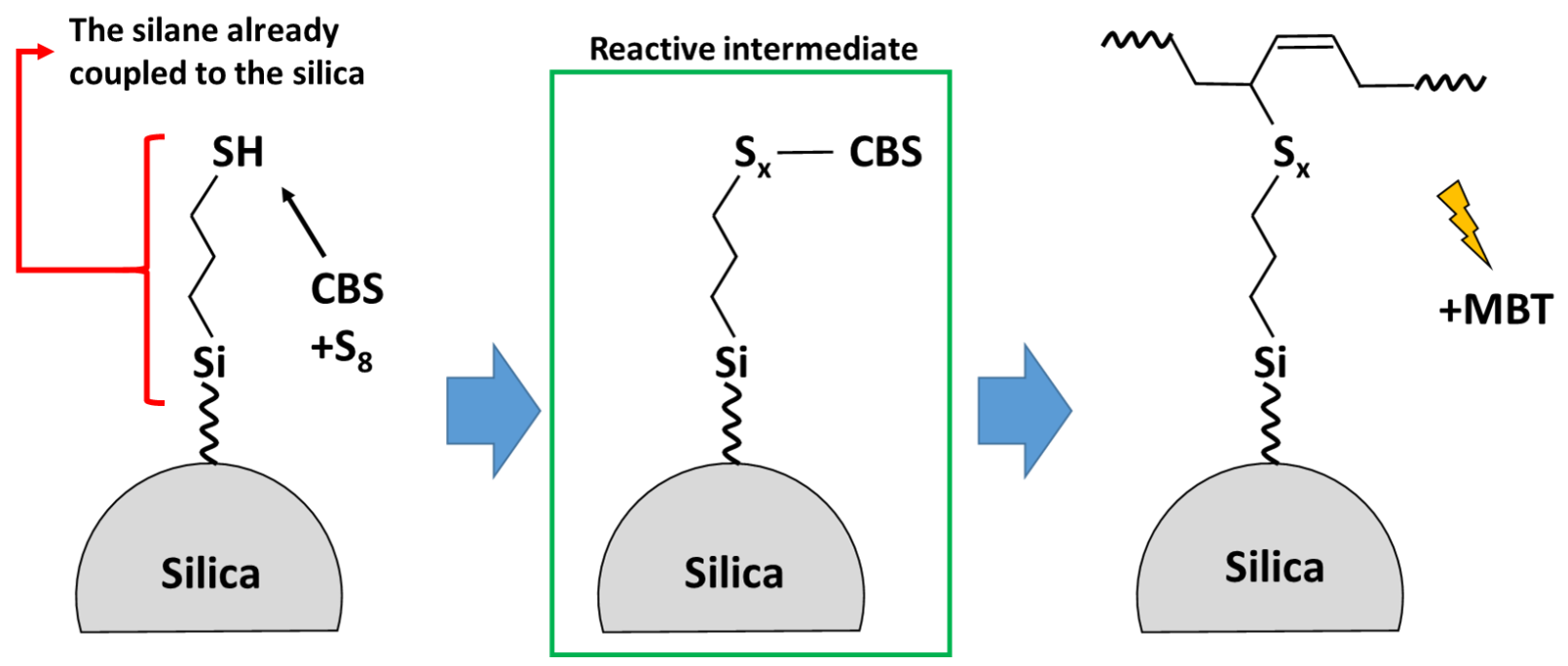

Figure 5.16 Immobilization mechanism of a mercapto-silane reacting with CBS preventing dimerization of the silane. ${ }^{[17,26]}$

Overall, these results indicate that the filler-polymer coupling via a silane during vulcanization of the final compound can differ from the reaction taking place during masterbatch mixing, as the latter contains the accelerators, which can interfere with the silane-polymer reaction. The vinyl group can react with the silane via an intermediate reaction with CBS; however, the reaction rate would be slower than the rate of the thiol-ene click reaction without CBS. Therefore the CR obtained from the masterbatch compound hardly affects the $\mathrm{MMI}$ in the latter case: Figure 5.15. 


\subsection{Discussion on the rheogram and marching modulus intensity at large strain $\left(\mathrm{MMI}^{\circ}\right)$}

As was seen in Figure 3.17, only filler-polymer and polymer-polymer interactions are active at a strain of 42\%: most of the filler-filler network will be broken when the filled rubber compound is subjected to such large deformations. Therefore, for the $\mathrm{MMI} 3^{\circ}$, a different approach than for the $\mathrm{MMI} 0,5^{\circ}$ (Paragraph 5.1.3.5.1) was required in order to interpret the results of the rheograms and marching modulus intensity. In the rheograms of the compounds measured at large strain (Figure 5.12(b)), an interesting point can be seen in the beginning of the vulcametry: a steeper slope at the initial part of the rheogram (maximum cure rate) is found when the SBR content decreases or the BR content increases. The maximum cure rate for the compounds and the time when the compounds show the maximum cure rate were calculated by differentiating the rheograms (Figure 5.12(b)) and are shown in Figure 5.17. A higher maximum cure rate is observed when the amount of BR increases. Additionally, the times at which the maximum cure rate is reached are longer when the amount of SBR increases. Dogadkin et al. ${ }^{[27]}$ compared the reactivity between sulfur and double bonds of the 1,4- and 1,2-configurations (vinyl) of BR and found that the reactivity of the 1,4-double bond is higher than the reactivity of the 1,2-double bond: Figure 5.18. Similarly, Marzocca et al. ${ }^{[1]}$ obtained a higher crosslink density when the amount of cis BR increases in the blend. 

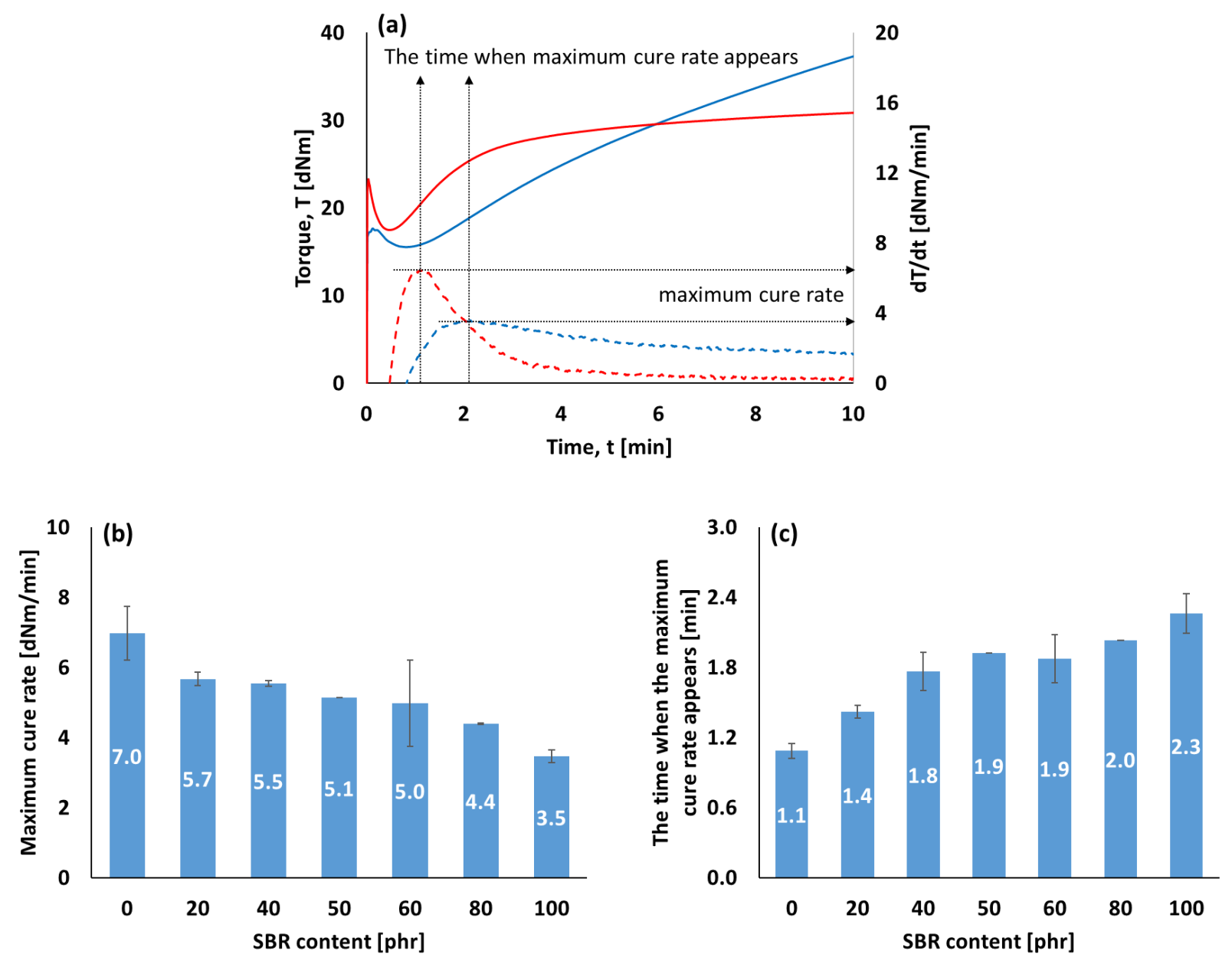

Figure 5.17 Calculated maximum cure rate and time to reach the maximum cure rate based on Figure 5.12(b); (a): example rheograms for SBR100 and SBR00 at large strain and their differential curves; (solid lines): Torque; (dotted lines): dT/dt; (_ ) SBR100; (_ ) SBR00; (b): maximum cure rate; (c): time to reach the maximum cure rate. 


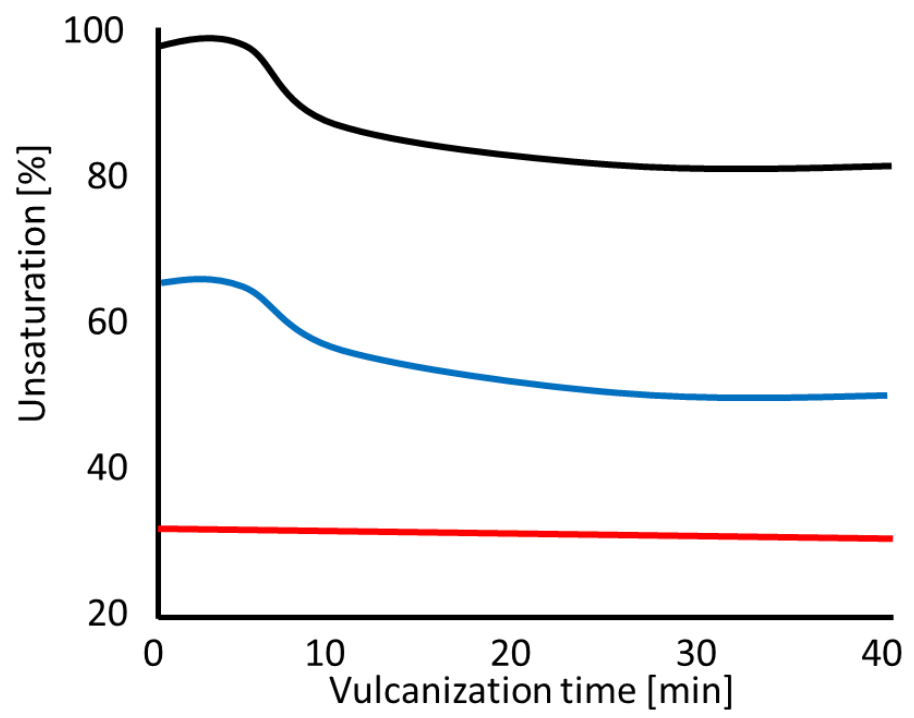

Figure 5.18 Variation of the unsaturation of rubber with $65 \%$ of 1,4 structural units during vulcanization in film form in a nitrogen atmosphere at $142^{\circ} \mathrm{C}$; ( $)$ ): total unsaturation; ( 1,4-structure; ( 1 ): unsaturation of 1,2-structure. ${ }^{[27]}$

In order to confirm this interpretation, the crosslink density of the vulcanizates with two different curing times - calculated curing time (listed in Table 5.4) and 30 minutes - were compared by measuring swelling ratios: Figure 5.19. As can be seen in this figure, the swelling ratios of the vulcanizates cured for the calculated time increase along with SBR content. As higher swelling ratio values correspond to lower crosslink densities, the SBR rich compounds have a lower crosslink density than the BR rich ones. Even though the SBROO compound (100 phr of BR) was cured for the shortest curing time (about half the time of the SBR100 compound), it shows the highest crosslink density.

Table 5.4 Calculated curing times

\begin{tabular}{cc}
\hline SBR content [phr] & Calculated curing time [min] \\
\hline 100 & 11.7 \\
80 & 11.6 \\
60 & 9.8 \\
50 & 9.2 \\
40 & 8.2 \\
20 & 6.5 \\
00 & 6.2 \\
\hline
\end{tabular}




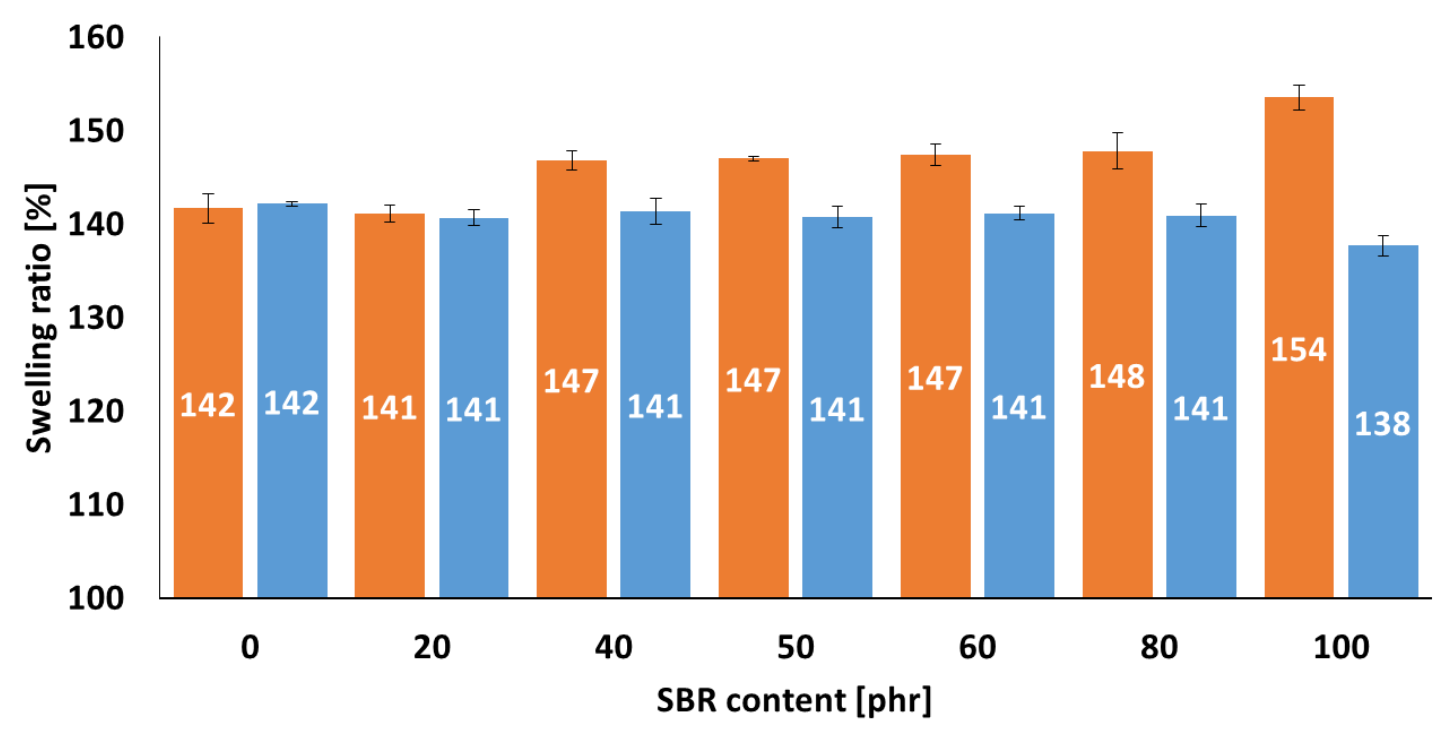

Figure 5.19 Swelling ratios of the vulcanizates with; $(\square)$ : calculated curing time; $(\square)$ : 30 minutes.

Finally the crosslink densities of all compounds become similar after 30 minutes of curing. Additionally, another interesting point can be seen in Figure 5.12(b): the maximum torque - taken as the torque at 40 minutes $\left(T_{40}\right)$ - becomes higher when the SBR content increases. This result indicates that more fillerpolymer coupling takes place in the SBR rich compounds on a longer time range. Limited polymer chain extensibility as evaluated by the Mooney-Rivlin equation, gives another evidence for more filler-polymer coupling of the compounds containing large amount of SBR. The inverse elongation ratio $\left(\lambda^{-1}\right)$ value at the upturn point can be used as an indicator for the intensity of filler-polymer interaction as well as the crosslink density, as stated in Chapter 3.1. As can be seen in Figure 5.20, $\lambda^{-1}$ at the upturn point increases along with the SBR content. In particular SBR100 shows a large difference in $\lambda^{-1}$ at the upturn point between the two curing times, which means that more filler-polymer coupling occurred during vulcanization. 


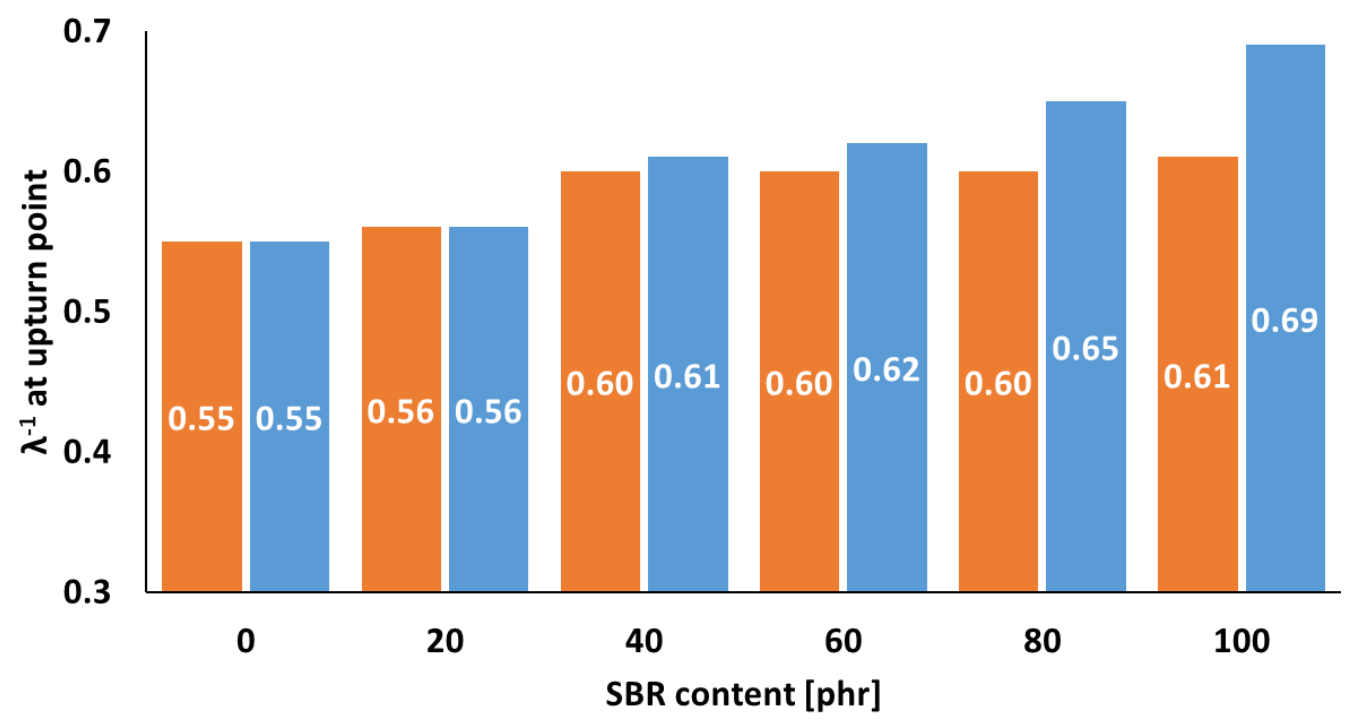

Figure $5.20 \lambda^{-1}$ at the upturn point of the vulcanizates with;

$(\square)$ : calculated curing time; ( $\square$ ): 30 minutes.

Taking into account Figures 5.10, 5.12(b), 5.13(b), 5.17, 5.19 and 5.20, the following can be concluded:

- The 1,4-structure double bond in SBR and BR is more reactive for sulfur curing than the 1,2-vinyl configuration. As a consequence, the compounds with a large amount of BR (having $96 \%$ of 1,4cis content) show a higher increment of torque in the beginning of the rheogram than SBR rich compounds;

- The presence of a curing accelerator such as CBS, suppresses the reaction between the sulfidic silane and the 1,2-structure double bond of SBR. However, the reaction still can happen on a long time range as indicated by a higher $\mathrm{MMI} 3^{\circ}$;

- The slow filler-polymer coupling reaction during the vulcanization process of SBR rich compounds results, however, in more crosslinks between filler and polymer: a higher torque level at 40 minutes of the rheogram measurement.

\subsubsection{Conclusions}

In this study, the effect of the polymer (SBR/BR) blend ratio on the marching modulus intensity of a silica filled compound was investigated. The polymer blend ratio turned out to be a strong influencing factor for the marching modulus as well as for the other curing characteristics. 
The MMI $0.5^{\circ}$ of the compounds having different SBR/BR blend ratios is mainly affected by the silica flocculation rate. Different from the results of Chapters 3.1 and 4.1, the FFR in the BR rich region is not affected by the amount of bound rubber, but influenced by the chemical characteristics of the rubber and silica. The silica tends to migrate to and be localized in the more preferred rubber phase (SBR). However, when the incorporation capacity of the favorable elastomer phase reaches its maximum, silica migration and, as a consequence, flocculation slows down.

The filler-polymer coupling reaction is strongly affected by the SBR content and the presence of an accelerator such as CBS. For the masterbatch compounds, increasing percentages of SBR lead to higher CR values due to a thiol-ene click reaction. But as soon as CBS is added to the compound in the final mixing step, the reaction rate slows down. Therefore, $\mathrm{MMI} 0.5^{\circ}$ of the compounds were not affected by the CR. The reactivity of the 1,4- and the 1,2-structure double bonds towards formation of sulfur bonds between the filler and polymer influences the rheogram and MMI at large strain. The 1,4-double bonds in SBR and $B R$ are more reactive than 1,2-double bonds in sulfur curing. Due to the reactivity of these types of double bonds, faster vulcanization is observed for BR rich compounds. However, the slow filler-polymer coupling reaction during the vulcanization process for the SBR rich compounds results in more crosslinks as the cure time increases: decrease in the swelling ratio and increase in $\lambda^{-1}$ at the Mooney-Rivlin upturn point of the vulcanizates along with cure time. As a consequence, a higher torque level after $\mathbf{4 0}$ minutes curing as well as $\mathrm{MMI} 3^{\circ}$ were obtained.

\section{References}

[1] A. J. Marzocca, S. Cerveny, J. M. Méndez, Polym. Int. 49, 216 (2000).

[2] Ch. H. Kang, M. S. Kim (Tread rubber composition and tire manufactured by using the same), Kor. Pat. KR 10-2013-0071620.

[3] A. Rathi, PDEng thesis: Process-Design for Tire Tread Compounds with Good Compatibility to Mineral-based Safe Process Oils, Univ. Twente, Enschede, the Netherlands (2016).

[4] J. M. Massie, R. C. Hirst, A. F. Halasa, Rubber Chem. Technol. 66, 276 (1993).

[5] J. E. Callan, W. M. Hess, C. E. Scott, Rubber Chem. Technol. 44, 814 (1971).

[6] T. Inoue, F. Shomura, T. Ougizawa, K. Miyasaka, Rubber Chem. Technol. 58, 873 (1985).

[7] H. H. Le, M. Keller, M. Hristov, S. Ilisch, T. H. Xuan, Q. K. Do, T. Pham, K-W. Stöckelhuber, G. Heinrich, H. -J. Radusch, Macromol. Mater. Eng., 298, 1085 (2013).

[8] M. J. Wang, S. Wolff, J. B. Donnet, Rubber Chem. Technol. 64, 714 (1991).

[9] H. H. Le, S. Ilisch, D. Heidenreich, K. Osswald, H-J. Radusch, Rubber Chem. Technol. 84, 41 (2011). 
[10] H. H Le, S. Ilisch, D. Heidenreich, A. Wutzler, H-J. Radusch, Polym. Compos. 31, 1701 (2010).

[11] K. W. Stöckelhuber, A. S. Svistkov, A. G. Pelevin, G. Heinrich, Macromolecules 44, 4366 (2011).

[12] L. Tadiello, M. D’Arienzo, B. Di Credico, T. Hanel, L. Matejka, M. Mauri, F. Morazzoni, R. Simonutti, M. Spirkovac, R. Scotti, Soft Matter 11, 4022 (2015).

[13] J. Fröhlich, W. Niedermeier, H. D. Luginsland, Composites Part A 36, 449 (2005).

[14] J. L. Leblanc, Prog. Polym. Sci. 27, 627 (2002).

[15] R. Scotti, L. Wahba, M. Crippa, M. D’Arienzo, R. Donetti, N. Santo, F. Morazzoni, Soft Matter 8, 2131 (2012).

[16] L. Wahba, M. D’Arienzo, R. Donetti, T. Hanel, R. Scotti, L. Tadiello, F. Morazzoni, RSC Adv. 3, 5832 (2013).

[17] M. Sato, PhD Thesis: Reinforcing Mechanisms of Silica / Sulfide-Silane vs. Mercapto-Silane Filled Tire Tread Compounds, Univ. Twente, Enschede, the Netherlands (2018).

[18] T. Posner, Ber. Dtsch. Chem. Ges. 38, 646 (1905).

[19] M. S. Kharasch, A. T. Read, F. R. Mayo, Chem. Ind. 57, 752 (1938).

[20] C. R. Morgan, F. Magnotta, A. D. Ketley, J. Polym. Sci. Polym. Chem. Ed. 15, 627 (1977).

[21] C. E. Hoyle, T. Y. Lee, T. Roper, J. Polym. Sci. Part A 42, 5301 (2004).

[22] M. Sumita, K. Sakata, S. Asai, K. Miyasaka, H. Nakagawa, Polym. Bull. 25, 265 (1991).

[23] M. Sumita, K. Sakata, Y. Hayakawa, S. Asai, K. Miyasaka, M. Tanemura, Colloid Polym. Sci. 270, 134 (1992).

[24] A. E. Zaikin, R. R. Karimov, V. P. Arkhireev, Colloid J. 63, 53 (2001).

[25] A. E. Zaikin, E. A. Zharinova, R. S. Bikmullin, Polym. Sci. Ser. A 49, 328 (2007).

[26] O. Klockmann, J. Hahn, H. Scherer, International Rubber Conference 2009, Nuremberg, Germany, June 29-July 2 (2009).

[27] B. A. Dogadkin, D. L. Fedyukin, V. E. Gul, Rubber Chem. Technol. 31, 756 (1958). 
Chapter 5.1 


\section{Chapter 5.2 The effect the dispersibility of silica filler on marching modulus}

\subsubsection{Introduction}

Within this chapter, the effect of dispersibility of a silica filler on the marching modulus is studied. This phenomenon, often observed in silica filled compounds, is strongly related to the degree of silanization and the dispersion quality of the filler within the compound. Therefore, different degrees of marching modulus depending on the dispersibility of silica can be expected.

The dispersibility of silica is defined as the ability of the filler to be homogenously distributed as small clusters in a rubber matrix. ${ }^{[1]}$ This ability of silica to disperse well makes the filler itself a strong influencing factor for the final dispersion quality in a rubber matrix. ${ }^{[2,3]}$ Blume and Uhrlandt ${ }^{[2]}$ compared the dispersion behavior of conventional (CV) and Highly Dispersible (HD) silicas by using laser diffraction after ultrasonic treatment of suspensions of these silicas, and found enhanced silica cluster fragmentation for HD silica. Compared to the CV silica, a relatively higher amount of HD silica clusters were converted into a smaller size of less than $0.5 \mu \mathrm{m}$ : Figure 5.21 .
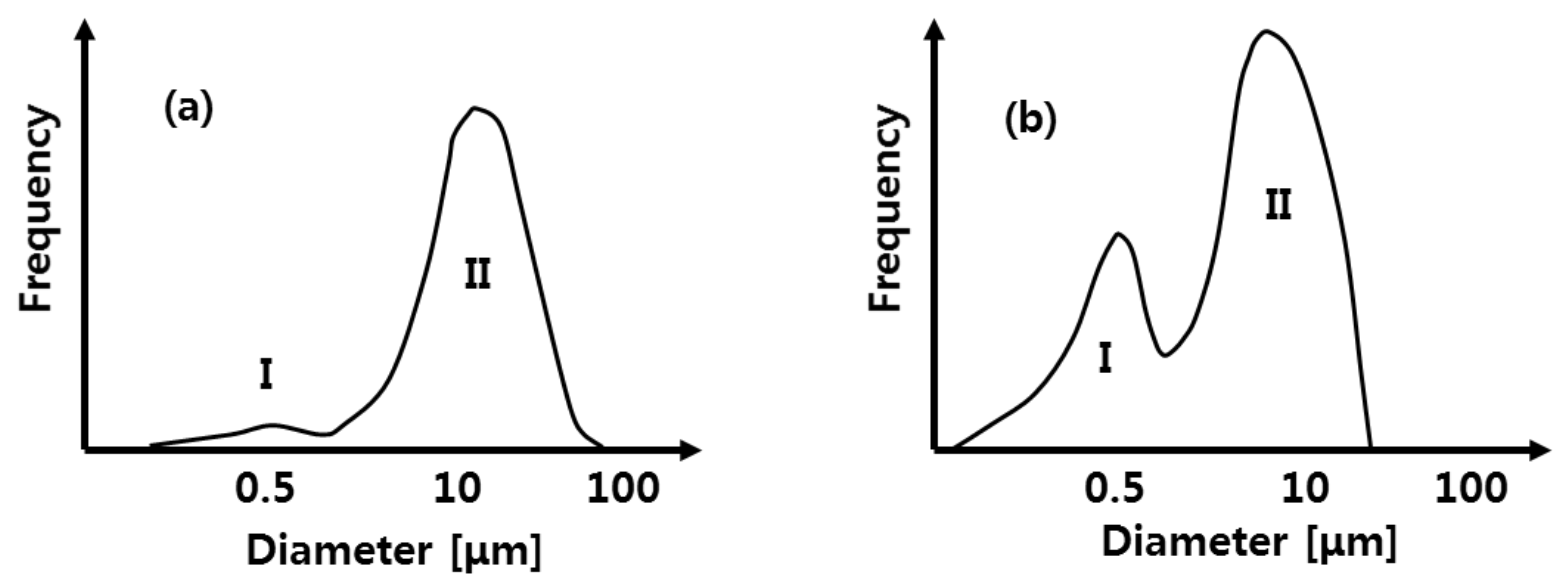

Figure 5.21 Particle size distribution after sonification; (a) Conventional silica; (b) HD Silica. ${ }^{[4,5]}$

In the present work, the effect of the dispersibility of CV and HD silica on the marching modulus intensity is investigated. The dispersibility of these silicas, with and without silane coupling agent, is compared in terms of macro- and micro-dispersion. Additionally, the factors affecting the marching modulus as well as the amount of bound rubber are evaluated and correlated to the former. 


\subsubsection{Experimental}

\subsubsection{Materials}

All series of experiments were done based on a tire tread compound as shown in Table 5.5. Blends of oilextended solution styrene-butadiene rubber (S-SBR, Mooney viscosity $\left(\mathrm{ML}(1+4) @ 100^{\circ} \mathrm{C}\right)$ of 65 and composed of $75 \%$ butadiene with a vinyl-content of $50 \%$ and $25 \%$ styrene-content, extended with 37.5 phr of TDAE oil), and high cis-1,4 polybutadiene rubber (BR, Mooney viscosity $\left(\mathrm{ML}(1+4) @ 100^{\circ} \mathrm{C}\right)$ of 44 and a cis-1,4 content of $96 \%$ ) were used in this study. The technical specifications of the silicas are listed in Table 5.6. Bis-(triethoxysilylpropyl)tetrasulfide (TESPT) was used as silane coupling agent. The amount of TESPT applied in the formulations of COM3 and COM4 were adjusted according to Equation 3.1, based on the CTAB surface areas of Silica 1 and 2 .

Table 5.5 Compound formulation

\begin{tabular}{|c|c|c|c|c|c|c|c|}
\hline \multirow{2}{*}{$\begin{array}{l}\text { Mixing } \\
\text { stage }\end{array}$} & \multirow{2}{*}{ Ingredient } & \multirow{2}{*}{ Product name } & \multirow{2}{*}{ Company } & \multicolumn{2}{|c|}{$\begin{array}{c}\text { Series } 1 \\
\text { (without silane) }\end{array}$} & \multicolumn{2}{|c|}{$\begin{array}{c}\text { Series } 2 \\
\text { (with silane) }\end{array}$} \\
\hline & & & & $\begin{array}{l}\text { COM1 } \\
\text { (phr) }\end{array}$ & $\begin{array}{l}\text { COM2 } \\
\text { (phr) }\end{array}$ & $\begin{array}{l}\text { COM3 } \\
\text { (phr) }\end{array}$ & $\begin{array}{c}\text { COM4 } \\
\text { (phr) }\end{array}$ \\
\hline \multirow{9}{*}{$\begin{array}{l}\text { Master } \\
\text { batch }\end{array}$} & S-SBR* & $\begin{array}{c}\text { BUNA VSL5025- } \\
2 \mathrm{HM}\end{array}$ & Lanxess & 110 & $\rightarrow$ & $\rightarrow$ & $\rightarrow$ \\
\hline & $B R$ & BUNA CB24 & Lanxess & 20 & $\rightarrow$ & $\rightarrow$ & $\rightarrow$ \\
\hline & Silica 1 & ULTRASIL ${ }^{\circledR}$ VN3 GR & Evonik & 90 & - & 90 & - \\
\hline & Silica 2 & Zeosil 1165MP & Solvay & - & 90 & - & 90 \\
\hline & TESPT & Si 69 & Evonik & - & - & 8.0 & 7.3 \\
\hline & TDAE oil & VIVATEC 500 & $\begin{array}{l}\text { Hansen \& } \\
\text { Rosenthal }\end{array}$ & 5 & $\rightarrow$ & $\rightarrow$ & $\rightarrow$ \\
\hline & Stearic acid & - & Merck & 1 & $\rightarrow$ & $\rightarrow$ & $\rightarrow$ \\
\hline & Zinc oxide & - & Merck & 2 & $\rightarrow$ & $\rightarrow$ & $\rightarrow$ \\
\hline & DPG & Perkacit DPG & Flexsys & 1.5 & $\rightarrow$ & $\rightarrow$ & $\rightarrow$ \\
\hline \multirow{3}{*}{ Final } & Sulfur & $S$ & J. T Baker & 0.7 & $\rightarrow$ & $\rightarrow$ & $\rightarrow$ \\
\hline & ZBEC & Vulkacit ZBEC & Lanxess & 0.2 & $\rightarrow$ & $\rightarrow$ & $\rightarrow$ \\
\hline & CBS & Santocure CBS & Flexsys & 2.2 & $\rightarrow$ & $\rightarrow$ & $\rightarrow$ \\
\hline
\end{tabular}

*27.3 wt\% oil extended 
Table 5.6 Analytical data of silicas corresponding to Table 5.5.

\begin{tabular}{ccccc}
\hline Sample code & Class & BET $\left[\mathrm{m}^{2} / \mathrm{g}\right]$ & CTAB $\left[\mathrm{m}^{2} / \mathrm{g}\right]$ & *OAN number $[\mathrm{ml} / 100 \mathrm{~g}]$ \\
\hline Silica 1 & CV Silica & 175 & 167 & 176 \\
Silica 2 & HD Silica & 155 & 152 & 205 \\
\hline
\end{tabular}

*Oil Absorption Number: the value represents the structure of silica: a higher number corresponds to a more developed structure.

\subsubsection{Mixing}

The compounds were mixed according to Mixing procedure B (Tables 4.2 and 4.3 ) with the same conditions as described in Chapter 4.1. The mixing fingerprints are depicted in Figure 5.22. In Series 1, both COM1 and 2 were mixed with 150 seconds of hold time for the silanization reaction to take place; in Series 2, COM3 and 4 were mixed with 50, 150 and 250 seconds of silanization time.
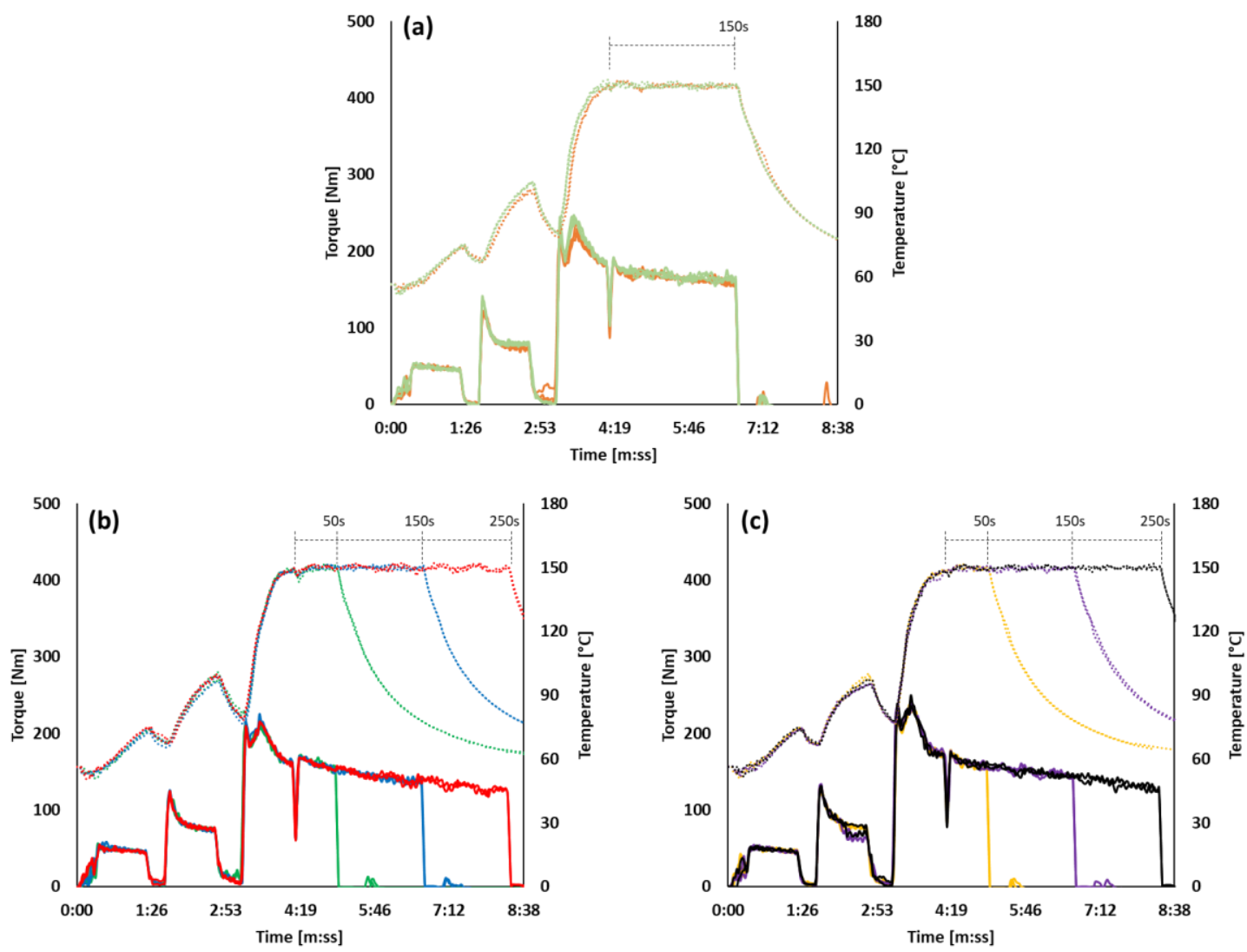

Figure 5.22 Mixing fingerprints of the compounds; (a): Series 1; (b): COM 3 in Series 2 with CV silica;

(c): COM 4 in Series 2 with HD silica; ( solid lines ): torque; ( dotted lines ): temperature;

$$
\text { ( }) \text { : COM } 1 ;(\ldots) \text { : } \operatorname{COM} 2 \text {. }
$$




\subsubsection{Property analysis and testing}

Payne effect, Filler Flocculation Rate (FFR), filler-polymer Coupling Rate (CR), cure characteristics, Marching Modulus Intensity (MMI), as well as bound rubber contents of the compounds were investigated according to the methods as described in Chapter 3.1.

\subsubsection{Macro-dispersion measurement: EVONIK topography test}

The EVONIK topography test was chosen for macro-dispersion assessment of the compounds. The images obtained from the measurement and the amount of undispersed silica clusters larger than $2 \mu \mathrm{m}$ were used for the indication of macro-dispersion. This method can measure the macro-dispersion of fillers by scanning a freshly cut surface of a cured rubber sample based on ASTM D2663 - Method C. ${ }^{[6]}$ In total, one hundred diamond tips having $5 \mu \mathrm{m}$ of radius slide over $5 \mathrm{~mm}^{2}$ of the cut surface and detect the irregularities, which represent the undispersed filler clusters. Subsequently, the surface roughness is analyzed by a special software, giving information on surface roughness, number of undispersed silica clusters and others. ${ }^{[3]}$ Additionally, a grey shades image can be obtained by converting the roughness of the cut sample surface. ${ }^{[2,3]}$ For this study, the measurements were done by the R\&D center of Evonik Resource Efficiency GmbH (Wesseling, Germany).

\subsubsection{Results and discussion}

\subsubsection{The effect of silica dispersibility on macro- and micro-dispersion}

In Series 1, the compounds without silane, the dispersibilities of the silicas are compared in terms of macro- and micro-dispersion after mixing, and the results are depicted in Figure 5.23. Surprisingly, the Payne effect levels were almost the same for both silicas: Figure 5.23(b). However, a significant difference was observed in the macro-dispersion. As can be seen in Figures 5.23(a), HD silica shows a much better dispersibility in terms of macro-dispersion. 


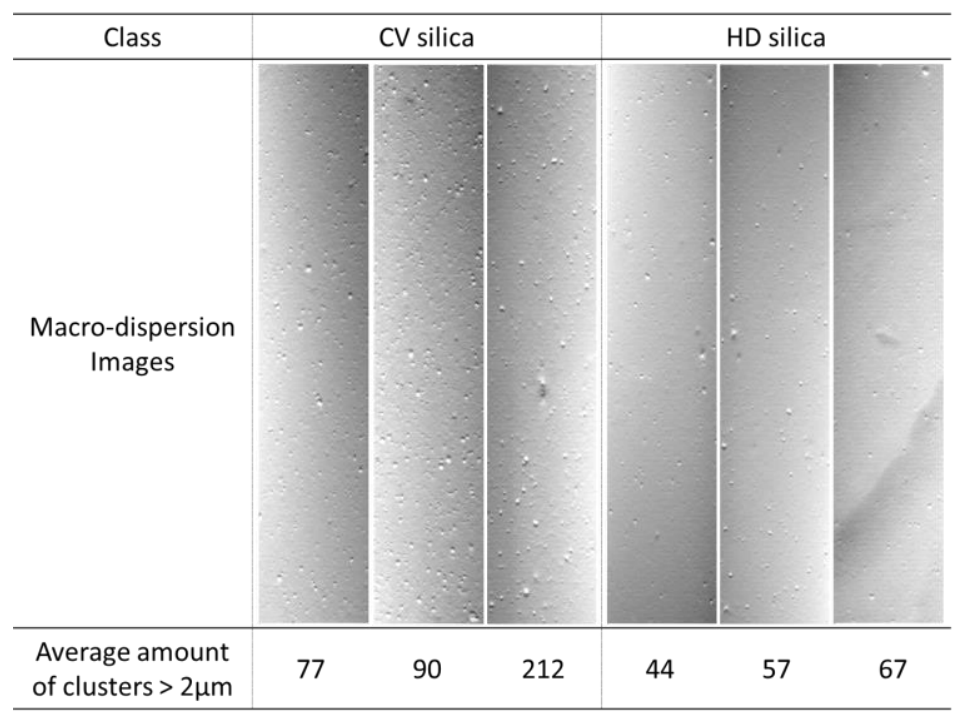

(a)

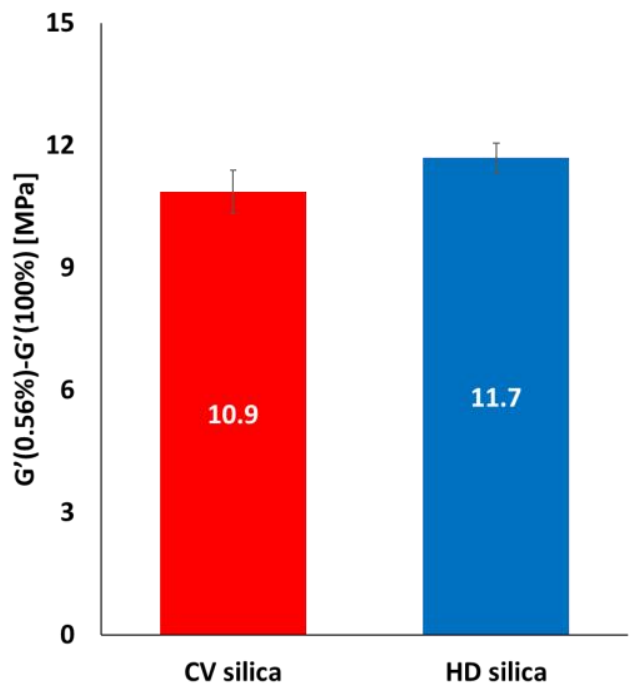

(b)

Figure 5.23 Dispersibility comparison of the silicas of Series 1; (a): macro-dispersion;

(b): Micro-dispersion represented by the Payne effect; ( $\square$ ): CV silica; ( $\square$ ): HD silica.

For the compounds containing silane (Series 2), similar micro-dispersion results are obtained. As shown in Figure 5.24, the levels of the Payne effect - representing the degree of micro-dispersion - is plotted as a function of silanization time. However, the type of silica does not affect the micro-dispersion.

Concerning the macro-dispersion level, a significant difference between the silicas is obtained (Figure 5.25), similar to the case when no silane is used as illustrated in Figure 5.23: A better macro-dispersion quality is obtained for HD silica. However, the presence of silane as well as a longer silanization time does not affect the degree of macro-dispersion. These results indicate the mutual independence of macro- and micro-dispersion of the respective silicas in the rubber matrix. ${ }^{[7]}$ To further elucidate this, a more thorough study is necessary, which is described and evaluated in Chapter 7. 


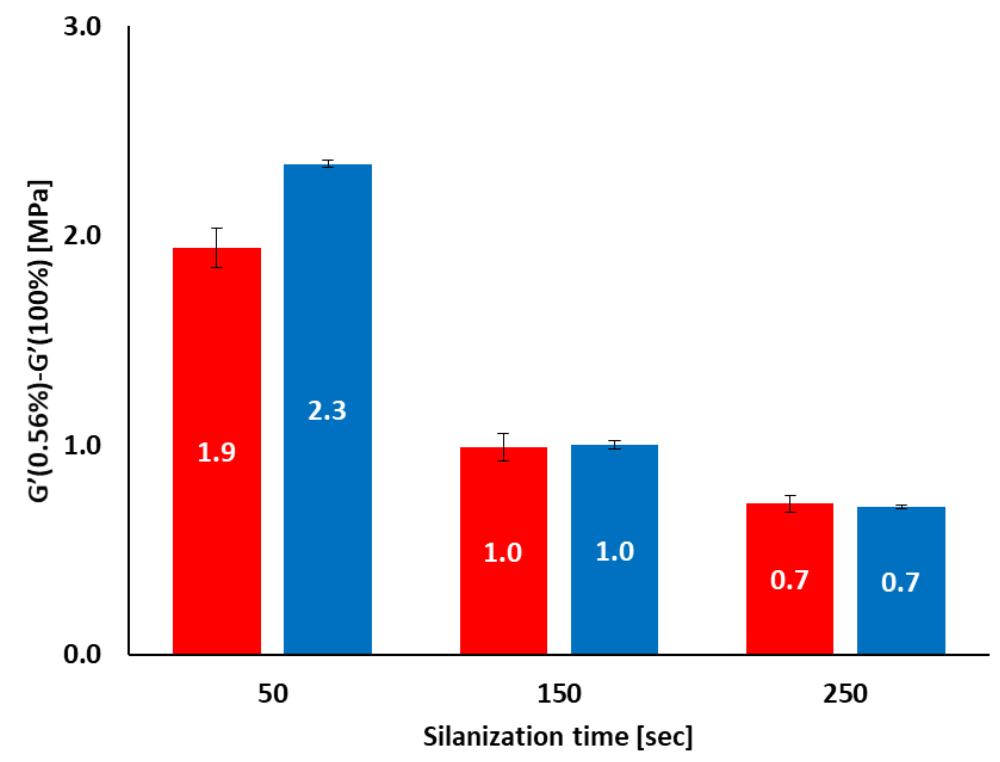

Figure 5.24 Micro-dispersion (Payne effect) results of Series 2 as a function of silanization time;

$(\square)$ ): CV silica; ( $\square$ ): HD silica.

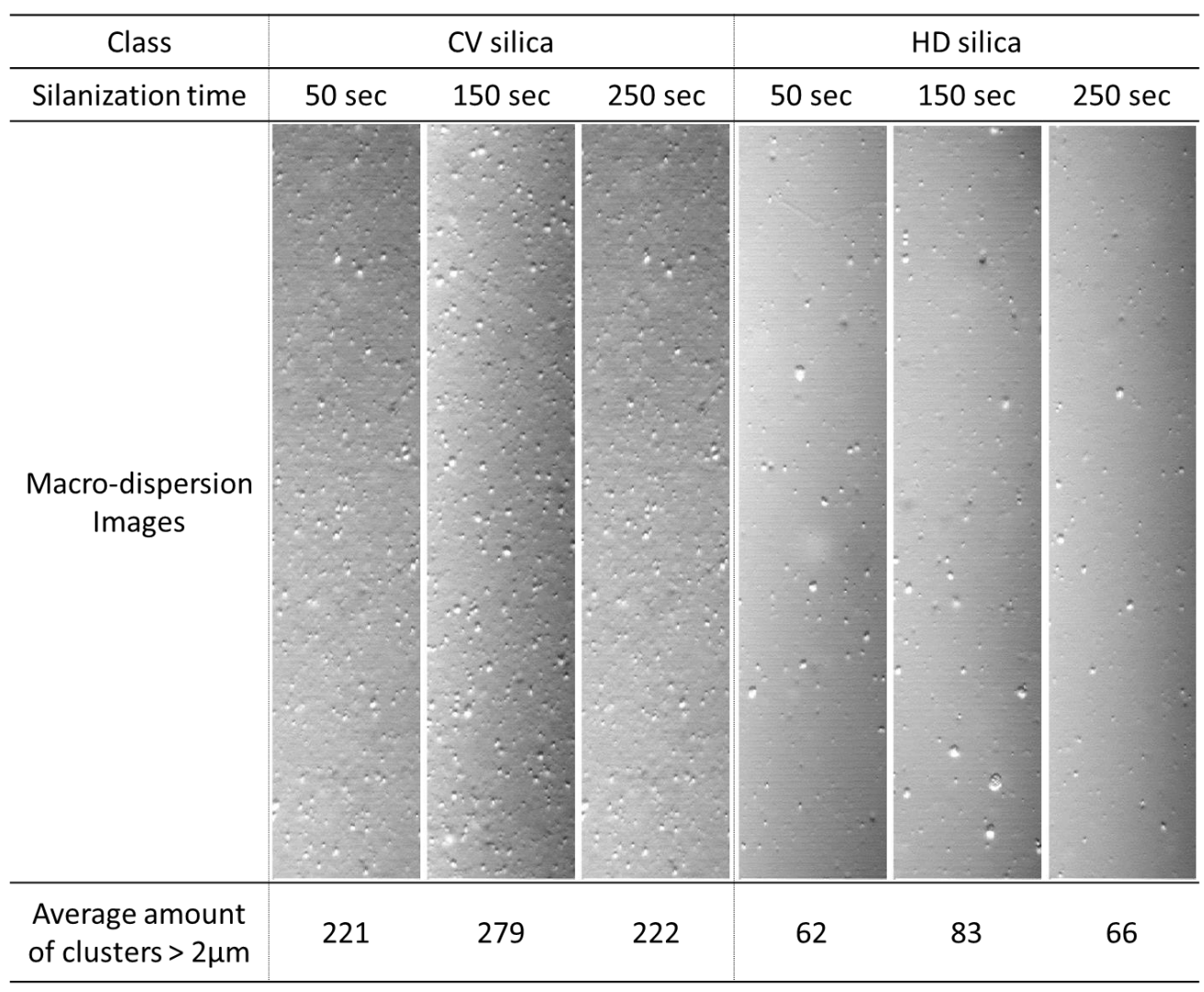

Figure 5.25 Macro-dispersion results of Series 2. 


\subsubsection{Bound rubber content as a function of silanization time}

The amount of bound rubber of Series 2 compounds is shown in Figure 5.25. As Series 1 compounds do not contain a coupling agent, no chemically bound rubber and just a small amount of physically bound rubber is formed: Figure 3.9(c) in Chapter 3.1.

In Figure 5.26, the amounts of bound rubber are plotted as a function of silanization time: a slightly higher amount of total and chemically bound rubber are obtained for the compounds with CV silica due to the slightly higher amount of silane according to the surface area: $0.7 \mathrm{phr}$ of silane was additionally added to the CV silica compound compared to the HD silica containing one.
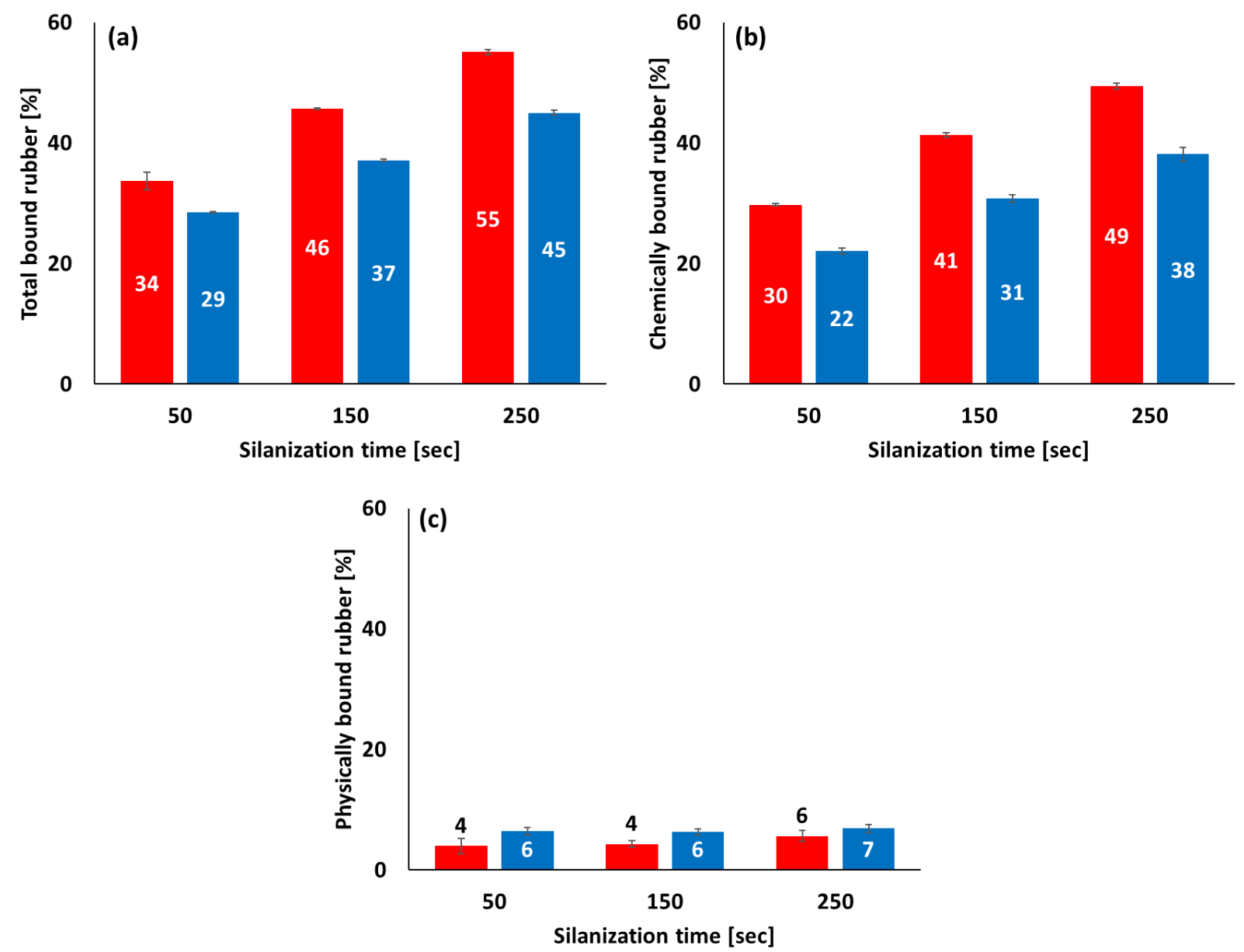

Figure 5.26 Amounts of bound rubber; (a): total bound rubber; (b): chemically bound rubber;

(c): physically bound rubber; ( $\square$ ): CV silica; $(\square)$ ): HD silica.

Figures 5.25 and 5.26 again give indication that the dispersibility of the silicas shows almost no effect on the amount of bound rubber. This was already elaborated in Chapter 4.1: the amount of bound rubber 
was mainly affected by the degree of silanization, and the silanization temperature and time turned out to be the key factors for the efficiency of the silanization reaction. Therefore, equal silanization temperatures and times lead to similar kinetics of silanization for both compounds, independent of the type of silica.

\subsubsection{Silica filler flocculation rate and filler-polymer coupling rate}

The silica Filler Flocculation Rates (FFR) and filler-polymer Coupling Rates (CR) decrease when a longer silanization time is applied for the compounds: Figure 5.27. When the macro-dispersion results (Figures 5.25) are also taken into account, it can be concluded that the FFR and CR are mainly influenced by the degree of silanization - regardless of the dispersibility of the silicas or the degree of macro-dispersion of the compounds: these rates decrease with increasing silanization time, whereas the macro-dispersion (Figure 5.25) is not influenced.
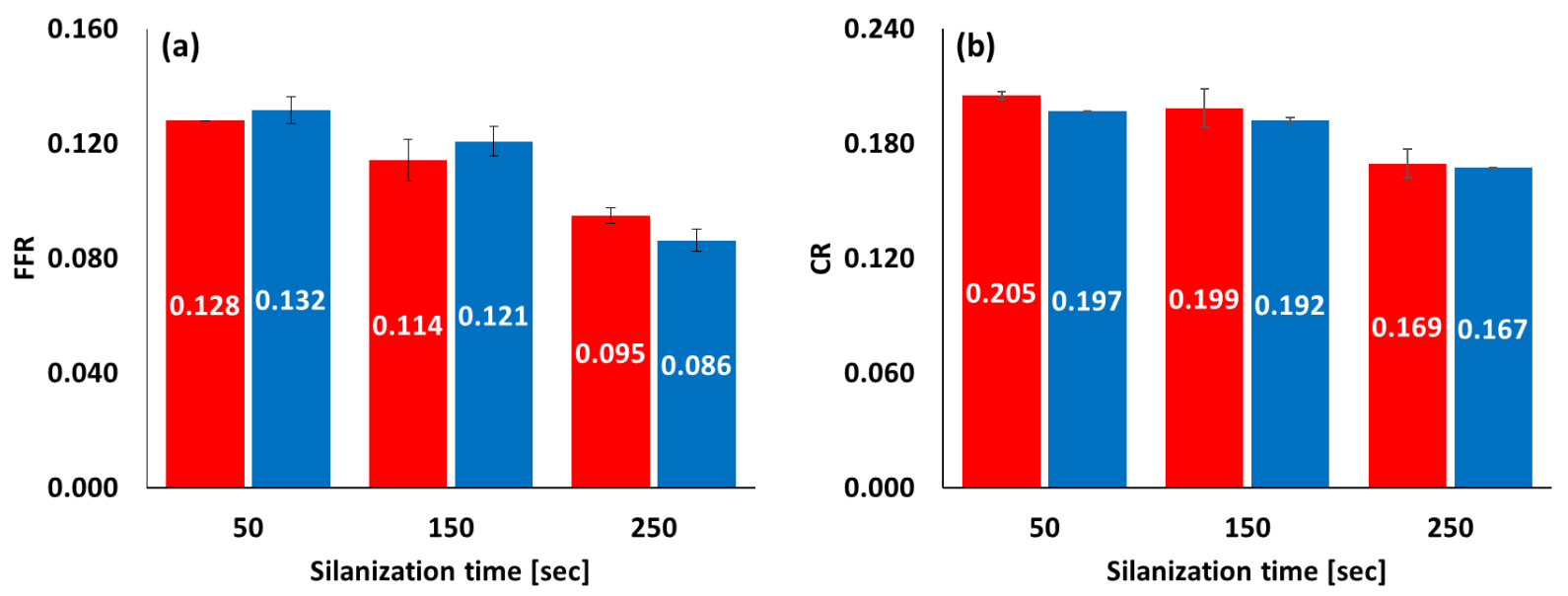

Figure 5.27 FFR and CR as a function of silanization time; (a): FFR; (b): CR;

( $\square$ ): CV silica; ( $\square$ ): HD silica.

Another interesting result is observed for the FFR values (Figure 5.27(a)): The flocculation of silica is mainly influenced by the small silica clusters; those in the range of micro-dispersion. Otherwise, COM3 (CV silica with silane) would show a higher level of FFR than COM4 (HD silica with silane), because a more than three times larger amount of undispersed large silica clusters were found in COM3 compared to COM4 regardless of the silanization time. However, due to the fact that equal silanization temperatures and times lead to similar kinetics of silanization for both compounds, similar FFR and CR values are obtained for $\mathrm{COM} 3$ and COM4. 


\subsubsection{The relation between dispersibility of the silica, macro-dispersion and marching modulus intensity}

The rheograms of the compounds and their Marching Modulus Intensity (MMI) measured at small strain are shown in Figure 5.28. Compared to CV silica, HD silica shows slightly lower MMI $0.5^{\circ}$ values due to a smaller specific surface area and thus a lower amount of silanol groups in this case. A similar tendency depending on the specific surface area of silica can be found in Mihara's ${ }^{[8]}$ work. As expected from Chapter 4.1, a longer silanization time will lead to a lower MMI $0.5^{\circ}$ due to a reduced FFR and CR. The dispersibility of the silicas as well as the degree of macro-dispersion of the compounds show no effect on MMI $0.5^{\circ}$.
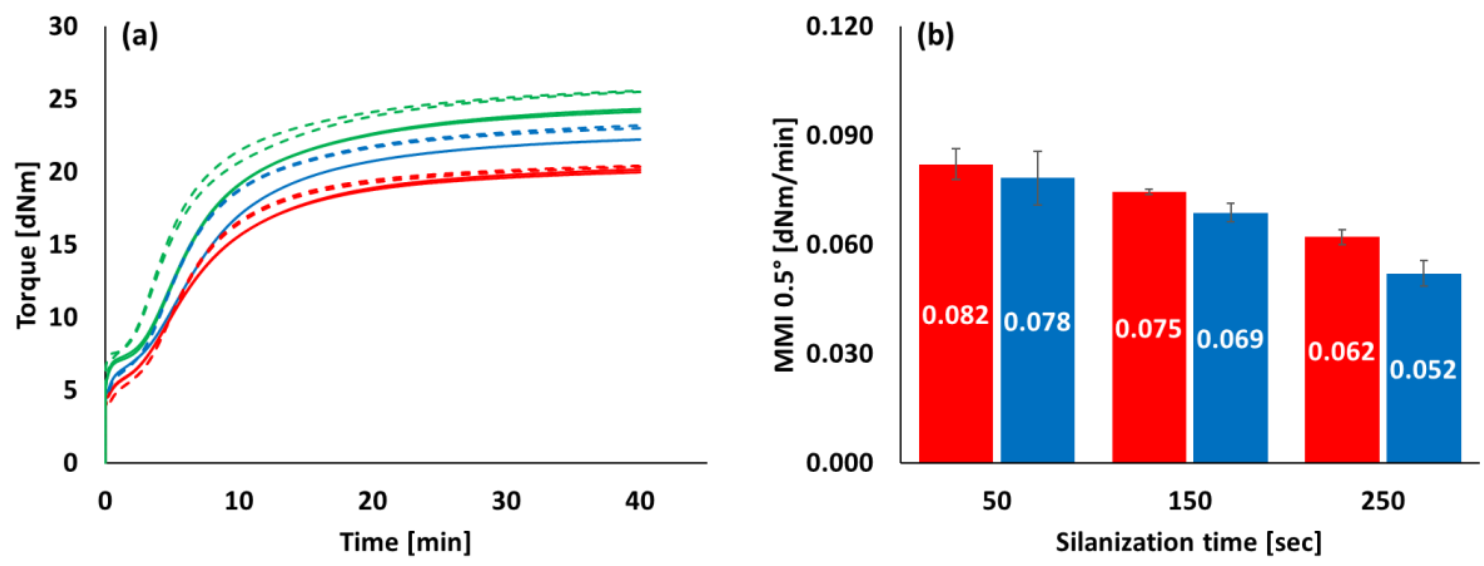

Figure 5.28 Rheograms and MMI $0.5^{\circ}$ ( 7\% of strain) values of the compounds in Series 2;

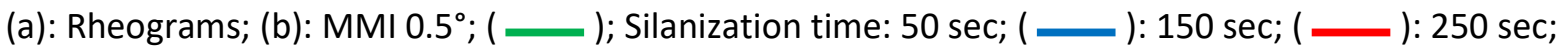

( solid line ): CV silica; ( dashed line ): HD silica; ( $\square$ ): CV silica; ( $\square$ ): HD silica.

\subsubsection{Conclusions}

In this work, the effect of dispersibility of two different silicas on the marching modulus phenomenon was investigated. The results indicate, that the dispersibility of silica has no effect on the curing behavior of silica filled rubber.

None of the factors related to the $\mathrm{MMI} 0.5^{\circ}$ - filler-filler interaction, the amount of bound rubber, FFR and $C R$ - was influenced by the dispersibility of the silicas or by the degree of macro-dispersion of the compounds. The specific surface area can affect the $\mathrm{MMI} 0.5^{\circ}$, however this effect was found to be small. The dispersibility of silica is strongly related to the degree of macro-dispersion of the compounds, but not to the degree of micro-dispersion nor to the efficiency of the silanization reaction. 


\section{References}

[1] F. Grunert, A. Wehmeier, A. Blume, 12th Fall DIK Rubber Colloquium, Hannover, Germany, 22-24 November, Paper No. 21 (2016).

[2] A. Blume, S. Uhrlandt, 157th meeting ACS Rub. Div., Dallas, Texas, Paper No. 32 (2000).

[3] F. Grunert, PhD thesis: Analytical Method Development to Predict the In-Rubber Dispersibility of Silica, Univ. Twente: Enschede, the Netherlands (2018).

[4] A. Voet, A, J. C. Morawski, J. B. Donnet, Rubber Chem. Technol. 50, 342 (1977).

[5] A. Blume, Kautsch. Gummi Kunstst. 53, 338 (2000).

[6] ASTM D2663-14, "Standard Test Method for Carbon Black - Dispersion in Rubber, Test Method C - Microroughness Measurement with Profilometer", ASTM International (2015).

[7] J. Jin, W. Kaewsakul, J.W.M. Noordermeer, W.K. Dierkes, A. Blume, International Rubber Conference, London, United Kingdom, 3-5 September (2019).

[8] S. Mihara, PhD Thesis: Reactive processing of silica-reinforced tire rubber: new insight into the time- and temperature-dependence of silica rubber interaction, Univ. Twente: Enschede, the Netherland (2009). 


\section{Chapter 6 Scale up: production scale mixing trials}

\subsection{Introduction}

The objective of this series of trials is to evaluate the effect of mixing parameters such as silanization time, temperature and rotor types (intermeshing vs tangential) on the marching modulus of silica filled compounds in a production scale mixer. Due to the fact that the scale of the mixer has a large impact on the mixing quality, the results acquired by changing the mixing parameters in a small (lab scale) mixer might different from the ones in a large size one (industrial scale). For example, the surface-to-volume ratio - which strongly influences the compound temperature, cooling efficiency and shear forces depends on the mixer size: ${ }^{[1]}$ Figure 6.1. Therefore, smaller sized mixers might be required when efficient heat transfer is required for particular rubber goods. ${ }^{[1]}$

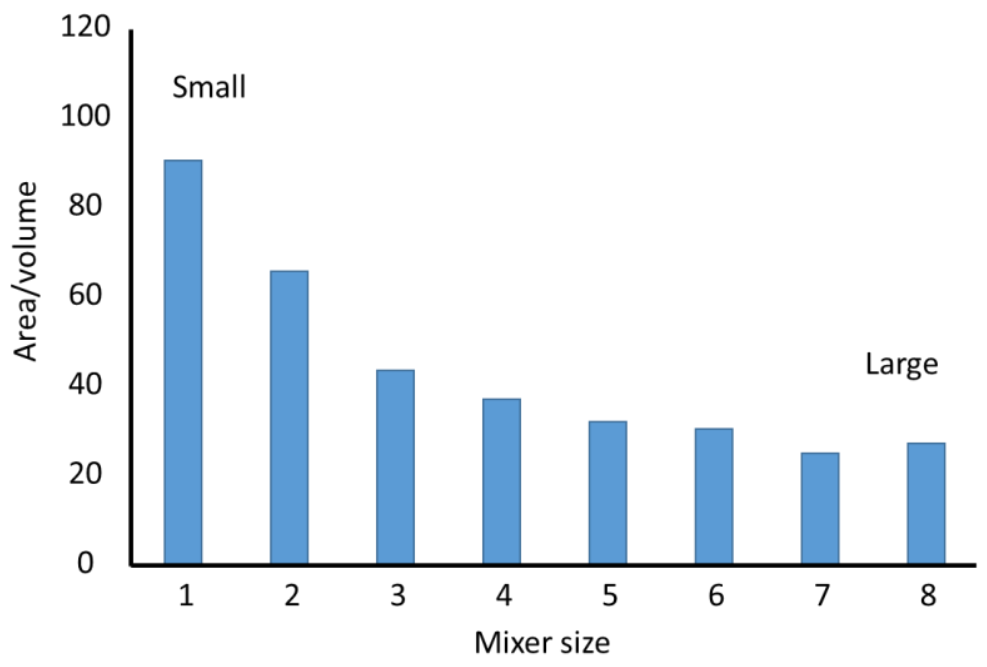

Figure 6.1 Surface-to-volume ratio vs. mixer size. ${ }^{[1]}$

In this work, compound mixing is performed using plant scale mixers in order to check if the basic mechanisms observed in previous chapters are still valid regardless of the mixer scale. The effect of silanization time (Series 1) and temperature as well as rotor geometry (Series 2) on compound quality are compared in terms of three influencing factors on marching modulus (FFI, FFR and CR) as well as on macrodispersion quality.

Studies were done on the origin and influencing mixing parameters for the marching modulus of silica filled rubber compounds and reported in Chapters 3.1 and 4.1. The results confirmed that three factors: 
the intensity of Filler-Filler Interaction (FFI), the silica Filler Flocculation Rate (FFR) and the filler-polymer Coupling Rate (CR) are the major influencing factors on the marching modulus phenomenon of silica compounds. All three are the consequence of improper silanization. These properties are therefore strongly influenced by mixing parameters such as silanization temperature and time, which have a large effect on the degree of silanization.

The geometry of the rotor is another parameter which affects the intensity of the fillerfiller interaction. ${ }^{[1-9]}$ Many studies were done on this topic; however, those were performed using lab scale mixers. In general, two types of rotors are commonly used for tire rubber compounding: tangential and intermeshing types. The strength of the tangential mixer is good feeding and high productivity. ${ }^{[2-5]}$ In contrast to this, the intermeshing mixer primarily offers better processability and quality of the compound: i.e. better cooling efficiency, lower compound viscosity and higher degree of filler dispersion. ${ }^{[1-3,6,7,9]}$

\subsection{Experimental}

\subsubsection{Materials and mixing}

All series of experiments were done based on a tire tread compound as shown in Table 6.1.

Table 6.1 Compound formulation

\begin{tabular}{c|c|ccc}
\hline Mixing stage & Ingredient & $\begin{array}{c}\text { COM1 } \\
(\mathrm{phr})\end{array}$ & $\begin{array}{c}\text { COM2 } \\
(\mathrm{phr})\end{array}$ & $\begin{array}{c}\text { COM3 } \\
\text { (phr) }\end{array}$ \\
\hline \multirow{3}{*}{ Master batch } & S-SBR & 90 & 85 & 90 \\
& BR & 10 & 15 & 10 \\
& Silica & 95 & 95 & 80 \\
& TESPT & 8.5 & 8.5 & 7.0 \\
& TDAE Oil & 45 & 45 \\
& Stearic acid & 1 & 1 & 2 \\
& Zinc Oxide & 2 & 2 & 2 \\
& DPG & 2 & 2 & 11 \\
\hline Final & Others & 16 & 16 & 3 \\
\hline & Curatives (sulfur+accelerator) & 3 & 3 & Series 2 \\
\hline
\end{tabular}




\subsubsection{Mixing}

The masterbatch compounds were mixed in the industrial scale mixer according to the variables described in Tables 6.2 and 6.3. The scale of the mixers is larger than $250 \mathrm{~L}$ net volume. For Series $1, \mathrm{COM} 1$ and COM2 were mixed at different silanization times with a fixed silanization temperature $\left(150^{\circ} \mathrm{C}\right)$. For Series 2 , COM3 was mixed at a fixed mixing time with two main variables: dump temperature and rotor type. Two or three batches were mixed for each set of conditions in order to check the reproducibility. The final mixing of all compounds was done on a lab scale two roll mill.

Table 6.2 Mixing variables for Series 1

\begin{tabular}{cccc}
\hline & Time 1 & Time 2 & Time 3 \\
\hline Silanization time $\left(@ 150^{\circ} \mathrm{C}\right)$ & $50 \mathrm{sec}$ & $100 \mathrm{sec}$ & $150 \mathrm{sec}$ \\
\hline
\end{tabular}

Table 6.3 Mixing variables for Series 2

\begin{tabular}{|c|c|c|c|}
\hline & & TG & IM \\
\hline \multicolumn{2}{|c|}{ Rotor type } & Tangential & Intermeshing \\
\hline \multirow{2}{*}{ Dump temperature } & Low & \multicolumn{2}{|c|}{$135^{\circ} \mathrm{C}$} \\
\hline & High & \multicolumn{2}{|c|}{$150^{\circ} \mathrm{C}$} \\
\hline
\end{tabular}

\subsubsection{Property analysis and testing}

Payne effect, Filler Flocculation Rate (FFR), filler-polymer Coupling Rate (CR), cure characteristic and Marching Modulus Intensity (MMI), bound rubber content of the compounds were investigated according to the methods as described in Chapter 3.1.

For Series 2, the dispersability of a tangential and intermeshing rotor were compared in terms of microand macro-dispersion. Micro-dispersion was evaluated by measuring the Payne effect; macro-dispersion was evaluated by using the DisperGRADER Alpha View (Alpha Technologies).

All properties are expressed as indices. The maximum value of each property was used as reference - set as $100 \%$ - for the index calculation. The indices of the chemically and physically bound rubber were calculated by using Equation 6.1:

$$
C B R(\text { or } P B R) \text { index }[\%]=T B R \text { index } \times \frac{C B R(\text { or } P B R)}{T B R}
$$


Where TBR, CBR and PBR correspond to Total Bound Rubber, Chemically Bound Rubber and Physically Bound Rubber, respectively.

\subsection{Results and discussion}

\subsubsection{Silanization time effect on marching modulus (Series 1)}

\subsubsection{Filler-filler interaction (Payne effect) as a function of silanization time}

The Payne effect values of the uncured compounds are plotted as a function of the silanization time for both COM1 and COM2: Figure 6.2. As was shown in Chapter 4.1, silanization time is one of the key mixing parameters for reducing filler-filler interaction, and this is still valid for a large mixer as seen in Figure 6.2.
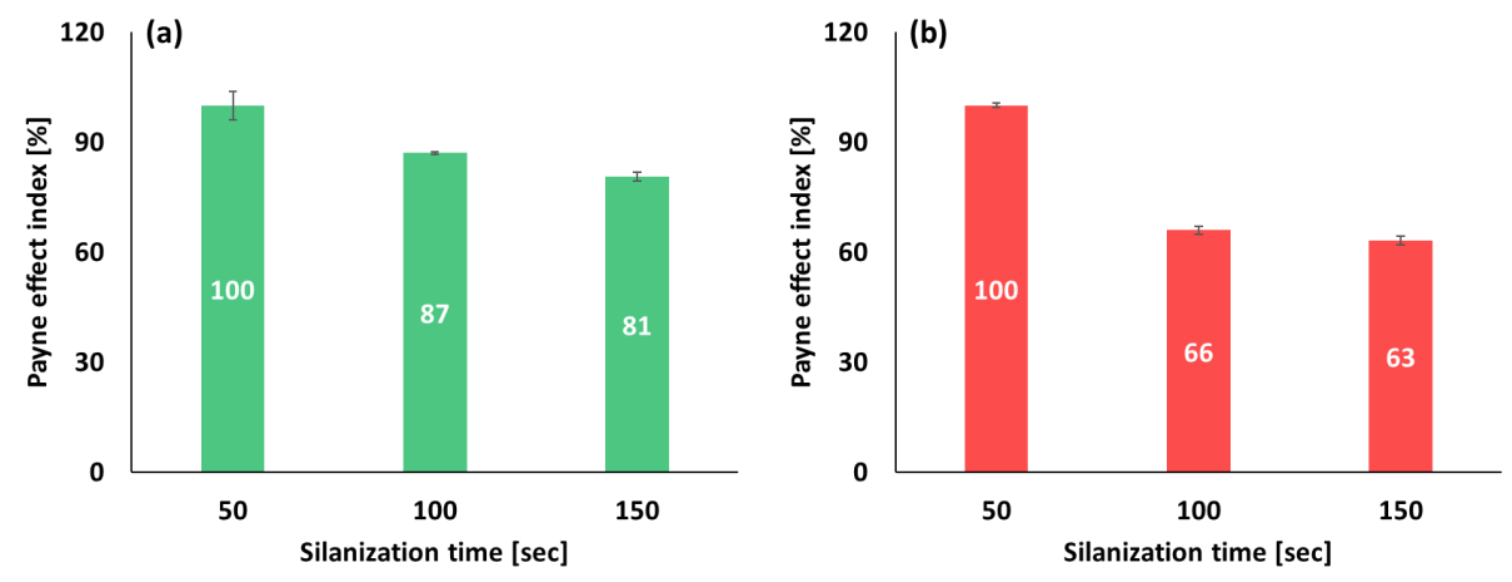

Figure 6.2 Payne effect as a function of silanization time; (a): COM1; (b): COM2.

\subsubsection{Bound rubber content as a function of silanization time}

Figure 6.3 shows the amount of bound rubber as function of silanization time. A higher amount of total and chemically bound rubber was observed when the compounds were mixed at a longer silanization time. As was shown in Chapters 3.1 and 4.1, the amounts of total and chemically bound rubber have a significant effect on reducing filler-filler interaction, and as a consequence on lowering the Payne effect. 

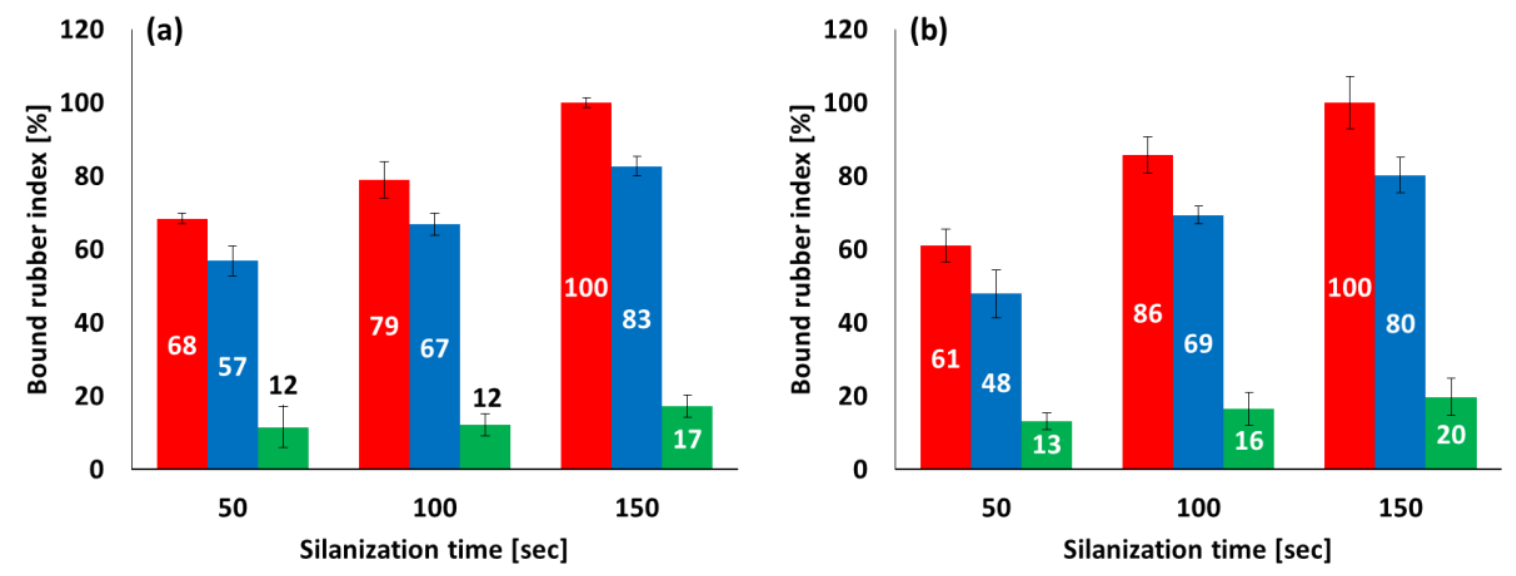

Figure 6.3 Bound rubber content as a function of silanization time; (a): COM1; (b): COM2;

$(\square)$ : total bound rubber; $(\square)$ ): chemically bound rubber; $(\square)$ : physically bound rubber.

\subsubsection{Silica flocculation rate as a function of silanization time}

The silica Filler Flocculation Rates (FFR) are shown in Figure 6.4. As a correlation between FFR and silanization time was found in Chapter 4.1, the FFR values are plotted as a function of silanization time.
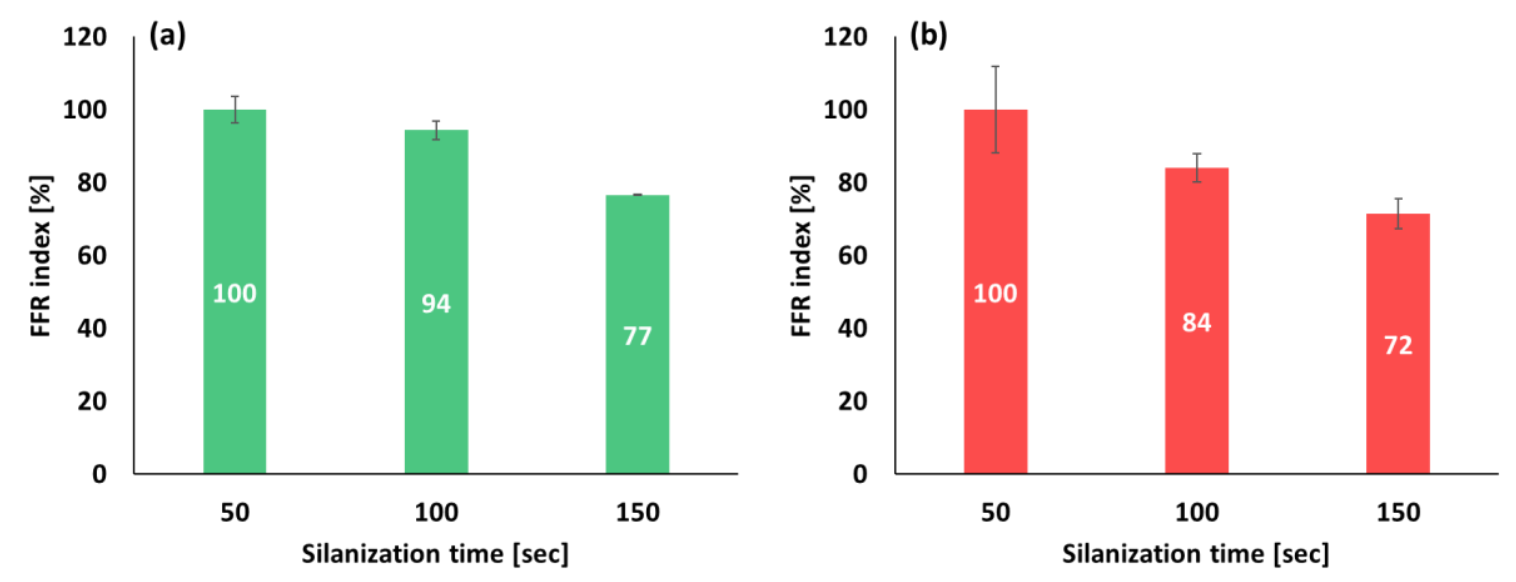

Figure 6.4 FFR as a function of silanization time; (a): COM1; (b): COM2.

It was already shown that a higher amount of bound rubber leads to lower filler-filler interaction and thus a reduced tendency for flocculation. Therefore, FFR indices of all batches are plotted against their total and chemically bound rubber content indices in Figure 6.5. The coefficient of definition $\left(R^{2}\right)$ values for the correlations between FFR and bound rubber are smaller than those obtained in Chapter 4.1 (0.94, see Figure 4.7), however the values seem still reasonable when the scale of the mixer for this work is taken 
into account: mixing in a large scale mixer is less controlled due to variations in weighing of the components and process control.
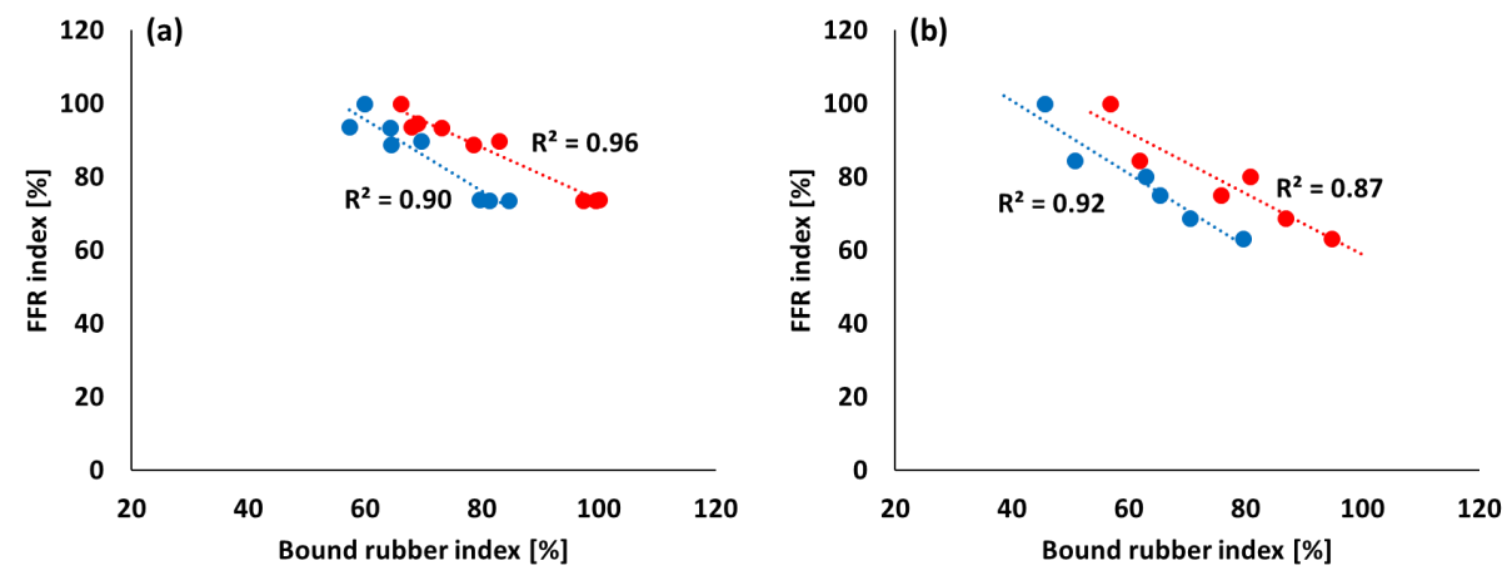

Figure 6.5 FFR as a function of total and chemically bound rubber; (a): COM1; (b): COM2;

$(\bullet)$ : FFR vs. total bound rubber; $(\bullet)$ : FFR vs. chemically bound rubber.

\subsubsection{Filler-polymer coupling rate as a function of silanization time}

The filler-polymer Coupling Rates (CR) are shown in Figure 6.6. The CR of the compounds plotted as a function of silanization time show a decreasing trend.
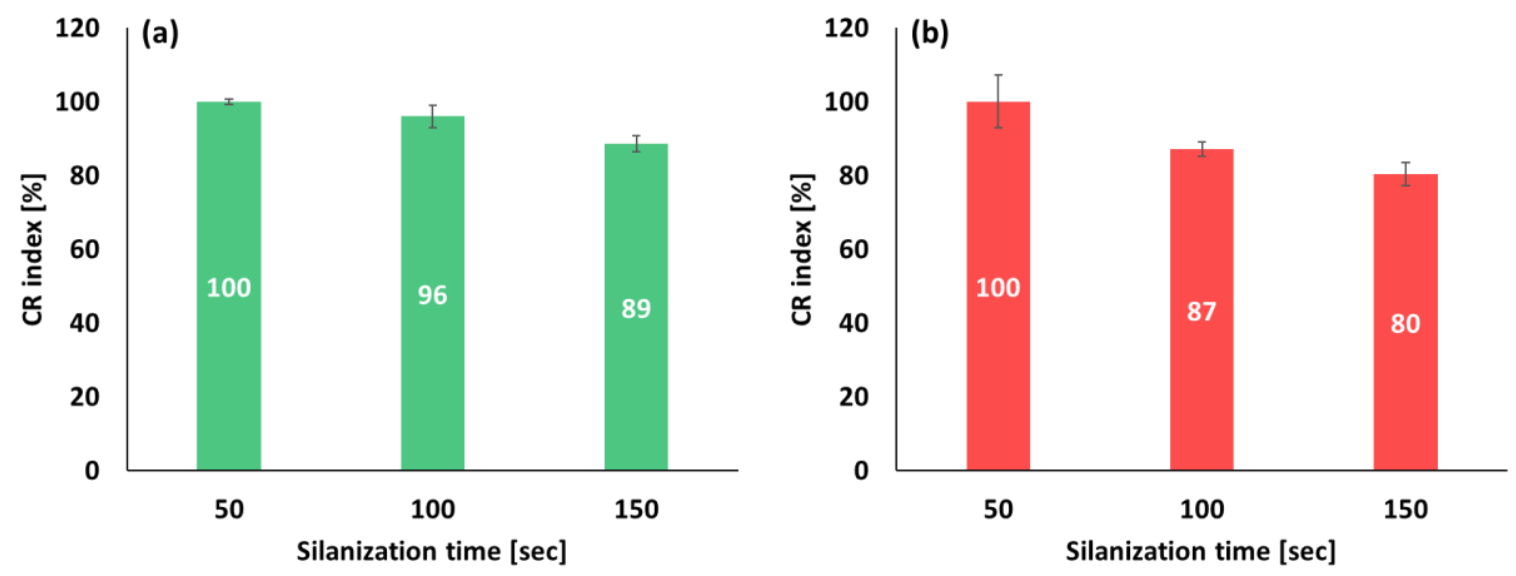

Figure $6.6 \mathrm{CR}$ as a function of silanization time; (a): COM1; (b): COM2.

Both types of bound rubber show a good correlation with CR as shown in Figure 6.7. CR decreases as the silanization time increases due to the lowered concentration of active sulfur and remaining free silane. 

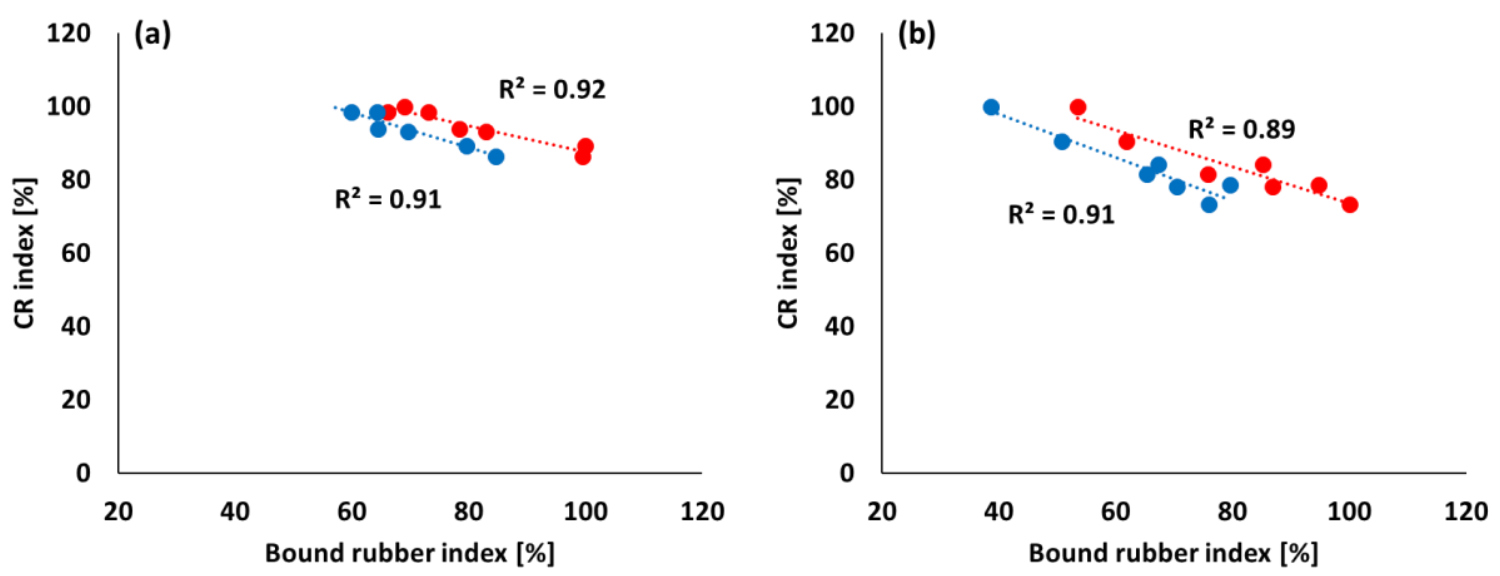

Figure 6.7 CR as a function of total and chemically bound rubber; (a): COM1; (b): COM2;

$(\bullet)$ : CR vs. total bound rubber; $(\bullet)$ : CR vs. chemically bound rubber.

\subsubsection{Marching modulus intensity}

The $\mathrm{MMI} 0.5^{\circ}$ and the correlation between $\mathrm{MMI} 0.5^{\circ}$ and FFR as well as $\mathrm{CR}$ are shown in Figures 6.8, 6.9(a) and 6.9(b), respectively. As expected from the results of Chapter $4.1, \mathrm{MMI} 0.5^{\circ}$ decreased with increasing silanization time. And the MMI $0.5^{\circ}$ show good correlations with FFR and CR for the reason as stated in Chapter 4.1: higher bound rubber suppresses the flocculation of silica and results in slower filler-polymer coupling due to a reduction of silane as well as released free sulfur concentration.
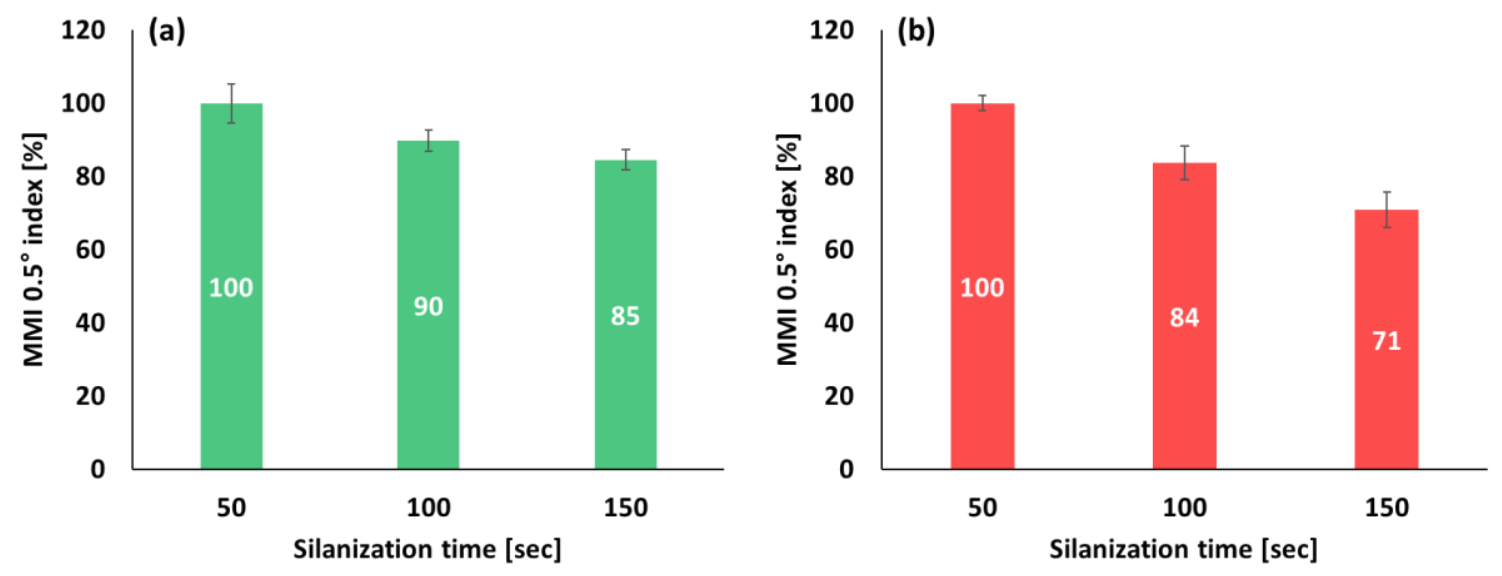

Figure $6.8 \mathrm{MMI} 0.5^{\circ}$ vs. silanization time; (a): COM1; (b): COM2. 

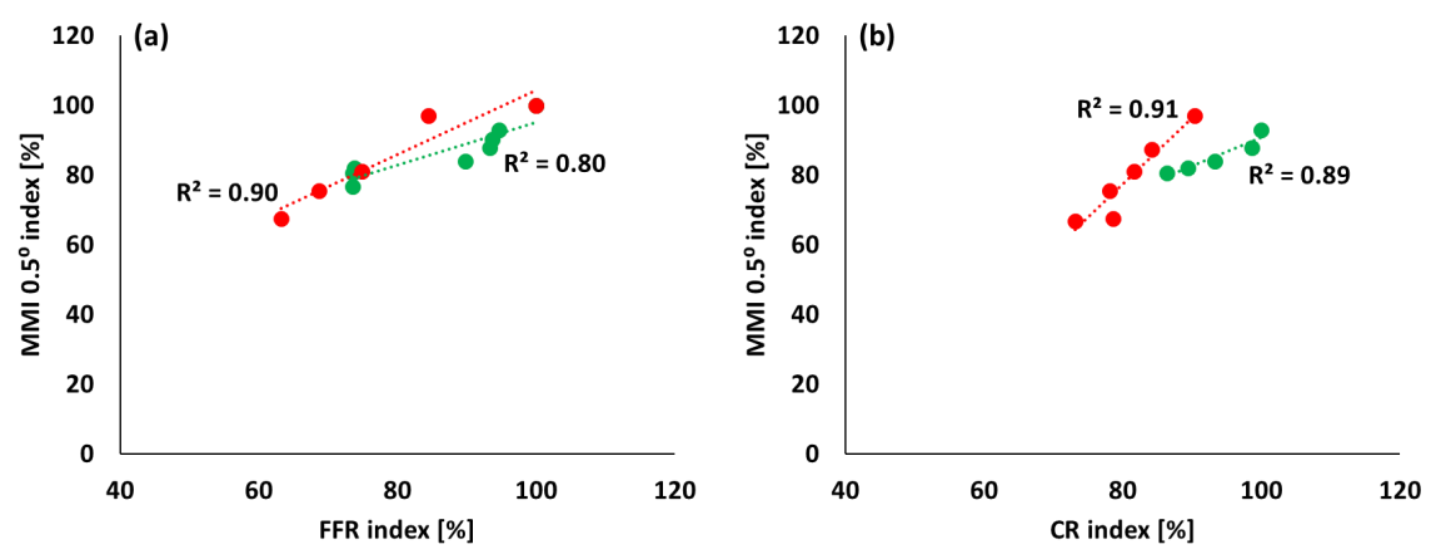

Figure 6.9 $\mathrm{MMI} 0.5^{\circ}$ as a function of FFR and CR; (a): MMI $0.5^{\circ}$ vs. FFR; (b): MMI $0.5^{\circ}$ vs. CR;

$$
(\bullet): \operatorname{COM} 1 ;(\bullet): \mathrm{COM} 2 \text {. }
$$

\subsubsection{The effect of rotor type and dump temperature on marching modulus (Series 2)}

\subsubsection{Filler-filler interaction (Payne effect)}

The Payne effect values of the uncured COM3 compound are depicted in Figure 6.10. The mixing temperature greatly affects the degree of silanization, therefore lower Payne effect values were obtained when the compound was discharged at a higher temperature. Besides, the intermeshing rotor (IM) shows better performance in micro-dispersion of silica due to its technical characteristics as stated in Paragraph 2.5.3.1. Overall, the effect of mixing temperature on Payne effect reduction was larger than the effect of rotor geometry.

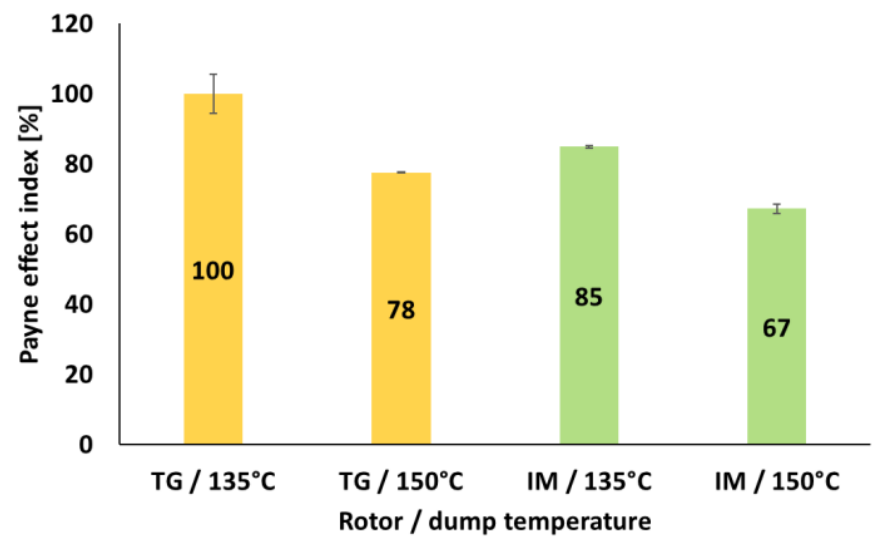

Figure 6.10 Payne effect in correlation to dump temperature and rotor type:

TG-tangential; IM-intermeshing. 


\subsubsection{Bound rubber content}

The amounts of bound rubber are shown in Figure 6.11. The amount of total and chemically bound rubber is mostly influenced by the dump temperature. Chemically bound rubber is a result of prior filler-polymer coupling during mixing and is sensitive to temperature. The amount of total bound rubber is mostly affected by chemically bound rubber. When the error ranges are taken into account, almost the same values are obtained for equal dump temperatures, regardless of rotor type. The amount of total and chemically bound rubber of all batches for both types of rotors are plotted with their actual dump temperature in Figure 6.12. This result clearly demonstrates that bound rubber content is mostly affected by dump temperature and less by rotor type.

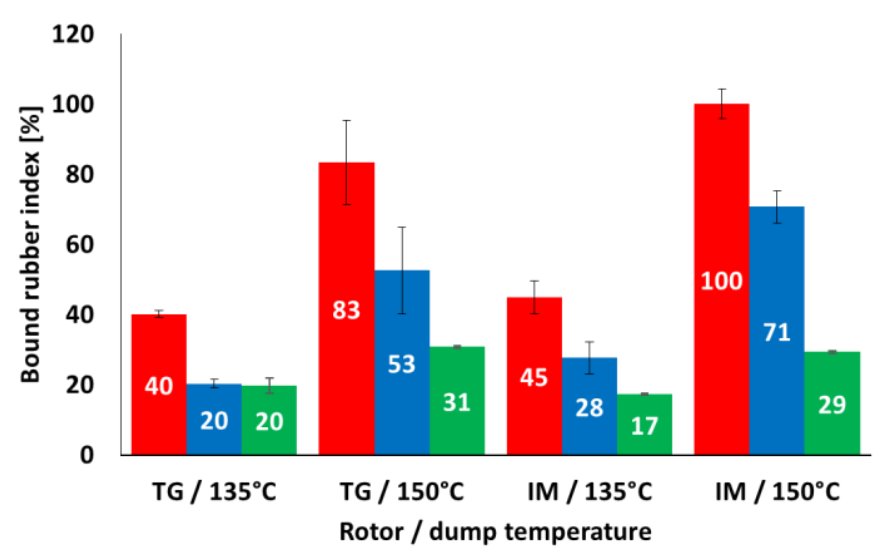

Figure 6.11 Bound rubber content as a function of dump temperature and rotor type;

$(\square)$ : total bound rubber; $(\square)$ : chemically bound rubber; $(\square)$ : physically bound rubber.

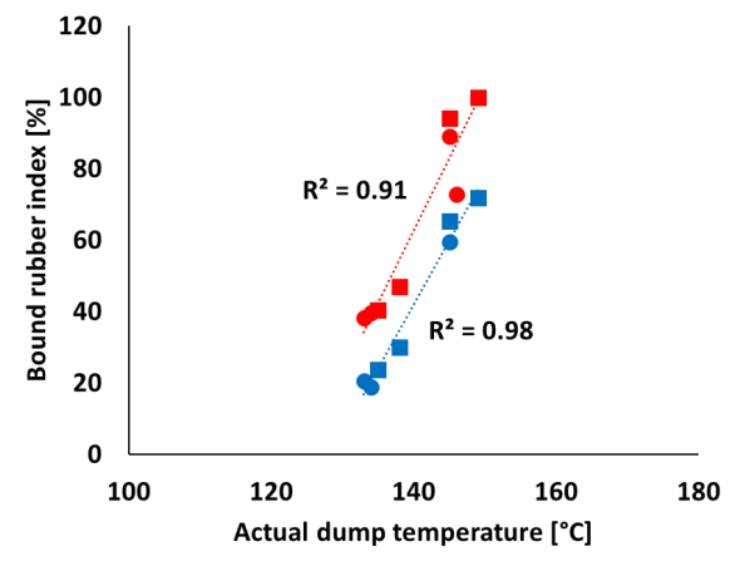

Figure 6.12 Bound rubber content vs. dump temperature; (red dots): total bound rubber; (blue dots): chemically bound rubber; ( $\bullet$ ): tangential; ( $\mathbf{a})$ : intermeshing. 


\subsubsection{Silica flocculation rate}

The silica Filler Flocculation Rates (FFR) are shown in Figure 6.13. The highest FFR value is obtained for the compound mixed with tangential rotors at a lower temperature due to the strong filler-filler interaction within this series: Figure 6.9. However, the effect of rotor geometry on FFR becomes less as the dump temperature is increased. The reason is, that the silica flocculation behavior is strongly related with the amount of total and chemically bound rubber. As seen in Figures 6.11 and 6.12, the largest amounts of total and chemically bound rubber were found when the compounds were discharged at a higher temperature, regardless of rotor geometry. In Figure 6.14, if the FFR values are plotted as a function of total and chemically bound rubber, and an effect of rotor geometry again cannot be seen: both rotor types fall on the same lines.

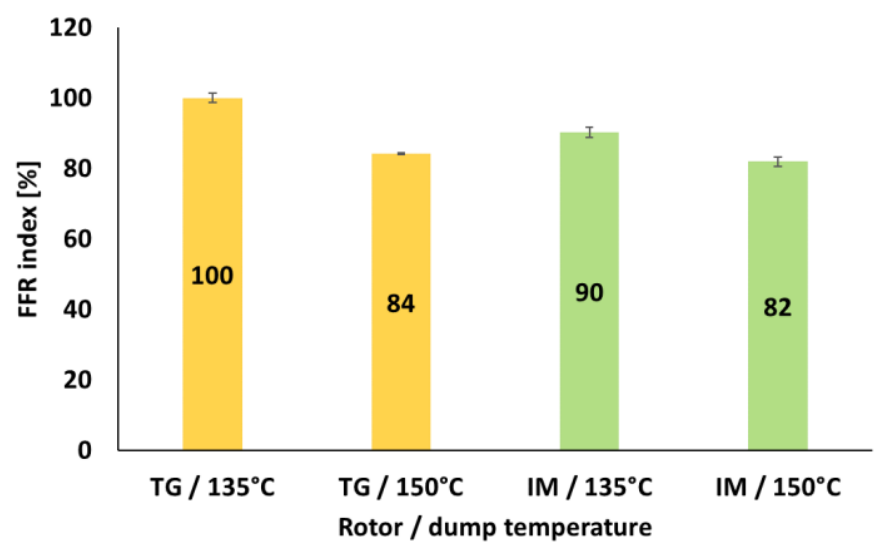

Figure 6.13 FFR according to the dump temperature and rotor type.

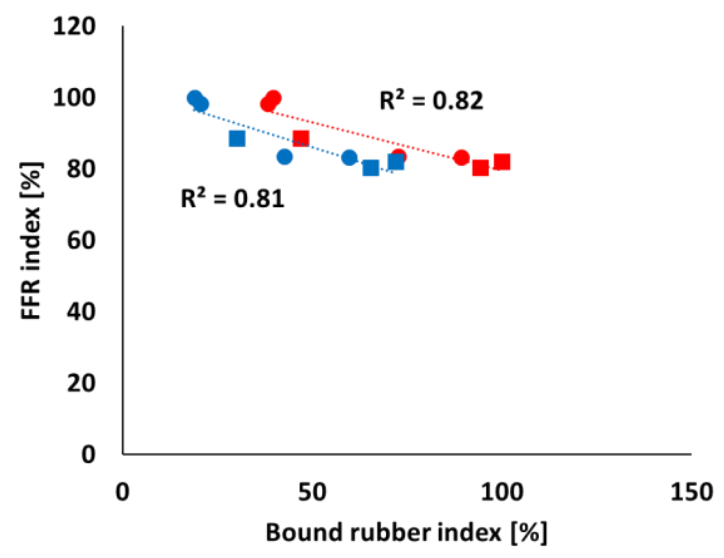

Figure 6.14 FFR as a function of total and chemically bound rubber; (red dots): FFR vs. total bound rubber; (blue dots): FFR vs. chemically bound rubber; ( • ): tangential; ( $\bullet$ ): intermeshing. 


\subsubsection{Filler-polymer coupling rate after mixing}

The filler-polymer Coupling Rates (CR) are shown in Figure 6.15. The rotor geometry effect on CR is small. Lower CR values were obtained for the compounds discharged at higher temperature. As expected from the results of Chapters 3.1 and 4.1, both types of bound rubber show a correlation with CR: Figure 6.16, irrespective of the rotor geometry.

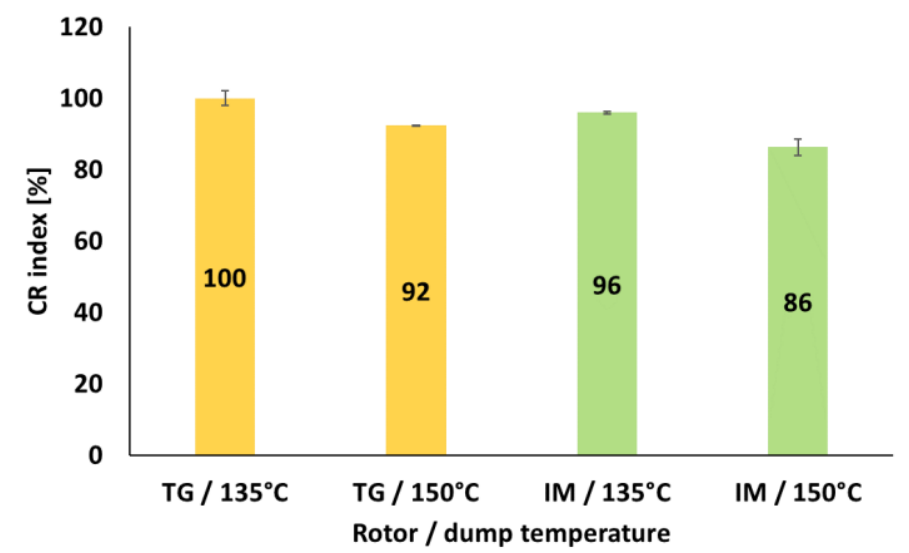

Figure 6.15 CR according to the dump temperature and rotor type.

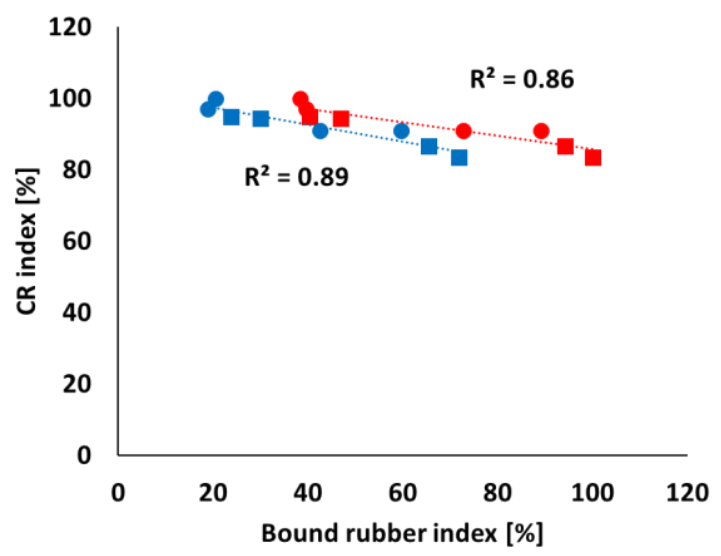

Figure 6.16 CR as a function of total and chemically bound rubber; (red dots): CR vs. total bound rubber;

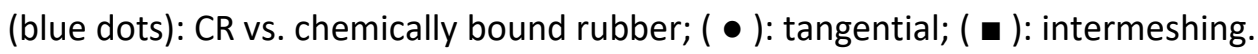

\subsubsection{Marching modulus intensity}

The MMI $0.5^{\circ}$ values are shown in Figure 6.17. Rotor geometry does influence the MMI $0.5^{\circ}$ level of the compounds, which were discharged at a lower temperature. As was reported in Chapter 3.1, the intensity 
of filler-filler interaction, FFR and CR are the major influencing factors on the marching modulus of silica compounds. Different from the tangential rotor, the intermeshing rotor is capable of lowering the fillerfiller interaction more (Figure 6.10) and as a consequence, a slower silica flocculation is found. However, the effect of rotor geometry on the $\mathrm{MMI} 0.5^{\circ}$ level becomes less in the higher dump temperature region. But still, a $5 \%$ lower $\mathrm{MMI} 0.5^{\circ}$ was obtained for the compound mixed with intermeshing mixer.

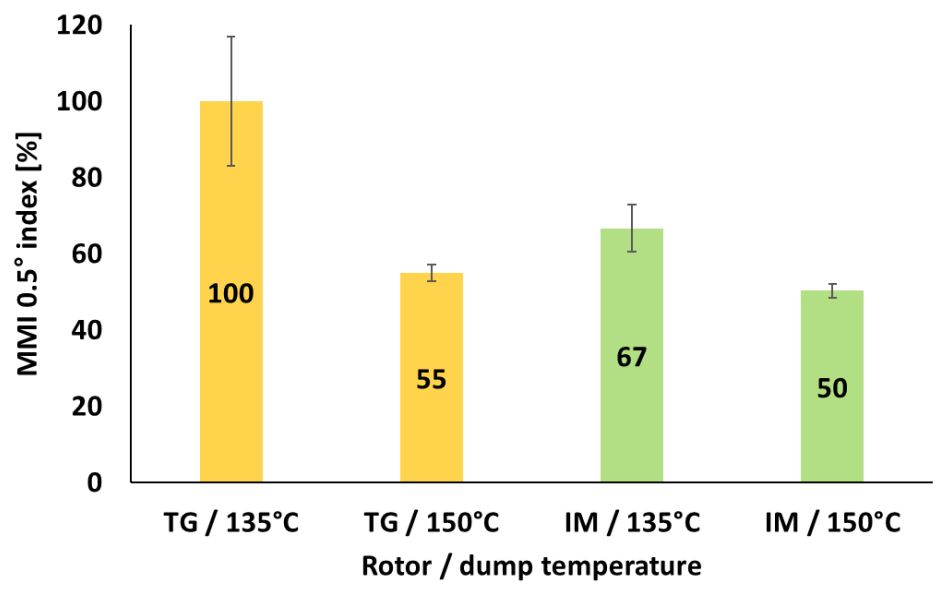

Figure 6.17 $\mathrm{MMI} 0.5^{\circ}$ according to the dump temperature and rotor type.

The correlations between MMI $0.5^{\circ}$ and FFR as well as CR are shown in Figures 6.18(a) and 6.18(b), respectively. $\mathrm{MMI} 0.5^{\circ}$ shows a good correlation with FFR and CR as expected from the previous results.
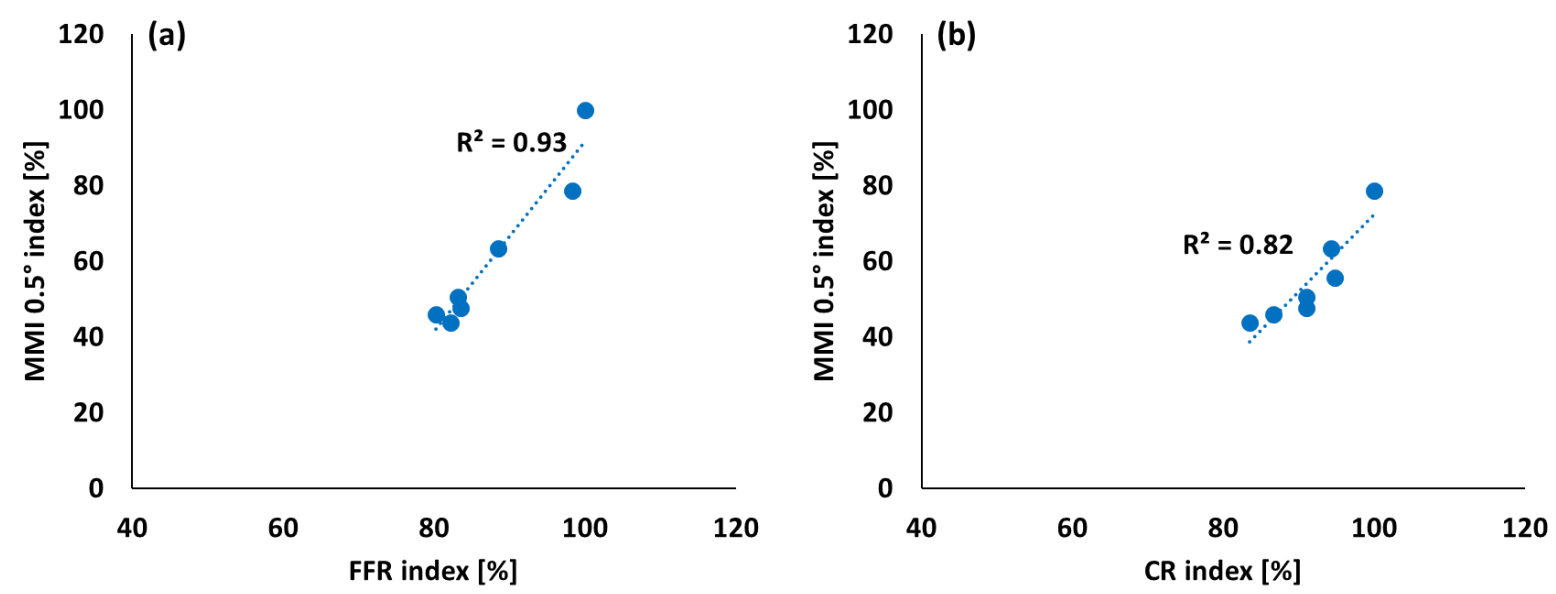

Figure 6.18 $\mathrm{MMI} 0.5^{\circ}$ as a function of FFR and $\mathrm{CR}$; (a): MMI $0.5^{\circ}$ vs. FFR; (b): MMI $0.5^{\circ}$ vs. CR. 


\subsubsection{Silica dispersability (tangential vs. intermeshing rotors)}

The silica dispersability of the two rotor types are compared in terms of macro-dispersion and illustrated in Figure 6.19. As can be seen in this figure, rotor geometry does clearly influence the degree of macrodispersion of silica. The effect of discharge temperature is less than the effect of rotor geometry. This result clearly indicates that intermeshing rotor geometry is better for compound quality in terms of dispersion due to its advanced technical characteristics as depicted in paragraph 6.1.

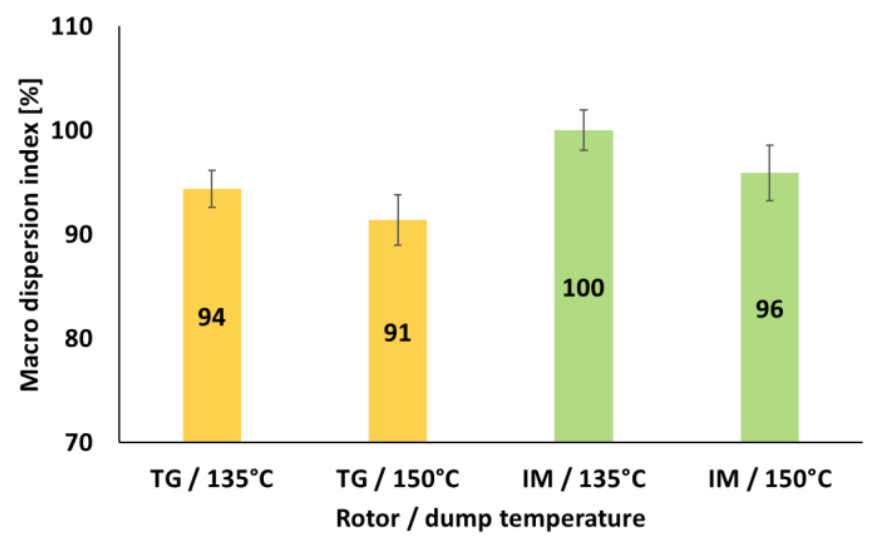

Figure 6.19 Macro-dispersion in correlation to dump temperature and rotor type.

An interesting point is that the macro-dispersion values slightly decrease with increase in discharge temperature. This tendency differs from the Payne effect results: the level of the Payne effect was reduced with increasing mixing temperature, Figure 6.10. No correlation was found between macrodispersion and Payne effect, as shown in Figure 6.20. A further evaluation and discussion of the relation between micro- and macro-dispersion will be done in Chapter 7.

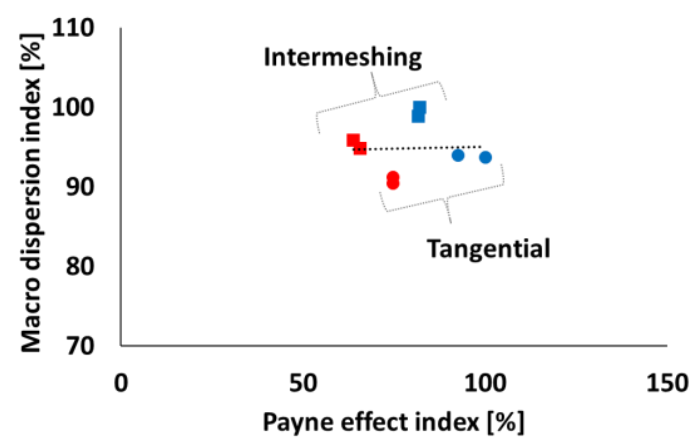

Figure 6.20 Macro-dispersion vs. Payne effect of Series 2; (red dots): high temperature; (blue dots): low temperature; ( $\bullet$ ): tangential; ( $\boldsymbol{\square}$ ): intermeshing.. 


\subsection{Conclusions}

In this study, the effect of mixing parameters such as mixing time and temperature as well as rotor geometry on the marching modulus of silica filler rubber are evaluated on industrial scale. The result of this work confirmed that the basic mechanisms for the marching modulus phenomenon of silica compounds still apply regardless of the mixer scale. The mixing parameters which lead to a proper degree of silanization - silanization time and temperature - reduce the FFR and CR of the compound and thus reduce the $\mathrm{MMI}$.

Rotor geometry turned out as a minor factor on $\mathrm{MMI}$, but a higher degree of micro- and macro-dispersion of silica filler can be achieved by using an intermeshing rotor. It is well known that intermeshing rotor geometry is more effective in distributive and dispersive mixing as well as compound cooling than a tangential one. Thanks to these advantages, an enhanced compound quality can be expected when mixing is done by using an intermeshing rotor geometry.

\section{References}

[1] F. J. Borzenski, Q. Hartley, Rubber World 248, 18 (2013).

[2] "Mixing of rubber compounds", A. Limper, Carl Hanser Verlag, Munich (2012).

[3] D. Berkemeier, W. Haeder, M. Rinker, 157th meeting ACS Rub. Div., Dallas, Texas, Paper No. 6 (2000).

[4] G. S. Donoian, E. L. Canedo, L. N. Valsamis, Rubber Chem. Technol. 65, 792 (1992).

[5] C. Koolhiran, J. White, J. App. Polym. Sci. 78, 1551 (2000).

[6] N. Yamada, K. Takahara, K. Inoue, 157th meeting ACS Rub. Div., Dallas, Texas, Paper No. 4 (2000).

[7] N. Nortey, 172nd meeting ACS Rub. Div., Cleveland, Ohio, Paper No. 124 (2007).

[8] N. O. Nortey, Rubber World 226, 32 (2002).

[9] P. S. Kim, J. L. White, Rubber Chem. Technol. 67, 880 (1994). 


\section{Chapter 7 The relation between macro-/micro-dispersion of silica in rubber matrix and their effect on rubber reinforcement}

\subsection{Introduction}

"Quality starts in mixing" is well known to all tire producers and quoted frequently. ${ }^{[1]}$ Mixing process engineers are regularly questioned concerning the mixing parameter control method in order to obtain a higher degree of micro- as well as macro-dispersion of the filler in the rubber matrix.

The dispersion of fillers such as silica can be divided into two categories: macro- and micro-dispersion. Macro-dispersion can be defined as dispersion of clusters which are in the size range of 2 to $100 \mu \mathrm{m}$. Micro-dispersion refers to dispersion of clusters of silica with a size smaller than $2 \mu \mathrm{m} .{ }^{[2]}$ Both dispersions are important, however, in order to achieve the best reinforcement of rubber, micro-dispersion is most crucial. ${ }^{[3,4]}$

Two basic mechanisms have been proposed, especially for carbon black, to describe the dispersion process. The first mechanism is the agglomerate rupture model: filler agglomerates cleave into two parts and further reduce their size by excess hydrodynamic forces to overcome the cohesive forces of the particles. Another dispersion model is erosion as was proposed by Shiga and Furuta via optical microscopy observations: the Onion peel model. ${ }^{[5-7]}$ They demonstrated that aggregates peel off from the surface of the agglomerates and form tails consisting of a large number of aggregates. Collin and PeuverelDiedier ${ }^{[8,9]}$ used a transparent counter-rotating shear cell which was capable of in-situ observation of carbon black dispersion induced by flow, and found two additional carbon black dispersion processes: debonding (sudden detachment of the matrix from the filler cluster) and collision (detachment of a fragment induced by collision of two or more parent clusters). ${ }^{[8]}$ But they also observed that erosion and rupture were the main mechanisms of carbon black dispersion. Additionally, they reported that the kinetics of erosion depend on the size of the agglomerates. Based on these studies, it is often assumed that micro-dispersion occurs only after the separation of large agglomerates of fillers (macro-dispersion), and that therefore those processes are strongly related.

Blume and Uhrlandt ${ }^{[10]}$ proposed a micro-dispersion mechanism of silica fillers as already shown in Chapter 3.1, Figure 3.8: First, the polymer penetrates into the voids of large clusters. When the polymer has penetrated, silica clusters are situated in a shearing condition and the polymer chains pull off smaller

Parts of the work described in this chapter were published in: J. Jin, W. Kaewsakul, J. W. M. Noordermeer, W. K. Dierkes, A. Blume, International Rubber Conference, London, UK (2019). Another part of this chapter is currently prepared for publication in Rubber Chem. Technol. 
entities from the large clusters. From this point of view, micro-dispersion of silica occurs regardless of the size of the silica cluster, therefore micro- and macro-dispersion seem to be independent.

Luginsland et al. ${ }^{[3]}$ have compared the Payne effect and dispersibility of three different silicas with different surface areas and inter-cluster structures represented by CTAB and DBP numbers, respectively Table 7.1 and Figures 7.1 and 7.2. The Payne effect is a measure of filler-filler interaction, and so indirectly - a measure of micro-dispersion. As can be seen in Figure 7.1, the Payne effects of those silicas do not differ much mutually when a silane - hexadecyltriethoxysilane (HDTES) or bis(triethoxysilylpropyltetrasulfide (TESPT) - is present, though the absolute value depends on the type of silane used. Without any silane, the macroscopic dispersibility results of the compounds with those silicas - measured by the Evonik Topography Test, Figure 7.2 - show significant differences.

Table 7.1 Analytical data of silicas ${ }^{[3]}$

\begin{tabular}{cccc}
\hline & Silica & CTAB $\left[\mathrm{m}^{2} / \mathrm{g}\right]$ & DBP number $[\mathrm{g} / 100 \mathrm{~g}]$ \\
\hline Silica C & (ULTRASIL ${ }^{\circledR}$ VN3 GR) & 167 & 175 \\
Silica D & (ULTRASIL $\left.{ }^{\circledR} 7000 \mathrm{GR}\right)$ & 163 & 210 \\
Silica E & (ULTRASIL $\left.{ }^{\circledR} 7005\right)$ & 177 & 220 \\
\hline
\end{tabular}

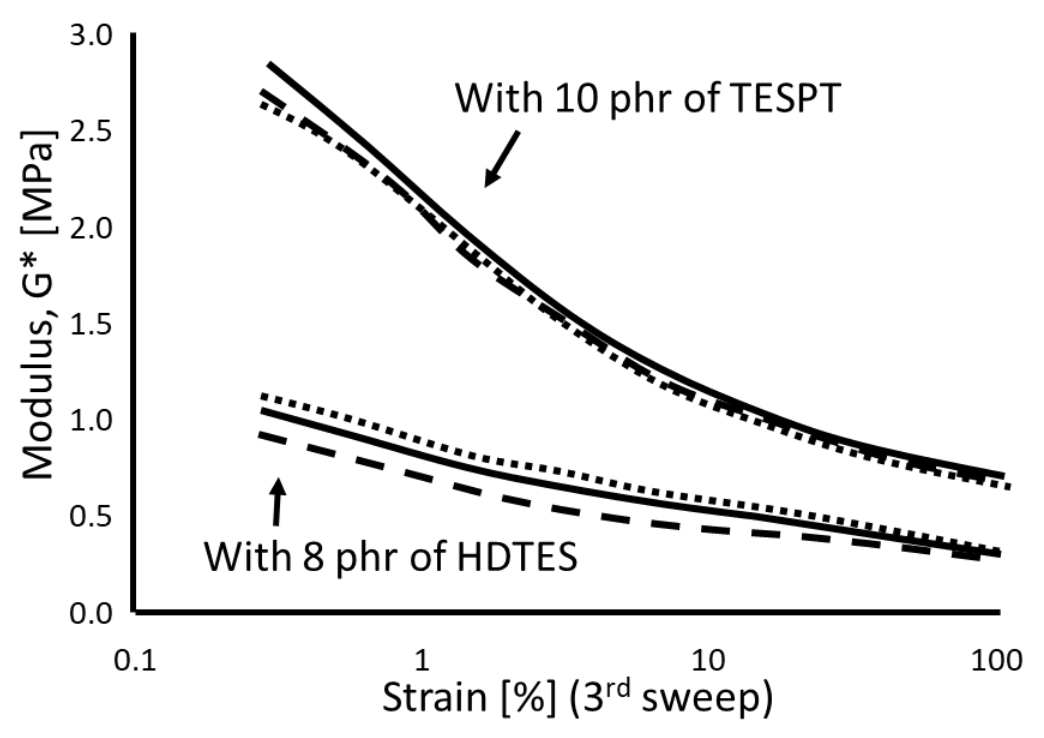

Figure 7.1 Vulcanizate: shear modulus G' vs. strain; compounds filled with 80 phr of silica and silanes in a blend of S-SBR (70 phr) and BR (30 phr); ( ......... ) Silica C; ( ..... ): Silica D; ( _ ) : Silica E. ${ }^{[3]}$ 


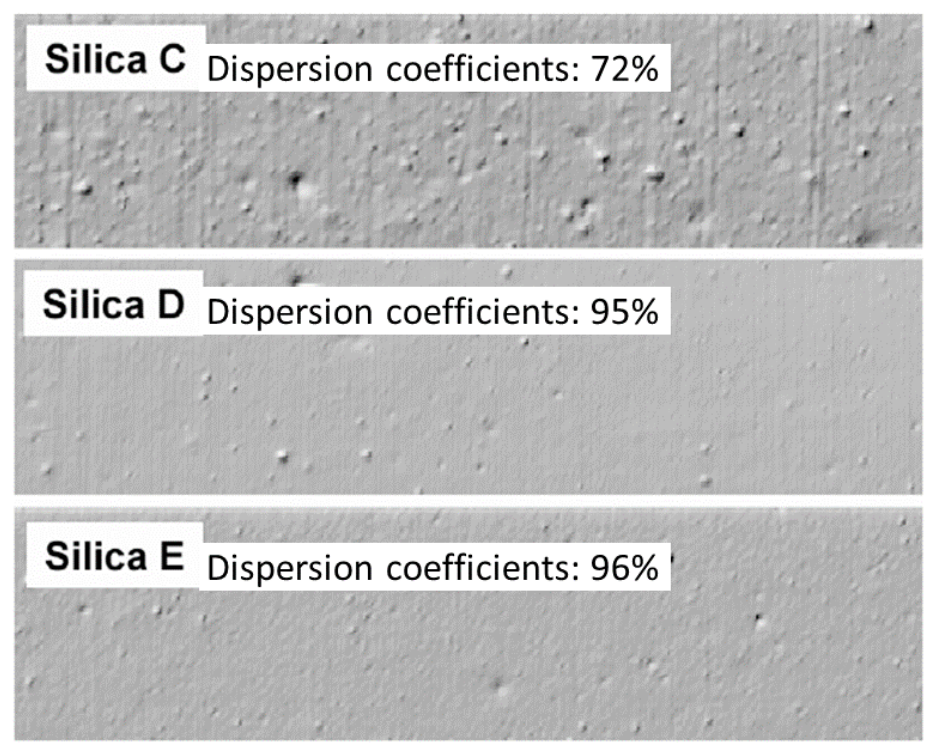

Figure 7.2 Dispersion behavior in E-SBR compound filled with 60 phr of silica C, D and E without silane. ${ }^{[3]}$

These results indicate that micro- and macro-dispersion are independent. In order to confirm the relation or independence between micro- and macro-dispersion of silica and their effect on rubber reinforcement effect, four series of mixing trials are performed. The details of the series of mixing trials are as follows:

- Series 1: The effect of silanization temperature $\left(130^{\circ} \mathrm{C}^{\sim} 160^{\circ} \mathrm{C}\right)$ and presence of silane;

- Series 2: The effect of silanization time ( $0 \mathrm{sec} \sim 250 \mathrm{sec})$;

- Series 3: The effect of the amount of silane at typical silanization temperature $\left(140^{\circ} \mathrm{C}\right.$, the temperature was selected based on the results of Series 1);

- Series 4: The effect of silica characteristics (surface area and structure).

The degrees of micro- and macro-dispersion for all series are evaluated by measuring the Payne effect and using the Dispergrader, respectively. The Payne effect of silica-filled rubber compounds is generally used as an indicator of the degree of filler-filler interactions. ${ }^{[3,11,12]}$ When a rubber is filled with a reinforcing filler, filler-filler interactions take place. Silica fillers have a high amount of -OH silanol-groups on their surface and thus form a strong filler-filler network in the rubber matrix via hydrogen bonding. ${ }^{[13]}$ In general, the storage modulus of such filled rubber compounds decreases with increasing strain amplitude due to the breakdown of the filler network. This effect is commonly known as the Payne effect, which is obtained from the difference in storage modulus at low and high strain amplitude. ${ }^{[11,12]}$ Sarkawi et al. ${ }^{[14]}$ and Seo et al. ${ }^{[15]}$ compared the results of Payne effect and the images obtained from Transmission 
Electron Microscopy (TEM) and reported that both results are comparable. Thus, the Payne effect was used as an indicator of silica micro-dispersion in the present work. Scanning Electron Microscope (SEM) is used for Series 1 for the visual analysis of micro-dispersion behavior of silica filler. TEM is used for Series 4 in order to obtain the general morphology images for ConVentional silica (CV) and Highly-Dispersible (HD) silica. For the last, the effect of micro- and macro-dispersion of silica on rubber reinforcement was investigated.

\subsection{Experimental}

\subsubsection{Materials}

All series of experiments were done based on a tire tread compound as shown in Table 7.2. Blends of oilextended solution styrene-butadiene rubber (S-SBR, Mooney viscosity (ML1+4@100 ${ }^{\circ}$ ) of 65 and composed of $75 \%$ butadiene with a vinyl-content of $50 \%$ and $25 \%$ styrene-content, extended with 37.5 phr of TDAE oil), and high cis-1,4 polybutadiene rubber (BR, Mooney viscosity (ML1+4@100 ${ }^{\circ} \mathrm{C}$ ) of 44 and a cis-1,4 content of $96 \%$ ) were used in this study. The classification of the different types of silica, as ConVentional silica (CV) and Highly-Dispersible (HD) silica, is listed in Table 7.3. Bis(triethoxysilylpropyl)tetrasulfide (TESPT) was used as silane coupling agent. The amount of TESPT applied in the formulations of COM4 and COM5 was adjusted according to Equation 3.1, based on the CTAB surface area of the Silicas 2 and 3: ${ }^{[16]}$ 
Table 7.2 Compound formulation

\begin{tabular}{|c|c|c|c|c|c|c|c|c|}
\hline $\begin{array}{l}\text { Mixing } \\
\text { stage }\end{array}$ & Ingredient & Product name & Company & $\begin{array}{l}\text { COM1 } \\
\text { (phr) }\end{array}$ & $\begin{array}{l}\text { COM2 } \\
\text { (phr) }\end{array}$ & $\begin{array}{l}\text { COM3 } \\
\text { (phr) }\end{array}$ & $\begin{array}{c}\text { COM4 } \\
\text { (phr) }\end{array}$ & $\begin{array}{l}\text { COM5 } \\
\text { (phr) }\end{array}$ \\
\hline \multirow{10}{*}{$\begin{array}{l}\text { Master } \\
\text { batch }\end{array}$} & S-SBR* & $\begin{array}{c}\text { BUNA VSL5025- } \\
2 \mathrm{HM}\end{array}$ & Lanxess & 110 & $\rightarrow$ & $\rightarrow$ & $\rightarrow$ & $\rightarrow$ \\
\hline & $\mathrm{BR}$ & BUNA CB24 & Lanxess & 20 & $\rightarrow$ & $\rightarrow$ & $\rightarrow$ & $\rightarrow$ \\
\hline & Silica 1 & ULTRASIL ${ }^{\circledR} 7005$ & Evonik & 90 & $\rightarrow$ & $\rightarrow$ & - & - \\
\hline & Silica 2 & Zeosil 1165MP & Solvay & - & - & - & 90 & - \\
\hline & Silica 3 & $\begin{array}{c}\text { ULTRASIL }{ }^{\circledR} \mathrm{VN3} \\
\text { GR }\end{array}$ & Evonik & - & - & - & - & 90 \\
\hline & TESPT & Si $69{ }^{\circledR}$ & Evonik & 8.0 & - & 4.0 & 7.3 & 8.0 \\
\hline & TDAE Oil & VIVATEC 500 & $\begin{array}{l}\text { Hansen \& } \\
\text { Rosenthal }\end{array}$ & 5 & $\rightarrow$ & $\rightarrow$ & $\rightarrow$ & $\rightarrow$ \\
\hline & Stearic acid & - & Merck & 1 & $\rightarrow$ & $\rightarrow$ & $\rightarrow$ & $\rightarrow$ \\
\hline & Zinc Oxide & - & Merck & 2 & $\rightarrow$ & $\rightarrow$ & $\rightarrow$ & $\rightarrow$ \\
\hline & DPG & Perkacit DPG & Flexsys & 1.5 & $\rightarrow$ & $\rightarrow$ & $\rightarrow$ & $\rightarrow$ \\
\hline \multirow{3}{*}{ Final } & Sulfur & $S$ & J. T Baker & 0.7 & $\rightarrow$ & $\rightarrow$ & $\rightarrow$ & $\rightarrow$ \\
\hline & ZBEC & Vulkacit ZBEC & Lanxess & 0.2 & $\rightarrow$ & $\rightarrow$ & $\rightarrow$ & $\rightarrow$ \\
\hline & CBS & Santocure CBS & Flexsys & 2.2 & $\rightarrow$ & $\rightarrow$ & $\rightarrow$ & $\rightarrow$ \\
\hline \multicolumn{4}{|c|}{ Used for the Series } & $1,2,3$ & 1,3 & 3 & 4 & 4 \\
\hline
\end{tabular}

Table 7.3 Analytical data of silicas corresponding to Table 7.2

\begin{tabular}{ccccc}
\hline Sample code & Class & BET $\left[\mathrm{m}^{2} / \mathrm{g}\right]$ & CTAB $\left[\mathrm{m}^{2} / \mathrm{g}\right]$ & *OAN number $[\mathrm{ml} / 100 \mathrm{~g}]$ \\
\hline Silica 1 & HD Silica & 180 & 177 & 223 \\
Silica 2 & HD Silica & 155 & 152 & 205 \\
Silica 3 & CV Silica & 175 & 167 & 176 \\
\hline
\end{tabular}

*Oil Absorption Number: the value represents structure of silica. A higher number corresponds to a more developed structure. 


\subsubsection{Mixing}

The compounds were mixed according to the methods as described in Chapter 4-1: Tables 4.2 and 4.3. The target hold temperature for silanization of all series are listed in Table 7.4. The mixing finger prints are depicted in Figures 7.3 - 7.6; In Series 1, both COM1 and 2 were mixed with Mixing procedure A; in Series 2, COM1 was mixed with Mixing procedure B; in Series 3, COM1, 2 and 3 were mixed with Mixing procedure $\mathrm{A}$, but with a fixed silanization temperature of $140^{\circ} \mathrm{C}$ for a purpose to be discussed later on. $\mathrm{A}$ short silanization time was applied for $\mathrm{COM} 3$, because the compound crumbled when the silanization time for $\mathrm{COM} 3$ exceeded 50 seconds. In Series 4, COM4 and 5 were mixed with Mixing procedure $A$ at a fixed silanization temperature of $150^{\circ} \mathrm{C}$. The reproducibility was good in all cases as judged based on the mixing fingerprints to practically coincide: Figures 7.3 - 7.6.

Table 7.4 Target silanization or hold temperature for the experimental series

\begin{tabular}{c|c|c}
\hline Series \# & COM \# & Target silanization or hold temperature \\
\hline 1 & 1,2 & $130,140,150,160^{\circ} \mathrm{C}$ \\
2 & 1 & $150^{\circ} \mathrm{C}$ \\
3 & $1,2,3$ & $140^{\circ} \mathrm{C}$ \\
4 & 4,5 & $150^{\circ} \mathrm{C}$ \\
\hline
\end{tabular}
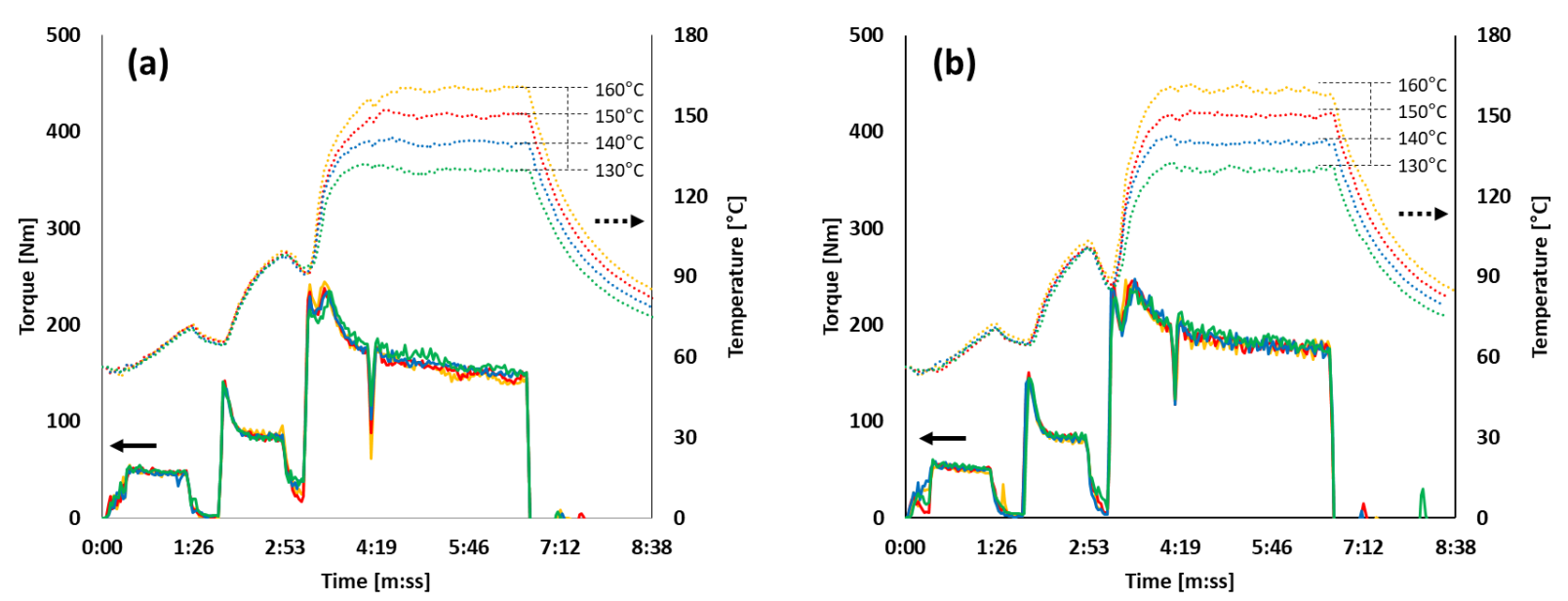

Figure 7.3 Mixing fingerprints of Series 1; (a): COM1; (b): COM2;

$$
(\square): 130^{\circ} \mathrm{C} ;(\square): 140^{\circ} \mathrm{C} ;(\square): 150^{\circ} \mathrm{C} ;(-): 160^{\circ} \mathrm{C} \text {. }
$$




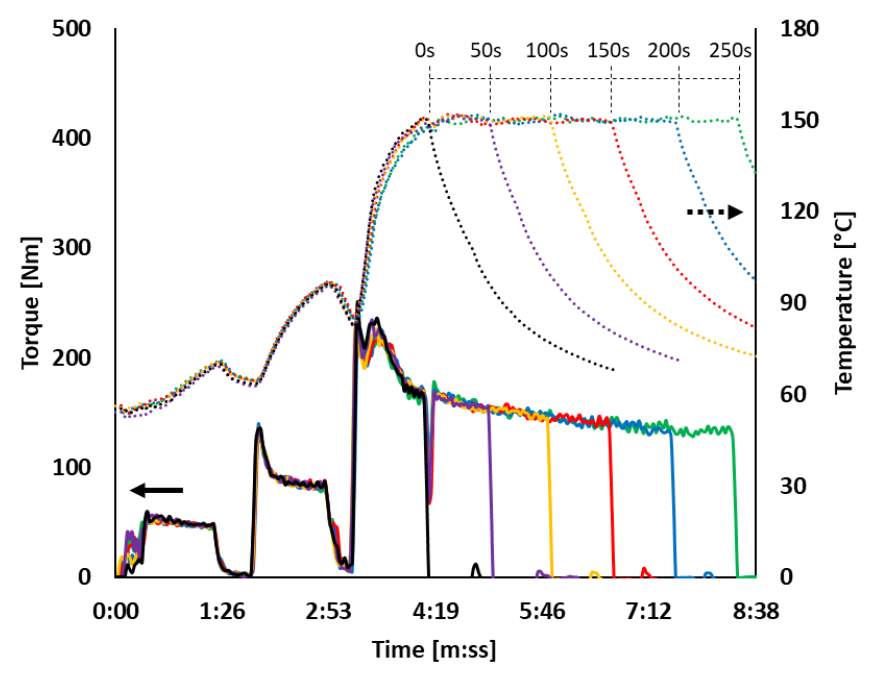

Figure 7.4 Mixing fingerprints of Series 2;

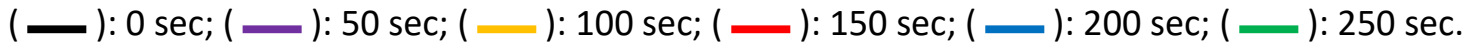

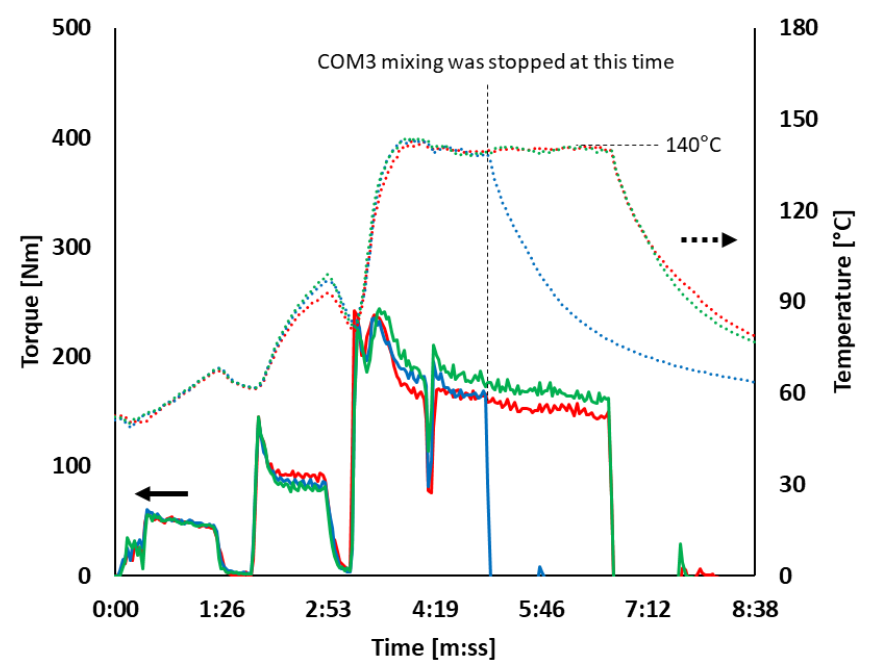

Figure 7.5 Mixing fingerprints of Series 3;

( ): COM1, 8phr of TESPT; ( 1 ): COM3, 4phr of TESPT; ( 1 ): COM2, 0 phr of TESPT. 


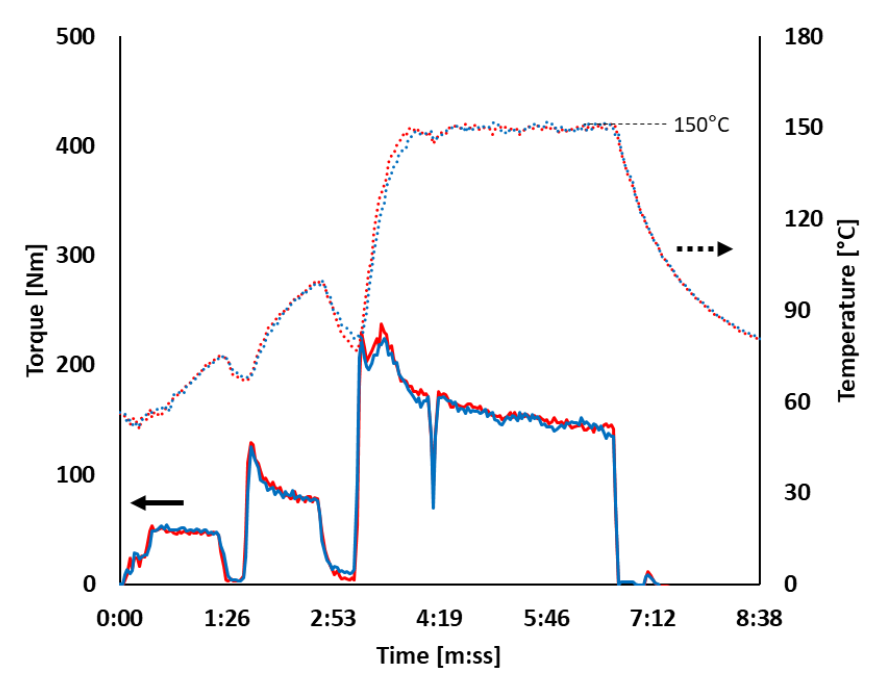

Figure 7.6 Mixing fingerprints of Series 4; (_ $)$ : COM4, HD silica; ( _ $)$ ): COM5, CV silica.

\subsubsection{Micro-dispersion of silica by Payne effect}

The Payne effect of the uncured rubber compounds was evaluated by using a Rubber Process Analyzer (RPA; RPA2000, Alpha Technologies for Series 1 / RPA Elite, TA Instruments for Series 2 - 4) according to the methods as described in Chapter 3.1.

\subsubsection{Macro-dispersion of silica by dispergrader}

A Dispergrader can measure the macro-dispersion of fillers based on ASTM D7723-11. ${ }^{[17]}$ The dispersion is assessed as the percentage of white area related to the total area of the scanned image. In the present work, the macro-dispersion of silica in the compounds was analyzed by using a DisperGRADER Alpha View (Alpha Technologies). The compounds were first fully vulcanized in order to obtain a flat and clear surface during sample cutting. The macro-dispersion values from five different points on each of the 3 similar samples were measured: in total, 15 measurements were averaged per mixing condition.

\subsubsection{Micro-dispersion mechanism visualization by Scanning Electron Microscope}

The dispersion behavior of the silica in a rubber matrix was visualized by using Scanning Electron Microscope (SEM, Jeol JSM-6400) with Energy Dispersive Spectroscopy system (EDS; configured with a Noran energy dispersive X-ray analyzer). The vulcanized sample of COM1 and COM2 for macro-dispersion measurement were extracted for five days in refluxing acetone in order to remove oils and remaining curatives. After the extraction, the samples were dried overnight using a vacuum oven running at $110^{\circ} \mathrm{C}$ 
in order to remove residual solvent. Subsequently, fresh surface of the cut samples was sputter coated with gold for three minutes under high vacuum. EDS was used in order to find the place where the silica clusters are positioned: Figures 7.7 and 7.8.

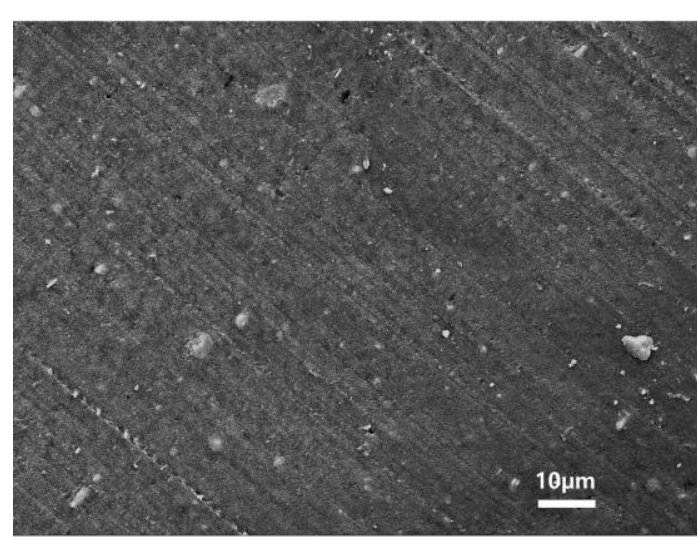

(a)
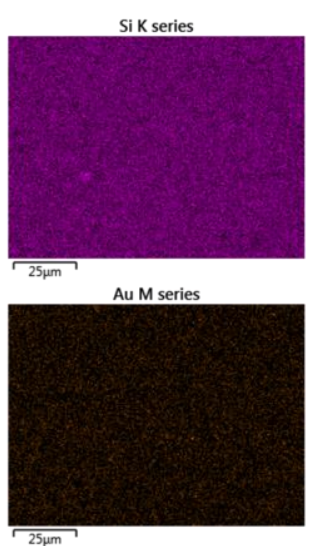
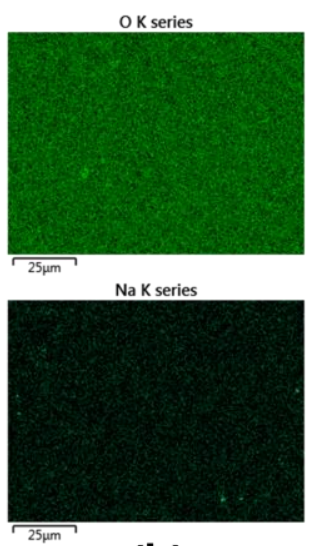

(b)
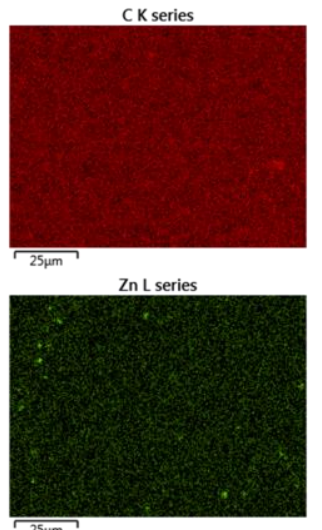

$25 \mathrm{~mm}$

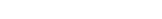

Figure 7.7 SEM and EDS images of COM1 (with TESPT) mixed at $150^{\circ} \mathrm{C}$ of silanization temperature;

(a): SEM image; (b): EDS images of (a).

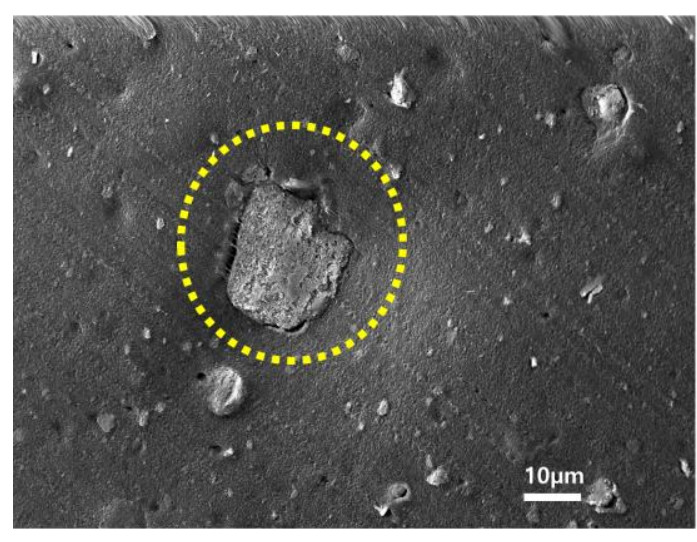

(a)

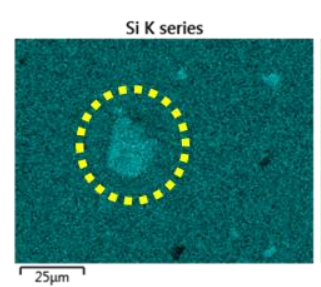

Au M series

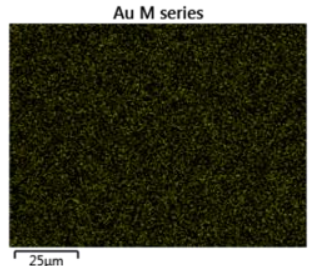

$\sqrt{25 \mu \mathrm{m}}$
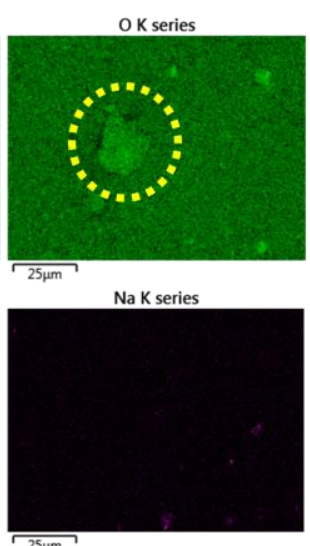

(b)

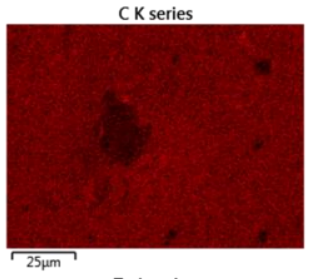

$\mathrm{Zn} L$ series

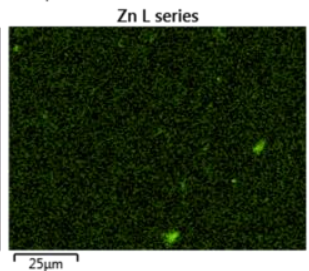

$25 \mu \mathrm{m}$

Figure 7.8 SEM and EDS images of COM2 (without TESPT) mixed at $150^{\circ} \mathrm{C}$ of silanization temperature;

(a): SEM image; (b): EDS images of (a).

\subsubsection{Observation of the silica morphology (TEM analysis)}

In order to observe the morphology of the HD and CV silicas for Series 4, Bright Field TEM (BF-TEM) analysis was performed in a Philips 300ST-FEG TEM at an acceleration voltage of $300 \mathrm{kV}$. Silica-ethanol 
suspensions were prepared by ultrasonification of $50 \mathrm{ml}$ of ethanol after addition of approximately 0.1 grams of Silicas 2 and 3. A droplet $(0.2 \mu \mathrm{l})$ of suspensions were placed on a holey carbon film on a TEM grid dried in order to remove ethanol. Images were recorded using the GATAN Ultrascan1000 $(2 \mathrm{k} \times 2 \mathrm{k}$ CCD $)$ camera.

\subsubsection{Vulcanization and tensile tests}

Tensile tests were performed only for Series 1 and 2 according to the methods described in Chapter 3. In this study, the compounds were cured with rather long and fixed times -30 minutes at $160^{\circ} \mathrm{C}-$ in order to vulcanize the samples properly because the optimum cure time calculation method used for Chapter 3 could not be applied for COM2: no clear tangential lines could be obtained (Figure 7.9). The reinforcement index was calculated in order to evaluate the effect of micro- and macro-dispersion on the reinforcement. The value of M200/M100 was used as a measure of reinforcement index instead of the more common $\mathrm{M} 300 / \mathrm{M} 100$, because some of the samples broke before they reached the $300 \%$ strain.

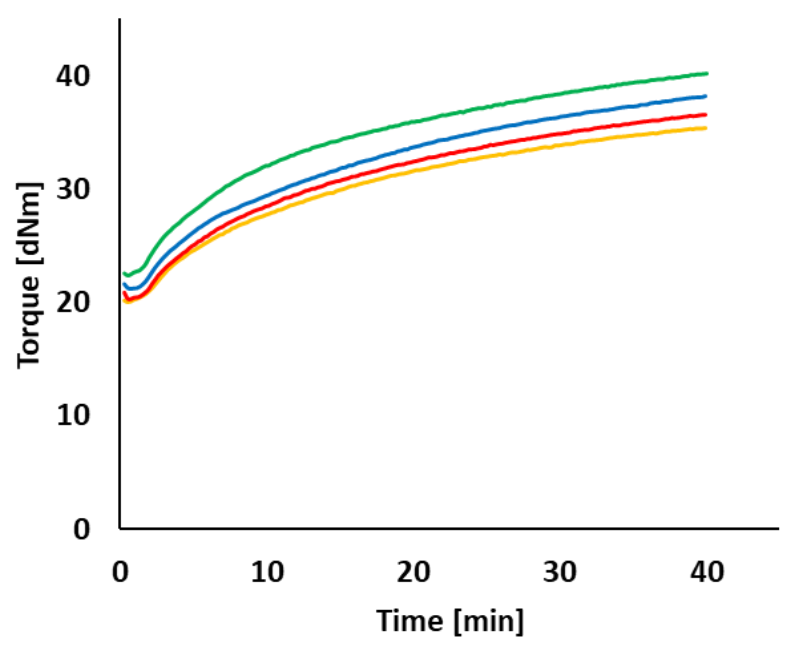

Figure 7.9 Rheogram of $\operatorname{COM} 2$ in Series $1 ;\left(\_\right): 130^{\circ} \mathrm{C} ;\left(\_\right): 140^{\circ} \mathrm{C} ;\left(\_\right): 150^{\circ} \mathrm{C} ;\left(\_\right): 160^{\circ} \mathrm{C}$.

\subsection{Results and discussion}

\subsubsection{Relation between micro- and macro-dispersion}

\subsubsection{The effect of silanization temperature and presence of silane (Series 1)}

The micro- and macro-dispersions of COM1 (with TESPT) and 2 (without TESPT) with different silanization (COM1) or hold (COM2) temperatures are compared in Figure 7.10. COM1 (with TESPT) shows a very low 
level of Payne effect for the whole range of silanization temperatures compared to COM2 (without TESPT). A decreasing Payne effect is observed in both series with increasing hold temperature which means for COM1 an increasing degree of silanization; $\mathrm{COM} 2$ does not contain silane. The fact that still some decrease in Payne effect is seen even in absence of TESPT can be explained by the micro-dispersion mechanism of silica: Figure 3.8. ${ }^{[2,10]}$
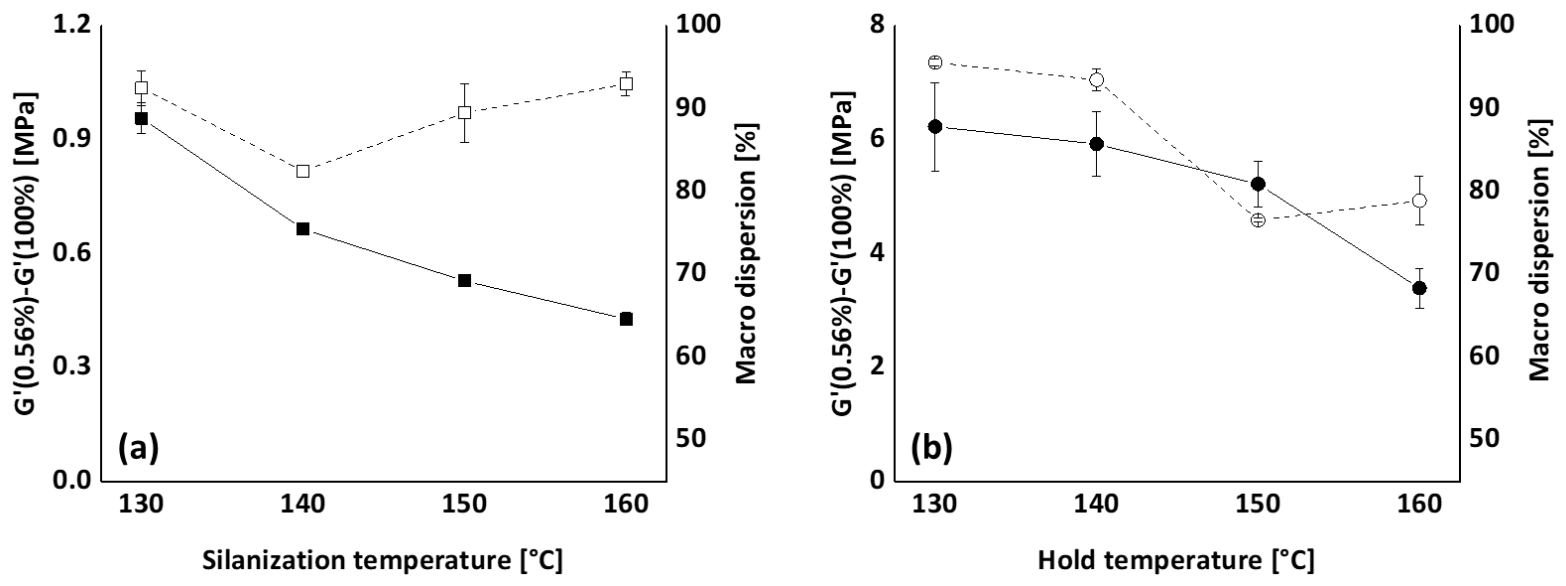

Figure 7.10 Relation between micro-dispersion (Payne effect) and macro-dispersion of a silica filled SSBR/BR compound mixed at different silanization temperatures; (a): COM1; (b): COM2;

$(\square$ ): Payne effect; ( $\square-\square, 0--O)$ ): macro-dispersion.

At higher temperatures, the polymer mobility is enhanced, either by lower viscosity or possibly by some polymer breakdown. This implies that the polymer can more easily penetrate into the voids of the large clusters. Consequently, clusters are better separated by the polymer, resulting in a better microdispersion of silica: Figure 7.11.

A higher degree of macro-dispersion for COM1 (with TESPT) and an increasing trend in macro-dispersion along with increasing silanization temperature were expected. However, the highest macro-dispersion is seen for COM2 (without TESPT) mixed at the lower temperatures of $130^{\circ} \mathrm{C}$ and $140^{\circ} \mathrm{C}$. 


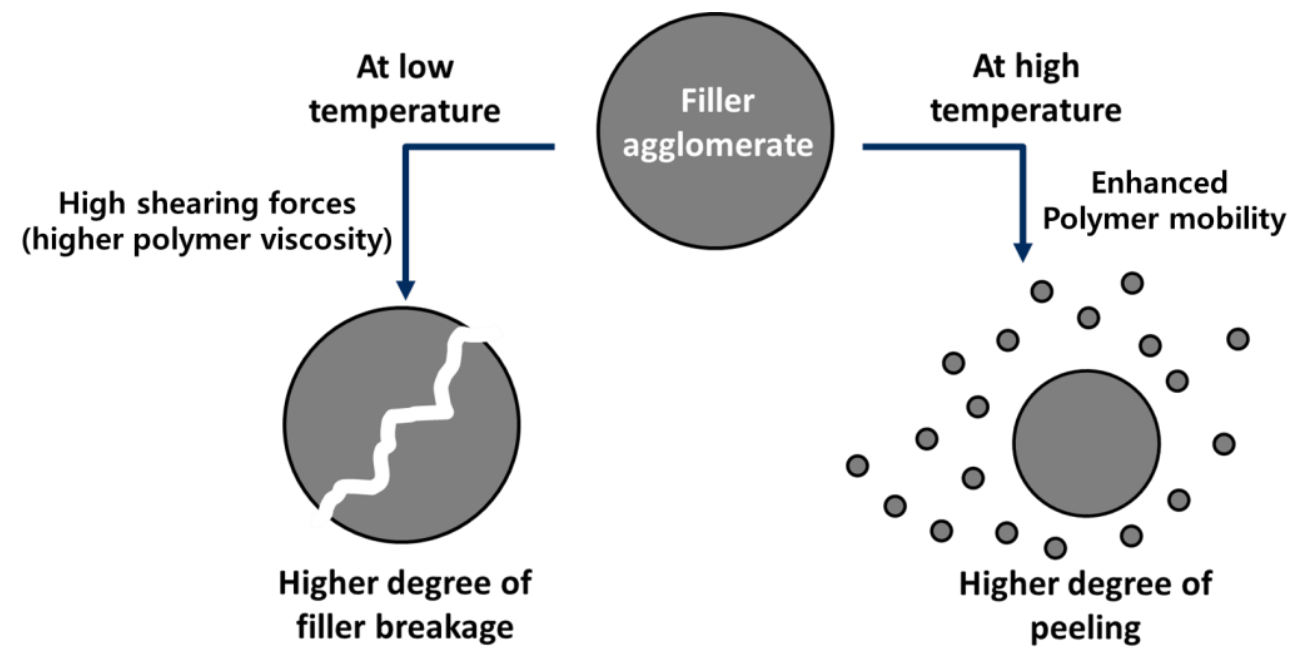

Figure 7.11 Macro- and micro-dispersion mechanism at different mixing temperature regions.

At lower mixing temperature the viscosity of the rubber polymer is higher, thus mechanical shearing is more enhanced, assuming that there are no other factors which affect the viscosity of the rubber polymer. ${ }^{[18]}$ Therefore, the macro-dispersion of the compounds without TESPT decreases with increasing hold temperature: Figure 7.10(b).

The curve progression is more complex for COM1 (with TESPT): Figure 7.10(a). The level of macrodispersion drops at $140^{\circ} \mathrm{C}$, after that gradually increases with increasing silanization temperature. In this case, the macro-dispersion of silica can be influenced not only by changes in the compound viscosity induced by temperature, but also by the silanization. When the silica is shielded by silane, the filler becomes more compatible with the polymer and this leads to a reduction of the filler network. This results first in a lower viscosity again. A silanization temperature of $130^{\circ} \mathrm{C}$ corresponds to the beginning of the silanization reaction with TESPT. ${ }^{[18,19]}$ So, even though the viscosity of the rubber at that temperature is relatively high, the degree of silanization is low. At $140^{\circ} \mathrm{C}$ the viscosity of the rubber polymer drops and results in a lower macro-dispersion like in the case of COM2, Figure 7.10(b).

At a higher temperature, the efficiency of silanization of the silica comes into play. Even though the polymer viscosity is reduced with increasing mixing temperature, the silica becomes more compatible with the rubber polymer due to the better hydrophobation of the silica surface induced by silanization. This does indeed enhance the macro-dispersion to some extent, together with the micro-dispersion. Macro-dispersion images for the compounds of Figure 7.10, obtained from the Dispergrader, are shown in Figure 7.12. As can be seen in this figure, the macro-dispersion of silica changes differently along the mixing temperature and presence of TESPT depending on the type of compound. 


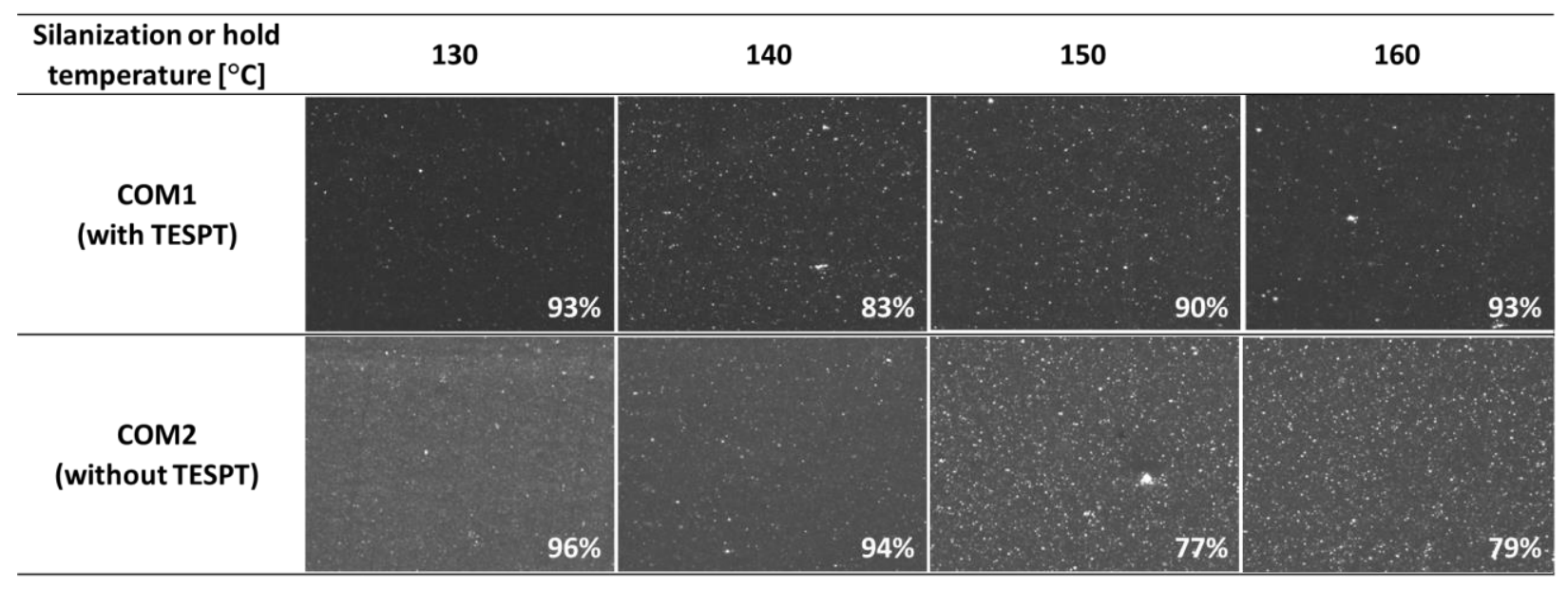

Figure 7.12 Macro-dispersion images and values for compounds of Figure 7.9.

\subsubsection{Visual analysis of undispersed silica clusters by SEM}

The penetration of the polymer into the voids of silica is observed for the SEM images of COM2 mixed at $150^{\circ} \mathrm{C}$; As was seen in Figure 7.7(a), silica clusters of COM1 are practically invisible because all are covered with polymer and all clusters are properly dispersed. However, although there was no silane at all in COM2, connecting filaments of rubber polymer were observed for the boundary between the silica cluster and the rubber phase (Figure 7.13(b)) as well as inside the cluster (Figure 7.13(c)): similar to stretched natto (Japanese food), Figure 7.14. With this model, a simultaneous process for macro- and microdispersion of silica is explainable. It is assumed that the pulling out of small or large clusters from the mother clusters will be enhanced by the presence of silane due to a higher intensity of polymer-filler interaction induced by filler-polymer coupling: higher silanization temperature finally results in better macro-dispersion: Figure 7.10(a). Additionally, these SEM images support the micro-dispersion mechanism of silica suggested by Blume and Uhrlandt (Figure 3.8): the more penetration of polymer into silica cluster void results in enhanced macro- and micro-dispersion of silica. 


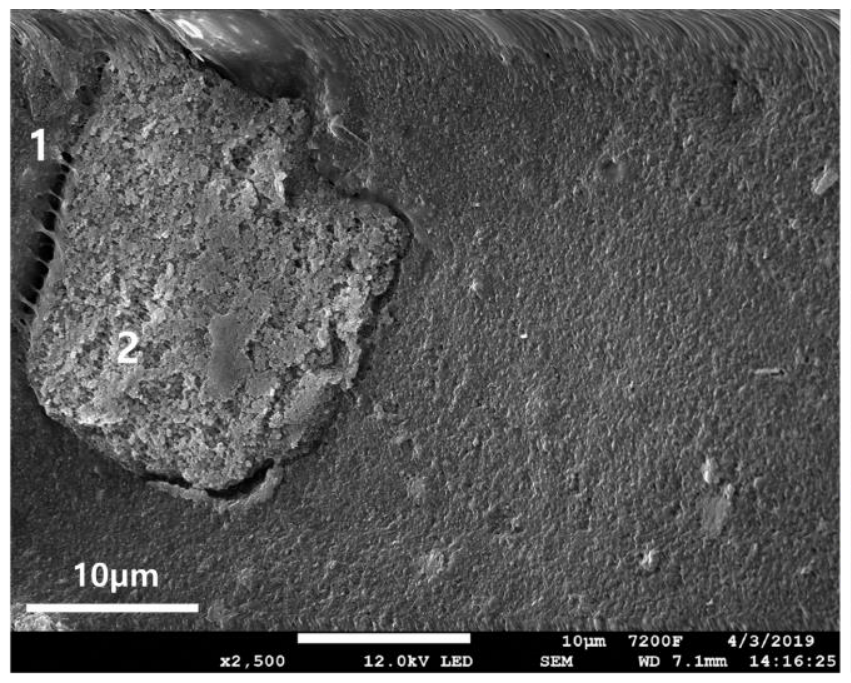

(a)

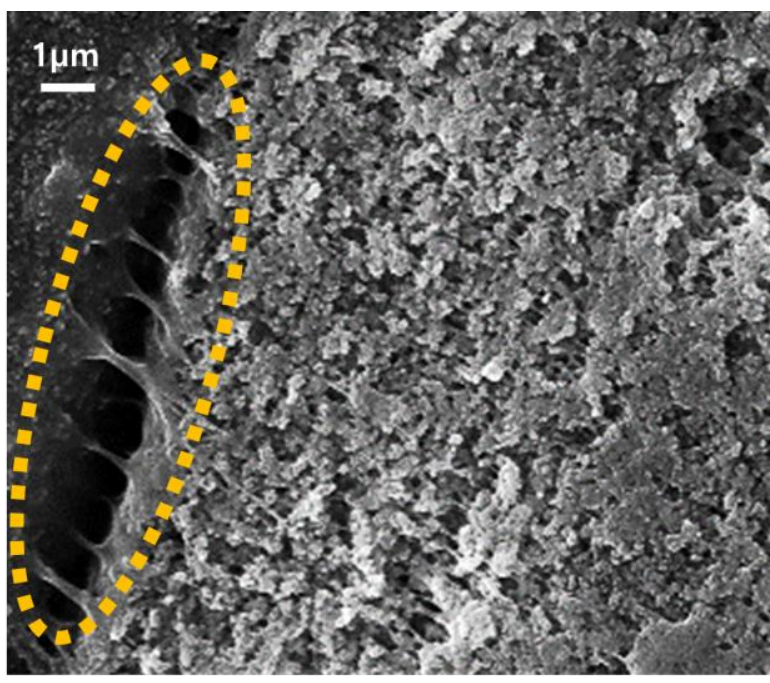

(b)

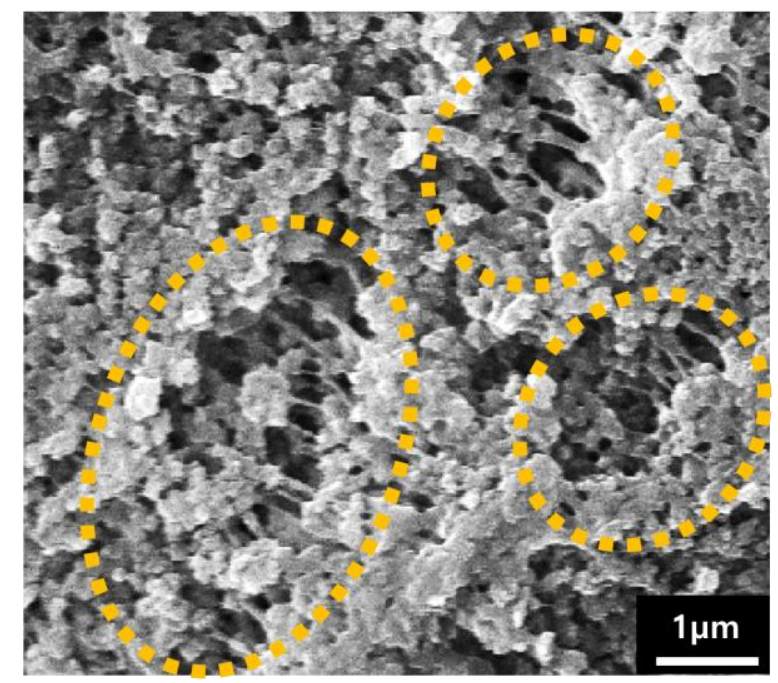

(c)

Figure 7.13 SEM images of (a) undispersed silica cluster of COM2 (without TESPT) mixed at $150^{\circ} \mathrm{C}$ of hold temperature; (b): magnified images of position 1 of (a); (c): magnified images of position 2 of (a); the scale bar magnification of (b) and (c) is adjusted according to the expansion ratio compared to (a). 


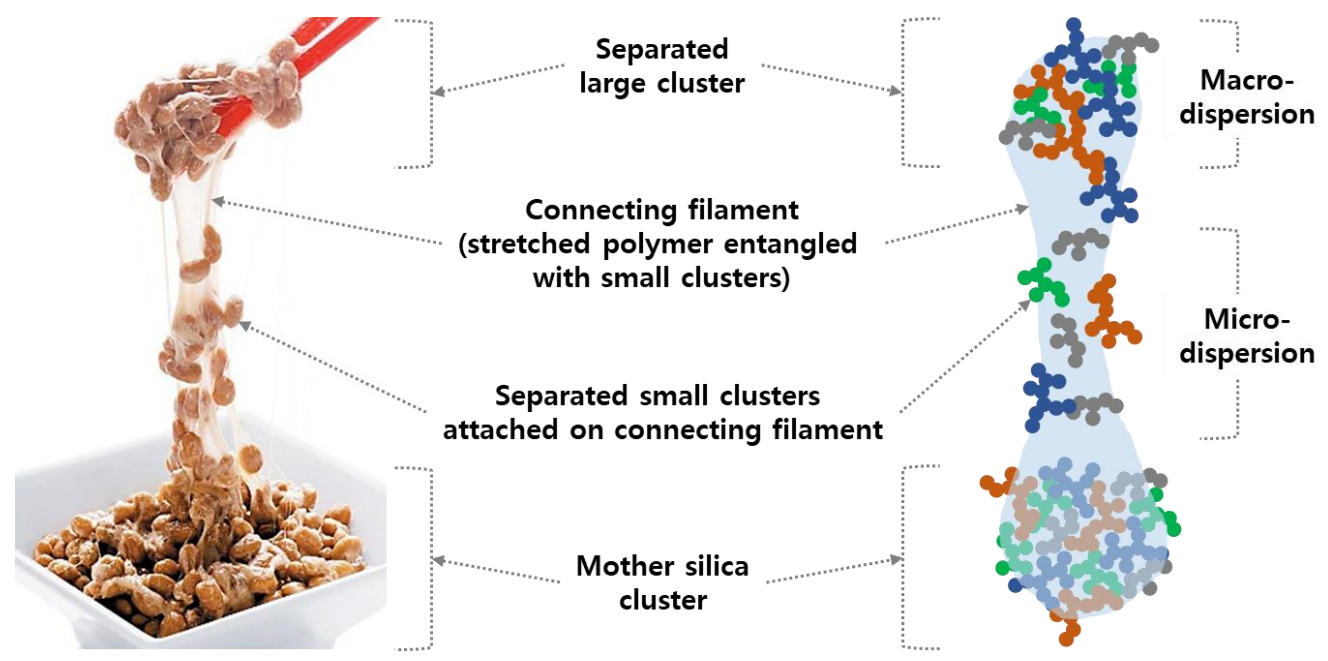

(a)

(b)

Figure 7.14 Stretched natto food effect; (a): stretched natto ${ }^{[20]}$; (b): model for silica cluster destruction during mixing process based on SEM analysis.

\subsubsection{The effect of silanization time (Series 2)}

In this part of the study, the micro- and macro-dispersions of COM1 (with TESPT) with different silanization times are compared: Figures 7.15 and 7.16. The Payne effect is strongly reduced with increasing silanization time, while the level of macro-dispersion stays almost the same. This result indicates that the macro- and micro-dispersion processes of fillers during mixing happen simultaneously, but independently.

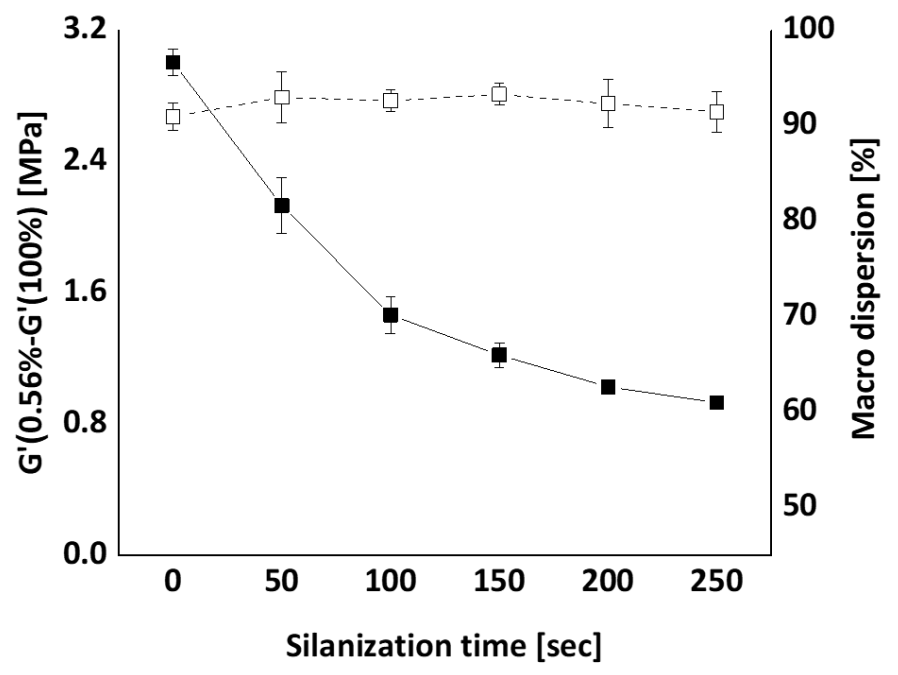

Figure 7.15 Micro-dispersion (Payne effect) and macro-dispersion of COM1 mixed with Mixing procedure B; ( $\square$ ): Payne effect; $(\square-\square)$ ): macro-dispersion. 


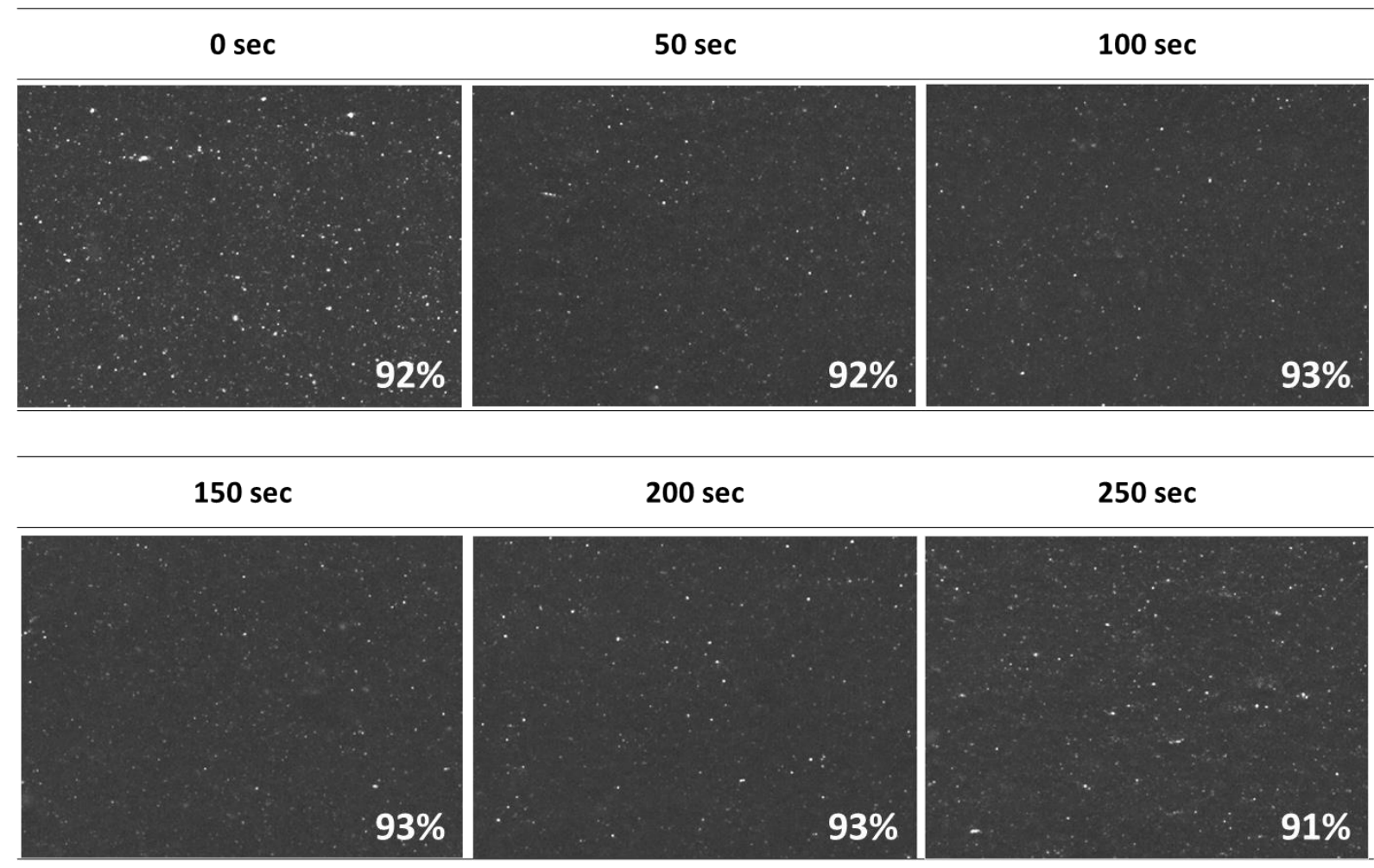

Figure 7.16 Macro-dispersion images and values corresponding to Figure 7.15.

\subsubsection{The effect of the amount of silane at typical silanization temperature (Series 3)}

COM1 (with 8 phr of TESPT), COM2 (without TESPT) and COM3 (with half (4 phr) of TESPT compared to COM1) are compared to quantify the effect of the amount of coupling agent TESPT in more detail. $140^{\circ} \mathrm{C}$ was selected for the silanization according to Figure 7.10, where COM1 (with TESPT) showed the lowest macro-dispersion level of all series, while the macro-dispersion of COM2 (without TESPT) was close to highest level. The results of Series 3 are shown in Figure 7.17, and the macro-dispersion images are depicted in Figure 7.18. The compounds with half silane (COM3) or no silane at all (COM2) show a higher macro-dispersion than the compounds with the full amount of TESPT (COM1). Especially for COM3, a higher level of macro-dispersion is obtained, even though less than 50 seconds of silanization time was applied, while the others had 150 seconds of silanization time. However, the micro-dispersion is still greatly enhanced - reduced Payne effect - with increasing amount of TESPT. 


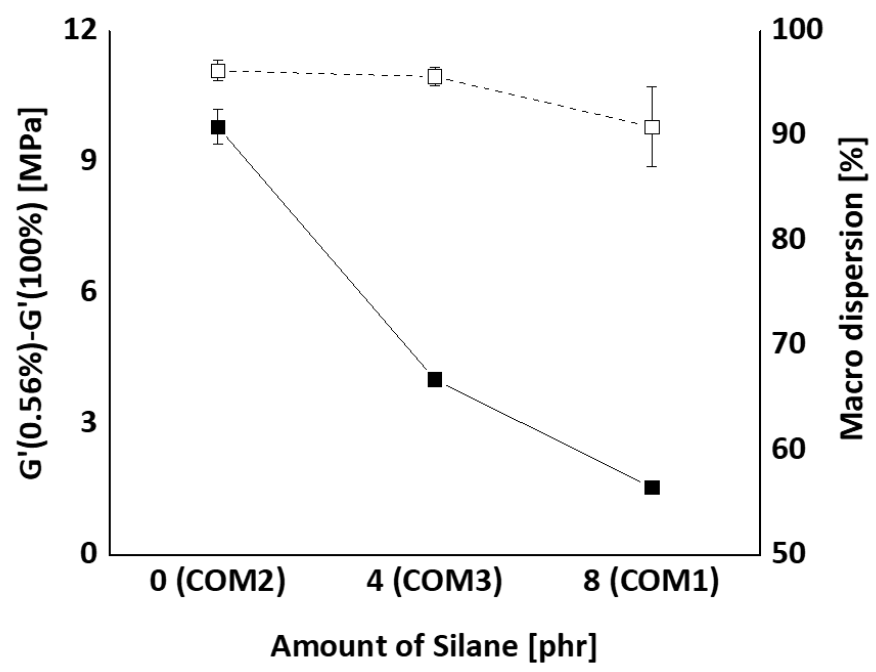

Figure 7.17 Relation between micro-dispersion (Payne effect) and macro-dispersion of compounds with different amounts of silane; ( $\square$ ): Payne effect; ( $\square--\square$ ): macro-dispersion.

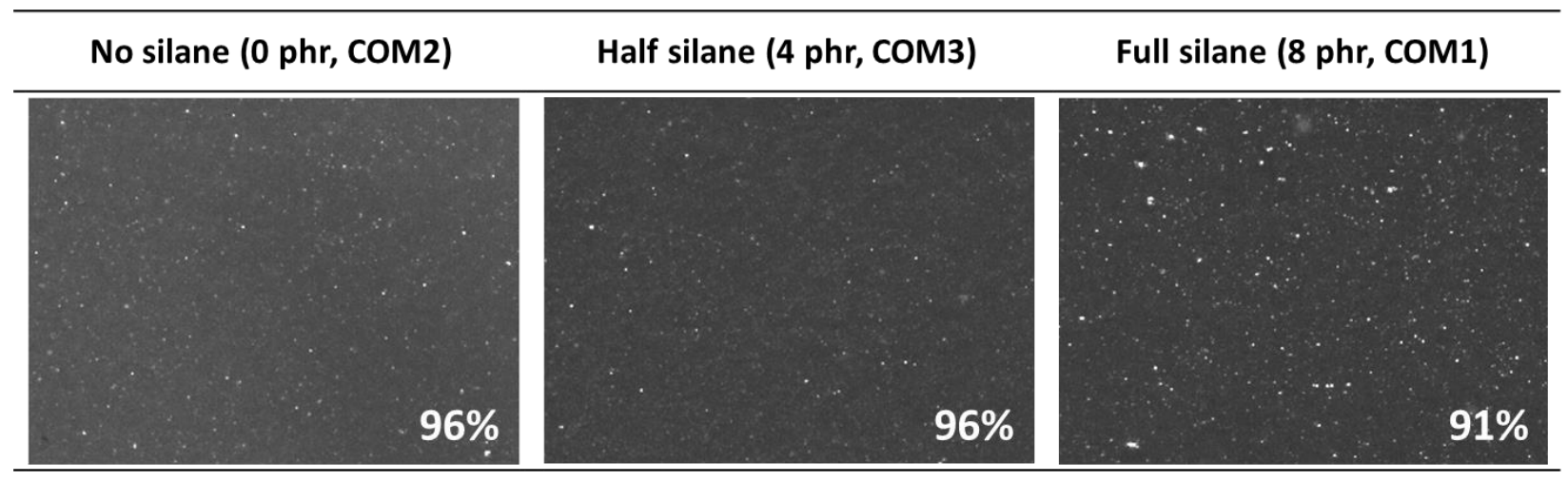

Figure 7.18 Macro-dispersion images and values corresponding to Figure 7.17.

\subsubsection{The effect of silica characteristics: surface area and structure (Series 4)}

In this series, the micro- and macro-dispersions of COM4 (HD silica) and COM5 (CV silica) mixed according to mixing procedure $A$ at $150^{\circ} \mathrm{C}$ are compared: Figures 7.19 and 7.20 . Only a small difference in the storage moduli $\left(G^{\prime}\right)$ at $100 \%$ strain -0.15 and $0.16 \mathrm{MPa}$ for COM4 and 5, respectively, Figure $7.19(\mathrm{~b})$ - is observed due to the structural differences of the silicas. ${ }^{[22]}$ Overall, the Payne effect is practically the same for both, COM4 and COM5.

A much better macro-dispersion is observed for COM4 (HD silica), as expected. These results again indicate that the micro-dispersion of silica is not related to the degree of macro-dispersion. Another important indication from this work is that the structure (OAN number) of the silica strongly influences 
the degree of macro-dispersion. The micro-/macro-dispersion results of COM4 can be explained as discussed in the previous paragraphs 7.3.1.1 and 7.3.1.2. In case of an HD-silica, when silane is present, both micro- and macro-dispersion are improved at $150^{\circ} \mathrm{C}$ mixing temperature due to an improved silanization reaction. However, this tendency is not observed for COM5: CV-Silica. This can be explained with the void or denser packing of the CV-silica and the details will be discussed later on.
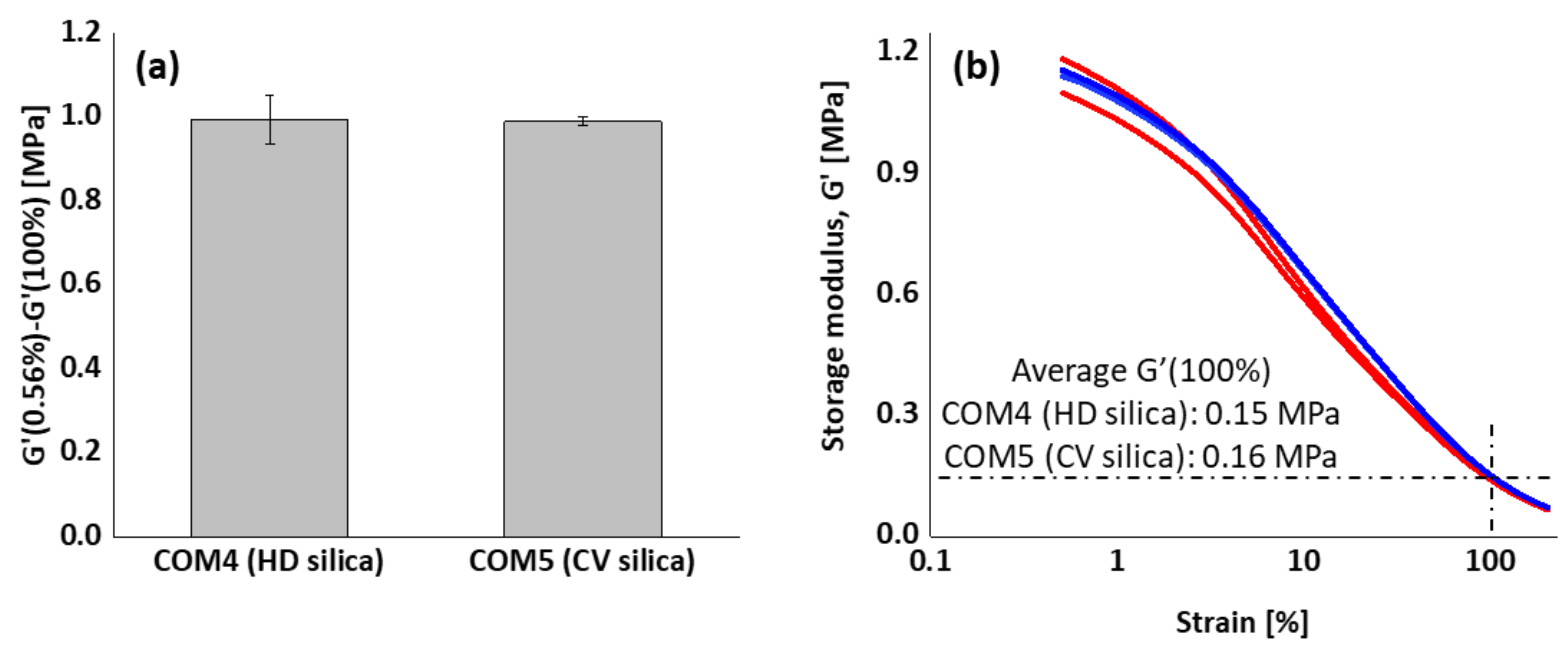

Figure 7.19 Micro-dispersion of COM4 (HD silica) and 5 (CV silica); (a): storage modulus G' vs. strain;

(b): Payne effect; ( _ ) : COM4; (_ )

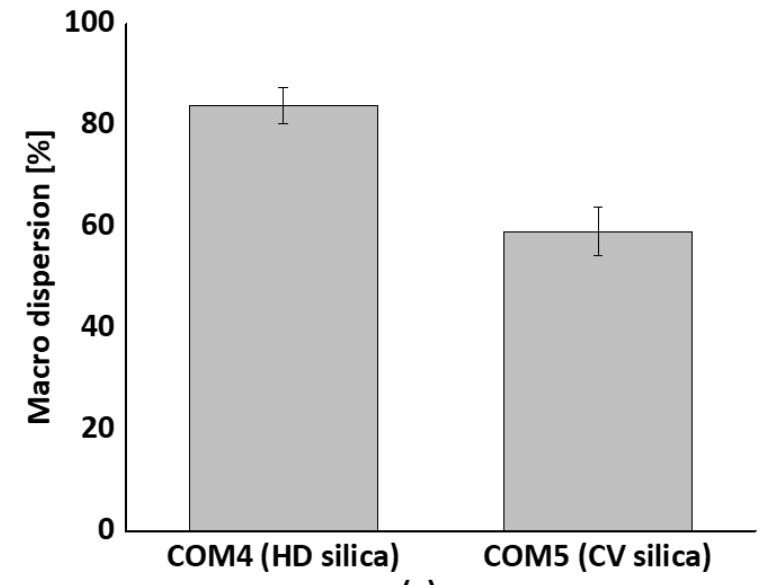

(a)

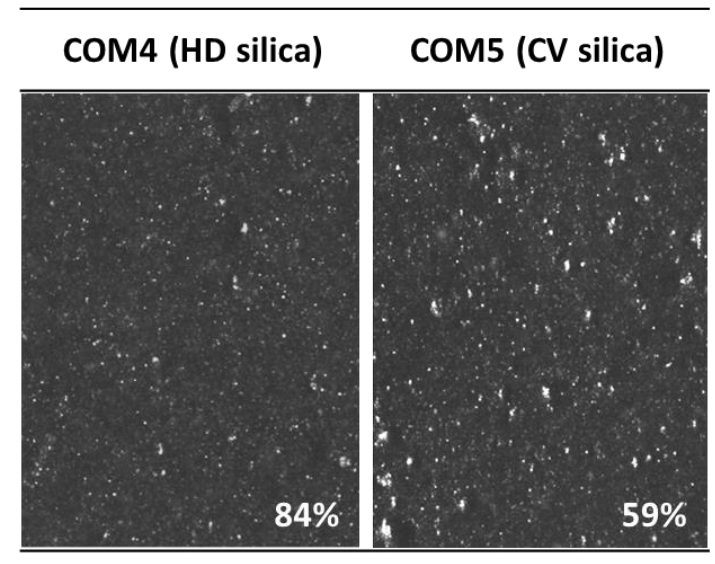

(b)

Figure 7.20 Macro-dispersion of COM4 (HD silica) and 5 (CV silica); (a): level of macro-dispersion;

(b): dispersion images and values. 
The surprising effect is that the micro-dispersion (Payne effect) is not influenced by this high-dispersion quality of the silica. In order to elucidate this phenomenon, theoretical qualitative silica cluster size distributions for HD and CV silica are depicted in Figure 7.21. The silica clusters might be distinguished into three groups according to their size and functions:

- $\quad$ primary particles: not detectable by a dispergrader and no effect on the Payne effect;

- $\quad$ small clusters: affect the Payne effect level but not detectable by a dispergrader;

- $\quad$ large clusters: detectable by a dispergrader but no effect on the Payne effect.

As shown in Figure 7.21, almost all HD silica is converted into small clusters and only a small amount is remaining as large clusters. For CV silica, a significant amount of silica is remaining in larger clusters. As a consequence, both silicas show similar level of Payne effect, but a different degree of macro-dispersion.

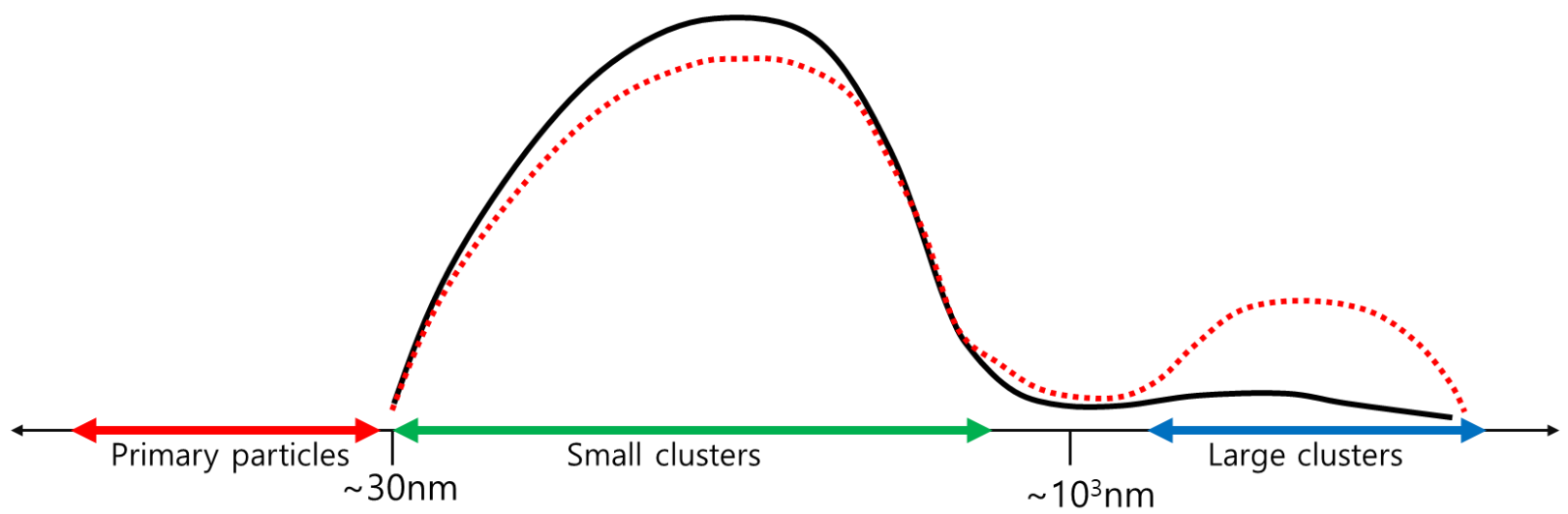

Figure 7.21. Theoretical silica cluster size distribution for HD and CV silica after mixing;

$$
\text { ( _ ) ): HD silica; ( . . . . . ) ): CV silica. }
$$

The different degree of conversion of large clusters into small clusters for HD and CV silica can be explained by their structure. A more developed structure can be seen for HD silica: Figure 7.22. The higher degree of complexity of these silica clusters would lead to a relatively open inter-cluster morphology Figure 7.23 - and thus the polymer chain can easily penetrate into the large cluster voids. Consequently, a higher efficiency on cluster separation can be obtained for HD silica. Due to its denser packing, the breakage of CV silica clusters is less effective than of HD silica, obviously because the latter was developed for that purpose. 


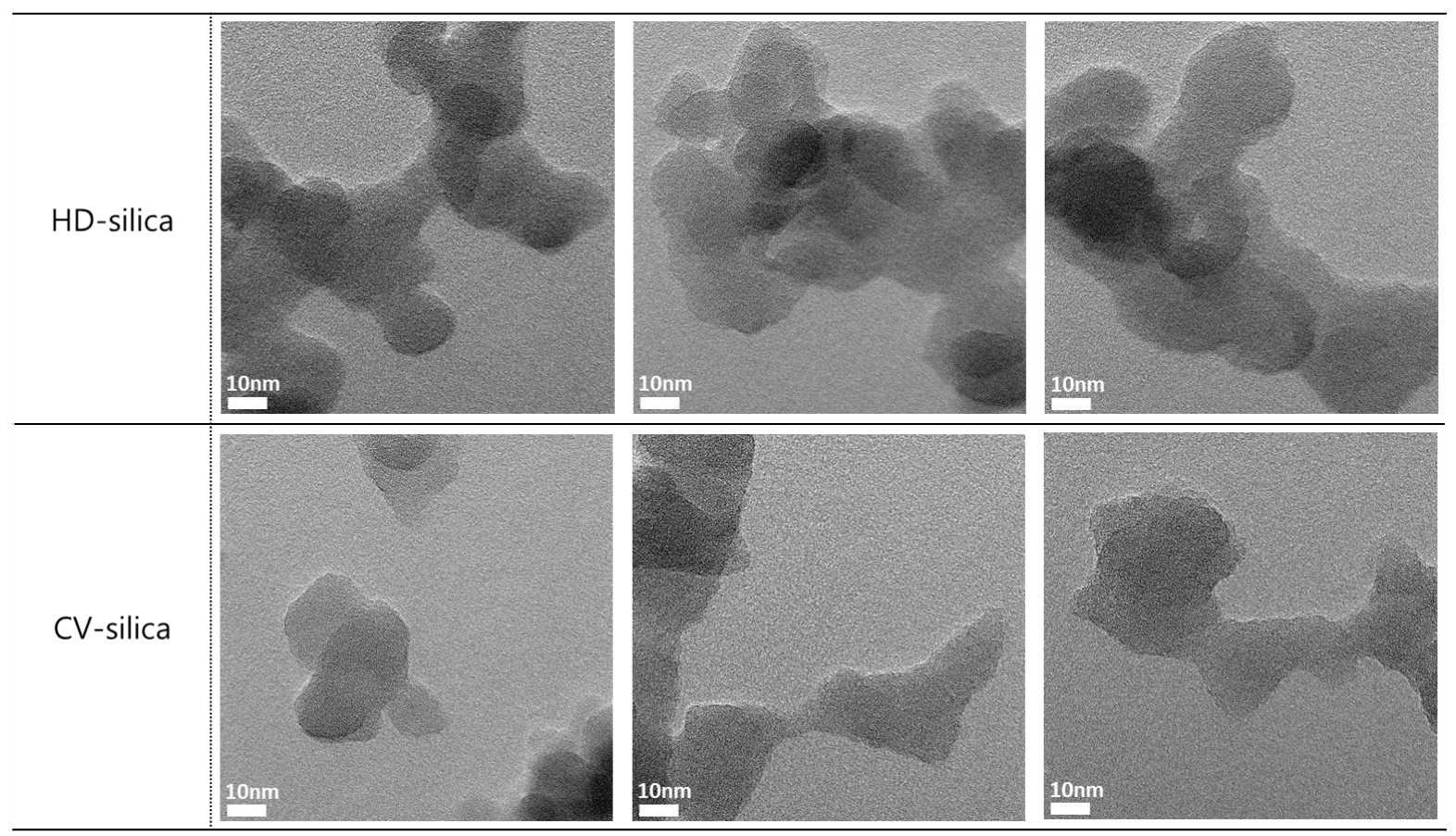

Figure 7.22 Silica cluster structure of HD and CV silicas.

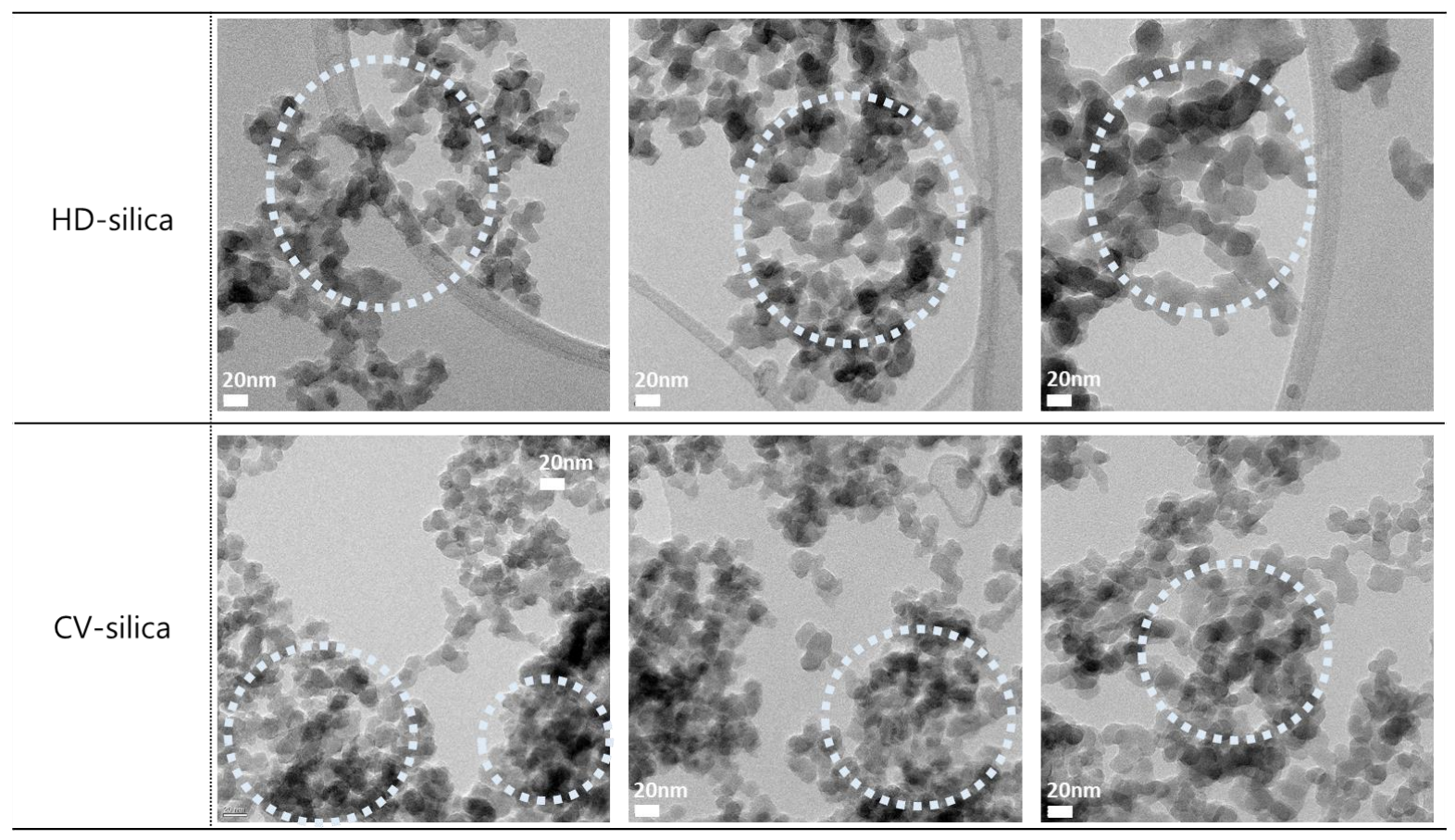

Figure 7.23 The inter-cluster morphology of HD and CV silicas. 


\subsubsection{The effect of micro- and macro-dispersion of silica on rubber reinforcement}

\subsubsection{The effect of silanization temperature and presence of silane (Series 1)}

The moduli at 100\% (M100), 200\% (M200) and the reinforcement index (M200/M100) of vulcanizates for Series 1 are shown in Figure 7.24. As was seen in Chapter 3.2, M100 is partly affected by the degree of micro-dispersion (Figure 3.30), and M200 is influenced by filler-polymer interaction (Figure 3.21). Which means these properties are not only influenced by total crosslink density but also by the aforementioned property. Additionally, calculating the reinforcing index (M200/M100) gives an overall information on the rubber reinforcement acquired by the filler induced by micro-dispersion as well as by filler-polymer interaction. Other properties such as tensile strength and elongation at break are more related to the total crosslink density and were not significantly influenced by micro-dispersion of the filler or fillerpolymer interaction (Paragraph 3.2.3.1), thus not taken into account for this study.

As expected from the compound formulation, a higher M100, M200 and reinforcement index are observed for COM1. It is obvious that the silane establishes a silica-polymer coupling, so that a reinforcement of the elastomer by silica can be achieved. Both compounds show a steeper decreasing trend for M100 along with silanization temperature, while M200 shows only a slight decrement.

The M100 trend (Figure 7.24(a)) was expected as and is already described in Paragraph 3.2.3.2.3: In case of a silica compound, the reduction of the storage modulus $\left(G^{\prime}\right)$ occurs at strains even higher than $100 \%$ of deformation strain due to a strong filler-filler interaction of the silica (see Figure 3.10 in Chapter 3.1). M200 values in the present work also show a - slightly - decreasing tendency with increasing silanization temperature (Figure 7.24(b)), which is opposite to the result of the Ref. compound in Chapter 3.2: Figure 3.24. It can again be explained with filler-filler interaction, when the silica content and the specific surface area of the silicas used for the Ref. compound in Chapter 3.2 and for COM1 in the present chapter are taken into account. COM1 contains 10 phr more silica than the Ref. compound in Chapter 3.2. Additionally, the silica used for COM1 (Silica 1 in Table 7.3, CTAB 177m²/g) has a larger specific surface area compared to the silica used for Ref. compound (same as Silica 2 in Table 7.3, CTAB $152 \mathrm{~m}^{2} / \mathrm{g}$ ). As a consequence, the filler-filler interaction is still present for COM1 due to a stronger filler-filler interaction compared to Ref. compound in Chapter 3.2. 

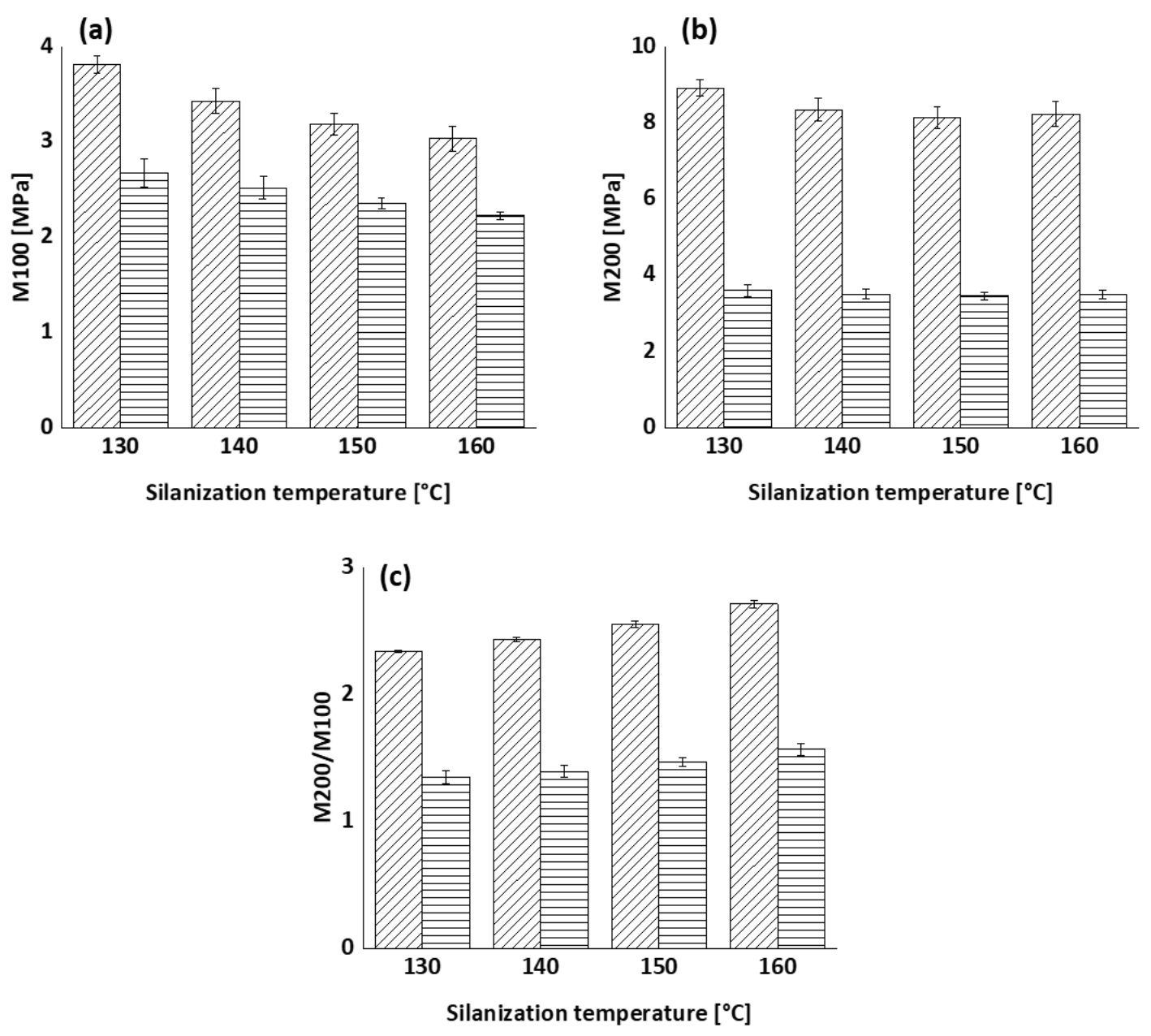

Figure 7.24 Tensile moduli and reinforcement index of Series 1; (a): M100; (b): M200; (c): reinforcement index; $(\mathbb{Z})$ : $\operatorname{coM} 1$; (击):COM2.

Another interesting trend can be seen in Figure 7.24(c): the reinforcement indices of COM1 and COM2 increase along with mixing temperature; for COM1 more than for COM2. When the results of micro- and macro-dispersion of these compounds are taken into account, it can be concluded that most of the reinforcement effect comes from the degree of micro-dispersion of the silica. The reinforcement indices correlated with the micro- and macro-dispersions are depicted in Figure 7.25. As can be seen in Figure 7.25(a), the reinforcement index values plotted as a function of Payne effect (micro-dispersion) show a correlation of $\mathrm{R}^{2}=0.99$ for both, COM1 and COM2. But no correlation is found between the reinforcement indices and the macro-dispersion: Figure 7.25(b). 

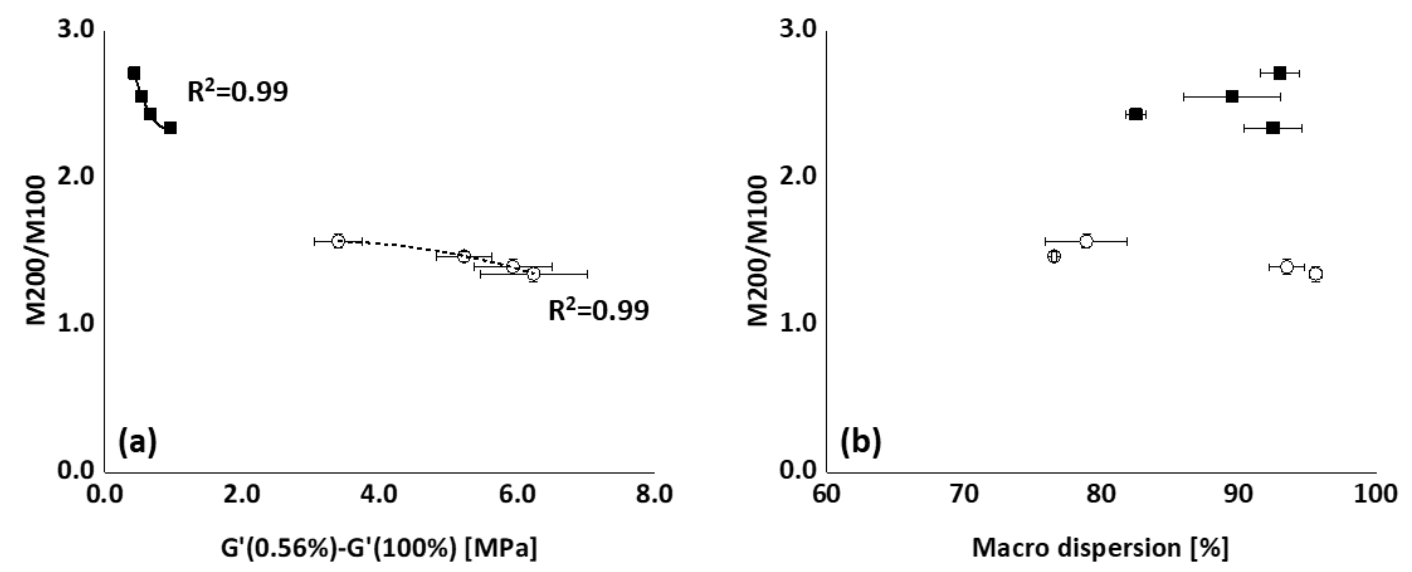

Figure 7.25 Correlation between reinforcement indices and micro-/macro-dispersion of Series 1;

(a): micro-dispersion; (b): macro-dispersion; ( $\mathbf{a}$ ): COM1; ( O ):COM2.

\subsubsection{The effect of silanization time (Series 2)}

The moduli at 100\% (M100), 200\% (M200) and the reinforcement index (M200/M100) of vulcanizates for Series 2 are shown in Figure 7.26. As silanization time increases, M100 and M200 decrease for the same reason stated in Paragraph 7.3.2.1: Figure 7.26(a), 7.26(b).

The reinforcement index values are almost the same but increase slightly along the silanization time: Figure 7.26(c). Correlations of the reinforcement indices with the micro- and macro-dispersion are depicted in Figure 7.27. As can be seen in Figure 7.27(a), the reinforcement index values show good correlation with the Payne effect (micro-dispersion): $R^{2}=0.94$. But the effect was small compared to the result of the silanization temperature: Figure 7.25(a). No correlation can be observed between reinforcement index and macro-dispersion: Figure $7.27(b)$. This again proves that micro-dispersion is the main governing effect on rubber reinforcement. 

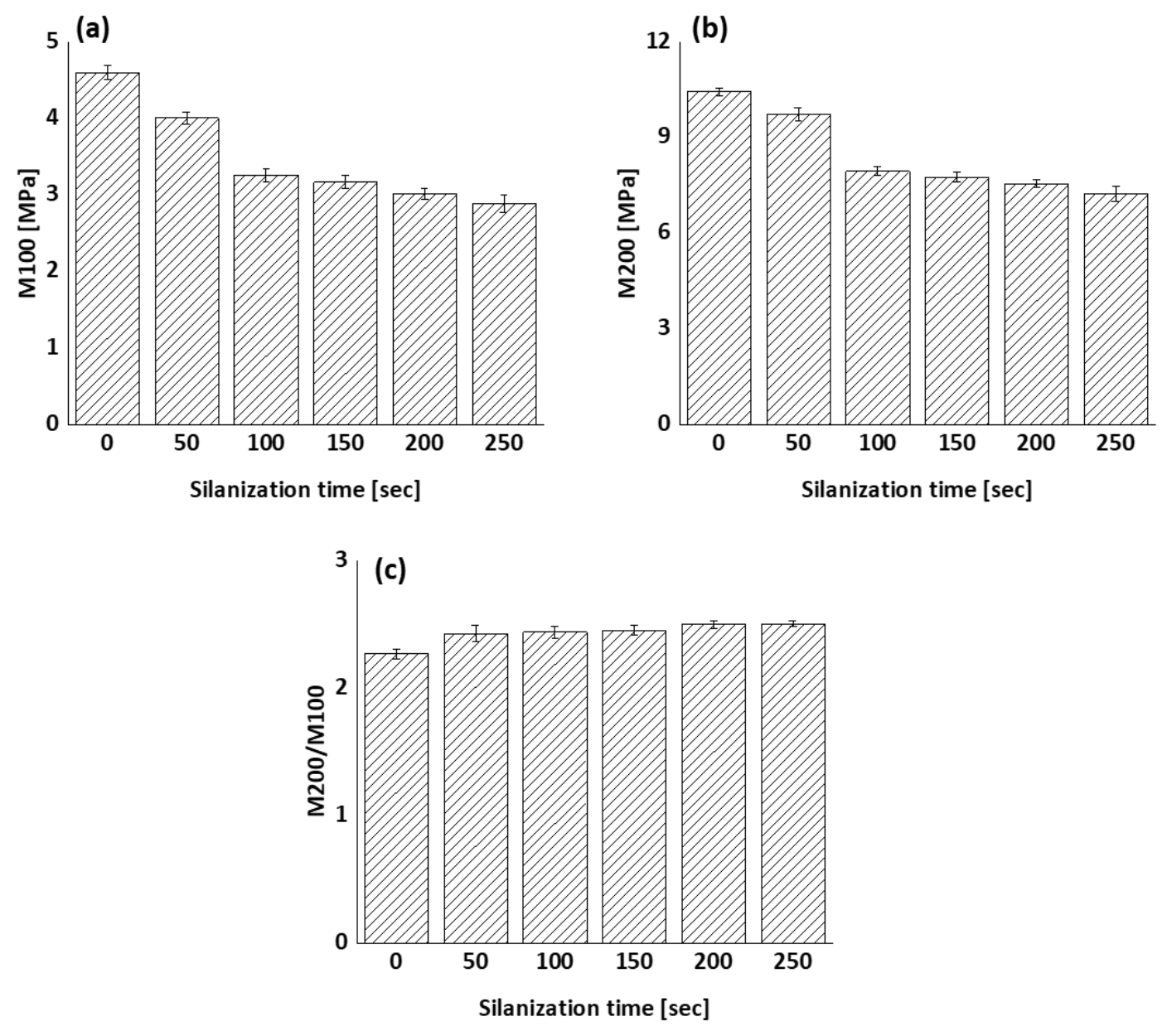

Figure 7.26 Tensile moduli and reinforcement index of Series 2;

(a): M100; (b): M200; (c): reinforcement index.
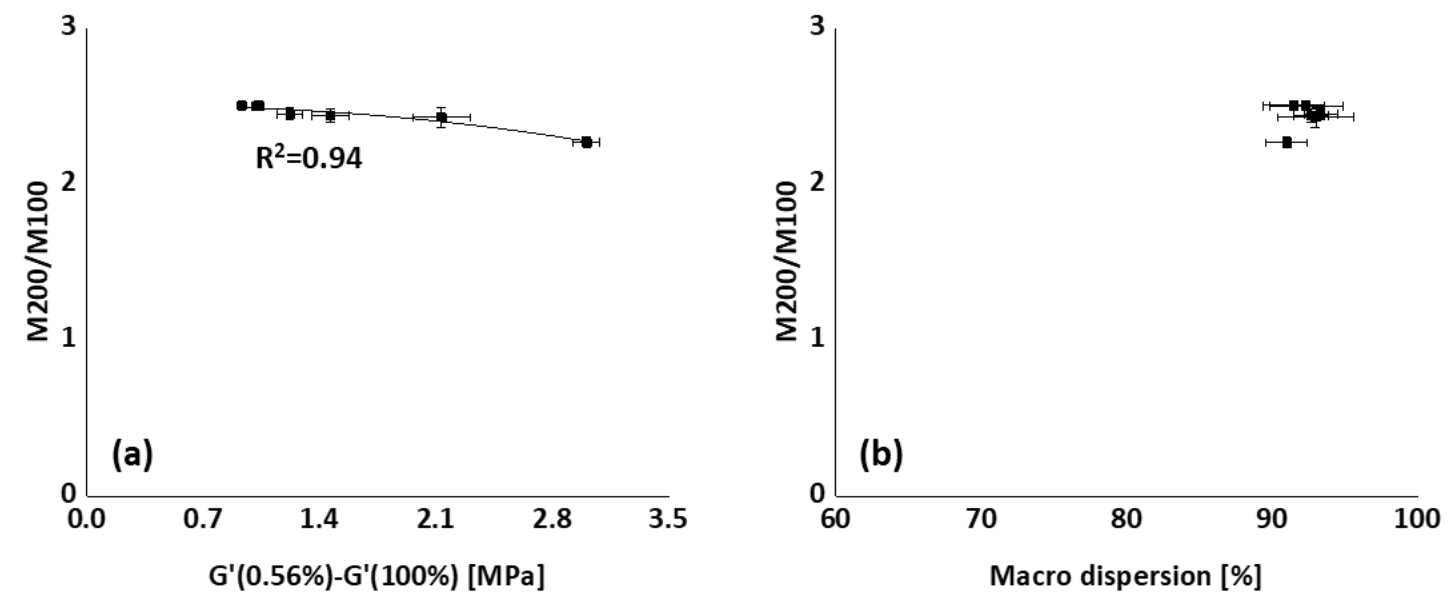

Figure 7.27- Correlation between reinforcement index and micro-/macro-dispersion of Series 2;

(a): micro-dispersion; (b): macro-dispersion. 


\subsection{Conclusions}

The focus of this study was to investigate the relationship between micro- and macro-dispersion of silica in rubber and its effect on reinforcement. No dependency could be found between these two types of dispersion. The results of this study confirm, that micro- and macro-dispersion processes of silica happen simultaneously, but independently.

The major influencing factors on micro- and macro-dispersion of silica are identified. All factors investigated within this work are summarized in Table 7.5. On the one hand, the macro-dispersion of the silica filler is better at lower mixing temperature, where the viscosity of the rubber polymer stays high. On the other hand, the micro-dispersion is better for a higher mixing temperature, because micro-dispersion is mainly influenced by the degree of silanization and the mobility of the polymer. The results of the SEM analysis strongly support the interpretation. The level of macro-dispersion is enhanced when the degree of silanization reaches a higher level, even though the viscosity of the polymer is reduced.

Table 7.5 Influencing factors on micro-/macro-dispersion of silica in a rubber matrix.

\begin{tabular}{|c|c|}
\hline $\begin{array}{l}\text { Dispersion } \\
\text { categories }\end{array}$ & Main influencing factors \\
\hline $\begin{array}{c}\text { Macro- } \\
\text { dispersion }\end{array}$ & $\begin{array}{l}\text { - Structure of silica } \\
\text { - Polymer viscosity especially when it is high (at lower mixing temperature) } \\
\text { - Degree of silanization (if silane is present) }\end{array}$ \\
\hline $\begin{array}{c}\text { Micro- } \\
\text { dispersion }\end{array}$ & $\begin{array}{l}\text { - Polymer viscosity when it is low (at higher mixing temperatures) } \\
\text { - Presence of silane } \\
\text { - Degree of silanization (if silane is present) }\end{array}$ \\
\hline
\end{tabular}

From a material aspect, the structure of the silica has not much effect on the micro-dispersion of silica, but strongly influences the degree of macro-dispersion due to the degree of packing of the silica clusters. The degree of micro-dispersion turned out as a key factor for silica filled rubber reinforcement. First indication was that micro-dispersion showed a good correlation with reinforcement index (M200/M100) obtained from Series 1 and 2, whereas their macro-dispersion was not. A higher level of reinforcement indices was obtained for the compounds showing lower Payne effect values regardless of the degree of macro-dispersion. Second, a higher degree of micro-dispersion leads to enhanced reinforcement unless silane is not present.

Overall, in case of silica-filled rubber compounds, the degree of silanization is crucial for both, macro- and micro-dispersion as well as rubber reinforcement. 


\section{References}

[1] D. Beach, J. Schroeder, Rubber World 222, 44 (2000).

[2] ASTM D3053-15, "Standard Terminology Relating to Carbon Black", ASTM International (2015).

[3] H-D. Luginsland, J. Fröhlich, A. Wehmeier, Rubber Chem. Technol. 75, 563 (2002).

[4] S. Mihara, R. N. Datta, J. W. M. Noordermeer, Rubber Chem. Technol. 82, 524 (2009).

[5] S. Shiga, M. Furuta, Nippon Gomu Kyoshikai 55, 491 (1982).

[6] N. Nakajima, E. R. Harrell, Rubber Chem. Technol. 57, 153 (1984).

[7] H. H. Le, M. Tiwari, S. Ilisch, H. J. Radusch, Kautsch. Gummi Kunstst. 58, 575 (2005).

[8] V. Collin, E. Peuvrel-Disdier, Elastomery 9, 9 (2005).

[9] V. Collin, I. Boudimbou, E. Peuvrel-Disdier, J. Appl. Polym. Sci. 127, 2121 (2013).

[10] A. Blume, S. Uhrlandt, 157th meeting Rub. Div. ACS Dallas, Texas, Paper no. 32 (2000).

[11] A. R. Payne, Rubber Chem. Technol. 39, 365 (1966).

[12] A. R. Payne, R. E. Whittaker, Rubber Chem. Technol. 44, 440 (1971).

[13] J. H. Bachmann, J. W. Sellers, M. P. Wagner, R. F. Wolf, Rubber Chem. Technol. 32, 1286 (1959).

[14] S. S. Sarkawi, W. K. Dierkes, J. W. M. Noordermeer, Rubber Chem. Technol. 88, 359 (2015).

[15] B. Seo, K. Kim, H. Lee, J-Y. Lee, G-H. Kwag, W. Kim, Macromolecular Research 23, 466 (2015).

[16] L. Guy, S. Daudey, P Cochet, Y. Bomal, Kautsch. Gummi Kunstst. 63, 383 (2009).

[17] ASTM D7723-11, "Standard Test Method for Rubber Property - Macro Dispersion of Fillers in Compounds", ASTM International (2011).

[18] "Rubber compounding; Chemistry and applications, 2nd Edition", B. Rodgers, CRC Press, New York (2016).

[19] W. K. Dierkes, J. W. M. Noordermeer, M. Rinker, K.-U. Kelting, C. van de Pol, Kautsch. Gummi Kunstst. 56, 338 (2003).

[20] https://news.jtbc.joins.com/article/ArticlePrint.aspx?news_id=NB11401104 viewed on 07-AUG2019.

[21] J. Fröhlich, W. Niedermeier, H. -D. Luginsland, Comp. Appl. Sci. Manuf. 36, 449 (2005). 


\section{Chapter 8 Summary}

The main objectives of this study were:

1) to investigate the physicochemical mechanism underlying the marching modulus phenomenon during the vulcanization of silica filled SBR/BR compound;

2) to evaluate the influence of mixing parameters, mixing protocols, compound formulation characteristics and mixing equipment;

3) to provide possible guidelines for a tailored mixing process and compound in order to achieve a better silica dispersion and rubber reinforcement.

In order to achieve the goals, the fundamental knowledge and actual practices to obtain a higher degree of silica dispersion and rubber reinforcement were reviewed concerning materials, mixing process parameters and mixing equipment in the beginning of this work: Chapter 2. Additionally, earlier studies relevant for the main topic of the present study - the cause of marching modulus of silica/silane compounds - were investigated as a starting point of the present study. Aspects looked at are:

- filler-filler interaction;

- filler-polymer coupling via silane;

- formation of additional crosslinks by released free sulfur from the silane.

With the knowledge from Chapter 2, the origin of the marching modulus phenomenon of silica compounds and its effect on rubber reinforcement were investigated in Chapter 3. Several series of silica filled tire tread compounds were mixed in order to clarify the origin of marching modulus in Chapter 3.1. Various silane coupling agents with different sulfur ranks and functionalities were used in the different mixing series varying the silanization temperature in order to obtain:

- different degrees of filler-polymer coupling;

- different amounts of sulfur donated by the silanes;

- different degrees of silanization. 
Commonly the marching modulus phenomenon was understood as a result of further formation of sulfur crosslinks, therefore the influence of crosslink density on marching modulus was also investigated.

The outcome of this study answers the first research question and confirmed that three factors: intensity of filler-filler interaction, silica Filler Flocculation Rate (FFR) and filler-polymer Coupling Rate (CR), turned out to be the major influencing factors on the marching modulus phenomenon of silica compounds, in order of decreasing importance. All three factors are related to each other (Figure 3.22) and are the consequence of the degree of silanization of the silica during mixing. A higher degree of silanization not only lowers the filler-filler interaction but also leads to formation of more chemically bound rubber, thus resulting in a lower FFR level. The formation of chemically bound rubber provides an additional reduction of the filler-filler interaction as well as the flocculation of silica particles. Additionally, a higher amount of chemically bound rubber indicates that there is a lower concentration of free sulfur - additionally introduced or released from the silane - as well as of remaining silane, resulting in a lower CR value. Finally, a higher degree of silanization will lead to a lower MMI. However, the total crosslink density was not a major influencing factor for the marching modulus phenomenon.

In Chapter 3.2, the effect of marching modulus on the consistency as well as on the level the of the mechanical properties and reinforcement of the silica filled rubber were investigated. The mechanical properties of the compounds in Chapter 3.1 were evaluated and compared to each other for three different curing times: calculated cure time, 20 minutes and 30 minutes. The expectation was a large alteration of tensile properties for the compounds having a higher Marching Modulus Intensity (MMI). However, it turned out that the MMI does not give any information about mechanical property variation along with the vulcanization time, as the crosslink density of the compounds were very similar regardless of the cure time. The level of tensile properties of silica filled rubbers are in first instance roughly determined by the total crosslink density, which includes not only polymer-polymer sulfur crosslinks, but also filler-polymer coupling established by the silane. The degree of micro-dispersion turned out to be another key factor for rubber reinforcement, as the level of the moduli at $100 \%$ of strain (M100) and the reinforcement index (M200/M100) were affected by the filler-filler interaction measured as Payne effect. Chapter 4 gives answers to a part of the second (evaluation of the influence of mixing parameters and protocols) and third objective (guidelines for a tailored mixing process). Based on the outcome of Chapter 3, the mixing parameters and protocols which can influence the degree of silanization were investigated in Chapter 4, in order to provide guidelines for minimizing the marching modulus of silica compound. 
In Chapter 4.1, silanization temperature and time turned out to be the crucial factors for the degree of silanization, FFR, CR as well as the amount of bound rubber. MMI was significantly reduced with increasing silanization temperature and time, as FFR and CR decrease due to a higher amount of total and chemically bound rubber. Therefore, applying an appropriate silanization temperature and time is crucial for silica/silane filled rubber mixing in order to obtain a higher quality of the compound: when silica is not properly silanized, the curing process cannot repair that. Additionally, a better processability in the last processing stage of tire manufacturing, the vulcanization step, can be obtained by minimizing the marching modulus phenomenon.

The results of Chapter 4.2 showed that the presence of $\mathrm{ZnO}$ - more precise, zinc stearate - influences the marching modulus behavior of silica filled rubber, but its concentration in the early mixing step had no effect at all. When $\mathrm{ZnO}$ reacts with stearic acid to form zinc stearate which can be adsorbed onto the silica surface, the two long hydrocarbon groups $\left(\mathrm{C}_{18}\right)$ are capable of blocking the access of the silane to the silica surface. Zinc stearate might provide a positive effect on reducing filler-filler interaction; however, as soon as $\mathrm{ZnO}$ is added in the early stage the amount of bound rubber is reduced and resulting in a higher MMI. The concentration of 1,3-diphenylguanidine (DPG) in the early or later mixing stage does influence the efficiency of the silanization as well as the filler-polymer coupling reaction via the silane coupling agent: Chapter 4.3. However, neither the presence nor concentration of DPG in the early mixing step turned out as an influencing factor for the marching modulus behavior of silica filled rubber. The concentration of DPG in the early mixing step influences the curing behavior of the compound as well as the crosslink density variation of the vulcanizates along with the curing time: the higher the DPG concentration in the early mixing step, the slower the curing rate. Besides, it reduces the crosslink density variation along with increasing the curing time.

However, the results of the mixing trials discussed in Chapter 4.2 and 4.3 did not fully confirm the wellknown role of $\mathrm{ZnO}$ (or zinc stearate) and DPG in silica compounding, therefore a model compound study was performed in Chapter 4.4. The direct reaction possibility between bis-(triethoxysilylpropyl) tetrasulfide (TESPT) and the mentioned ingredients was analyzed using High Pressure Liquid Chromatography (HPLC), Liquid Chromatography-Mass Spectrometry (LC-MS) and ${ }^{13} \mathrm{C}-$ Nuclear Magnetic Resonance $\left({ }^{13} \mathrm{C}-\mathrm{NMR}\right)$. The outcome of this part confirmed another functionality of DPG in silica/TESPT filled rubber, which was proposed by Mihara: DPG can react with TESPT, and both are thus partly consumed during the mixing process. However, $\mathrm{ZnO}$ or zinc stearate did not affect the release of sulfur from the TESPT molecule. 
The other parts of objective 2 (evaluation of the influence of the compound formulation) and objective 3 (guidelines for compound design) are addressed in Chapter 5. The effect of the polymer and silica characteristics on marching modulus of a silica filled tire tread compound were investigated in this Chapter. Compounds were mixed with different polymer blending ratios (Chapter 5.1) and using two types of silica having different dispersibility (Chapter 5.2) and their properties were further analyzed.

Based on the results of Chapter 5.1, the SBR/BR blending ratio turned out to be a strong influencing factor for the marching modulus as well as for the other curing characteristics. Silica tends to be localized preferably in the SBR phase or at the interphase of SBR and BR domains due to the physical affinities of these rubbers towards silica. When the volume of the SBR decreases, the population of silica in SBR increases leading to faster silica flocculation (higher FFR) due to the short distance between the silica clusters. However, when the incorporation capacity of SBR reaches its maximum due to a lower SBR volume, the remaining silica is distributed in a higher volume of BR therefore the flocculation again slows down (lower FFR). Consequently, the MMI varies due to the silica flocculation rate varying with the SBR/BR blending ratio. Besides $\mathrm{MMI}$, other curing characteristics such as maximum vulcanization rate are influenced by the total amount of 1,2- and 1,4-structured double bonds in the polymer. The 1,4-double bonds in SBR and BR are more reactive in sulfur curing than the 1,2-double bonds. Due to this difference in reactivity of these types of double bonds, faster vulcanization is observed for the compounds having a higher amount of 1,4-double bonds.

The dispersibility of silica turned out as an influencing factor for the degree of macro-dispersion, but showed no effect on the marching modulus behavior of silica filled rubber: Chapter 5.2. Compared to the Highly-Dispersible (HD) silica, ConVentional (CV) silica showed poor macro-dispersion. However, due to the fact that the amount of chemically bound rubber, CR and FFR are strongly affected by the degree of silanization, HD and CV silica showed similar values in these properties when the compounds are mixed at the same silanization temperature and time. It is interesting to note that there was no relation between the degree of micro- and macro-dispersion of silica, and this phenomenon is further evaluated in Chapter 7.

The influence of mixing time and equipment (the remaining part of the second objective) on $\mathrm{MMI}$ and silica dispersion is evaluated in Chapter 6. Besides, a guideline for silica compound mixing equipment, which is related to the third objective, was prepared. In this chapter, compound mixing is performed using plant scale mixers in order to check, if the basic mechanisms observed in the previous chapters on laboratory scale are valid regardless of the mixer scale. The silanization time, temperature as well as the rotor types were selected as the main variables to obtain different degrees of silanization and micro- 
dispersion of silica. The result of this work confirmed, that the basic mechanisms for the marching modulus phenomenon of silica compounds still apply regardless of the mixer scale. The mixing parameters which lead to a proper degree of silanization - silanization time and temperature - reduce the FFR and CR of the compound and thus reduce the MMI.

The intermeshing rotor is generally recommended for silica filled rubber mixing, but the rotor geometry turned out as a minor factor on MMI. However, a higher degree of micro- and macro-dispersion of silica filler can be achieved by using an intermeshing rotor.

Chapter 7 dealt with the investigation of the relationship of micro- and macro-dispersion of silica in rubber and its effect on reinforcement. No dependency could be found between these two types of dispersion. The results of this study confirm, that micro- and macro-dispersion processes of silica happen simultaneously, but independently. Based on Scanning Electron Microscope (SEM) analysis, a silica dispersion model which can explain the simultaneous process of micro- and macro-dispersion is suggested: the stretched natto food model (Figure 7.14). When mixing at a lower temperature and without silane, macro-dispersion can be enhanced due to a high compound viscosity. However, at higher temperatures and with silane, macro-dispersion can also be improved depending on the degree of silanization. The presence of the silane enhances the degree of micro-dispersion, even more when the compound viscosity becomes lower at a higher temperature. As was seen in Figure 3.8, the penetration of polymer chains into the voids of silica clusters is a main factor for the micro-dispersion of silica. A higher mixing temperature will lead to an enhanced mobility of the polymer chains, therefore a higher degree of polymer penetration into the voids of silica clusters is possible. Additionally, the degree of silanization is strongly influenced by the mixing temperature as was seen in the previous chapters.

Regarding the material, the structure of the silica has not much effect on the micro-dispersion of silica, but strongly influences the degree of macro-dispersion due to the degree of packing of the silica clusters. The degree of micro-dispersion turned out as a key factor for silica filled rubber reinforcement. A first indication was that micro-dispersion showed a good correlation with the reinforcement index (M200/M100), whereas macro-dispersion did not. A higher level of reinforcement indices was obtained for the compounds showing lower Payne effect values regardless of the degree of macro-dispersion. Secondly, a higher degree of micro-dispersion leads to enhanced reinforcement when silane is present. All in all, in case of silica-filled rubber compounds, the degree of silanization is crucial for both, macroand micro-dispersion as well as rubber reinforcement. 
Overall, this thesis confirmed the importance of the mixing process for tire manufacturing. The last stage in tire manufacturing - vulcanization - is still influenced by the first process - mixing - even though there are several steps in-between.

In a nutshell, the degree of silanization turned out a key factor for:

- reducing marching modulus;

- a higher degree of silica dispersion in terms of micro- and macro- scale;

- a higher reinforcement effect of for a silica filled rubber compound. 


\section{Hoofdstuk 8 Samenvatting}

In deze studie zijn de volgende doelstellingen behandeld:

1) Het fysisch-chemische mechanisme, dat verantwoordelijk is voor het 'marching modulus' fenomeen tijdens de vulkanisatie van silica-versterkte SBR/BR mengsels;

2) De invloed van mengomstandigheden en mengsel-receptuur op de 'marching modulus';

3) Het samenstellen van een handleiding voor een aangepaste mengprocedure en mengselsamenstelling, om de verdeling van silica in de rubber matrix en het versterkingseffect te optimaliseren.

Om deze doelen te bereiken is allereerst bestaande literatuur bestudeerd ten aanzien van fundamentele en praktijkgerichte kennis met betrekking tot grondstoffen, mengprocedures en -apparatuur ter verbetering van silica dispersie en materiaalversterking: Hoofdstuk 2. Daarnaast is bestaande literatuur met betrekking tot de hoofdvraag - de oorzaak van de 'marching modulus' van silica mengsels bestudeerd als beginpunt van deze studie. De volgende aspecten kwamen daarbij aan de orde:

- Vulstof-vulstof interactie;

- Vulstof-polymeer binding via een silaan;

- $\quad$ Additionele polymeer-polymeer bindingen door zwavel vrijgekomen uit de silanen.

Met als uitgangspunt de kennis uit Hoofdstuk 2 is de oorzaak van de 'marching modulus' en het effect op de versterking van de rubber in Hoofdstuk 3 onderzocht. Hoofdstuk 3.1. was gericht op het onderzoeken van het 'marching modulus' fenomeen. In dit onderdeel van de studie is de reactiviteit van silanen met verschillende zwavelketenlengtes en andere functionele groepen onderzocht in verschillende mengseries, met variatie van de silaniseringstemperatuur, om de volgende invloedfactoren te bestuderen:

- Verschillende mates van vulstof-polymeer binding;

- Variatie in de hoeveelheid zwavel vrijgekomen uit de silanen;

- Variatie in de silaniseringsgraad. 
Een verklaring voor de 'marching modulus', welke vaak in de literatuur wordt gegeven, is een verder voortschrijdende vorming van zwavelbruggen (crosslinks); daarom is de invloed van de netwerkdichtheid op de 'marching modulus' ook onderzocht.

De uitkomst van deze studie beantwoordt de eerste onderzoeksvraag en laat zien, dat de vulstof-vulstof interactie, de silica uitvlok-nelheid (FFR) en de vulstof-polymeer bindingssnelheid (CR) - met afnemende belangrijkheid - de hoofdoorzaken zijn voor de 'marching modulus' van silica mengsels. Deze drie factoren zijn van elkaar afhankelijk (Figuur 3.22), en zijn het gevolg van verschillen in de silaniseringsgraad van het silicamengsel: hoe hoger de silaniseringsgraad, hoe lager de vulstof-vulstof interactie. Dit leidt op z'n beurt tot een hoger gehalte aan chemisch gebonden rubber, zgn. 'bound rubber' en minder uitvlokking. Daarnaast wijst een hoger chemisch 'bound rubber' gehalte op een lager gehalte aan vrije zwavel - óf toegevoegd met de vulkanisatieadditieven óf vrijgekomen uit de silanen - en een lager gehalte aan silaan, wat op z'n beurt een lagere CR tot gevolg heeft. Een niet verwachte uitkomst van dit onderzoek was, dat de netwerkdichtheid op zichzelf weinig invloed heeft op de 'marching modulus'.

In Hoofdstuk 3.2 is de invloed van de 'marching modulus' op het niveau en de constantheid van het versterkingseffect van silica op de mechanische eigenschappen van de rubber onderzocht. De materialen uit Hoofdstuk 3.1. zijn geanalyseerd en qua mechanische eigenschappen vergeleken. Voor vulkanisatie van deze mengsels zijn drie verschillende tijden gebruikt: t90 afgeleid uit het rheogram, en twee vaste tijden: 20 minuten en 30 minuten. Verwacht was, dat de mengsels met een hoge 'marching modulus' intensiteit (MMI) grote verschillen zouden vertonen afhankelijk van de gebruikte vulkanisatietijd. Tegen de verwachting in zijn er geen grote verschillen in netwerkdichtheid en mechanische eigenschappen gemeten met variatie van de vulkanisatietijd. De trek-rek eigenschappen van rubber worden globaal beïnvloed door de netwerkdichtheid, waaraan polymeer-polymeer zwavelbruggen maar ook vulstofpolymeer bindingen door het silaan bijdragen. Daarnaast is de microdispersie een belangrijke factor voor de versterking van rubber door silica: de modulus bij 100\% rek (M100) en de versterkingsindex (M200/M100) worden beïnvloed door de vulstof-vulstof wisselwerking gemeten als Payne effect.

Het onderzoek in Hoofdstuk 4 geeft antwoorden op de tweede (invloed van mengcondities en protocollen) en derde (richtlijnen voor verbeterde mengprocedure) onderzoeksvragen. In dit hoofdstuk is gebruik gemaakt van de metingen en ervaringen uit Hoofdstuk 3, de invloed van de mengprocedures en werkwijzen op de silaniseringsgraad is onderzocht om uiteindelijk richtlijnen te formuleren voor een verlaging van de 'marching modulus' van silica mengsels.

In Hoofdstuk 4.1. bleken de silaniseringstemperatuur en - tijd de hoofdfactoren, die de silaniseringsgraad, FRR, CR en 'bound rubber' bepalen. Door verhoging van mengtemperatuur en - tijd wordt de MMI 
significant verlaagd, als gevolg van een lagere FFR en CR, die wederom veroorzaakt worden door een hogere totaal en chemisch 'bound rubber' gehalte. Een goede keuze van silaniseringstijd en temperatuur is daarom uitermate belangrijk voor een goede materiaalkwaliteit; een lage silaniseringsgraad kan tijdens vulkanisatie niet meer worden gerepareerd. Bovendien wordt het vulkanisatiegedrag verbeterd door het verminderen van de 'marching modulus'.

In Hoofdstuk 4.2 werd duidelijk, dat de aanwezigheid van $\mathrm{ZnO}$ - of beter gezegd zink stearaat - invloed heeft op de 'marching modulus', maar dat de concentratie ervan in de eerste mengstap geen effect heeft. Zink oxide reageert met stearinezuur tot zink stearaat, en dit kan worden geadsorbeerd aan het oppervlak van silica. De twee lange C18 ketens belemmeren zo de toegang van silaan tot silica, maar hebben ook een positief effect door tegelijkertijd ook de vulstof-vulstof interactie te verlagen. Het gevolg van de aanwezigheid van $\mathrm{ZnO}$ in de eerste mengstap is een reductie van 'bound rubber', resulterend in een verhoging van de MMI. De concentratie van 1,3-diphenylguanidine (DPG) in de vroege of latere mengstadia heeft invloed op de silanisering zowel als op de vulstof-polymeer koppelings-reactie via de silaan coupling agent: Hoofdstuk 4.3. Echter, noch de aanwezigheid noch de concentratie van DPG in de vroege mengstap bleek een invloedsfactor voor het 'marching modulus' gedrag van silica-gevulde rubber. De concentratie van DPG in de eerste mengstap heeft wel invloed op het vulcanisatie-gedrag van het mengsel, zowel als op de vernettingsdichtheid als op de vulkanisatiesnelheid: hoe hoger de DPG concentratie in het begin van het mengproces, hoe trager de vulkanisatie en hoe lager de netwerkdichtheid met toenemende vulkanisatietijd.

Omdat de uitkomst van de mengstudies in Hoofdstukken 4.2 en 4.3 de rol van $\mathrm{ZnO}$ - of zink stearaat - en DPG zoals beschreven in de literatuur niet helemaal kan verklaren, is dit in een modelstudie verder onderzocht: Hoofdstuk 4.4. De reactie tussen bis-(triethoxysilylpropyl) tetrasulfide (TESPT) en de genoemde activatoren is geanalyseerd met behulp van High Pressure Liquid Chromatography (HPLC), Liquid Chromatography-Mass Spectrometry (LC-MS) en ${ }^{13} \mathrm{C}$-Nuclear Magnetic Resonance $\left({ }^{13} \mathrm{C}-\mathrm{NMR}\right)$. Uit deze studie bleek nog een andere functie van DPG in rubber met silica en TESPT, die eerder ook al door Mihara is geopperd: DPG kan zelf met TESPT reageren, waardoor de TESPT-concentratie verlaagd wordt gedurende het mengen. Verder is aangetoond, dat ZnO en zink stearaat het vrijkomen van zwavel uit TESPT niet beïnvloeden.

De resterende vragen van doelstelling 2 (invloed van de mengselsamenstelling) en 3 (richtlijnen voor mengselsamenstelling) worden in Hoofdstuk 5 beantwoord. Hier is de invloed van de silica- en polymeereigenschappen op de 'marching modulus' van een bandenloopvlak-mengsel onderzocht. De verhouding van de verschillende polymeren (Hoofdstuk 5.1) in het mengsel is gevarieerd, en twee silica- 
types met verschillende dispersiekwaliteit (Hoofdstuk 5.2) zijn vergeleken qua invloed op verwerkings- en materiaaleigenschappen.

Hoofdstuk 5.1 laat zien, dat de SBR/BR verhouding een belangrijke factor is voor het fenomeen 'marching modulus' en het vulkanisatiegedrag. Omdat silica een grotere fysische affiniteit tot SBR heeft vergeleken met BR, is het met name te vinden in de SBR fase en in het SBR/BR grensvlak. Als de hoeveelheid SBR verlaagd wordt ten bate van BR, wordt de silica-concentratie in SBR hoger. Omdat de afstand tussen de silica-clusters dan kleiner wordt, treedt er meer uitvlokking op (hogere FFR). Wordt de opnamecapaciteit van SBR overschreden doordat het SBR gehalte verlaagd wordt, gaat silica naar de evenredig toenemende BR fase met als gevolg een lagere uitvloksnelheid. Daardoor verandert de MMI met de polymeerverhouding. Naast MMI worden ook andere vulkanisatie-eigenschappen zoals maximale vulkanisatiesnelheid door de verhouding van 1,2- en 1,4-dubbele bindingen beïnvloed: 1,4-dubbele bindingen in SBR en BR zijn reactiever tijdens vulkanisatie dan 1,2-dubbele bindingen. Deze hogere reactiviteit manifesteert zich in een snellere vulkanisatie van mengsels met een hoger aandeel 1,4dubbele bindingen.

De dispergeerbaarheid van silica resulteerde in verschillen in macro-dispersie, maar had geen effect op de 'marching modulus': Hoofdstuk 5.2. Gebruik van 'ConVentionele silica' (CV) gaf een lagere mate van dispersie dan gebruik van 'Highly-Dispersible' (HD) silica. Omdat chemisch 'bound rubber', CR en FFR sterk bepaald worden door de silaniseringsgraad, is er geen verschil met betrekking tot deze eigenschappen tussen CV en HD silica, als de mengsels met vergelijkbare temperatuur en gedurende dezelfde tijd gesilaniseerd zijn. Een andere onverwachte uitkomst is dat micro- en macrodispersie onafhankelijk zijn van elkaar. Dit fenomeen is verder onderzocht in Hoofdstuk 7.

Resteert nog een vraag open bij doelstelling 2, de invloed van mengtijd en -apparatuur op MMI en silica dispersie: dit is onderzocht in Hoofdstuk 6. Daarnaast is in dit hoofdstuk een richtlijn voor het type mengapparatuur voor silica mengsels aangemaakt. In deze studie zijn mengproeven op productieschaal doorgevoerd om te onderzoeken, of de kenmerken van silica mengsels zoals uitgewerkt op laboratoriumschaal ook op grote schaal van toepassing zijn. Silanerings- en microdispersiegraden zijn gevariëerd door instellen van mengtijd, -temperatuur en rotor type van de menger. De uitkomst van dit onderzoek was, dat de invloedfactoren voor 'marching modulus', die op kleine schaal gevonden zijn, onafhankelijk zijn van de schaal van de menger. Mengparameters, die bepalend zijn voor de silaniseringsgraad: silanisatietijd en -temperatuur, verlagen ook op grote schaal de FFR en CR en daardoor de MMI. 
'Intermeshing' rotoren van de mengers worden algemeen aanbevolen voor het mengen van silica mengsels, maar in dit onderzoek bleek de rotor-geometrie van ondergeschikt belang voor de MMI. De 'intermeshing' rotor presteerde wel beter qua micro- en macrodispersie.

In Hoofdstuk 7 is in detail gekeken naar de verhouding van micro- en macrodispersie van silica in rubber en de invloed daarvan op de versterking. De uitkomst was, dat deze twee materiaaleigenschappen onafhankelijk zijn van elkaar, maar wel tegelijk tot stand komen. Gebaseerd op een Scanning Electron Microscopy (SEM) studie is een dispersie-model ontwikkeld voor deze twee onafhankelijke maar gelijktijdige processen: het 'stretched natto food' model (Figuur 7.14). Wordt een silica mengsel bij lage temperatuur en zonder silaan gemengd, dan kan een goede macrodispersie bereikt worden door de hoge viscositeit van het mengsel. Wordt bij hoge temperatuur en in aanwezigheid van silaan gemengd, dan wordt eveneens een goede macrodispersie bereikt door een hoge silaniseringsgraad. De aanwezigheid van silaan verbeterd de microdispersie, in het bijzonder bij hoge temperatuur en lage viscositeit. Zoals in Figuur 3.8 te zien is, is de penetratie van polymeerketens in de vrije ruimte in silica clusters een belangrijke factor voor microdispersie: bij hogere temperatuur zijn de polymeerketens flexibeler en vinden deze gemakkelijker hun weg in de silica structuur. Daarnaast is de silaniseringsgraad sterk afhankelijk van de mengtemperatuur, zoals in de eerdere hoofdstukken geconcludeerd.

De structuur van silica heeft weinig effect op de microdispersie, maar wel een duidelijke invloed op de mate van macrodispersie door de compactheid van de vulstof. De microdispersie bleek een sleutelrol te spelen met betrekking tot het versterkingseffect. Een eerste indicatie daarvoor was een goede correlatie tussen de microdispersie en de versterkingsindex (M200/M100), die bij macrodispersie niet te vinden was. Mengsels met een laag Payne Effect hebben doorgaans een hogere versterkingsfactor, onafhankelijk van de macrodispersiegraad. Daarnaast vertoont het materiaal een hogere versterkingsfactor als de microdispersie beter is door aanwezigheid van silaan. Samengevat is de silaniseringsgraad van silicamengsels cruciaal voor de macro-en voor de microdispersie en zo voor de versterking van de rubber. En algemene conclusie van deze studie is dat het mengproces uitermate belangrijk is voor bandenproductie. De laatste stap in het productieproces - vulkanisatie - ondervindt nog steeds de gevolgen van de eerste stap in het proces - mengen, zelfs al zitten er nog verschillende stappen tussen. De silaniseringsgraad is een uitermate belangrijke invloedsfactor voor:

- $\quad$ het verlagen van de 'marching modulus';

- $\quad$ een hogere dispersiegraad van silica op macro- en microschaal;

- $\quad$ een hoger versterkingseffect van silica. 
Hoofdstuk 8 


\section{Bibliography}

\section{Journal Articles}

1. J. Jin, J. W. M. Noordermeer, W. K. Dierkes, A. Blume, The origin of marching modulus of silica-filled tire tread compounds, Rubber chem. Technol. in press. (2019) https://doi.org/10.5254/rct.19.80453

2. J. Jin, J. W. M. Noordermeer, W. K. Dierkes, A. Blume, The Effect of Silanization Temperature and Time on the Marching Modulus of Silica-Filled Tire Tread Compounds, Polymers 12. 209 (2020) https://doi.org/10.3390/polym12010209

3. J. Jin, W. Kaewsakul, J. W. M. Noordermeer, W. K. Dierkes, A. Blume, Macro- and micro-dispersion of silica in tire tread compounds: are they related?, Rubber Chem. Technol. (2019), under revision.

4. J. Jin, A. P. J. van Swaaij, J. W. M. Noordermeer, A. Blume, W. K. Dierkes, On the various roles of 1,3diphenyl guanidine in silica/silane reinforced SBR/BR blends, European Polymer Journal, in preparation.

5. J. Jin, A. P. J. van Swaaij, J. W. M. Noordermeer, A. Blume, W. K. Dierkes, Effect of SBR/BR elastomer blend ratio on filler and vulcanization characteristics of silica filled tire tread compounds, Macromol. Mater. Eng., in preparation.

\section{Presentations}

1. J. Jin, W. K. Dierkes, J. W. M. Noordermeer, A. Blume. Factors reducing the marching modulus of silica filled tire tread compounds. Poster session presented at Deutsche Kautschuk Tagung, DKT 2018, Nürnberg, Germany, July 2-5 (2018).

2. J. Jin, J. W. M. Noordermeer, W. K. Dierkes, A. Blume, The Origin of Marching Modulus of Silica Filled Tire Tread Compounds, Paper presented at Fall 194th Technical Meeting of the Rubber Division, ACS 2018, Louisville, United States, October 9-11 (2018).

3. J. Jin, W. Kaewsakul, J. W. M. Noordermeer, W. K. Dierkes, A. Blume, The relation between macroand micro-dispersion of silica filler in rubber matrix, Paper presented at International Rubber Conference, IRC 2019, London, United Kingdom, September 3-5 (2019).

4. J. Jin, J. W. M. Noordermeer, W. K. Dierkes, A. Blume, Key factors influencing marching modulus in silica-silane compounds, Paper presented at International Rubber Conference, IRC 2019, London, United Kingdom, September 3-5 (2019).

5. A. Blume, J. Jin , A. Mahtabani, X. He, S. Kim, Z. J. Andrzejewska, New Structure Proposal for Silane Modified Silica. Paper presented at International Rubber Conference, IRC 2019, London, United Kingdom, September 3-5 (2019). 


\section{Acknowledgement}

This project was carried out within four years under the contract between the University of Twente (UT) and HANKOOKTIRE Co., Ltd. Due to the fact that countless number of people have supported and helped me to enable this project finished. Therefore, I would like to express my appreciation to them.

First of all, I would like to express my huge sincere gratitude to my promotor and supervisor: Associate Prof. Dr. Wilma K. Dierkes. You kept me motivated and always spent your best effort for me. I am sure that I could not finish the project without your effort and kind support. According to my memory, I could not convince you at the beginning of the project - I understood your word "literature study" literally, so I just had read the papers but did not start to write Chapter 2 Literature Review - but you waited me in patient and kindly encouraged me. You were very busy with other businesses while I was writing approximately 250 pages of thesis, but you never missed a small typos. You are a superwoman. Thank you very much for very nice Dutch translation of Chapter 8.

I want to extend my sincere gratitude to Prof. Dr. Jacques Noordermeer, who is not only one of the best and the most passionate professor in elastomer technology but also being the best mentor. It is a great honor that I shared 4 years of time and the same place with you during my PhD life. With your guide and encouragement, I could finish writing 5 papers in total for the journal publication. You might be suffered and bored because you had repeated reading of all my papers at least more than 5 times per each to make those papers perfect. Now, I know the way how the cookies crumble because of you.

I would like to express my huge appreciation to another superwoman Prof. Dr. Anke Blume, the captain of Elastomer Technology and Engineering (ETE) as well as an European mother for all students in ETE. You gave me a great opportunity: doing PhD at the University of Twente. You have always helped and encouraged me very kindly by technically and mentally. Additionally, you took a lot of materials - in particular several types of white solid powder and yellowish liquid with smell of sulfur - through the backdoor (?) of your company to provided me. And all of them was for free.

Assist. Prof. W. Kaewsakul, a friend of mine, thank you very much for your golden tips for Equations 3.3 and 3.4 and all of the insightful discussions. You had motivated me to proceed Chapter 7: one of the chapter which I like the most in my thesis. I wish your success in the Netherlands.

I could not survive in the Netherlands without the help and support of Madam. Ceciel H. E. ter HorstStrootman, the best secretary in the Netherlands so far as I know. I could settle down in the Netherlands easily because of your help. With your very nice and perfect arrangements, I could travel Europe and the USA for conferences and lectures without any problem. Thank you very much. 
Dear Andries P. J. van Swaaij, so called Sir. Dries, a highly intelligent technician and the most effective experimental problem-buster, thank you very much for keeping all the devices in the lab always in the best condition. Because of your effort, I could finish all my experiments within time. And Thank you for being my chemistry consultant.

I would like to appreciate and express my regards to Dr. M. de Rooij, Prof. Dr. J. E. ten Elshof, Prof. Dr. I. Hudec, Prof. Dr. C. Kummerlöwe, Dr. J. Kiesewetter for accepting to be my graduation committee.

I would like to also express my gratitude to Mr. Hasse (Evonik, Germany) and Mr. Wehmeier (Evonik, Germany) for the material and technical support and Mr. Guy (Solvay, France) for the material support. To all of my post and current colleagues in ETE group; Fabian (a beer monster who always take the most hard tasks), Akansha (a friendly coffee breaker who wants to talk a lot), Kumar (a good Indian dancer), Nadia (straightforward Dutch lady), Hans (diligent old PhD), Jacob (friendly gentle grandpapa), Masaki (senpai hakase, yasashi aniki), Chesidi (shy Thai guy), Marzieh (attractive Iranian lady with high and clear voice), Neven (just like heaven, silent stealer, watch your watch), Ayush (plastic-man who can make friends everywhere), Indria (warm-hearted Indonesian lady), Gina (perfect and best English user in ETE came from USA), Marcel (not a walking dead but a working dad and sometimes walking dad), Suppachai (the only one who can teeth his brush), Rafal (Starwars-addict, traveler, scientist, who is not allowed to enter spring water bath in Japan), Amir (who-insane but not crazy, best movie director in ETE), Xiaozhen (supermom), Arqam (so called Amir, another working dad and sometimes walking dad), Zuzanna (totally cool Polish lady), Dorota (the environmentalist, leader of team Poland), Carmela (silent Italiano who can do psychometry for coffee tasting), Anna (the best smiler came from Poland), Andrea (smoking Italiano who informed me there is no bolognaige pasta in Italy), Łukasz (prison breaker), Maja (movie distributor), Patrik (who wants to have metal supports for his presentation), Randalf (a highschool teacher, the alchemist), Sunkeun (another Korean as well as a working dad), Marcin (foodfighter equipped with attractive(?) laughter), Priyanka (hard-working Indian lady appears with a motorcycle sound), Chigusa (an alien, thousand grass, who owed me PlayStation 4), Rounak (paparazzi of ETE), Anmol (Indian chef), Pilar (who fell in love with a beer monster), Rick (the youngest), Jan-Willem (who is in metal stable status always), Marnick (a rower equipped with wide shoulders, who asked me hard questions during the coursework but finally lead me to have high grades), Stefan (sulfuric researcher), Eva (ECC Viol's Assistant), Dr. Viol (who brought the best chocolate cake for SinterKlaas party in 2019), Dr. Kannika (boss of Chesidi and Suppachai), Dr. Reuvekamp (who did not allow me to say Heineken in Enschede, remember me!). Thank you very much for your sincere friendship and the best wishes. All of you made my life in the Netherland as a precious treasure. My special thanks to Arqam and Sunkeun for being my paranymphs. 
To my parents, parents-in-law, my brother and his family and aunts. Thank you very much for all of your sacrifices and helps. I could concentrate on my PhD project because of you all. I and my family could stay in the Netherlands without any worries. There is no possible way to express how much I appreciate you all. To my family, my dearest wife Ji Eun Choi, my dearest daughter Chae Eun Jin. You spent hard time in the Netherlands because of me. Everything was unusual and strange at the beginning but finally we managed it. I cannot imagine the life without all of you. Dear my wife, Ji Eun Choi. Without your sacrifice, I could neither complete my PhD nor survive in the Netherlands. I love you so much. Now, it is my turn. I will do my best to become a good husband. Dear my daughter Chae Eun Jin. I remember the day when you came to us. Since that day, your presence became a huge healing for me. You kept me happy always. Thank you being my precious daughter. I hope you to visit Enschede, a lovely city in the Netherlands, where you born, with me and your mom after you grown up. It will be great if you become a student of the University of Twente in the future.

I would like to extend my appreciation to HANKOOKTIRE Co., Ltd. for the financial and material support during this project. I would like to thank the people in HANKOOKTIRE by using my mother language.

먼저 저에게 해외 연수의 기회를 주신 조현식 부회장님, 조현범 사장님, 구본휘 전무님께 감사 인사를 드립니다. 학업에 지장이 없도록 항상 신경써주신 본사의 HR 부문장 김종윤 상무님, $\mathrm{HR2}$ team 의 이홍석 팀장님과 장형찬님, 연구소 기획 담당 오상현 상무님, R\&D HR 유근만 팀장님, 항상 믿어주시고 학업을 장려해주신 김학주 전무님 감사드립니다. 저에 대한 무조건적인 믿음을 주시고 항상 격려, 동기부여 및 조언을 해주신 이형재 상무님 (상무님의 가르침이 박사과정을 진행하는데 있어서 특히 많은 도움이 되었습니다.), 미국 학회 기간 동안 3 시간 거리를 달려오셔서 격려해주신 전일환 상무님과 박동명 상무님, 헝가리 공장에서 아낌없는 지원과 격려를 해주신 황성학 전무님, 민경신 팀장님, 송동현님께도 감사 인사드립니다. 항상 응원 및 격려와 지원을 해주신 연구소 가족분들인 박일용님, 이상걸님, 강현욱님, 권대익님, 전재후님, 김연환님, 김홍원님, 신세희님, 김찬연님과 그 외의 모든 동료 직원분들께도 감사 인사 드립니다.

I would like to extend my appreciation to my international colleagues in HANKOOKTIRE Co., Ltd. - Mr. Olah Szabolcs, Mr. Peter Kertesz, Ms. Agnes Jakso, Mr. Cialone Massimo - for the supports and encouragements during this project.

For the last, I would like to thank to Korean people I met in the Netherlands and my friends in Korea by using my mother language.

진상은 사무관님 가족분들, 오준철 사무관님 가족분들, 노훈석, 양윤선 부부 박사님 가족분들, 김제복 박사님 가족분들, 김두리 박사님, 작년에 엄마가 되신 김찬미님께 감사드립니다. 이우제님과 
가족분들, 같은 연구 그룹에서 유일한 한국인이자 동료이자 든든한 페이스 메이커가 되주신 김선근님과 그 가족분들께 감사드리며, 순탄한 학업의 종료와 네덜란드에서의 생활에 항상 행운이 함께하길 기원합니다.

새벽에 인천공항까지 데려다 준 가장 오래된 친구 송기훈, 항상 한국에서 응원해준 고등학교 동창 이영섭, 최경원, 제상우, 지금은 비록 사이버 친구상태이지만 재수 학원 동문인 박시형, 대학 동문 용석이형 (엄용석), 호영이형(조호영(상환)), 이종석, 서영화-권지연 부부, 네덜란드 거주 기간동안 한국 제품을 보급해주신 내 배우자의 친구분이신 고예원님, 차예리님 모두에게도 감사인사 드립니다. 
